- 


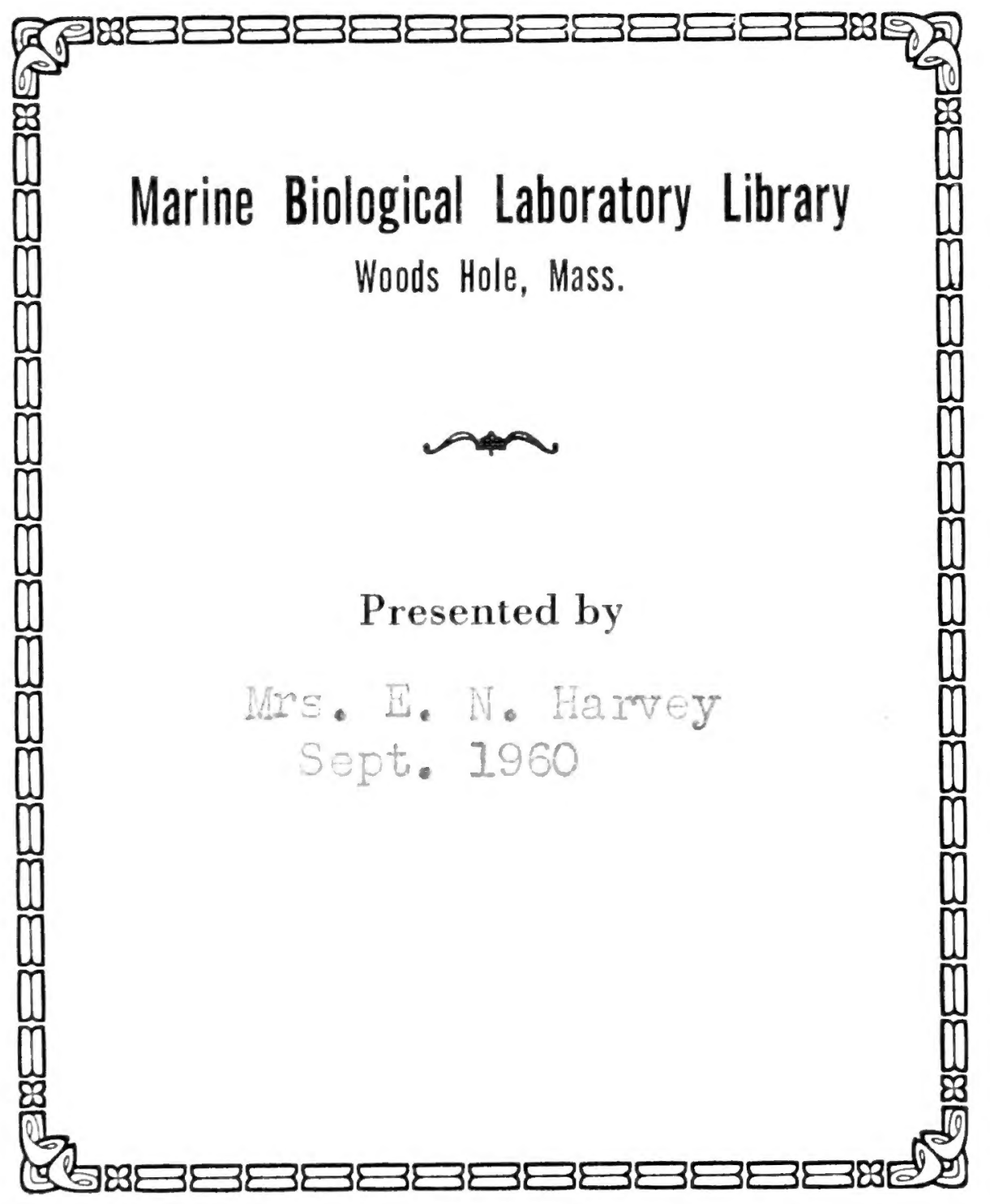


foseph mehollin S cotK. Dypantment Biologen. Mount Unoón College. 1920. 


THE CAUSES AND COURSE OF ORGANIC EVOLUTION 


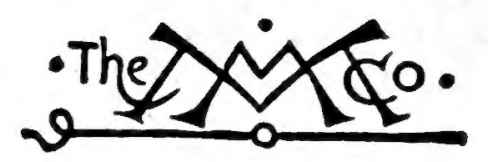

THE MACMILLAN COMPANY NEW YORK - BOSTON - CHICAGO SAN FRANCISCO

MACMILLAN \& CO., LIMITED LONDON - BOMBAY - CALCUTTA MELBOURNE

THE MACMILLAN CO. OF CANADA, ITD. TORONTO 


\title{
THE CAUSES AND COURSE OF
}

\section{ORGANIC EVOLUTION}

\section{A STUDY IN BIOENERGICS}

\author{
BY \\ JOHN MUIRHEAD MACFARLANE, D.SC. \\ PROFESSOR OF BOTANY \\ DIRECTOR OF THE BOTANIC GARDEN \\ UNIVERSITY OF PENNSYLVANIA
}

Nien 照ark

THE MACMILLAN COMPANY

I9I 8

All righes reserved 
COPYRIGHT, I9I8,

BY THE MACMILLAN COMPANY

Intelligenter fregs

Steinman \& Foltz, Lancaster, Pa. 


\section{PREFACE}

In sending out the present volume as an addition to the literature of evolutionary science, the writer feels that a few words of personal explanation may be appropriate.

As a university student he was deeply impressed, during the epochal period from 1875 to 188 , by the varied -often conflicting-views on evolution presented by his teachers. So he early formed a resolve to investigate quietly, but as extensively as possible, the problems presented by organisms from the simplest types up to man himself. Later as a teacher and investigator of biological and specially of botanical problems, during a period of well nigh forty years, he has had considerable, and in some respects exceptional, opportunities for becoming acquainted with organisms over extensive fields of the Old and New World; for studying the structure and behavior of these in laboratory, in experimental grounds, and in cultural houses; as well as for quiet study of biological literature personally and in conjunction with his graduate students.

Though a student and teacher of zoological as well as botanical science for about ten years of his earlier academic career, he would in many ways have preferred to confine attention to the latter science, that has been his chosen field for specialized investigation. But questions constantly presented themselves from the side of plant life, that seemed to demand continuous study and solution from the animal side as well. If one such case may be cited here, reference might be made to the past and present geographical distribution of plants as eminently favoring the view that these originated over fresh-water areas, and only secondarily spread into the sea. Is the truth of this became increasingly evident, the writer was confronted by the universally arcepted though opposing view of zoologists that animal life originated in the sea, and thence spread landward. Here then was a seeming biologieal anomaly that called for investigation. The results of such are shortly pre- 
sented in succeeding chapters of this work, and though the writer's conclusions are at variance with past current opinion, he trusts that the facts advanced will be weighed in that spirit of fairness and impartiality that should characterize all scientific method.

But, as has often been emphasized during the past quarter century, it is impossible to bring together a book like the present, with pretension at the same time on the part of the author to the knowledge of a specialist in every department of biological inquiry. The facts of Botany and Zoology are now so detailed and elaborated that few can hope to cover more than a very limited field with the knowledge and discerning keenness of an extreme specialist. This need not militate however against the making of an effort to link together isolated facts or groups of facts, if thereby the bounds of biological knowledge can be extended. But in saying this the writer is deeply conscious that not a few shortcomings or mistakes may be revealed in the succeeding pages, when these are perused by the specialist. For, as a specialist himself in some departments of botanical science, he has ever found the above to be true of the efforts even of the most distinguished investigator. He would therefore ask a degree of consideration on the ground that his whole aim has been to advance truth and knowledge, even though at the cost of cherished opinions or convictions of the past.

1)uring the progress of his investigations he has been increasingly impressed by three outstanding characteristics of organisms. First and of extreme importance is the principle of continuty as traced from the simplest organized body to the highest expressions of moral and religious organization in man. Second, as the subtitle of this work and practically every chapter of it emphasize, the outstanding phenomenon of organic existence is not passive inert matter but energy, that continuous'but ever transformable and subtle agency that starts and effects all cosmic changes. So energy, continuity, erolution may be said to constitute the triune basis of existence, and together form the keynote to this volume. 
The work as now presented was practically completed in June, 1914, and in that month a sealed copy was deposited in the vaults of one of the learned societies, where it now remains. But some statistics and results bearing on plant and animal distribution, as well as some questions dealt with in Chapter $\mathrm{X}$, had to be supplied later. These were added to and deposited with the above in July, 1916. Since that time only a few footnotes have been appended in small type and while the work was passing through the press. So the conclusions presented in Chapters XXI to XXX have been in no way determined by the iniquitous international struggle of the past three years. The writer cannot but hope, however, that some of his views may find the more ready acceptance, in virtue of the melancholy spectacle presented by the nations in this Twentieth Century of Christ's Era.

The writer would now make hearty and grateful acknowledgment of the kind help and encouragement extended by his friends Dr. Henry W. Cattell and Mr. Roberts Le Boutillier, for without their sympathetic aid this volume could not now have seen the light. He also expresses sincerest thanks to his friend Dr. Joseph Hepburn, who willingly volunteered to revise proofs, and by his expert chemical knowledge has furnished not a few valuable corrections and suggestions from the chemical standpoint.

In conclusion the writer desires to state that he would gladly welcome suggestions from every source, whether these may differ from or conform to the views now expressed. For, if strength, length of days, and needed leisure are granted him, he trusts in future volumes to amplify and further extend present conclusions.

Philadelphia, December, 1917. 



\section{CONTENTS}

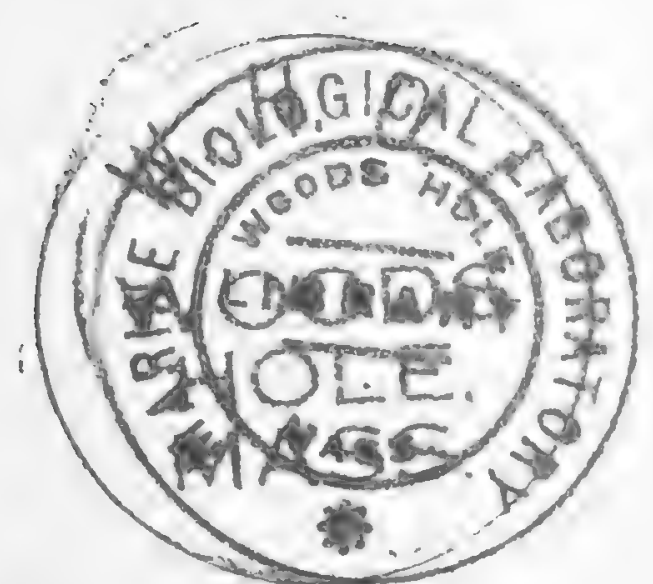

I. Ether and Energy in the Evolution of Matter.......... 1

II. The Relations and Transformations of Energy during the

Evolution of Matter.

III. The Relation of Inorganic to Organic Bodies............. 48

IV. The Energies of the Organic World-the Biotic, the Cognitic, the Cogitic - and their Relation to Organisms......... 68

V. The Biotic State of Energy..................... . 97

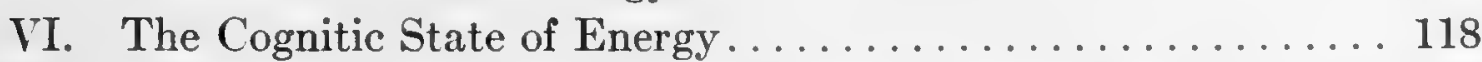

VII. A Review of the Formed Constituents of Organisms.........147

VIII. Pentamorphogeny, or the Five Cooperating Causes in Organic Evolution................................ 17t

IX. The Law of Proenvironment................... . 205

X. Cell Multiplication, and the Origin of Sexuality in Plants and

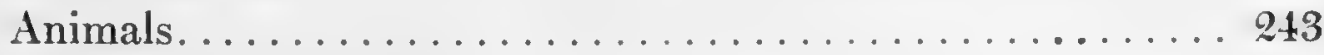

XI. The Evolution of Plants...................... 300

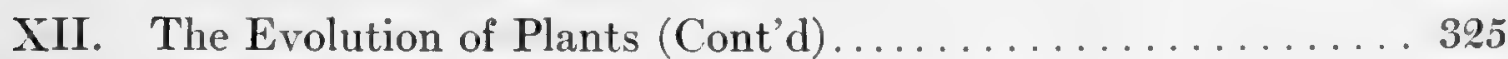

XIII. The Evolution of Plants (Cont'd).................. 349

XIV. The Evolution of Animals....................... 374

XV. The Evolution of Animals (Cont'd) . . . . . . . . . . . 411

XVI. The Evolution of Animals (Cont'd)................. 453

XVII. The Evolution of Animals (Cont'd)...............475

XVIII. The Phylogeny of Animals.................. 504

XIX. The Relation of Higher Animals to Man............... 5 54t

- XX. The Physical Evolution of Man................... 570

XXI. The Mental or Cogitic Evolution of Man in Relation to that of the Higher Animals. . . . . . . . . . . . . . . . . . 598

XXII. The Operation of the Law of Proenvironment in the Evolution

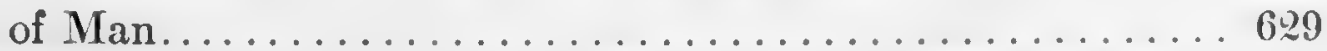

XXIII. Morals as a Factor in Organic Evolution, and their Biological Origin............................. 652

XXIV. Religion as a Factor in Human Evolution..............675

XXV. Religion as a Factor in Human Evolution (Cont'd)........ . 0 02

XXVI. Religion as a Factor in Human Evolution (Cont'd)......... 7 74

XXVII. The Competitive System Amongst the Lower Animals and Man................................

XXVIII. The Cooperative or Social System Amongst Lower Animals and Man........................... 6 . 68

XXIX. Human Organization in Relation to Environment.......... 798

XXX. Probable Future Advances in Fuman Evolution........... 895

Bibliography. Index.................... 851 



\section{CONTENTS}

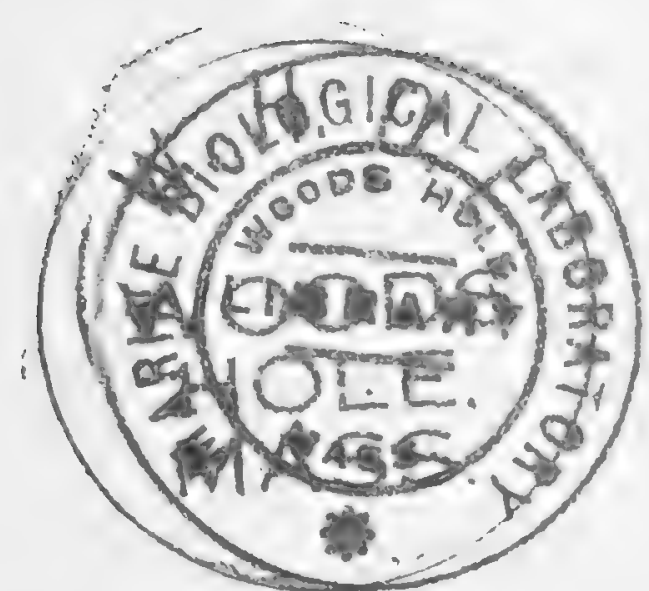

I. Ether and Energy in the Evolution of Matter........... 1

II. The Relations and Transformations of Energy during the

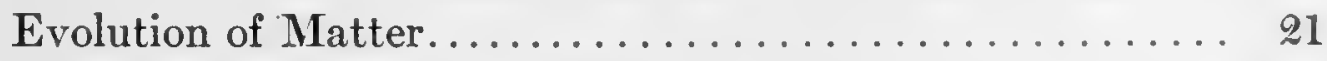

III. The Relation of Inorganic to Organic Bodies............ 48

IV. The Energies of the Organic World-the Biotic, the Cognitic, the Cogitic - and their Relation to Organisms......... 68

V. The Biotic State of Energy................... 97

VI. The Cognitic State of Energy................. 118

VII. A Review of the Formed Constituents of Organisms......... 147

VIII. Pentamorphogeny, or the Five Cooperating Causes in Organic Evolution............................ 174

IX. The Law of Proenvironment.................. 205

X. Cell Multiplication, and the Origin of Sexuality in Plants and Animals..............................243

XI. The Evolution of Plants..................... 300

XII. The Evolution of Plants (Cont'd)................. 325

XIII. The Evolution of Plants (Cont'd)................. 349

XIV. The Evolution of Animals.................... 374

XV. The Evolution of Animals (Cont'd)................ 411

XVI. The Evolution of Animals (Cont'd)................ 453

XVII. The Evolution of Animals (Cont'd) .............. 475

XVIII. The Phylogeny of Animals.................. 504

XIX. The Relation of Higher Animals to Man............... 544

- XX. The Physical Evolution of Man................... 570

XXI. The Mental or Cogitic Evolution of Man in Relation to that of the Higher Animals...................... . 598

XXII. The Operation of the Law of Proenvironment in the Evolution of Man............................. 629

XXIII. Morals as a Factor in Organic Erolution, and their Biological Origin.............................. 652

XXIV. Religion as a Factor in Human Evolution............... 675

XXV. Religion as a Factor in Human Evolution (Cont'd)......... . r02

XXVI. Religion as a Factor in Human Evolution (Cont'd)......... 794

XXVII. The Competitive System Amongst the Lower Animals and Man................................. 751

XXVIII. The Cooperative or Social System Amongst Lower Inimals and Man ............................ 768

XXIX. Human Organization in Relation to Environment.......... 798

XXX. Probable Future Advances in Human Evolution........... 825

Bibliography. Index..................... S51 



\section{The Causes and Course of Organic Evolution}

\section{CHAPTER I}

\section{ETHER AND ENERGY IN THE EVOLUTION OF MATTER}

In many recent treatises on physical and physiological phenomena, all cosmic changes have been considered to be due to the existence and interrelation of two great factors, matter and energy. From the discoveries mainly of the past century the former was viewed as being composed of discrete particles or atoms that belonged to some one of about 70 elements, that were truly ultimate and elemental in their nature. Hydrogen, oxygen, mercury, potassium, carbon, and iron might be cited as examples. Such were regarded as passive or inert in themselves, but, owing to the all-pervading presence and activity of some form of energy, the atoms were supposed to be in constant motion. Their relation to each other and degree of motion, however, might vary greatly according as the atoms were in a gaseous, a liquid, or a solid state.

Further, such atoms might unite with each other in definite combining proportions, and with definite interchanges of energy, to form more complex bodies or molecules. Water $\left(\mathrm{H}_{2} \mathrm{O}\right)$, carbonate of lime $\left(\mathrm{CaCO}_{3}\right)$, and sodium phosphate $\left(\mathrm{Na}_{2} \mathrm{HPO}_{4}\right)$ might be named as types.

The energy that gave rise to such activities and changes was considered to exist under several conditions, such as heat, light, chemical affinity, or electricity. These energies, however, 
as proved by Mayer, were convertible into each other under appropriate relations, and so "the transformability of energy" was a fundamental law in physics.

But the epoch-making discoveries of the past decade, and especially the recognition of radio-active bodies, promise to revolutionize many of the views that physicists, chemists, and biologists have alike founded on in the past. So it can truly be said that we live in a period of transition (1: 182), and that no one can predict what the ultimate views - not to say established facts - as to the constitution and relation of matter and energy may be.

The ancient opinion that a diffused and inert ether filled the world and equally sidereal space was revived during the past century, and it has been nidely accepted that this ether is a necessary constituent of the world's upbuild. It has been viened as composed of separate entities or units of extreme elasticity. These units have been supposed to act as centers round which charges of energy - of varying velocity according to the substance studied-revolve at varying rates. So by some the ether units were viewed as interpenetrating the atoms of matter, and acting as vehicles for the transmission of energy between the atoms.

Le Bon (2:13) says: "The ether is doubtless a mysterious agent which we have not yet learned to isolate, but its reality is manifest, since no phenomenon can be explained without it. Its existence now seems to several physicists more certain than even that of matter. It cannot be isolated, but it is impossible to say it cannot be seen or touched. It is, on the contrary, the substance we most often see and touch. When a body radiates the heat which warms or burns us, what constitutes this heat, if it be not the vibrations of the ether? When we see a green landscape on the ground glass of a camera obscura, what constitutes this image, if not the ether?"

But the past five years have brought to our notice some remarkable and far-reaching views. From the researches of the Curies (3) and later of Rutherford and of Ramsay (4) into the properties of radium and its direct relation to other bodies, 
it has been proved that at least some of the so-called material elements can be further split up, and so transformed into simpler elements. Thus the metal uranium gradually changes into ionium, and later into radium, while it again can be changed into helium. But experiment and atomic weight alike suggest strongly that the scarce element polonium becomes transformed into helium and likewise into lead. Furthermore, since it has been found that the characteristics of all of the so-called elements can be expressed in terms of their atomic weight, this would suggest that possibly a continuous and definite relation exists between all of the elements. Accordingly, some physicists from Prout's time (1815) onward have regarded hydrogen -with atomic weight 1 -as the sole element, from which, by increasingly complex unions and transfers of energy, the other now known 82 elements have resulted.

In reaching this conclusion, however, physicists have been almost insensibly carried another step backward, so as to suggest that the world-and possibly all sidereal space-is the arena for only two primitive and primeval entities, ether and energy. The former consists of highly elastic particles, absolutely inert in themselves, but when charged with definite amounts of energy they have properties conferred on them that have caused us in the past to name them ultimate atoms, of which hydrogen might be regarded as the simplest.

But the development of electro-chemistry has introduced a still more fundamental conception as to the ultimate material unit. From the researches of J. J. Thomson and of Larmor $(\tilde{5}, 6)$ it has been shown that even the unit-element hydrogen, as well as others of higher atomic weight, when rendered incandescent, gives off extremely minute negatively electrified "corpuscles" or electrons. The radius of each of these is about the ${ }_{100,000}^{1}$ part of that of a hydrogen atom, and its mass about the $\frac{1}{1700}$ part. So it may be said that the ultimate physical unit is the electron or corpuscle, that is immensely smaller than the hydrogen atom. This probably has an inert but elastic ether core or center, and carries around it a charge of negative electricity. When brought in contact with a body 
charged with positive electricity, union occurs with formation of an atom. The atoms again, when electrified to varying degrees, or when acted on by other forms of energy, e. g., heat or light, unite into more complex particles or molecules, of which most inorganic and all organic bodies consist.

Such views as the above have weighty experimental facts to support them, but it seems difficult if not impossible at present to say whether they will be fully sustained, or whether some new discovery may not modify the viewpoint, for the future. Meanwhile, it may be accepted as a working hypothesis that facts suggest the probable primitive and universal existence of only two constituents, energy as a moving, impelling, molding agency, and ether particles, elastic but inert. The two together constitute an electron, and when groups of them vibrate or rotate at definite rates and in definite relations they give rise to those bodies that we have hitherto spoken of as the physico-chemical elements.

Striking features of these elements that suggest an ultimate association and possible origin in common are: that they exhibit stages of transition from gaseous through liquid to solid bodies; that when two or more unite they form a compound of invariable and predicable proportion (Dalton's first law); that when two or more bodies unite in several proportions the combining weights of these always bear an exact relation (Dalton's second law); also that the law of proportional numbers of Berzelius, the law of specific heat of Dulong and Petit, all confirm a definite progressive relationship between the so-called elements.

Of these elements the gaseous body hydrogen is lightest and has been accepted as the unit element.

In order to obtain, if possible, a correct picture of molecular potentialities amongst living bodies, it is necessary that we look into the fundamental qualities of those elements that enter most largely and importantly into the constitution of living matter. Of the 83 known elements, some like hydrogen, oxygen, and nitrogen exist free and abundantly in nature, but only in the gaseous state. Others like argon, krypton, neon, 
and helium agree thus, but they are scarce. Only two elements, mercury and bromine-both of infrequent occurrence-are liquid at ordinary temperatures. The majority are solids, and are either abundant and widely diffused, like carbon, sulphur, silicon, and many of the metals, or are relatively scarce, like the greater number of the metals, and in some cases are strikingly related to living bodies, like sulphur, iodine, and phosphorus.

By far the most abundant of the elements in organic bodies are carbon, hydrogen, oxygen, and nitrogen, all characteristic non-metallic bodies. As pointed out by H. Spencer ( 7 : 4-10), the first of these shoms a remarkable relation to the other three. Carbon is not only allomorphic as occurring naturally in the forms of charcoal, graphite, and diamond; it is also solid at ordinary and even high temperatures. It likenise shows a striking degree of molecular stability and cohesion, since it unites with oxygen at a rather high temperature, it unites with difficulty with hydrogen, and still less readily with nitrogen. It might thus be termed the passive element of fundamental strength and tenacity, which acts as the basis for most organic molecules, and which caused organic chemistry, at an earlier stage of its history, to be spoken of as "the chemistry of the carbon compounds."

Hydrogen, oxygen, and nitrogen, in marked contrast to carbon, are gaseous, mobile, and amongst the lightest of elements. But even these are diverse in behavior. Nitrogen is extremely inert chemically, and only when united with oxygen, or to a less degree with hydrogen, does it show a marked capacity for chemical unions with other elements. But, given the formation of these, the resulting bodies are often of most unstable equilibrium, or even readily and violently explosive. Hydrogen, though less inert than the last, only shows high chemical activity when in presence of oxygen, but then takes part with it, and by aid of it and carbon forms many substances, particularly those of organic origin. Oxygen, while gaseous, light, and mobile in physical relation, exhibits on the other hand extreme chemical activity, and might well be re- 
garded as the element that starts energizing action, and that passes on and distributes energy to the other three, so as to cause these to link up in ever-increasing complexity.

Of the metallic elements, potassium, calcium, magnesium, and iron are most abundant in living tissues. They exist there in various degrees of combining complexity.

We may now briefly glance at the views generally held by physicists and geologists of the present day, as to the evolution of the earth's crust; the disposition of materials in it as gradual cooling took place; the relation of land, water, and air to each other as these became differentiated; also the environmental relations which probably prevailed when conditions had become favorable for the appearance of primitive organisms on it.

In the gradual condensation and cooling of the earth from an originally nebulous or gaseous and then incandescent planet, it seems possible and even probable from the studies made by Arrhenius (8) that the most abundant or the densest elements such as platinum, gold, bismuth, and iron tended to gravitate toward the center, to form there a gaseous core composed very largely of iron, mixed in much smaller proportions with other and rarer metals such as platinum and gold, but all in a highly compressed though gaseous state. According to some, as this metalliferous core continued to condense, and to radiate off heat, it became a solid mass round which the lighter metals, and some of the nonmetals-notably aluminium, carbon, and silicon-collected either in pure state or in varying chemical combinations between themselves and the heavier metals within, or with the more gaseous bodies, such as oxygen and hydrogen.

But the metal iron, from its preponderating abundance in the earth's core, and from the strains taking place between the core and exterior, must have continually welled up, been ejected, or exposed in large amount. Thus the rocks of archæan or proterozoic age contain large deposits of iron, probably derived in part from decomposition of igneous rocks, in part owing to activity of primitive plant organisms (p. 16). Iron, therefore, in all subsequent ages up to the present, has been the leading 
metal of the earth's surface layers. So there originated an investing zone or crust round the earth's metallic core, which, in virtue of its own heat energy, in virtue of the heat radiated out to it from the core, in virtue also of the active chemical affinity existing between many of the condensing bodies themselves, gave rise to extensive masses of carbonates, silicates, and other compounds of aluminium, iron, or manganese, which, when rearranged or erupted later, formed the igneous or foundational rocks of the earth's crust.

But on the still hot, often it might be incandescent, surface crust, and aided by abundant chemical reactions that were then proceeding, union of the light hydrogen and oxygen of the gaseous envelope gave rise to water vapor, that probably long remained as a dense shroud, and that was constantly added to by new formations.

Owing to continued radiation of heat into space, owing also doubtless to condensation and conversion of heat round the ether particles into intra-atomic energy, probably of an electric nature as Le Bon has pictured, the gaseous and vaporous envelope became condensed and cooled. But, during transition from a gaseous and highly-heated state to that of commencing formation of solid surface materials, the earth doubtless passed, as Arldt has well indicated (9: 553), through those successive phases of appearing among the other planets as a white star, later as a yellow star, still later as a red star, this last phase inaugurating formation of the beginning solid crust. As this crust condensed it must have been subject to considerable strains and displacements. So it would tend to be ridged up into evolving higher lands, and to collapse into depressions which, when the water vapor had sufficiently cooled or condensed as water, would start denudation action. This also, considering the active agencies then at work, would proceed comparatively rapidly, and would tend still further to mix up the already-formed constituents, and to evolve new chemical compounds in addition.

Contrary, however, to the older views of geologists, and in line with the suggestive studies of Arldt (9:564-60t), we now 
recognize that these elevations and depressions were fundamentally different in distribution, situation, and connection from those of the present day, or even of the mesozoic period.

Gradually, therefore, through intimate union of the cooling materials of the crust, there formed upon the igneous rock masses that extremely complex layer of fundamental solid rock that we know as granite. From all geological evidence, this is the primitive crystalline zone on which all the more recent strata have been deposited, and even from which in great part they have been derived, by subsequent decompositions and denudations. It may therefore be of value here to give a tabular view of the composition of granite in nine of its most abundant components, that together make up about 99.75 per cent. of its composition.

\begin{tabular}{|c|c|c|c|c|c|c|c|c|c|}
\hline $\mathrm{SiO}_{2}$ & 70.60 & 72.24 & 74.82 & 73.38 & 71.90 & 73.27 & 66.83 & 71.62 & 71.83 \\
\hline $\mathrm{Al}_{2} \mathrm{O}_{3} \ldots$ & 16.40 & 14.92 & 16.14 & 14.86 & 14.12 & 15.51 & 15.24 & 14.99 & 15.27 \\
\hline $\mathrm{Fe}_{2} \mathrm{O}_{3} \ldots$ & 1.52 & 1.63 & & 0.10 & 1.20 & 0.33 & 2.73 & 1.27 & 1.25 \\
\hline $\mathrm{FeO} \ldots$ & 0.36 & 0.23 & 1.52 & 1.64 & 0.86 & 1.14 & 1.66 & 1.01 & 1.05 \\
\hline $\mathrm{MnO}$ & 0.48 & 0.32 & & & 0.05 & trace & 0.10 & 0.17 & 0.22 \\
\hline $\mathrm{MgO}$. & 1.00 & 0.36 & 0.47 & 0.23 & 0.33 & 0.15 & 1.63 & 0.74 & 0.73 \\
\hline $\mathrm{CaO}$. & 2.47 & 1.68 & 1.68 & 0.89 & 1.13 & 2.74 & 3.59 & 1.33 & 2.06 \\
\hline $\mathrm{Na}_{2} \mathrm{O}$ & 4.14 & 3.51 & 6.12 & 3.94 & 4.52 & 4.79 & 3.10 & 3.62 & 4.21 \\
\hline $\mathrm{H}_{2} \mathrm{O}$. & 4.29 & 5.10 & 3.55 & 3.89 & 4.81 & 1.66 & 4.46 & 4.81 & 4.07 \\
\hline
\end{tabular}

From the above table it will be seen that the most striking peculiarities of granite are: (1) the presence in it of all the elements needed for complete nutrition of plants and animals except those three fundamentally diverse elements already referred to, carbon, hydrogen, and nitrogen. But undoubtedly the three existed in abundance in the surrounding gaseous envelope that constituted the atmosphere of the evolving earth. For $\mathrm{CO}_{2}$ has all along played an important part in uniting with bases to form carbonates, while the masses of graphite that occur in the older crystalline rocks prove that it must have been abundant. Nitrogen must also have been a widely diffused gas in the atmosphere, but its presence otherwise is demonstrated by its frequent occurrence in cavities of granite, gneissose and basaltic rocks. The same is true for hydrogen. 
The element phosphorus, in many respects as typical for organisms as carbon, though present at times in minute quantity, is also present in all typical granites but only to the extent of $\frac{1}{10}$ to $\frac{1}{20}$ of one per cent. and so has been omitted from the above table.

A second feature of granitic composition is the important part which oxygen plays, in that it is a constituent of all of the granite components, and is the energizing element that would further cause these to enter into new combinations.

As bearing on the formation alike of crystalloid and of colloid bodies, and their subsequent presence in organic tissues, it may be convenient here to present, in tabular form, data regarding the earth's crust. From comparative study of the substances entering into its composition, it is found that oxygen and silicon make up by far the largest percentage proportion. Thus oxygen, with atomic weight 16 , constitutes about 50 per cent.; silicon, with atomic weight 28, constitutes about 27 per cent. of the entire mass; or these two together represent about 77 per cent. of its bulk. The subjoined table of the six most abundant nonmetals and of the six most abundant metals, with the atomic weight of each, graphically sets forth the preponderating importance of the twelve in the construction of the earth as a whole, since together they make up fully 99 per cent. of it.

\begin{tabular}{|c|c|c|c|c|c|}
\hline Nonmetals & $\begin{array}{l}\text { Atomic } \\
\text { weight }\end{array}$ & $\begin{array}{l}\text { Proportion in } \\
\text { outer crust } \\
\text { including } \\
\text { crust, sea, } \\
\text { atmosphere }\end{array}$ & Metals & $\begin{array}{l}\text { Atomic } \\
\text { weight }\end{array}$ & $\begin{array}{l}\text { Proportion in } \\
\text { outer crust } \\
\text { including } \\
\text { crust, sea, } \\
\text { atmosphere }\end{array}$ \\
\hline Oxygen & 16 & 50 & Aluminium & 27.30 & 7.45 \\
\hline Silicon & 28 & 26 & Iron & 55.90 & 4.20 \\
\hline Hydrogen & & 0.90 & Calcium & 39.90 & 3.25 \\
\hline Carbon & 11.97 & 0.20 & Magnesium & 23.94 & 2.35 \\
\hline Phosphorus & 30.96 & 0.08 & Potassium & $39.0 \pm$ & 2.35 \\
\hline Sulphur & 31.98 & 0.06 & Sodium & 22.99 & 2.40 \\
\hline
\end{tabular}

These again, as already set forth for granite, enter primarily into union with oxygen to form oxides. The next table sets forth F. W. Clarke's average results of the analysis of 830 
typical samples of the primitive part of the earth's crust; and from this it will be seen that the group of oxides given makes up about 99 per cent. of its total mass.

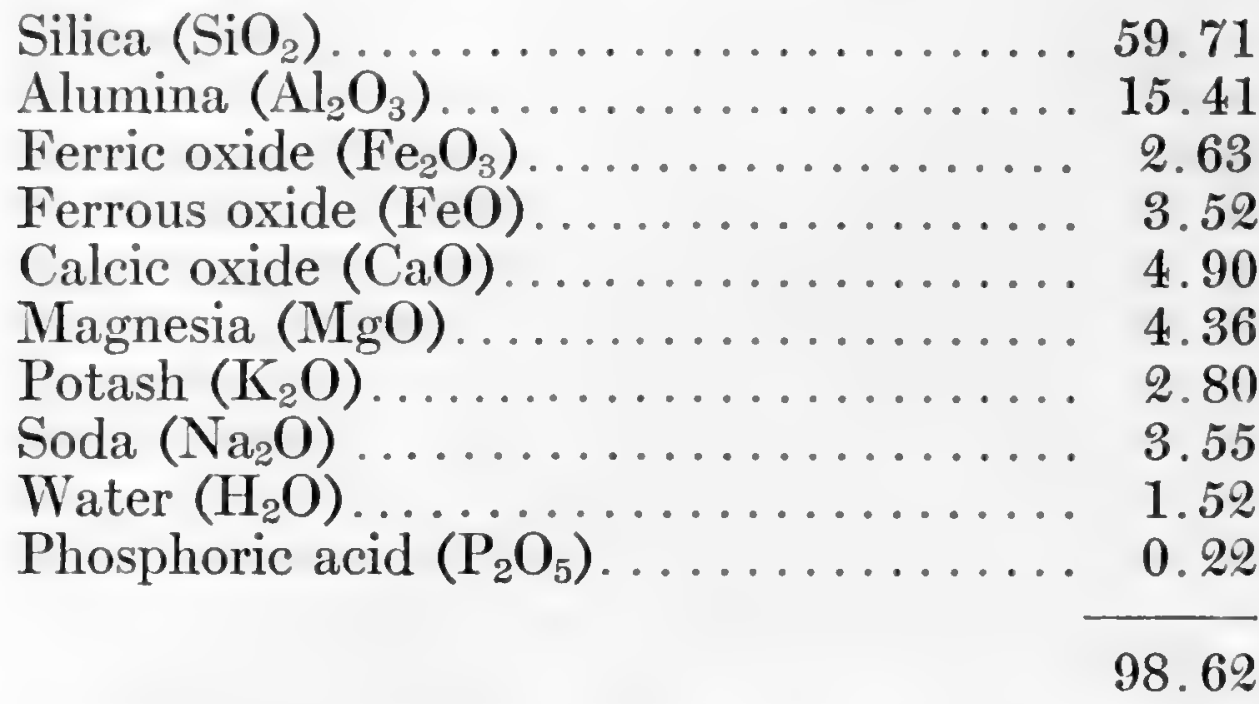

It is generally conceded that, during the early formation of this shell or crust, abundant and widespread volcanic activity on the one hand combined with increasing cooling of the crust on the other were proceeding, so that, with evolution of a definite enveloping atmosphere, subaerial denudation would, on the one hand, cause steady attrition, disintegration, and removal of the previously formed masses, while new accumulations of molten material, thrown out from the earth's interior, would cause, by gradual cooling of these, steady upbuilding or integration of the crust.

At the close then of what may be termed the igneous or volcanic epoch of the earth's history, the internal masses of gaseous iron and rarer metals, along with smaller quantities of other elements that now formed, on Arrhenius's view, the earth's core, were bounded by a transition layer of viscous and unstable material, that again was enveloped by a granite shell. Or to picture it from without inward in Geikie's words: "The interior of the earth, therefore, with the exception of a solid crust about 40 kilos thick, consists of a molten magma 100 to 200 kilos in depth, which shades continually inward into a gaseous center. The liquids and gases in the interior possess a viscosity and incompressibility such as permit them to be regarded as solid bodies." 
The granite shell or crust contained either in its own constitution, or enclosed as graphite masses (in the case of carbon), or included as gaseous cavities, all of the elements that make up organic bodies. Furthermore, abundant and varied combinations had already taken place among the elements, so as to produce granitic compounds, from which, by subsequent alterations, disintegration, and reintegration, all of the more recent rock constituents could be derived.

If we now compare the views of physicists and geologists, as to the probable age of the earth's crust from the time when the granitic rocks had been formed up to the present day, a considerable diversity of opinion is encountered. Kelvin, in his earlier calculations, based his estimates on three physical factors, and concluded that a period of from $20,000,000$ to $100,000,000$ of years has elapsed since subaerial denudation and deposition began to take place. But the more recent studies of G. Darwin, Woodward, and Perry, on the physical side, have caused them to concede at least $100,000,000$ of years as a likely time-minimum. In this they have agreed with the earlier claims of geologists, who alike from the geodynamical and the palæontological standpoints claimed a greatly extended period.

From the geological standpoint J. Joly (10: 23) has estimated the amount of sodium annually carried to the ocean, since its earliest formation, in relation to the total quantity dissolved there, and concludes that the process of transfer has proceeded for 90 to 100 millions of years. Sollas (11:24) again is inclined, also on geological grounds, to accept a time limit vaguely intermediate between the minimum and maximum of Kelvin.

Arldt in treating of the phases of the earth (9: 553) apportions the time limits as follows, beginning with the period when the earth had just passed from a nebulous state into that of a white star, and onward till recent date.

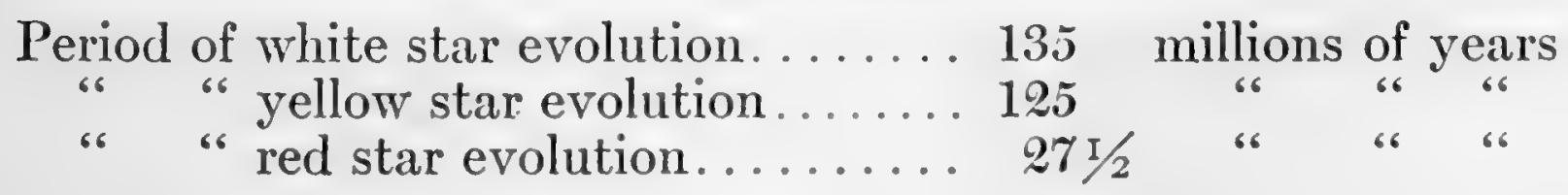




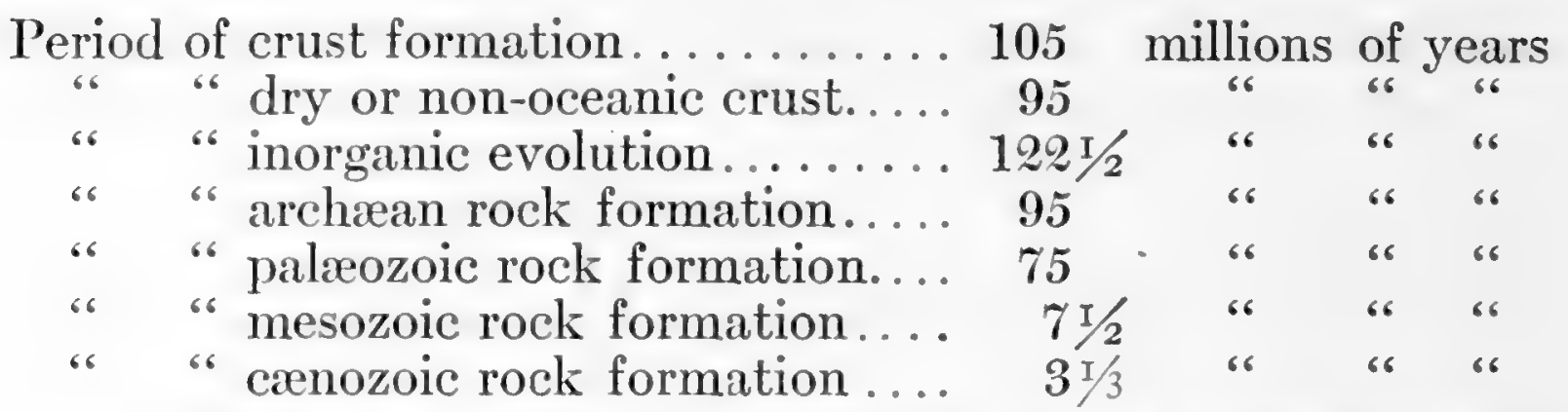

This calculation-confessedly vague -would give about 180 millions of years for formation of the sedimentary crust.

The oldest stratified or semi-stratified rocks of the earth's crust have variously been called the metamorphic, archæan, proterozoic, or pre-cambrian, and it is to the early or midarchran epoch that all evidence tends, as the starting point for the dawn of organisms on the earth. The rocks then formed are usually quite unconformable with the masses of primitive granite on which they rest, thus indicating that a considerable - even extended-period of time had elapsed between deposit of the two systems. Only rarely do they seem to be continuous, but even then the composition of the older igneous or granitic and newer or archæan rocks differs markedly (12:863). Often also the older archæan beds are broken up, distorted, and copiously interbedded with intrusive granitic masses, that indicate active and extensive alterations in the crust.

But, while the archæan strata or masses consist very largely of schistose and micaceous gneissic materials that have been highly metamorphosed by contact with granitic or other intrusive igneous flows, in the middle or later beds of the series there occur zones of graphite that may have had a vegetable origin; cherts and iron ores probably also of vegetable origin, as Geikie has suggested (12: 87\%) and as the writer has further elucidated (13: 257); as well as limestones that may have had an organic, possibly even a regetable, origin; and all frequently interbedded with schists, serpentines, and other rocks.

The deposits of this age are enormously developed over extensive areas of North America, Europe, Asia, and Australia, possibly also of Africa. Their vertical thickness, over the regions north of the Great Lakes, has been calculated to be about 65,000 feet or 12 miles. They evidently resulted in 
large part from exposure of the more primitive granitic masses to alteration, denudation, and removal, with subsequent redeposit; so that active wearing domn of land areas and simultaneous formation of lacustrine or sea deposits were now established. Thick beds of sandstones, slates, and conglomerate rocks enclosing rounded water-worn pebbles are fairly frequent. Such facts clearly indicate that during the archæan epoch terrestrial conditions must have resembled those of the present day to a considerable degree. But as Arldt has suggested, and as the writer has indicated from the standpoint of plant life (13:259), the temperature very probably was much higher than now, ranging from $80^{\circ} \mathrm{C}$. down to $60^{\circ}$ or $65^{\circ} \mathrm{C}$; ; the atmosphere in many places must have been warm, moist, and stimulating to molecular change; diverse chemical substances must have undergone active integration and disintegration; while copious supplies of thermic, lumic, chemic, and electric energy must have favored widespread chemical action.

While there seem to have been ocean carities of wide extent but shallow depth, the active volcanic and denudation changes proceeding must have resulted in formation of extensive swamp, lacustrine, and shore areas of no great depth, but which would become the arenas for diverse chemico-physical activities. Not a few of these areas must have shown conditions that at the present day are only paralleled by those now limited regions where geysers, hot springs, and mud volcanoes are active. Even though our knowledge of these is still limited, the chemico-physical and biological problems they present, as set forth by Weed (14: 619), Davis (15: 145), and others, suggest many interesting parallels with what probably was archæan environment. So the writer has pointed out that the rich protophytic life of present-day hot springs which flourishes at $60^{\circ} \mathrm{C}$. to $80^{\circ} \mathrm{C}$. may have had its origin amid like archæan environment (13: 259). When we reflect on the rertical thickness of the deposits of this epoch, and compare these with the total mass of the later sedimentary rocks, it is not surprising that some geologists, as well as palæontologists, have demanded for deposition of the former a time-interval 
as great as for all of the latter, or in rough numbers a period of 50 millions of years. Even if a shorter time interval be conceded, the period must have been a long-extended one.

All evidence then points to the conclusion that during the mid- and later archæan epoch extensive areas of land and fresh water existed; that many and varied inorganic compoundsin which could be traced all of the elements now entering into organic bodies-were undergoing extensive and manifold processes of synthesis and analysis; that stimulating conditions for molecular union, due to frequent volcanic activity, to favorable atmospheric surroundings, to abundant chemical compounds, and to rich supplies of energy, were nidespread and in active operation; and that the epoch represented a distinct phase in evolutionary progress which formed a linkalike physical and chemical-between a more primitive and a more evolved state, in a continuous line of progressive change.

It is important also to emphasize here that in the process of transition from the nebulous through the astral to the solid state of the earth, while some elements like hydrogen, oxygen, and nitrogen persisted largely during the archæan and on even to the present day as gaseous elements, gradual cooling and condensation of others, and of the earth's mass, had given rise to compound gases, to the abundant liquid water, and still further, as well as gradually, to numerous solids. These were built up, stage by stage, of binary, ternary, and to a less extent of quaternary unions of elements, that not infrequently suggest a neat and orderly progressive evolution of them with advancing age of the earth.

We may now appropriately study the minute arrangement of the atoms and molecules, which by gradual chemical combination gave rise to the earth's constituent bodies, since they will have most important relations to subsequent studies.

Almost all of the substances that made up the foundational or igneous, and the later or archæan, rocks showed that arrangement of the atoms and molecules which has been termed crystalline. This is evidently the most primitive, as well as the most stable, condition of inorganic bodies, and crystalli- 
zation is effected with least expenditure of energy, though it requires considerable expenditure of energy for its disruption. By such action practically all of the earth's crust was formed, so that such rocks as the granites, gneisses, schists, and sedimentary strata show crystalline structure.

When subjected to chemical, physical, or microscopic examination, such crystalline compounds are proved to be made up of crystal constituents, each of which shows definite faces, angles, and affinities that confer definable characters on the mass. These constituent crystals again ore such properties to the molecules of which they are built up. From their behavior again these molecules are evidently of small size, are incapable of resolution even by the highest powers of the microscope, are relatively stable when combined with each other, can all pass by dialysis - though with varying degrees of rapidity-through parchment membranes when in solution, have a relatively high osmotic pressure, and show a relatively small degree of mobility.

But, from the biological standpoint, special interest attaches to the group that was first carefully investigated by Graham, and which is known as the non-crystalline or colloid bodies. Though our minuter knowledge of them dates back only for 50 years, it is not too much to say that their study gives promise of most fruitful results, in the explication of organic compounds and of their life phenomena. Each passing year also adds to their number, and to the information gained regarding them. Such "elementary" metals as gold, silver, platinum, bismuth, lead, and iron, when electric energy is spent on them in presence of water or other appropriate liquid, become changed into a substance that has a uniform aspect, and relatively thin consistence, if the liquid be in sufficient quantity. But with increasing condensation the mass becomes a jelly that may vary from dull white -like white of egg-to dark brown as in colloid bismuth (17:425).

Again the colloid sesquioxide of iron-abundant in waters of the later archæan or laurentian period, as it still frequently is in recent lagoons - has the dark red color of venous blood, 
when in 1 per cent. aqueous solution. This solution is coagulated at ordinary temperature, by sulphuric acid, alkalies, alkaline carbonates, sulphates, and neutral salts in general. It then is a deep red jelly resembling a clot of blood, but more transparent.

The extensive prevalence of such colloid iron oxides in waters of archæan age seems exactly to explain the origin and abundant deposits of bog iron ore, of hæmatite, and of magnetic iron. At this time, as nill be emphasized later, a simple but abundant vegetable and animal life must have been evolving. Thus Geikie (12: 612) says: "In marshy flats and shallow lakes, where the organic acids are abundantly supplied by decomposing plants, the salts of iron are attacked and dissolved. Exposure to the air leads to oxidation of these solutions, and consequent precipitation of the iron in the form of hydrated ferric oxide, which, mixed with similar combinations of manganese, and also with silica, phosphoric acid, lime, alumina, and magnesia, constitutes the bog-ore so abundant in lowlands of North Germany and other marshy tracts of North Europe. On the eastern seaboard of the United States, large tracts of salt marsh, lying behind sand-dunes and bars, form receptacles for much active chemical solution and deposits. There, as in European bog-iron districts, ferruginous sands and rocks containing iron are bleached by the solvent action of humus acids, and the iron removed in solution is chiefly oxidized, and thrown down on the bottom. In presence of the sulphates of sea water and of organic matter, the iron of ferruginous minerals is partially changed into sulphide, which on oxidation gives rise to precipitation of bog-iron. The existence of beds of iron ore among sedimentary formations affords strong presumption of the existence of contemporaneous organic life, by which the iron was dissolved and precipitated."

Compounds of silicon, arsenic, and other nonmetals can also assume the colloid state. Thus Ostwald says (18:429), "in nature silicic acid occurs very often in such a form. It gets into natural waters from the silicates when these are decomposed by $\mathrm{CO}_{2}$." It may remain fluid for days or weeks in a sealed tube, but is sure to gelatinize and become insoluble at last. Such colloid silica may be precipitated by the action of constituents of decaying plants or animals, when it may take the form of extensive siliceous deposits over the bed of the ocean, or round the edges of geysers (14), as in the Yellowstone region and New Zealand, amongst many others. 
While some of the simpler metallic colloids, like platinum, gold, and silver, may be comparatively stable, and may remain in solution for months, others, like lead and iron, soon change into new compounds from contact with the atmosphere. This tendency to undergo change and decomposition is, of course, associated with interchanges of energy, and is true of the great majority of colloids, as contrasted with the usually slower alteration of crystalloids. Hence Spencer, quoting Graham, (7: 16) says: "The colloid is, in fact, a dynamical state of matter, the crystalloidal being the statical condition. The colloid possesses energia. It may be looked upon as the primary source of the force appearing in the phenomena of vitality."

With such considerations before us, it behooves us to look further into colloid phenomena. As to size, investigators agree that colloid molecules are greatly larger than the crystalloid; they are incapable of passing, or only slowly pass, through parchment and similar membranes; this incapacity seems to be wholly due to the large size of the molecules, which are stopped in front of the minute interstices of the membrane. Their osmotic pressure is very low; and, beside being relatively unstable, they often start catalytic changes in liquids with which they come in contact.

One triumph achieved by the ultramicroscope has been the resolution of various colloid substances into discrete molecules floating in the solvent liquid. As the water or other solvent is evaporated, these molecules gradually approach each other until, in the firm gelatinous or even hard stage of silicic acid, they may only be separated by water jackets from each other. Measured by the ultramicroscope, the colloid particles of platinum are about $45 \mu \mu$ in diameter according to Walker (19: 221).

As compared, therefore, with true crystalloid solutions, which are perfectly clear, homogeneous, and devoid of visible particles, all colloids show a finely granular or coarse molecular state. But even colloids present themselves under two phases, that have been called the suspensoid and the emulsoid. The former show definite suspended and finely divided floating 
particles, the latter appear as a very fine liquid emulsion; the former become coagulated and precipitated when any electrolytic substance is added to the solution, the latter are not coagulated by small quantities of electrolytes, but they can be coagulated by large amounts of neutral salts; the former are highly mobile, the latter viscous and inclining readily to gelatinize. The above precipitation action is evidently due to the comparatively large colloid molecules falling together into larger aggregates of greater density.

As to the possible relation of these two to each other, results have been obtained that are highly suggestive for living tissues. Thus if traces of the emulsoid gelatine be placed in a gold solution, and an electrolyte be then added, the gelatine prevents precipitation of the gold particles by apparently forming a protective coat round the latter, that shields these from electrolytic action.

Added features of interest from the standpoint of organic bodies are, that the molecular weight of colloids is always higher, often greatly higher, than that of crystalloids; and that, while crystalloids can diffuse themselves through colloids nearly as rapidly as through water, colloids can scarcely diffuse at all through other colloids. "So we must regard the colloid compounds of which organisms are built as having, by their physical nature, the ability to separate colloids from crystalloids, and let crystalloids pass through them with scarcely any resistance." Graham further pointed out that even the colloid body, if made up of constituents that are of feeble affinity, may undergo splitting up if passed through a dialyzer. Thus the hydrochloride of sesquioxide of iron $\left(\mathrm{Fe}_{2} \mathrm{Cl}_{6} \mathrm{H}_{24} \mathrm{O}_{12}\right)$ separates into hydrochloric acid and the colloid sesquioxide of iron.

Again attention has been drawn to the similarity in action of some inorganic colloids to the organic ferments. Thus the dark brown liquid that is the colloid state of platinum has been found by Bredig and Von Berneck to decompose "hydrogen dioxide like organic ferments, and resembles the latter in many other particulars. A gram atomic weight of platinum in 
$70,000,0001$. of water decomposes hydrogen dioxide appreciably, thus resembling organic ferments, where a very small quantity can effect a large amount of decomposition. An even more striking analogy between the action of the colloidal platinum and organic ferments is to be found in the effect of certain poisons upon both of them. A very small amount of certain substances will entirely destroy the activity of organic ferments. Exactly the same was found to be the case with the colloidal solution of platinum. A gram-molecular weight of hydrocyanic acid in 1,000,000 l. of water diminished quite appreciably the activity of the colloidal platinum toward hydrogen dioxide; and a gram-molecular weight of hydrogen sulphide in 345,000 l. water greatly diminished the activity of the platinum. A gram-molecular weight of hydrogen-sulphide in 34,500 l. almost destroyed the activity of the platinum." (16: 252) Finally from the standpoint of molecular energy Burton (1\%: 443) states that "there is practical unanimity in the opinion that these particles in colloidal solutions are enclosed by a double electric layer, the electricity of one sign on the surface of the particle being in equilibrium with an equal amount of electricity in the layer of liquid immediately surrounding the particle." In a later chapter we shall have occasion to return to this statement.

When, therefore, we compare crystalloids with colloids and again compare inorganic colloids with those obtained from an organic source, it does not seem to be an overstepping of the bounds of prudence if we affirm that our most fundamental knowledge of living bodies and of life itself will result from a continued study of these colloid groups in their chemico-physical behavior.

If we now attempt to sum up the conditions and relations shown by ether atoms when united to form the material elements and their molecular compounds, it may be said that, while most inorganic bodies are of crystalloid nature and behavior, a considerable and ever-increasing number that range from comparatively simple states of elements to complex compounds are non-crystalloid or colloid. These latter, when 
sufficiently swollen or dissolved in water, alcohol, etc., preseni marked resemblances to gelatine, white of egg, blood clot, and other organic bodies of colloid nature. The differences between the crystalloid and the colloid groups are specially interesting biologically. For, while each ultramicroscopic molecule that makes up a crystalloid body shows regular and neat facets that fit into neighbor facets of other units to build up a solid relatively stable whole or compound crystal, in colloid bodies the primary molecules are surrounded by a jacket of liquid, and the groups or compound molecules of these are so surrounded and separated by liquid that they fail to fit closely together.

Each group or compound molecule then-that by Nägeli and others has been called a micella-is enveloped by a liquid film, while crystalline bodies, formed by direct apposition of simple molecules, are devoid of enveloping water. The greater irregularity and plasticity of the former also give to such two important qualities: (a) that of readily absorbing and holding water or other liquid in the areas between the complex molecules, and (b) that of permitting a labile motion of the micellæ between each other. It thus follows that colloids are more liable to alteration and to undergo decomposition changes than crystalloids. In other words they are more readily affected by, and respond to, environal action. 


\section{CHAPTER II}

\section{THE RELATIONS AND TRANSFORMATIONS OF ENERGY DURING THE EVOLUTION OF MATTER}

Since every ether particle, and so every resulting atom or molecule of matter, is constantly charged with some form or forms of energy, and since it is by utilization of such energy that the more complex combinations of matter result, we may next appropriately treat of the relations and transformations of that energy.

In the early nebulous condition of our earth, heat was the abundant and active type of energy. This originated in part as the intra-atomic heat of the gaseous particles that made up our planet when thrown off from the sun; in part it was absorbed subsequently as solar heat radiated out into space. This heat, associated with elastic ether particles, caused rapid motion of these. In this gaseous state of the earth, a temperature of at least $15,000^{\circ} \mathrm{C}$. may have existed according to Arldt. But, as seems to be the history of every planetary mass, a gradual condensation of heat took place round each particle, so as to increase its rate of motion and also the sum total of stored intra-atomic energy. This then may be regarded as the primary or initial phase of earth evolution in relation to energy flow and storage.

The above condition was gradually succeeded by one in which the elemental atoms began to pass into a liquid state at or toward the surface of the mass, and so into a condition in which the energy-charged particles described a more restricted but more intensely energized pathway of motion. The atomic energy now manifested itself chiefly as light, which is at once a more evolved and perfect form of energy than heat, though transformed from it by increased condensation of energy units. 
But even now, though heat was rapidly being absorbed as such, and also modified as light, into the atoms, and so a cooling of the surrounding surface regions $\pi^{*}$ as proceeding, the temperature probably ranged from $10,000^{\circ} \mathrm{C}$. down to $4000^{\circ} \mathrm{C}$. The earth then appeared successively as a white, next as a yellow, and later as a red star.

But with increasing absorption of intra-atomic energy over the evolving crust, with resulting rapidity of atomic motion and reduced amplitude of the field of movement between the energized ether particles, there was next established an increased viscosity, and so the possibility of intimate union of atoms between themselves, to constitute molecules. The energy resulting from this union represented in turn a still higher and more perfect type than that of light, and so greatly higher than that of heat, and is what we term chemical affinity. This period rould approximately correspond, in Arldt's table already given (p. 12), to his "crust-formation" and in part his "anhydrate" periods, and so may have extended over more than 100 millions of years.

The next progressive stage in energy transformation and molecular evolution seems to have been the electrical. Since gases are bad conductors of electricity-theoretically nonconductors in the complete absence of radio-active bodiesthe abundant formation and free transfer of it during the gaseous period of the earth would have been small. Only after the liquid and specially the solid state was assumed would pathways for its flow be easy, and atomic centers for storage of it be appropriate. So the period of its increasing evolution and transformation would extend from Arldt's crust-forming, on to his inorganic, epoch. Much of this electricity may have been derived from the more primitive type chemical energy, owing to the active chemical reactions proceeding between metals and electrolytic substances; in part it may have condensed from light transformations, but not a little may have been derived on the one hand from radiated internal heat and on the other from absorbed solar radiations. During this period, therefore, those extensive magneto-electric disturbances 
and flows over the earth's crust may have become pronounced that to our day have continued on gigantic scale.

Fauvelle has strongly advocated the view (20: 107) that electricity is a highly condensed or latent heat. Whether we consider the ease with which electricity can be reduced to heat, the possibility during the reduction process of utilizing some of this energy in effecting chemical dissociations, or the development of light in the process, there is much to be said alike in favor of Fauvelle's view, and of that now advanced which regards the four forms of energy - heat, light, chemical affinity, and electricity - as condensing and accelerating modifications of a common state of motion amongst ether particles.

So the successively evolved physical states of the gaseous, the liquid, the viscous, and the solid would be a result of, and paralleled with, the transition from thermic to lumic, from lumic to chemic, and from chemic to electric conditions of motion.

The apparent climax of this process for inorganic bodies was reached, when various of the elements or inorganic compounds united into complex colloid molecules, at times of large size. As already stated (p. 19), these are, according to Burton, charged with a double jacket of electric energy, each with different sign from its neighbor. It is this double electrified state, probably, which gives to colloids their peculiar properties as contrasted with crystalloids. The relation of it again to possibly higher and more condensed types of energy will be emphasized later, from the standpoint of organic bodies.

But, with increasing intra-atomic condensation of energy, there seems to have resulted, pari passu, a more and more complex union of atoms and later of molecules. Thus, when the earth-like the sun at present-was in the state of an incandescent body, the gaseous mass would consist only of elemental substances. But as condensation and cooling occurred there would be formed simple unions of the more stable elements like carbon, silicon, aluminium, etc., at the same time that the heavier metals became condensed as such in the core, or occasionally themselves formed simple oxides. Later, when 
the liquid state was assumed, not only binary but ternary combinations would become abundant, through the combined action of thermic and of the higher chemic energy. The complexity of the action would be still further advanced, and the variety of compounds increased, when electricity combined with heat to produce thermo-electric effects, such as our present-day thermo-electric furnaces illustrate, but only feebly imitate in power.

One result of this was the production of the progressively more and more complex igneous rock masses, the great majority of which are of highly complex composition, but are all crystalloid in structure. Such masses, like the granite already referred to (p. 8), may be said truly to represent the culmination of crystalloid energy-condensation activity.

A noteworthy feature of such solid bodies - that has only begun to be fully recognized since Le Bon (2: 85) drew attention to the subject of intra-atomic stored energy, and since radio-active bodies have been the subject of continued investigation-is that they all possess enormous stability of molecular or structural relation, in virtue of the enormous amount of potential energy stored up. So, though the pathway of free motion between the atoms or molecules may be extremely circumscribed, the relatively enormous amount of stored energy in them causes them to occupy a very constant and stable relation to each other. Therefore, as a physicist has well remarked, the visible relation of the particles in many solids may remain unaltered for millions of years, and so give to such solids a definite character and morphology by which they are constantly recognized.

But that the energy may gradually be again given up or dissipated - in part as electric, in part as chemic, in part as lumic, and in part as thermic energy - is now recognized to be true in the history of such elemental metals as uranium, ionium, radium, and helium, that represent stages in the devolution of bodies which have existed for millions of years as definite crystalloid compounds, but are now undergoing gradual transformation by degradation and in the process are giving rise to simpler bodies. 
The formation then of ternary and quaternary chemical compounds of crystalloid character, which might again become mixed by fusion with other compounds to form the granitic and other igneous rocks, seems to represent the highest and most stable result of electric, of electro-chemical or electrothermal action. If then a further advance rere possible, alike in the more perfect condensation of energy and in the upbuilding of more complex molecules in relation thereto, such advance would only seem likely by the production of some more concentrated but tenacious kind of energy, and of a more elastic type of molecule, that the energy might traverse and be held in, in large part, as an intra-molecular reserve. Such seems to be presented in the capacity for colloid formation possessed by some elements, and for the upbuilding of the colloid molecule already dealt with (p. 15).

Reference has been made to some of the inorganic colloid bodies. Since they are all formed only in presence of water or other light liquids, they can only have originated long after complex crystalline bodies had been formed in the earth's crust by electric or by lower forms of energy. Thus, during Arldt's anhydrate period (p. 12) in the earth's history, they must have been entirely absent, and only during the inorganic period succeeding, that Arldt roughly estimates as extending through 120 millions of years, would necessary conditions at times occur for their formation.

From the experiments of Muck and Tommasi the colloid red and yellow ferric hydrate can be obtained by treating ferric salts with alkalies, or by oxidation of ferrous salts. The former process must have occurred in nature before, or apart from, the presence of terrestrial organisms, but, as will be stated later, much of the colloid iron that has produced the beds of bog-iron ore or limonite, as well as hæmatite, was due to the oxidizing energy of plant organisms amid swampy marshes, into which iron oxides had been washed. From the red ferric hydrate Ruff has also obtained like iron ores by heating it in presence of water under a pressure of 5000 atmospheres.

Colloid silicic acid must also have been formed in nature apart from-though more abundantly by-organisms, as must 
other of the colloids referred to on p. 16. But our knowledge of them, and of their molecular composition, is still comparatively limited. It is through these, however, we believe, that a continuous and direct passage can be had from the so-called "inorganic" to the "organic" compounds.

In contrasting inorganic crystalloid and colloid bodies it can be said that, while the former are abundant, the latter are comparatively rare; the former are usually binary or ternary, rarely quaternary, compounds, the latter even at ordinary temperature range from elemental to binary, ternary, or quaternary compounds; the former have a relatively simple molecular structure, the latter a greatly more complex one; the former can hold little intermolecular water or other liquids, the latter show exceptional capacity in this respect. But, as we advance from the more complex binary and ternary inorganic colloids, in search of others with an increasingly complex structure, we invariably reach so-called "organic" compounds.

Now, had we lived in the early part of the archæan epoch, and, with the knowledge we now possess, had we surveyed the procession of inorganic advance from the thermic- and the lumic-charged gaseous state, through the thermo-lumic and chemically charged liquid or viscous state, to the chemicoelectric and electrically charged solid state of the earth's crust, the question might naturally have occurred: Can there, or does there, exist a more condensed, perfect, and powerful type of the all-pervading energy than even electric, and if so can it exhibit its potentiality by building up greatly more complex ternary and quaternary colloid molecules than any now existing? Can it even further elaborate quinary, hexary, or heptary bodies, that shall exhibit a greatly more complex composition and interrelation than do the varied and mixed constituents of granite or related rocks of the crystalloid? Such to the mind of the archæan surveyor might have seemed difficult, even impossible, of realization or projection. But he, in his bodily upbuild, would have been an impersonation of the fulfilled hope, at least so far as the molecular complexity is concerned. As regards the energizing factor that accomplished such striking 
results, we propose to speak of it as the "biotic" type, and in succeeding chapters of this work reasons will be advanced for accepting its existence.

We would therefore regard the colloid state of many inorganic bodies as that which evolved out of previously anhydrous matter, such as alone existed in pre-archran times. This colloid matter differed from the inorganic liquids and solids in being built up of large hydrated molecules, whose size and molecular upbuild were rendered possible by special and evolving condensation of electric energy, so as to form a double charge round each molecule. But by further condensation of this doubled energy, so as to constitute a new type or phase of it, biotic energy gradually evolved, and in the process began to energize colloid molecules of increasing size and complexity, till the albumen molecule, and later the highly complex and labile protoplasmic molecule, was upbuilt. This became the fundamental organic energizing basis on which all subsequent elaboration of more complex energized ether particles took place, in the development of progressively evolving plants and animals.

But, if such complex molecular energizing took place, it is unquestionably to the archæan epoch that we should turn for evidence in favor. It is universally conceded by geologists that in the lower schistose strata of the period no direct traces of life exist; also that the same is true of the middle period. During those periods enormous and extensive volcanic activity proceeded; huge deposits of volcanic rock were poured out over, forced between, or overturned the sedimentary strata then forming; atmospheric temperatures probably ranged from $85^{\circ} \mathrm{C}$. to $100^{\circ} \mathrm{C}$. over at least a considerable part of the earth. Toward the close of the lower archaan period, however, and in the dawn of the mid-archran, the conditions stated in last chapter seem to have become established (p. 12), and the bands or masses of chert, of limestone, of iron ore, and of graphite all strongly attest commencing organic activity.

We may now try to ascertain how such activity originated in its simplest expressions, by linking together such present- 
day facts as will guide us. While fully accepting uniformitarian principles as governing the changes then proceeding, it seems absolutely necessary and appropriate to accept that the rate and intensity of change on the earth's crust-surface were considerable more accentuated than now, in fact formed an average between the earlier "anhydrate" and "inorganic" periods when granites and other rocks nere forming, and our own day with its restricted and slow crustal changes. So between the active volcanic outbursts that the rocks of this period attest to; the extensive geyser, mud volcano, and hotspring deposits that the cherts and limestones of the period suggest; the denudation of the more loose volcanic material; its subsequent rolling and conversion into conglomerates; the setting free of many chemically active products in steaming lacustrine, and shallow inland lake areas with formation there of bog-iron deposits; and the abundant disengagement of electrolytic bodies; conditions existed that favored progressive colloid elaboration.

From recent studies on colloids ne now accept it that these microscopically may closely resemble foamy mixtures such as Bütschli (21) and later Krafft have carefully investigated, and which in some cases may simulate complex protein compounds, as described by Mann (22: 36). Furthermore, Traube and Leduc have demonstrated by experiment that, where two different liquids such as 4 per cent. potassium ferrocyanide and a granule of cupric sulphate, or a liquid and a colloid body such as tannic acid and mucilage, come in contact, chemical changes are set up which cause formation of an enclosing membrane or pellicle, and included materials that may be powerfully osmotic. To quote Leduc's statement as given by Le Bon (2: 359) "a granule of sulphate of copper of $1 \mathrm{~mm}$. to $2 \mathrm{~mm}$. diameter, composed of about two parts of saccharose, one of sulphate of copper and water to cause it to granulate, is planted in an aqueous solution containing 2 per cent. to 4 per cent. of ferrocyanide of potassium, 1 per cent. to 10 per cent. of chloride of sodium and other salts, and 1 per cent. to 4 per cent. of gelatine. It germinates in a space of time which varies 
from a few hours to a few days according to the temperature. The granule surrounds itself with a membrane of ferrocyanide of copper permeable to water and certain ions, but impermeable to the sugar enclosed within it, which produces in this artificial seed the strong osmotic pressure which determines its absorption and growth. If the liquid is spread over a glass plate, the growth takes place on the horizontal plane; if the culture is made in a deep basin, it takes place at once horizontally and vertically. One single artificial seed may give 15 to 20 stalks sometimes as high as $25 \mathrm{~cm}$."

At times osmotic absorption through the membrane proceeds to such an extent as compared with tension resistance of the membrane that rupture of the latter occurs. Then new healing membranes are formed, continuous with the old one, or minor buds arise, after the manner of budding yeast. But here we must take exception to Le Bon's commentary on Leduc's results, when he says: "We may think we have here the image of life; but there is hardly any more connection between these artificial plants and real ones than there is between a living man and his statue. Their production merely shows that osmotic equilibria may condition certain external forms." We would rather suggest, and we hope to show, that these are the necessary stages and phenomena that carry us from inorganic colloids with complex molecular composition, and a high degree of intra-molecular energy, to the varied series of organic colloids in which a still higher or biotic energy resides.

Thus the numerous colloidal osmotic growths that Leduc, following out and extending Traube's studies, has described and figured (23:193) are, as he truly remarks, structures, each of which is "completely analogous to that which we meet with in a living organism." Again he says, in summing up their characteristics, that each "osmotic growth has an erolutionary" existence; it is nourished by osmosis and intussusception; it exercises a selective choice on the substances offered to it; it changes the chemical constitution of its nutriment before assimilating it. Like a living thing it ejects into its enriromment the waste products of its function. Moreover, it grows and 
develops structures like those of living organisms, and it is sensitive to many exterior changes, which influence its form and derelopment. But these very phenomena-nutrition, assimilation, sensibility, growth, and organization-are generally asserted to be the sole characteristics of life."

Nay more, under the conditions that must have pertained during the early archæan epoch, such actions and reactions must often have occurred, in presence of the considerable colloid masses that must then have existed. But further the fact deserves to be emphasized that the transition from a crystalloid to a colloid body, or vice versa, is often rapid and continuous, as already indicated for iron and silicon colloids. This is equally true for "organic" compounds. Thus the crystalloid sugars can be changed quickly in plant cells into the colloid starch. Cellulose and inulin can be quickly transformed into sugars. The amides and even the albumen compounds can be resolved into crystalloid constituents.

But such considerations would form a feeble means of explaining the formation of complex plant and animal proteids, still less of protoplasm itself. We may, however, attempt to analyze primitive plant structures and activities into their apparently primary factors, so as thereby to reach the nearer to inorganic relations. So when we study the structure and life phenomena of such simple non-nucleate plants as Gloocapsa and Chroococcus, apart from the colloid cellulose wall, three fundamentally important but intimately cooperative constituents are recognized. First, a colorless colloidal synthetic protoplasm, which in virtue of its intra-molecular energy, and the contact relation between the energized molecules, can build up complex food substances into proteids allied to itself; second, a colorless metabolic colloidal constituent or set of constituents which can transform or split up formed foods into assimilable-and usually simpler-bodies, that are then utilized by the protoplasm; third, a green substance, chlorophyll, which can absorb sunlight, and, by its energy in presence of the protoplasm, can link up simple inorganic constituents - carbon and water-into the primary food constituents. 
Now the last of these, chlorophyll, can readily be separated from the first and second, but still exhibit all its properties, except that isolated from them it ceases food formation according to most, it still may continue its action according to Priestley and a few other observers. The second constituent seems to be by no means single or simple, but evidently consists of a series of colloid bodies held in equilibrium, but each capable of performing a certain metabolic or digestive action on food compounds. These are the zymogens and enzymes or ferments, and each of them can, as experiment has shown, perform its work as perfectly in a test tube as in a living cell. The first constituent - the most complex evidently in composition-is a colloid or more probably a set of colloids of great molecular complexity, and which, if we may judge by the highly complex bodies which it readily builds up, is traversed by and results from currents of energy of enormous power, condensation, and yet perfect quality. It is well also to note that the above three constituents act cooperatively in building up or changing the numerous and varied substances encountered in their tissues.

The important question then is-and for many decades has been: can we trace or construct fragments of a bridge that once connected the inorganic crystalloid and specially colloid bodies that we have postulated for the mid-archæan epoch, with those highly complex compounds of the lower plants indicated above? Or to express it more directly, as has often been done before: is there a possibly continuous and graded passage, from dead or inorganic to living or organic matter?

Leduc in his most suggestive work (23: 147) well says: "Considering the impossibility of defining the exact line of demarcation between animate and inanimate matter, it is astonishing to find so much stress laid on the supposed fundamental difference between vital and non-vital phenomena. There is in fact no sharp division, no precise limit where inanimate nature ends and life begins; the transition is gradual and insensible, for, just as a living organism is made of the same substances as the mineral world, so life is a composite 
of the same physical and chemical phenomena that we find in the rest of nature."

At the outset it must be candidly confessed that the stages are few and extremely imperfect, but they are greatly more abundant and helpful than in the days of "the evolutionary fathers."

A first requirement for the successful formation of the above organic constituents in steadily increasing complexity is some type or phase of energy that can so energize even the most complex inorganic crystalloid or colloid that it will continue to integrate or build up into more complex states. Without such, all aggregations of matter must have remained inert.

In other words, while we accept Leibnitz's view as to force or the exhibition of energy being the most fundamental and important phenomenon in the universe that alone explains the structure and actions of inorganic and organic bodies, we would emphasize, as a further necessity of the case, not only the existence of such phases of energy as we have hitherto associated with inorganic bodies, but further such phase or phases of energy, of more condensed quality, as will explain to us the capacity for linking together even the most complex colloid molecules of organic kind, such as the molecules of the gray brain substance of man.

It is well known that green plants absorb large amounts of lumic energy, but such is not directly used in splitting up the $\mathrm{CO}_{2}$ molecules, and in uniting the freed carbon with water constituents, for the heat evolved in the process would burn up the tissues. It is tacitly accepted that electric energy is the active agent in the work. In such a case, or even according to the view that some other type of energy is concerned, the chlorophyll may be regarded as an energy transformer. Evidence in favor of this will be advanced later.

If electric, chemic, lumic, or thermic energy, separately or together, have started life conditions, then it might seem at least likely that these energies could continue to build up numerous quaternary, quinary, and other more elaborate products, without life being necessarily attached to or a result 
of their formation. No evidence of this exists. The view already given for the simpler inorganic colloids, viz., that their molecules are invested by a double charge of electricity, a positive and a negative, suggests that new and different energizing conditions were evolved for their upbuilding. Meanwhile, therefore, we will accept it as a working hypothesis, to be expanded later, that transition from the inorganic colloid to the organic colloid body was gradually accompanied by the evolution of a new and more condensed phase or modification of energy, the "biotic."

Accepting such, we can next inquire as to the conditions - necessary for the advancing synthesis of colloid compounds. Four requirements, probably five, at least are called for. First, time, which is now readily conceded alike by physicists and geologists. Second, abundant formation and intermixture of many kinds of crystalloids and colloids of inorganic nature. Third, long continued action and reaction of these on each other, and amid favorable liquid media. Fourth, the gradual establishment between these - as being distinct yet intermixed and cooperative bodies - of a molecular equilibrium, which would only be upset when some appropriate extrinsic or intrinsic stimulus acted. Fifth, the capacity for multiplication and continued renewal of such equilibrated bodies.

Under the first caption it is agreed, alike from the thickness of the strata, their striking deformation and modification, as well as their relation to subsequent rock formations, that a period of 20 to 30 millions of years would be a moderate estimate for the duration of the mid-and late archæan periods.

Under the second caption it may suffice if we recall attention to the ample series of chemical compounds furnished by decomposition of granitic, of schistose, and of gneissic rocks. Also, if one consult such analyses as those given by the author (1.3: 255) for the waters of various geysers and hot springs, it becomes evident that these may not unfrequently form extensive colloid masses, if oxygenating agencies are at work.

Under the third requirement one obtains a vivid picture of the probable - the almost assured-terrestrial conditions over wide areas, by perusal of the accounts given by Weed. 
He says: "Regions of solfataric activity have always been of peculiar interest to scientific observers, not only on account of the curious and often extremely beautiful hot springs and the rarer occurrence of geysers in such districts, but also from the varied phenomena of rock decomposition and of mineral formation and deposition which always accompany such hydrothermal action. It is in these natural laboratories that we are permitted to see in operation processes which have produced important changes in the rocks of the earth's crust and afford a key to many of the problems of chemical geology.

"There is perhaps no other district in the world where hydrothermal action is as prominent or as extensive as it is in the Yellowstone National Park. In this area of about 3500 square miles over 3600 hot springs and 100 geysers have been visited and their features noted, and there are also almost innumerable steam vents."

As to general aspect and relation of the water, he says: "The water of the Mammoth Hot Springs is remarkably clear and transparent; the temperature varies at different springs from $80^{\circ} \mathrm{F}$. to $165^{\circ} \mathrm{F}$., exceeding $130^{\circ}$ in all the larger springs. While hot, it generally possesses a sulphurous odor, the intensity varying greatly at different springs, but always being strong if the temperature exceeds $140^{\circ}$, when sulphur is found incrusting the Algæ filaments growing near the vent of the spring. When cold, the water is not peculiar in taste, or in odor, but it is considered unfit for drinking, owing to the large amount of carbonate of lime which it holds in solution."

The comparative analyses given by him (on the opposite page) are instructive: (Constituents grouped in probable combination. Grammes per kilogram.)

Now, in spite of the widespread havoc to organisms that volcanic and hot-spring activity often causes, we would still insist that it is to regions that chemico-physically were similar to water areas located in the midst of such hot-springs that we have to look for organismal origin. In their waters all or most of the elements are present that enter into organic composition; these elements show marked tendencies to colloid organization; in.these waters also interaction of the different types of colloid would have freest play.

Most of the springs were bordered extensively with colloid siliceous deposits or "sinter." But in the northwestern corner of the park the "Mammoth Hot Springs" were purely cal- 


\begin{tabular}{|c|c|c|c|c|c|c|}
\hline & $\begin{array}{l}\text { Asta } \\
\text { Spring }\end{array}$ & $\begin{array}{l}\text { Splendid } \\
\text { Geyser }\end{array}$ & $\begin{array}{l}\text { Grand } \\
\text { Geyser }\end{array}$ & \begin{tabular}{c|} 
Old \\
Faithful \\
Geyser
\end{tabular} & $\begin{array}{l}\text { Great } \\
\text { Geyser, } \\
\text { Iceland }\end{array}$ & $\begin{array}{c}\text { White } \\
\text { Terrace } \\
\text { Geyser, } \\
\text { N: Zealand }\end{array}$ \\
\hline 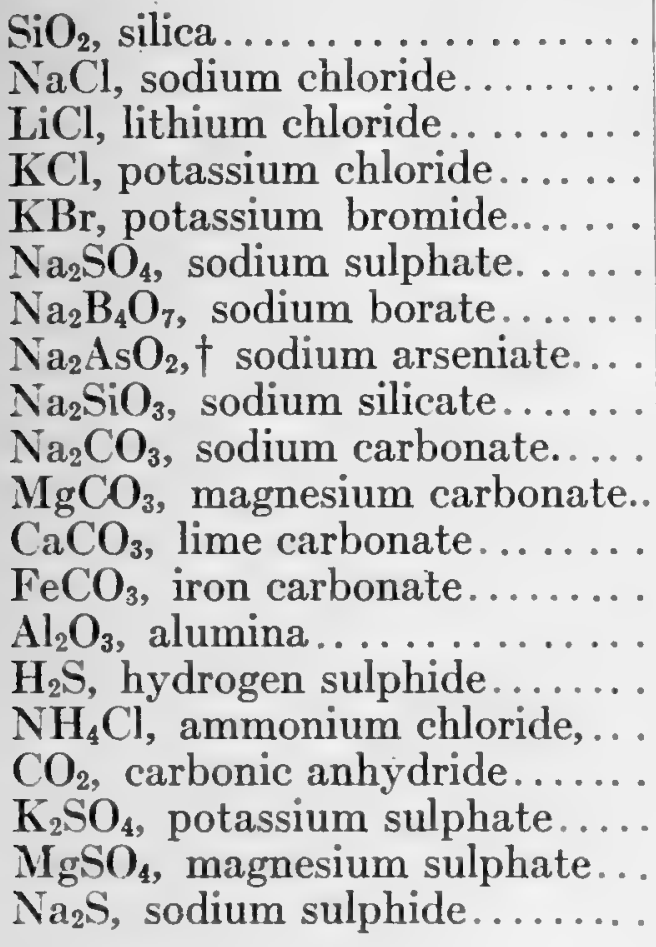 & $\begin{array}{l}.1463 \\
.0035 \\
.0295 \\
.0112 \\
.1045\end{array}$ & $\begin{array}{l}.2964 \\
.4940 \\
.0140 \\
.0231 \\
\\
.0281 \\
.0335 \\
.0025 \\
.5286 \\
.0018 \\
.0075 \\
.0001 \\
.0051 \\
.0002 \\
.1989\end{array}$ & $\begin{array}{c}.3035 \\
.5643 \\
.0218 \\
.0319 \\
\text { Trace } \\
.0387 \\
.0350 \\
.0014 \\
\\
.3209 \\
\text { none } \\
.0070 \\
\\
.0061 \\
\text { Trace } \\
.0012 \\
.0587\end{array}$ & $\begin{array}{l}.3961 \\
.6393 \\
.0340 \\
.0478 \\
.0051 \\
.0270 \\
.0213 \\
.0027 \\
.0279 \\
.2088 \\
.0021 \\
.0038 \\
\text { Trace } \\
.0017 \\
.0002 \\
\text { Trace }\end{array}$ & $\begin{array}{l}.0180 \\
.0091 \\
.0088\end{array}$ & $\begin{array}{l}.2290 \ddagger \\
\text { Trace } \\
.025 \\
.005\end{array}$ \\
\hline Total. & .6764 & 1.6340 & 1.3905 & 1.3908 & 1.2305 & 2.6570 \\
\hline Specific gravity.. & & 1.00132 & $\mid 1.00108$ & $|1.00096|$ & $|1.000205|$ & 1.00077 \\
\hline
\end{tabular}

${ }^{*} \mathrm{CaCl}_{2}$.

$\ddagger \mathrm{Na} 2 \mathrm{O}$.

$\uparrow \mathrm{Na}_{2} \mathrm{HAsO}_{4}$ probably is intended here.

careous in relation, and deposited extensive beds of colloidal "travertine."

All of them had simple cellular plants or protophytes actively growing in, and forming, the colloidal deposits, in similar manner to that first studied and elucidated by Cohn for the Carlsbad mineral springs. As explained in the next chapter the protophytic organisms, that cause chemical precipitation of the deposits, live at temperatures of $50^{\circ} \mathrm{C}$. to $85^{\circ} \mathrm{C}$., and seem, from world-wide evidence, to be most active in depositing the sinter or travertine between these limits.

Though such regions are now restricted to comparatively limited areas of the earth's crust, their occurrence every here and there over every one of the continents is significant. We believe we do not overstate it if from direct geological 
evidence we suggest that in the mid-archæan epoch such regional conditions must have been very frequent, and that a large part of the then dry-land formed an extensive "Yellowstone" picture.

This picture suggests a world-wide natural biochemical laboratory, in which synthetic and analytic activity unceasingly went on for at least 25 to 30 millions of years probably. And with what result? This leads us to the fourth requirement already stated, the gradual formation and establishment, between distinct yet intermixed and cooperating chemical bodies, of a molecular equilibrium.

Here, it must be acknowledged, our experimental data are sadly deficient, but fresh and valuable contributions are daily being made that help not a little. If we compare the actions and reactions of the most complex inorganic crystalloids and colloids yet studied with the behavior of living self-perpetuating substance, the following features impress one:

1st. The former bodies are largely uniform, simple, and direct in chemical reaction; the latter are varied, complex, and indirect, or we might rather say complicated and overlapping in chemical reactions.

and. The former show their energizing activity by integrating merely molecules that resemble and add to their own substance; the latter not only effect this, they are able to synthesize or to analyze bodies diverse from themselves.

3rd. The former only show the colloid phenomena of reversible or irreversible molecular action to a limited degree; the latter exhibit this in practically indefinite manner, and are self-regulatory in securing discharge of superfluous products by the respiratory process.

4th. The former at any one moment show only catalytic or analytic activity; the latter may simultaneously show both in action.

It would be impossible here to take up these differences in detail. In some cases, as will be pointed out below, the differences are not invariable or in all cases fundamental. For information along these lines one can consult the valuable 
works of Czapek (24), Höber (25), and Duclaux (26). But the labors of biochemists of the past quarter century have been so fruitful that we have little hesitation in predicting the gradual removal of all those distinctions, fundamental though they may now appear.

We will shortly touch on some of the evidences which incline us to make the assertion. It is little more than half a century since the chemist had to confess that no primary organic compound-even the simplest, such as sugar-had been continuously and synthetically built up from purely inorganic bodies and by expenditure of thermic, chemic, electric, or other form of energy.

But when Wöhler in 1828 synthesized urea; Kolbe, in 1843, obtained tetrachloride of carbon $\left(\mathrm{CCl}_{4}\right) ;$ Melsens, in 1845 , methane $\left(\mathrm{CH}_{4}\right)$; Kolbe, in 1845 , bichloride of carbon $\left(\mathrm{C}_{2} \mathrm{Cl}_{4}\right)$; Melsens, in 184t, acetic acid $\left(\mathrm{C}_{2} \mathrm{H}_{4} \mathrm{O}_{2}\right)$; Kolbe, in 1849, ethane $\left(\mathrm{C}_{2} \mathrm{H}_{6}\right)$; and then other investigators obtained acetone, chloroform, formic acid, ethylene, and oxalic acid, the barrier between inorganic and organic bodies was remored, and the way was paved for future biochemical study of all living compounds.

When further, from 1860 onward, indigo, sugars, alizarine, caffeine, theobromine, and other valuable "organic" products had been synthesized, such formed a welcome advance from the chemical side, that ran parallel with the simultaneous acceptance of evolution, as the principle of cosmic as well as biologic change.

It is well however here, at once, to confess and to emphasize that the above are all crystalloid bodies, and that while Fischer and his coworkers have synthesized colloids even of complex composition, this has been by using simpler organic compounds in the process.

Thus E. Fischer's synthesis of soluble polypeptides, similar to peptones, by the union of complex molecules of several amino-acids represents perhaps the most advanced step in organic synthesis. But the complicated methods employed, and the relative complexity of the amino-acids from which the initial start was made, prove how different are artificial 
and natural syntheses of organic compounds, from the energizing standpoint.

In view, nevertheless, of accomplished results it seems almost assured that investigation will gradually solve for us the problems: (1) what energy transformer or transformers have been evolved, for use in the evolving cell; (2) what equilibrated combination of diverse colloid molecules can regenerate or sustain themselves and also act as synthesizers of added food; (3) what colloid bodies can effect transformation or digestion of food materials without themselves undergoing great decompositions?

To each of these we can give as yet only very imperfect reply. But a partial-even if slight-answer may be got by study of some simple living organisms, and to these we shall now turn.

Did we think only of the mode of nutrition pursued at the present day by green plants and by animals, it might be considered that all organisms have originated from a monophyletic ancestry, in which by formation of a primitive and later of a specialized pigment, chlorophyll, the earlier process of food formation took place, through elaboration of the simpler carbohydrates, such as sugar. But results of the past quarter century have fundamentally modified our conceptions.

Amongst the Acaryophyta (p. 51) or Protobiota at present existing, those forms which have been termed the sulphur bacteria, the nitrogen bacteria, and the iron bacteria seem all to derive food products or stores of energy, not through the intervention of chlorophyll, but by direct utilization of constituents of various inorganic salts dissolved in water. In other words, all evidence points to the conclusion that they can form complex organic molecules from simple inorganic salts in solution, and that the energy needed for the life of each organism is obtained from some salt absorbed. Furthermore, it is highly important to note that such work can be performed perfectly, as by Beggiatoa, in the dark, since it utilizes not sunlight but sulphur compounds as an internal source of energy.

True, this still leaves us far removed from an exact knowledge as to how inorganic or-if we may use such an expression- 
semi-organic molecules can continuously absorb all constituents needed, and utilize these, for their orn increase and for energy expenditures. But in the behavior alike of emulsoid to suspensoid colloids ( $p .17$ ), and of different emulsoids in being coagulated by neutral salts, and again undergoing reversible solution in pure water (19: 229), we have examples, made up of many known cases of colloid interactions, which, though relatively simple, all point to wide possible variations in action and reaction, as well as of solution growth.

But Macallum (27: 436) has well observed that, in seeking "to explain the origin of life, we do not require to postulate a highly complex organism such as we can see even with the low power of the microscope, as being the primal parent of all, but rather one which consists of a few molecules only and of such a size that it is beyond the limits of vision with the highest powers of the microscope. Such an organism would be the smallest unit of life and it might be supposed that protoplasm arose from aggregation of such units, each more or less differentiated from its fellows, just as the higher or multicellular forms of life have arisen by aggregation of cells which have differentiated more or less, thus giving rise to differences of function in the different parts."

But some of the osmotic growths described by Leduc are as striking as they are indicative in character, for they demonstrate a capacity for absorption, for growth, and for formmodification that only finds a near parallel in cellular plants. Thus if a fragment of $\mathrm{CaCl}_{2}$ be placed in a flat glass vessel that contains a solution of potassium carbonate ( 76 parts), sodium sulphate (20 parts), and tribasic potassium phosphate ( 4 parts): "the calcium chloride surrounds itself with an osmotic membrane; water penetrates into the interior of the cell thus formed, and a beautiful transparent spherical cell is the result, the summit of which soon emerges from the shallow liquid. 'The cell continues to increase by absorption of the liquid at its base, and may grow up out of the liquid into the air for as much as one or two centimeters.

"This is a most impressive spectacle, an osmotic production, half aquatic and half aerial, absorbing water and salts by its 
base, and losing water and volatile products by evaporation from its summit, while at the same time it absorbs and dissolves the gases of the atmosphere." So Leduc further adds: "Like a living being, an osmotic growth absorbs nutriment from the medium in which it grows, and this nutriment it assimilates and organizes."

With such phenomena before us it will be conceded as likely that our knowledge of primitive organismal evolution will best be advanced by study of those forms of life which consist of simple and mostly non-nucleate cells. Further, if we learn that these exhibit rather varied or even distinct modes of vegetative existence, that conduce to perfect support and multiplication of the organisms, this will afford proof that not one, but several and varied, modes of regetative life-action were primitively evolved. Thanks to the researches of Winogradsky, of Nathansohn, of Beijerinck and others, we now know that such truly proceeded.

Some of the sulphur bacteria are quite colorless, or only slightly yellow from presence of glistening sulphur granules in the tissue. As already stated, they can live in the dark, while their supplies of energy are got from sulphur compounds. The mode of nutrition of some of these may well be said to confer on them the title of prototrophic, as suggested by $\mathrm{A}$. Fischer (39). Free hydrogen must often have been generated during volcanic changes in the archæan period. This at temperatures of $250^{\circ}$ to $300^{\circ} \mathrm{C}$. could unite with free sulphur or with sulphide salts to form hydrogen sulphide, $\mathrm{H}_{2} \mathrm{~S}$. The bacteria then absorb this into their colloid protoplasmic substance, and there oxidize it, setting free the sulphur in the protoplasm as minute yellowish granules visible to the eye. In the act of union of the hydrogen and oxygen, heat energy is evolved. At the present day the earlier stages of the process are started by the liberation of nascent hydrogen gas from decomposing plants. This can readily unite with sulphate salts, and by reduction action set free the hydrogen sulphide.

So it may well be that we have, in existing sulphur bacteria like Beggiatoa, Chromatium, and Thiothrix, surviving types 
of very ancient forms that originated amid the ceaseless physico-chemical changes of the archæan epoch. They seem, however, to have remained a distinct group and to have given rise to very limited lines of evolutionary advance.

Again, in the nitrogen bacteria still existing, we have types which are capable of fixing loose inorganic nitrogenous compounds even in the dark. In presence of such a salt as carbonate of ammonia the organism oxidizes the nitrogen constituent and in the process sets free energy, which is utilized in splitting up the carbon dioxide molecule, the nascent carbon then uniting with other constituents to form elaborate food products. But, in spite of the detailed and fruitful studies of Winogradsky along this line, we are only at the beginning of what will in time constitute important results. These organisms, however, seem to represent a second group that have failed to evolve complex organic types. But the possibility of their having primitively been colorless forms, which by slow degrees elaborated pigments that might erolve into yellow, yellow-purple, purple-green, blue-green, and green energyabsorbers or transformers, has to be kept in view. This view is favored by all we know at present of the succeeding series. Further, it seems highly probable that from primitive members of this group ancestral animal forms may have been derived.

A considerable group of rod-shaped bacteria of pink, pinkpurple, and purple color has been studied during the past thirty years with great care. These are mainly found, often in great abundance, in marshy or brackish expanses, and at times, as the writer has observed, coloring wide expanses of mud-flats. Engelmann has shown that they can absorb and utilize the dark heat or infra-red rays, and also to a minor degree the yellow rays, through the presence of the contained pigment. These rays are then utilized for the upbuilding of complex food molecules, but whether the pigment definitely acts as an energy-transformer is still undecided.

Now, at the present day, in all of the important geyser regions the beautiful color of the water and of the siliceous colloid deposits is observed to vary from almost white or pale yellow 
that is associated with organisms living in the warmest waters $\left(60-75^{\circ}\right.$ C. $)$, through pink and pink-purple toward browngreen, blue-green, and olive-green; the last being formed in waters only slightly, if at all, above maximal land-plant temperatures $\left(35-40^{\circ} \mathrm{C}\right.$.). These species are all ranked as Bluegreen Algæ, Protophyceæ, or Cyanophyceæ. Since it has been shown also that the purple bacteria, like green plants, set free oxygen during daylight, it may be that these are the forms which yield us the stages in gradual elaboration of plant chlorophyll, a purely chemical substance, but by whose activity alone green plants are able to decompose carbon dioxide and to so link up the carbon with elements of water $\left(\mathrm{H}_{2} \mathrm{O}\right)$ as to form therefrom sugar, the fundamental "organic" crystalloid, that is the first step in, and the starting point for, elaboration of all higher plant foods.

According to the above view, then, we might consider all green or chlorophylloid plants to have originated from primitive colorless cells, evolved by slow degrees during the archæan epoch. These, like the nitrogen bacteria, may at first have been colorless, and simultaneously utilized nitrogen compounds in the process of protein elaboration, as well as nascent carbon for formation of carbohydrates like sugar. By increasingly perfected development of a pigment through yellow, pink, purple, purple-blue, and blue-green stages a definite green substance - chlorophyll-eventually became so important an energizing material that the first steps in linking up of inorganic into organic compounds was effected wholly through its activity. So may have originated the great monophyletic group that culminated in the abundant ramifications of the dominant or green plant series.

But a fourth group of primitive plants now seems to survive in localities like those favored by the purple bacteria. The iron bacteria of the present day form a distinct physiological series, in that they obtain their supplies of energy by utilizing and oxidizing iron salts. As already stated (p. 16) the bogiron ores of nearly every stratified rock group probably had their origin in connection with these iron bacteria. But it 
should be borne in mind that iron is an essential element for the formation of chlorophyll, though probably it acts there rather as a conductor of energy, or as an aid in energy transformation. It is even possible that the purple and iron bacteria had an origin in common, and early evolved along diverging lines, the former becoming in time the important and advancing group, morphologically and physiologically.

A comparison of the four groups now reviewed would suggest that living substance very gradually originated amid chemically acting and reacting inorganic compounds in aquatic or subaquatic situations, and where colloid as well as crystalloid bodies tended to arise through environal agency. From longcontinued interaction of emulsoid and suspensoid types of colloid compound, a chemically sensitive and yet stable mixture of several colloids resulted, that we now call protoplasm, which as in Traube's cells threw off an investing wall. The most important activities of this mixture were surface chemical interactions with surrounding liquid molecules; in time a utilization of certain definite salts of these liquids, in part as a constant source of constitutive or intra-molecular energy, and in part as material for replacing broken down particles. Of the four possible modes of accomplishing these two ends, the one in which a definite compound became evolved by stages to constitute chlorophyll proved physico-chemically the most continuous, efficient, and beneficial in its relation. This then became gradually selected and dominant among the colloid masses or organisms that were to carry forward advancing molecular aggregation, namely as green or chlorophylloid plants.

If we are to accept the concurrent views of physicists and chemists, which call for a constantly increasing expenditure of energy for the linking together of increasingly complex molecules, then, for effecting such molecular combinations as the formation of sugar, chlorophyll, or proteids, an enormously high expenditure of thermo-electric energy, or a moderate expenditure of some even more perfect and condensed energy, seems absolutely called for as a physico-chemical necessity. 
Such considerations as the above cause us now to close the present chapter with a comparison of inorganic energies in relation to the five great physiological activities that have generally been looked on as typical of and peculiar to organisms. These five are irritability, nutrition, respiration, growth, and reproduction. If direct evolutionary continuity has existed in world processes, from the simplest to the most complex combinations of energy and ether, these terms must either have an arbitrary and artificial limitation, or-if our knowledge untrammeled by our hereditary prejudices enables us-they should be extended to include a wider range of phenomena than has hitherto been accepted. The latter seems to be the more accurate attitude to assume, and we shall now try to ascertain how far and how appropriately such terms can be applied to phenomena of the inorganic world.

Here the writer may be pardoned if he suggests that the treatment of organic activity in many current physiological text-books is by no means helpful to a consistent and consecutive study of the subject. Only when organic bodies are viewed from the five successively ascending phases above indicated do we secure a concrete picture of physiological continuity.

Irritability has usually been accepted as a term peculiar to the biologist's province. Without attempting to give or to discuss the many current definitions of it, that which seems alone to cover all conditions in the organic realm would be "molecular response to environal stimuli, that may be visible or invisible to the naked eye." Now we do not need to adduce endless instances to show that all material bodies conform to such a principle. We would rather say that if we attempt to limit the term to living bodies, with our increasing knowledge of physical chemistry, we are constantly landed in unending inconsistencies, as we hope to show in later chapters. We are compelled to accept it that living and non-living bodies are alike irritable - the chemist says that they act and react-and that the range of it may be from the infinitesimally sluggish response by a crystal of silica locked up for millions of years in some igneous rock, up to the delicate and rapid response of 
a saturated or electrolyzed solution, the movements of a Mimosa leaf, or the contraction of an animal muscle.

Verworn has equally realized the necessity for such an extension of the term in the realm of animal physiology. For in his lectures on "Irritability" (p. 1) he says: "Irritability is a general property of living substance but not exclusively so. Irritable systems also exist in inanimate nature. What characterizes living substances is not irritability as such, but an irritability of a specific type."

The consideration of Nutrition and Growth presents a more complicated problem. But what would have seemed absolutely discontinuous processes before Graham's discoveries are steadily becoming steps in a consecutive chain of events. For, while crystalloids show no phenomenon comparable to organic nutrition in adding to their substance, the group of colloids presents some remarkable and exact similarities. Thus inorganic and organic colloids alike consist of complex molecules of varying size, but each surrounded by a jacket of water. Both show a high degree of mobility and elasticity, according to the amount of water present at any moment. Both show capacity for formation of membrane pellicles round enclosed contents-either crystalloid or colloid-of more or less active osmotic capacity. Both show elastic expansion of, and capacity for, forming additions to such membranes, through continued absorption from surrounding liquids of substances that add to the amount of the enclosed materials. Other homologies might be mentioned, but for present purposes such would be superfluous. We therefore consider with Leduc (p. s9) that exact cases of nutritional and growth continuity have been established in both. So to speak of the nutrition and growth of some inorganic colloid mixtures or unions is as appropriate as if we spoke of those in a yeast cell or an amœeba.

Respiration is an unceasing and important phenomenon of organic types that seems, with our present knowledge, scarcely if at all referable to inorganic bodies. If the student of colloid chemistry could demonstrate that in growths like 'Traube's cells, or in mixtures of several nearly equilibrated bodies, a 
steady interchange of two constituents or groups of them took place, one beneficial to the mass and absorbable by it, another waste and disintegrating in action, and that was steadily removed from it, then added proof would have been secured of physico-chemical and biochemical continuity. The nearest approach to such is the experimental result described by Leduc, who says that calcium chloride growing in a solution of potassium carbonate is transformed as follows into calcium carbonate, $\mathrm{CaCl}_{2}+\mathrm{K}_{2} \mathrm{CO}_{3}=\mathrm{CaCO}_{3}+2 \mathrm{KCl}$. Thus an osmotic growth can make a choice between the substances offered to it, rejecting the potassium of the nutrient liquid, and absorbing water and the radical $\mathrm{CO}_{3}$, while at the same time it eliminates and excretes chlorine, which may be found in the nutrient liquid after the reaction. So far as we are aware, the above is the nearest proof that has even been suggested. Respiration, therefore, is one of the partial barriers between inorganic and organic bodies that has yet to be removed, or more perfectly explained, in its true significance.

The terms reproduction and multiplication have been used in so wide and varied a sense for organisms that, for purposes of comparison with the inorganic bodies, use of the term must be restricted and its meaning defined. Since sexual differentiation and reproduction are entirely absent, at least in the simpler Acaryota, and since these are devoid of a nuclear mechanism that is evidently a necessary constituent of sexual reproduction, we do not take account of it at present. Of asexual multiplication the varieties that we call division, budding, and endogenous formation may either or all have been primitive, though the first has become the successfully surviving type. But, from the inorganic side, the changes that may occur in Traube's cells are most instructive. That experimenter showed that, if continued inbibition and swelling of a walled-in colloid mass proceeded, a stage was reached where rupture of the wall occurred, but new and minor masses resulted therefrom, which started to grow and to absorb surrounding materials. Leduc has added to and extended these results (23: 124). 
If then we are to be logically and scientifically fair and consistent in our use of terms, irritability, nutrition, growth, and reproduction are all such as should be made to include continuous and related phenomena from the inorganic to the organic stage of cosmic evolution. As yet respiration alone seems to be largely typical of organisms, and is correlated with the highly complex, self-adjustive, and equilibrated colloids that make up these bodies.

With all the evidence-physical, chemical, geological, and biological - that we now possess of archæan conditions, and of plastic evolving continuity from the inorganic to the organic phase, we would reiterate the already expressed opinion of not a few scientists, that living bodies have arisen in continuous and graded fashion from an inorganic stage, while the same laws that govern inorganic bodies are directly applicable to the organic, except that the latter show more perfect exhibitions of some kind of energy, and probably in connection therewith exhibit conditions of environal interchange or respiration that have not yet or only imperfectly been demonstrated for the inorganic. 


\section{CHAPTER III}

\section{THE RELATION OF INORGANIC TO ORGANIC BODIES}

In past discussions on the relations between the inorganic and organic kingdoms, and the possible mode of origin of life on the earth, one of five possible methods of treatment has generally been adopted by writers. Special creationists of bygone centuries considered that an impassable barrier or gap separated the two, and so they regarded the phenomena of life as a mystery that must forever be beyond human explanation, since successive organic bodies appeared on the earth by separate creative acts.

Others, in tracing minutely the erolution of living forms, were impressed by the apparent absence of eren approximate continuity between inorganic and organic bodies. So they did not attempt to push their inquiry into an apparently fruitless field. Again, while impressed by the same lack of continuity, others tried to apply a semi-uniformitarian method, by suggesting that primitive life was imported into our world from some other member of the solar system.

Still others, after consideration of recent riews on the structure, composition, and mode of growth of inorganic bodies, had little hesitancy in passing by a very abrupt and extended step from the more complex inorganic compounds to nucleated masses of protoplasm. For to them it seemed impossible that any break in uniformitarian continuity should exist, even though the recognized gap was a serious one. Finally, others have patiently tried to elucidate, step by step, the possible transitions from inorganic to organic evolution, by aid of every bit of direct or collateral evidence that might aid them. Even though compelled at times to imitate the second last group of inquirers, we shall strive to follow the methods pursued by the last. 
But it must be acknowledged-even demanded-in such an inquiry that immense gaps are to be expected in our accepted record of evolving continuity. If we grant for present purposes-and no one probably will deny it-that insensibly graded and advancing types once connected the Algæ with the Characeæ, and equally with the Bryophyta or moss alliance; also that some higher type of alga, or possibly primitive type of bryophyte, united these with primitive members of the Pteridophyta or fern alliance, it will at once be evident that whole families, of connecting organization, have been entirely swept out of existence, or have been preserved to us very rarely in fossil form, owing to a fortunate combination of circumstances.

If such be true for these higher and often resisting groups, it must be immensely more so for primitive organizing bodies, and for all the earlier evolving plants and animals that were composed wholly or largely of soft perishable tissues. These, moreover, from their first organization up to the present day have been exposed to the tremendous and often widespread destructive environal changes that have blotted out whole cohorts of comparatively recent plants and animals. If, therefore, the continuity relation be often faintly and imperfectly traceable, such is entirely appropriate to the nature of the case.

In the previous chapter it was shown that a stage was reached in the world's history when environal conditions were suited to the formation and continued erolution of organisms. 'The specific stimulating environal areas we considered to be geyser basins, hot springs, warm shallow fresh water lagoous, and brackish marshes. The - on first thought-somewhat attractive view has often been expressed that still ocean depths were the favorable regions. But slight reflection will persuade every one that it was not there, where stagnation, dreary uniformity of inorganic compounds, want of raried and interchanging energies, as well as absence of light, prevailed. Rather in those areas above stated, where exactly opposite conditions were typical, the combination of circumstances 
existed that must have been suited-even were requisite-for such beginnings.

Though only fragmentary indications of the pathway pursued remain to us, these we believe plainly show that colloid molecules, of increasing complexity and diverse composition, aggregated into equilibrated molecules of high mobility, but extreme stability and tenacity of union. These, during transition from the inorganic to the organic state, differentiated primarily in each mass into three inter-related lines of molecular complexity. First into molecular aggregations which, by long continued contact action and interaction, alike renewed its own substance and effected upbuilding of diverse intrinsic chemical products - both crystalloid and colloid - that together constituted the substance protoplasm, and its accessory products. Second, other colloid aggregations arose that are now identical with, or related to, nucleo-proteins, and which, by analytic contact action, altered or split up elaborated colloids and crystalloids, thereby setting free food products and energizing compounds for use of the organism. Third, that a pigment slowly evolved from, but remained in contact with, the protoplasm, which probably passed through successive color changes and elaborating advances, from pale yellow or yellow-purple through orange and orange-green to the stable green substance chlorophyll, that is now a heritage of nearly all higher plants.

Such molecular aggregations would, from exposure for millions of years to varying and often rapidly changing environal conditions of liquid tension and temperature, of gaseous discharges, of liquid chemical stimulation and other modifications, undergo very distinct and diverse modification as to shape, size, consistence, and relation to surrounding media. So reaction to such environal actions would give rise in the most primitive cases to spherical mass-aggregations like Synechocystis and Glococapsa, or again into oval or elliptic bodies like Aphanothece or Gloeothece, or to such bodies in aggregate colloid gelatinous masses as Aphanocapsa, to loosely united individuals as in Onchobyrsa, or into elongate thread forms as in Beggiatoa, Oscillatoria, Crenothrix, etc. 
A great variety of such, that present many modifications in shape, size, structure, and consistence, all survive with us in the Acaryota.* They may be said to consist of a group of colorless or yellow or purplish plants, the Protomycetes, Schizomycetes or Bacteria; or of species referable to a pigmented group of plants, the Protophyceæ, Schizophyceæ, or Cyanophyceæ; and to a colorless assemblage of forms that are usually regarded as primitive animals which we will term the Acaryozoa.

Now, if any or most of these are direct, and possibly little altered, descendants from archæan types, we should expect them to show-greatly more than do the more evolved typesrelatively strong resisting capacity to high and low temperatures; to changing environal liquid media; to drying and dessicating agencies; to light, heat, and chemical stimuli. They should also, in their apparently primitive species, exhibit a simple cell structure that advances by degrees to increasingly complex examples. The Acaryota as a living group exactly fulfill these requirements, and so we will now look into their comparative morphology, physiology, ecology, and taxonomy.

Though, as suggested in the last chapter, certain colorless types may be most primitive in structure, it will be convenient for us here to begin with the Protophyceæ, or popularly the

* Without desiring to invent or multiply names or systems of classification, the writer considers that some simple table is needed to set forth the relationships suggested in this rolume. He therefore puts forward the following for approval:

\section{ACARYOTA}

(Primitive non-nucleate organisms)

Acartophyta (Protobiota)

(Primitive plants)

1. Protomycetes

(Colorless non-nucleate plants)

2. Protophyceae

(Blue-green non-nucleate plants)
ACARYOZOA

(Primitive animals)

1. Proteomyxa

CARYOTA

(Nucleate organisms)

Caryophyta

(Nucleate plants)

1. Thallophyta

2. Bryophyta, etc.
Caryozoa

(Nucleate animals)
1. Protozoa

2. Metazoa 
Blue-green Algæ. The writer would here refer the reader who desires greater detail to such monographic accounts as those of Zopf (29), Hansgirg (30), Gomont (31), West (32), and Tilden (33), specially also to the excellent condensed description by Kirchner in "Pflanzenfamilien" (34).

In structure each living cell is bounded by a mucilaginous mall of material related to cellulose, as well as in some cases an additional film related to cutin. Both of these, according to Hegler, can resist chemical agents much as cutin does. Within is a protoplasmic mass, that shows under $1000^{\circ}$ magnification a finely granular, but doubtless profoundly complex, hereditary structure, that faithfully hands down exact molecular details to succeeding derivative cells. The outer spherical or cylindric zone of this protoplasm usually has a semifoamy, semireticular aspect, and this has the bluish green, brownish green, or reddish green pigment distributed through its substance. Hence the entire outer zone has been called the chromatophore. It is of interest to observe-alike in itself and in its connection with animal cells-that this often includes small masses of glycogen, which seem to be formed in the zone as a carbohydrate food. Within, the clear protoplasm in some cases fills the central part of the cell, but in the multicellular and so more erolved thread-forms clear granules begin to appear. These assume rarying degrees of union and complexity, until a loose open netrork or skein of chromatin is formed (35: 296) which eridently is the evolving nucleus that appears in perfected state in all the Caryota. Further, the protoplasm of neighboring cells is often connected by delicate tags that pass through the partition walls.

While our physiological knowledge of the group is still too imperfect to permit of important conclusions, Kützing (36:181) has shown that morements toward or away from heat and light are made by some, that suggest a sluggish response to thermic and lumic stimuli.

But a wide field exists for comparative physiological investigation of the degree and rapidity of response to environal stimuli, shown by the simplest and the most complex blue- 
green algae. Its importance as a field of study appears when we remember that it is amongst these, rather than amongst the nucleate green plants, that definite and invariable response to environal stimuli first became a permanent phenomenon and hereditary condition of each species.

Ecologically the group is of exceptional interest, and merits much investigation in the near future. As to temperature range about forty of the species (13: 253) might appropriately be called "hot spring Algæ" or "thermophilic Algæ." Thus while Brener overestimated in giving $94.5^{\circ} \mathrm{C}$. as the temperature of the water in which some grew, Setchell (37), Tilden, and others have carefully shown that it runs up to $75^{\circ}$ or $76^{\circ} \mathrm{C}$. in the Western American geyser regions. At least half of the above number of species are found habitually and normally in siliceous and carbonated hot springs, at temperatures of $45-65^{\circ} \mathrm{C}$. As Cohn, Weed, and others have proved, they not only form an "abundant regetation in gelatinous (colloid) silica and carbonate of lime, they precipitate these by chemical action and deposit extensive tracts of these minerals."

As already pointed out (p. 27 ) it is extremely probable that extensive rock masses, which greatly resemble these sinter and travertine minerals, and which are met with in all the great rock formations back to the archæan period, had their origin in like manner. It seems, therefore, justifiable to conclude that if forty or more living species of Protophycer, now left to us, can thrive and multiply abundantly at temperatures from $50^{\circ}$ to $75^{\circ} \mathrm{C}$., many primitive protoplasmic structures or species must have had an equally high resisting capacity, and may even have formed a temporarily predominant type of vegetation, over extensive thermic areas of the world.

Equally true is it that at least some resist low temperatures well. For while Kuitzing states that the cold waters of high mountains are not suited for their growth, it is true that in situations where they are often exposed during winter to at least $-10^{\circ} \mathrm{C}$., as on rock faces and amongst boulders of hill regions in the United States and in Switzerland examined by the writer, they still multiply and persist. 
Some show great capacity for resisting dessication at high temperatures. But, from the standpoint of physico-chemical relations to environment, they along with species of the Schizomycetes are unique, in that they can grow and abundantly multiply amid siliceous, calcareous, ferruginous, and sulphurous waters where other organisms are rare or entirely absent.

But the natural habitats of most existing species also indicate extreme adaptibility. Thus the writer has calculated that about 524 are purely fresh water or soil and rock growers, 24 live in brackish rater, and 105 species in salt water.* Many of the genera, like Calothrix and Rivularia, include species that may be fresh water or sea dwellers: some species of Dermocarpa are epiphytic on sea weeds, while others are normally symbiotic on higher plants. In the genus Nostoc, one species, Y. punctiforme, regularly inhabits, and forms a bluish green zone, in roots of Cycadaceæ or Sago Palms, an ancient group of flowering plants, whose genera are at present distributed over the globe. The writer and his students have studied eight of the genera that now make up the group. These came from Australia, Japan, China, India, South Africa, Florida, Central and South America, and the Pacific. In all of them the Nostoc organism was abundant. An allied species $N$. sphoricum is equally widely distributed, and is associated with genera of the Scale Mosses or Hepaticæ.

Again in Azolla, an aquatic ally of the ferns, Strasburger has traced the Blue-green Alga, Anabcena azolloe, in leaves from specimens native to America, Africa, Asia, and Australia. Such facts, like all of those above given, conspire to stamp the group as of remote antiquity.

Excepting some of the Schizomycetes or Bacteria, no other living group presents such evidence of primitive origin or of graded and evolving modifications up to a certain stage. The thermal-spring forms we regard as descended from and still representative of archæan ancestors, while the other species

\footnotetext{
* This reckoning has been made on the basis of Hansgirg's, Kirchner's, and Tilden's works. Gomont reduces the number of species considerably, but the ratio would be about the same for his species.
} 
in their extreme adaptability have undergone slow subsequent modifications to suit changing terrestrial environal conditions.

It must be admitted that, between even the simplest of these and the most perfectly adjusted colloid substances or mixtures that we know, the difference is great. But this difference we can only regard as one of degree, for nearly all of the physico-chemical and physiological peculiarities are shared in common, though to varying extent in different bodies.

The Schizomycetes, or in general language Bacteria, show many resemblances to the last group, but are devoid of bluegreen pigment. As already indicated (p. 40) good reasons can be adduced for considering that they may have evolved along at least two or three separate lines by distinct methods of energy utilization. In contrast to the fairly large size of schizophyceous species, the present are nearly all small, though Hillhousia mirabilis, first described in 1909 by West and Griffiths (Proc. Roy. Soc. 81: 398. 1909), almost rivals the algoid species. Much can be said in favor of the view that the more primitive types of animal branched off from them, and such is accepted as likely in this work. Most animal forms, however, have assumed a more active and rapidly multiplying mode of life than is seen in the former group, and so the formation of mucilaginous walls or masses of colloid jelly is rarer. But further, except for the purple pigmented cylinder of the purple bacteria, each cell in the Schizomycetes is devoid of the chromatophore of the Schizophycex. The protoplasm is either dense, finely granular, and fills the cell cavity, or often one or more central vacuoles may arise. There has been much discussion as to the presence of a nucleus. Often the methods of preparing and staining preparations of the species seem accountable for what looks like a nucleus. Most observers are agreed, that here as in the former group no nucleus exists, but that evolving chromatin granules or chromidia may represent a rudimentary nuclear substance. Vejdorsky, however, considers that he has satisfactorily demonstrated its existence in some species. 
The iron, the sulphur, and the purple bacteria seem all to be able to utilize inorganic substances for their nutrition and for supplies of energy. But a physiological group of immense importance and widespread distribution at the present day is that of the saprophytic species. These utilize decaying products of other organisms for their support, and so probably branched off early from some one of the above autotrophic lines, as plants and animals became increasingly abundant and in time underwent decay.

A fifth group, derived almost certainly from some one of the above four, attracted chemotactically by the rich food and body tissues of these, gradually assumed a parasitic mode of life. The numerous species of bacterial parasites now living is proof that in past stages of organic evolution they may have been at least equally abundant, and doubtless then, as now, were responsible for widespread destruction of higher plants and animals. Later we shall adduce evidence that favors the origin of primitive animal forms from either the saprophytic or parasitic bacteria.

While many of the species or even genera like Sarcina are inert, passive, and permanently embedded in mucilaginous wall substance, graded transitions can be traced to types that are highly active and responsive physiologically. It is also amongst the latter forms that a cell nucleus, or its fairly formed rudiment, has been asserted to exist.

Irritable or chemotactic response to the presence or absence of oxygen, carbon dioxide, and many other chemicals is similarly graded in its manifestations. Equally true is this for thermic, lumic, and electric stimulation, as the works of Engelmann (38), M. Ward, Jennings (42), and others demonstrate. It need scarcely be added then that we know much more regarding them than the Schizophyceæ as to physiological response. This, however, is in many respects a matter for regret, as the latter if sufficiently studied would give us in all likelihood valuable and fundamental results.

In geographical distribution the bacteria excel again, for it may well be said that they are omnipresent and abundant, 
except at very high elevations, and at great depths in the earth and sea.

Ecologically they present striking and almost exactly parallel phenomena as do the Protophyceæ. As regards temperatureendurance Setchell (37: 936) has pointed out that in the Yellowstone geyser region, while the Protophyceæ do not seem to be able to live and grow at a higher temperature than $75^{-}$ $76^{\circ} \mathrm{C}$, various bacteria thrive up to $81^{\circ}$ and $83^{\circ} \mathrm{C}$. We may rest assured that, when a critical bacteriological investigation has been made of the chalybeate, the warm marsh, and the moist decaying regetation areas of the world, rich scientific results will be secured. Here again then we would consider that these hydrothermal species still exist amid environal conditions like those of their primitive ancestors, which also represented the primitive organisms of the rorld.

But in the norldwide group of the "thermophilic bacteria" (B. thermophilus, etc.) that occur in manures, amid fermenting and putrefying plants, sewage channels, and even in the alimentary canal of animals, we have a physiological series that flourish at $55^{-}-75^{\circ}$ or even $80^{\circ} \mathrm{C}$. Their entire life history suggests strongly that they were originally hydrothermal types which have often become adapted to cooler environment, while retaining adaptability still for high temperatures. The rounded spore cells also of these and of many other bacteria exhibit a degree of vitality against heat injury that is perhaps unexcelled by any other group. For not a few can successfully resist boiling for some time, and equally resist a cold state of $-200^{\circ} \mathrm{C}$. Here, therefore, are organisms that during one stage at least in their continuous existence can resist a temperature range of at least $300^{\circ} \mathrm{C}$. So Chodat's observation seems amply justified: "Les forces qui les mettent en jeu peuvent etre des forces tout physiques."

Every believer then in evolution-continuity will readily accept that the pure rich protoplasm of primitive simple plants, as of their living descendants, has had and still uncuestionably has a labile potentiality that we seldom credit it with. This we believe to be in marked contrast to nucleated protoplasm, when actively vegetating, as will be pointed out later. 
The spores and even the active cells of some bacteria resist drying dessication for not merely months, but also it may be for years.

In their action alike on crystalloid and on colloid bodiesinorganic and organic-the bacteria show themselves to be minute but highly complex colloid mixtures, which, in virtue of their own definite chemical structure, act on definite chemical bodies, so as to change their composition. In the process, food materials and supplies of energy are thus secured by the bacteria, which then utilize these supplies for their own growth and multiplication.

The rapidity with which they undergo division at one stage, and spore formation at another stage of their history, is often amazing, and recalls in final results the rapid formation of chemical products on an extensive scale. Thus it has been calculated that, since the cholera organism divides its cell in 20 minutes, were food supplies and other needed environal conditions suitable, it would have produced 16 hundred trillions of cells in a day, and the resulting mass would represent 100 tons of solid residue (39: 17).

The delicate wall of each cell individual consists in part of cellulose, or, as in the zooglœa stage, of a mucilaginous modification of this in which the cells are embedded, exactly as in many Schizophyceæ. But most are agreed that the cell-wall of the higher bacteria consists largely of a nitrogenous substance - probably albuminous - as is observed in animal walls. So here, as in most details of structure, the present group may well have included forms from which a few of the higher plant types started, and equally the primitive types of animal.

While the simple spherical or coccoid bacteria are devoid of cilia, many of the rod forms have one to many cilia. These may vary from a single terminal whip to a terminal bunch and again to diffusely ciliate examples. Such organisms can move with extreme rapidity in their appropriate liquid media, and recall vividly types like Herpetomonas and various Coccidia amongst the unicellular animals.

On the whole it may be said that, while the simpler Schizomycetes and the Schizophyceæ have gradually elaborated a 
complex chemical activity or a complex cell pigment that permits of food supplies and energy being absorbed and utilized while the organism remains fixed or sluggish, in the higher Schizomycetes more active modes of life and of securing food have been evolved, by the formation of cilia, and so has resulted the varied diet of living or decaying vegetable or animal substance now absorbed by and largely typical of them.

But in addition to the Schizophycex and Schizomycetes a third group, that deserves well to be classed amongst the Acaryota, includes species and even larger families that are viewed as simple animals. Some of these are as yet so imperfectly known in their complete life history that they can only tentatively be discussed. But there remain others which are better known, and which present many points of agreement with the above groups, specially the Schizomycetes.

This somewhat heterogeneous assemblage in recent zoological plans of classification is known as the Proteomyxa. This includes genera which at some stage in their history show an irregular amoeboid condition of the protoplasm, that is deroid of any recognizable membrane. But, when the resting or encystment stage is reached, a bounding membrane is formed. In some genera no cell nucleus is discoverable, in others scattered chromatin granules suggest an evolving nuclear body, while in Vampyrella and Plasmodiophora evident nuclei can be traced at all stages in the life history. The capacity these have of absorbing either liquid or solid substances, and of digesting them, suggests that they are more highly evolved derivatives from one of the two last groups. This view is further favored by their methods of multiplication, and by the possession in some of them of a definite nucleus.

The Mastigophora is a group of unicellular animals that seems always to have aggregated chromatin gramules (Herpetomonas) or a nucleus commonly. Though the species are now distinguished by their manifold adaptations to other and higher animals as hosts, it seems by no means unlikely that they may have been derived from some higher nucleate lines of the Schizomycetes. 
If we now focus up the leading features of the Acaryota, it may be said that it includes organisms whose protoplasm responds to diverse chemical stimuli, much as would a complex but equilibrated mixture of different colloids; that it shows high adaptability to extremes of temperature, which may range from at least $100^{\circ} \mathrm{C}$. to $-200^{\circ} \mathrm{C}$. under certain conditions; that it can resist prolonged dessication; that it is affected by all forms of energy to a greater or less degree, and reacts to these correspondingly; that it shows extreme adaptability to fresh, to brackish, and to salt water, or to a moist aerial environment; that it absorbs and radiates out supplies of energy either of a thermic, lumic, chemic, or electric kind; that in all of these features it resembles many well-known colloid bodies of inorganic origin. But in that it shows varied means for reconstituting its molecules, or in other words of continuously nourishing itself, and simultaneously of throwing off waste substances, it show's a marked degree of advance on even the most complex or perfect colloid mixture at present known.

With such intrinsic protoplasmic characters before us, the question may next be asked, whether, as we rise in the scale of diverging plant and animal life, we can still trace such as more or less perfectly persistent hereditary characters, even when alongside and interunited with such more delicately organized and sensitive conditions as we hope later to show have been introduced through evolution of the chromatin or nuclear substance of the cell, and possibly other constituents of more complex nature associated with the chromatin? We consider such to be the case, and to constitute the explanation for many rather puzzling biological phases in plant and animal life.

To pursue this inquiry somewhat further now, it is a wellknown fact that, while most plants and animals show a greater sensitivity in their life relations during the actively growing or vegetative phase, they often tend to reach a stage where, either from direct environal agency-as in intense insolation and dessication of fungoid spores, lichens, etc., amongst plants, or rotifers amongst animals - or at a definite stage in their 
life history, they pass into a dormant or resting period. During this time they persist in a state of "dormant life" or reduced vitality, or as Preyer expressed it "anabiosis."

This state may persist for a few days, months, years, or even decades. As a preliminary to this the protoplasm gradually parts with a large amount of its water, and becomes condensed in the process into the state essentially of a hydrogel solution. Then, the nuclear chromatin being-as we hope to show later-in a dormant and inert state, the protoplasm exhibits those characters above summarized for the Acaryota.

Such is typical for many spores and bud cells as well as seeds of plants; for the mature plants of many lichens and some fungi; of some mosses, ferns, selaginellas, and even flowering plants; for some corms, bulbs, tubers, aerial stems, and woody cuttings of flowering plants; for dessicated infusors and rotifers; for hibernating fishes, amphibians, reptiles, and mammals; and more rarely or to a limited degree for various animal eggs.

We by no means desire to assert here that the protoplasm and all of its accessory substances are in all or in any one group of the above, absolutely identical with that of other groups. But we do claim that the fundamental substance or admixture that we call protoplasm, and which shows certain fundamental similar staining and other peculiarities, undergoes a temporary physical or physico-chemical change, that makes it more nearly resemble that of the ancestral Acaryota.

Instances of such protoplasmic resistance are given in many of the well-known works that deal with plant and animal life; while the writer refers to some of these in a paper already noted (13: 265). Now it can quite accurately be claimed that what is the condition or characteristic of any plant or animal at any one phase of its history might well have been its normal condition in its past genealogical history. So it need not surprise us if the Caryota, at any stage in their evolution, or in their individual life cycle, show features which carry us back to the Acaryota, as being ancestral forms.

The conclusion can warrantably be reached, then, that at the basis of all plant and animal cells there exists a funda- 
mentally stable, mobile, highly complex molecular mixture of colloid constituents called the protoplasm. This may form, and have associated with it, various accessory chemical compounds as food materials or as sources of added energy, and it carries additional accessory compounds for the chemical alteration or metabolism of the food materials.

But it should not be forgot that all of this molecular complexity fundamentally represents rotatory motion of ether particles, in which the varying degrees of rapidity of motion, the amount of vibratory or constitutive energy, the lines of flow of that energy, and the areas of the fields of energy that the particles or the groups of particles possess have determined the exact morphological complexity, size, relation, and resistance capacity of the constituents that make up the whole. So, whatever changes occur in one or more constituents of that whole, these are to be explained in terms of some alteration in the energizing currents. This may seem an extremely fugitive and unstable foundation on which the whole superstructure of plant and animal morphology and physiology has been reared. But it may be possible to show that it is an amplification merely of views expressed fully a quarter century ago, and that it alone affords us a reasonable and exact basis on which to explain the evolving complexities of organic bodies, up even to their most intricate phenomena in man.

Now in a survey of the Acaryota, even from the systematist's or taxonomic point of view, it is evident that the entire classification of the group rests on the oft-confirmed view that each species and genus continuously and hereditarily hands down certain definite characters by which such a species or genus can be distinguished from its neighbors. These characters may be said to depend on one of two possible conditions. First, two species of a genus, or two related genera, may contain exactly the same materials or compounds, but in one of the two these may be different in amount, or be disposed along different lines of energy than in another. Many species or even genera seem often to differ in such connection alone. Second, in one case a definite chemical substance or substances 
may be formed by the protoplasm, or by some ferment excreted by the protoplasm, and such may be entirely absent in the other.

Naturally the former of these methods is the most easy and gradual in operation for the evolution of new species, since it merely involves a very slow change in the distribution and activity of centers of intra-molecular or constitutive energy. The latter represents a totally new combination and distribution of ether particles with their constitutive energy charges.

But in this connection, were we dealing with either a simple or a complex colloid mass, we know that very similar results could be secured. Thus, in some of Leduc's colloid structures, were the chemical action stopped at a certain stage, a simple spherical body might result; were it continued so as to ensure added supplies of the same substances, a branched or arborescent body might ensue. On the other hand, were one or both of the osmosizing colloids substituted for another, a structure would arise that would be physico-chemically different.

We are definitely and accurately aware, then, that the only method of fundamentally altering any inorganic body as to its molecular constitution is by the process of chemical substitution, in which by exact and predicable action and reaction one or more molecules of some one compound are substituted by others or may even be removed. In the inorganic kingdom the total number of compounds of the elements capable of being formed by natural process has been variously given at from 500 to 800 . In contrast to this, the organic chemist has already made known about 50,000 definite compounds (26:127) as coming under his eye, and the number is constantly being added to. All of the latter are either natural products that are built up in plant and animal tissues, or so related by molecular structure and decomposition change that they form part of an organic whole.

In any one series of these, such as the methane or benzene, one body may differ from another only by the substitution of one atom for another in each compound. But, when even minute substitution change is made, it usually confers new characters, new reactions, and new potentialities on each body 
treated. Similarly, if any one group of molecules in a living structure be altered even to the minutest chemical degree in one out of many of its constituents, such must confer new specificity on the structure itself.

Just as in inorganic and organic compounds, then, to which the chemist has applied exact names, these remain stable and are acted on and react precisely, so long as they are exposed within like physico-chemical limits or environment, so for living bodies-highly complex and delicately equilibrated though their molecules may be - each within precise physicochemical limits is recognized by a specific name, that connotes it as truly as does the name given by the chemist for each substance.

It will readily be seen, however, that, even with only 10,000 to 20,000 possible combinations on which to work, the capacity which plants and animals possess for linking-up combinations or for decomposition actions is practically endless. But, while such is largely true, we hope to show in a future chapter that the supposed limitless variations which some evolutionists have considered that organisms possess are after all fairly restricted, and work along certain fairly exact grooves.

Reference has already been made (p. 63) to the steadily increasing list of "organic" compounds which the chemist has been able to synthesize. The list is an imposing one if note be made only of the synthesis of a certain compound from a simpler one that is also of organic origin. But, if one select those only which have been built up step by step from what were inorganic compounds to start with, the list becomes reduced to small proportions. Furthermore, practically all of those synthesized are crystalloids, and colloids are conspicuously absent from the list. Thus, to select one from many cases, while several of the sugars have been built up from simpler bodies, neither vegetable starch nor cellulose, both of them abundant and typical plant colloids, has been so produced.

If one take note further of the laboratory means employed, to effect synthesis of the organic crystalloids already secured, one is impressed by the elaborate expenditures of either thermic, 
chemic, or electric energy needed to effect the change. But, in order to reach correct biological conclusions as to the evolutionary origin of the organic compounds, one has to lay emphasis on very different substances from those most fully treated in manuals or text-books of organic chemistry. Even the designation and definition given is often misleading biologically. Thus organic chemistry has been described shortly as the chemistry of hydrocarbons and their products. But all evidences point very clearly to the view that the hydrocarbons alike of plants and animals are amongst the ultimate products, if they are not actually the extreme end products, of decomposition or analytic changes.

Therefore, were the attempt made to write what might be called "a biological text-book of organic chemistry," all present knowledge points to the treatment first of sugars and other crystalloid carbohydrates, next of the starch, cellulose, gum, and other colloid carbohydrates; thereafter the oils, fats, and fatty acids would have their origin traced; next the amides and amino-acids; then the proteins, ferments, and protoplasm; since all of these represent advancing biochemical bodies synthesized by successive upward steps. In descending series the protein derivatives, the glucosides, alkaloids, organic acids, crystals, and finally the hydrocarbons, including rubbers, resins, and volatile oils, would receive consideration. Want of knowledge as yet prevents such treatment, while the subject itself is one of enormous extent. But the beautiful and often continuous series of organic compounds already constructed by the chemist lead one to expect even more striking advances for the future, and not least along the lines at present indicated.

Now it may well be asked whether the plant, in elaborating even its simpler substances like sugar and oils, effects the unions by intricate means and highly lavish expenditures of lower types of energy, or whether such are built up by more direct action, and by the energizing agency of some more perfect energy than we have hitherto recognized, or have only surmised the possible existence of. In partial reply, it may not be inappropriate here to quote shortly a few of the laboratory 
methods for artificial synthesis of well known organic compounds.

Thus Benzoic aldehyde $\left(\mathrm{C}_{7} \mathrm{H}_{6} \mathrm{O}\right)$ is formed in plant tissues such as 'seeds of bitter almond, when the glucoside amygdalin is split up by the ferment emulsin or synaptase. Fisher $(40: 59)$ gives its artificial preparation as follows: Benzyl chloride and copper nitrate are boiled in water for five to eight hours. The resulting oil is extracted with ether, the ether is evaporated, and the oil, shaken with sodium bisulphite, yields crystals of aldehyde-bisulphite. The crystals are warmed with dilute sulphuric acid, and the aldehyde, now set free, is extracted with ether.

Quinoline or quinoleine $\left(\mathrm{C}_{9} \mathrm{H}_{7} \mathrm{~N}\right)$ is obtained from alkaloids of the quinine (Rubiaceæ) and strychnine (Loganiaceæ) families. Skraup has synthesized it thus: Nitrobenzene, aniline, glycerine, and concentrated sulphuric acid are cautiously mixed in a flask, and heated till reaction starts. The mixture is then boiled for two hours, diluted with water, the nitrobenzene is distilled off, and an alkaline remainder is distilled with steam, when quinoline appears.

Grape-sugar $\left(\mathrm{C}_{6} \mathrm{H}_{12} \mathrm{O}_{6}\right)$, a crystalline carbohydrate of wide occurrence in plants, has been obtained by Soxhlet through mixing hydrochloric acid and alcohol; this when warmed has cane-sugar added to it, and the whole is kept hot till the sugar is dissolved. When cold a small quantity of grape-sugar is added, and the mixture, frequently stirred, should stand for several days at room temperature, when the grape-sugar crystallizes out as small colorless crystals.

Dulcite $\left(\mathrm{C}_{6} \mathrm{H}_{14} \mathrm{O}_{6}\right)$, another and related carbohydrate to the last, occurs in such families as the Celastraceæ and Scrophulariaceæ. It is obtained by dissolving the allied carbohydrate galactose in ten times its volume of water, and adding sodium amalgam, the mixture meanwhile being neutralized by dilute sulphuric acid. The solution, separated from the mercury of the amalgam, is poured into hot 95 per cent. alcohol, and on concentration over a water bath the dulcite separates as crystals.

In contrast to the above, relatively large quantities of each can be produced in plant cells that show no marked indication of chemical reactions proceeding; that do not evolve extra amounts of heat, even when, as in large flower clusters that are actively metabolizing, a few degrees of rise in temperature 
is carefully recorded; and that show, even by fine galvanometric records, only moderate electric disturbances. So the only explanation, or mode of escaping the difficulties of the question, has been to say that all is due to "that mysterious something we call life." In the next chapter an effort will be made to analyze life, even in its higher phases, as being an expression of several highly perfect forms of energy in active cooperation to build up increasingly more complex molecules or energized ether particles, and thereby start increasingly complex adjustments to environal agents and factors. 


\section{CHAPTER IV}

\section{THE ENERGIES OF THE ORGANIC WORLD-THE .BIOTIC, THE COGNITIC, THE COGITIC- AND THEIR RELATION TO ORGANISMS}

As science from the times of Buffon, Lavoisier, Lamarck, Goethe, Berzelius, Spencer, and Darwin began to shake itself free from religious dogma, from the vagaries and errors of alchemy, or from traditions of crude cosmogonies, observation, experiment, collation of facts, and deductions therefrom were increasingly accepted as necessary methods of study for the extension of scientific knowledge.

But in acquisition of such knowledge the physicist, the chemist, the botanist, the zoölogist, and the palæontologisteach concerned with his own branch, or with some small field of it-has often failed to grasp the significance of discoveries in one department that throw important light on another. In the progress of chemistry, the successive discoveries of Ohm's law, of Joule's, of Dalton's, of Bertholet's, of Richter's and others, have given immense impetus to that science, that will ever continue to bear fruit.

But, when chemists began to compare purely inorganic substances with those obtained from plants and animals, the extreme complexity of the latter, and the apparent impossibility of building them up after the manner that many inorganic bodies had been, so impressed them that they spoke of these substances as the "organic compounds." Further, while pursuing rigorous scientific methods most of the distinguished chemists at the close of the Eighteenth and in the earlier part of the Nineteenth Century claimed that there was or must be a "vital force," while others in less explicit terms spoke of "that mysterious thing called life." Others again accepted 
the action only of the recognized forms of energy, but confessed the difficulty of using these so as to build up organic compounds.

Thus Liebig says: "The capacity of elementary bodies to form special combinations which are produced by plant or by animal life is nothing else than chemical affinity, but the cause which prevents these from uniting and from yielding to attractions which under other circumstances would carry them the one toward the other, the cause then which arranges them in living beings and assigns to them a special form, that is vital force."

Berzelius says: "If in the phenomena of organic chemistry one understands by vital force something other than a particular combination of different circumstances that bring into play the normal forces of organic nature, in short if one means by that a special chemical energy inherent in living bodies, one commits an error Electricity, light, heat, and chemical reactions unequally distributed, such are the conditions which excite the elements to give rise to varied organic compounds; art seeks to imitate the vital action by making use of these conditions, although their combinations in this case would not be as perfect as in living nature."

But when electricity became more and more prominent as a highly active and comparatively perfect form of energy; when Wöhler, Kolbe, Melsens, Davy, Kuhlmann, Liebig, Dumas, and others succeeded-even by expenditure of much energyin building up some of the simpler crystalloid organic bodies; when it was recognized that some of Graham's colloid substances greatly resembled-even though greatly simpler thansome organic colloids; and when substitution transformations and their formulæ in chemistry became more perfectly understood, a closer bond was felt to exist between the inorganic and organic kingdoms. It is fair to say also that the past quarter century has greatly strengthened the bond. So during the past forty to fifty years chemists and physicists have generally agreed that the recognized forms of energy are those which can sufficiently account for all biochemical changes.

In harmony with such a view it might well be said that the varied analytic changes which go on in plant and animal tissues, such as breaking down of glucosides into sugar and other sec- 
ondary products, or of sugar into alcohol, glycerine, and carbon dioxide, are explicable, if ne consider the change to be started by a ferment and continued by supplies of chemical energy derived from the decomposing body. But the problem becomes a very difficult one - as most of our text-books openly acknowledge - when the effort is made to synthesize, from a truly inorganic source, even one of the simplest organic crystalloids such as sugar or inulin. The methods are complicated; the amount of energy expended for the result attained seems in striking disproportion to the ease with which such bodies are formed in plant tissues; while the resisting apparatus employed is such as no living tissue can boast of. Examples are described on p. 66 of the last chapter.

Furthermore, when one observes the great amount and perfect quality of energy displayed by a minute ciliated bacterial organism, a motile Pleurococcus cell, a fern spermatozoid, or a small ciliate infusor, in which reserve food products seem scant, the question almost forces itself upon the attention as to whether one or more definite organic energies of high quality, transformation capacity, and energy-utilization may not after all exist.

The following are some of the older views of well-known plant and animal physiologists.

Johannes Müller says ("Physiology," Eng. ed., 1838, p. 23): "The harmonious action of the essential parts of the individual subsists only by the influence of a force, the operation of which is extended to all parts of the body, and which does not depend on any single part; this force exists before the harmonizing parts, which are, in fact, formed by it during the development of the embryo." And again (p. 25): "The formative or organizing principle, on the contrary, is a creative power modifying matter, blindly and unconsciously, according to the laws of adaption." Also (p. 27): "Into the composition of the organic matter of the living body, there must enter an unknown principle, or the organic matter must maintain its properties by the operation of some unknown forces. Whether this principle is to be regarded as an imponderable matter, or as a force or energy, is just as uncertain as the same question is in reference to several important phenomena in physics. The mobility of this principle is certain. Its motion is evident in innumerable 
vital phenomena. From all these facts the existence of a force which is often rapid in its action, and which moves through space, or of an imponderable matter, is evident; nevertheless, we are by no means justified in regarding it as identical with the known imponderable matters, or general physical forces, caloric, light, and electricity, a comparison which is refuted by a close examination. . . . . Life, therefore, is not simply the result of the harmony and reciprocal action of these parts, but is first manifested in a principle or imponderable matter which is in action in the substance of the germ, enters into the composition of the matter of this germ, and imparts to organic combinations properties which cease at death."

But Verworn, in commenting on the above (General Physiol., Eng. Tr., 1899, p. 23) says: "In the year 1828, Wöhler gave the theory of vital force its death wound by his epoch-making synthesis, out of purely inorganic substances, of urea, a body produced only in nature by organisms." Now, as a matter even of logical continuity, the view may well be advanced that if it is produced only in nature by organisms, or artificially by the organism man, the energy ordinarily needed or used to produce it can not be an inorganic one, but such as is typical of organisms. Further the very means which Wöhler used to obtain it are such as no organism could exist under.

The substance, moreover, being a crystalloid one, and so not most typical of the organic or higher colloid bodies, it is of minor import in any fundamental consideration regarding the life-role of organisms. And exactly the same argument applies to all of the synthesized organic products hitherto obtained by man.

Maudsley in "Body and Mind" (p. 172 app. "On vitality") expresses himself thus: "It is certainly extremely unphilosophical, in the present condition of knowledge, to refuse to accept vitality as a special mode of manifestation of force; the special character of its phenomena demands that, whatever its real nature may be, vital force should for the present be received as a distinct force, on the same terms as chemical force or electrical force. The facts of observation as well as a priori considerations unquestionably demand also that it should be regarded as subject to the laws of the correlation and conservation of force." 
Kerner clearly recognizes this when he expresses himself thus: "In former times a special force was assumed, the force of life. More recently, when many phenomena of plant life had been successfully reduced to simple chemical and mechanical processes, this vital force was derided and effaced from the list of natural agencies. But by what name shall we now designate that force in nature which is liable to perish while the protoplasm suffers no physical alteration and in the absence of any extrinsic cause; and which yet, so long as it is not extinct, causes the protoplasm to move, to enclose itself, to assimilate certain kinds of fresh matter coming within the sphere of its activity and to reject others, and which, when in full action, makes the protoplasm adapt its movements under external stimulation to existing conditions in the manner which is most expedient?

"This force in nature is not electricity nor magnetism; it is not identified with any other natural force, for it manifests a series of characteristic effects which differ from those of all other forms of energy. Therefore I do not hesitate to designate again as vital force this natural agency, not to be identified with any other, whose immediate instrument is the protoplasm and whose peculiar effects we call life. The atoms and molecules of protoplasm only fulfill the functions which constitute life so long as they are swayed by this vital force. If its dominion ceases, they yield to the operations of other forces. The recognition of a special natural force of this kind is not inconsistent with the fact that living bodies may at the same time be subject to other natural forces. Many phenomena of plant life may, as has been already frequently remarked, be conceived as simple chemical and mechanical processes, without the introduction of a special vital force, but the effects of these other forces are observed in lifeless bodies as well, and indeed act upon them in a precisely similar manner, and this cannot be said of the force of life."

Reference has already been made to the view that, in the simpler colloid bodies, a double electric charge, each of separate sign to its neighbor, invests the colloid molecule. Such might explain, in part at least, the synthesis of the inorganic colloid bodies. But it leaves untouched the question of the formation of colloid polymeric carbohydrates like starch, not to say of the greatly more complex amides, glucosides, and proteids.

But we have already suggested that-no matter how we view heat, light, chemical affinity, or electricity, as related 
to or transformable into each other-they seem to have had a graded connection and climax for manifestation of their activity, that corresponds broadly with the gaseous, the liquid, the viscous, and the solid states. These again seem correlated, from the energy standpoint, with binary, ternary, and rarely quaternary molecular aggregations. Thus heat in the rarer gaseous state, light in the dense gaseous and liquid state, chemical affinity in the liquid and viscous states, electricity in the solid state, and finally doubled electricity in the colloid state, seem all to suggest graded relationships in condensing kinetic or vibratory energy, and its passage toward potential or constitutive energy.

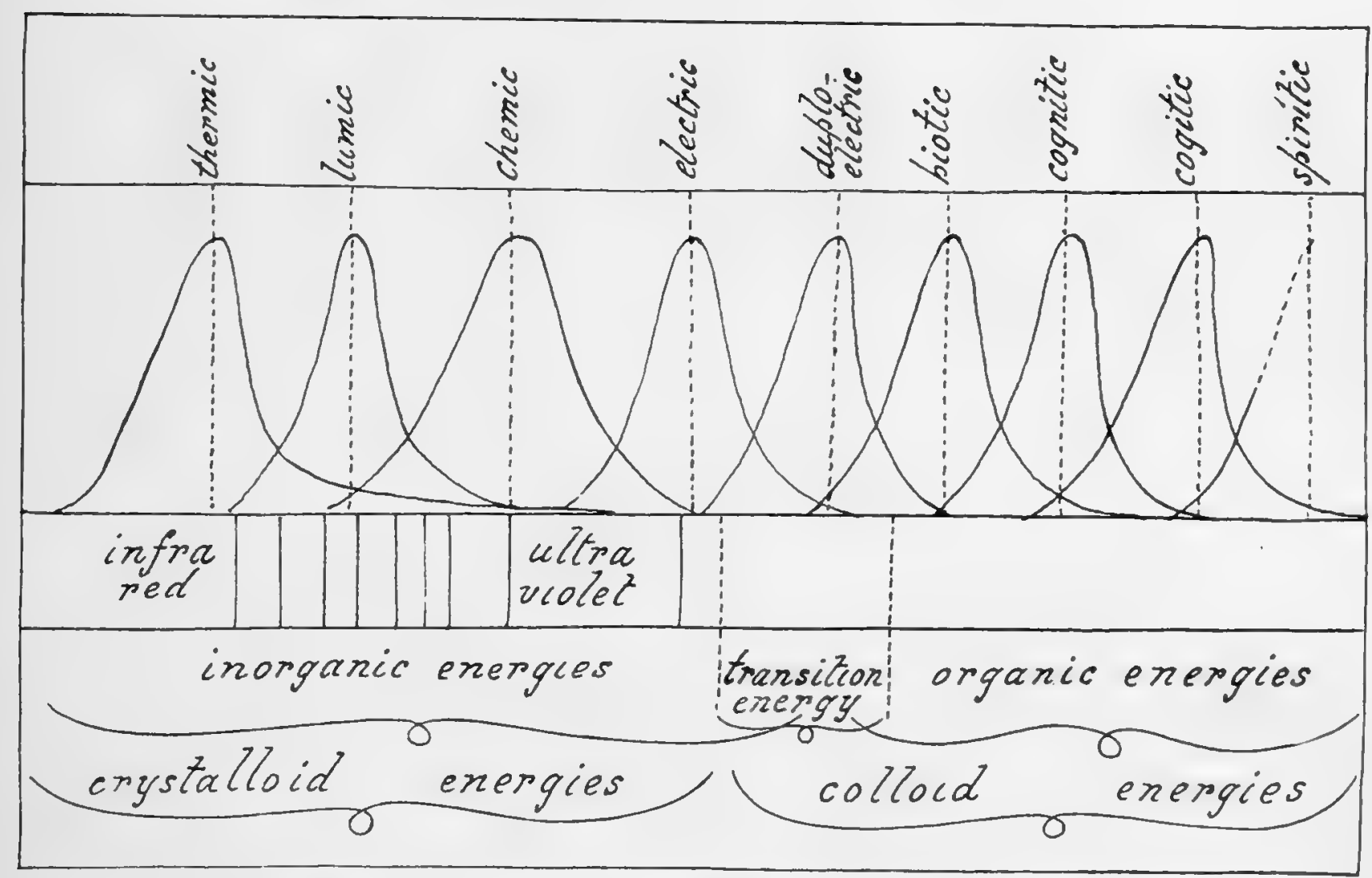

FIG. 1.-Diagram to illustrate supposed relations of the inorganic and organic energies.

The accompanying diagram, the left-hand portion of which the writer owes to the kindness of Professor Dahlgren, sets forth the relation of the recognized energies to each other, beginning with the simplest and ultimate exhibition of energy, namely heat, and passing to the most perfect, electricity. By the activity of heat, light, chemical affinity, and electricity, 
the varied inorganic compounds are built up which we meet with in nature, and which may be binary, ternary, or rarely quaternary in complexity. These, however, in their comparatively simple composition, are in striking contrast to the greatly more complex molecules that can be linked together in an aqueous matrix as colloid "organic" molecules; though a transition connection seems to exist in the rather numerous inorganic colloid bodies that are now known to the chemist. The right-hand portion of the diagram, therefore, is that which requires further consideration.

In riew, then, of the greatly more complex molecular combinations that are typical of nearly all synthetic organic compounds; in view also of the highly striking peculiarities that these show, and which cause us to speak of them as living bodies, it may well be questioned whether there may not exist one or more forms of energy of advancing condensation, that as perfectly characterize such bodies as are the recognized energies typical of the inorganic kingdom.

In favor of such inquiry it may be observed: (a) that the capacity for transformation of a given weight of food stuff or fuel into available work is greatly superior in organisms to that of the most perfect machines; (b) that the most elaborate attempts hitherto made to convert even the most complex inorganic body into one of the simpler crystalloid or colloid organic compounds have been limited; (c) in such efforts, the complicated methods employed are in striking contrast to the ease with which, in a day, the product of one bacterial cell could absorb, metabolize, and build up 100 tons of material (p. 58); (d) even granting in the last case that the food supplied rwas itself complex or organic, the raising of this to a more complex chemical state renders the process the more noteworthy; (e) if four or more different modifications or condensations of energy have all been evolved for advancing elaboration of elemental gases into elemental compounds, and of these into ternary or quaternary compounds, it might well be likely that some still more perfect modifications of energy must have originated for elaboration of the very complex ternary, quater- 
nary, quinary, and higher compounds that are everywhere met with in the organic world; (f) the existence of energytransformers in the tissues of many animals, possibly too in the chlorophyll of plants, for the conversion of muscular or other energy-whatever that may mean-into electricity (electric eel, torpedo, eté.), or light (firefly, glow-worm), or chemic energy (animals generally), or heat (very frequent), strongly suggests that an energy or energies higher than they can be rapidly and economically let down into them; (g) the extremely varied, complicated, and delicate reactions to environal stimuli that most plants and animals may show simultaneously find very remote resemblance in inorganic bodies; $(h)$ in all changes, proceeding in living bodies, kinds of energy are needed that can stimulate to the building up and breaking down of food products, give out sufficient body heat but yet distribute the latter gradually, enable the organism to respond to diverse extrinsic stimuli, and start reactions that will place the organisms most favorably in relation to such stimuli.

Elaborate classifications have been devised in works on plant and animal physiology, setting forth the relations of energy in organisms, but in most cases such treat merely of stores of food, and take little account of the primary question as to how and by what kinetic energy the complex molecules have been reared, that give to organic bodies their specific and outstanding qualities:

Unless we admit and demonstrate the existence and activity of one or more organic energies, even while accepting the continuity of inorganic and organic processes, we are constantly led into inconsistencies. Thus Leduc (23: 147) says: "Considering the impossibility of defining the exact line of demarcation between animate and inanimate matter, it is astonishing to find so much stress laid on the supposed fundamental difference between vital and non-vital phenomena. There is in fact no sharp division, no precise limit where inanimate nature ends and life begins; the transition is gradual and insensible, for, just as a living organism is made of the same substances as the mineral world, so life is a composite of the same physical 
and chemical phenomena that we find in the rest of nature. All the supposed attributes of life are found also outside living organisms. Life is constituted by the association of physicochemical phenomena, their harmonious grouping and succession. Harmony is a condition of life."

But on the succeeding page, after referring to living bodies as being made up of the same chemical elements as inorganic, he says: "Hence life is but a phase in the animation of mineral matter; all matter may be said to have within itself the essence of life, potential in the mineral, actual in the animal and vegetable. The flux and reflux of matter is alternate and incessant, from the mineral world to the living, and back again from the living to the mineral world."

But here the "animation of mineral matter" constitutes the crux. Were we to say and know that "life is but a phase in the thermification, or in the lumification, or in the electrification, of mineral matter, or in all of these combined," we would know the exact lines along which alone experimental study should be carried. But it is the higher and more perfect step of primary animation of matter that is the fundamental need.

Possibly a more correct and graphic picture of the contrast in energy needed for linking up and holding together inorganic and organic molecules can be obtained if we compare the number of heat calories as related to their chemical formulæ, in some well known inorganic bodies, with the chemical formulæ of certain important organic bodies. On page 103 a set of the former is given. Though, in virtue of their labile and viscous character, organic colloid molecules may not represent relatively the same expenditure of heat calories, their extremely complex chemical composition, and consequent great amount of stored intra-molecular energy, can readily be estimated by selecting a few examples from well-known plant and animal products. Thus wheat gluten or artolin has the formula:

$$
\mathrm{C}_{185} \mathrm{H}_{288} \mathrm{~N}_{50} \mathrm{SO}_{58}
$$

The serum albumin of vertebrate blood has, according to Kurajeff's analysis (57: 355), the formula:

$$
\mathrm{C}_{450} \mathrm{H}_{720} \mathrm{~N}_{116} \mathrm{~S}_{6} \mathrm{O}_{140}
$$


A product of the thymus gland known as nucleinate of histone is built up as follows:

$$
\mathrm{C}_{255} \mathrm{H}_{390} \mathrm{~N}_{84} \mathrm{SP}_{12} \mathrm{Ca}_{3} \mathrm{O}_{114}
$$

Finally hæmoglobin of the blood is represented by:

$$
\mathrm{C}_{753} \mathrm{H}_{1203} \mathrm{~N}_{195} \mathrm{O}_{218} \mathrm{FeS}_{3}
$$

To the writer it has seemed that, in passing from the inorganic crystalloids and colloids to those composing organic bodies, the fundamental need of the case was the evolution and increasing activity of an energy that would as far excel electricity in its perfect quality as does the latter excel chemical affinity, and it again heat. Many evidences can be adduced to favor such a requirement, apart from those above suggested.

In this connection $\pi$ saw in the last chapter that amongst the simplest forms of which we now have living representatives - the Schizophyceæ-a pigment was evidently gradually evolved through etiolin, purpurin, carotin, and chlorophyllin stages, each still represented in definite species of the group, that became the special center for formation of carbohydrates. Such carbohydrates, in the algæ proper and in higher plants, may appear even as the complex polymerized substance starch. But starch, it is now agreed, is a greatly more complex body than sugar that is first formed, and which the chemist by great effort has synthesized. Now it is proved that definite rays of sunlight, absorbed by the chlorophyll, supply much if not all of the energy needed for such union. But no one probably will assert that the light as such, or the heat as such, of the absorbed rays, is directly utilized, for this, as Pringsheim and others well recognized, would cause immediate dessication of the tissues. Partly on this account originated Pringsheim's view, that the chlorophyll acted rather as a protective screen.

But if we view the chlorophyll as an energy absorber and transformer, that converts certain light rays into a much higher and more condensed phase of energy, nearly every difficulty disappears. Naturally the possible existence of one or more types of organic energy does not preclude, but rather presupposes, the frequent presence alongside it of electric (Dionaa), lumic (as in fungi, etc.), or thermic energy (spadix of aroids), 
since in analytic processes the higher may constantly become degraded into lower phases. Equally true is this for such inorganic bodies as a colloid or crystalloid solution, or a bar of iron.

During the past quarter century increasing attention has been given to the phenomena of surface tension, as exhibited between substances of different chemical composition. Thus, since Bütschli, Berthold, and Quincke explained the streaming movements of cell protoplasm as being fundamentally due to surface tension between the protoplasm and enclosed sap, other writers have tried to extend this inter-relation between many organic compounds. But surface tension and the internal changes in substances that are due thereto do not represent a type or species of energy traversing such substances. They merely indicate the relative rapidity with which a definite energy or energies are working to effect molecular changes in diverse substances, when the molecules of these are brought within suitable fields of interaction. The high surface tension, therefore, thit may be demonstrated to occur between different organic bodies, or between these and surrounding fluids, would be due to the complex character of the molecules, and correspondingly to the high discharges of electric, biotic, or other forms of energy set free during the process of interaction.

In the last chapter we showed that the Acaryota are wholly or largely protoplasmic, and that only in highest genera of the group a diffuse nucleo-protein body was observed. Such simple organisms should aid us then by yielding simple material. But it was also stated that, even in the highest plants and animals, at least temporary life-phases might be passed through when only protoplasmic activity - and that usually sluggishmight be traced. In the past fifteen years, however, the view has been widely held by biologists that nuclear material is essential for most life processes. But consideration alone of the Acaryota would suggest that in the lowest or most sluggish life actions nuclear material may be entirely dispensed with.

As a first example of energy distribution we may now examine Beggiatoa alba, one of the common sulphur bacteria. Living 
in pools, lagoons, and mud-flats, where hydrogen sulphide $\left(\mathrm{H}_{2} \mathrm{~S}\right)$ is generated, the plant according to Winogradsky absorbs this along with free oxygen, according to the formula $\mathrm{H}_{2} \mathrm{~S}+\mathrm{O}=$ $\mathrm{H}_{2} \mathrm{O}+\mathrm{S}$. By oxidation action it sets free the sulphur, which accumulates in its tissues, so as even to color it yellow. Next it is stated that the sulphur combines with carbon dioxide $\left(\mathrm{CO}_{2}\right)$ and other products absorbed only in minute quantity from the water, to form necessary plant foods, and also the necessary heat of respiratory interchange. Now if the required plant foods, the carbohydrates, amides, and proteids, are as readily elaborated as the above reactions indicate, it should be possible to effect the same result in the chemist's laboratory. But even a near approach has hitherto failed. If on the other hand we accept it that the already existing molecules of Beggiato a represent ether centers of a constitutive energy greatly more condensed than electricity, this might steadily expend a part of its intra-molecular energy in building up new molecules, and in recouping its onn temporary loss. Thus me get a bridge built, so to speak, between inorganic and organic bodies.

In the purple bacteria it is found that slight traces of chlorophyll exist, somewhat masked by the pink-purple pigment that gives them their name. In contrast, therefore, to Beggiatoa they make for and luxuriate in the light. But further they live and flourish amongst concentrated amounts of $\mathrm{H}_{2} \mathrm{~S}$ that would be fatal to other plants. They are able to oxidize the sulphur, by utilizing the oxygen set free in the splitting up of $\mathrm{CO}_{2}$, and this sulphur, instead of accumulating as in Beggiatoa, is used up directly as a partial source of food and energy. Simultaneously, however, additional material is built up from union of carbon and water.

We therefore have, in these simplest types, a double mode of formation of the primary food products, which combines the method in Beggiatoa with that seen in green plants.

In the Schizophyceæ or Blue-green Algæe the sulphur of Beggiatoa is replaced, as a source of energy, by a reddish green or bluish green chlorophyll compound. As in all bright green plants, this is intimately associated with the protoplasm, and as in them we would regard it as a light absorber and transformer, which starts organic synthesis by the presence, as we aim to show, of biotic energy. Chloroplyyll then mould stand in relation to the protoplasm, much as does a water-dynamo 
to the electricity which it generates. That is, it seems alike to alter the vibratory character and the power value of the absorbed rays, so that a higher and greatly more perfect energy results. It is now fairly well agreed that the iron or in some cases manganese of chlorophyll acts as a metallic conductor of energy, but, instead of actually entering into the molecular constitution of the chlorophyll, it seems rather to aid the latter in giving a labile and conducting character to the molecules of it. Various observers have shown that along with the chlorophyll there is a dense oily substance to which several names have been given, and as to whose functions various opinions have been expressed. It does not seem inappropriate to suggest that the oil constituent of chlorophyll may have been evolved as an insulation substance. The green pigment, therefore, and the associated protoplasm of a chloroplast, may play the part equally of an absorber of lumic energy, a transformer of it, and an accumulator specially of carbohydrate supplies.

But, as in the most perfect electric transformers, so it has been found by Duclaux for chlorophyll (26: 70) that the total lumic energy which falls on the surface of green plants is greatly in excess of the amount of elaborating work done by the plant.

With such results in view, and seeing as above noted that diverse organisms belonging to the Acaryota, and exhibiting a plastic capacity for surviving under wide ranges of temperature, in diverse liquid chemical surroundings, under different light relations, and forming their most complex food constituents from simple but diverse food beginnings, yet show fundamentally similar life conditions, in that they build up extremely complex and largely colloid carbohydrates, amides, proteids, and protoplasmic substances, it follows that all of this action and reaction necessitates expenditure of some perfect quality of energy. The actions and reactions also are performed in presence of the-in itself inert and yet most complex-constituent protoplasm, that shows throughout the whole range of the organic world fundamentally similar aspect, structure, staining reaction, and energized activity. To perform the 
upbuilding work, that is to energize the inert ether particles that form the centers of the atomic and molecular structures, we have suggested that heat, light, chemical affinity, and electricity, as phases of energy, are unequal to the task in hand.

We consider, then, that during that extended period of the archæan age, when conditions suited to, and stimulating for, the origin of organisms had evolved, a more perfect concentration of energy occurred, as a part of and a result of many coöperating factors of advancing cosmic evolution. This seems to have had its first beginning, probably during the earliest part of the archæan epoch, in the formation of hydrated bodies from more primitive crystalloids which started that peculiar type of inorganic body, the colloid. In this, distinguished chemists consider that a redistribution of electric energy can be traced round each molecule, that we consider was probably the forerunner and anticipator of the biotic energy that we regard as the basic energizer of organisms.

By a process of action and reaction that we will later picture as proceeding through the entire organic scale, the colloidal molecules of ancient thermal areas became increasingly charged with greater and more condensed amounts of energy, and in utilizing this became themselves linked up into more and more complex and inter-related molecules, at the summit of which we place protoplasm, so far as the activity of biotic energy acted.

One outstanding quality of this energy is that it can effect highly intricate molecular combinations, and yet give off a relatively small amount of heat. This characteristic alone would indicate its superior nature as an energizer, and equally indicates that it can absorb enormous amounts of constitutive energy during elaborative work.

But at this stage the question might well be asked: Can such energy be conducted or conveyed from one place to another, as in the conduction or convection of electricity, light, or heat? Though anticipatory to our treatment of the subject, it may be replied that, in all cases of transplanting, grafting, transfusion, parasitism, and related phenomena in plants and animals, conduction or convection of biotic energy is an essential requisite of the process. 
In turning from the Acaryota to the Caryota or nucleated organisms, for evidence as to the possible existence of biotic energy, it should be again emphasized that in undoubtedly non-nucleate genera, such as the simpler Schizophyceæ and Schizomycetes, active and delicate response to environal stimuli, as mell as a determinate growth action in a given direction as resulting therefrom, are comparatively feeble. So they usually have a spread-out or irregular or botryoid growthoutline as in masses of Glœocapsa, Nostoc, Sarcina, Micrococcus, etc. But in higher types like the Oscillatoriacece and Rivulariacec, where evolving chromatin or nuclear substance is present, the degree of response is greatly more exact and delicate. Again amongst the higher bacteria, that live amid rich inorganic or organic media, delicate and often rapid response to thermic, lumic, chemic, and electric stimuli can be traced (41:34, 42:38). It is in such forms again that chromatin or even a definite nucleus has been asserted to exist.

Therefore, it seems generally true that evolving chromatin organization confers added and more condensed energy activities. To this we shall return later.

In the whole of the Caryota, the evolving nucleo-protein chromatin granules, chromidia, or chromatin skein already spoken of for the Acaryota, have assumed definite form, at least during some stage of the organism's existence, and it constitutes the nucleus. The appearance of this structure then, in the evolution of organisms, is of exceptional interest, for not only does it give to the whole of the Caryota those important characters which we now associate with nucleated cells, it is the concomitant of, and is we believe the expression of, a more complex and condensed organic energy than even the biotic, and which we propose to name in future discussion the cognitic energy.

From the studies of Hansgirg (30), Hegler (43), Phillips (35), and Olive (44), as well as other observers since on the Schizophyceæ, from observations made directly by the writer, and from more doubtful statements regarding the higher bacteria, it seems assured that granules gradually appear 
in increasing number and in more and more intimate connection with each other that give definitely recognizable chemical and staining reactions in common. These further react like the refractive chromatin substance of the nucleus and nucleolus of caryotic cells. In some species or genera they are few in number, small in size, and comparatively scattered; in others they are fairly large, aggregate, and uniform; or again they are united by delicate chromatin threads so as to form a central connected skein or network of granules of loose open pattern.

Between such arrangements and the fully formed nuclear structure of all caryotic cells, there seem to be the most perfectly graded examples and phases. So from nucleo-protein granules to granule loops, from these to an open network, from this to a closed network with bounding chromatin membrane, and finally to a nuclear netrork or basket that encloses and hangs a special nucleo-protein mass or nucleolus, the transitions are still left to us and can be followed stage by stage.*

As to the molecular and chemical constitution of the substance chromatin, most biochemists and physiologists are agreed that it is a highly complex protein compound, which is excelled only in organized complexity by Nissl's granules (which will importantly concern us later) of animal nerre cells. Unlike the simpler proteids of protoplasm, it is deroid of sulphur, but is rich in phosphorus. By compounds of the latter it holds iron in its substance, either as an integral constituent or more probably to promote conductivity of energy currents. As explained later we consider that it exists in most concentrated state in the nucleolus, whence it extends outward as a sponge network whose threads are intimately fused as a nuclear network, in the interstices of which is protoplasmic or nucleoplasmic substance, and again processes of it - the kinoplasmic threads of some cytologists - extend into and throughout the general cell protoplasm as a fine network.

* The above conclusions, as well as those set forth in an already quoted article (13: 252) by the writer, have been confirmed in a recent valuable paper by Miss Acton (Ann. of Bot. 28 (1914) 433), who has given detailed attention to several of the unicellular Cyanophyceæ. 
As compared even with protoplasm and its accessory constituents, therefore, in dealing with the Caryota, we have now to recognize a greatly more complex cell-substance, whichforeshadowed morphologically and physiologically by the chromidia in Acaryota-now becomes an integral and invariable factor of every living cell of the entire series. But though exact, stable, specific in its molecules, and in their inter-atomic arrangement, as was the substance of the protoplasm, it must be acknowledged, as in line with all modern views of energy and ether relation, that the constituents of the chromatin substance are absolutely inert and passive unless molded and guided by currents of energy. Are its constituent molecules then built into position by biotic or some one of the inorganic energies? Or has its upbuilding been associated with, and been the outcome of, a special nuclear or chromatin energy?

We hope to show by degrees that the latter seems not only to be true, but that the existence and influence of such an energy as we have proposed to name the cognitic goes far to solve many of the most difficult problems in the organic kingdom.

But, if such energies or phases of energy as the biotic and cognitic exist, some explanation needs to be offered as to their relation to the lower or inorganic energies. Such seems to present itself when we try to follow the origin of each. Currents of thermic, chemic, and electric energy constantly traverse the living tissues of plants and animals. The varying amounts or strengths of these currents have been estimated by Burdon Sanderson (46), Kunkel (47), and very delicately by Waller in his galvanometric studies on plant $(48: 6)$ and animal tissues (28). If it be true for inorganic colloids that a special double condensation of electric charge (pp. 19, 23) exists, such at least should be true for the greatly more complex organic colloids. But the intrinsic molecular capacity for building up, for breaking down, and for metabolic change from colloid to crystalloid and vice versa, shown by organic colloids, is so greatly superior to anything shown by the inorganic that we postulate at once a graded energy transformation and condensation toward the biotic exhibition of energy. This 
becomes typical of, and is the fundamental combination for, the varied rates of ether-particle vibration that together constitute protoplasm throughout the entire organic realm. This then, working in harmonic relation with the pigment chlorophyll, and the sunlight that the chlorophyll absorbs and transforms into biotic energy, enables the chlorophyll to link up carbon and hydration elements into sugar, and again by further expenditures of intra-molecular biotic energy to change the sugar into starch or into some still higher chemical substance, that in turn is used as an energizing chemical food for the protoplasm by being successively linked up into amino-acids, peptides, proteids, and finally protoplasm.

As already stated then (p. 80) we consider that what might be called accumulators or Leiden Jars of biotic energy are to hand, and these of a very perfect kind. In dormant seeds that retain vitality for 10-50 years, in dormant bulbs that retain it for 1-10 years, in dormant cycadeous trunks that retain it for 12-24 months, as well as in many similar structures that will occur to every one, stores of some perfect energy are bottled up, and only very slowly expended.

Similarly the prolonged periods of environal dessication and dormancy undergone by various infusors, rotifers, tardigrades, fishes like Protopterus, and higher forms up to the hibernating mammals indicate the existence of like reserves of biotic energy amongst animals.

Now there is absolutely no physical nor biological proof that either electricity or chemical energy can be stored in accumulated amount in living organisms, and then on appropriate access of oxygen water and suitable temperature start intrinsically to expend itself in multiplying cells, in enlarging colloid walls, in increasing the amount of protoplasm, and in gradually transforming the stored foods. Not only so, were such a mass of matter as a trunk of Cycas revoluta, two feet long, charged to fullest capacity with heat, this would be radiated into space in a few hours at most, till the trunk became of surrounding temperature; were it stored with light from any ordinary source of combustion this would be radiated out and 
used up in a few days; were it charged with chemic or electric energy such would be exhausted or radiated out into space in a few months. But such a trunk may be left alive in a slowly metabolizing state for at least a year. No proof has yet been adduced that electric or chemic energy separately or together are capable of effecting such energy radiations from like linkedup elements. All details of the general phenomenon point to some more perfect form of energy. So we would define protoplasm as a definitely correlated rotatory motion of variously energized (or linked) and highly complex groups of ether particles of colloid nature, in which the specific rates of motion between the groups are an expression of biotic energy. Such energy of intra-molecular storage or reservation is continuously expended in causing synchronous motion of other and less complex or less energized ether particles that may come within its field of action, and these gradually become absorbed into the same rotatory or protoplasmic system, to replace that which is being expended or broken down.

But such considerations lead us to a very important and interesting connection that exists between protoplasm and the nucleus, that is; between biotic and cognitic energy. Why, it may be asked, do we speak only of biotic energy in dormant plant parts such as seeds, bulbs, etc., and not equally of bioticcognitic energy, since all of its cells are nucleated? Because abundant experimental evidence can be, and later will be, advanced to prove that nucleo-protein substance, alike amongst plants and animals, is greatly more susceptible to environal change than is the protoplasm. So when, by gradual elevation or lowering of temperature beyond a sub-maximum or subminimum, by gradual evaporation of water to a sub-minimal or dessication point, by gradual shutting off of light action by formation of pigmented cell walls, the cognitic energy seems less and less perfectly to flow and to energize each of the associated cells. So the nucleo-protein substance of each cell passes into an extremely reduced state of energized activity that may be termed suspended vitality. Numerous illustrations of such will be cited later from the vegetable and animal kingdoms. 
Contrariwise, the nucleus in relation to cell size is always proportionately large in young active bio-cognitic cells of plants and animals; it remains so in cells that continue long in activity, as in gland cells, stomatic cells, apical cells, and cambial cells of plants, and in dividing epithelial cells, in gland cells, etc., of animals; while in nerve cells, and specially in multipolar nerve cells, that are to a minor degree biotic, are to greater degree cognitic, and are specially cogitic in energizing relation, the nucleus and particularly the nucleolus are very large.

We shall meanwhile accept it then for further discussion, that in the simpler Acaryota, protoplasm that is energized by biotic energy alone is evolved; that, in the higher forms of these, rudiments of nucleo-protein bodies associated with condensing cognitic energy are traceable; and that, in the Caryota, protoplasm and nucleo-protein in the form of definite nuclei are energized by and correlative with biotic and cognitic energies.

Accepting the action of biotic and cognitic energies as the fundamental agents in building up and placing ether particles for the Acaryota and the lower Caryota - that would include higher plants and the simplest groups of invertebrate animalswe turn next to the higher groups of the invertebrates, viz., from the Coelenterata upwards, and to the rertebrates, as illustrating a still more perfect degree of molecular organization, that is superposed on the protoplasmic or biotic, and the chromatin or cognitic. This, if we use the somewhat popular name, we may designate as the phenomenon of mentality. Such is always associated with the activity of those special cell unitsthe nerve cells-as the abundant studies of the past thirty years have demonstrated.

The nerve cells exist in their simplest state, either as isolated cells (Anemone, etc.), or as groups of such cells united by connecting fibers as in some medusa, echinoderms, etc. It is in these groups then that Haeckel, Lauder-Lindsay (49), and Romanes, as well as successors have looked for the most primitive expressions of mental activity; that activity which in several of the higher animals assumes a marvelous degree of 
complexity, but reaches its most striking climax in man, and which is characterized throughout by definite reflex actions.

When we study all of the life phenomena of these higher Caryota up to man himself the question may again be asked: Can we trace only biotic and cognitic energies in their highest degree of evolution and most perfect cooperation or action? Or is there a higher type of energy associated with, and a causal explanation for, erolution of the nerve cell, and which we in general language have commonly called mind? We propose in the remaining part of this chapter to indicate that such exists. As being euphonious with the other energies, and expressive of its function, we will speak of it as the cogitic.

Further, though such may seem highly illogical in treatment, we propose first to study phases in man's history, for possible verification of the position taken up. Having then obtained some details as to the coöperation of biotic, cognitic, and cogitic energy, as associated with and stimulating to formation of the protoplasm, the chromatin or nucleo-protein, and the Nissl substance or neuratin-as we will term it-of the nerve cell, in the succeeding chapters we shall consider the two first in detail, and shall leave to a later chapter like detailed consideration of cogitic energy.

Throughout such human study it will be necessary and appropriate to view the organism alike in health and disease; in developing, in growing, and in mature state; in the possession of full life-vigor and in the successive stages up to death; in normal activity and when affected by chemical agents; and under average past environment, as well as amid changed environal states.

When we study the developing, growing, and mature stages of man, we learn that distinct and successive phases or accretions of structure and activity are superadded on each other. These are now minutely described in standard physiological works, or in special treatises as those of Preyer (51), Romanes (52: 213), Hobhouse (184), Parmelee (53), and others.

From the time that the human egg becomes a multicellular structure, up to 1-4 days after birth, it performs functions 
that are purely or almost wholly protoplasmic or biotic. That is, in passively absorbing food, in digesting it, in respiring, and in excreting, the child shows phenomena that do not differ biologically from those functions seen in the primitive Acaryota. As Preyer and others also have shown, there is at least up to the close of the first day - possibly the third or fourth day after birth-no perceptible active response of the organism to environal stimuli such as light, heat, sound, chemic, mechanic, or electric stimuli. Fundamentally it constitutes that passive inert stage where food absorption and digestion are proceeding; where respiratory change is effected; where tissues are being repaired and even rapidly built up; but in which response to environal stimuli is absent or as in the higher Acaryota only is in slow process of evolution. As Preyer well puts it, though in too personal and appreciative language for the child, "We note very early that the foreground is occupied by nourishment. The hungry child is indifferent to everything else until his hunger is appeased." The cell nuclei are present, as at every point in the history of the organism, and so far as one can distinguish well developed. But their function as irritable centers is as yet undeveloped or extremely feeble. The gray matter of the brain, that is, the special mental centers, is still lacking in the newly born child, or is present only in very small quantity whereas after six weeks its presence can be more easily demonstrated.

But from the third or fourth day after birth, onward till about the close of the second or even into the third year, the sense of taste is first awakened, thereafter that of smell, of touch (cutaneous sense), of hearing, and of sight at varying intervals apart. Further, some of these senses, like those of hearing and of seeing, are acquired to a fairly perfect degree at a relatively slow rate, and in what might be called "installment" quantities. For, while a diffuse sight perception is acquired after a few days, the ability to distinguish between colors may be lacking till the third year or longer. Or as Preyer again well puts it: "All the organs of sense are organs of time. . . Two sensations belonging to different depart- 
ments of sense can never come into consciousness at exactly the same time. Never simultaneously, but only successively, can two impressions on precisely the same place in a sentient surface be perceived as such."

The above then is evidently the cognitic stage, where, as in all nucleate plants and in animals, a capacity of more and more rapidly perceiving, correlating, and responding to environal stimuli unfolds.

The gray matter of the brain, however, is at this time rapidly forming and increasing in quantity, though as yet its functional activity is feeble or wanting, as proved in cases of idiotic or imbecile children at two or three years of age. In such, though the cognitic activities may be fairly or quite active, the cogitic are lacking, as is the needed gray substance. But the biotic activities are now vigorously established, and act as a helpful regetative basis for the cognitic activities, as these in turn are a fundamental need for the later establishment of the cogitic capacity for perception and response.

But in time a third step is made, and a higher stage is reached. For, with increasing cognitic ability, sensations or perceptions of external stimuli become linked together and reasoned on. So "a sensation defined as to time and space, i. e., a perception which becomes through the accession of the cause an object of knowledge we call a representation, or an idea, or a thought. Ideas are the exclusive contents of the whole higher intellectual life" (Preyer ut sup. 37). Such ideas in the average child begin in the second or early in the third year, and steadily strengthen thereafter up till maturity, when the brain-the last and most complex organ of the body to mature- has ceased to grow. This then, specially associated with the nerve cells in the gray matter of the brain, as a material basis, represents the flow of that most complex condensation of energy that we have named the cogitic.

If comparison be now made of the human organism in health and disease, it might seem at first sight, when we study works on nervous diseases or on psychopathology, that we are face to face with tangled and inexplicable biological phenomena. 
But a case which came minutely before the writer's attention nearly trelve years ago furnished what seemed to be a key to many of the nervous derangements described in man and the higher animals.

An extremely active, healthy, and intellectual married lady received a sudden mental shock at a delicate period in her life. The entire organism showed complete collapse, except that the vegetative or biotic organs-the alimentary canal, the heart, the glands, and the lungs - continued to perform their work, as if in an otherwise dead inert subject. The eyes remained closed, hearing and smell seemed gone, the voluntary muscles were passive, for weeks. Biotic energy alone continued to flow fairly actively; the cognitic and cogitic centers were practically passive.

Under careful nursing, a time-stage was reached when very slow but steady return of the cognitic capacity was indicated. The eyes opened; the fingers and skin generally became responsive to pressure and temperature changes; sounds gradually seemed to become correlated with slow movements of the eyeballs; motion of the limbs became more and more marked.

But the entire mental organism was still a tabula raza. No movement of the lips or power of speech was shomn; father, mother, and all nearest friends were unrecognized; no facial indication was given that even an elementary intellectual impression was being framed or conveyed. This state continued for nearly nine months. Then a child-like cogitic capacity began to reestablish itself. Some friends were recognized by active expressions of the eyes; others were only imperfectly or not at all recognized. Eventually a fair general recognition of them, and connection of them with past events, as well as developing power of speech returned.

Here protoplasmic activity and continued flow of biotic energy were the only indications of life, in the most depressed and inert stage. After months of such truly proto-biotic: existence, an active nucleo-plasmic and so cognitic state was reestablished above and upon the last. Only after nine months were the gray nerve cell material and the flow of cogitic energy 
so reestablished and inter-related that mental expressions began to appear.

Guided in part by such a human indication, the writer has perused with interest many text-books and papers on nervous diseases and abnormal states. He has in many cases succeeded in giving these a fair or even perfect biological interpretation. But to such return will be made later.

If the evolutionary stages or platforms that we have attempted to lay down for the human organism are correct, it might well be expected that in the transition from a state where all the bodily faculties are in action but have commenced to degenerate, and on to the period of death, a reverse relation in the organism would manifest itself. Such is the testimony of all human physiologists. Thus Ribot (54: 122) says: "The functions acquired last are the first to degenerate." And again more exactly he says (p. 114): "Since dissolution always follows the inverse order of evolution, it results that the more complex manifestations of will must disappear before the simpler ones, and the more simple before the automatic activity. In order to give to the statement of the law its exact form, treating volition, not as a singular event, but as the highest manifestation of activity, we will say: Dissolution pursues a regressive course from the more voluntary and more complex toward the less voluntary and simpler, that is to say, toward the automatic."

When finally we compare the normal human subject with one who has been subjected to various drugs or chemical agents, like biological relations are traceable. Thus in an acute alcoholic attack mental impressions-that at first are slightly quickened through general stimulation of the biotic and reflexly of the other two energy flows-and the power for high mental correlation first become lost; though the subject can still recognize and pass from a darkened to a lighted room, will recognize musical and other sounds, will retain for a time the use of voluntary muscles, etc. Thus, the cogitic state having become inert, the cognitic state is for some time retained but gradually becomes reduced. This, however, in turn is almost 
wholly subdued and the inert passive biotic state succeeds. If the alcoholic indulgence has been excessive, then, after a longer or shorter period, disintegration of the complex energyconducting protoplasmic molecules takes place and death ensues.

The action of poisons on the cells both of plants and animals seems also to fall under the three categories of biotic or protoplasmic poisons, of cognitic or nuclear-chromatin poisons, and of cogitic or nerve cell-Nissl-substance-poisons. As all are aware, our knowledge of poison or of drug action has been most fully studied, alike experimentally and observationally on animals and especially man. And, in shortly naming a few apparent cases out of many, we desire it to be understood that each group scarcely can restrict its action to one of the three living substances named, since all three substances are so intimately and organically inter-related. But mineral acids like arsenious or antimony, and glucosides like digitalin or strophanthin, seem to act mainly on the first; chloroform, ether, etc., on the second; and organic acids or their allied compounds such as prussic acid and potassium cyanide, oxalic acid and oxalate of potash, also many organic alkaloids, on the nerve-cell substance.

In closing this chapter it may not be inappropriate to compare the above phenomena in the human subject, and to learn how far they conform to, or seem contradictory to, the possible presence and interaction of biotic, cognitic, and cogitic grades of energy. At least on first consideration it might be asserted that all human energy merely varies according to its amount or degree of concentration in each cell, or in each chemical constituent of that cell. From the manner in which the various physiological responses, of increasing complexity, seem often to grade into each other, such a view looks at first feasible. But it in no way explains the striking and often sharply demarcated triple stages or platforms of advance indicated above, and which have been more or less recognized as stages or degrees by various writers, amongst others by Ribot and Romanes (55: 152), already quoted. Again it might be considered that 
the difference in the rapidity and complexity of response result is solely due to, and determined by, the molecular structure of each body constituent. But, while it may be granted that this has an important indicative significance on the static or morphological side, it in no way explains the dynamic or physiological differences observed.

In this connection reference might here be made to the substance or "granules of Nissl," considered by animal physiologists as being typical of cerebral and other nerve cells. These in normal condition, or when under sub-maximal stimulation, are abundant and are densely granular in aspect. But from combined observation and experiment it has been shown that with increasing mental effort the granules become less in quantity, possibly extremely minute, while after an ensuing rest period they again increase in amount. But this would simply suggest that their material is gradually used up in generating cogitic and, possibly by analytic transformation of this in turn, so much cognitic energy. Detailed consideration of this is made on a later page.

Again in relation to developmental evidence it has been noted that, at birth and for some time after, the constituent parts concerned in sight are embryologically imperfect and unconnected with the optic area of the brain (50:9). So in this and like cases it might be said that the optic-cognitic faculty was not shown, because the conducting apparatus was imperfect.

Each of the three types of energy then that we have outlined abore is capable of being absorbed by, conducted by, and of energizing its appropriate molecular substance, that itself seems to consist of an intra-molecular charge of like energy, around ether particles. Each also acts along the entire biologic scale through which it extends, from its earliest condensation and evolution. Thus the biotic we consider started with the most primitive Acaryota during probably the early mid-archæan epoch, and has extended up through the ages since, passing into the Caryota, and there energizing and composing the cell protoplasm up to man himself. The cognitic probably evolved 
gradually in the late middle or early last archæan epoch, and was continued as nuclear material through the higher Acaryota and on through the entire range of the Caryota. The cogitic probably originated feebly toward the close of the archæan epoch amid the higher colenterates, the simpler echinoid, and the simpler rotiferan types. It has since gone on elaborating increasingly complex linkings or combinations of its cell-substance up to the thousands of ganglionic cells of the human cerebrum or cerebellum.

Additional evidence for and against the present view mill be presented in succeeding chapters, but we would again assert that the recognition in the higher animals of three distinct energies that are alike graded and inter-related, that can gradually be transformed into each other as with the "inorganic" energies, that in ascending grade of energizing complexity are correlated with a like advance in molecular morphology, and that may yet under varying life conditions show surprisingly sharp separation in their phenomena, is alike warranted and promises to unravel for us many intricate life problems.

Naturally, if it can be demonstrated that the amount of any of the inorganic energies needed theoretically for the synthesis of the albumens or nucleo-proteids, not to say neuratin or Nissl substance, can be expended in upbuilding the enormously complex molecules of these substances, and yet remain there in balanced state as intra-molecular energy of an electric, a chemic, or a thermic kind, without causing constant disruption or burning of the tissue aggregate, then the need for any more perfect or concentrated form of energy is superfluous. Absolutely no evidence of such a possibility is as yet forthcoming.

So for shortness and exactness in our future descriptions we will speak of the following varieties of energy, in what re regard as their progressive scale of relationship and condensation. 


\author{
thermic \\ lumic \\ (tonic) \\ chemic \\ (molic) \\ electric \\ biotic \\ cognitic \\ cogitic
}

For reasons which need not be given here the highly important exhibitions of energy that we have termed tonic and molic (or gravic) will be considered later. A more detailed study of the characteristics of biotic and cognitic energies will next be made. 


\section{CHAPTER V}

\section{THE BIOTIC STATE OF ENERGY}

If the attempt be now made to determine further as to the possible existence and action of a biotic energy, it will be at once accepted that a distinct difference exists, between the simplest Acaryota at present known to us and inorganic bodies, in that the former possess what we call life. Now whatever life be, and no matter what extension or intension we give to the term, it must be conceded that life is an exhibition of energy. True, it is always and only associated with a definite complex of molecules, but even "when life has fled" these molecules remain, though they at once begin to break down into simpler compounds. Or rather one might say, from all the evidence to hand, that at the same instant that life is extinguished the molecules disrupt, and contrariwise, as life becomes increasingly evident in even the minutest cell, the molecules are in process of being built up and multiplied.

Whether it be the sluggishly growing and dividing cells of Sarcina or Gloeocapsa, the slowly gliding and waving cells of an Oscillatoria thread, the energetic, delicately chemotactic and actively multiplying cell of a Bacillus, the fundamental law evidently is that inert ether particles charged with an intra-molecular supply of energy are as exactly and accurately linked to each other as are the molecules in a crystal, and all available evidence indicates that this combination alone gives them their living characters. Life then might be defined as: "Relatively similar complexity and synchronism of motion of quinary, hexary, and heptary compounds, that represent similar complex definiteness of structure and similar lines of flow of biotic energy."

When the life-energy ceases to flow, even though abundant supplies of electric, chemic, and thermic energy be applied, 
the protoplasmic molecules at the same instant start to disrupt, and give rise to "secondary products of decomposition," which are readily recognized by every biochemical student.

But in the Acaryota, as compared with the Caryota, we deal only with one life substance-protoplasm - that exhibits all the fundamental regetative qualities associated with the protoplasm of even the highest of the Caryota. Life energy then, in its simplest and yet perfect expression, is primarily associated with protoplasm, as was generally accepted between 1870 and 1900.

By this we do not mean to assert that biotic energy is only associated with one definite chemical substance, but rather that the complex of constituent materials, which collectively we term protoplasm, is the special field for its activity and conductivity.

But the too exclusive attention given to the life histories of the Caryota, during the past fifteen years, has caused us to misinterpret or overlook the importance of protoplasm, in favor of the role played by nuclear material in the Caryota. So it seems of importance to emphasize that by far the larger number of unicellular organisms now living are in no sense acaryotic. Though still unicellular they have evolved a nuclear organization that confers on them greatly higher properties, in addition to those shown by the protoplasm that surrounds the nucleus. In a sentence, these exhibit the biotic energy of protoplasm plus the cognitic energy that traverses the nuclear substance. It is the failure to recognize this exactly which has, we believe, caused Binet in his otherwise excellent work (56:106) to attempt to discover "psychic" phenomena in unicellular non-nucleate forms. But equally his strictures on Richet's laws of irritability as drawn from nucleated muscle studies seem at least in part appropriate, when they oppose somewhat the over-confident laws there expressed.

The fundamental phenomena then of regetative life we regard as exactly and intimately bound up in and with the protoplasm. Additional evidence therefore will now be advanced for considering that a biotic energy resides in, traverses, 
and constitutes protoplasm when associated with correlated ether particles.

A peculiarity that deserves more than passing notice in the Acaryota, and in the Caryota that are subaquatic or aquatic in habit, is the tendency toward formation of gelatinous sheaths or even extensive gelatinous coats as in Gloocapsa and Aphanocapsa, or in the mycoprotein masses of many bacteria. This has often been regarded functionally as a protection against protoplasmic death by dessication. But in many of the forms that show it the chances for such are reduced to a minimum, while it is a striking fact that when allied types to these, as well as the higher plants, evolve as land dwellers the organism becomes dry, firm, and non-mucilaginous. Yet it is not that the gelatinous coat might be considered of special advantage. If we bear in mind that energy of all kinds is more easily conveyed through a liquid medium like water than through a gaseous medium like air, the function of the gelatinous coat as an energy-insulator becomes more likely. Such therefore we incline to regard it as.

This would satisfactorily explain the practically negative results obtained by Waller during study of various seaweeds and some samples of frog spawn, in his electric blaze experiments. For in both cases the thick mucilaginous coats may have prevented direct contact of the terminals or have absorbed the slight currents of electric energy passing out to them from the contained protoplasts.

If it be true even in the Acaryota that the cell energy which stimulates to nutritive metabolism of the protoplasm, to growth, and to division of the protoplasm be either chemical energy alone, or electric energy, or both combined, we might expect to find that, apart from organic tissues and by expenditure of an equal amount of energy as is given off in the burning of a definite organic mass, equally intricate bodies as the proteids could be built up. As previously emphasized the linking up from inorganic constituents even of the simplest organic colloid starch has hitherto failed, even under application of abundant and diverse charges of energy. 'Therefore, so far 
as our present knowledge leads us, the kind of energy needed for elaborating protein compounds and even simpler colloid bodies such as cellulose and starch can only reside in, and is conducted by, protoplasm.

Against such a view is the important fact that all colloid bodies, up to the most complex albumins, seem to be constantly associated with electric synthesis and analysis or are iso-electric; and further that Mann even regards them (5\%: 264) as electrolytes in action. But this in no sense militates against the continuous existence of a greatly higher and more condensed energy, into which electric energy is steadily being converted. For similarly light is constantly being changed into higher energy in green plant cells, and conversely electricity is oftenperhaps constantly-being changed into heat. So the high degree of electric energy indicated by active ionization changes may merely be an index to greatly higher energy storage or energy expenditures on the part of colloid organic molecules.

If we return to another phase of the situation that has already been referred to, it can be said that many of the simpler Schizophyceæ, as well as the simpler Schizomycetes, can be dried and dessicated for months, but we still have to deal with so much living organic matter in a highly complex though dormant molecular state. In other words, we have stores of some energy possessing orbital or oscillation movements round ether particles, that retain their potential energy and to a slight degree their kinetic energy in so continuous a state that even after long dessication when moistened in presence of oxygen of the air, at a suitable temperature, rapid outflows of electric, chemic, and thermic energy are established, and pronounced vegetative changes ensue. But none of the inorganic energies seems capable of continuing and accentuating such changes.

In the above connection, however, statements by the distinguished animal physiologist $\mathrm{A}$. Waller seem to the writer mistaken from all experimental results. We refer to the classification of organic matter into living and non-living, and the further placing of dormant seeds under the latter category as "formerly living," He speaks as follows (48:5): "Dry seeds 
kept for long periods in hermetically closed vessels have not been found to manifest any evidence of the most fundamental and general chemical change occurring in living matter, viz., a production of $\mathrm{CO}_{2}$. Their chemical reply to the question, 'Are you alive?' has been 'No.'

"But does this negative answer 'not-alive' imply that such seeds are dead? Evidently not, as may be seen if, under suitable conditions of temperature, moisture, and so forth, they are found to germinate and grow into plants. So that a seed, insofar as it does not manifest chemical change, is not proved to be living, and, inasmuch as it germinates, is proved not to be dead. Evidently, here is a dilemma; in the absence of an objective chemical sign of life, we have no right to say that a seed is alive; it is, as far as we can tell, not-alive; in the presence of its subsequent germination we are assured that it is living, and that therefore it was not dead. And the usual manner of escape from this dilemma of the seed which is neither living nor dead is to say that it is in a state of latent life, during which there is a complete suspension of chemical changes characteristic of the living state."

To escape from the above dilemma he considers that one of two explanations might be offered, "firstly, that our means of chemical investigation are not refined enough to reveal to us the smallest and most infinitesimal changes that may be going on in an apparently dry and perfectly dormant seed; and, secondly, it is possible that chemical change may be completely and absolutely arrested (e. g., by low temperature) without that arrest being of necessity final and definite.

"I will place evidence before you of this point, which I have investigated at some length. As to the second, which I have not myself investigated, I will only mention that it appears to be established by the observations of Horace Brown, who found that dry seeds kept for 110 hours in closed vessels at a temperature of $-183^{\circ}$ to $-192^{\circ} \mathrm{C}$., i. e., seeds in which the arrest of chemical change must be considered to have been absolutely complete, germinated quite normally when they were placed under suitable conditions of temperature and moisture." 
Instead of the above accepted explanation, we would suggest that such seeds possess in their richly protoplasmic and foodstoring cells-greatly as have schizophyceous cells-a locked up mass of intra-molecular biotic energy that very slowly undergoes letting down, it may be through long years, but that we have no special and exact test for recognition of this energy as yet, unlike the means that can be adopted for recognition of electric and chemic activity. So for each species or family of seeds very different lengths of time may elapse for dormant phenomena, but a maximum period is reached after which death occurs. If it be asked equally for dessicated schizophyceous masses, for species of bacteria, or for dormant seeds, what change occurs to bring about this maximum, we would reply that our present information would incline us to consider that steady loss of water gradually converts certain of the colloid constituents of the protoplasm, or possibly constituents of the reserve food, into irreversible hydrogels.

From the standpoint of energetics we do not yet know what exact physical change occurs when thermic energy is converted into lumic, nor when lumic or thermic is converted into chemic, nor when any one of these is converted into electric. So, granted the existence of a biotic energy, it need not be regarded as a point of weakness if we say that we do not know how any of the lower energies become converted into it.

But when we prove experimentally that a definite and moderate amount of thermic energy will cause union of binary bodies which contain the same elements as are needed for organic structures, such as $\mathrm{CaO}$ and $\mathrm{CO}_{2}$ into calcium carbonate $\left(\mathrm{CaCO}_{3}\right)$; that a definite amount of chemic energy expended on two bodies, one a simple the other a binary substance such as oxygen and ammonia, will cause formation of the ternary compound nitrite of ammonia, according to the formula: $4 \mathrm{NH}_{3}+3 \mathrm{O}_{2}=2 \mathrm{NH}_{4} \mathrm{NO}_{2}+2 \mathrm{H}_{2} \mathrm{O}$; that, when a ternary compound such as ferrous sulphate $\left(\mathrm{FeSO}_{4}\right)$ is dissolved in a solution of potassium cyanide (KCN) and heated, the combined thermic and chemic effect is to link up constituents of these into potassium ferrocyanide according to the formula: $\mathrm{FeSO}_{4}+6 \mathrm{KCN}=$ 
$\mathrm{K}_{4} \mathrm{FeC}_{6} \mathrm{~N}_{6}+\mathrm{K}_{2} \mathrm{SO}_{4}$; that a definite amount of lumic energy will cause union of binary or more complex bodies, as when mercuric chloride acts on ammonium oxalate to form ammonium chloride, according to the formula: $2 \mathrm{HgCl}_{2}+\left(\mathrm{NH}_{4}\right)_{2} \mathrm{C}_{2} \mathrm{O}_{4}=$ $2 \mathrm{NH}_{4} \mathrm{Cl}+2 \mathrm{CO}_{2}+2 \mathrm{HgCl}$, we obtain a faint notion of the amount of energy required to combine even the simpler ternary organic bodies, such as sugar or starch.

For the formation of the complex protein constituents of protoplasm, necessarily an enormously greater absorption of energy must be effected. Now, whether it be the cell of a cyanophyceous alga or the protoplasm of a human gland-cell, the energy needed to build up additional protein or protoplasm from simpler foods must be great.

An approximate estimate of the enerzy absorbed by an organism in order to continue its life processes is got, if we compare first the number of heat-units or calories needed to combine some simple inorganic bodies. Thus in the formation of hydriodic acid (2HI) from two atoms of hydrogen $\left(\mathrm{H}_{2}\right)$ and two of iodine $\left(\mathrm{I}_{2}\right)$ 12,200 calories are absorbed. The heat of formation of anhydrous zinc sulphate $\left(\mathrm{ZnSO}_{4}\right)$ from zinc ( $\left.\mathrm{Zn}\right)$, sulphur (S), and oxygen (O.) is 14,637 units. In striking contrast to these is the typical simple organic substance cane sugar $\left(\mathrm{C}_{12} \mathrm{H}_{22} \mathrm{O}_{11}\right)$ which is represented by $1,354,000$ calories. For an amide or proteid, therefore, composed of four to six elements in complex combination, the number of calories would be greatly increased. Reference to page 76 will illustrate this.

Now, in the autotrophic nutrition of the sulphur and allied bacteria, the continued existence of the cell, and its continued multiplication, are dependent on energy obtained from a chemical source. But chemical energy as such could not explain the linking together of all the protoplasmic constituents needed for continued life and multiplication of the cell, for in the process an amount of heat would be evolved that would char the already existing protoplasm. But if we accept that the chemical energy is gradually changed into a much more perfect biotic energy, which during condensation and accumulation is ab- 
sorbed into and energizes the increasingly complex colloid molecules, such fulfills the apparent needs of the case.

Again, in those plants that have reached a still higher and more complex condition by the gradual evolution of chlorophyll, if we consider the cylindrical green screen or chromatophore of Blue-green Algæ to be, as already suggested, at once an absorber, a transformer, and an accumulator of lumic energy, that is there condensed into biotic energy and in accumulating could be expended for the upbuilding of glycogen within the chromatophore, while a quantity of the same energy, conveyed into the protoplasm, could be used for repairing its waste and for the formation in higher members of the group even, of elementary or evolving chromatin substance, we get a connected and explicable view both of the morphology and of the physiology of such cells.

If we now accept it as a good working view that chlorophylloid protoplasm is an energy transformer, the question at once arises: are there other cases of such transformation amongst plants or animals? Our knowledge in regard to plants is still too limited to verify such, though luminous bacteria and higher fungi suggest the rapid conversion of biotic into lumic energy. But some striking cases occur amongst animals. The electric eel and the torpedo fish both have enormous demonstrable stores of energy, which can be discharged as powerful electric shocks. But such accumulations of energy are not exhibited or discharged by the unirritated fish, which then differs in no way from other species; nor after successive discharges have weakened them does their body differ from that of other fishes, except that they have special "electric organs." Now these consist of connective tissue in which ramify rich supplies of nerves and of blood vessels. The rapid generation of electric energy by them is usually ascribed to transformation of chemical substances brought by the blood and decomposed by nervous stimuli. But this involves a rapid transfer of definite compounds by the blood, and the chemical transformation of these from a potential chemical into a kinetic electric state.

In line with other evidences adduced, we would consider, rather, that the electric organs store complex organic molecules 
that are charged with the condensed and perfect form of biotic energy. On stimulation from the brain or spinal cord, the nerves act on and break up these biotically-charged molecules, with discharge of energy in the form of the lower or electric type.

A similar explanation would apply to the luminous organs of insects and of some fishes, to such thermic organs in plants as germinating seeds or flower spathes, and to various portions or organs of animals. Naturally in all of these cases we premise constant chemic transformation, but we regard this as a process essentially subsidiary to a physical energizing activity that primarily involves flows of energy from a definite point, that becomes a center of stimulation, due to the action there of an external stimulus, or, following Eimer, of an internal stimulus that originally represented external stimulation.

Chlorophyll and protoplasmic substance then in themselves seem to be mainly upbuilders and accumulators of biotic energy, and as already stated (p. 77) show in their simpler organic personalities such as the cells of Chroococcus or Aphanothece rather sluggish response to environal stimuli such as gravity, light, heat, or chemical agents. So they appear as irregular, diffuse, and uncorrelated patches of cells. But, as chromatin granules, threads, and skeins become increasingly evolved in higher members of the group, corresponding delicacy of response can be traced. To this, however, we can return in succeeding pages.

As regards the capacity for resistance of such protoplasm to extremes of environal action, we show in another section (p. 57) that many simple organisms now living can endure without injury exposure to temperatures that range from $-200^{\circ}$ C. up to $+100^{\circ} \mathrm{C}$., particularly when enclosed in mucilaginous or similar coverings that prevent over-rapid flows of energy. Elsewhere and in the present work (p. 27) the writer gives reasons for believing that primitive organisms evolved when the average water temperatures of the earth varied between $75^{\circ} \mathrm{C}$. and $85^{\circ} \mathrm{C}$. Resistance to other environal agents will be considered later. 
But, if bacterial or nostacaceous or like material be gradually dried and kept in such state for some months, we deal continuously with so much matter in a highly complex though passive molecular state. This however retains the capacity, when moistened and exposed to suitable temperature, of exhibiting in a short space of time currents of energy that start transformation of the molecules and reëstablish all the phenomena of life. Now this surely means one of three things: either (a) that some kind of energy remained potential but latent in such organisms during the entire period of dessication; or (b) that the molecular constitution of the matter itself was such that, when wetted, it acted as an absorber from without of, and conductor for some kind of, energy; or (c) that some "mysterious" property resided in the matter that might be called the "life property" and which was capable of causing energy to flow through the dormant matter under suitable conditions.

The last of these alternatives is a meaningless refuge of ignorance when no better explanation can be got. The second alternative is unsatisfactory since it takes for granted that such organic matter is able to exist for long periods of time in a dormant state, and yet, when suitable environal conditions presented, that it could become active again, and assimilate afresh. But, if there is any one fact in relation to such dormant organic matter that is more clear than another, it is that all such matter becomes dead and inert after a measurable period due evidently to the dissipation and removal of some energizing constituent that it formerly possessed.

If therefore we now turn to the first of these alternatives, we must confess that such energies as the thermic, lumic, chemic, or electric would, according to our present knowledge of these, be dissipated or reduced to a uniform heat level, long before the expiry of the time named above. Possibly to this statement the only exception might seem to be chemic energy. But it is well known that though we mix somewhat dry eggsubstance, starch, sugar, or other organic bodies, that might all seem to contain abundant chemic energy, these only decay -.her moisture, oxygen, and appropriate heat are supplied. 
It is remotely supposable that electric energy might, by specially condensed action, build up quinary and hexary molecules, and after their formation continue to renew them, if sufficient like energy were added to replace that which is constantly being dissipated. But absolutely no proof of such exists. As already suggested, however, from the relative dissipation ratio. of thermic, lumic, and electric energies, there is strong warrant for assuming that dried protoplasmic substance is the seat of a slowly dissipating and highly perfect energy that can only travel along complex protoplasmic molecules as conductors, but which after a measurable time dissipates therefrom, unless means be adopted for increasing its quantity, and in the process for adding to the matter along which it travels. This we have called the biotic energy.

When we pass in review the Acaryota as a simple and primitive group that is possibly traversed by a biotic energy, the feature that rises before the mind is a material or morphological series of forms possessed of certain common characters and also of certain recognizable specific differences. And in our monographs, text-books, and memoirs these are the details that are alone emphasized. But, while this is rightly so, when we think only of visible or descriptive evidences of evolutionary modification, if we attempt to reach the basic cause or causes of such morphological details, we perceive and must acknowledge that such is all due to varying energizing action. For every atom of carbon in the mucilaginous wall of a blue-green alga, every molecule of glycogen that is contained in the cavity, every constituent of the chlorophyll and blue pigment that is associated with the production of the glycogen, every complex molecule of the protoplasm that is the highest product of the cell, have each and all been linked together and lifted into a position of porer and complexity not by any inherent quality or capacity which they as such possess, but purely and solely in virtue of the energy that has guided and placed each. So, while the different molecules, according to their relative complexity, are gauges, indicators, or measures of the amount and quality of energy that has been spent in lifting, 
linking, and holding them together, the energy that has done this is the truly important agent, no matter though we may have difficulty in isolating it, or studying it apart from its material consort and conductor. Now, since work of high complexity is done in such cells with comparative ease and by high utilization of energy in the process, we believe that the recognition of a biotic energy as typical of the Acaryota will help us greatly toward a true interpretation of their life changes.

We may now attempt to secure evidences for the existence of a biotic energy in nucleate or chromatin-forming plants. Here, however, if our views are correct, we must ever bear in mind that the union of protoplasm and chromatin represents the correlative display of biotic and cognitic energy. So for demonstration of the continued action of the former energy we must either be able to observe nucleate plants under conditions where one of these energies mainly is in evidence, or to analyze up each, apart from its constituent energy. Both methods seem helpful and possible.

In nearly all land plants, and particularly in those which are exposed to marked seasonal changes of hot and cold weather, or of dry and moist states, there are alternate periods when each plant shows what might be called an active and a passive phase, a growing and a dormant seasonal condition. Thus in temperate regions, at the close of the growing season of the year, the more actively responsive parts, such as the leaves, the flowers, and even young twigs, fall to the ground. Throughout the winter and early spring, active response to environal stimuli has ceased, but the plant is not dead. Nor is it merely a passive inert mass of chemical material, for the protoplasm and reserve food do not decay; they are not readily invaded by fungi as masses of inert chemical food would be; on warm days even the protoplasm may show "vital" activity and start sap-flow. In other words there is some reserve of energy in the comparatively sluggish or dormant cells, which causes continued energizing of the protoplasmic and protein molecules; which may set free and start, on short exposure to suit- 
able temperature, water and oxygen supply, the metabolism of stored food; which also, in contrast to the shed leaves and flowers, can resist extremes of temperature up to $60-65^{\circ} \mathrm{C}$., or as low as $-30^{\circ} \mathrm{C}$., and yet, on return of favorable conditions, start into full activity of growth.

Now if such plants be kept so environed that they are exposed not for a few months but for years continuously to such dormant states, a stage is reached-as at times happens in hot countries that for years may have no appreciable rainfallwhen the protoplasm fails to remain sufficiently energized, and the organism disrupts in its living machinery.

Throughout all the phases of this process there is a continuous flow of some form of energy, as is proved by the slow utilization of reserve starch or oil during the process. Were it electric energy, the plants nould reveal such in disrupting their reserve supply, unless we suppose that it is steadily discharged into the earth. Or, were it chemic energy, the plants would be denuded of their reserve food supplies, which often is by no means the case. But the abundant supply, the labile character, the resisting capacity, and the persistent flow for months or even years of the kind of energy here involved, all indicate that we have to deal with a more perfect and condensed exhibition of energy than those of inorganic bodies, but which after expending itself for a certain period becomes exhausted, and then, though reserve food be still present in abundance, death of the plant occurs.

Again in not a few tissue-cells of higher plants, that are alive and perform mainly a vegetative function, the nucleus may be absorbed, the protoplasm may alone remain, and yet vegetative functional activity may continue. Such are seen in sieve-tubes, in many latex cells, apparently also in some food-storing cells of tubers and seeds. In the case of sievetubes even, a seasonal variation in formation of plate-callus towards the close of each season may alternate with digestion and resorption of the callus substance at the beginning of the next seasonal growth. In all of these cases a slow and steady vegetative activity is shown by the protoplasm, at the same 
time that it is starting chemical changes in the contained substances of these cells. For such changes again, which involve? the liberation and action of ferments, we would suggest the existence of a biotic energy.

But it is when we study and experiment with dormant spores, seeds, or tubers, and with cuttings, specially of a woody nature, that highly suggestive confirmation of the existence of such an energy is got. As for some protophytic plants, so for spores and seeds, it has been shorn that many of these will live for years in dormant state, if merely removed from normal water supply. Similarly they can be exposed to decidedly high and low temperatures. But here also a fairly, or even exactly, definite time-interval is reached, when the protoplasmic molecules begin to disrupt and death ensues.

If it be here asked why some seeds, e. g., those of Graminaceæ, retain vitality only for one to five years, those of Malvaceæ for five to ten years, and those of some Leguminosæ for ten to seventy-five years, this seems in part to be due to the nature of the reserve food, but is probably in large measure due to the intimate relation of the embryo proper to its large food-storing cotyledons, in which, apart from the stored starch and protein, there is a large amount relatively of protoplasm in the Leguminosæ. On the other hand, in Graminaceæ research has shown that much of the starch may be stored in cells devoid of protoplasm, while those seeds in general which show short vitality have the embryo and stored food as separate structures.

The spores of many fungi are known to have a high temperature-resistance and age-resistance. Thus yeast according to A. Mayer continued to bud up to $53^{\circ} \mathrm{C}$. and when air-dried its cells will survive after heating to $100^{\circ} \mathrm{C}$. Cells of pressed yeast, at optimum temperature, will remain alive for two years. The stored energy of such minute cells must be great and of a perfect quality, to permit of survival for so long a period.

The possibilities for survival of woody cuttings or shoots, and of the aerial parts of desertic plants such as cacti, euphor- 
Dias, und stapelias, are also striking. Thus cuttings or slips of many well-known fruit-trees, willows, poplars, etc., may be kept for months, or in some cases even for years, if only water be withheld, though under conditions of temperature and oxygen that are normal. But during all of this period the protoplasm is slowly transpiring, and retains a reserve of energy that enables the shoot when placed in soil to form rapidly roots below and leaves above. In this connection the writer knows no more marked case than that of the Carolina Poplar or Cottonwood. A terminal shoot with stem ten to twelve feet in length, and that is as thick as one's arm, can be cut from a tree, allowed to lie dormant for weeks at rather low temperature $\left(1-5^{\circ} \mathrm{C}\right.$.), can then be planted in spring, and from the base of the stump abundant rootage will start, that in a season may reach to five or more feet in each root, while above ground vigorous leafy shoots will form. Naturally a large part of such luxuriant growth is effected at expense of the abundant reserve food stored in the tissues of the stem and branches, but alike in the primary growth-start, and as a continuous radiating energy flow, there can only be accepted an expenditure of some perfect quality of energy that greatly excels those of the inorganic world.

It may now be suggested that, if such an energy as the biotic exists in the manner indicated, it should be possible to lead it along appropriate conductors from one center of higher biotic tension to one of lower, or vice versa, just as electricity can be conducted rapidly along a silver or platinum wire, less perfectly along a copper one, and only slowly along a zinc wire. Such conduction and also convection currents are well known. Though, owing to the imperfect condition of our knowledge at present, we can state nothing regarding acayrotic types, it is now well established for higher forms that currents of electric, of chemic, and of thermic energy traverse the protoplasm. But in the case of the Carolina Poplar described above, and many similar examples even amongst herbaceous plants, the constant effort or energy-expenditure put forth by the tissues to push out as roots or leaves indicates a greatly more - 
condensed and subtle energizing activity than either osmotic pressure or any of the energies named above, while the continued energizing of the most peripheral cells also indicates continued flow of a highly complex form of energy.

In ordinary graft unions, and even more in graft hybrids, union of the living cells seems to be correlated with currents of biotic energy. We would not deny in the above, nor in many similar cases, that a still more complex or cognitic energyflow is also concerned, but to such we shall return later.

In passing from the plant to the animal world, the rarity or doubtful character of non-nucleate or acaryotic types prevents study of purely protoplasmic masses amongst the lower animals. But the continued vitality of the Amøabe, of many Infusoria, and of some Sporozoa, when dried and kept for months or even years, again indicates stored energy of an organic grade of complexity. Also the striking studies of Maupas (58:165), verified and extended by subsequent observers, show that after a definite period of growth and division the animals become smaller, senile, and then die. But the rapid movements of these infusors, the amount of energy displayed during division and in elaboration of added protoplasmic substance, all powerfully indicate again the existence and conduction through the protoplasmic substance of streams of biotic energy.

As in cases also of plant grafting, so in skin grafting, in transplantation of limbs and even of body segments of lower animals, likewise in blood transfusion, some condensed and yet readily transformable energy seems alone to explain the perfect manner in which union of the former and reactivation of tissues under the latter treatment occur. For while in blood transfusion the more perfect oxygenation of the patient's tissues may be aided by infusion of the healthy blood corpuscles into the blood vessels, and so give an exhilarated and strengthened stimulus, this alone does not seem to explain the rapid resultant benefit. When we remember also that the chemical constitution of the hæmoglobin of blood is $\mathrm{C}_{758} \mathrm{H}_{1203} \mathrm{~N}_{195} \mathrm{O}_{218} \mathrm{FeS}_{3}$ $(5 \%: 465)$, we get a faint idea of the enormous number of heat 
calories that it represents, and so of the enormous amount of intra-molecular energy that is stored in the hæmoglobin molecule.

Naturally here we would not neglect the protective, the metabolic, and the destructive action of the various blood corpuscles in relation to the tissues through which they are carried. But we would regard such actions as contributory to or associated with the direct activation of tissue, effected by increased or regenerated blood-flow, that increases supplies of biotic energy.

The remarkable experiments also of Carrel and of Ross Harrison, in temporary excision or transfer of vegetative organs in animals, receive a basic explanation, as we would consider, from the constant flow and energizing action of a biotic energy.

We still lack an exhaustive set of morpho-physiological studies on the action of various poisons on the cells and the entire individuals of the Blue-green Algæ. A comparison also of such action on the lowest non-nucleate types such as Chroococcus with like action on evolving chromatin types such as Oscillatoria would be most instructive. But, from the standpoint of infection-resistance against various pathogenic bacteria, extensive experiments have been made. These indicate that such mineral acids or salts as arsenious acid and copper sulphate, also various organic acids, and hydrocarbons such as menthol and eucalyptol, have a destructive action. This is possibly explained through withdrawal of needed oxygen from the bacterial to the protoplasmic molecule, as suggested by Binz and Schulz for arsenious acid. The formation of ozone by eucalyptol seems to suggest a similar relation. Various alkaloids, also, such as quinine and nicotine are bactericidal, and seem likewise to owe this property to their connection with normal oxygen supply.

In all of the above cases then, and many similar ones, disturbance of the normal oxygen supply probably breaks up the protoplasmic molecules, and so sets free the stored energy of the bacteria with their resulting death. It might here be objected that such substances would also seriously injure the 
living organism on which the bacteria feed. But the greatly more complex tissues of the former often secrete or set free products that are neutralizing to a greater or less degree to many chemical agents.

Other substances, however, than those mentioned above, if in sufficient concentration, act similarly on the protoplasm of higher plants and animals as on that of bacteria. Thus Loeb (59:21) says: "Budgett has observed that in many infusorians visible changes of structure occur in the case of lack of oxygen; as a rule the membrane of the infusorian bursts or breaks at one point, whereby the liquid contents flow out. Hardesty and I found that Paramccium becomes more strongly vacuolized, when deprived of oxygen, and at last bursts. Amœbo likenise become vacuolized and burst under these conditions. Budgett found that a number of poisons, such as potassium cyanide, morphine, quinine, antipyrine, nicotine, and atropine, produce structural changes of the same character as those described for lack of oxygen. As far as $\mathrm{KCN}$ is concerned, Schoenbein had already observed that it retards the oxidation in the tissues, while Claude Bernard and Geppert confirmed this observation. For the alkaloids, W. S. Young has shown that they are capable of retarding certain processes of autoxidation. This accounts for the fact that the abovementioned poisons produce changes similar to those observed in the case of lack of oxygen."

The action throughout in higher animals is apparently one in which the vegetative cells - those of the alimentary canal and of the unstriped muscle, of the associated glands of the canal, of the blood corpuscles, of the heart and the lungsbecome disturbed and in time death ensues, through disruption of the energized molecules that compose the living substance of them. Several suggested explanations might be given; but we here propose that such chemical bodies may have a special affinity for some such constituent of the protoplasmic molecule as oxygen, and by union of these a breaking up of the protoplasmic molecule ensues, thereby stopping or setting free the rhythmic flow of the biotic energy. 
So we conclude that protoplasm is the complex energized substance that is the fundamental seat of all metabolic or vegetative changes, and that the energy which binds together its complex molecular organization is of a greatly more perfect type than the lower energies, though next higher in quality to the electricity of colloids.

The two striking physico-chemical features, however, of protoplasm are its great stability on the one hand and its great lability on the other. Now, as Herbert Spencer long ago pointed out clearly, the former seems to be due to the carbon atom, the latter to the nitrogen atom, while oxygen is the gobetween element, that is alternately aiding in building up, alternately in breaking down. If steady and harmonic integration of carbon and oxygen with other elements predominates over nitrogen and oxygen integration, increase and multiplication of the protoplasm are effected; if integration of nitrogen and oxygen over the other two predominates, disintegration and waste ensue.

In regard to the great stability of protoplasm, as represented by any one plant or animal, we too seldom realize its character, even though familiar with the fact. Thus a Sequoia tree of 1500 years age, a Sea Anemone of 50 years, a man of 100 years, all represent organized continuity of protoplasm in each case. But, during the entire period of each individual's existence, a constant change in the elements that make up the protoplasmic molecules, even in the molecules themselves, has been effected. The one continuously uniform factor is the energy that binds together the whole. Localized parts may alter or die, leaves may drop or branches may be torn off by storms in Sequoia; fresh food may be ingested by Actinia that in weight may much exceed its own in a few months, and which is again visibly excreted to be replaced afresh; a man may sustain many injuries or local deaths of cells, but these may be and often are satisfactorily replaced. All of this we would consider to be due to continuous flows of biotic energy.

As to the great lability of the nitrogen atom, this is equally evident, and has often been discussed. So the living proteid 
or group of proteids possibly, that we term protoplasm, has a highly unstable character in virtue of the $\mathrm{N}$ atom. Now, in the decomposition products of protoplasm and of proteids generally, a constituent part of these seems always to be the radical cyanogen $(\mathrm{CN})$. Verworn, therefore, in commenting on and quoting from Pfluger's studies on this subject, says: "This points strongly to the probability that living proteid contains the radical cyanogen, and thus differs fundamentally from dead or food proteid. Pfluger therefore says: 'In the formation of cell substance, $i$. e., of living proteid, out of food proteid, a change of the latter takes place, the atoms of nitrogen going into a cyanogen-like relation with the atoms of carbon, probably with the absorption of considerable heat. That considerable heat is absorbed in the formation of cyanogen follows from the fact that, as calorimetric investigations show, cyanogen is a radical possessing a great quantity of internal energy. By the addition of cyanogen to the living molecule, therefore, there is introduced into the living matter energetic internal motion.

"Accordingly the great property of decomposition possessed by living proteid is explained as the result of the absorption of oxygen; for, since the atoms of cyanogen are in active vibration, the carbon atom of the cyanogen at the approach of two oxygen atoms will pass out of the sphere of influence of the nitrogen atom into that of the oxygen, and will unite with the latter into carbonic acid. Thus the cause of the formation of carbonic acid, i. e., of the decomposition of the living substance, lies in the cyanogen, and the condition is the intra-molecular introduction of oxygen." "'

The last sentence deserved special consideration, for in connection with poisoning of the protoplasm we have suggested that withdrawal of oxygen from it seems to be the main cause of its molecular disruption. The immediately succeeding formation of nitrogenous cleavage products such as creatin and lecithin, in which cyanogen-like groupings occur, suggests in this as in other protoplasmic changes that withdrawal of needed oxygen causes splitting of the cyanogen, or of an allied nitro. 
genous substance, with accompanying exhibition of electric or thermic phenomena, through degradation of the biotic energy. The oxygen atom therefore seems to be that which, by its vibratory action and capacity for becoming attuned or harmonized with other elemental atoms, brings needed supplies of energy to the protoplasmic molecule, and in part uses this with $\mathrm{C}$ and $\mathrm{H}$ of the molecule for formation of carbon dioxide and water.

Now such combining or oxidizing action and splitting or catalytic action seem to be constant phenomena of living protoplasm, and proceed often at a relatively rapid rate. They are probably due to the action of peroxides, or of a group of ferments, the oxidases in the former case, and to the action of a great group of cleavage ferments in the latter case, both of which are themselves products of living protoplasm. But the oxygen absorbed by any plant or animal seems in considerable part to be used in continued energizing of the protoplasmic molecule, so that the organism can carry on increased growth and cell division, while only half the amount is probably used in the combustion of organic compounds revealed by the respiratory exchange. And it is the continued autoxidizing action, along with resulting growth and division, that represents a kind and quality of energy different from those hitherto generally recognized, and which we have designated the biotic. 


\section{CHAPTER VI}

\section{THE COGNITIC STATE OF ENERGY}

We shall now inquire as to chromatin substance, and its possible relation to a cognitic energy. Thereafter cases will be considered in which intimate union of protoplasmic and chromatin substance gives to most plant cells and simpler animal cells a combined bio-cognitic energy.

It has already been pointed out that non-nucleate cells differ from nucleate ones in the feeble and sluggish response of the former to environal agents. But the more rapid response of the latter means a more perfect and quickly discharged energyactivity. So in the more evolved bacteria and in Blue-green Algæ, like Oscillatoria, Lyngbya, and Tolypothrix with their chromidial granules, threads, or skeins, marked and even at times rapid sensory response to environal stimuli is seen.

But, to appreciate fully what this means, we must bear in mind that nearly all simpler chromatin or nucleated organisms show definite response to heat, to normal light, to intense illumination, to gravity, to rotation, to chemic agents, to water, to tactile stimuli, and to electricity. So, in passing from the simplest nucleate organisms up to man, the writer would consider that account has to be taken of nine senses or stimulation areas for reception of environal stimuli. These are the thermotactic or heat-perceptive, the heliotactic or light-perceptive, the geotactic or gravity-perceptive, the chemotactic or substance-perceptive (that in higher animals becomes separated into the olfactory and gustatory senses), the hydrotactic or moisture-perceptive, the thigmotactic or mechano-perceptive (that in higher animals becomes separated into the touch and sound senses), the gyrotactic or circumnutating, the electrotactic, and the paraheliotactic or supra-light-perceptive, senses.

The questions then arise as to how these areas or centers hare evolved. what is their most primitive expression, what 
evolutionary stages can still be traced in ascent from the simplest to the highest plants, also from the simplest to the highest animals, and finally what connection they may show with chromatin and a possible cognitic energy. For none of these questions can be separated from each other.

If we start with the view that the primitive and simplest spherical bacteria were composed only of protoplasm that was mainly vegetative and autoenergizing in function, in virtue of their biotic energy-and all observational evidence is in favor of this-such cells would show sluggish and also equisymmetrical response to environal stimuli. But the environal stimuli that would be called into play would be first the chemotactic and next the heliotactic. For, if the view already put forth here (p. 40) be correct, primitive spherical plants that had the same mode of nutrition as have the sulphur and iron bacteria now would be mainly, perhaps wholly, chemotactically equisymmetrical.

But, with the gradual evolution of the peripheral chromatophore, the centrally placed protoplasm would retain its function, but glycogen food would be formed in the former, and on accumulation would appear as the globules of Hegler and others. In process of slow evolution, however, from the blue-green to simpler green algæ (Chap. II), the glycogen evidently became changed into the more complex oil or starch, now met with in the green algæ as the reserve oil or carbohydrate. This necessarily involved a greater expenditure of energy for oil formation, and still more for starch formation (26:62).

So, in the peripheral chromatophore-centers of highly energized substance, the pyrenoids or chloroplast centers arose, which by their high intra-molecular energy could transform the crystalloid sugar or glycogen, into the greatly more complex colloid, starch (p. 154). These pyrenoids or plastids we regard as chromatin masses, alike from their structural relation to cell-nuclei, their stainability, their connection with the cell-nuclei (p. 128), and their mode of multiplication.

But, as we pass to the higher groups of bacteria and of the Blue-green Algæ, increasing polarity in relation to the earth 
is shown, till it becomes a striking and inherent characteristic in nearly every one of the higher species. The question may well be asked then: Are there special chromatin or sense perceptive centers for perception and focusing up of the heliotactic, geotactic, and other stimuli? During the past sixteen years, the writer has placed it before his students, as a working hypothesis, that the different tropisms shown by living organisms can alone be satisfactorily explained if we accept it that definite plastids or energids show a special sensitivity and polarity to environal stimuli. Such bodies he termed geoenergids, helioenergids, etc.

Thus when one placed, as he first did eighteen years ago for this purpose, roots of pea or broad bean at right angles to gravity, and after 12 to 24 hours removed, fixed, cut, stained, and mounted sections of the now curved root in balsam, it was found repeatedly that a much larger number of minute stainable chromatin-like bodies were in the shorter and more richly protoplasmic cells of the lower root surface than in the longer, paler, and more vacuolated cells of the upper. More recently Haberlandt seems also to have recognized these.

The manner in which ciliated algoid and fungoid cells, as well as plant spermatozoids, move by their clear anterior end towards any area of chemotactic stimulation indicates either that the protoplasm is specially chemotactic in that region or even that some definite plastid or plastids may there be located, unless indeed the frequent thickening at the base of the cilia does not represent such a chemotactic center.

We know, however, from the investigations of Meyer and others, that leucoplasts are of wide occurrence in plant cells, that they can transform sugar into starch, and may themselves at times become chloroplasts. When we add to this that chloroplasts can usually orient themselves in most algæ and in higher green plants to incident light rays, there seems considerable warrant for the view that chemoenergids, photoenergids, and geoenergids have evolved amongst the higher members of the simple algæ in the order indicated, and have persisted throughout the vegetable kingdom. 
Reasons have been advanced in another part of this work for the view that animal forms have been derived from plants (chap. 14). Now, while corresponding plastids or energids to those above cited in plants have not yet been identified, the often restricted area by which food is perceived and held, or other particles rejected, amongst most protozoa, would suggest that in addition to the general cell-protoplasm there are special chemotactic centers for food perception. Thus Jennings regards the anterior end of the body in Amœba as the specially chemotactic region, and remarks: "It is sometimes said that these Amœbøe take food at the wrinkled posterior end. This, if true at all, is certainly rare; the author has never observed it, though he has seen food taken in dozens of cases. The essential features of the food reaction seem to be the movement of the Amoba toward the food body (long continued in some cases), the hollowing out of the anterior end of the Amoba, the sending forth of pseudopodia on each side of and above the food, and the fusion of the free ends of the pseudopodia." Amongst infusors also the same is suggested. For Jennings has proved that they show, like definite plant parts, positive or negative chemotropism, according to the strength or nature of the solution in which they are, and also that there seems to be a fairly exact location of the chemotactic area. Thus he says, speaking of repellent chemical stimuli: "The direction toward which the animal turns in the avoiding reaction does not depend on the side of the animal that is stimulated, but is determined by internal relations. The animal always turns toward the aboral side. It is true that with chemical stimuli the stimulation usually occurs on the oral side, so that the animal turns away from the side stimulated."

As we ascend in the animal scale, however, chemotaxis becomes located mainly or wholly in the olfactory and oral areas, which represent a correspondingly concentrated energizing of the chemotactic cells. But these areas are the first usually of the sense centers that unfold in the embryo.

Heliotactic stimulation and response are markedly shown by many ciliated algoid cells. Thus, no matter whether we 
regard Euglena as a free-swimming plant, as a transition type from such to the animal side, or as a flagellate infusor, the presence in it, as in many other motile algæ, of a red "eye-spot" distinct from the chromatophore, is proof that the chlorophylloid areas are not the only heliotactic centers. Engelmann's experiments (38) with this organism make it "clear that the anterior end is more sensitive to light than the remainder of the body."

But that even plant parts which are colorless are highly heliotactic is proved by the aerial growths of Pilobolus, Mucor, etc., though as yet we are unaware whether definite helioplasts are the cause of the movement. According to the experiments of Jennings (42:133), most infusors that were studied seem to be heliophobic, or may possibly be heliotactic to feeble light. He says: "Every time that the anterior end swings into the light, the avoiding reaction is caused; the animal therefore swings its anterior end in a large circle, trying many directions. Every time it swings its anterior end away from the source of light into the shadow of its body, on the other hand, no reaction is produced; the position thus reached is retained. This process continues, the animal trying new directions every time its anterior end swings toward the light, until in a short time the anterior end must inevitably become directed away from the light. In this position the anterior end is no longer subjected to changes in illumination, for the axis of the course coincides with the axis of the light rays, and the body maintains a constant angle with the axis of the course. The amount of light received by the anterior end therefore remains constant. Hence there is no further cause for reaction, and the organism retains the position with anterior end directed away from the source of light."

While in higher algæ and still more evolved groups the heliotactic sense becomes as widespread or diffuse as are the chloroplast centers, in the main lines of evolving animals it becomes increasingly restricted to one, two, or more anterior heliotactic organs, the eyes. So, in the Rotifera, the Turbellaria, the Nemertinea, and upward through the main vertebrate line 
(Chap. XV) to man, the pigmented eye-spots or eyes are anterior structures that develop embryologically after the chemotactic or olfactory and oral areas.

The geotactic region in plants is the basal or fixing region. In the free-swimming cells or zoospores of the unicellular, as well as the multicellular, algæ, the clear anterior ciliated end that is devoid of chloroplasts is the fixing region. This seems to be determined, in part at least, by molecular constituents of the protoplasm, that are photophobic. But, combined with these or aided by them, there evidently is a definite set of constitutent molecules that show a positively geotropic and geotactic irritability. In the formation of embryo plants from the spore also, amongst the Hepaticx, the Musci, and the Filices or ferns, the first division wall laid down nearly always serves to demarcate a colorless geotactic cell from one that contains chlorophyll, and that is both apogeotropic and heliotactic.

Thanks to the experiments of Darwin, Czapek, and successors, we now know also that the growing tip of phanerogamic roots is geotactic, and this over a neatly restricted area. Further the propagation backward of a stimulus from the tip to an older root area that is geotropic indicates that there is $a$ definite linking up of molecules, whether after the visible manner described by Nemec may be left open for the present. These seem so to affect the protoplasmic sacs of the geotropic cells that they lose water on one side and become more turgid on the other, so as to cause bending in the root as a whole.

In multicellular animals the geotactic sense appears to be so intimately blended and associated with the gyratory that a common sense-center for both is alone suggested by the morements executed under natural and experimentally modified states. But the definite geotropic responses secured by Loeb for some actinians and echinoderms $(60: 57)$ are proof that specialized sense-centers exist in these. The constant effort also, on the part of most animals, to assume a definite satisfied position in relation to gravity is strong suggestion alike of a geotropic sense and of geotactic centers. But our 
knowledge of structural and experimental details is still of the most meager nature for animals.

A fourth sense is the gyrotactic or rotatory, regarding which we still know far too little. It was one of Darwin's rich contributions to biology, when he showed that the organs of many and great plant groups showed a gyrotactic or circumnutating movement. It would be impossible here even to touch briefly on all the views that have been expressed in explanation of the phenomena. But when we find such movements exhibited in pronounced manner by many motile bacteria, by swarmspores generally, by the spermatozoids of plants and animals, by Spirogyra filaments, as well as other rather simple but multicellular algæ, and increasingly developed as we pass to the highest plants, this is partial proof of its far-reaching character. That the phenomenon is mainly shown by living elongating cells, that it proceeds in root-tips buried in the soil, as well as in stem-apices exposed to light, that some flowering plants such as the "stem twiners" practically owe their capacity for survival to its activity, are proofs of its relation to the living cellsubstance, and its tendency to be located in active free cells, or in terminal growing areas of an organism.

But we are ignorant as yet of any definite structure or plastid of the cell that may govern or aid rotatory or circumnutation movements. All are agreed, however, that such movements are only effected in living and even young tissues, and are due to varying turgidity of cells, and ultimately to physico-chemical changes proceeding in the protoplasm.

Direct continuity however of such sense-movements from plants to animals has not, so far as the writer is aware, been traced. Yet it is now experimentally demonstrated that "the sense of rotation" is typical for vertebrates. The writer also suggests the existence (chap. 15) of rotatory sense organs in nemerteans. For in these a portion of the auditory organ that seems exactly to correspond to the organ of rotation in lower vertebrates is present. The question therefore presents itself as to whether such a sense and such a sense-movement exist in simpler and specially in unicellular animals. The observa- 
tions of Jennings (4\%: 44,47,62,85) supply an apparently helpful answer in the case of the infusor Paramocium, for in causing the typical constant movement he says: "The stroke of the cilia is always somewhat oblique, so that in addition to its forward or backward movement Paramœcium rotates on its long axis. . . . The swerving in any given direction is compensated by an equal swerving in the opposite direction; the resultant is a spiral path having a straight axis." Again he says: "Since in its spiral course the organism is successively pointed in many different directions, the samples of water it receives likewise come successively from many directions. Thus the animal is given opportunity to 'try' the 'various different conditions supplied by the neighboring environment. Paramœcium does not passively wait for the environment to act upon it, as Amœba may be said, in comparison, to do. On the contrary, it actively intervenes, determining for itself what portion of the environment shall act upon it, and in what part of its body it shall be primarily affected by the varying conditions of the surrounding water. By thus receiving samples of the environment for a certain distance in advance, it is enabled to react with reference to any new condition which it is approaching, before it has actually entered these conditions."

Further, in the highly interesting reactions to electric stimuli, according to the strength and direction of the current, the organisms show an extended or restricted rotation. The same author also says: "Practically all the infusoria agree with Paramøcium in swimming in a spiral when passing freely through the water, and in the fact that when stimulated they turn toward a certain side defined by the structure of the organism." Chilomonas, Euglena, Colpidium, and other unicellular types there described likewise show rotatory movement, not under one but varied forms of stimulation.

The group of the Rotifera is, according to the writer's estimate, one of prime importance as forming the metazoan starting point for most invertebrate groups (chap. 18). Since the time of Ehrenberg, various careful observers have described the rotatory movements shown, and these may be shortly 
summed up in Jenning's recent statement (p. 242): "In many free swimming Rotifera the chief methods of movement and reaction are similar even in details to those of the free-swimming Infusoria. Like the Infusoria, these rotifers swim by means of cilia, revolve on the long axis, and swerve toward one side (usually dorsal) as they progress."

But as indicating an increased tendency to localization of rotatory sensation at the anterior end of the organism, as compared with the diffuse sensation in infusors, he adds: "It is interesting to observe that in the Rotifera, owing to the concentration of the cilia at one end of the animal, there is no such incoherence and lack of coordination, in the reaction to the constant electric current, as is found in Infusoria. The rotifer (Anurcea cochlearis) becomes oriented with anterior end to the cathode by the same method as in reactions to light and other agents."

The researches of Pearl on the flatworm Planaria, also of several authors on the earthworm and leech, suggest that, with increasing complexity and correlation of the nervous system, the circumnutating or rotatory movements tend to become somewhat restricted to an ellipse or even side to side movement. But these differ little from zigzag ellipses or circles that our students often record on glass for the growing apices of plant stems and roots. The movements of the head in caterpillars, of the antennæ and eyes in lobsters, as well as other related actions, seem all to form a consecutive whole, that is explained on the basis of the existence, from lower nucleate plants to highest animals, of a rotatory sense. This in vertebrates becomes evidently so poised or equilibrated to average environments that it is only when the equilibrating nerves are serered that want of correlated movement in a straight line becomes evident. But it suggests also that such movement in a straight line has resulted from evolving condensation of the primitively spiral rotatory course.

But, as discussions on circumnutation in plants and as the constant intimate connection between the geotactic and gyrotactic sense-areas in animals demonstrate, there seems to be a 
close bond between both senses that may yet prove to be due to mass-perception response, in the widest sense of the term. In other words graviperception and rotation may represent physiological phases of one fundamental energy and matter relationship. Circumnutation and animal rotatory movement also are preeminently the proenvironing sense, in that such enable each organism to sweep round, "survey the country" in circles, and finally enable it to determine on the most satisfying line of movement.

The hydrotactic sense is so delicately and yet powerfully shown by the rhizoids of simpler plants, and by the roots of those higher, that it has greatly attracted the attention of botanists from the time of Sachs's publication. But, while we know much as to the physiological results, we still are largely ignorant as to the cell-conditions that start hydrotropic movement. But probably most are agreed in regarding it as a physico-chemical action, that is fairly distinct from the chemotactic stimulation. As to its occurrence in animals, it is well known that many land or amphibious genera "perceive" and move toward a water supply. Most human beings also can distinguish relative variations in the atmospheric moisture, while some can estimate with wonderful exactness the hygrometric state of the atmosphere when they pass from one green house to another. But our almost complete ignorance regarding the details of the process prevents further consideration.

The last statement is largely true for the thigmotactic or mechano-perceptive, and for the paraheliotactic or blue-purple light stimuli. It may now be safely said, however, in view of the experimental results of the past twenty years, that both play an important part in the life-movements of plants and animals, from some of the simplest to the most complex. The importance of the latter in determining the movements of animals has been largely overlooked or misunderstood, even where recorded, by zoologists, but has received much more attention from botanists since the time of Darwin onward.

We are still largely ignorant, however, as to the more minute causes of both movements. The writer has suggested (61: 183) 
that paraheliotactic movement in sensitive and in more sluggish plants is associated with the pulvinus of the leaves, and is probably due to molecular changes in the aggregation bodies. But much added observation and experiment are needed before exact statements can be made.

To sum up shortly the foregoing considerations, it may be said that definite chromatin masses or plastids evolved in the probable order: first, of leucoplasts or chemoenergids that are chemotactic (or olfactory and gustatory in higher animals) in function; second, of helioplasts (chromoplasts and chloroplasts) or photoenergids, that may be red or green in most plants, but seem to exist as colorless bodies in fungi, and possibly in animals; third, of geoplasts or geoenergids that have been recognized in root cells of flowering plants but may be much more widely distributed; fourth, of gyroplasts or gyroenergids that we know only in their functional activity, not in their morphological identity; fifth, of thigmoplasts or mechanoenergids that are in the same category as the last so far as plants are concerned, but which in the touch bulbs or touch corpuscles and in the auditory organs of higher animals seem to find an expression; sixth of parahelioplasts or parahelioenergids, which again are only known in their energizing results, not as yet as definite structures. The hydrotactic sense finally requires further elucidation.

Now as the writer and also his former student Watson have pointed out (62:336) the typical plastid-whether leucoplast or chromoplast - is linked to the cell nucleus by fine chromatin threads. Further in minute structure and in stainability the plastids and the cell nucleus agree. But cells of the filamentous Blue-green Algæ show small bodies embedded in the peripheral chromatophore which have been called "granules" by Zacharias, "red granules" by Bütschli, "cyanophycin granules" by Borzi, and "cyanoplasts" by Hegler. These according to some of the above-named authors are connected with the evolving central nuclear skein by fine threads. It is to be regretted that we still have little precise information as to nuclear conditions in the Tetrasporacex, the Mycoidacex, the Pleuro- 
coccaceæ, and the Protococcacex, which we accept, in a subsequent chapter, as transition groups from the blue-green to the higher or nucleate green algæ. But many facts indicate that the evolutionary history of cell-chromatin is probably as follows.

Through increasingly complicated action and reaction between the higher Blue-green Algæ and their environment, due to increasingly marked fluctuations and changes in this environment, these have evidently become traversed by a more and more condensed and perfect form of energy than even the biotic, though starting from it and interlinked in energizing changes with it. This manifested itself in the linking up of more complex molecules than even the protoplasmic. In these molecules phosphorus became an outstanding element.

In its first differentiation, as seen in Nostocacer and related algx, as also in some middle types of bacteria, this substance appeared as minute scattered refractive granules. By their highly energized activity these conferred added rapidity and delicacy of response to the foam strands of the viscous cell. But in process of their evolution, and owing doubtless to slight chemical or physico-chemical differences in their constitution, some became first chemoenergids, in relation to the continuous chemical action proceeding in the cell, others later became photoenergids, and still later others differentiated as indicated in previous paragraphs.

But, differentiated from, continuous with, and embedded in the protoplasmic foam substance, these remained linked up with each other by a fine system of chromatin threads - the kinoplasm threads of some authors. Thus started the striking peculiarity of chromatin cells, viz., their capacity, not only to perceive or be stimulated by two, three, or more external stimuli, but to so compound these as to start a single resultant response, that represented the most satisfied response possible to such environal stimuli. Or, where one external stimulus and intrinsic resulting response exceeded one or more simultaneous or nearly simultaneous stimuli, growth or motion in that direction would be proenvironed by the cell substance and followed accordingly. 
Such action and reaction between different and at times diverse stimuli on the one hand and diverse chromidia on the other necessitated the gradual evolution of a central correlating chromidial or chromatin mass, that would focus up and energize the different peripheral or the more deeply placed chromidial factors. Such we see in Oscillatoria and Lyngbya with their nuclear skeins, while in the simpler green algx the process has largely or wholly been completed, through formation of the chromatin center or nucleus. So, as to the functional as well as morphological significance of this, and in light of all cytological papers that have appeared in recent years, the writer can only repeat the explanation given by him nearly twenty years ago (63:279): "We would consider that the nucleolus is the special chromatic (chromatin) and cell center; that it sends out fine radiating processes - the intra-nuclear networkwhich partially fuse externally to constitute the nuclear membrane, the interspaces of the network being occupied by nucleoplasm concerned in metabolic changes; that radiating continuations of the chromatic substance pass out beyond the nuclear membrane, and form a network in the protoplasm, while we would suggest, for further proof or disproof, that they further may be continued through wall pores to form an inter-cellular chromatic connection." Also that chromatin "threads can be traced to radiate out through and beyond the nuclear membrane, and across the cell-cavity to the pyrenoid centers," while "extremely fine chromatic threads stretch across to, and connect, the pyrenoid centers" in the chlorophyll bodies.

So, evolutionarily, functionally, and morphologically, we can only regard the chromidia, the chromatin skeins, and the chromatin nucleus-nucleolus as evolving expressions of one fundamental substance, that is intimately associated with a cognitic energy. This energy brings each organism into rapid and delicate connection with environal stimuli. The origin of the chromatin substance then seems only explicable, as due to long continued environal stimuli, and previously absorbed intra-molecular biotic energy acting and reacting along complex colloid molecules, so as to build up a sensitively receptive 
correlating and responsive system, the chromatin or cognitic, that now presides over and in part directs the more sluggish protoplasmic activity. Like the central nervous system therefore of greatly more complex significance-whose evolution fortunately we can still trace, step by step, from surface origin to embedded safety, in a fish, an amphibian, even man; and whose still more complex actions and reactions can be explained through the steady flow of cogitic energy; the entire chromatin system seems to represent a long-drawn exposure to stimulatory environal acts, and resulting intra-molecular energized responses, that ran through millions of years of archæan activity, and that now are stamped on each organism as the structures and functions of the nucleated cell.

If then at this stage it be granted that a biotic energy traverses the protoplasm; that by its activity this substance is stimulated to perform those acts-nutrition, respiration, growth and protoplasmic division-which together constitute the vegetative activities; that by its rather sluggishly irritable response to environal stimuli it is enabled to continue such functions as tolerably distinct phenomena; then in view of the active and delicate irritable response of nucleated or chromatin-bearing cells to environal stimuli; in riew of the exact manner in which several stimuli are perceived, correlated, summated into a resultant impression, and by proenvironal action responded to; in view also of the greatly more sensitive and restricted endurance by nuclear chromatin of temperature changes when in the active state, as compared with protoplasm, we may now try to secure evidence in favor of the existence of a cognitic energy.

As to range of temperature endurance for chromatin during the active growing period, this varies from $2^{\circ} \mathrm{C}$. to $40^{\circ} \mathrm{C}$, though averagely it is between $8^{\circ} \mathrm{C}$. and $35^{\circ} \mathrm{C}$. But, as already indicated, seasonal periods of dormancy may gradually be prepared for, when, enclosed within the surrounding protoplasm, it can survive through wide ranges of temperature that extend from $-100^{\circ} \mathrm{C}$. to $+65^{\circ} \mathrm{C}$. Such possibilities must always be kept in view, when study of nuclear life phenomena is made. 
As to the more immediate chemical composition of cell nuclei and associated structures, these are now usually regarded as composed of three complex organic bodies, plastin, nuclein, and chromatin. Of these plastin seems most nearly if not exactly to resemble protoplasm, and would be explained as the nucleoplasm that surrounds the most complex constituents and that nourishes them. Nuclein seems to be an intermediate substance between it and chromatin, while the last-named is the most complex in organization or composition. So on the above riew the nuclear plastin is the regetative constituent, that is, the absorptive and feeding part of each nucleus or of each plastid; the nuclein is a product which is gradually passing from a plastin state to the still higher condition of chromatin, and so acts as an intermediary in the linking up or condensation of it; while the chromatin is molecularly the most complex and physically the most highly energized of the three constituents.

Such a riew also is farored by staining results like those described by Miss Huie, specially when one compares these with the staining of cells in the resting stage. For, during the latter stage in plant cells, the nucleolus, the radiating nuclear threads, as well as the nuclear membrane that are all richly chromatin-bearing, stain, by use of saffranin and gentian violet, a deep brown-red; various constituents within or alongside these stain of a reddish-purple; while the more liquid and bathing constituent stains of a purplish-blue color. The variations in staining observed by Miss Huie point to a sharper and more active temporary and visible differentiation in the three constituents connected with the more active substancetransformations proceeding during division.

A marked sensitivity then to environal stimuli, and the rapacity to link up the action of several of these stimuli, so as to form a resultant proenvironal response, we would regard as fundamental peculiarities of chromatin or the cognitic substance. But it may now reasonably be objected that, even if we do not regard chemire or clectric energy as sufficient for such response, hiotic energy of the protoplasm might amply 
suffice. To this it can be replied that during the past quarter century all experiments made-confessedly rough in many cases - to isolate the cell nucleus from the protoplasm and then cause the latter to increase, or to divide, or to metabolize food material for , any length of time, as well as the efforts of Boveri and Delage to fertilize the enucleate protoplasm of an animal egg by a spermatozoid, have in large part failed. Not unfrequently, in the former cases, the protoplasm has lived and moved for days, but what might be called the correlated capacity for stimulation-perception and cumulated response, so as to energize the protoplasm rapidly and continuously, has gone."

Objection might next and very forcibly be raised, that the varied and active responses shown by many of the flagellate bacteria are as rapid and sensitive as are those of the highest nucleate plants. This is so, but we would place these members of the Schizomycetes on a level with the higher Blue-green Algæ, in part because of the oft-reported presence in them of chromidia, in part because of the specialized and energetic character of the cilia, which seem often, if not always, in higher plants to be formed from and in connection with chromatin substance, and even as in spermatozoids to be intimately united with the nucleus.

In the simpler evolving nucleate algæ, such as the Pleurococcaceæ, Tetrasporaceæ, and Protococcaceæ, growth and environal response in the adult organism are usually sluggish, the protoplasm as in higher Cyanophycex being predominant in action. Only when ciliate swarmspores arise does active and summated response to environal stimuli occur. But, in the Volvocacex, Desmidiacer, and higher groups, experiment shows that response is active and well directed. Particularly true is this again when motile swarmspores or ciliated gametecells develop. The incessant movements then seen under the microscope, the extremely rapid viluration of the cilia in correlated manner, and the rapid response shown to gravity, heat, light, chemical agents, and injurious orer-intense blue-riolet rays, all indicate the existence, in the nucleolus, nucleus, kino- 
plasmic threads, and their finest peripheral terminations, of a highly condensed and continuous flow of energy that stimulates and directs the whole, at the same time that it is receiving and being affected by stimuli from without, most of which acting through definite energy-transformers-the plastidsstart thereby definite proenvironal movements in the chromatin substance.

But division of such cells proceeds on the indirect or karyokinetic plan, in which previous careful sorting out and division of the chromatin material into apparently symmetrical halves occur, prior to division of the protoplasm. Thereby, after each division, as has been universally accepted during recent years, a redistribution of all the hereditary substance is constantly effected. This then may approximately raise the entire question of how division proceeds, and what is the significance of it. We have referred to such in another part of this work (chap. 10) but, so far as the mechanics and so far as the energetics of division are concerned, the valuable observations of Buitschli (21 passim) and Leduc show that exactly such shapes, patterns, and distributional strains may be developed in inorganic liquids, such as salt water, a drop of the same pigmented with India ink and on either side of the latter a hypertonic drop of salt solution of lighter color.

But, while such experiments show that definite inorganic substances of relatively simple chemical composition can form what we may call inorganic karyokinetic figures, from the standpoint of life energetics it does not touch the question of how highly complex colloid molecules are split asunder in profoundly exact manner, and are carried to definite distances apart, there to start a new and definite role of behavior. It is, we claim, for the accomplishment of this that a highly condensed and economically work-transformable energy such as the cognitic is needed.

Here, however, it should at once be acknowledged that perfect division of the simpler cyanophyceous protoplasmic cell proceeds, though unfortunately we know only imperfectly regarding the minuter details of the process. In the higher 
members of that group Hegler, Phillips, and others have described and figured apparent exact division of the chromidia or chromatin skeins. Therefore, in the former and simpler of these two cases, intra-molecular biotic energy is probably alone expended in separating and redistributing the complex molecules, while in the latter case the same energy, along with an already active cognitic energy, may more or less cooperate to effect the change. But more is said regarding this on another page (chap. 10).

In the higher green and other algæ, where division of labor and variety of form in cells are equally shown, it is unquestionably true, and accepted by all, that, as each plant unfolds from the spore or the fertilized egg, what appears like equal and homogeneously uniform division of the chromatin substance may at times actually be such, if like daughter cells are to be produced. But, where two systems or physiologically distinct types of cell are to be produced, the sorting out and subdivision, in mathematically exact manner, of the chromatin molecules must be profound. But that there is a morphological and physiological homogeneity or similarity or nompolarity of molecules in cells that normally and eventually indicate behavior otherwise, is strongly suggested by experimental studies on young algoid seedlings, on gemmæ of Marchantia, and on early segmenting animal eggs.

But even in the last cases an exact polarity is soon established, in all probability through migration of all or of the majority of the geoenergids and hydroenergids in one direction so that such an area becomes geotropic, while a simultaneous distribution of photoenergids and chemoenergids in another direction would promote heliotropic growth.

Here it cannot be too strongly emphasized that the fundamental determining feature in all such morements consists in exact lines of energy-flow. Now, if it cam be shown that electric or any other hitherto known energy can, at temperatures suitable for chromatin activity, effect such exact divisions, segregations, and steadily continued functionings as are alosolutely required by the phenomena of nucleated organisms, then 
it would be superfluous to inroke the aid of a cognitic or other delicately stimulus-perceptive type of energy. But from all that we know even the biotic fails to meet this requirement, while the failure to explain the energetics of cell-division, the exact inequality that at times characterizes such divisions, and the future role that the derivative cells play, forces us to the acceptance of a more perfect and energy-utilizable kind than either the biotic or the electric.

Want of space prevents us doing more than glance at the life-phenomena of other rather simple nucleate organisms. But some of the Myxomycetes like Badhamia utricularis are highly instructive, and have been fairly often and carefully watched by the writer and his students. When in the "streaming" state its protoplasm is richly studded with nuclei, each of which encloses one, two, or even three nucleoli. At this time the riscous foamy yellow mass shows marked chemotactic response to appropriate food, also marked response to gravity, to light, and to moisture at least. It absorbs food particles in quantity, and churns these up in its endoplasmic substance into fine material that is digested and assimilated so as to cause pronounced gromth. All of these energized responses are balanced and combined into resultant action that is most satisfying for the entire organism.

It also frequently shows nucleo-nucleolar chromatin division with resulting multiplication of nuclei. Though we are still in doubt as to what may constitute sexual fusions, such can be left aside in view of the many other points that raise $\mathrm{Bad}$ hamia above the Acaryota, alike in structure and energyresponse.

In this soft viscous state, then, it continues to show alike vegetative or biotic phenomena, and irritable chromatin or cognitic phenomena. But if water be gradually withdrawn, and all the better if the temperature be kept at $2-10^{\circ} \mathrm{C}$., the mass dries gradually, till it may appear as a fine dry film on Stereum or other nutritive fungus, and then it can be kept as a scarcely recognizable streak for months. Here the cognitic activity has been reduced we believe to its lonest ebb, and the biotic also is about as much reduced. But that a reserve of highly perfect energy lingers there, sufficient to again energize the organism, is shown when it is once more placed in moist and appropriately warm environment. For then the film starts to swell, to flow, and, within eight to ten 
hours, to spread over the nutritive substratum. It is for the present purpose a matter of minor moment, whether we should view such an organism as a plant or an animal.

When we turn to the highest plants, cursory study of the irritable phenomena might indicate a simple physico-chemical explanation, such as the mere upsetting molecularly of a definite organic substance, and, after the effect of the stimulus has ceased, a gradual reestablishment of the molecular equilibrium. But the conditions are much more intricate and fundamental. Thus, when the tip of a leaflet of Mimosa pudica, the tentacle of a Drosera leaf, or the hair of Dioncea is stimulated by mechanic, thermic, chemic, or electric energy, the stimulated cells respond, as the writer has pointed out elsewhere (81:205), by change in the protoplasmic-chromatin pellicle, and almost immediately thereafter by undergoing chemical decomposition changes in the protoplasmic substance, so that the enclosed "aggregation body"-itself a complex lipoproteid-squeezes out water and contracts.

Such changes are particularly well followed in Mimosa and Drosera, in part by the naked eye, in part by the microscope. Now one might readily suppose that the entire change centered in, and was determined by, the aggregation mass within the nucleo-protoplasmic sac, and not at all by the latter. But we cannot then explain why the energy applied as a stimulus passes first through the enclosing and living sac; second, why this living sac becomes porous to the passage of liquid; third, why, after the effect of the stimulus has passed, the sac again passes liquid inward to the aggregation mass, so as to cause resorption by it of the liquid; fourth, why in time when the mass has reestablished its former size and equilibrium the sac becomes closed to liquid exudation and even shows high tension-conditions.

Thus the rapidity with which a fallen Mimosa leaf will, within five to eight minutes after stimulation, rise against gravity and reexpand its leaflets is proof not merely of tension strains between cells, but much more importantly of definite flows and redistributions of energy in the chromatin and the 
protoplasm, as the first requirement. A chemical explanation will not suffice, a physico-chemical-and that of high work transformability capacity -is needed to explain the case. When we bear in mind further that all of the above-named plants have been demonstrated to possess intercellular protoplasmic continuity - and possibly most plants have suchthis enormously complicates the question from the purely chemical standpoint. But such the more claims from us the acceptance of a physico-chemical explanation, in which definite lines of energy-flow, or lines of force, constitute the ruling and directive factor.

The existence of a high quality of chromatin or cognitic energy, associated hereditarily with definite lines of force, as affected by and responsive to external stimuli, would also explain many hitherto obscure cases of irritable stimuli and growth reaction. Thus, while the main stem or occasionally also some sideshoots of plants are usually upright, others are oblique or even transverse - rarely as in Yucca filamentosa directed vertically downward. If in this case we consider that a certain number of geoenergids have become hereditarily massed in, and are physically repulsive to, a definite number of photo- or helioenergids, in the branch cells, such would start exact protoplasmic aggregations and growth actions in the cellwalls, that would explain the resulting naked-eye conditions.

Again such striking cases as those cited by my former student, Dr. Schively, for Amphicarpcea (65:270), and as have been described also for the coltsfoot (Tussilago Farfara) and the ivy-leaved toadflax (Linaria Cymbalaria), where growth reversals occur, would equally represent changed lines of force. The case of Amphicarpcea, the common hog peanut, is worth quoting. This leguminous plant forms delicate branches in the axils of the lowermost foliage leaves. These have been demonstrated-alike by observation and reversing experiment - to be positively geotropic, and so as compared with ordinary branches may be considered to have an excess of geoenergids massed in their cells. But if, while these branches are still an inch or two in length, the main axis be cut off, the hitherto 
geotropic shoots curve round and grow upwards into the air, or become apogeotropic. Here, therefore, chemoenergids, or photoenergids, or both combined probably in the side-shoots, overcome the action of the geoenergids after removal of the upper part of the main axis.

In turning to animals for evidence as to cognitic energy, the old familiar dictum that animals move and feel at once indicates their high cognitic sense and their decided advance on the whole in rapid response to environal stimuli over most plants. But the motile stage of many algæ and fungi, as well as motile plant spermatozoids with their demonstrated keen chemotactic perception and response, are proof that many unicellular plants or plant phases may closely resemble animals.

Mention of spermatozoids at this transition stage in our discussion of plant and animal response causes us to observe that their mode of origin and growth show them to be largely naked motile chromatin or nucleolar-nuclear bodies. Now each carries, locked up in its molecular substance, not merely sufficient energy to enable it to spin forward in continuous gyratory fashion, for from thirty to sixty minutes at least, but, if one of them reaches and fuses with an egg, its absorbed substance so completely permeates, reenergizes, and molds the substance of the egg as to cause the latter to be recognizably affected and modified in every future cell of the organism that the egg gives rise to on segmentation.

This phenomenon is exactly and strikingly shown in the minute structure of plant hybrids, such as the writer first elucidated in all of their cell details. It has also been shown since to be visibly true at least in the young stages of such animal hybrids as Loeb, Boveri, Morgan, and others have investigated, while the often closely intermediate character of known fish and mammalian hybrids demonstrates a like permeation of sperm characters though the entire cell lineage of each new organism.

In this case again we are compelled to accept physico-chemically that the spermatozoid, or minute male chromatin mass, either introduced so complex and condensed a mass of chemical 
molecules that they, in virtue purely of chemical affinity, influenced every future cell of the descendant organism, or that the spermatozoid introduced a so condensed and perfect amount of some kind of energy that this started the complex molecules of the egg to vibrate and coalesce in such rhythmic unity that exact lines of force were established between the two as to cause all subsequent complex molecules to exhibit blended characters.

Equally true too would it be that, when the complex molecules of the sperm and egg are derived from parent forms that are widely apart systematically and so morphologically as well as physiologically, minute differences in the rhythmic vibrations, and in the physico-chemical relation of the chromatin in each to the other, may be such as to cause, in the attempted fusion of both, a complete disorganization of their substance. For in sperm and egg maturation and subsequent fusion we have to deal with enormously more complex phenomena than any mere mixing, or action and reaction, of purely inorganic bodies.

Fusion of a free sperm nucleus then, with an encysted egg nucleus, we would regard as an excellent exhibition of cognitic energizing activity, and of the establishment of lines of force that permeate every part of the resulting organism. Some of the exhibitions of this that the writer has called attention to (63: 250) are marvelously exact. Thus in the formation of starch grains, that are a purely secondary product of cell activity, these show the blended peculiarities of the starch grains of both parents to a minute degree. Now the upbuilding of each such grain is effected by agency of a chromatin plastid, that expends energy not only in linking together the highly complex colloid molecules, but in so accurately molding and fitting these on an exact stereo-chemical plan that the fundamental character of the grain of each parent is accurately reproduced, in blended fashion, in that of the hybrid. The recent studies of my colleague Reichert are a valuable confirmation and demonstration of this principle, as applied to starch grains in general. 
But such stereo-chemical fitting or combination would be entirely impossible, were it not that a subtle and perfect exhibition of stereoenergesis is expended along definite lines, so as to place molecule beside molecule as accurately as are the bricks fitted by a bricklayer's skill (which is itself a highly complex stored energy that becomes kinetic in the process), in the rearing of a house.

If it can be demonstrated approximately that electric, chemic, or even biotic energy can link together molecules of so complex a nature in so exact and highly complex a manner, there is no need for a higher energy. The failure hitherto to do this causes us to accept the cognitic type of energy as a necessity of the case.

In the paper on plant hybrids already quoted the writer has shown that such a complex and far-reaching feature as the time of blooming of plant hybrids is in many cases an exact, or nearly exact, mean between that of the parents. Now we have ample phytophenologic statistics to prove that time of blooming depends annually on many factors, chief of these being light and heat units. But minter freezing, direction of winds, amount of rainfall, and other factors seem to combine in determining the recurrence of this event. To all of these then we prove that the plant responds accurately, when we note that the hybrid flowers unfold at a time that is intermediate between those of the parents. And this throughout is not a mere question of chemical combination. It is throughout a question in which we deal with definite external stimuli that affect the plant, and to which it responds by exhibition of lines of energy-expenditure that cause placing of molecules at an exact periodic ratio, and in exact growth-position, so as to culminate in an exact time of unfolding of each flower.

We might equally refer to the comparative hardihood of hybrids as elucidated by the writer, and since extended by Webber and others. But, without lingering orer such, the remarkable cases of unisexual and bisexual hybridity described and figured by the writer form a cogent proof of the need for some labile but tenacious and high work-transforming energy 
such as the cognitic. For, in the cases of unisexual hybridity cited (63: 273) as well as in many others that could be adduced, structures that resulted only from definite local expenditures of energy, by the placing there of definite molecules characteristic of one parent, and which are absent in the other, appeared of about half the size and number of those in the former.

Again, in cases of bisexual hybridity, two structures of diverse morphological nature were both reproduced, though reduced about half from those of two parents. Such is purely a molecular expression of the activity of lines of energy-flow, that here were reduced hereditarily by about one-half in intensity.

Amongst unicellular animals, the need for recognition of a cognitic energy is even more claimant than for most plants. Thus the responsive streaming movements of an amœba or foraminifer, and the directive movements of a heliozoon, as detailed in zoological literature, may be started by a chemic, a lumic, a mechanic, an electric or other stimulus from without. But the correlated streaming of the protoplasm, or bending of the stiff pseudopodium when a food particle is caught, represents a greatly more complex energized perception on the part of the organism, that results in seizure, in absorption, in digestion - the two latter largely biotic - and in assimilation, that in their correlated or resultant interdependence proclaim an adaptive distribution of lines of subtle energy-action and reaction.

Amongst the Infusoria such displays of energy are greatly more intensified, and a capacity is shown on the part of each organism for "perceiving" several diverse stimuli in rapid succession, for summating these into a resultant whole, and rapidly thereafter for pursuing a line of motion or action that is most satisfying to it for the time. All of this again represents the incidence, it may be, of several stimuli, such as lumic and gravic, the action of each of these on some special irritable substance, the linking of such "perceptions" into a common sensation, and the proenvironal arrangement of molecules by energy expenditures, whose summated aggregation results in proenvironal response. 
We shall not at present consider the higher animals in which nerve-cells are evolving or have attained a high degree of complexity, since these will be studied in relation to cogitic energy in a subsequent chapter.

But, before closing the present one, short account may be taken of some well-known phenomena, in which biotic and cognitic energy seem to play a conjoint part, as must in the great majority of cases be true, could we only analyze them up in a sufficiently accurate manner. The case above cited for Infusoria is to the point. For while the chemotactic perception of food, the mechanotactic ingestion of it, and the digestive changes that are effected in it are all, we would consider, correlated expenditures of biotic energy, the increasingly rapid and alert movements that are correlated and directed to the above end, the manner in which such activities become reduced by age as shown by Maupas's experimental cultures, the slow but steady modification of structure so as to constitute variational and at length specific differences, and the transmission of such by sexual heredity we would regard as due to chromatin or nucleo-nucleolar structures, that are traversed by cognitic energy.

Some cases however, in which temporary reduction or dormancy of the cognitic flow of energy, and continued action of the biotic, deserve notice in the present connection. Thus the widespread phenomenon of sleep, that in plants we designate nyctitropism, in animals slumber, equally represent a state in which at fairly regular intervals, and particularly on the approach of darkness, the entire cognitic energizing network becomes gradually more flaccid, irresponsive, and passive. In nearly all such cases definite positions are naturally assumed, as for example, fall or rise in leaflets or leaves, horizontal position in many animals, erect perching position in many birds, hanging position in bats, etc. The senses become almost or wholly incognizant of surrounding stimuli, unless these be of greater than normal intensity.

But the flows of biotic energy, though reduced in intensity, still proceed in even manner; digestion, respiration, growth, 
and cell-divisions all continue their course; while changes preliminary to, if not actually culminating in, formation of asexual cells go on, as in spore-formation of Pilobolus and Mucor. Now, on account of the food-transformations and conveyances that we know to proceed then, as well as from the manner in which, succeeding to this rest period, plants soon after attain to their maximum of diurnal activity, animals to their most active and excitable phase, it seems almost assured that such sleep movements represent stated periods, when the centers of most active cognitic energy-expenditure become dormant and passive, when by transformation of supplies of chemic, possibly electric, and specially of biotic, energy, there is gradually elaborated a potential or intra-molecular store of future kinetic cognitic energy, which, on reaching a definite post-nocturnal optimum, causes "waking" of the plant or animal, accompanied by a sense of renerred activity and alertness for reception of, correlation of, and response to environal stimuli.

A closely similar condition is that already treated of in the annual or perennial dormancy of plant-parts and of many animals. The carefully prepared for winter dormancy of temperate and sub-arctic plants, and almost as strikingly of many xerophytic or sub-xerophytic tropical plants, represents a steady reduction of the cognitic capacity for response, and a sluggish continuance mainly of the biotic.

The wavering periods of dormancy, however, that at times alternate with growth and sensitive response during mild winters, prove that the entire event is not that of a "not living" state, but of dormancy that varies according to the specific organism studied, or the degree of environal "rigor" to which the organism is exposed.

So also the frequent hibernations of animals, specially of fresh water or land types, like the rotifers, snails, spiders, many insects, a few fishes, many amphibians, reptiles, and mammals, all represent an adaptation of the chromatin substance to the varying environal changes that the animal has to pass through. Here however, unlike sleep, since the period 
of dormancy is often a long one, and since there is a slow continuance of respiratory and other biotic conditions involving waste, unattended by times when fresh supplies of food are absorbed, the animal emerges from its rest period greatly reduced in tissue constituents, and so in its capacity for active cognitic response.

The case of such fishes as Lepidosiren and Protopterus is of interest, since we have an entire group that for long ages has been fresh water in habit, and that now lives amid environal states which change from a shallow fresh water to a dried mud condition. Through environal action and proenvironal reaction, these fishes have become perfectly adapted for survival through periods of from at least two to four months.

All such conditions as those above described demonstrate at once the demarcation line that can clearly be traced between the biotic and the cognitic states, at the same time that they indicate the intimate and dovetailing character of the protoplasmic and of the chromatin substance. Could the biochemist show that transition materials like plastin and nuclein link together both substances, such would be a helpful step toward proof that a steady transformation and condensation from biotic to cognitic energy is also a typical phenomenon of nucleated plants and animals.

So instead of saying, as has often been done in the past, "we do not understand the nature of living matter," and yet of declaring that living matter may be defined as "a certain protoplasmic architecture, which implies essential relations between nucleoplasm and cytoplasm," we would consider that it had its commencing foundation in colloid materials; that, under the action of inorganic energies and later gradually of biotic energy, it became synthesized into complex molecules; that these gradually assumed alternating condensation and expenditure of this biotic energy, in an autotrophic, autorespiratory, and autoauxanotic capacity, also in a power of multiplication by expansion or division of the colloid membrane, through osmotic pressure action and growth strengthening.

Later, and for the great majority of organisms now living, a striking advance was made, through increased complexity 
of molecular structure being correlated with a still higher condensation and more perfect work-transforming form of energy, the cognitic. Such by traversing a complex and yet labile molecular structure, and by its high energized capacity, enabled intricate and yet rapid proenvironal reaction to be made in response to one or more environal stimuli, at the same time that, when such stimuli became different in intensity or quality, this slowly effected molecular change in the organism, through alteration in the amount, and in the lines of flow, of cognitic energy passed to the regions involved. 


\section{CHAPTER VII}

\section{A REVIEW OF THE FORMED CONSTITUENTS OF ORGANISMS}

In bringing together the materials for the present chapter during the past fifteen years, the writer grouped these under the title, "The stock-in-trade of plants and animals in relation to their environment." And such seems in many ways a more apt title than the heading given above. The contents of the chapter will explain the line of investigation and argument pursued.

During the past half century, the steady spread of evolutionary views has given rise to such expressions as "the production of new characters," "the inheritance of acquired characters," "definite or indefinite variation of species," "the influence of the somatoplasm and of the germplasm in descent," "the breeding true of unit characters," and many of kindred import. Widely divergent and often conflicting views have been put forth as to these and related questions of evolutionary trend.

In all such studies it has been either tacitly or openly demanded and accepted that many new and highly important morphological or physiological characters appear in organisms, as we ascend in the organic scale. But, granting an apparently new character to have appeared, say in animals, in the form of nerve cells and associated fibers, that are absent as such in lower animals and in all plants, the question is still worth looking into, as to how far and how fundamentally new characters appear, and what is the sum total of the substances or compounds that all living bodies tend to possess in common.

This inquiry suggested itself to the writer about sixteen years ago when he was endeavoring to secure accurate data as to "acquired characters." The results seemed both striking and 
unexpected, and as now presented they may possibly suggest to others a revisal or modification of their views on this subject.

It was previously stated (p. 63) that the organic chemist has already recognized and named about 50,000 compounds that are either naturally present in organisms, or are obtained from them artificially in the laboratory. Amongst so large a number, the possibilities for new combinations and for molecular changes in such complex and condensed laboratories as plant and animal cells might well be regarded as enormous. But, while it seems undoubtedly true that many of these-let us roughly say 5000 at least-are actually produced in living cells, the great majority of them are closely related compounds of various chemical series, which differ from each other only in the replacement of one or a few atoms or radicals, for another, in a highly complex molecule. Given therefore the formation of any one of a definite chemical series, as say grape sugar in the glucose group, the formation of the other members of the series would probably be a gradual but easy effort on the part of the organism.

The definitely recognized and named constituents of animal tissues, moreover, often are identical with, or closely allied to, those of plants; in rarer cases constituents are peculiar, so far as we know, to one of the two great living groups. We shall therefore for convenience consider plant and animal cell contents together.

But, in considering the possible origin or distribution of the organic bodies, an important question at once presents itself, which has not apparently received due consideration. It is the following. Confining our attention meanwhile only to plants as a field of study, even cursory examination shows that many constituents encountered amongst the simplest Caryota seem to be again lost or obliterated, only to reappear higher up in the plant scale. Thus the red pigment of Chlorococcum seems to be identical with that of many other unicellular algæ or algoid spores, though in the adult plants that these spores may form the pigment entirely disappears from view, as in Ulothrix zonata, etc. But it again reappears in 
the oogonial or antheridial wall cells of Nitella, in the capsular wall cells of some mosses, in the seed-coat cells of cycads, in the red fruits of many of the higher seed plants.

Again mannite is met with amongst such algæ as Laminaria, Halidrys, and Fucus, is widely distributed amongst the fungi, is found in roots of celery and carrot, in tubers of aconite, in the liquorice (24, 1:361), and in the rind of several widely separated groups of flowering plants (p. 377).

Oxalate of lime crystals occur in species of Spirogyra, in many conifers, in some monocotyledonous and dicotyledonous families or genera, though not at all in other of these groups. In crystallization shape, also, they are equally diverse in their characters. Even the relative distribution of the needle crystals or raphides is often highly suggestive.

Again the brown pigment of indurated walls is frequent in fungoid spores, in the spores of liverworts, mosses, and ferns, in the cells of many seeds, and in the strengthening tissues of seed plants. But amongst soft and shade loving plants, in aquatic plants, and in many xerophytes it is absent or rare.

Similar cases might be cited as to the sporadic occurrence of certain glucosides, of certain alkaloids, and other bodies. Scores of examples that illustrate like appearance and disappearance of cell constituents can readily be demonstrated by all, with aid of such works as those of Wiesner (66), Zopf (29: 386-464), or Czapek (24).

But, even if we confine attention to the higher flowering plants, many equally striking results are obtained. Thus it is true of nearly every large--and of not a few small-families of the dicotyledonous Apopetalæ and Sympetalæ, as of the higher monocotyledons, that, though showing little affinity often in taxonomic value of their flower, they all start in their primitive genera or species with greenish yellow or yellow floral coverings, but show steady advance to yellow-red or pink-red, then to pink-crimson or pink-purple, and from such to purple-blue, while many of the families advance to pure blue. Though minor variations at times seem to cause exceptions to the rule, the author has proved by calculations that 
a real advance exists along each family, as was beautifully shown by Huxley for the genus Gentiana (67:101). Such advance in floral color nearly always is correlated with advancing morphological specialization of the flower as a whole. We need only cite such families as Liliaceæ, Iridaceæ, Orchidaceæ, Ranunculaceæ, Papaveraceæ, Caryophyllaceæ, Malvaceæ, Rosaceæ, Fabaceæ (Leguminosæ), Myrtaceæ, Asteraceæ, Campanulaceæ, most families of the Bicarpellatae in the widest sense, amongst many others that could be given.

Now the question arises: are such appearances and disappearances, or are such frequent appearances at different stages of the evolutionary process, to be explained by our supposing that certain substances are evolved as totally new and "acquired characters" at certain points along the main lines and the by-paths of the great evolutionary road, to be again lost in some cases, or to be replaced in other cases by different but related substances? Or is there not rather a large series of bodies common to all plants, and specially to the Caryota, which can at any time be gradually reproduced by the joint action on, and reaction of, protoplasm and its related ferments, under the more fundamental action of appropriate environmental stimuli? For, further, as will be accepted later, if such stimuli change in quantity or quality, so might the reproduced bodies change, even to the extent of seeming to be again absorbed, or possibly represented by ultra-microscopic particles.

The latter of the two above alternate views is that which the writer in his lectures has advocated during the past fourteen years, as being the one which is abundantly and widely supported by observations and experiments, some of which will be presented in a later chapter.

If such be the condition of affairs, varietal, specific, generic, and wider characters resolve themselves into the waxing or waning of definite substances, according as environal stimuli act on certain constituents of the cells. It follows also that most of the discussion on the possible acquisition of new characters, on the hereditary transmission of such, on dormant and recessive factors, have mainly been of value in stimulating 
research. If then it can be shown experimentally that "characters" vary when subjected to changing environal stimuli, this will be a first step toward a true aspect of the question. To the consideration of this we shall proceed in a future chapter (chap. 8).

An examination can now be made of the "stock-in-trade" of the Caryota, in order to ascertain how far and to what extent similar cell substances occur throughout the entire series of plants and animals.

The cell protoplasm-as histological text-books generally show-consists of a viscous material with properties in common alike to plants and animals. Thus it usually is divisible into ectoplasmic and endoplasmic layers; its substance is traversed by a fine fibrillar network; and the whole has an alveolar or foamy texture. It absorbs or rejects stains according as these show or do not show an affinity for it in chemical action and reaction (22). It nearly always invests itself with a wall or membrane that is of isolichenin, fungus cellulose, cellulose, or is infiltrated largely with nitrogeneous constituents as in the animal wall. All of these varieties of wall material show transitions from one to another.

This protoplasm seems in all cases to represent an intricate mass of interacting ether particles of complex interunion that together are traversed by, energized by, and that are built up or broken down by biotic energy. This biotic energy is constantly being replenished by supplies of electric and chemic energy, that become transformed into it as intra-molecular biotic energy, while the last is constantly dissipating or breaking down into the simpler types of energy.

The protoplasm often shows continuity from cell to cell by intercellular threads, and shows like irritable response to thermic, lumic, chemic, electric, and other stimuli, though to varying degrees and according to specific differences. Accordingly Waller truly notes (48: 12) "the essential identity between the excitatory responses of vegetable and of animal protoplasm"; while a like refrain comes from many biologists.

Cilia are definite outgrowths of the living plasma, that may appear and disappear alike amongst plants and animals, according to the functions and environal activities of the exposed cells. Phillips, working under the writer's direction, very clearly showed primitive and slowly moving cilia in some of the Schizophyceæ (35:318). Amongst the bacteria they attain 
to great fineness and agility of action. The sperm cells of all the "cryptogams" and of some gymnosperms possess them, but clearly they have been absorbed in the sperm nucleus of the highest flowering plants. Their formation on various motile spore-cells of algæ is frequent also. Amongst animals the more active life of the cell individual, as in Infusoria and Rotifera, is accompanied by ciliary growth, but in the Metazoa cilia appear and disappear again according to environal relations. Thus all acknowledge that the varying degree of ciliation of invertebrate larval forms represents transition phases from a common ancestral holotrichous state.

As to the minute structure and innervation of cilia, it seems probable that these arose primitively, as in the Schizophyceæ noted above, from simple protoplasmic extensions. But their origin from a blepharoplast at least in some cases, and the chromatin nature of that body, as the writer would consider it, are in keeping with the view that possibly all cilia of the Caryota are connected with and innervated from cognitic energy in chromatin substance.

The nucleus and nucleolus will receive detailed consideration later (chap. 10), but here it may be said that in structure, composition, staining capacity, changes undergone during division, and to a large measure also during fertilization activity, the phenomena are fundamentally alike for the Caryota, though varying minor specific modifications are at times shown. In all of the great groups sexual phenomena, alike in the narrowest and the widest sense, appear with, and remain associated with, the fundamental constituent of the nucleus and nucleolus known as the chromatin, which remains the same throughout the plant and animal kingdoms; but which develops specific peculiarities in forming varying numbers of chromatin segments during cell-division, in the pathways these pursue during division, and in their connection with the nucleolar mass.

Pure chlorophyll of green plants agrees throughout in structure, spectroscopic characters, behavior to agents, functional activity, and decomposition changes. Equally true is it that, at different evolving stages in the plant world, groups of plants -flowerless and flowering - tend to become saprophytic or parasitic, owing to gradual accustomment to absorption of organic food from other living or dead plants or animals. In the process the chlorophyll gradually ceases to form and ultimately pale yellow or perfectly white plants result. The successive stages in this process shown by about a dozen families of flowering plants are instructive from the common standpoint of devolution. 
Cellulose is a substance that plays an important part in plant tissues alike phylogenetically and structurally. 'The pure cellulose of most primary cell walls is largely retained in tissues throughout the plant series. But, with the transition from a primitive aquatic to a subaquatic and eventually to a terrestrial mode of life, three important modifications of it have been laid down by the protoplasm, viz., the pectin, the lignin, and the cutin types. The two former will mean while engage our attention.

Pectin seems to occur as an ordinary wall substance in even the lower algæ, but it and lignin become set apart as the strengthening materials for cell walls of terrestrial plants. Deposited as successive fine layers from the protoplasm, and on the interior of the pure cellulose membrane, they give to the latter, when they are of a pectin nature, great tenacity and flexibility as in bast fibers, e. g., of flax; or, when of a lignin nature as in wood fiber, they give at once strength, hardness, and considerable tenacity.

Now from the group of the liverworts or Hepatica, where they first appear conspicuously as secondary deposits, upward to the highest plants these appear and disappear wholly according to environal relations. Thus in climbing terrestrial aroids the cell walls in roots, stems, and petioles may often be heavily thickened by pectin or lignin deposits; in soft and short-lived shade-loving species it may be small in amount; while in such aquatics as Orontium only feeble deposits are locally formed. Comparison of Polygonum amphibium in its aquatic variety with others like $P$. lapathifolium that are upright resisting types, or of Ranunculus aquatilis in its stem tissues with such an upright species as $R$. bulbosus, reveals like striking differences. But scores of such examples might be cited from allied species or genera of a family. From the alga upward for pectin, from the moss alliance upward for ligin, then, these definite allies of cellulose may appear and disappear from tissues according to environal conditions.

Cutin. The nearly related and highly important cutin is of prime value as a protection of surface tissue cells from over evaporation. So while doubtfully present in some of the Schizophyceae this substance becomes a wall modification that appears and disappears in amount and even in recognition, in practically every group from the Hepaticae upwards. Here again also one notes that in aquatic species or genera of a family it may scarcely if at all be recognizable by chemical test, while in xerophytic plants of perennial growth, like the leaflets of cycads, or the tissues of yuccas, of aloes, of agaves, of eucalypts and many others, it may attain great thickness. 
Cort is formed beneath or by epidermal and cutinous tissue as a cell layer, but as indicated in detail by Czapek (24, 1: $572-580$ ) it seems nearly related to, if not identical with, cutin. The manner and degree in which it may arise amongst isolated genera of a family, as in the stem of Draccena and Yucca amongst Liliaceæ, in Agave amongst Amaryllidacer, on the' air roots of aroids like Philodendron, on the perennial roots and stems of some species and yet absent from other annual species of dicotyledon, are all features that we consider to be correlated with environal stimuli, as calling forth or obliterating lines of energy that at one time become well marked, at another sink from observation.

Passing next to the formed products or foods, the group of sugars can only be glanced at.

Glycogen is formed according to Hegler (43: 229) in various of the Schizophyceæ. In many fungi it has been minutely studied (24: 1: 233). It is frequent amongst the Myxomycetesorganisms formerly classed as plants, but now placed by some amongst animals. Its wide distribution amongst animals under the name of animal starch is well known.

Glucose or grape sugar has wide distribution amongst green plants, and seems to be the first "organic" product of food elaboration in sunlight. In fruits it is always associated with the allied fructose. But glucose also is formed amongst higher animals in the pathological state known as Diabetes mellitus. Cane sugar or saccharose of many plants and milk sugar or lactose of animals are nearly related bodies, and are frequent though not invariable constituents of many plants and animals.

Starch is one of the most typical and widely distributed reserve foods of green plants, though it seems to be absent in animals. But the somewhat simpler substance glycogen represents it in Blue-green Algæ and in animals.

The fixed oils and fatty acids are a large and very natural group common to plants and animals. A neat and continuous series of glycerides of the fatty acids is that which includes such well-known bodies as butyric and stearic acids These illustrate well the principle of chemical substitution or addition - in this case the addition of $\mathrm{CH}_{2}$ in the advancing seriesas regards molecular constitution, and, since all have been definitely obtained from plant or more rarely from animal substances such as the fats or tallows, we give them in continuous molecular relation. 


\begin{tabular}{|c|c|c|c|c|c|}
\hline ormic & acid & $\mathrm{CH}_{2} \mathrm{O}_{2}$ & Undecylic & acid & $\mathrm{C}_{11} \mathrm{H}_{22} \mathrm{O}_{2}$ \\
\hline Acetic & & $\mathrm{C}_{2} \mathrm{H}_{4} \mathrm{O}_{2}$ & Lauric & “ & $\mathrm{C}_{12} \mathrm{H}_{24} \mathrm{O}_{2}$ \\
\hline Propionic & 6 & $\mathrm{C}_{3} \mathrm{H}_{6} \mathrm{O}_{2}$ & 'Tridecylic & “ & $\mathrm{C}_{13} \mathrm{H}_{26} \mathrm{O}_{2}$ \\
\hline Butyric & “ & $\mathrm{C}_{4} \mathrm{H}_{8} \mathrm{O}_{2}$ & Myristic & “ & $\mathrm{C}_{14} \mathrm{H}_{28} \mathrm{O}_{2}$ \\
\hline Valeric & “ & $\mathrm{C}_{5} \mathrm{H}_{10} \mathrm{O}_{2}$ & Pentadecatoic & "๘ & $\mathrm{C}_{15} \mathrm{H}_{30} \mathrm{O}_{2}$ \\
\hline Caproic & 66 & $\mathrm{C}_{6} \mathrm{H}_{12} \mathrm{O}_{2}$ & Palmitic & 66 & $\mathrm{C}_{16} \mathrm{H}_{32} \mathrm{O}_{2}$ \\
\hline Heptoic & " & $\mathrm{C}_{7} \mathrm{H}_{14}$ & $\mathrm{Ma}$ & “6 & $\mathrm{C}_{17} \mathrm{H}_{34} \mathrm{O}_{2}$ \\
\hline Caprylic & 66 & $\mathrm{C}_{8} \mathrm{H}_{16} \mathrm{O}_{2}$ & Ste & “6 & $\mathrm{C}_{18} \mathrm{H}_{36} \mathrm{O}_{2}$ \\
\hline Pelargonic & & & Nondecylic & "6 & $\mathrm{C}_{19} \mathrm{H}_{38} \mathrm{O}_{2}$ \\
\hline apric & “ & $\begin{array}{l}\mathrm{C}_{9} \mathrm{H}_{18} \\
\mathrm{C}_{10} \mathrm{H}\end{array}$ & & & \\
\hline
\end{tabular}

Now if a few be selected from the above list it can be stated that formic acid has been detected in pine tips, in the stinging cells of nettles, in the muscle of animals, in some animal secretions, and in red ants as the stinging substance, according to older authors. But the more recent researches of Bergmann (68:731) show that it may appear in tissues from the algæ and fungi upward to the flowering plants where it is frequent. In all probability then it may be detected in a wider variety of animal tissues. According to Bergmann acetic acid is of equally wide distribution amongst plants, and, though it is not met with in animals, acetone that occurs pathologically in the blood and in urine is a related substance that on oxidation splits up into acetic and formic acids.

Butyric, valeric, caproic, palmitic, and stearic acids are all common to both organic kingdoms, and in both form reserve food substances. Other of the fatty acids like lauric, myristic, and arachidic seem to be peculiarly vegetable products. It should further be noted that, in the more complex acids of the above list, those which contain an odd number of carbon atoms are not natural but artificial products, so far as known.

The oils and fats into which the above and related bodies enter in composition appear as rounded, or at times when fatty as almost angular, masses in the cells that store them. In the algæ and fungi they occur alike in the vegetative and spore cells; in the Hepaticae they often appear as large dull spherical or oval masses crowding the leaf cells; amongst mosses they are met with in the protonemal threads and in the spores; while in higher groups they become increasingly abundant and varied. But for us at present the important fact is that they may arise at any evolutionary stage in the entire plant series. Though our comparative knowledge is more limited, the same seems to be true for the animal series.

The large group of the amides and amino-acids have variously been regarded biologically as intermediate steps in the elaboration of complex albumens from carbohydrates and nitrogen 
compounds, or as dissociation products of albumins. Much can be said in favor of both views, but, without entering into this at present, it might shortly be said that leucin, iso-leucin, aspartic acid, glutaminic acid, lysin, guanidin, arginin, phenylalanin, tyrosin, and others of the amino-acid group are common to plants and animals, though found only in definite genera, or families, or even in certain tissues, and at definite periods in the history of the individual organisms.

The comparative study of proteins as found in plant and animal cells has been so fully elaborated by Mann (5\%:346-424) that reference need only be made to his work. But as set forth there, alike by Cohnheim and the author (pp. 349-352), much difficulty is experienced in making accurate comparison owing to the great variety and extreme complexity of the albumin compounds. Mann further adds: "We may safely assume that in their coarse chemical framework all groups of plants and of animals agree, and that those characteristics which are peculiar to each individual species are produced by transformations and substitutions in 'side-chains'; and, further, that this change is only possible by a new arrangement of the different amino-acids both as regards the relative quantities in which each acid is present and also the order in which they are linked to one another."

Ferments. The remarkable group that most physiologists are inclining more and more to regard as protein compounds, namely the ferments or enzymes, are common in many cases to the vegetable and animal kingdoms. These enzymes seem at least in some cases to be formed in cells by transformation of certain protein granules known as zymogens or ferment producers. The longest known and best investigated of these ferments is diastase. We know accurately that it is present throughout the entire vegetable and animal scale, in order to effect the chemical transformation of starch into sugar. Thus amongst the Acaryota its secretion by various bacteria enables them to liquefy and change starch into sugar, which is then absorbed in nutrition. The yeast plant and many other fungi form it, while algæ like Spirogyra that produce abundant starch change this by diastatic action. From the moss alliance upward the relation of stored starch and of transforming diastase seems invariable and intimate. Space considerations prevent us doing more than to mention the two varieties, both of which show well-marked peculiarities, and which may be called solution and corrosion diastase (69:31).

Diastase has been detected even in unicellular organisms although from their structure it exists side by side with other 
enzymes in the general protoplasm. In all the important groups of the Invertebrata and Vertebrata, it is secreted either by special glands - the salivary glands - when it has often been called ptyalin; or it may be secreted along with other ferments from some part of the alimentary canal, or of glands associated with the mid region of the canal.

Inulase, trehalase, invertase, glucase, and cytase are all closely related ferments to diastase, and effect digestion of inulin, trehalose or fungus sugar, cane sugar, cellulose, etc. All of them occur in at least some of the fungi, and even two or three may be secreted by the same plant for different lines of digestive action. They have been little looked for as yet amongst the moss and fern alliances, but flowering plants frequently yield all of them, except perhaps trehalase.

For the digestion and utilization of the oils that are elaborated directly, or often subsequently stored in quantity both in plants and animals, a ferment that may appropriately be called lipase is secreted. It has been separated from some of the fungi, and is traceable up into the flowering plants, where it abounds in germinating oily seeds like those of castoroil, hemp, and poppy. It is the active agent for oil digestion from the sponges up to man. As shown by the subjoined equation it causes stearin fat to take up water (as is true of nearly all cleavage ferments), and then splits the whole into glycerin and stearic acid.

$$
\underset{\text { stearin }}{\mathrm{C}_{3} \mathrm{H}_{5}\left(\mathrm{C}_{18} \mathrm{H}_{35} \mathrm{O}_{2}\right)_{3}}+\underset{\text { water }}{3 \mathrm{H}_{2} \mathrm{O}}=\underset{\text { glycerin }}{\mathrm{C}_{3} \mathrm{H}_{5}(\mathrm{HO})_{3}}+\underset{\text { stearic acid }}{3\left(\mathrm{C}_{18} \mathrm{H}_{35} \mathrm{HO}_{2}\right)}
$$

Mention is made below of that widely distributed group of chemical bodies, the glucosides, which probably result from splitting up of proteids. But the glucosides are in turn decomposed by a series of ferments, and yield glucose as one of the decomposition products. Hence the name given to the series. Emulsin or synaptase acts on a range of related glucosides, and splits these as follows in the case of amygdalin contained in seeds of the almond:

$$
\underset{\text { amygdalin }}{\mathrm{C}_{20} \mathrm{H}_{27} \mathrm{NO}_{11}}+\underset{\text { water }}{2 \mathrm{H}_{2} \mathrm{O}}=\underset{\substack{\text { benzoic } \\ \text { aldehyde }}}{\mathrm{C}_{7} \mathrm{H}_{6} \mathrm{O}}+\underset{\text { prussic }}{\mathrm{HCN}}+\underset{\text { acid }}{2\left(\mathrm{C}_{6} \mathrm{H}_{12} \mathrm{O}_{6}\right)}
$$

In salicin, the simpler glucoside contained in willows, the action is as follows:

$$
\underset{\text { salicin }}{\mathrm{C}_{13} \mathrm{H}_{18} \mathrm{O}_{7}}+\underset{\text { witer }}{\mathrm{H}_{2} \mathrm{O}}=\underset{\text { saligenin }}{\mathrm{C}_{7} \mathrm{H}_{8} \mathrm{O}_{2}}+\underset{\text { glucose }}{\mathrm{C}_{6} \mathrm{H}_{12} \mathrm{O}_{6}}
$$

It has already been found in many fungi, and in diverse families of flowering plants, so that its presence in many others 
may confidently be looked for. Its capacity to split up a variety of glucosides of varying chemical composition, and equally varying occurrence throughout the plant world, is alone highly suggestive. Indications of its presence amongst animals have been made, but more careful verification is needed.

The most complex group of ferments is the proteinaceous or proteolytic. Regarding these Green says: "We find that certain proteids, such as albumins and globulins, can be split up in various ways by different reagents, and that as a result of such splitting other proteids are formed, which we have reason to think have a simpler composition than either albumin or globulin. We find further that, on very profound decomposition, certain of these can give rise to crystalline bodies which are not proteid, but which belong to the group of substances known by chemists as amides. All these bodies occur naturally in the vegetable and animal organism."

The changes undergone in transformation need not be traced minutely here, further than to indicate that thus the proteids are split into simpler and soluble compounds that can be readily assimilated by the organism. Like the glucoside ferment emulsin, such proteolytic ferments as pepsin can act not on one but on several distinct protein compounds, and even on gelatin. While exact knowledge of pepsin is confined almost wholly to the animal kingdom - throughout which it is widely encountered-trypsin is a related type that has been traced with great exactness from the lowest plants (bacteria, yeasts, etc.) upward through the higher plants, where it may occur in quantity in such fruits as the pineapple, cucumber, and papaw. It is met with in the simpler as well as in the higher invertebrates, and hence can be traced upward to man. Chittenden inclines to regard vegetable and animal trypsin as allied but distinct. It may well be, however, that the nitrogen difference on which he bases conclusions may result from the activity of another ferment that has not yet been isolated.

The clotting ferments, pectase, thrombin, and rennet, at present occupy a position that may well make us hesitate in accepting present-day results as final. For the first is considered to be peculiar to plants and the second to animals, while the third is common to both.

We can now treat in succession the widely distributed cell contents that seem to be produced by analysis of the more complex protein bodies by ferment action. These are the glucosides, allialoids, dissolved pigments, crystals, tannin com- 
pounds, and the hydrocarbons that include resins and rolatile oils. It is from amongst this large aggregation of compounds that we might well look for substances that could very appropriately be called "acquired characters" or, better, "acquired chemical compounds." But further they rarely, if ever, enter into the upbuild or permanent constitution of the plants. They thus constitute the large group that we may designate as accessory cell-contents. Their function is a more or less temporary one. For though some, like the glucose derived by decomposition of glucosides, seem at times to become true foods, they most frequently act as defensive compounds that ward off the attack of parasitic fungi, or of the many and diverse animals that might browse on and exterminate the plants that form them. These compounds therefore constitute, in a very real sense, the defensive equipment of the plant world. They include also a very large proportion of the definite chemical bodies that seem to be present as such in living plant tissues, but which at the same time may chemically or electrolytically act and react on each other. Their absence or rarity in animals seems to suggest the difference between a fixed and a motile organism in regard to defensive methods.

It would be impossible to treat, even cursorily, the extensive series of each group. But their often closely related and yet chemically distinct characters, that are undoubtedly due to gradual substitution changes, evolved during the evolution of one species or genus from another, are well illustrated in some of the glucoside and alkaloid groups, that have been carefully investigated during the past half century by different distinguished "organic" chemists. The rapidly increasing size of the volume, and the condensing type used, in recent works on organic chemistry, testify to the rapid advance in our knowledge of these, as well as of allied compounds, that are only artificially produced.

Though in the above statement we have attached-and justly so-preponderating importance to the accessory plant compounds, it should clearly be noted that various parallel, and at times closely related compounds have developed in 
animals. Thus the alkaloid xanthin is met with in the blood and in various glands of animals, as well as in some flowering plants. But since animals, by action of and reaction to environal stimuli, by struggle for existence, and other cooperating causes, have in most cases become highly active so as to escape from devourers, have developed efficient defensive scales, plates, shells, secretions, or colors, so as to resist, or be overlooked by, other animals as attackers, and have used these coverings also as protection against over-rapid changes of temperature, light, and other physical environal agents, the result has been that the variety of accessory chemical substances is not nearly so extensive amongst animals as amongst plants.

A comparative chemico-biological study of them that admirably supplements the more purely chemical descriptions of the organic chemistry text-book is given in the works of Wiesner, Czapek, Tschirch, and Fischer. Here also the fact cannot be too strongly insisted on that all of these secondary and accessory compounds are as exactly chemical bodies in their composition as are such inorganic substances as chloride of sodium or common salt, carbonate of lime or marble, and sulphate of magnesia or Epsom salts. Like these also they are in nearly all cases crystalloids, and so stand in marked contrast to the carbohydrate, amidated, and albuminous colloids. But a significant feature is that so far few of them have been built up synthetically, and these few have been obtained only by elaborate chemical or electric laboratory methods. This is another argument we consider in favor of the activity of combined bio-cognitic energies in the tissues.

The glucosides, as at present known, are vegetable products, and are mainly yielded by the higher flowering plants, since by far the larger number are peculiar to and obtained from the tissues of dicotyledons The exact chemical formula of each has been determined in most cases, and this shows that some like amygdalin $\left(\mathrm{C}_{20} \mathrm{H}_{27} \mathrm{NO}_{11}\right)$ may contain nitrogen and be quaternary. But the majority consist of compounds of carbon (C), hydrogen $(\mathrm{H})$, and oxygen $(\mathrm{O})$, that are combined in rather complex proportions. Like the succeeding group they mainly act as protective substances against animal and 
possibly fungoid attack, but as they always yield sugar on analysis they may further act as plant foods.

Now, while, so far as at present known, each may be peculiar to one or to a few related species of plant, it is often the case that the same glucoside appears in genera or even families with no special affinity. Thus, to cite only a few cases, hesperidin $\left(\mathrm{C}_{50} \mathrm{H}_{60} \mathrm{O}_{27}\right)$, found in ripening fruits of the orange genus, is also met with in the somewhat related genera Barosma, Skimmia, and Toddalia. But it is also reported by Vogel from Scrophularia nodosa, a widely different type. Syringin $\left(\mathrm{C}_{17} \mathrm{H}_{24} \mathrm{O}_{9}\right)$ occurs not only in the related lilac and privet genera, but also in the white locust (Robinia Pseudacacia). Coniferin $\left(\mathrm{C}_{16} \mathrm{H}_{22} \mathrm{O}_{8}\right)$ is not only largely typical of genera belonging to the family Coniferae; it has been recognized in Asparagus, in sugar beet, and in one of the Compositæ. Quercitrin $\left(\mathrm{C}_{36} \mathrm{H}_{38} \mathrm{O}_{20}\right)$ as such, or as a nearly related substance, occurs in genera of many families of dicotyledons. Are we then to regard each case of occurrence of these as a long drawn hereditary or as an acquired character?

Of the alkaloids, upwards of 200 have already been isolated almost wholly from the higher flowering plants, but these probably represent a smaller number than will in time be recognized. They are all nitrogenous compounds, and a continuous serial relation can at times be traced between groups of them, that indicate chemical substitution action. Thus xanthin that occurs alike amongst plants and animals, methylxanthin, caffein that is frequent amongst plants but is specially rich in the coffee and cacao plants, also theobromine obtained from the cacao plant, have an advancing formula-relation thus:

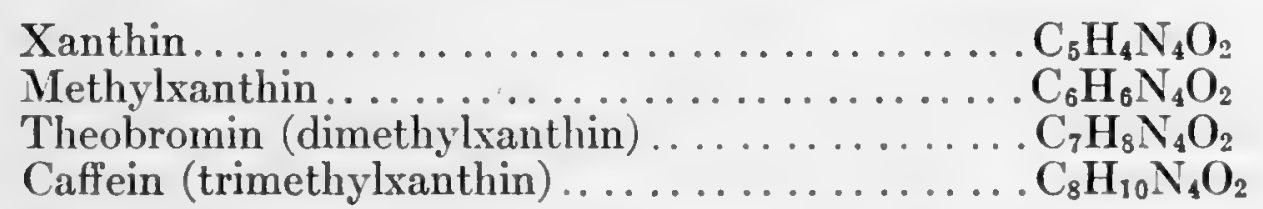

Similar but more complex relations can be traced in the morphin-atropin series of vegetable poisons, as well as in others. It will probably remain true that these compounds can correctly be called acquired characters for dicotyledonous plants, since few have been observed in more primitive groups, and they are also rare amongst animals.

That these compounds have not suddenly and wholly developed however amongst dicotyledons is well brought out, if one review the information given by Czapek (24: II, 259-316). He there points out that a few occur amongst the fungi, which seem frequently to show a higher and more intricate chemical 
activity than do the algoid or moss alliances. They are occasional in the fern alliance and the gymnosperms, about twenty occur amongst the higher monocotyledons, and the remainder amongst dicotyledons. Further, they are almost absent amongst members of the Incompletæ, are fairly abundant and varied amongst the apopetalous groups, but are most frequent in the sympetalous or highest dicotyledons.

Present facts would lead us to consider that they have been evolved as accessory or by-products, that have by natural selection remained in the plant tissues, to act there for defensive protection, or have again been largely or wholly absorbed. But that this action is limited in its range ecologically is proved by the capacity which various fungi possess of causing widespread havoc amongst coffee, tea, and similar crops, and which either have become immune to definite alkaloids or have formed an antitoxin in their tissues that neutralizes the alkaloidal poison of the host. The condensed account given by Czapek (24, 2: 927-28), regarding this line of inquiry, should be consulted.

The volatile oils and resins form a very large group of compounds, that are shortly distinguished from the two previous groups by being insoluble in water. They are generally contained in special cells or intercellular spaces of plant tissues. Their average chemical composition shows that they are simpler than the alkaloids or glucosides, but functionally they act like these as defensive substances. They are rare amongst the simpler plants up to and including the fern alliance, are widely distributed, though not varied in number, amongst the Coniferæ, and are specially characteristic of higher dicotyledons. Some plant families like the Pinaceæ or Coniferæ, the Aurantiaceæ, Myrtaceæ, and Lamiaceæ are specially rich in them, and so resist fungoid and animal attack rell. But the micro-fungi, and the boring insects that ramify through the tissues of some of the above, again prove that immunity against destructive environal agents has been a prolonged and fierce struggle on the part of surviving members of the sedentary plant world.

Very few volatile secretions have been met with amongst the lower animals, but amongst mammals they are frequent, castoreum of the beavers being one example. The tail, foot, facial, and other glands of different mammals all yield volatile products.

The tannins and trumin compounds with approximate formula $\mathrm{C}_{14} \mathrm{H}_{10} \mathrm{O}_{4}$ can next be reviewed, for like the three last groups they have the same approximate distribution in the plant 
world, and we believe are primarily defensive. They occur here and there amongst the algæ and fungi, are fairly abundant, in the moss and fern alliances, become at times very abundant in the cellular cortex of many gymnosperms, they are fairly frequent, sometimes even rich, in the aerial and subterranean parts of monocotyledons, but attain their richest and most abundant formation in the dicotyledons, whose underground and aerial stems, leaves, and fruits may contain relatively large quantities. Czapek has presented (24, 2: 587-591) the views held by different investigators as to the functions of the tannins. According to some they are end-products in analytic action; others consider that they aid in food transformations; or again that they unite with definite bodies to form new compounds; or, as we would most fully accept, they are primarily protective compounds, that are usually massed in or near the surface tissues, where attack by other organisms is often made.

There now remain for consideration certain structural and physiological conditions that can best be treated in succession to what has gone before.

Alike in plants and animals glandular secreting structures are identical morphologically, and may range from a single cell, with richly granular protoplasm and a well-formed nucleus, through every grade of complexity till we reach the elaborate sunken glands that are formed on the stem and leaves of the pitcher-plants or Nepenthes (78:17), and the similar glands along the alimentary canal of animals $(70,7: 270 ; 10: 12)$. But amongst the highest animals, owing to the softness of surrounding tissues, the rich nerve supply, and the abundant secretion of some glands, these have advanced in branching complexity, though by no means in fundamental relation, far beyond the condition shown by the simpler animal glands.

That the secretion of such glands, whether of a simple or of a complex nature, is a restricted and locally evolved feature is shown on adjoining faces of the same tissue. Thus in Nepenthes one may at times observe a sugar-excreting gland on the outer side of the pitcher wall, and a tryptic-excreting gland on the inner side, both being connected with a common bundle that ramifies through the separating tissue. In animals from the cyclostomes upward, and even amongst many inverte- 
brates, a still more varied functional diversity may be seen, as in the skin glands and those of the alimentary tract (chap. 15) of nemerteans, etc.

Again epidermal coverings, such as hairs, cutin, and cork layers in plants, are as exactly comparable in primary origin as they often are in function to the hairs, scales, and plates of animals.

When we review physiological responses similar results are reached. Thus enough exhaustive work has been done on nearly every group of plants as to enable us to say that, from such unicellular organisms as Chlamydomonas or Pilobolus up through all intermediate groups to the highest flowering plants, there is a definite set of environal stimuli, and as definite a response by each organism to these. For example, the combined geotropic and apoheliotropic growth of the mycelium of Pilobolus is in contrast to the delicately heliotropic growth of the sporangiophore. The apoheliotropic, chemotropic, and hydrotropic mycelium of Polyporus igniarius is continuous with the delicately geotropic spore tubes of it. But, in experimental study alike of motile and of fixed plant parts amongst the Caryophyta, we have no evidence for considering that any of these show "trial and error" relations. Already much lower in the scale of evolving formation of inorganic colloid substances and in cells of the most primitive acaryophytes, such stimulation responses have already been fully determined. The physiological responses then that are seen in the simplest Caryophyta are those also which characterize the highest plants. The intensity and the localization of these may vary even in related species; their presence is fundamental.

It would be impossible in a work like the present to advance more evidence on the comparative "stock-in-trade" of plants and animals. Enough we consider has been adduced that will enable us to discuss intelligently the position taken in the beginning of this chapter. The present brief review, fortified by abundant additional evidence that all can collate, warrants the writer in accepting a widely extended and closely uniform series of hereditary characters that remain typical 
and common throughout the entire organic world, and which may be called forth or may be absorbed, according to the action or the cessation of environal stimuli.

The protoplasm, the nucleus and nucleolus, the cell wall, the chlorophyll, the food substances from sugars up to proteids, the ferments, various accessory compounds, the cilia, the glandular relations, the physiological responses are a common and continuous inheritance from the simplest nucleated plants up to man; except that for plants - which in virtue of chlorophyll development have become primarily food elaborators, and in virtue of their sedentary habits have utilized many defensive substances - characters that remain uniform throughout for them are not carried into and evolved by animals, owing to their dependence on green plants.

But we have already seen that, in the Acaryota, evolving stages of various of the above can readily be traced. The nucleus and nucleolus, the chlorophyll, the red and blue dissolved pigments, the greatly more limited variety of food products including frequent absence of starch, the apparently limited number of ferments - so far at least as present knomledge helps us - and the gradual evolution of cilia observable, all suggest that below the nucleated Caryota, whether they are unicellular or multicellular, we must look for the development and fixation of those characters that are typical and potentially hereditary for the entire series.

Similarly the formation of surface protections in response to environal stimuli, as well as the hereditary transmission of all the tropisms and of other response characters throughout the plant and animal series, is in uniformity with, and is correlated with, morphological constancy, from the simplest nucleate organisms upward.

On the other hand our study of the glucosides, alkaloids, oleo-resins, tannins, etc., which exist in the sedentary plant organism, rarely in the active animal organism, and that are more often formed from progressive and destructive analysis rather than from synthesis, has revealed that hundredspossibly thousands-of definite chemical bodies have evolved 
in the plant laboratory by varying actions and reactions without and within the cells. It is highly significant, also, that they are preponderatingly abundant amongst the flowering and eren more exactly the most evolved or sympetalous flowering plants, where their number is as perplexing as is their constitution varied.

But few will deny that these are mainly accessory chemical agents, which by their presence may intrinsically influence cell life, or aid in its survival; they are in no sense a fixed hereditary characteristic of the entire plant world.

If now, in view of the above conclusions, we try to approach the question, wherein has evolution of the Caryota or of one of them consisted? an answer may best be got if we endeavor to picture, as some writers have already done, the gradual process of upbuilding of a complex multicellular plant or animal from its egg. Such an egg can quite truly be called the unicellular or temporary or starting evolving stage in the organism's existence, that corresponds to the entire life of such a unicellular type as Chlamydomonas or Paramocium.

It may conduce better to future consideration if we limit attention meanwhile to the vegetable world. The unicellular plant Chlamydomonas may well be called a microcosm of those great hereditary characters of the Caryota already spoken of. It has abundant protoplasm surrounding a well defined nucleus and nucleolus, specialized and highly energetic cilia during the motile stage, chlorophyll that surrounds and aids the protoplasm in carbohydrate elaborating action except at the ciliary end where the clear protoplasm alone is present, a sap vacuole or two that permit diosmotic and possibly electrolytic changes between the protoplasm and contained materials, a small mass of red or reddish yellow pigment that during the motile stage is aggregated into the "eye-spot." It has a cell wall that during the resting state is a firm and easily recognizable cellulose investment but is extremely delicate like the animal wall during the motile state. It elaborates sugar and starch in its chlorophyll; it shows often globules or granules that give a proteinaceous reaction. We may almost certainly conclude 
then that, like similar cells, it contains two or more ferments for food transformation.

As experiments in test-tubes and on live slides show, it exhibits long-acquired and hereditarily fixed physiological responses to environal stimuli. Thus, when unilaterally illuminated by optimum intensity of white light in a small test tube, it after being simultaneously stimulated by gravic action and showing to this negative or apogeotropic response, by oxygen and $\mathrm{CO}_{2}$ and showing positive chemotactic action, exhibits no "trial and error" movement, but, after placing itself so as to allow these stimuli jointly to act, starts, after an excitation period, on a compounded line of movement that is the resultant of the lines of stimulation that have acted on it. But, should an intense light fall directly on the tube, the irritant blue-violet rays start a paraheliotropic stimulus, and then a line of movement is executed that is again a resultant pathway representing the compounded action of all the stimuli alike as to form and intensity of energy applied. Each stimulus acts directly as in a sluggish vegetating acaryophytic alga, but in this simple nucleated caryophyte all of the stimuli are linked up or compounded so as to give a new resultant pathway (p. 129).

If we suppose our unicellular Chlamydomonas then to undergo gradual evolution, histologically and physiologically, into a higher plant, the result would be, not the appearance of many new morphological or physiological "characters" in the vaguely understood sense of the word, but the septation or membering of it into many cells, to one or to groups of which would be allocated the "characters" and functions that existed together in its unicellular state. Thus, as happens in the growth of many multicellular algoid sporelings, the clear ciliary and fixing end, becoming septated, might derelop the rudiment of a holdfast, and in still higher plants the rudiment of a root, that would show restricted geotropic and apoheliotropic localization. The green protoplasmic end, continuing to enlarge and septate, might gradually withdraw the chlorophylloid substance into the deeper placed cells, and thus the mass would originate a colorless epidermis-rarely chlorophylloid in higher plants- 
and an inner green multicellular mass. The former would be exposed mainly to, and would most strikingly respond to, environal stimuli, by forming hair growths, or protective pellicles or glands. The inner zone, largely deroted to nutritive and respiratory metabolism, would septate off a series of cells that would act as the chlorophylloid cells, would septate others as food storing centers, and would, with increasing growth in length and longitudinal sap flow, evolve long narrow sapconducting cells. Such conducting cells or zones, at first primitive and soft when the organism was small, would become increasingly large, varied, resistant, and complex in function as the plant attained considerable size. So, for support of the mass, thick walled cells of pure cellulose in many lamellæ would first appear, as is still true of many algæ and fungi. But, with increasing exposure to aerial strains and stresses, lignified cells would in time more efficiently replace or strengthen the related substance cellulose. Instead of continued and diffuse division and redivision into separate individuals as in Chlamydomonas, continued division would become restricted to definite spaces or intercalated regions amid older tissue, or be restricted to rings. So the original oval or spherical cell would become, as even still is seen in some Acaryophyta, a thread form, next a branching growth, and later a complex plant body.

Furthermore the same environal responses to stimuli as are seen in Chlamydomonas, continuing to act, would become centered in definite regional cells, but would collectively function in the organism as a whole, similarly to those in the single cell. So geotropism and apogeotropism, heliotropism and apoheliotropism, paraheliotropism, hydrotropism, rheotropism, chemotropism, .and mechanotropism would all be distributed in cells, that would most perfectly contribute to survival of the organism.

Though some may vigorously object to such a conclusion, for reasons that need not now be given, we emphatically affirm that the above is not a fanciful imagining, but an exact picture of what has occurred in plant evolution. For every step in 
the process is happily preserved to us still, in forms left alive at the present day. But, since some of the members in the direct line of ascent have been blotted out, we are compelled to seek for their restoration by piecing together information yielded by remotely connected species, genera, or even tribes.

Now in the whole of this evolving process we witness almost entirely two changes proceeding. First, we observe an increasingly discrete and expansive placing of substances already in existence, including the exact number and position of each new cell-partition; second, this discrete placing causes a corresponding localization of functional activities. In other words we would clearly assert that, between a Chlamydomonas individual and one of the highest algæ, the only possible differences might be a more detailed distribution of cell constituents, through changes in lines of energy-flow, therefore of growthactivity, and so of material distribution; while attendant on this and even inaugurating it is a like distribution of responses to environal stimuli, or to these when they have reflexly become intrinsic stimuli. The fundamental conception, back of the changes, consists in changing lines of flow, and changing intensities in the lines of flow, of energy distributed throughout the organism. The molecules that register this openly for us need not be altered in a single atomic detail. The quantity and kind of molecules placed constitute the register of the activity and distribution of energy flow.

But if - as in the transition from aquatic to land plantsa definite new chemical compound appears, e. g., lignin, cutin, or possibly a glucoside or alkaloid, such represent "new characters" in the strictest sense of the word.

So here an important consideration presents itself. If we are to reach an accurate and measurable estimate of evolutionary change and modification, account must be taken minutely of every minute change in cell form, size, substance, and environal action along with resultant response. The writer has already emphasized this as a prime necessity (64, 2: 202), and was deeply impressed by it in his microscopic study of plant hybrids (63: 271). But in ordinary taxonomic descrip- 
tions of plants and animals, in morphological comparisons of races from the hereditary standpoint, in physiological responses by allied individuals, the relative length, shape, size, and color - very rarely the rapidity of response-are recorded when such appeal to the eye. Hitherto, however, we have largely overlooked the slight commencing bulging or thickening of a cell wall, the redistribution of color from one set of cells to another, the increasing strain-action placed on one cell or group of cells through wind, rain, running water, snow, or other organisms, and the reaction shown in modification of length, or changed wall resistance of the cells thus acted on. These and many related problems have yet to be studied and minutely estimated.

Now in connection with the question already raised (p. 150) as to whether certain supposed "characters" appear that seem to have been absent in related species or genera, but which are an evident heritage of plant or animal tissues lower down and higher in the scale of life, we already have amply sufficient evidence to show that some such cases undoubtedly are, and probably all are, merely due to a temporary cessation in chemico-metabolic activity of, or energy distribution to, a constituent of the protoplasm, either through distribution elsewhere of the appropriate food materials needed for their formation, or through absence or changed cooperation of the environal stimuli, that would have called forth-if acting-the temporarily suppressed "character." When the proper flows and proper combinations of energy are again reestablished, the living substance, or some chemical constituents of it, so acts as to cause reappearance of the temporarily suppressed "character." The instances of this are now so overwhelmingly abundant and exact that to deny such is to refuse the evidence of immediate chemical demonstration.

All changes, all alterations, in the organic as in the inorganic kingdom are due to the changed conditions, kinds, and relations of energy that place or remove, that build up or break down, variously energized molecules, which themselves are energycharged ether particles. Relative distribution and relative 
condensation of energy then, we would suggest, are the important factors at the foundation of all organic as of all inorganic change.

But the quite remarkable evolution of hundreds-possibly thousands - of secondary chemical products, the glucosides, alkaloids, acids, tannins, crystals, hydrocarbons like terpenes, resins, and volatile oils in plants, seems in marked contrast to the above continuous uniformity of substance. As already indicated, however, we believe that the explanation is not far to seek. Green plants have evolved along a sedentary line, where fixation for life alongside predatory fungi or animals means either extermination, or varied and effective means of defense for warding off attack. Animals on the other hand have evolved along a motile line, where increasing alertness and activity, combined with protective coverings of a defensive -more rarely color-protective or warning-kind have served best in the struggle for existence.

No one who has wandered over the Alps of Europe or other lands, over the southern savannahs or the prairies of the west, has failed to observe how severe is the struggle between evolving plant life and predatory animals-including therein insects, slugs, birds, and mammals - of herbivorous habit. When we add to these the increasingly severe attacks of parasitic fungi, as these have passed more and more from a facultative to a holoparasitic stage, and have branched off into new raceforms, varietal forms, and specific forms, we begin to appreciate how the individuals of a species, and the species of a tribe, have selected and conserved every accessory and at first fortuitous by-product of the cell laboratory that the better enabled it to practise selective survival. Even the by-products of food elaboration in animals could be better expended in movements, in surface coverings, or in surface pigments. Hence we believe the relative poverty of animals in diffused accessory chemical constituents.

We would by no means deny that in some cases these accessory products in plants may also have been utilized either as food formers or transformers, or as contact bodies in effecting metabolic change. It would seem decidedly peculiar if the 
large group of glucosides existed in plant tissues, and there readily split up, in presence of acids or ferments, into glucose and other products, if such glucose were not utilizable by the plant, at least in times of emergency. But such a relation in the struggle for existence would not at all act in so efficient a manner for continued formation, and selective retention, of accessory products, as when a plant is directly saved from extinction by the presence of a protective substance.

If a short summary be now given of the contents of the present chapter it might be said that: (1) a comparative review of caryotic or nucleate plant and animal tissues, from the simple unicellular forms to the most complex multicellular ones, shows that there is direct continuity and uniformity in all the important cell substances, and in all the cell reactions to environal stimuli; (2) that gradual but increasingly fundamental change in environal relations and stimuli may start the production of a few new substances, as for example cutin, lignin, or inulin in plants, and muscular, cartilaginous, or osseous tissue in animals; (3) that, during the diversified chemical actions and reactions involved in the above, accessory products may be formed that may primarily be purely waste substances, as with crystals and hydrocarbons in plants, or some pigments and odorous substances in animals. These may be broken down and excreted as is usual in animals, or if helpful as defensive or attractive agents may be conserved and even added to by natural selection, as has largely occurred for sedentary plants; (4) that the appearance and in turn disappearance of some accessory product, or even of essential substances, in places, quantities, and relations that formerly possessed them, results from gradually changed lines of energyflow, which may again be reestablished or may continue the new lines of flow with resulting metabolic activity in the new centers involved; (5) that such changes usually start through microscopically small alterations in the size, shape, quantity of substance involved, and energy stimuli of the cell or cells in question, and so start at first imperceptible progressive evolutionary changes, as happens to most organisms. Or the altera- 
tions may be on a macroscopic scale, through sudden change of environment, or by hybridization action, when mutational evolutionary changes like those elaborately advocated by de Vries may be started; (6) that all changes, even of the most minute kind, are due primarily to changing or changed lines of energy-flow, which start and gradually establish changed positions, relations, and amounts of the energized molecules which such lines of energy determine and build into place. 


\section{CHAPTER VIII}

\section{PENTAMORPHOGENY, OR THE ACTIVE CAUSES OF ORGANIC EVOLUTION*}

From the time when Buffon and Lamarck first pointed out the fallacies of "special creation" as an explanation for the origin of living bodies, many master minds, aided by willing contributors, have built up the great principles of organic evolution on an imperishable basis. In accepting their verified results as practically tested by his own experience, the writer stated (71:54) nearly two years ago (1909) that "heredity upbuilds, struggle for existence stimulates, environment carves and chisels, reproduction blends and continues, selective survival sits as the final arbiter of life or death for the individual and at length for the species." But the writer further concluded: "Speaking for himself the writer would say that this one factor, 'environment,' greatly outweighs all of the others in importance, and that possibly it is capable of further and wide extension to a degree that even the Neo-Lamarckian does not go." The above "factors of organic evolution" will now be considered, while a "further and wide extension" of environal relation will be dwelt upon, as a great law of organic progress, that we propose to designate the law of proenvironment.

* Since the above was written Osborn's able paper on "Tetraplasy, the Law of the four inseparable factors of Evolution" has appeared in the "Journal of the Academy of Natural Sciences of Philadelphia" (S. 2, Vol. 15, 1912). The very thorough exposition there given of evolutionary factors and of their minor branches should be carefully studied by all. The four factors which Osborn accepts are Heredity, Ontogeny, Environment, and Selection. In the present chapter the writer differs from Osborn in regarding Ontogeny not as a cause or factor, but as a cumulated effect that results from the interaction of all the factors or causes. The writer also regards Reproduction as a highly complicated factor, while he presents the Law of Proenvironment as a new factor of prime importance.

So the writer would regard "Pentamorphogeny" or the cooperative action of Heredity, Environment, Proenvironment, Selection, and Reproduction as the combined cause by which the typical structure of any organism is effected or built up. 
If, in beginning our survey, we view plants and animals simply as bodies which have evolved from inert ether particles and active molding energy, which are subject to the same cosmic laws as other bodies around them, which now consist largely of labile colloid substances of high complexity, and which show a combined variety and delicacy of response that places them in the most advanced or evolved rank amongst such bodies, then, in virtue of the continuity relationship that we have already accepted as clearly traceable, in passing from the inorganic to the organic world, we should expect to find similar agencies operating in the process of evolutionary unfolding, though possibly becoming somewhat altered in degree or in intensity, as we advance to increasingly complex bodies.

(1) Heredity. In chapter I (p. 15) we referred to the steady uniformity with which the relatively few and simple inorganic bodies preserve their molecular structure, and this, for reasons that we consider fully warranted, we term their heredity. This exact hereditary likeness of one crystal to another of the same kind could only be altered by the interchange of so much energy, and the resulting addition or displacement of one or more particles throughout the mineral. Putting aside for the present the latter result, it is to the static or hereditary condition that we will first attend.

But at the outset the postulation of inorganic heredity might raise the objection that heredity in inorganic and organic bodies represents quite distinct phenomena, to which the same term is inapplicable. This would be true were we to attempt arbitrarily to limit the term heredity to transmission by sexcells of bi-parental peculiarities, as some have done. But, were such an attempt made, we would be landed in the alike illogical and unscientific position of ruling out heredity amongst the primitive and asexual Acaryota. Yet these for long ages have transmitted structures and functions hereditarily, that gradually culminated in sexual differentiation. We accept it then as necessary that heredity existed amongst the Acaryota. But these, in growing and multiplying, divided or budded off parts of their cells, and each part separated as an new organism, 
that continued the hereditary characters of the original organism. In like manner the halves or smaller parts of a crystal, that have been separated by ice action or by chemical change, may be carried to a distance, then recrystallized by accretionary addition of particles from an appropriate solution or pabulum, so as to regain their original size. But even more exact hereditary relations are traced when we study the growth and multiplication of Traube's cells or other inorganic colloid mixtures.

Now the molecular sameness of carbonate of lime crystals, of colloid gelations, or of Traube's cells remains absolutely and hereditarily true so long as they are retained in exactly like environal conditions. But if, by chemical substitution of even one molecule at some point in these, a new body chemically is formed, then a new and different hereditary combination ensues. If however we merely vary the degree of concentration of the colloid substances used, in such experiments as those of Leduc (23:125), different sizes and shapes of colloid films will be obtained, that will remain hereditarily true to the new concentration states. So three possible states hereditarily may be traced in inorganic colloids. First, given a definite concentration of two or more substances that can start Traube's phenomena, and given an exactly unaltered play of chemical or other energy on these substances, like growth will ensue, and like multiplication by division and formation of new masses will be carried out. Second, if the substances remain the same but the degree of concentration of one or more of the substances be altered, and so also the lines of energy-flow be thus changed in intensity, direction, or otherwise, different sizes or forms of film cells will arise, which as in the first case will remain hereditarily true. Third, if the chemical composition of one or more of the substances be changed, then new forms will be built up that will differ from the first not merely as does the second, but which, in chemical composition, in osmotic relation to other substances, and in response to environal stimuli, will show fundamentally different characters, that will be transmitted to new masses arising from such, if continued in the same chemical environment. 
When a review is made of the general assemblage of organic bodies, it can truly be said that botanists and zoologists have been profoundly impressed by the apparently unerring manner in which the minutest details of plant and animal structure were handed down for each species. Unquestionably also, it was this impression which caused the early cosmologists to propound the view of special creation They were frequently also medicine men, astrologers, and priests to their nations. So, as will be more fully traced later, special creation became a part of international religious belief. Though now ne give to heredity its appropriate place as one factor amongst several in evolutionary history, that place deserves to be first.

But the marvelous exactness with which even the most microscopic parental details of both parents are handed down, in sexual heredity, from generation to generation in plants, was first demonstrated by the writer from a study of plant hybrids and their parents (63 passim). Thus as shown there in Plates I-VII the forms, the sizes, the numbers, the degree of wall thickening of cells; the number, size, and shape of hairs and of their cells; the stomata over a given area; the size and shape of starch grains; and every other detail of cell life were a blended and faithfully reproduced picture of parental details, except that these were each reduced either exactly or approximately by one-half. Though some observers have tried to obscure or cover over such exact results as applicable to parental heredity between two species, the evidence is overwhelmingly in favor of the correctness of such a conclusion. Some years previously Galton had reached a like conclusion for man, by statistical study of many naked-eye details (79: 12).

But, as the writer clearly and frequently emphasized throughout his work, the hereditary details are by no means always evenly and equally blended, but show at times a marked tendency to swaying more in the direction of one parent than of another. And here the subject of variation in individuals of common parents, and in individuals of a common species, at once arises in connection with evolutionary descent. It can be accepted fully, however, that in organisms, as in inor- 
ganic bodies, continuity in form, in size, in molecular constitution, in response to environal stimuli, will persist through successive new formations or generations, so long as the relation of the organic or inorganic body to its environment, or to environal agents that have gradually become intrinsic factors, remains the same. The growth by accretion of any crystal, as compared with the growth by intussusception of any inorganic or organic colloid body, represents the physical difference of crystalloid and colloid aggregations. Heredity, or like molecular structural continuity, is equally exhibited however in both. But it still remains a moot point with physicists as with physiologists whether colloids may not also grow by accretion to their molecules.

This hereditary principle explains why crystals of fluorspar, formed during the silurian period in some drusy cavity of a rock mass, exactly resemble others formed under like surroundings during the eocene period, and why when exposed in our day to subaerial environal agencies, side by side, both will undergo analytic change. And it equally explains why fossil examples of Lingula from the liassic formation exactly resemble those of the present day, and also why, when such are placed in a keenly competitive or in an unfavorable physical environment, they show analytic change and death. That the fluor crystal is a solid crystalloid body, while Lingula is a mixture mainly of labile colloid substances, is renewed proof of Graham's contention-since often demonstrated and extended - that matter may assume a crystalloid or a colloid state under certain environal conditions.

We can only regard disturbance of heredity then, or variation as we generally designate it, to be due to changed environal conditions, that act as stimuli to start responses or motions of energized particles. The admirable illustrations of this, marshaled and explained by Cope (73: 398-430) mainly from the palæontological side of animal life, are a few of many like illustrations which the studies of the past thirty years have brought together.

Numerous definitions of heredity have been given, but most of these apply mainly or only to man. H. Spencer well says: 
"The law is, that each plant or animal produces like kind with itself." His definitional statement further is wonderfully expressive: "Inherited constitution must ever be the chief factor in determining character." But we have claimed - and nature claims - a wider field for heredity than the above include, and so we would suggest "the like continuity of molecular structure in relation to like outgoing and incoming currents of energy, so long as a body is exposed to the same environment, or to an environment that, within definite limits, fails to alter its average constitution."

(2) Environment. The emphasis placed on environment in the above definition indicates that we would place this Lamarckian factor as second to heredity in logical relation, and to a large degree also in importance. And its deep significance consists in its dynamic potentialities. But acceptance of such a factor at once raises many and still fiercely debated questions, in connection with which the terms Lamarckians, Neo-Lamarckians, Spencerians, Darwinians, Weismannians, Mendelians, unfortunately are freely flung about. Without lingering over the merits or demerits of these "schools of the prophets," however, the question may be asked: Can we or can we not prove that environment "carves and chisels" every organism?

If we go back to the inorganic world we are forced to accept that, unless the environment be a hermetically sealed one like a drusy cavity or a closed rock fissure, environal action and inorganic body-reaction are everywhere seen. The crystalline masses-whether existing as isolated or clustered crystals, as baked igneous rock masses, or as granitic compoundsare broken up and worn away by frost or sun action, then washed down by snow, rain, or water currents; the colloid masses of thermal springs, of ferruginous marshes, and of volcanic areas, are and have been altered by thermal, by chemotactic, and by electric action, or by organic agency. By such agency they may be altered in their chemico-physical composition, so that a new body may be formed, which differs little molecularly in its composition from the parent substance, as 
when a nitrite becomes a nitrate. But the new body accurately transmits definite crystallizing and growth potentialities that differ from those of the original substance. If this be true for all inorganic bodies, and not equally so for all organic bodies, then some law or principle must be acting that shelters organisms from its action.

In the rork above quoted, Cope says (p. 476): "Herbert Spencer has defined evolution as a process of 'integration of matter and dissipation of motion'; 'the absorption of motion and the diffusion of matter' he terms dissolution (First Principles, Ed. 2, 1873, p. 542). If by evolution Mr. Spencer referred only to that of inorganic bodies and masses, his definition must be accepted; but the evolution of organic bodies, since it has proceeded in a direction the opposite of the inorganic, cannot be so characterized. Organic evolution has passed beyond the domain of the inorganic, and the terms applicable to the latter process cannot be correctly applied to the former. In organic anagenesis there is absorption of energy; dissipation of energy is only known in the functioning of organic structures, which is catagenetic; not in their progressive evolution, which is anagenetic."

If Cope's contention be correct, then much of the present volume is incorrect and superfluous. But, if we examine one or two of the expressions in light of exact scientific results, it will be seen that these are the expressions which largely have retarded scientific advance. Thus when he says "organic evolution has passed beyond the domain of the inorganic" we believe he would have correctly expressed his own views as a Neo-Lamarckian had he said "organic evolution has risen upon and rests on the domain of the inorganic." So, also, instead of adding "the terms applicable to the latter process cannot be correctly applied to the former," had he said "the terms applicable to the latter process can often accordingly be correctly applied to the former," he would have expressed the true position. Not only so the larger part of every chemical text-book can simply and accurately be termed the environal actions and reactions of cosmic bodies. 
When further he remarks "in organic anagenesis there is absorption of energy; dissipation of energy is only known in the functioning of organic structure, which is catagenetic; not in their progressive evolution, which is anagenetic," he failed to realize that, up to the first appearance of organisms in the world, the entire process of cosmic evolution had been one great anagenetic procession, while at the same time dissipation of energy was known in the functioning (a perfectly exact inorganic word) of inorganic structures, as when hydrogen and oxygen were split asunder amid volcanic changes; or through the functioning of hot iron and water vapor; or by the chemotactic functioning of some metals on acids; or by the mutual functioning of magnesium or related alkali and water.

Environal action then is a great cosmic function that is ever in play. When environment ceases to act, and when bodies amid an environment cease to respond, we have reached an inert dead world, where inorganic and organic agencies alike have no play. It would be hard to imagine such, however. In the consideration of organic environal action, we would consider it necessary that the principles reached in the last chapter be kept ever in view. For, if several organisms have branched off along lines of evolutionary modification, and some show at repeated points along those lines repeated capacity to form red pigments, multicellular hairs, thick or thin cuticle, and other modifications of cell detail, while others show no such tendency, such is proof, along with the many other confirmatory cases cited, that every organism inherits not merely protoplasm or a nucleus, but also the inherent chemical capacity to develop or to suppress many compounds, according to the stimulating or retarding nature of the environment.

The calling forth of the response as a fixed hereditary character seems always to be slow in action, and, where an organism, or group of organisms, suddenly becomes exposed to an unaccustomed environal surrounding, death may ensue. Even where the response occurs by pigment formation, wall thick- 
ening, glucoside secretion, these may readily be suppressed, or called forth for a long period in wavering manner. And only after long generations of growth, amid like surroundings, does the "character" become "fixed" for the time being, or permanently if like environment be continued.

As a case in point we might cite coloration of the pitchers in the seven species of Sarracenia that are all native to North America, and which the writer has studied, in their native haunts, over thousands of miles of territory. The most primitive species unquestionably is $S$. minor, whose pitchered leaves are most usually of a pale green, more rarely of a brownish green, hue. S. flava and S. Sledgei rank next in advancing specialization, are both bright green, with veinings of crimson behind or along the throat of the pitchers, or in a frequent variety of $S$. flava the entire back of the throat is of a rich crimson hue. But, in sunny meadows that are wet in winter, and whose soil becomes hard and dry in summer, all rarieties of both species may be gathered near at hand or in distinct localities, in which the color spreads from the throat, first along the vascular bundles of the pitcher, and in others even throughout the tube generally, so that this becomes of a deep crimson-purple color, and attracts the eye at many yards distance. The writer has often removed numerous groups of these to greenhouses where they have been watched, and where natural conditions could be imitated or altered.

He has clearly proved that the amount of pigmentation is determined by light intensity, combined with relative dryness or moistness of soil, the smallest, firmest, and most richly pigmented being those that were simultaneously exposed to the brightest light and to moist soil conditions at one period, becoming dryest soil conditions at another. Even by sufficient shade, it is possible to grow leaves of a uniformly green color, that in the previous season were crimson-purple.

But S. rubra, S. Drummondii, S. psittacina, and S. purpurea show steadily advancing specialization, alike in pitcher and in floral details. They show like advance also in pitcher color, so that plants of S. purpurea, growing as they usually do in 
sunny situation, are of a purple-crimson tint, while a variety of it, that is not unfrequent in some of the Gulf States, is uniformly of an intense deep crimson-purple color. But even S. purpurea when it grows-as it occasionally does-in the rather deep shade of swamp thickets is always uniformly green, or shows only purple lines along the back of the throat. Like changes can be produced with almost measurable exactitude under cultivation.

We conclude therefore that red pigmentation is being slowly developed by Sarracenias; that the capacity for formation of it resides in the cell contents; that in S. minor it is not perceptible as a rule, and in any case is feeble; that owing to environal and mainly light action it is being more and more richly formed, and as aiding in insect attraction is being selected; that such color may have existed in greatly more primitive ancestors of the group, belonging to the Incompletæ, if we may judge from analogy with other groups, but the environal stimulus may have waned, and the color may have receded to the disappearing point, to be again called forth as above, when life relations were favorable.

As John Bartram well observed nearly 150 years ago, the Venus fly-trap (Dioncea muscipula) shows equally sharp color changes, according to natural or artificial environal states. For it may vary from pale yellow-green, in deep shade, to a deep uniform purple, in hot savannah situations.

Another case might be cited that greatly impressed the writer. After knowing and experimenting with the Sensitive Plant for fully a decade in greenhouses, where it always was of bright delicate green color, with occasional faint traces of brown color in the stem, specimens were planted out in his garden twenty years ago during early August. These were exposed to atmospheric conditions for about seven weeks that were decidedly different from those of a slightly shaded hothouse. They often were exposed to hot bright light during day, and rather cold night states from the latter part of August. All of the plants developed a dark reddish pigment in their stems and leaf stalks, while the plants generally in continuing 
to grow became more dense and reduced in internodes, leaf stalk, and leaf-size. Since then the experiment has been frequently repeated, and with uniform results.

We again conclude that the capacity for color production existed in the plant, but only manifested itself when definite environal stimuli started definite chemical changes that produced the red color. Many similar illustrations that the writer has observed for color effect alone have been noted by him or recorded by others.

Similarly, were we to deal with cuticular thickness, with cell-size, with relative development of indurated tissue, with plant habit in relation to growth, abundant and exact evidence could be adduced to show that heredity includes not merely characters and potentialities that may alone be evident under certain environal conditions, it includes many others, for which the protoplasmic machinery, or some substance under its control, is perfectly adapted for its production, but which appear only as material evidences, when the appropriate stimuli or energy expenditures act on the tissues.

The experimental studies of Lothelier (74:480), of Bonnier (75:217), of Schively (65:220), and of many others since on plant species, and the corresponding studies of zoologistsnot least those recent ones of Beebe ( $\% 6$ ) and of Tomier on animal species-point the way to a future accurate estimation of environment as a factor.

The degree of variability of such animals as the Brine Shrimp (Artemia salina) or the Axolotl (Amblystoma tigrinum) - to use rather extreme cases - so as at different periods of their lives to simulate distinct genera is similarly explicable we believe. Therefore it is that in any attempted definition of heredity we believe it to be absolutely necessary that it should include the expansive clause "or to an environment that, within definite limits, fails to alter its average constitution." On this principle, therefore, every species of plant or animal shows a mobile or elastic adaptibility, that is at times as striking as it is unexpected. It is this adaptibility, also, which enables it, when in the egg state, to fuse with a complemental sperm 
nucleus from another species or even genus of plant or animal, and so to build up a dual organism that may, as the writer has demonstrated (63 passim), either blend exactly all of the parental peculiarities, or exhibit these as distinct morphological structures side by side with each other, as in the accompanying illustrations, where the large honey gland of one parent species of liliaceous plant, Lapageria (Fig. 2, a, b, and c), is entirely absent in the allied genus Philesia, but is reproduced of about half size in the hybrid "genus" Philageria, developed under cultivation. The writer has cited or illustrated various similar cases amongst flowering plants.
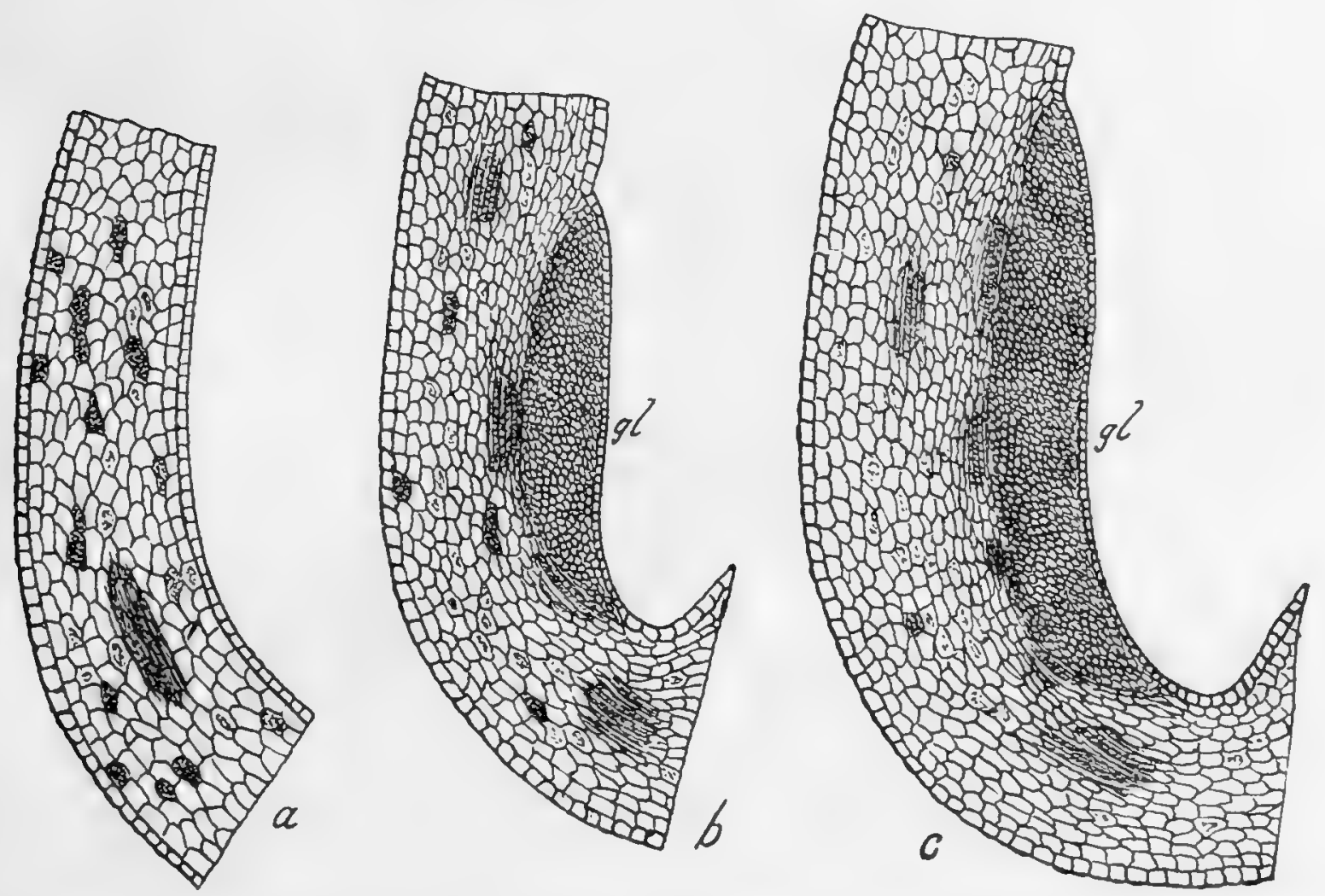

FIg. 2-Long. sect. through base of sepal of (a) Philesia, (b) Philageria the hybrid, (c) Lapageria. gl. gland.

If then it be asked wherein one species can be surely separated from another in hereditary relation, we would reply that, if by any environal stimuli some one or more characters or responses to energy be so replaced or modified by energizing action of another kind or type that the original character or response fails to reappear in the immediate descendants, while the new kind or type does, a new species results. But this 
would in no way prohibit the possibility of the former character reappearing in the future - and it might be remotely removed - descendants, were the environal stimuli such that the character or type of energy response was again called forth. But by that time many other alterations or replacements might have taken place, so that, when the resubstitution has been effected, it no longer is reformed into the exact type of organism that before showed it, combined with other characters.

In other words, every plant and animal possesses a common heritage of a stock lot of energy-combinations with ether particles of varying complexity, that we term material characters. These undergo definite permutations and combinations, according to environal stimulations and relations that definitely affect them. With the gradual evolution of the earth, since organic life first appeared on it, many environal agents have been successively called into play, which have added to the series that we might call the inorganic agents and which include varying intensities of light, heat, and other energies, chemical and physical characters of the soil for plants, or of plant and animal food for animals.

But the organic agents that often cause keen "struggle for existence" on the one hand or progressive evolution on the other are too much overlooked biologically. The parasitic fungi that infest plants and animals, the flowering parasites like dodder (Cuscuta) and broomrape (Orobanche), the animals that prey on plants or other animals, all cause either mechanical or irrito-chemical stimulation and analysis that the infested organism must either counteract, overcome, or die before.

Again the varied intimate beneficial adaptations between the flowers of higher plants and visiting insects would have been superfluous during the devonian epoch, when, according to all present knowledge, neither the groups of insects nor of plants that are adapted to each other had evolved. But evidently during the permian age both were slowly being evolved, and by environal action and reaction through millions of succeeding years the stimulating action of insects so molded 
and adapted flowers that now the climax is seen in such families as Orchidaceæ, Lamiaceæ, and Asteraceæ. We may not yet be able, in the biological realm, to apply Newton's law strictly, which declares that "action and reaction are equal and opposite"; but in antiserum action and prophylactic agents; in changed chemical food stimuli, and the exact modifications these effect, as cited (p. 285) for Saprolegnia; in the varying polarity of the gemmæ of Marchantia under varying light stimuli; in the exact stereo-molecular action of the sperm nucleus of one plant species when it fuses with the egg of another species, we see a few of very many proofs that environal agents act on organisms, and that these respond at first physiologically, but later and surely we nearly always find that the physiological responses start and continue to produce definite morphological results, that then become the measuring line by which the degree and intensity of environal energizing agents can be gauged.

It will readily be noted, then, that the above views recognize and involve the constant presence of exact and continuous lines-or to use Faraday's expression "tubes"-of energy, that traverse every organism, and every cell of each organism, in a manner that has become continuous, uniform, and hereditary. These so act that they exactly place molecules, or groups of energized particles, of appropriate chemical composition for each cell or organ concerned, in exactly the same relation to each other and to the organisms as a whole. Like all colloid bodies, these molecules are at times capable of mobile adaptation amongst themselves to a limited degree. But, when any of the tubes of energy gradually increase in strength, increased formation of molecules along these lines will occur. When, however, the flow of the tubes becomes by degrees modified to the extent of substituting a new atom, atoms, or molecules, a new character thus gradually is given to the organ or organism, in which the energy has thus effected change.

We are well aware that not a few eminent erolutionists still reject Lamarck's Law as alike unproven and superfluous. But if environment does not constantly affect and mold every 
body-inorganic and organic alike - then all nature is a lusus naturae, a gigantic scientific deceit. If, on the other hand, extremely complex molecules, that are unquestionably energized-as every chemist of the colloid groups acknowledgesby extremely condensed amounts of energy, possess a mobility and yet stability such as Graham emphasized for all colloids; and if these, like all inorganic compounds and many crystalline products of organic bodies, are influenced by environment so as to pass even from the crystalloid to the colloid state, from the condition of sugar to that of starch, and so on, then surely environment acts on all organic bodies, if these show, in every detail of evolutionary progress, peculiarities that become increasingly explicable as we the more fully and precisely use the environal key to explain the often tangled details. The beautiful studies of Ryder on strain distribution and digit modification in mammals ( $7 \%: 607$ ), and those of Cope on mammalian dentition, can only be interpreted as progressive modification through reaction to the action of mechanical or molic stimuli.

If now a definition for environment be sought for in current dictionaries, that from the "Century" is long, and owes its value more from its explanatory fullness than its epigrammatic neatness. "The sum of the agencies and influences which affect an organism from without; the totality of the intrinsic conditioning to which an organism is subjected, as opposed to its own intrinsic forces and therefore as modifying its inherent tendencies, and as a factor in determining the final result of organization. It is an expression 'used' in explaining that at a given moment a given organism is the resultant of both intrinsic and extrinsic forces, the latter being its conditions of environment, and the former its inherited conditions."

The above definition is admirable for organic structure, but would be practically perfect for the inorganic as well, were the words "a body" to be substituted for "an organism." For with our knowledge now of intrinsic atomic and molecular energy, of colloidal molecules and their degree of organization, 
as well as the rapid changes in some cases, and slow changes in others that various metals as well as other inorganic bodies undergo, the above definition, if modified as suggested, would exactly and appropriately include all "bodies." Shortly defined the law might be expressed as: the summated action of all external agents on any body.

(3) Proenvironment. We pass now to the consideration of an evolutionary factor that hitherto has been entirely overlooked, but which takes high rank alongside the above two. When we say that it has been entirely overlooked, we mean for the organic world as a great whole. But Kidd, in his two most suggestive volumes, "Social Evolution" and "Principles of Western Civilization," has closely approached the factor for man, though he has failed to trace its gradual evolutionary advance from plants up through all stages of animal life to Man, and has also failed in reciting the fundamental phenomena that start and continue the factor. We propose to term it the law of proenvironment or prosynergy.

Its far-reaching importance can perhaps scarcely be estimated as yet, owing to our imperfect knowledge of some of the simpler organisms, notable the Blue-green Algæ. We can select first, for experimental illustration, some germinating plant embryos, and conveniently those of the common broad bean or pea, whose roots are pushing out through the seed coat. The colorless radicles first protrude, and, in virtue of their being influenced or stimulated hereditarily by gravity, by moisture, by soil contact or friction, and by negative lumic relation at least, these all grow directly into the earth. But suppose three seeds to have been purposely so placed under shaded bell-jars (Fig. 3, a, b, and c) above plates filled with water that their radicles are illuminated from one side, and two are placed transversely; further that one of the two is exposed to the uniformly moistened atmosphere of the jar interior, while the other has a steadily moistened sponge hung near and above it. As all who have had even an elementary course in practical plant physiology know, three totally different pathways will be determined on and accurately pursued, by the 
three roots studied. While each one of those in the soil will grow directly downward as above indicated, that away from the sponge will take the course indicated in (a) while that adja-

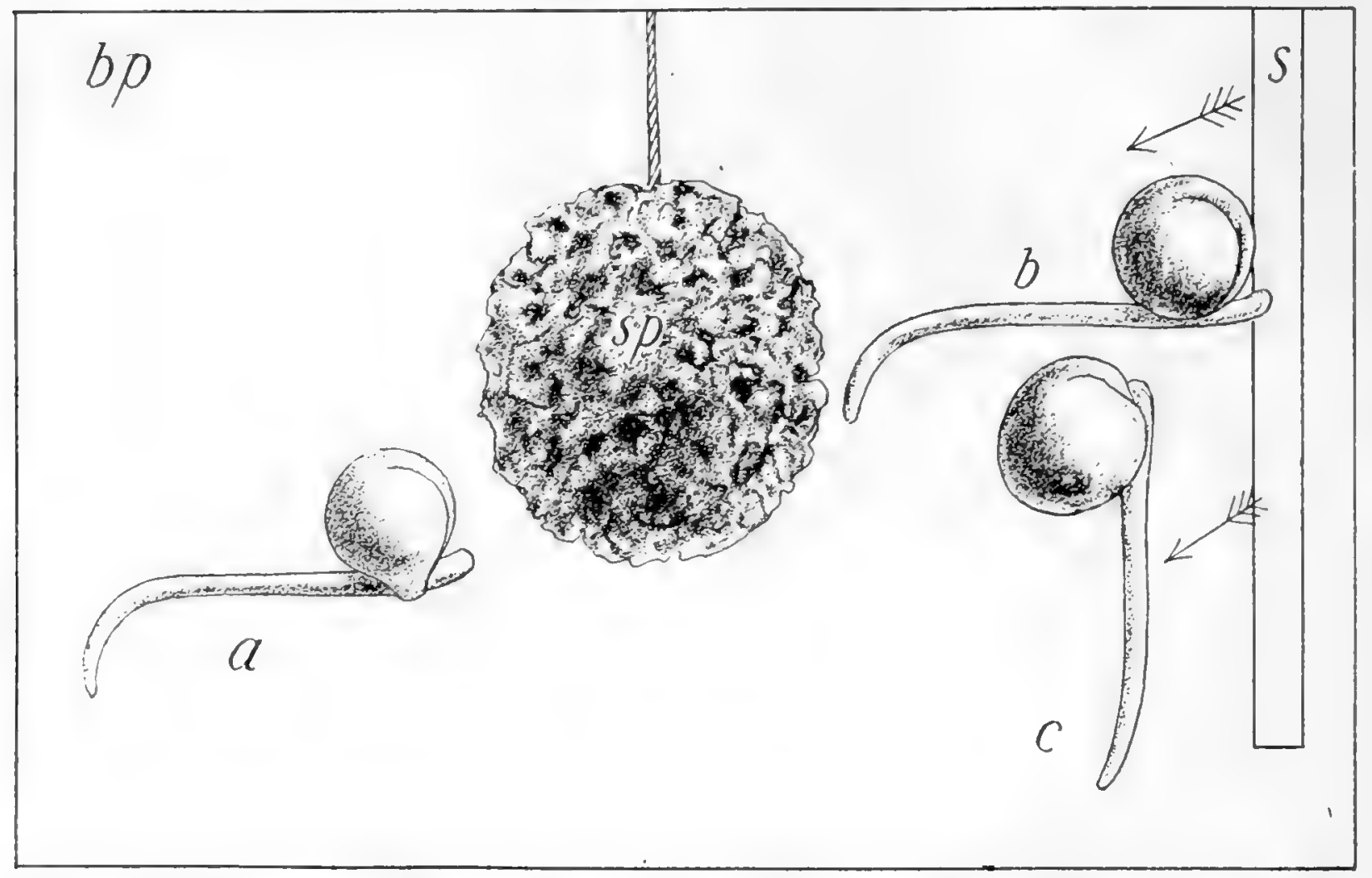

FIG. 3.-Young seedlings of pea. $a$, radicle when placed transversely has responded directly at its tip to gravity by bending downward; $b$, oblique position of tip due to hydrotropic action of moist sponge, $s p$, that has slightly counteracted geotropic action; $c$, downward growth of tip, as due to geotropism, and hydrotropism from moisture in dish below, both slightly counteracted by apoheliotropic effect of light from slit $s$ in belljar covered with black paper, $b p$.

cent to the sponge will grow as in (b). The third grows nearly downward in virtue of gravity acting powerfully in a straight line upward, and equally of moisture that rises upward. But the lumic stimulus is sufficiently powerful to cause the root to bend away from the light, and to a slight degree therefore to bend away from the downwardly vertical, that indicates the response to gravic and hydric stimulus. The second takes the course shown, because the hydrotactic stimulus of the wet sponge plus the negative lumic stimulus greatly overcome the gravic and the weaker diffuse hydric stimulus.

Here then in the above three cases the same type of radicle, when acted on by the same stimuli, applied at different angles, starts an intrinsic molecular machinery in order to plan out a pathway that is the most satisfying for each to pursue, but that yet differs in all three, and that is the compounded resultant 
of all three environal forces, acting on or stimulating the young cells of the radicle. The three radicles proenviron (to coin a suitable verb) exact and yet different pathways, before even the slightest external indication is shown. As every plant and animal physiologist knows, the length of time needed for proenvironing determination has been termed the excitation or latent period. The future "fate for weal or woe" of the plant may largely depend on the proenvironed pathway projected by the protoplasmic or chromatin molecules that constitute the energids.

But let us pursue the investigation further. As the plumule or young stem disengages itself from the seed coat, in an open exposed situation, it at once plots out a proenvironal course

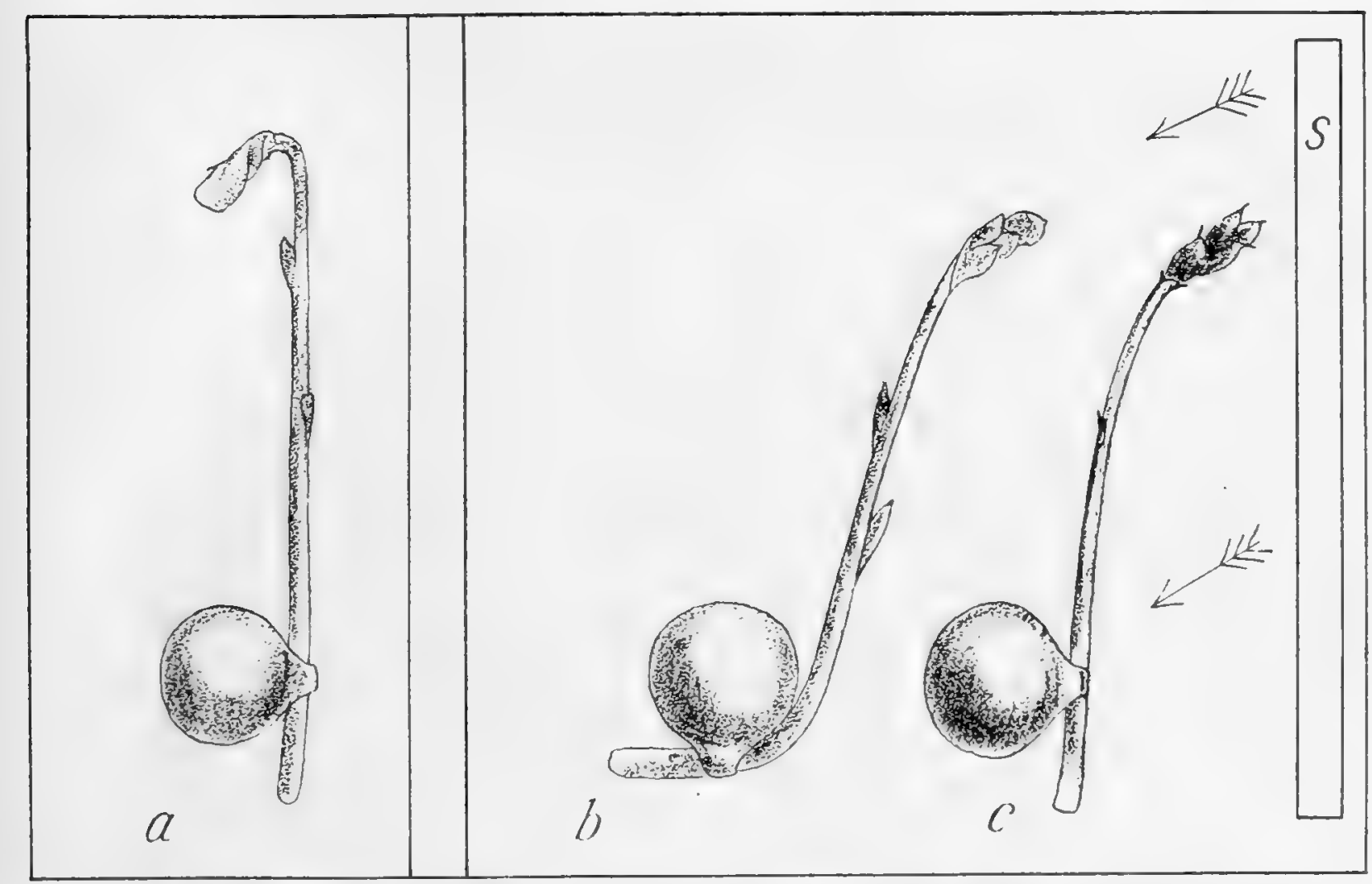

FIG. 4.-Seedlings of pea. $a$, plumule uniformly exposed to light; $b$, plumule of plant placed transversely in dark box with light admitted by slit $s ; c$, plumule of upright plant in box. The shoots of $b$ and $c$ show resultant response, in varying degree, to apogeotropic, apohydrotropic and heliotropic stimuli.

that ordinarily is apogeotropic, positively heliotropic, and apohydrotropic. So the actual pathway pursued is as seen in Fig. 4, a. But the second, that was placed under a belljar, pursues the course of Fig. 4, b, while the third develops a direction as in Fig. 4, c. Further, when the fully formed 
green leaves have arisen on each, it can readily be shown that these move their surfaces, or even in many flowering plants their entire leaves, through pathways that are compounded resultants due to optimum lumic stimulus (heliotropism), to gravic action (diageotropism), to supra maximum light action or intensity (paraheliotropism), even in some cases to mechanic and chemic stimuli. Each leaf then, so long as it is in a growing state (and in many "sensitive" leaves long after growth has ceased) is constantly proenvironing a course that later is accurately taken.

It is scarcely overstating the case to say that the greater number of movements performed throughout the entire range of the Caryota are not simple movements due to a single direct environal stimulus, but are resultants of linked together responses, while as will be shown below they become, by progressive and easily traced stages, of great complexity in the higher insects and mammals. The far-reaching value and increasing importance of proenvironment then, as an evolutionary factor, becomes more and more apparent as one rises in the organic scale, while it receives its most remarkable illustrations in the responses of superman during the decades that are now passing. Added illustrations will be presented in the succeeding chapter, and in other chapters of this work. But the writer hopes to issue a separate volume in which graded phases of this law will be treated of from all the leading groups of the Caryota.

Our scanty physiological knowledge of blue-green Acaryota and to some extent even of the colorless ones, such as the bacteria, prevents us speaking in certain terms as to proenvironment amongst these. The mode of growth and the lines of growth response, the often irregular shapes assumed, and the irregularly heaped up, coiled, or aggregated states of the simpler Blue-green Algæ from Chroococcus and Merismopedia through Nostoc and Wollea to Mastigocoleus and Fischerella or Stigonema would suggest that they show single, simple, and slow responses to separate types of stimuli. But, as we pass from the last to Schizothrix, Lyngbya, and Oscillatoria, the growth 
of these and especially the manner in which they radiate out in definite lines and threads over the surface of water and on to firmer lighted substrata indicate probable resultant, even though sluggish, correlations of two or more responses. But a wide field for experimental study remains open here.

The exact and predicable proenvironal courses taken however by practically every unicellular and multicellular member of the Caryota, at least during some stage in its history, form a factor of vast importance in organic evolution. Furthermore, when we reach the higher fields of mind and memory, it conducts us along pathways that hitherto have been closed, or nearly so, to continuous practical investigation.

The proenvironal response acts usually-perhaps alwayswith extreme accuracy, so as to plot out a course or pathway that may ultimately guide the organism along the same path as that already pursued by it, or by its ancestors for generations. This occurs if environal agents or stimuli remain continuously uniform in strength, direction of application, and otherwise. But the environal stimuli may become changed, and cooperate to carry the organism along an ascending and evolving line that conduces to betterment of the individual and in time of the species. Or again one or more of the separate stimuli may give rise to a resultant response that conduces to an analytic, descending, or devolving condition, and this will surely, though it may be slowly, lead to extinction of the individual and in time of the species, if such analytic stimuli exceed the catalytic.

We would now define this factor as "the resultant response of an organism to the sum-total of all the environal agents that act on it or on any part of it, and which causes the organism to proenviron a course or pathway that is temporarily satisfying to it, and that can alone be taken in virtue of the action of the several environal agents, and the reaction to each of these by appropriate organismal molecules," or in more condensed language we may define the law as "the correlated resultant response by any body to the summated correlation of stimulatory action, that leads to a temporarily satisfied state." 
During the past half century many writers have drawn attention to the great "betterment" process or steadily progressive principle that seems to run, as a guiding thread, through the entire plan of evolutionary unfolding. For they have realized vividly that, as well in the ontogeny of any plant or animal individual as in the progressive advance of any great organic group, there often is shown an apparent intrinsic tendency toward improvement, betterment, or advance, which if understood or recognized would largely explain to us the entire evolution process.*

The law of Proenvironment furnishes the needed principle. From abundant and direct experiment we know that every nucleate organism, and many non-nucleate organisms, show an exact response to each environal stimulus. This is evidently due to certain molecular changes wrought by the stimulating agent on the living substance, and later it may be on certain substances accessory to it, as can be observed during movement in leaves of Drosera, Dioncea, or Mimosa, amongst many others. These molecular changes inevitably cause a definite course or pathway of movement to be pursued, which is determined during that measurable period of time that we now call the latent period or period of excitation. At the close of that period-amounting, it may be, in some plants or plant tissues to a quarter second, in others to several minutes, but in most animals of much shorter duration - the organism has plotted a definite line of response or a pathway, even though as yet it may be motionless, that is as exact for the future result as if the response action had already taken place. Or in other words a definite mode or line of response has been determined on, that will cause the organism to reach out to or occupy a definite environal relation.

\footnotetext{
* One of the last to refer to this is Osborn (45: 282), who says: "This mutability, or evolution movement, or whatever it is that is in progress in the blastos, is the chief and most difficult matter either to explain or to form any conception of.

"Emphasis may be laid on the words adaptive necd or necessily because there is distinct evidence in palrontology that the blastos is not evolving alone through its internal forces independently of the external forces of ontogeny and of environment, but that there is some harmony or form of interaction, the nature of which we do not understand."
} 
As already indicated for the simple non-nucleate plants, each stimulus and kind of stimulus seems to act separately, and to be separately responded to. And this may in large measure be explained through these organisms living in an environment that during archæan times was relatively monotonous and unvarying.

But with advancing evolution of nuclear chromatin or cognitic substance, and the correlative incidence during every twenty-four hours of varied stimuli, each organism or part of it acquired an increasing capacity to link together the summated actions of several stimuli, and to project therefrom a response or pathway of action, that was a resultant of all. Examples of such we have cited in the roots and shoots of plants, the movements of infusors, etc.

The extreme complexity of some proenvironal movements in plants is graphically shown by experimentation with Mimosa or any other amongst hundreds of sensitive species. Each movement also may involve the action, the summated correlation, and the organismal reaction, connected with four to seven distinct kinds of energizing stimuli. Amongst the higher insects and mammals - where a nervous system with associated cogitic power exists-the rapidity of correlation and response are such that the original separateness of the component stimuli fails to impress the observer. It is this fact, coupled with a failure to realize the existence in the above animals of a condensed cognitic and cogitic energy, that has hitherto prevented a correct analysis of acts of intelligence, reason, and selection. In the next chapter and also in chapter 21 the se are more fully discussed.

(4) Selection. A fourth factor that we believe to be cooperative with the above three is selection, natural selection, or selective survival. If one watch the life career of an individual, or the species career of a group of organisms, the impression will be gained that, for success or continued existence alike of the one and the other, three requirements have to be fulfilled, viz., successful vegetation, successful defense, and successful reproduction. Under the first of these we include 
in their proper order (p. 44) the physiological activities of irritability, nutrition, respiration, and growth. These four, when in balanced and regulated activity, give health to the organism, and ensure its ontogenetic growth. They also represent the combined activity of heredity, environal action, and proenvironal response or reaction.

But amid plant and animal competitors, as well as amid destructive physical agencies, the most successful or perfect means of defense or resistance that the organism can evolve will best insure its and its posterity's continued existence. Every chemical substance therefore which may give strength and resistance, particularly over an exposed surface; every line of energy-flow that will conduce to transfer of defensive material; every waste product of the cell-laboratory that would in itself be useless as food, but which would, without being deleterious to the organism, defend it against destructive environal forces; every mode of growth that may be developed to overcome denudation, laceration, dessication, burial, or asphyxiation by inorganic agents; such will tend to be, and are, perpetuated as helpful factors in the life of each organism. Hence the origin, we believe, of the greatly varied cell contents of plants (p. 158), as well as of plant and animal cuticles, of crystals, and of not a few secretions amongst plants and animals. Hence also the varied armature of many animals, their coloration in part, the attitudes they often assume, and the associations they may establish.

So when successful regetation and defense or resistance coexist, so also will the best conditions be for perpetuation of the life of the individual or of the group. A full recognition of their joint importance in the history of each organism enables us the better to appreciate the value of the factor which Wells, Natthews, but specially Wallace and Darwin, emphasized, and which we may shortly call "selection." This, like the previous two factors, involves incessant energy-transfer or dynamic change. So to speak, as some have done, of "a theory of dynamic evolution" is alike superfluous and tautological. The very term evolution - a rolling out or unfolding - expresses steady dynamical action. 
To realize vividly, however, the exact connection of it with each factor already noted, and its cooperative working with another factor yet to be named, let us for a moment picture to ourselves such often dormant bodies as a bacteroid spore, a moss or fern spore, a seed of the sensitive plant, a gregarine cyst, or a bird's egg. When taken from an environment that favored or arrested vitality to another that started growth, the first and leading result would be that in virtue of heredity each would begin to unfold or grow out, and would continue to do so according to details of the parent organisms, and through hereditary capacity for absorbing or utilizing hereditary substances. But scarcely will the wall have burst than the outgroming contents will be acted on by environal agents, such as winds, rains, light-rays, heat, or other organisms, to mention only a few, and in due time by proenvironal activity the unfolding body will react to them. But some of the agents are helpful, others are detrimental; and the latter, as well as the former, very soon make their effects visible. Growth results from the action of the helpful agencies, and then the struggle becomes a successful one; arrest, reduced growth, or it may be decay and at length death, result from the action of the detrimental. Then the struggle for existence becomes an unsuccessful one.

Since the days of the "Evolutionary Fathers" many have traced carefully and graphically the action and often the effects of such struggle; while, now that we know-as with Columbus and his egg-what to look for, all are becoming recorders of the phenomena.

"Selection" then is a factor that may start early in the life of an organism, as when a few out of many in a batch of seedlings or in a hive of insects survive. But it often becomes conspicuous in later life after the three preceding factors have mainly held sway in each organism. As an active factor in organic evolution then, it deserves fourth rank, for the decay or death of an organism is often ensured more by the possession of certain inherited qualities which under slightly changed environment or defective proenvironal adjustment act pre- 
judicially. It does not therefore hold the important place which its earlier advocates claimed Thus Herbert Spencer entirely overestimated when he wrote "while yet organisms had small ability to coordinate their actions, and adjust them to environal actions, natural selection worked almost alone in molding and remolding organisms into fitness for their changing environments; and natural selection has remained almost the sole agency by which plants and inferior orders of animals have been modified and developed" (7:552). We have yet to learn of even the simplest individual of the Caryota that does not exhibit clearly heredity, environal action, proenvironal response, as well as selectivism in every minute and every day of its life. The above also traverses the rule which he so emphasized later thus: "the coordination of actions is a definition of life."

But to emphasize the destructive at the expense of the constructive equally obscures issues. Thus to say, as Mivart has done, that by Natural Selection "is meant the result of all the destructive agencies of nature, destructive to individuals and to races by destroying their lives or their powers of propagation" (Lessons from Nature, 1876, p. 300) is to present only a part of the negative side of a great law. Without attempting to coordinate, or eliminate, or express the often divergent views of authors during the past half century, we would simply indicate that the facts advanced by Darwin alike on the positive and negative sides in chapters III and IV of "The Origin of Species" are as suggestive as they are indicative.

So one regrets to read Uexkiull's pronouncement, in relation to Darmin's views on the origin of species, that "a positive enrichment of our knowledge has not resulted. The whole enormous intellectual labor was in vain" (Zeit. f. Biol. 50 p. 168). This surely expresses the viewpoint of one who has become obsessed by the importance of his own observations.

But in subsequent discussions authors have at times indulged in a vague or imperfect application of terms. Thus so-called "artificial" or human selection has been regarded as distinct from "natural selection." But the domestication and culture 
of special plants and animals by man in no way differs fundamentally from the selection of a special host-root by a saprophytic fungus; or of a particular host tissue - e. g., roots of the beech by the beechdrops (Epiphegus virginiana)-through action of an invading parasite; or the concealment of one species of animal by another that is more perfectly provided with defensive apparatus; or the housing and rearing of aphides by ants as a means of nourishment for the latter.

The difference in degree here, though not in kind, is merely due to man's more highly organized cogitic activities, as compared with those even of the highest apes. To speak therefore of artificial and natural selection as distinct lines of inquiry simply obscures the advancing continuity of a great law, by raising false barriers of nomenclature.

Darwin in formulating and expanding the law of Selection spoke of it as "the survival of the fittest." This expression "the fittest" has often been criticized or explained by succeeding investigators. In the writer's estimate the expression "survival of the best adapted" "rould more exactly designate alike the static and the dynamic ramifications of the law, in its positive as in its negative phase. For selective survival is very largely dependent on the sum total of environal action and of proenvironal response. Organismal adaptation therefore is usually intimately bound up with selective survival.

We would define the law thus: "Continued organismal survival in an individual or in its progeny, due to the sum-total of conditions possessed by such, imparting sufficient integrating or resistant reaction to environal agents as to prevent disintegration or death, while other organisms not so conditioned are exterminated."

On its positive side the law may operate to effect one of two results: (1) uniform continuity of existence, or (2) progressive advance; both of which conduce to the survival of the individual and of the species. On its negative side the law only effects degeneration, and, if such be progressive, it effects ultimate destructive elimination. The consideration of one example of each of these may not be inappropriate here. 
The first of these occurs when the integrating agencies as a whole are practically balanced by the disintegrating, alike in the individual up to the period when reproduction has been effected, and in its progeny through successive generations. In this case the continuously regenerated group of individuals that makes up the species will remain unaltered. This is the condition that applies to by far the largest number of individuals of any species, when studied at any one place amid like environal surroundings, and not through long continued periods of time or over wide and varied areas.

It was this fundamental and exact similarity in millions of individuals that had intercrossed over wide expanses of country that caused the earlier systematists to believe in species-immutability. Only as the world was ransacked and viewed by observant naturalists like Lamarck, Darwin, Hooker, Wallace, Mueller, and others before or since, did the idea of speciesmutability dawn, and the causes for it become discussed. Then since "extermination" stood out as an apparent and universal cause for disappearance of the connecting links, selective survival stood out as a dominant factor in the minds of many evolutionists. It is to the lasting credit of Lamarck that he looked beneath the surface struggle, and recognized environment as a potent cause of change, and often even directly of extinction.

But when one reflects on the profound and extensive balance, and yet continuance, of existence that may prevail for thousands or it may be millions of years between species-organisms and the environal agents around, one realizes how complicated may be the factor of Selection, even though of subsidiary value to the other three. So a balanced selective survival-or panmixia of Weismann-usually represents accessory or collateral combinations of conditions that the three factors, heredity, environment, and proenvironment, have largely established. Though less active then as an originating cause, it may effect long persistence of individuals and of a species, if environal conditions remain uniform.

The second exhibition of Selection, that we have called progressive advance, differs from the last in that combinations 
of conditions arise mainly, as we would consider, by environal action, and proenvironal response, though in part also by utilization of accessory developments, which in their summated effect on the organism cause it to advance in physiological and in morphological detail, and in the process to vary or depart from other individuals that it once resembled, but which fail to show like progressive advance.

If such advance gives added dominance to the organism it will tend to survive alongside its former neighbors, which will tend to be eliminated. It was this phase of Selection that Darwin emphasized thus: "If variations useful to any organic being ever do occur, assuredly individuals thus characterized will have the best chance of being preserved in the struggle for life; and, from the strong principle of inheritance, these will tend to produce offspring similarly characterized. This principle of preservation, or the survival of the fittest, I have called Natural Selection. It leads to the improvement of each creature in relation to its organic and inorganic conditions of life, and, consequently, in most cases, to what must be regarded as an advance in organization."

In this case, then, each such creature shows, not a balanced state alongside summated environal forces, but it so rises above or excels the possible balanced, not to say disintegrating, actions of an environment that advance or new integration is effected. Instances of such are everywhere quoted and discussed in works on evolution.

The third or negative or eliminating side of Selection has been greatly discussed. For the writer no more striking instance can be cited than the connection and slowly disintegrating action to the point of elimination, of ectotrophic or endotrophic fungi, on those groups of flowering plants with which these fungi are associated. Here species of the higher fungi penetrate the roots of many flowering plants, evidently by chemotactic action. Then, as the writer would interpret the phenomena, they live not a purely parasitic but rather a symbiotic existence, in which both organisnins are more or less helpful to each other. But by extremely slow degrees 
the fungus absorbs more than it gives, so that the flowering host is steadily impoverished, till the individuals of a species, and the species of a genus, and even the genera of a family, slowly verge to extinction.

Thus amongst Orchidaceæ the steady degeneration in species of such genera as Goodyera, Aplectrum, Limodorum, Neottia, Corallorhiza, and Epipogon, as they become increasingly saprophytic; the like change in genera such as Pyrola, Chimaphila, Allotropa, Schweinitzia, and Monotropa amongst Ericaceæ in the widest and only natural sense; that in Canscora, Obolaria, Bartonia, Voyria, and Leiphaimos amongst Gentianaceæ; and the striking degeneration proceeding in all genera of the family Burmanniaceæ; form a graded set of examples of the highest value.

Now while on the part of the symbiotic fungus, in each case a definite environal stimulus was originally started, doubtless between it and nutritive products of the host plant, that led to proenvironal and invading response, the continued persistence, extended invasion, and adaptation of the fungus, as well as the gradual reduction to the stage of disappearance of chlorophyll in the host; the reduced size and simplified character in the stems and specially the leaves; as well as the simplified flowers and embryos of the host plants; all furnish perfect evidence of eliminating selection.

Parasitism between one group of flowering plants that gradually pass from a vigorous green to a simplified degraded and ultimately colorless state and another group of flowering plants that are parasitized on furnishes equally striking illustrations, but cannot be enlarged on now.

Sexual selection has been so fully debated that we need only to recall it here.

(5) Reproduction. The writer would now cite this as a fifth and closing factor in evolution. It is one, however, that has scarcely, if at all, been insisted on, by students of the subject, as a factor that deserves to be ranked with the preceding four. But, if Darwin's famous aphorism "Nature abhors perpetual self fertilization" be correct, then it might be expected 
that at least some phenomena of reproduction have a far-reaching value, and even dynamic significance. A brief consideration of some of the more important facts will alone be attempted here. And in this the history of asexual spore cells, as well as sexual gamete cells, will shortly be included. Ample reason for such a course is given on pp. 252-256.

The formation amongst algæ of akinetes, aplanospores, and swarmspores often occurs at exact seasonal periods, and represents phases of energy transformation, of cell metabolism, and of species dissemination that are alike vital and helpful to each organism, but which in no way fall under heredity, environment, proenvironment, or selection, though more or less influenced by each of these. Furthermore, since these undoubtedly transmit characters that have been slowly acquired by each parent form, the dissemination and wide propagation by water ensures rapid spread of the species, and so the opportunity for increased exposure of individuals of the species to new environments, which might in time again start new and rapidly acquired variations. Furthermore all known facts lead us to conclude that the act of setting free of all such entire or divided-up cell masses, and the existence of these for a time free in the water, produces a greatly more rapid growth capacity than if such masses had remained inside the parent walls.

But, with the gradual evolution from motionless vegetative cells (Zygnema, Cosmarium, etc.), or the evolution from swarm spores, of complemental sex-cells, a highly important and accelerating factor in evolution was established. For the principle involved in Darwin's aphorism above quoted evidently carries with it also a profound advantage to each resulting organism formed from a cross-fertilized egg. In no way perhaps does this appear more strikingly than in diverse crosses of some flowering species.

Thus the writer has drawn attention to hybrids of the genus Nepenthes (78:446) that exhibit combined characters of both parents which make the hybrid more virile than either parent. The same is true of some hybrid sarracenias, orchids, hippe- 
astrums, violets, and other groups. It is quite fair to conclude that, if definite and recognizable advantage accrues when rather diverse species are crossed, the more vigorous offspring that Darwin and many observers since have recorded, as resulting from crossing of varieties or even distinct individuals of a species, clearly indicates that sex-differentiation and sex-cell fusion constitute an important evolutionary factor. Some possible explanations are set forth in chapter $\mathrm{X}$. But, while this fifth factor has a profound importance as an agent in evolution, we still are so ignorant relatively of the more exact details involved that we must look to the future for its fuller development.

If now one postulate the factors of heredity, environment, proenvironment, selection, and reproduction as cooperative agents in organic evolution, it necessarily follows that the result or effect will be the gradual unfolding from the spore cell, or from the fertilized egg of a plant or animal body, of a mathematically exact structure, except that the second, third, and fourth factors may more or less modify it during the unfolding process, and in time we believe modify its descendants. Such plant or animal, moreover, represents an absolutely continuous line of organic development, it may be through millions of years, during which changing environment, changing proenvironal response, changing degrees of selectional strain, and changing reproductive blendings have all cooperated to alter the organism along an evolving or devolving line, or these factors have so acted in balanced relation that the type of organism retains a stationary state.

So, from the time when a spore-cell is set free, or when two complemental sex-cells fuse to form a fertilized egg, and onward through the life of each organism, the combined action of the above five factors as a great cause gives rise to the gradual unfolding or ontogeny of the organism as an effect. Such a summated cause or cooperative agency then might be termed pentamorphogeny, or the action of the five organic factors that are form-producing, in the history of every organism. 


\section{CHAP'TER IX}

\section{THE LAW OF PROENVIRONMENT}

It is a principle of general application for all organisms that fixed or sedentary forms are less quickly responsive to environal stimuli than are motile ones. So fixed Blue-green Algæ like Nostoc are less quickly responsive than free forms like Oscillatoria; both of these are less responsive than a flagellate Bacillus, in which a special part of the protoplasm has become modified and energized for locomotion. Plants again as a group are greatly less responsive than most animals, since they usually lead a fixed life. But that parts of their tissues can become highly irritable is shown by the movements of the leaf in Dioncea and Mimosa being more rapid and powerful than the muscular movements of many animals. Plants therefore, as a group, are greatly less specialized than animals, though, when such a tissue as that of Diona becomes highly sensitive, it exhibits many of the fundamental responses that even the higher animals do. Continuity in stimulation action and in response phenomena, is therefore unbroken from the lower plant types up to the higher animals. The recognition of this fact is of prime importance, when we attempt to learn how all organisms have evolved.

Many naturalists, in surveying the trend of erolutionary advance, have recognized a principle or method of improvement or of betterment as linking together the whole. Alongside this, as indicated in the last chapter, may be an evidently static state of the terebratuloid kind, in which neither progression nor degradation occurs. Not unfrequently howerer marked degeneration or devolution can be observed; specially in saprophytic and parasitic plants, and in sedentary or parasitic animals.

But the first of these three stages has been the most attractive phase of evolution, and so has received most attention. 
There has been no more attractive side to it, also, than that which attempted to explain why an evidently steady upward advance was made. Was there a great unseen guiding handa Paley's inspiring mechanician? Or did the whole result from natural forces or agencies common to all plants and animals? We shall not attempt here and now to review the many suggestions that have been propounded as sufficient explanations. Synopses of these can be found in Thomson's "Heredity" (79).

The author believes that in the Law of Proenvironment the agency is secured that would fully furnish an explanation. As already defined and shortly explained, it is that capacity which all caryotic or nucleate organisms and undoubtedly many of the acaryotic or non-nucleate show, of being stimulated by and then positively growing or moving, in part or in whole, toward an environment that represents the satisfying resultant or mean between all of the environal stimuli by which they are surrounded.

Proenvironment and Struggle for existence though often conducing toward the same biological result, viz., selective survival, are totally distinct factors. Thus the former is an inherent physiological capacity of the organism, that manifests itself when one or more new stimuli, or differing strengths of stimuli, are applied. The latter may be and often is connected with stimulation response, but may equally secure its results by rapidity of growth, or of motion unconnected with the stimuli; or by formation of localized defensive products that primarily are waste products. Proenvironment again is an expression of what the chemico-physical organization of each organism responds to, as conducting to the most satisfying environment for it or some part of it that such an organization can secure; struggle for existence is most often the chemico-physical effort to survive amid an environment that the organism is already exposed to. The former is an exactly predicable relation of the organism to environal stimuli, so that, if the molecular sensitivity of the cells and the direction as well as the strength and kind of each stimulus were measured, 
the direction, degree, and amount of resultant response called forth could be precisely foretold. The latter is often fortuituous, non-predicable, and adaptive on the moment to temporary conditions of the organism. Proenvironment always anticipates and is associated with an ultimately expressed energizing polarity of the organism; struggle for existence is often passive or resisting or non-polar in expression.

As in our treatment of the organic energies, so here we propose to follow the somewhat illogical, but yet highly practical, method of proceeding from the most complex-but in many respects best known-to the simplest but as yet obscurely known.

All who have surveyed Milan Cathedral from its surroundings - unfortunately however all too constrained-, who have moved alongside the outer walls, who have passed in under the marvelously detailed and finished entrance gateways, who have wandered along the inner walls and aisles, who have ascended the stairways to the galleries, and who finally have explored the carved and elaborately finished nooks and recesses of the outer roof, must surely have asked themselves the question: Could all this possibly have been planned and thought out by a single human brain?

The history of the conception of the cathedral is fairly well known, and in light of such knowledge, and of our knowledge of similar edifices, we may fully accept it that such was truly the case, even though the building itself took centuries for completion, and called forth the energies of many other minds and hands for its construction.

Similarly when one stands by a modern newspaper printing machine that is receiving, distributing, printing on both sides, drying, folding, and delivering the rolls of paper as finished newspapers, that are spread over with the world's items, one can scarcely conceive on first thought that the complicated interacting machine was planned and worked out in the brain of one man, while as yet no metal nor paper were around.

But in both cases, and in many like conceptions of the human intellect, we know that both constructions gradually arose 
in the minds of their inceptors as concrete mentally visible pictures, that lived for them as truly as though they had been already finished and visible to the naked eye. In each case, as a result of many previous isolated stimuli of a lumic, a mechanic, a gravic, a chemic nature, that had gradually been summated into an energizing whole, there was formed in the inceptor's mind a resultant proenvironal response of a highly complex character, that enabled each structure truly to stand forth in mental perspective, when as yet no material indication of it existed. This brought into play all those higher phenomena of living cells that we designate as memory, reflection, intelligence, constructiveness, and so on.

So, by the exercise primarily of a wide series of distinct sense impressions, several summated proenvironal responses originated. These again were compounded by cells of the highest brain centers into complex resultant responses, and gave rise there to the mental picture that slowly projected itself in marble as the classic cathedral, or in metal as the elaborate printing machine.

But by selecting an example here and there, along the entire scale of caryotic or nucleate life, we hope to show that, from the simplest to the most complex, the proenvironal law is constantly at play, and in fact is a fundamental law of all nucleated cells.

The origin of it is to be sought for, in those primitive responses of living substance that caused such constantly to grow or move toward a position or region that would prove most satisfying to the organized molecules, when these were acted on by thermic, lumic, gravic, chemic, or electric stimuli. This again carries us backward to the more primitive inorganic relation of "satisfied" or "unsatisfied" molecules or atoms, in connection with other chemical or chemico-physical conditions, as has been so often and ably urged by J. Loeb.

Thus one of the simplest and most delicate exhibitions of it consists in the movements of aerobic bacteria to centers where oxygen supply is amply sufficient to their needs, as demonstrated by Engelmann. In this case the presence of 
an infinitesimally greater amount of oxygen on one side than on another, of each bacterium, causes it to plot out a pathway for itself that is accurately determined stage by stage according to the relative amount of oxygen in its surroundings.

Now, in the above case selected from the Acaryota, the stimulus and the succeeding proenvironal response are alike single, simple, and direct. The extreme delicacy of the stimulus, and so of the perceptive response in this case, is proved by Engelmann's estimate that the $\frac{1}{100,000,000}$ of a milligram of oxygen will suffice to start movement. So far also as we at present know them physiologically, it seems true for many of the Acaryota that their proenvironal responses are simple and direct for each type of stimulus. But it may be that at least the higher types, with rudimentary nuclear structures, like Oscillatoria and many motile bacteria, may yet be shown to have the power of perceiving and of compounding two or more energizing stimuli, and thereafter of plotting an environal pathway that is the resultant mean of these several stimuli, and that will lead the organism along a satisfied resultant environal course.

Thus, if it could be clearly demonstrated in regard to Massart's experiments on gravitation movements of bacteria ( 80 : 158) that these bacteria, when exposed to gravic stimulus in one direction and to oxy-chemic stimulus in another, pursued a pathway that was a resultant between the two stimuli, direct proof of resultant proenvironal response would be secured. A very wide field for experimental study is here open amongst the Acaryota. An excellent summary of results already obtained is given by Jennings (42: 37-40).

Our knowledge of the unicellular Caryota is much fuller and more exact. Their possession of a chromatin nuclear and nucleolar mass evidently confers more complicated and quickly adjusted capacity for linking together, and responding to, two or more stimuli. The motile or zoogonidial stage of such waterweeds as Sphorella and Chlamydomonas are highly favorable plant objects, while Euglena, Stentor, and Paramœcium are equally good animal forms. Reference has already 
been made to the proenvironal capacity of Chlamydomonas ( $\mathrm{p}$. 166). We may therefore study unicellular animal responses.

In nearly all such cases it is observed that not the entire organism, but some polar center or line of stimulation, is the receptive area, and that, when one or more stimuli act on this, a period of excitation elapses during which the living molecules are plotting a proenvironal pathway, that eventually is pursued. Further, from the experiments of Jensen, Jennings, and others, we learn that if the organism is free swimming and is exposed to a single new stimulus, even though this be favorable or satisfying, it may during the excitation period retreat slightly. From the experimental evidence at hand we would interpret this as being to permit of time for action of the stimulant energy on the molecules of the organism, and so for determining whether favorable or unfavorable response to the stimulant energy should be made. Or in other words such constitutes what the plant physiologists term the perception phase in the latent or excitation period.

Abundant evidence in favor of proenvironal response, when a single stimulus acts, is to be found in many works, and some of these are synopsized by Davenport (41), Jennings (42), and J. Loeb (59). But results for two or more stimuli are often as yet uncertain or even contradictory. Thus Jennings (42: 92-98) considers that, where two stimuli act, any one of four possibilities are shown for Paramœcium, namely either: (1) that if a stimulus $a$ is acting and another stimulus $b$ be applied it will react to $b$ in the usual way; or ( 2 ) it will continue to react to $a$; or (3) it will form a compromise "between the usual reactions to the two agents"; or (4) it will react in a new way, different from the usual reactions to either $a$ or $b$. But in several of these cases many experiments were not made to test whether a definite optimum, as well as maximum and minimum, for each kind of stimulus existed. This however is necessary for every physiological action.

But one could well explain 1, 2, and 4, that seem uncertain, as being truly resultant effects. Thus, if in 1 stimulus $a$ be rather weak and applied at a different angle from $b$ that is 
strong, there would truly be a linking up and resultant response as stated, but owing to the direction and strength of $b$ it would in appearance cancel $a$. Again under $\mathcal{2}$ the converse result might be true. An explanation of 4 as being in reality a resultant response might be given in several ways. Thus, if the stimulant used so acted on some constituent molecules of the organism as to cause the formation of a new chemical compound, the presence of this might entirely alter the resultant response that might otherwise have been given. It would scarcely be necessary now to cite other possible explanations.

But, in reviewing all the observations made on Infusoria, Jennings's conclusion (42:109) is of interest in our present inquiry. For he says: "Why does the organism reject certain conditions and retain others? We find that the animal rejects, on the whole, such things as are injurious to it, and accepts those that are beneficial. There are perhaps some exceptions to this, but these are rare, and only noticeable because exceptional; in a general view the relation of rejection and acceptance to injury and benefit is evident. It results in keeping the animal from entering temperatures that are above or below those favorable for the life processes, in causing them to avoid injurious chemicals of all sorts, in saving them from mechanical injuries, and in keeping them in regions containing food and oxygen. Clearly the animal rejects injurious things, and accepts those that are beneficial." In other words, as we have already expressed it, the organism plots a satisfied course.

But all such movements are undoubtedly caused by one or more external stimuli so acting on the protoplasm, or on a localized part of it or of the cell contents, that these undergo molecular changes. In some cases the general cell protoplasm seems to be stimulated through the nucleus; since non-nucleate cells are less often responsive, owing, as we would consider, to the latter being traversed only by biotic, the former by biotic-cognitic, lines of energy. In other cases perception and proenvironal response are located wholly or most strongly in a special center. Thus Engelmann (38:387) showed this to be true for the anterior third of Euglena, and possibly even 
phototactic perception resides wholly in the "eye-spot" that is located there. The molecular changes then, established there, are the first indications of a definite role of events that will culminate in motion of the animal in a definite direction.

Amongst the multicellular Caryota, not only is proenvironment exhibited on every hand; in many cases each proenvironal response is a resultant of two, three, or more compounded stimuli. Oring also to size of the parts and ease of observation the results are already of great value, and are recorded in text-books on plant and animal physiology. Further, as has been pointed out already in treating of the "stockin-trade" of plants and animals (p. 164), the chief proenvironal factors can be traced to act throughout the entire scale of higher organic life, and most of them are known as the tropic or tactic stimuli. Some of these, however, are often treated of in text-books as simple stimuli, when in reality constituent rays of lumic energy may cause very diverse results. Thus we often speak of natural or white or mixed light as causing either positive or negative response phenomena, when strictly the red or red-orange constituent may start one response, the green another, and the blue-violet a third; or these may all cooperate in producing one satisfied result up to a certain degree of intensity, and thereafter the blue-violet rays may become highly irritant and hurtful, while the red-orange may continue to exercise a beneficial effect.

Short reference has already been made to the fungus Pilobolus. As this develops its spore bearing stalk, if the manurial mass on which it is gromn be illuminated equally from above, the fungus will proenviron a course that is directly upward, since the stalk is apogeotropic or is negatively responsive to the earth's mass, while it is also heliotropic to light, and thus proenvirons a course that is in line with the light rays and gravic stimulus. But, if the well-known experiment of illuminating a crop of it through a minute orifice on one side of an otherwise darkened chamber be arranged, then the heliotropic resconse is so great that the apogeotropic one is canceled or modified to an angular degree that accurately brings the axis 
of the sporangiophore in line with the incident rays. So in such an illustration as that of Fig. 5 each individual plots and pursues a proenvironal path that is different in angular relation to every other one. But all execute such movement that the most beneficial result is secured to the species, in resulting growth and dissemination of the spores. The surprisingly accurate manner in which the sporangia hit a circumscribed light-orifice is a striking proof of lines of energy-distribution and of cell tensions in relation thereto.

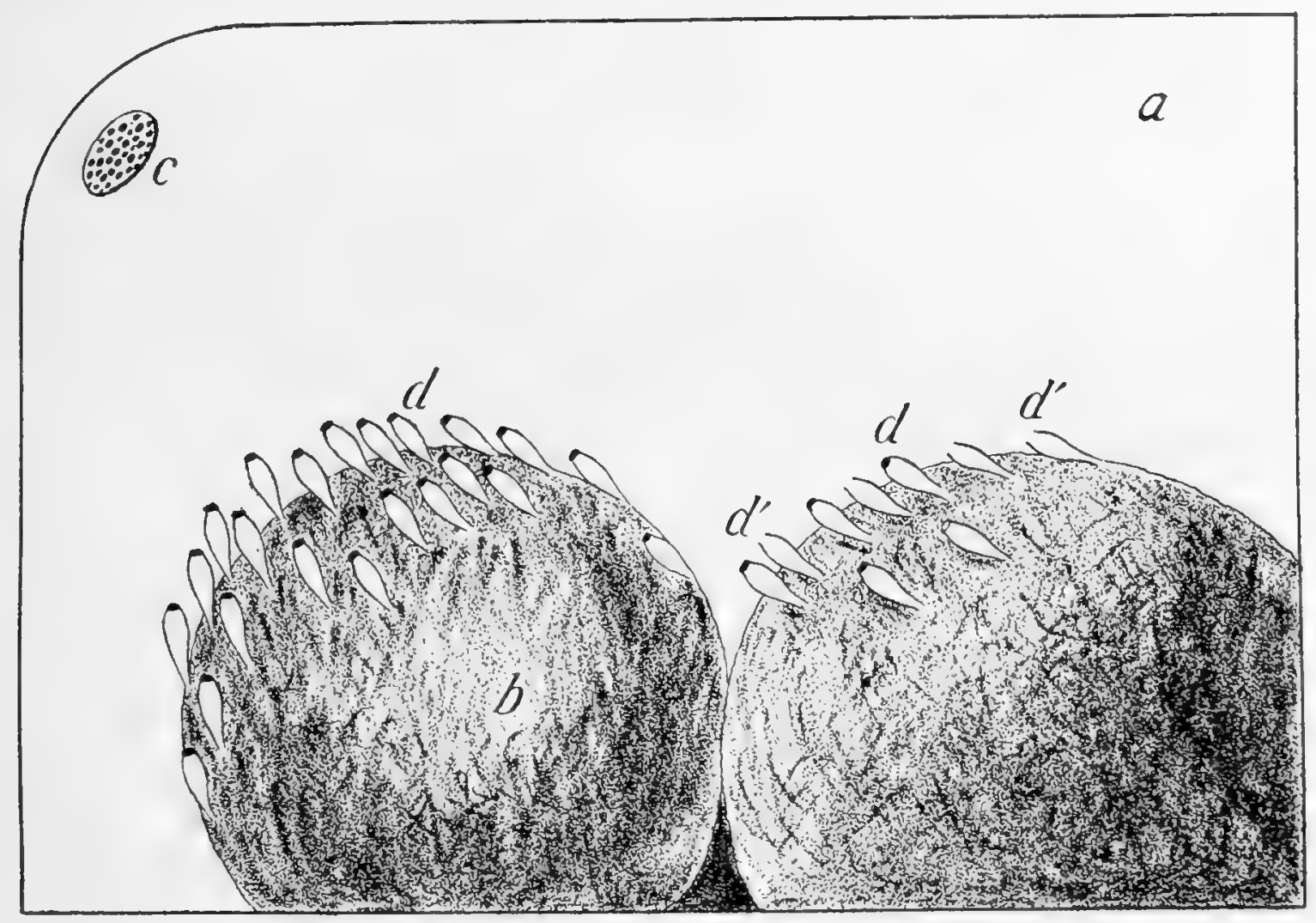

Fig. 5.-Growth of the Fungus Pilobolus on horse manure, $b$, placed under belljar, $a$, the exterior of which was lined with black paper except for orifice at c. From resultant action of apogeotropic and specially of heliotropic stimuli the black sporangia are discharged accurately against the orifice.

But the feeding or absorptive threads of this fungus, which are a continuation of the same elongate plant cell that produced the spore masses, exhibit proenvironal responses of a totally opposite kind, though equally beneficial for the species. Thus these grow either directly or obliquely downward into the manure through geotropic action; they at the same time grow away from the light or are apoheliotropic; they also are influenced hydrotropically by the moisture of the manure and so grow toward it to absorb food material. Ali three of these 
separate responses are usually compounded in a resultant line of growth, that is nearly or quite the same as for any one individually. We can prove further, however, that at the very earliest stage of germination from the parent spore and on to maturity a fourth stimulus - the chemotropic one-is in action and is responded to. For, when we attempt to grow such spores on moist bread, potato, or sugar solution, only failure is recorded. The exact kind of food that can chemotactically stimulate the young plant, and cause it to respond in satisfied manner, is absent in the latter substances, but is present in the horse manure.

But, while such proenvironal action and response usually remain constant for definite plant parts, some structures show an elastic adaptability of response that strongly suggests profound and exact influencing of the living cell substance by environal stimuli. Thus the case might be cited of the gemmæ of Marchantia, that are flat bodies developed on a stalk, and that appear almost uniform with each other till they are washed or blown out from their cups. As one side or the other of the flattened body happens to fall next to the soil, so is the future proenvironal response. For it causes the lower side to form absorptive rhizoids and remain pale, the layer above to elongate into interlaced cells, and the superior zone to derelop tissues in a still more complicated manner, amongst which green or chlorophylloid cells arise.

Compounding of diverse stimuli, and resulting proenvironal growth, may be said to be the general rule for the organs of vascular plants, though at times also simple stimuli may be effective. Roots generally behave much in the manner already described for the mycelial threads of Pilobolus, and no better examples for experimental study could be selected. By such simple experiments as are ordinarily performed in botanical laboratories, it can be clearly demonstrated that each primary root tip, say of the pea or bean, is simultaneously affected by molic, chemic, lumic, hydric, and mechanic stimuli, as pointed out in the last chapter. Further, when these stimuli are caused to act not in the same direction, as is commonly true for roots 
in nature, but at different angles with each other, the proenvironal course pursued is an exact resultant between the lines of action and the intensity of stimulation of the different stimuli applied.

But even the same quality of stimulus, when applied at varying distances, may cause proenvironal deviations that are highly suggestive. Thus the writer had under observation for nearly two years a plant of the screw pine, Pandanus caricosus. The strong thick aerial roots descended (Fig. 6) from parts

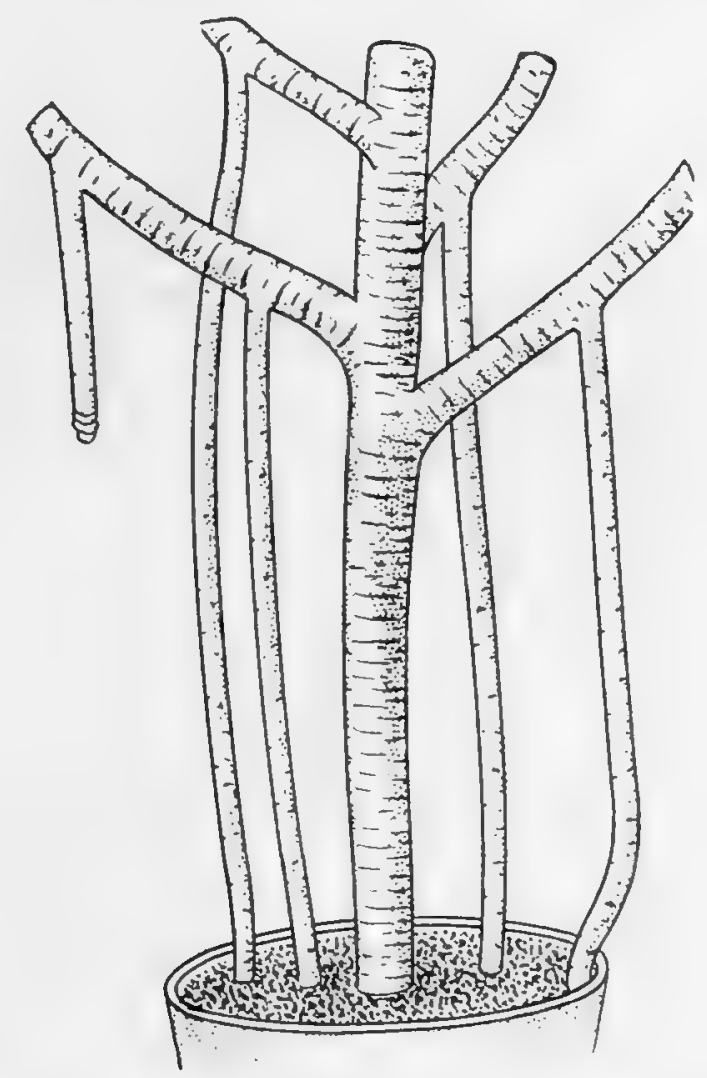

FIG. 6.-Stem with branches of Pandanus caricosus that bear aerial roots. The root to the right has bent, hydrotropically, so as to reach and penetrate the soil of the pot.

of branches that had spread out considerably beyond the circumference of the large flower-pot below. These roots grew almost vertically downward in virtue of geotropic, apoheliotropic, and weak hydrotropic action. Somewhat subdued light reached the roots through a mass of regetation, and faintly but perceptibly caused uniform growth of all away from that side. But when three and a half inches above the level of and outside the circumferential range of the pot, and fully fifteen inches above the cinder-covered soil below, the hydro- 
tropic stimulus of the pot-soil caused a bending aside of the apparently firm and thick roots, from the geotropic and the more distant hydrotropic soil stimulus of the ground beyond on which the pot sat, so that these reached and grew into the pot.

Many related examples of root stimulation and proenvironal response could be given and later may be quoted in detail in a special volume. All prove that linked-up or resultant proenvironal action is often wholly determined by the relative strength, proximity, or quality of stimulus. But some highly suggestive reversals in root response are known and have been investigated, not a few of which the writer has examined practically. Thus the roots of a considerable number of plants, from ferns like Osmunda cinnamomea up through gymnosperms like the swamp cypress (Taxodium distichum) and monocotyledons like Phoenix sp. on to various dicotyledons, are capable of growing against gravity, unlike other roots on the same individual. These show normal root caps, root hairs, and internal structure as in ordinary roots. In other words their general morphology seems unaltered from the normal. Their energizing relation however is altered, and this produces vertically upgrowing roots. Now the change is regarded by most botanists as being due to an important chemotactic response of roots, namely their perception of and response to oxygen supply in the air above, since the amount seems to be insufficient in or insufficiently absorbed from the surrounding water or water-logged soil in which these plants grow. So, while some of the roots continue to be stimulated by, and to respond to, gravic, apoheliotropic, and mechanic forms of energy, these aerating ones have so canceled such in favor of the - to them-more important aerotropic or oxytropic growth response. In all such cases, therefore, proenvironal effort and intrinsic linking up of several lines of most satisfied response have resulted in growth of parts of the same organ morphologically in two opposite directions.

A somewhat analogous and yet different case is seen in parasitic roots of the mistletoe. Here the geotropic stimulus 
has wholly or largely been canceled, by the chemotropic or food searching stimulus. Thus, whether a seed be placed with the embryonic radicle directed toward the bark or toward the earth, the radicle in elongating bends toward and penetrates the bark, while showing no perceptible gravic response. In this case the apoheliotropic and hydrotropic may possibly aid the chemotropic stimulus in determining the proenvironal pathway that is most satisfying.

It would be impossible in the present work to enter into a detailed description or discussion of the perception of gravity by root tips and the propagation of the stimulus to the responsive region further back where growth and bending occurs, in cases where the root has been placed transversely or obliquely downward toward gravity. The papers by Charles and Francis Darwin, by Czapek, Haberlandt, Noll, Newcombe, and others, are all quoted in recent works on plant physiology. While the writer believes that mechanic stimulation by sliding starch-grains in cells of the root cap or tip may aid in geoperception, histological studies he has made cause him to regard fundamental changes in constituents of the chromatin, that he has termed geoenergids, as the prime determining cause (p. 120). The study is one of especial interest from the standpoint of cell energetics.

Stems and branches of vascular plants have been minutely studied in their tropistic responses by Sachs, Darwin, Wiesner, and other more recent authors. On page 191 a short reference was made to responses in seedling stems of bean or pea. The rapidily growing extremities of stems of many flowering plants are exceptionally favorable for proving that linked up resultant responses are very frequent, almost invariable for them. Only a few added cases can now be cited briefly.

If the growing end of a French bean, a sensitive plant, or Cuphea be grown in a pot in optimum and generally diffused light, it will grow vertically upward under the apogeotropic and directly overhead heliotropic stimuli (Fig. 7, a). But, if the pots be placed in a dark box into which light enters by a narrow slit from a position to the right of Figure $7, b$, the formerly cooperating stimuli that linked up to give a resultant 
vertical direction of growth will now be separated into distinct factors. So the shoot will place itself as in Fig. 7, b, since the vertically upward apogeotropic or apogravic action will be modified by the unilateral lumic one.
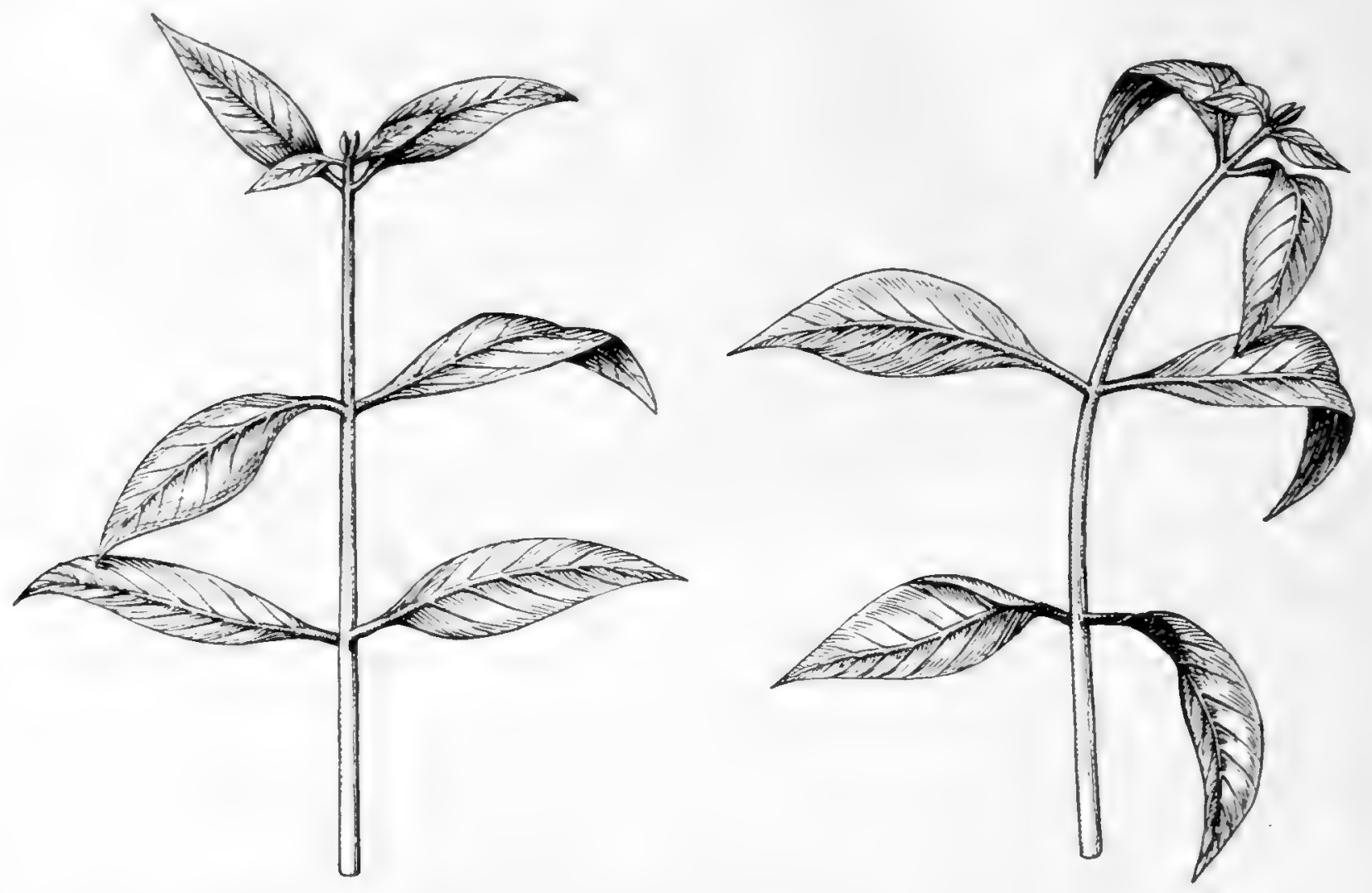

FIG. 7.-Terminal shoots of Cuphea platycentra. The left-hand figure (a in text) grew under vertical illumination, the right hand figure (b in text) grew under unilateral illumination.

All sensitive plants-such as many of the Oxalidacex and Leguminosæ - are exceptionally valuable for our present study. As illustrations of stimulation and resultant response alike for stems and leaves we may select the sensitive plant (Mimosa pudica), since compounding or separation of response are quickly performed in it or in its ally $M$. Spegazzini. Photoillustration 8 a, is taken from a pot-plant that grew in a stove house with bright diffuse illumination. The stem therefore shows compounded or resultant apogeotropic and heliotropic position response. The leaves are placed transversely or are diageotropic, and all, whether on the stem side toward the incident light or on that away from it, are so tilted at varying angles that the incident rays fall at right angles to the surface. So we may say that these are diaheliotropic. But, if a plant be inverted, the leaves inside an hour or two have undergone a striking angular rearrangement. But, for our present purpose Fig. 8 , b that was made two days after inversion, but other- 


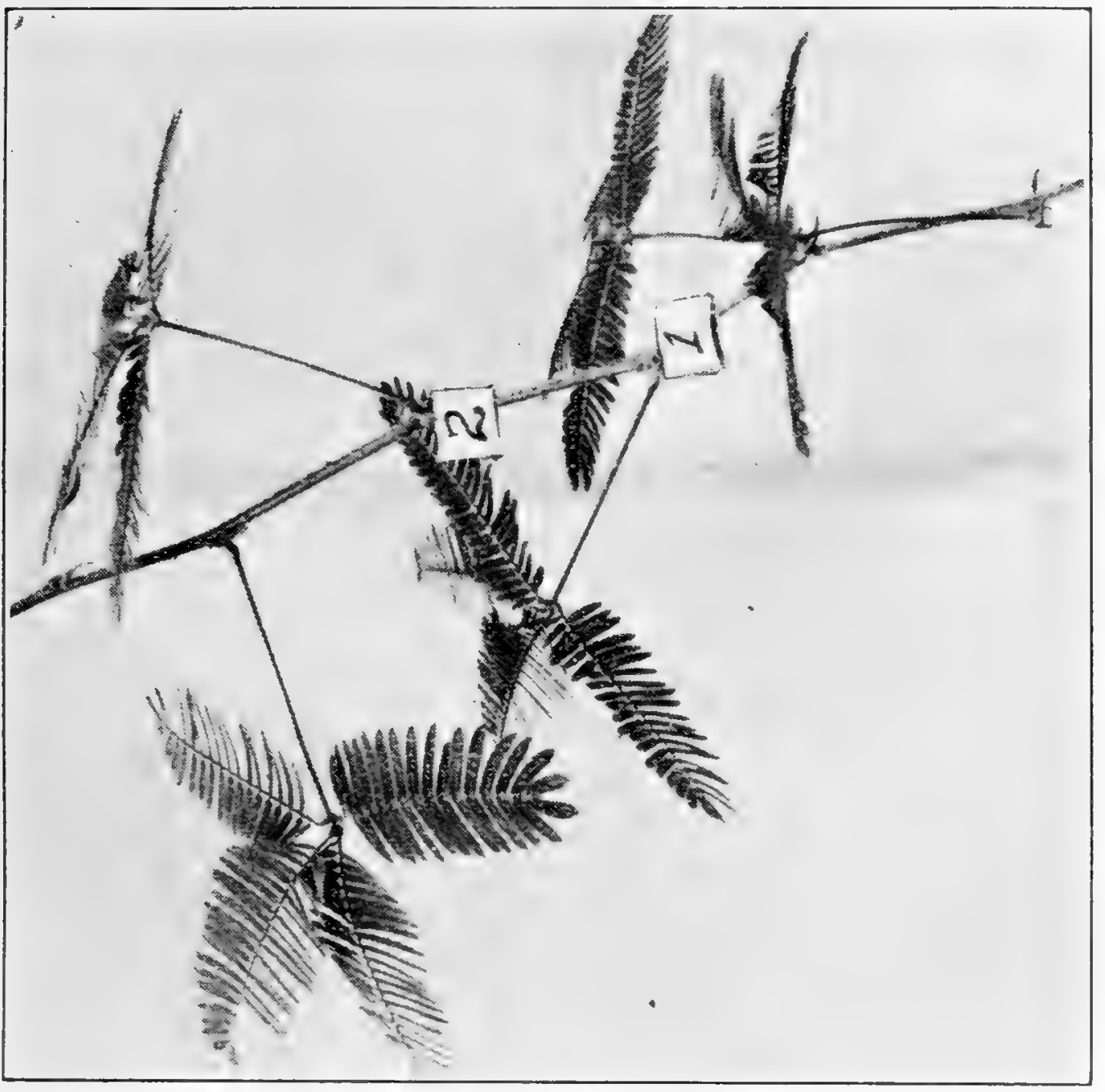

ن

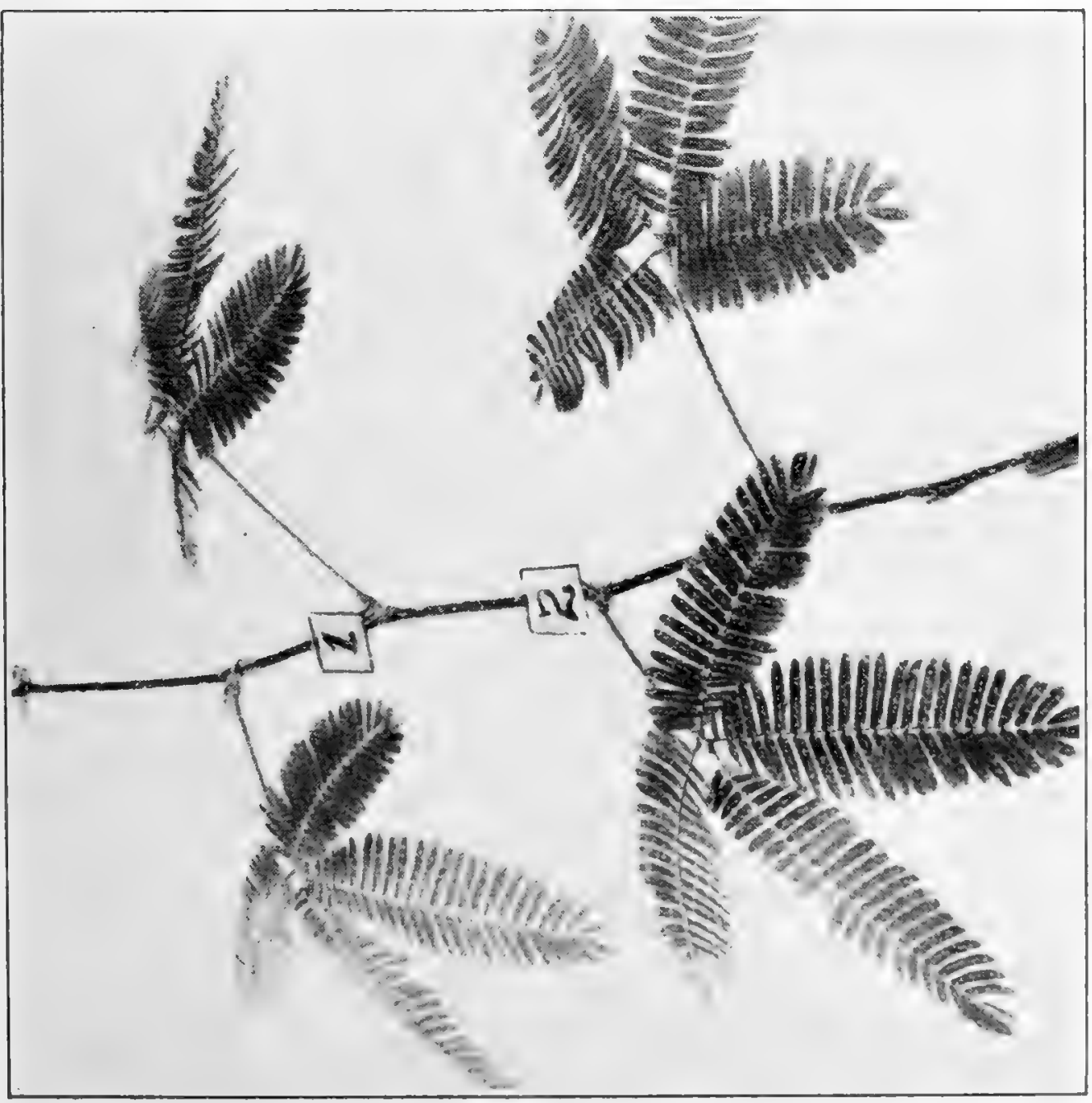

క్

$=\frac{\pi}{\infty}$

$+\infty$

도.

원

Еี

:

.5

先

ธ

范

13

$\infty$

这䓂 
wise in the same position or relation, is more instructive. Here the growing stem apex, in elongating further, has responded apogeotropically by curving upward, but not vertically so, for heliotropism has also been at work, so the resultant direction seen in this figure has been taken. The varying angular positions, assumed by the leaf stalks as well as the primary and secondary leaflets, are most instructive, as exhibiting every phase of resultant response between diageotropism and diaheliotropism, in which however the more important latter phase for the leaf surpasses the less important former one.

But on bright hot days a third factor may be made evident in resultant movements of the leaflets, namely paraheliotropism, or the effort on the part of leaflets to so tilt upward that the intense illumination by blue-violet rays may be minimized. Thus when the sky is clear and the light intensityas roughly estimated by shade temperature-accompanies a temperature of $32-35^{\circ} \mathrm{C}$. the secondary leaflets become slightly moved upward. With increasing light of blue-violet quality the upward angle increases until a resultant between diageotropism, diaheliotropism, and extreme paraheliotropism is attained, and in which the leaflets have risen through an angle of $40-45^{\circ}$. Then a temperature of $38-40^{\circ} \mathrm{C}$. is usually recorded.

Again the same leaflets show varying degrees of response to thermic, mechanic, and chemic stimuli, in which each may act separately, or one may, as in Jennings's results, overpower another, or all by skillful adjustment to degree-intensity of stimulation may form a resultant response.

As the author also pointed out eighteen years ago (81), and has greatly extended since in his lectures on "Plant Irritability," the species of each genus of sensitive plant exhibit graded specific differences in perception of and in response to environal stimuli, that cause corresponding differences in the resultant reactions. Thus Mimosa Spegazzini is the most sensitive plant known to him at present. M. pudica is slightly less sensitive, M. lupulina, M. latispina, M. sensitiva or albida is each less sensitive than its predecessor in the list, till through a series of other species we reach $M$. argentea that would in no way be regarded as a sensitive plant in ordinary parlance. Similar graded lines of sensitivity have been constructed by the writer for the species of Cassia, of Desmodium, of Biophytum, of Oxalis and other genera. Comparative studies of the relative sensitivity of such genera will form a valuable physiological and possibly phylogenetic contribution for the future. 
With such results before us as above presented, if a wide survey be made of general plant stimulation and response, many resultant phenomena seem to present themselves, though regarding most of them our knowledge is still so elementary that one can do no more than suggest the possibility, in some cases the probability, of their existence. Thus the compounded or resultant forward and also gyratory motions of many spermatozoids; the ascending spiral relation of the successive segmentcells cut off from the apex of most multicellular cryptogams; the successive segmentation of these and equally of the young tissue cells of flowering plants along definite lines, until a definite amount of tissue is built up; even the correlative development of a certain color, size, shape, degree of insertion on the receptacle, and degree of nectar secretion in certain flowers; seem all to be the product of several stimulant factors united into a resultant response.

Such responses, in which lines of energy are constantly being set free along definite tracts or pathways, cause a flow of energized molecules toward certain centers and a flow away from other centers. So the functional activities slowly but gradually determine and mold the structural outlines. Structure therefore seems to be the temporarily static expression of many resultant actions and reactions of energized particles, that have become more or less definitely placed in hereditary positions, through proenvironal response to environal stimuli.

Before passing from such plant studies to those of animals, we might summarize our position so far by stating that the law of proenvironment seems to have its simplest organic expression in single, simple, and successive responses of the simplest bacteria and Blue-green Algæ to the varied environal stimuli by which each is surrrounded. This action, we have suggested, probably owes its origin to the chemical principle of satisfied or unsatisfied chemical molecules, the presence of the latter state in any body constantly causing flows of energy and resulting molecular integration or disintegration.

But in the higher Acaryophyta, such as the Oscillatoriacese and Bacteriacese, evident intrinsic compounding of two or more 
stimulation responses are indicated or definitely recognizable. In the Caryophyta, while simple and direct responses to like simple stimuli may occur, it often happens that a compounding of two to five separate and successive stimuli, that all act on some definite constituents of the living cell, gives rise to a direct resultant response or rearrangement of the molecular machinery of the cell, so that a pathway or line of action or motion is proenvironed intrinsically, before the organism exhibits any evident intrinsic or extrinsic change. This may be a resultant compromise or mean line, between the lines of stimulation action, if the separate stimuli are all balanced in strength relatively to each other, or it may in part or in whole be in line with one only of the stimulation lines, if this exceeds the others in strength.

Throughout the animal series of the Caryota (Caryozoa) we already have many interesting cases of resultant proenvironment up to the Spongida and the Hydrozoa. But in the more evolved genera of the latter, such as the Hydromedusæ, as well as in the Anthozoa, the Echinodermata, most worms and higher groups, there begin to appear new and special cell units, the nerve cells, which become increasingly abundant and complicated in their connections till we reach a climax in man. These introduce, we would consider, a new and greatly more complicating condition which we can now stay to consider, before treating of the responses of the Metazoa* as a whole.

It has been already explained (p. 88) that three probable forms of life-energy already dealt with, the biotic, the cognitic, and the cogitic, seem to be located respectively in the protoplasm, the nuclear-nucleolar substance or chromatin, and the Nissl substance of the nerve-cell or neuratin, respectively. so in the simplest living Acaryota the biotic energy of the protoplasm acts, as above stated, so as to produce simple proen-

*Here and subsequently we use the term Metazoa as including all nucleate multicellular animal organisms; and its relation to unicellular and to nonnucleate types is set forth in classification manner on p. 51 thus:

(a) Acaryozoa-Unicellular animals without cell nucleus.

(b) Caryozoa-Unicellular or multicellular animals with cell nucleus.

1. Prolozoa-Nucleate unicellular animals.

2. Metazoa-Nucleate multicellular animals. 
vironal responses. But in all nucleate or chromatin-bearing plants, and in the simpler non-ganglionic animals that are also chromatin-bearing, the perceptive or cognitic energy that we believe to be associated with and to traverse the chromatin substance acts intrinsically so as to link up or combine into one resultant response the separate responses of two or more energizing stimuli, and so to proenviron a course that is the resultant of all, and that is satisfying to the organism.

But, with the gradual formation and appearance in nerve cells of ganglionic substance or neuratin, the still higher cogitic energy that we consider to be associated with and to traverse its substance acts intrinsically so as to absorb two or more sets of distinct resultant responses, and then link up or combine these into one compound resultant response that is satisfying to the ganglionated organism. Hence result, as already stated, the responses that we associate with memory, mind, intelligence, intellectuality, and morality, that are due to combined complex action and reaction of biotic, cognitic, and cogitic energies acting in an increasingly more and more complex manner through the protoplasm, chromatin, and neuratin (if for convenience we may adopt such a term) so as to exhibit (a) simple protoplasmic responses, or (b) resultant chromatin responses, or (c) compounded resultant ganglionic or reflex responses.

An attempt may now be made, therefore, to trace the possible gradual segregation and evolution of nerve cell or ganglionic substance, beginning with the more specialized Protozoa and the simpler Metazoa, and then passing upward through the higher groups of the animal kingdom. Two of the higher groups of Protozoa seem to furnish a key to initial conditions.

Amongst the Hromoflagellata, according to the researches of Schaudinn, Leger, and Prowazek, each young cell-individual shows a single nucleus. But during cell maturation the nucleus divides, and one of these redivides, so as to give rise to three nuclei. One of the three has been called the trophonucleus, as it seems to preside over or govern the general sensory and trophic functions of the cell. But without desiring to coin new words unnecessarily, the writer will call it the cognito- 
nucleus. Another, that has been called the kinetonucleus, seems to gather up or condense in it all the important hereditable characters of the cell, and later when sex-differentiation and maturation of the cells are progressing it again fuses with the cognitonucleus. We propose therefore to term it the gametonucleus. A third, that is sister-nucleus to the last in origin, becomes extended along the ectoplasm of the anterior body-part, and forms there, along with a membranous expansion of the ectoplasm, the characteristic sense-responsive or correlating membrane of the group, from one or both ends of which starts the lighly sensitive flagellum. We propose therefore to term this the cogitonucleus.

The researches of the above named observers show that these three nuclei all remain connected with each other by fine chromatin threads, and so definite and common tubes of energy-flow must constantly pass between and influence each. But such differentiation of nuclear substance clearly suggests the formation of three fundamental though interrelated body masses, namely a somatic mass associated with the cognitonuclear apparatus, an irritoreceptive and sense-correlating mass associated with the cogitonuclear apparatus, and a hereditary as well as reproductive mass associated with the gametonuclear apparatus.

Here then, in a unicellular organism, are outlined the initial morphological and physiological differentiations that correspond to the general protoplasmic and nuclear or the bio-cognitic, to the nervous or cogitic, and to the reproductive or hereditary substances.

An investigation of the Infusoria may next be made. As outlined in the succeeding chapter, there occur in most if not all genera of the group two nuclear constituents, the micronucleus or micronuclei, and the meganucleus. Both are composed largely of chromatin substance, but they differ much in size as well as in number usually. Thus the micronucleus may be single and small, but in genera like Spirochona and Stentor three to twenty micronuclei may occur. The meganucleus is usually large, and may at times become branched, elongated or otherwise modified. It evidently presides over the general somatic or cognitic functions of the cell, for during cell-life it is the most evident nuclear part. But during and soon after conjugate fertilization it becomes broken down and absorbed, to be again developed in the new individual. 
Therefore the writer would suggest here, as before, that it might appropriately be called the cognitonucleus. The micronucleus may lie against or be considerably removed from the mega- or cognitonucleus. When conjugation of two individuals has been effected "the micronucleus of each swells up and undergoes division by mitosis into two micronuclei. This is immediately followed by a division into four. Of these four nuclei three degenerate, and are either absorbed or ejected from the body. The remaining one, which behaves as a sexual nucleus, undergoes division into two nuclei. One of these crosses the line of junction of the two conjugating individuals, and enters the cytoplasm of the other individual, and may be called the migratory nucleus. The other remains in the cytoplasm of the parent, and may be called the stationary nucleus. Thus, as a result of the divisions of the original micronucleus of each of the conjugating individuals, five nuclei are formed: three degenerate and disappear, one migrates, and the remaining one is stationary. A fusion of the migratory nucleus of one individual and the stationary nucleus of the other then takes place to form the cleavage nucleus, and soon after this has occurred the two individuals separate. The cleavage nucleus then divides into two, and generally a second time into four, or a third time into eight. Ultimately, however, one of the halves of a division gives rise to the new meganucleus, and the other to the micronucleus of a new individual. The new meganucleus can therefore be traced back to its origin from micronuclear elements, and to this there is no exception." (82: 389.)

Here then the micronucleus behaves as a special hereditary or sexual or reproductive nucleus. It would, further, be of extreme interest to ascertain whether fine connections do not exist between the mega- or the micronucleus and the peripheral ectoplasm, which usually is highly sensitive and even differentiated into two or more layers. Of these, one, known as the neuroneme layer, has been regarded as nervous in function, and, if derived from or connected with the meganucleus or micronucleus, might well be homologized with the ectoplasmic cogitonucleus of the Homoflagellata. But, even in want of such recognizable nuclear demonstrations, it can be accepted that a cogitonuclear, a cognitonuclear, and a hereditary substance are all segregated in some Infusoria.

These epitomized studies then definitely suggest that, in two of the highest groups of Protozoa, four specializations in cellsubstance have been effected, viz., the protoplasm or sub- 
stance for display of biotic energy; the cognitonucleus for condensation and distribution of cognitic energy; the cogitonucleus in hrmoflagellates, or the neuroneme substance in some ciliate Infusoria, for condensation and distribution of cogitic energy; and the gameto- or micronuclear substance that gathers up, condenses, and hereditarily hands down to future generations the peculiarities of the species.

These however exactly represent the four fundamental celltypes or cell substances that are characteristic of metazoan animals. And of the four, the cogitonuclear or the nervous and the gametonuclear or reproductive are of special significance. We defer study of the latter till the succeeding chapter. But a distinct evolution and segregation of nervous substances is of high value amongst Protozoa, for it anticipates and explains the origin of the ganglionated or cogitic system of Metazoa.

As compared with the chromatin or cognitonuclear substance of plants and of simple Protozoa, this more complex neural cell substance initiates that striking peculiarity of animals that we call reflex action. Such action seems to be entirely absent from even the highest and most sensitive plant organs, whether these be the actively motile cells of Chlamydomonas, or the delicately responsive tissues of Mimosa pudica-the sensitive plant-and Dioncea muscipula-the Venus fly-trap. We may therefore essay, at this stage of our inquiry into proenvironal reaction, to learn how such concentrated nerve substance originated from simpler substance. This mill carry us back to a consideration of the nature and distribution of chromatin or cognitic substance.

If chromatin be truly a sensory or cognitic material, its fundamental function would be to condense and then to concentrate inwardly tubes of energy that would represent the fundamental energies of the world that environs each organism. That is, the nine fundamental sense-centers that we have already postulated, the thermotactic, the heliotactic, the chemotactic, the geotactic, the paraheliotactic, the hydrotactic, the mechanotactic, including the touch and sound 
senses of higher animals, the gyrotactic, and the electrotactic, which all play more or less on organisms, might be considered to be condensed in the chromatin masses or chromosomes of division figures. Each such chromatin mass of the eight observed in division of some hæmoflagellates, and in some other plants and animals, might be regarded as made up of definite, minute, even ultramicroscopic, constituents which we have already called energids. If an excess number of such energids be distributed during chromatin and cell-division to certain cells, say those of the root, that we would then call geoenergids-some of which energids are distributed in the protoplasm as minute geoplastids, and others connected with these in the nuclear chromatin as nuclear geoenergids-, these latter might start tubes of energy-flow from the nucleus to the peripheral protoplasm in such direction as to cause a greater accumulation of and growth by the protoplasm, later still growth of the wall more on one side than another, if the root has been placed in unsatisfied position. By such energized growth the root would steadily be stimulated to assume a satisfied - that is, a normal-position in relation to gravity.

A like explanation could well be given for the distribution and action of other types of energy. Furthermore, in connection with those fairly numerous cases of reversing tropic or tactic response, now well known in certain plant parts, such as the aerating roots of some sub-aquatic plants, the shoots of hog peanut (65), of Kenilworth ivy (Linaria Cymbalaria), of Sarracenia, and others, we might consider that here, before reversed stimulation started, either a migration of energids occurred, or that a few formerly present multiplied, and then received and distributed more powerful tubes of energy in definite directions.

Such actions and reactions, however, we well know are limited mainly to growing plant parts, or to restricted intercalary growth-areas amongst the higher plants. Now, with increasing specialization and activity in animal organisms, the gradual evolution of a nuclear and later of a cell factor, that would condense all sense-impressions, link up or correlate 
these, and then radiate definite tubes of energy to definite and appropriate areas of response in the organism, became by degrees an established and ever more complex feature of evolving animals.

In seeking now for a morphological and physiological continuity between higher Protozoa and simpler Metazoa, from the proenvironal standpoint, the writer believes that such can be established if it be accepted that during mid-archæan times a group of organisms existed that were somewhat intermediate between a ciliate infusor and a primitive rotifer. This probably resembled much the embryonic stage of Callidina as described and figured by Zelinka.

Here extremely delicate division walls early differentiate the egg into a set of biotic-cognitic cells that functionally correspond to the trophonuclear constituent in hæmoflagellates, and the meganuclear constituent in infusors, with their associated protoplasms. Such cells become the main mass of the epiblast and hypoblast, including the alimentary canal, the muscular tissue and gland cells. Next, and almost simultaneously, the nervous system originates as an anterior multiplication of epiblast cells, that in position and future energizing relation recall the anterior motile nuclear part of a hæmoflagellate. Further the deeper hypoblast forms a medioposterior cell-mass that becomes the primitive gametic constituent of the organism.

From later mid-archæan days on to the present time myriads of types doubtless lived, that must perfectly have filled up the gap between existing ciliate infusors, on the one hand, and embryonic rotiferan types, on the other, and which in graded manner must have united unicellular organisms possessing bio-cognitic, cogitic, and gametic or hereditary constituents, with simple multicellular ones in which these became loosely separated entities as in rotifers now.

Alike the cogitic or nerve cell substance and the gametic or hereditary cell substance of primitive metazoans probably represents greatly more complex and energized chemical compounds than do the biotic or protoplasmic, and the cognitic 
or chromatin substance. Thus the gradual formation, within ganglionic cells, in increasing richness up to man, of the Nissl granules that we have termed neuratin is partial evidence of this.

In correlation with this we find that protoplasm yields greatly more complex analytic constituents of the albumen groups than are presented or even distantly approached by any inorganic body; that chromatin contains nucleo-protein compounds of still more complex constitution than the protoplasmic substance; while neuratin or Nissl substance becomes so steadily used up during intense mental effort, and slowly replenished again after such effort has ceased and perfect nutrition has been reestablished, that the substance itself suggests a high molecular complexity. Unfortunately our knowledge of its exact chemical composition is still practically negative.

The remarkable advance effected therefore in transition from higher Protozoa to simpler Metazoa consists we believe: (a) in the aggregation of energids within special ganglionic cells, that represent all kinds of energotactic chemical constituents; (b) in the capacity that these ganglionic cells have for linking up the diverse resultant stimuli which they receive into one complex resultant; and (c) in the discharge of this resultant energy, so as to cause change or even motion in some part of the organism, correlative with or apart from the regions of initial stimulation. Thus in all probability originated that characteristic of the Metazoa that we term reflex action.

But in all complex proenvironal reactions there are gradually added on to reflex action those advancing phenomena that we speak of as associative memory, mentality, intelligence, intellectuality, and morality, that are treated in other chapters.

Now, in evolution of the cogitic or complex correlating nerve cell substance, condensation of all of the raried sense-resultants of different kind, and formation of a complex resultant response, may have been aggregated amongst simplest Metazoa in one or a few cells. But, with increasing rapidity of response to different stimuli, special areas or groups of nerve cells would become associated, in one or more nerve centers, with each 
kind or type of sensory stimulus. Tubes of varying energizing stimuli would pass to these, and, on becoming linked within them into a satisfied resultant response or responses, would be radiated outward to appropriate centers of action. Such is seen in diffuse state in several of the simpler metazoan groups.

But, in the great line of evolutionary advance that in a later chapter we trace from Infusoria through the rotifers, prototurbellarians, turbellarians, protonemertines, nemertines, protocyclostomes, and cyclostomes, we observe that from a small multicellular brain in simpler rotifers we advance to such a type as Callidina, in which an anterior olfactory and lateral optic areas can be distinguished from the remainder of the nervous or brain mass, that, from its nerve-thread connections, evidently combines a mechanotactic, a thermotactic, and other sense functions. With increasing specialization and aggregation in nerve substance the brain of a nemertean like Eupolia is reached (p. 433). Here the superior or dorsal brain lobes are primarily concerned with correlation of the higher cognitic stimuli, in that they are connected with strong olfactory or chemotactic, optic or heliotactic, and combined audito-geotactic nerves. So the varied stimuli from without that are passed by these nerves to the brain centers are there combined into a resultant response.

The above or dorsal again are connected with the ventral brain lobes by transverse commissures. These ventral lobes are primarily concerned with the reception of diffuse mechanotactic, thermotactic, and gustatory or chemotactic stimuli. Such a nemertean brain therefore, from the standpoint of proenvironment, is a complex sense-receptor, to which definite sensory or energotactic nerves pass, which is itself composed of definite sensory receptors and correlators, in which a definite resultant response is built up, and from which a definite compounded stimulus passes to a peripheral center or centers. This stimulus then causes the animal to behave in an exact and correlated manner to all of the incident stimuli.

From such a brain to that of the elephant or ape the transition is gradual and progressive. Such transition involves 
also the segregation of increasingly large brain areas for reception of stimuli, and, much more so, for increasing formation of accessory correlating centers, where compound resultant responses can most quickly and perfectly be started, that stimulate the organism to rapid satisfied action.

In applying the above views to proenvironal reaction in the simpler Metazoa we may first cite the detailed studies of v. Uexkuill (83) on the sea-urchins. He has demonstrated the presence of a diffuse nervous system external to the shell, and of a radial internal system. Each little ganglionic area of the former system, that is associated with a movable spine or a pedicellaria, can so stimulate either of the latter that one of them can respond individually, and proenviron a line of movement even when isolated from the shell. In other words each minute nerve-center for a spine, and each nerve center for a pedicellaria, can act as a definite receptive and responsive area. But further, these individual centers are all linked up, so that "they cause the various organs to work in harmony, usually assisting to perform certain necessary functions."

Thus, if debris falls upon the sea urchin, the pedicellariæ seize it, break it into bits, and with the aid of the spines and the cilia remove it from the body. Small animals coming into contact with the sea urchin are seized by the pedicellariæ and held, till they are grasped by the slowly moving tube feet and spines, and by them carried to the mouth and eaten. When the sea urchin is attacked by an enemy, the spines all bend toward the region of the attack, presenting a serried array of sharp points to the advancing enemy.

V. Uexkuill has therefore designated this animal "a republic of reflexes," and so it can well be quoted as a transition type between the lower and the higher animals. But such transition involves more complicated results than are seen in plants or in lower animals, and suggests first a simple summation of resultant stimuli. Thus the presence of particles of debris start chemotactic, geotactic, and thigmotactic stimuli, all of which become compounded into a resultant response. But weight of the particles to be removed and their relative hardness start apogeotropic and thigmotropic stimuli that together become a resultant response, and this along with the former resultant constitute a compounded resultant that effects breakage of the particles and the casting free of them. Definite quantities or tubes of energy therefore, of varied quality, acting on the ganglionic centers, are gradually compounded 
into one complex resultant, that exhibits itself in orderly motion in one given direction.

As illustrations of animals with more aggregate or condensed nervous system, we may next select Thysanozoon and also planarians that Loeb has studied. His sectioning experiments (60:72) indicate that the anterior or richly ganglionated and correlated head part, as well as the posterior or poorly ganglionated and correlated part of the nervous system, can receive, correlate, and summate into resultant proenvironal response several environal stimuli that act on it. But the posterior part is more sluggish and less perfectly linked together than is the anterior. Such is partial proof that the evolving brain ganglia are condensed and equilibrated summators of all afferent stimuli, while the body ganglia are less perfect. The results got by Loeb with Planaria torva would suggest that it has a more diffuse capacity for reception and correlation of stimuli than has Thysanozoon, and so that it is intermediate between the latter and the sea-urchin in physiological elaboration.

At this point consideration may shortly be given to the evolution and relative action of individual nerve cells, and further of motor and inhibitory nerve centers, or ganglia, a subject that is further treated in a later section (p. 238).

The fundamental peculiarity of a nerve cell is that it can receive a stimulus from one center of cell stimulation, and reflect or direct it to some other center, so as to cause change in the latter, or also in other cells around. But a further and highly significant function of such a nerve cell is that, when two or more stimuli reach it, simultaneously or in rapid succession, from different centers, the summated action of these may all be cumulated into one resultant that causes a definite proenvironal response in the same or some other centers. This inherent and unique capacity we have considered to be due to the action of a highly perfect and transformable exhibition of energy, the cogitic, which effects a linking together of highly complex colloid molecules, so as to start a compounded reaction along proenvironal lines.

But it must further be noted that along with such compounded reaction there is involved, in increasing complexity, as we ascend the scale of animal life, the explanation of mem- 
ory, of instinct so-called, of intelligence, and of reason, all of which are evidently associated with and center round nervecells.

The question therefore at once presents itself: Wherein does such a nerve-cell differ from other nucleated body cells, all of which we would class as bio-cognitic? In any attempted explanation graded stages of developmental or evolutionary complexity should probably be traceable, that lead insensibly upward to the most complex nervous or mental acts. At the foundation of all such action we would propose a principle that might be expressed as "the recurrent periodicity of definite organic molecular vibrations." The beginnings of such seem to be traceable in some phenomena of plant life, and are wholly and invariably originated by changes in environal stimuli. Thus the experiments of Darwin, Pfeffer, and Ulrich demonstrate that in plants which show nyctitropic movements, if the plants be kept beyond the normal period in darkness, rhythmic change occurs about the time when the normal lumic stimulus that is now lacking should have acted. So plants kept continuously for two to four days in the dark will "wake up" about the normal period when light should have reached them. But, if the periodic waves or rhythms of alternate light and darkness be upset, after six to eight days the plants become unhealthy and decay.

Again the annual or even more frequent seasonal formation of reproductive cells appears often to proceed with rhythmic regularity, even though some of the primary exciting stimuli are absent. In other words there is registered in the living cells such a rhythmic time relation that, in absence of some of the factors that constitute the complete stimulus, response action may be shown. Even in the simpler metazoan animals the rhythmic recurrence of hunger and of satisfied states, of active and of passive existence, of accumulation of waste material and of expulsion of it, of mating and of alternating isolated life, as well as other phenomena that will occur to all, including the "habits"--so-called - in higher animals, are similar exhibitions of rhythmic stimulation. 
The gradual localization, in a few cells, of substances that undergo such rhythmic transformations, under the action of even slight environal change, would constitute, we believe, one initial cause for origin of nerve cells.

Another initial cause for such origin would be the cumulative association of two or more distinct stimuli in cells of a localized area, and the subsequent gradual separation of sister cells from these, or the linking up of these with separate cells or groups of cells, that would act as the response-discharging cells.

Still another cause that has been recognized and discussed by Loeb, Thorndike, and others is association and associative memory. In this case, as a result of the utilization and cumulation of sereral simple sense impressions, a resultant response is started; or even several resultants, from groups of simple stimuli, may be compounded, and may then give rise to a satisfied response. Repetitions of such actions, at first rather imperfectly, become more perfect and easy, as a definite association and succession of colloid molecular combinations is effected in rhythmic relation. Such doubtless are in turn connected with definite molecular disintegrations and combinations in definite constituents of the nerve cells. So, when even a few of the primary stimuli-that with others for the moment may be absent but which ordinarily make up the complete stimulus-become appropriately combined, these tend to lead up to, and work out, the ultimate resultant; owing to tubes of energy-flow along lines of least resistance.

So chronic or rhythmic vibrational excitation in a cell or group of cells, cumulative association of several distinct resultants in definite nerve cells, and passage of a compounded resultant response to other cells detached from or linked up with these, also associative flows of tubes of energy along connected molecular lines, and impression of these more and more deeply on the molecular organization, seem all to be primitive causes for origin of the highly complex nerve-cell.

With such increasing complexity in the cumulation of distinct stimuli, it might be expected that certain probably recognizable bodies should characterize the nerve cells; should 
be least permanent and abundant in simpler metazoan nerve cells, but should increase in aspect and quantity along the metazoan scale; should be most regular and fully formed during a rest period; should be reduced in number, in size, in regularity, or in density during fatigue; and should show certain micro-chemical characteristics. The small bodies that have been called Nissl's granules, and whose substance we propose to term neuratin, answer to all of the above requirements.

During the past ten years an increasing literature on these has appeared, and study of this literature may now be summarized. The investigations of Nansen, Pfluicke, McClure, Rhode, Legendre, and Smallwood amongst others on invertebrate nerve cells, and those of Fleming, Cajal, Held, Marinesco, Gehuchten, Ewing, Carrier, Lugaro, and Ranson on the nerve cells of vertebrates, show that definitely arranged and stainable bodies occur in the protoplasm of the nerve cell around the cell-nucleus. These have variously been called tigroid, sigroid, basophile, chromophile, and Nissl granules or substance. Amongst invertebrates various worms, nemerteans, molluscs, crustaceans, and insects have mainly been studied. In such worms as the Nereidce the granules are usually aggregated in the protoplasmic spongework, and in somewhat irregular manner; a similar disposition seems to occur in the nemerteans studied by Montgomery; in the crayfish and other crustaceans they are often very abundant, oval in shape, and definitely arranged in concentric rows round the nucleus of each nerve-cell, though at times they are prolonged into the uniting ends of the nerve-fibers. In Mollusca, specially as studied by Smallwood and Rogers alike histologically and experimentally, the granules appear as diffuse or aggregate masses around the nucleus of the ganglionic cells. The above observers conclude (84:63) "that these structures will be found to fill the same place in the economy of the invertebrate nerve-cell as does the Nissl body of the vertebrate nerve-cell." Experimentally also it was found that, alike during progressive starvation and exhaustion by fatigue, the granules are gradually reduced in number, or size, and at length disappear. 
McClure had earlier reached the conclusion, for the nervecells of Gasteropoda, that the granular substance "is homologous with that found in the nerve-cells of vertebrates (Nissl bodies)"'(85:13).

Various and important papers have appeared on the Nissl granules of vertebrates, some of which deal with histological details, others with histo-physiological changes. Under the latter head several observers have reached the common conclusion that, after section of ganglionic cells, degenerative changes occur in one to four days, that result in "a progressive solution of the tigroid (Nissl) substance, beginning either near the nucleus or at a point intermediate between the nucleus and the periphery of the cell. The cell becomes swollen, and the nucleus more or less displaced toward the periphery." $(86,18: 101 ; 19: 125$.$) "When in time repair may occur such$ consists of a restoration of the Nissl bodies and a return of the nucleus to the center of the cell."

Ranson (supra, also V. 18) not only agrees with his predecessors Lugaro, Cox, Warrington, and Griffiths, as to the existence of several types of nerve-cell, specially the "small" and "large" cells, but also concludes that in the former the most rapid and extensive changes occur, while in the latter the changes are slower. He has also traced the successive integrative developments toward regeneration in young rats "that began on the eighth or ninth day and was almost complete in twenty days." During reformation of the Nissl granules these gradually increased in number and size, so that "by the seventeenth day the central portions of the cells contain Nissl-bodies of considerable size, and by the twentieth day the distinction between the coarsely granular peripheral ring and the rest of the cell has disappeared."

But the observations of the above-named, and of other investigators such as IIolmgren, Hamaker, and Rand, suggest that each ganglion cell is traversed by a fine network system of threads, as well as by a system of canals, which seem both to be intimately related to the Nissl or neuratin substance. There are thus represented in each nerve cell. apart from the 
cell protoplasm or biotic substance, and the nuclear chromatin or cognitic substance, (1) a definite material, the neuratin or cogitic substance, that is steadily used up during intense mental effort, and is then regenerated slowly during nutritive anabolism; (2) a canal system that permits steady absorption into it of secondary or waste material from the neuratin substance during active effort, as well as transfer later of rich constituents that can rejuvenate the neuratin centers; and (3) a system of threads that can efficiently conduct tubes or currents of cogitic energy generated during metabolism of the neuratin granules.

The objection may here be made that the Nissl or neuratin granules have by Smallwood-Rodgers as well as others been viewed merely as "some sort of a fat." The investigators just named say. $(84,18: 72)$ that "most of the tests used require a long time for their action, and in some cases even failed to act at all." . . . "Osmic acid blackens the bodies after a long time. In many of the specimens the blackening was merely superficial, indicating that the substance is highly resistant or that it is not a fat but some substance which may break up into a fat and some other substance." The slow and varying reaction given in different animals strongly favors the view that it is an extremely complex proteid, that has been built up through the action of highly complicated currents of energy and which breaks down very slowly during discharge of cogitic flows of energy. The fatty constituent here, as in all highly energized cells, we would regard as an energy insulator, and-according to its position-as a path-distributor for the outgoing currents of cogitic energy.

We would hazard the thought, therefore, that the most important cytological study for the future, in the realm of evolving animal life, will be that pertaining to the individual nerve cell, and its comnection with others in evolving nerve ganglia.

The studies already published show us that the number, position, relation, size, and behavior to chemical agents of the neuratin granules vary greatly in different cells, or in different ganglia, or even in different animal groups. Such may well 
indicate fundamental differences that are associated with different mental functions. Furthermore, as one rises in the animal scale from the simplest to the highest types, the increasing number of nerve cells, of groups of these as nerve ganglia, of groups of such ganglia that constitute nerve or brain centers, and finally the enlarging size of the brain in the highest animals, all demonstrate a steady and advancing complexity of the individual cells, that may be correlated with like advance in the neuratin substance, as to its quantity and correlation. So on the principle of environal action and of proenvironal response, as animals have become exposed to increasingly complex environal stimuli, these seem to have started the formation of new and more intricate combinations of the neuratin substance, that have very slowly resulted in the formation of new and ever more complex as well as rapid compounded resultant responses, which we designate habits, instincts, intelligence, and intellect amongst others.

But that such acquisitions are only slowly started might be expected from the highly condensed and difficultly soluble character of neuratin substance. This would be in entire accord with the view held in the past decade by many, viz., that heredity is greatly more uniform and persistent than is environal action, in the history of chains of organisms. Such, however, should not blind us to the fast accumulating evidence which demonstrates that mental associations and capacities are gradually developed and persisted in by individuals.

So in all afferent stimulatory acts that affect nerve cells, and in all succeeding compounded resultants that start proenvironal response amongst the higher invertebrates and vertebrates, we accept it as proven that acquisition of new lines of molecular motion which are satisfying to each organism can occur. When moreover such is beneficial to the life and successful reproduction of each individual, persistence of such lines of motion develop into "tendencies," and ultimately into what have vaguely been called instincts. Thus the studies of v. Uexküll (87), of Allen (88: 293), of Spaulding (89:49), of Yerkes (90), of Edinger (91) and many others in recent 
years are too decisive and indicative to cause serious doubt that new impressions or stimuli give rise to new and exact lines of energy-flow, that bear along and locate material particles in new relation to such as formerly existed, and which confer thereby new proenvironal capacities on the organism.

To reach such a result we do not necessarily need to assume the formation of any new substances. A more advantageous placing or linking together of already formed material, or a steady increase in the amount and complexity of connection throughout that material, owing to environal stimulation and proenvironal response, would amply explain the formation of new tendencies, habits, and instincts. Moreover, structural details are evidently steadily established, so as to extend the spheres of action and reaction. For, as C. L. Herrick (92: 23) has pointed out, accessory cells surrounding nerve endings may take up and distribute currents of energy from the nerres. Thus he says, speaking of some Amphibia and reptiles: "A nervous end-organ is so connected with a meshwork of vast extent as to suggest a very extensive somatic influence of a nature similar to nervous reaction over vast tissue areas."

The gradual evolution then among metazoans of an increasingly more complex nervous system, as these animals increased in size and in time passed from an aquatic to a land life, was necessary. So correlating sensory-motor areas or clusters of nerve cells, that formed brain masses, became steadily enlarged and subdivided into sense-correlating and memory centers. These permitted more and more complicated inflows of varied kinds of energy from without; the transformation of these into discrete currents of bio-cognitic energy, and later, when linked up through passage into the nerve cells, of cogitic energy. This again, through complicated interlinking of molecules in the nerve cells, might produce efferent lines of cogitic energy-flow, that would start definite proenvironal responses.

So, by extremely slow but steadily progressive multiplication, interconnection, and condensation of nerve cells, such brain centers originated and were built up gradually as have 
been diagrammatically but graphically figured by Edinger for some groups of vertebrates, and which are here reproduced (Fig. 9). In these diagrams the evolving neencephalon can
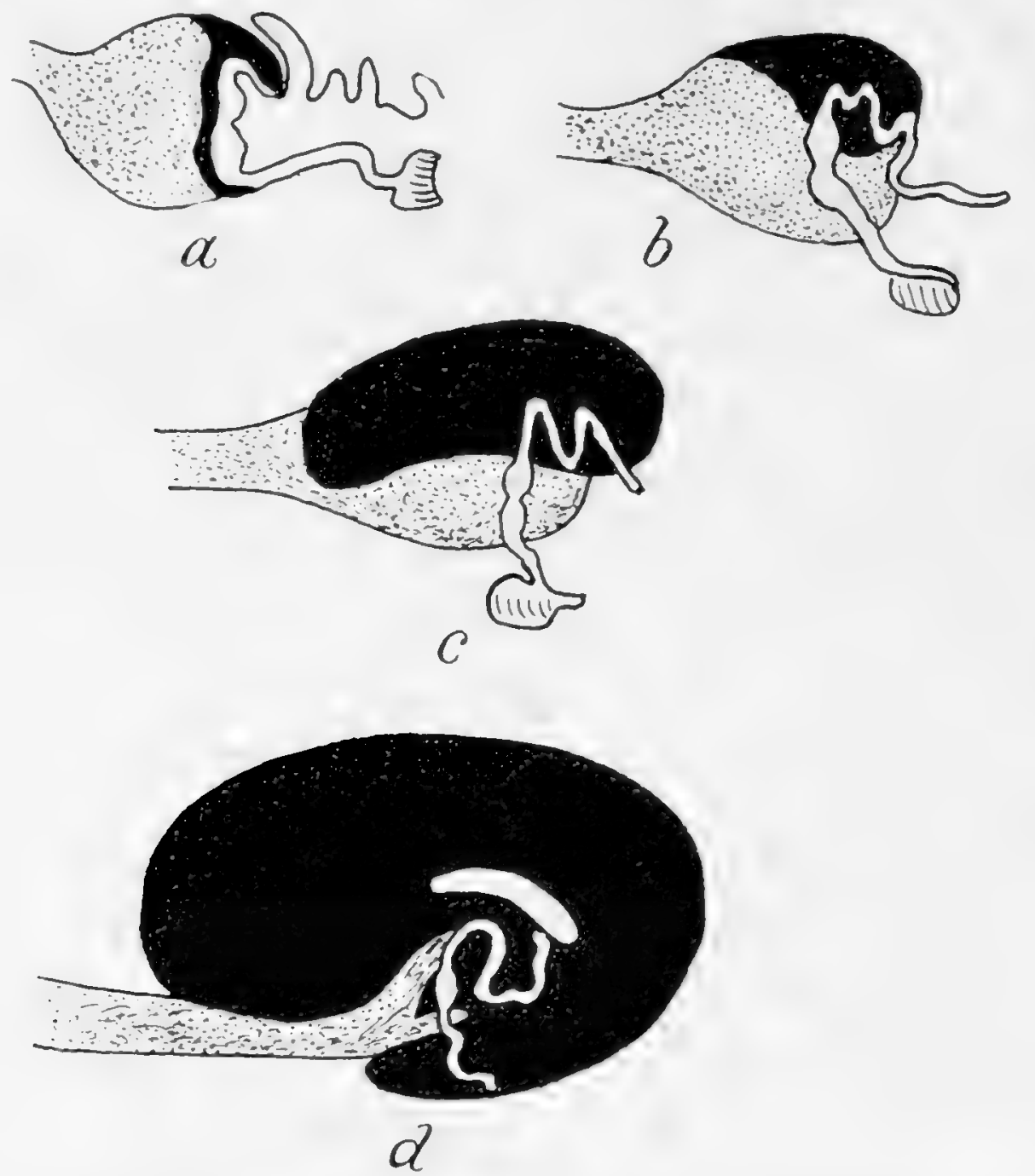

FIG. 9-Comparative diagrammatic stages in development of the front brain or neencephalon, in (a) Selachian, (b) Amphibian, (c) Reptile, (d) Mammal, in each case shown in black. (After Edinger.)

truly be regarded as a receptor for discrete simple or resultant stimuli, as a condensor and correlator of these into complex or summated perceptions, as a storage center for these perceptions, and particularly as a great proenvironal activator, from which extremely varied and often complexly summated efferent stimuli proceed.

It is not necessary therefore at present that an attempt should be made further to trace examples of proenvironal reaction as a great evolutionary factor that has worked through- 
out the entire series of the higher Metazoa. For most of the responses or actions shown by such animals are wholly due to efferent proenvironal stimuli which start as summated responses to simpler stimuli that have passed in from without, and which guide - we might almost say impel-each animal along definite pathways.

But in all such proenvironal nerre responses the gradual evolution of a motor and of an inhibitory nervous system must constantly be kept in view as a great evolving zoological principle. Accordingly all afferent stimuli from without that affect any nerve cell have been termed receptory; all stimuli that originate from such receptories either as direct or as summated responses toward action effectory; and all stimuli that are reducing, canceling, or contrary factors to the last inhibitory.

It would be impossible to deal with the comparative relation of the effectory and the inhibitory now. But, on the grounds already advocated (p. 208), namely that each proenvironal act brings about a temporarily "satisfied" state in an organism, if we may judge from frequent human behavior and from not a few acts of the lower animals, it must often happen and have happened that a certain summated act, resulting from several environal stimuli, may have originated a "satisfied" state, but yet that one or more of the constituent stimuli may have been helpful and upbuilding, another more or less injurious and disintegrating. By frequent and slow responses, in which all such acts were tested, a course or pathway would be ultimately plotted, so that different groups of animal organisms would gradually reach a phase of development in which the "satisfied" but disintegrating stimuli would be subsidiarized to the "satisfied" but upbuilding, as these were compounded into summated action. The converse as well as a balanced state would equally hold true.

If we may borrow an illustration at this stage of our studies from human history, it can truly be said that the surviving and even dominant nations of today are largely those who have subsidiarized "satisfied" but disintegrating responses or unsat- 
isfied responses to those that are more lastingly "satisfied" and also are upbuilding.

Proenvironment then we would view as that great and everexpanding law of organic life, by which varied environal stimuli are linked into a summated and unified response, that brings each organism into satisfied relation to its environment. Such satisfied relation may upbuild, may only prolong, or may even start degeneration and ultimate death, specially if combined with unsatisfied molecular states, to each organism and to groups of organisms. 


\section{CHAPTER X}

\section{ELL MULTIPLICATION AND THE ORIGIN OF SEXUALITY IN PLANTS AND ANIMALS}

Many valuable books, memoirs, and papers have appeared during the past quarter century on the above subject. These have been freely drawn on by the writer, in addition to observtions of his own, in the preparation of the present chapter. In study of the theme, if his methods or conclusions are more or less divergent from results given in the publications referred to, it will merely be that he has striven to interpret facts and theories in a manner that will conduce the more to further advance according to his thinking. If he is mistaken in his views, the steady onward march of discovery will gradually demonstrate such.

The study of sexuality and of sex-evolution is one of the basic requirements of biological progress. For no process in life is more striking and regular to even the casual biological observer. The tangled floating masses of conjugating Spirogyra, the orange-yellow oozing spermatial material on the tips of Fucus thallus, the stellate or umbrelloid growths on the liverworts and mosses, the flower clusters and pollen clouds that appear on exact days often of each year, even though on separate shrubs or trees, are all familiar objects in plant life. Equally also the floating medusoids, the bee or ant flights, the migrations of the salmon and other fishes, the frog-spawn of spring time, the nesting of the birds, and many other equally expected events are seasonal exhibitions of sexual development, or are prophetic of such.

Now the marvelous exactness in time relation, shown equally during sex-cell conjugation and spore-dissemination preliminary to such conjugation, indicate that definite waves of energy, definite lines of energy, and definite transformations 
of energy mainly characterize the process; these being in turn very delicately regulated by environal conditions. Thus not only does each species of Spirogyra conjugate at definite seasons, the cells in opposite filaments mature within a few hours or days apart each year. The same is true for gatherings of desmids, diatoms, and related algæ. In Oedogonium, Nitella, and Chara definite growth-waves and maturation-waves of energy traverse the plant from below upward, till the motile sperms have been shed, and the eggs have been fertilized.

In hepatics and mosses generally, the maturation of antheridia and archegonia proceeds within surprisingly exact time limits, as does also that of spore formation in alternating relation. In ferns the period for maturation of the antheridia and archegonia on each prothallus can be very accurately predicted for each species, if note be taken of light, moisture, and temperature conditions.

But it is amongst the flowering plants that these phenomena are most pronounced, though this is probably due to such being most readily observed and conspicuously shown. The writer has noted, for about 14 years, the comparative flowering period of Ginkgo biloba round Philadelphia, and there for several staminate and pistillate trees some distance apart. While the period of blooming has been as early as April 25th during warm seasons and as late as May 20th during cold seasons, the staminate and pistillate trees have been synchronous often to a day, while anthesis and pollination have largely been limited to about four hours, namely between 7 and $11 \mathrm{a} . \mathrm{m}$. of one day.

Equally exact results have been recorded for the staminate and pistillate trees of the white poplar $(P . a l b a)$, the Carolina poplar, ( $P$. monilifera), and subdioecious trees of the silver maple (Acer saccharinum) as also for the hermaphrodite showy flowers of the catalpa tree (C. bignonioides), and many others.

But the views regarding, and mode of presenting, the question of sex differentiation are often as varied as they are illogical. For in not a few evolutionary treatises that discuss the subject, instead of any patient attempt being made to start 
with primitive asexual forms, and to work upward to others that become gradually more complex, the student is suddenly plunged into the most complicated questions of inheritance amongst highest plants and animals. The aim in this chapter will be to trace evolutionary progress step by step, so far as possible with the fragmentary plant and animal record of the present day. But, for a correct understanding of the phenomena of sexuality, it is absolutely necessary that we approach the subject by the pathray of cell-division, as the succeeding context will demonstrate.

In such Acaryota as Chroococcus, Sarcina, many of the simple bacteria, and in Proteomyxa, each organism is a simple cell that shows no apparent trace of nuclein or nuclear rudiment, and that multiplies by simple division of the protoplasm. But as already emphasized (p. 98) such a process is not to be regarded as a mere haphazard separation into equal or unequal halves of a homogeneous substance. Every new fact gained adds to the view that the protoplasm is an extremely complicated chemical foam that is made up of very definitely related compounds arranged into definite lines, groups, or areas of complex molecules, that are traversed by and constantly altered by definite currents of energy. Slow responses also are made to environal stimuli, and in the process food and oxygen are absorbed, which in time set up intrinsic metabolic and stimulatory changes.

But, in simple plants like Chroococcus, Aphanocapsa, and others, there seems to be little if any polarity, so that successive divisions are successively at right angles to each other, as if the whole mass were a uniform-eren though molecularly complex-substance, that in attaining a certain size begins-from surface-tension, gravity, and other relationsto fall into halves, each of which again may grow in size. But, even in dividing along definite rectangular planes, we have evidence that definite lines of energy-flow are traversing the protoplasmic substance, and are transporting materials to localized areas in exact manner. How then can such phenomena be led up to, from conditions that are purely inorganic? 
In reply it can be said that during the past half century the physical necessity for division to occur in any steadily growing colloid vesicle, if it is to preserve its vesicular character, has been fully recognized since Herbert Spencer first drew attention to the physics of the act.

Hartog (9\%: 23) has fully emphasized and illustrated the case thus: "Growth of an organism which retains its shape alters the ratio of the surface area to the whole volume, so necessary for the changes involved in life. For the volume of an organism varies as the cube of any given diameter, whereas the surface varies with the square only. Without going into the arithmetical details, we may say that the ratio of surface to volume is lessened to roughly four-fifths of the original ratio when the cell doubles its bulk. As Herbert Spencer and others have pointed out, this must reduce the activities of the cell, and the due ratio is restored by the division of the cell into two. This accounts for what we must look on as the most primitive mode of reproduction, as it is the simplest, and which we term "fission" at Spencer's "limit of growth." In a footnote the above named writer then works out some exact examples arithmetically which demonstrate the correctness of these views.

Now the multiplication here, that is coextensive with formation of new individuals, is a process that probably wholly is due to osmotic relation of the cells and of their contents, to the liquids by which they are surrounded. Thus Leduc describes (23: 124) the artificial formation of colloid vesicles through the dropping of "a fragment of fused calcium chloride into a saturated solution of potassium carbonate or tribasic potassium phosphate." As a result a vesicle or cell is formed by the calcium chloride. Then "the first cell gives birth to a second cell or vesicle, and this to a third, and so on, so that we finally obtain an association of microscopic cellular cavities, separated by osmotic walls-a structure completely analogous to that which we meet with in a living organism.

The membranogenous substance, the chloride of calcium, diffuses uniformly on all sides from the solid nucleus, and forms an 
osmotic membrane, where it comes into contact with the solution. This spherical membrane is extended by osmotic pressure, and grows gradually larger. Since the area of the surface of a sphere increases as the square of its radius, when the cell has grown to twice its original diameter, each square centimeter of the membrane will receive by diffusion but a quarter as much of the membranogenous substance. Hence, after a time, the membrane will not be sufficiently nourished by the membranogenous substance, it will break down, and an aperture will occur through which the interior liquid oozes out, forming in turn a new membranous covering for itself. This is the explanation of the fact that all living organisms are formed by colonies of microscopical elements, although we must not forget that Nature often produces similar results in different ways."

In the primitive plant cell, as in nearly all higher ones, a phase in such osmotic tensions has been reached where the living substance of the cell secretes an interior new strengthening pellicle, between halves of the distended mass, before rupture of the proceeding one occurs, as the researches of Schimper and Strasburger have demonstrated. All known facts then seem to indicate that division was, of necessity, the simplest and most primitive mode of multiplication. As Leduc's account also would suggest, budding, as seen in the yeast and other plants, is a variety of the same process fundamentally.

When repeated divisions in one plane succeed each other, and the resulting cells remain in contact, a filament is formed, and then, as often happens with the higher or thread types of the Cyanophycex, multiplication may be due to breaking across of a part of the thread at some point where weight of part of the thread causes bending and compression of a cell with resulting rupture. So, in simple unicellular Cyanophycex and even in thread forms, division in some way is the only method of multiplication known.

But, when we reach the Nostocacex, Chamaesiphonacex, and Stigonemaceæ of the Blue-green Algæ, a most interesting 
adrance is shown. For here, in addition to the above details, there gradually appear in the protoplasm, and often rather diffusely scattered through it, minute spots of nucleo-protein or chromatin substance, that give the characteristic chromatin reactions. These seem often to be isolated, but evidently in some cases, and probably in all cases, they are linked together by fine connecting viscous threads (p. 52). In the higher Blue-green Algæe specially (Oscillatoria, Lyngbya, Rivularia, etc.), these form a very definite skein or loose thread-coil on which chromatin granules are disposed. It becomes important then to know how these behave during division. While Hansgirg and Fischer have doubted the existence of, and formation of, phases in chromatin division of a rudimentary cell nucleus, Hegler, Wager, Olive, and Phillips have all traced division changes of a fairly definite nature. These consisted in aggregation and rearrangement of the previously diffuse chromatin masses, in a splitting of the constituent aggregation material into halves that retreated towards the poles of the cell, in the presence of connecting threads that temporarily united these, and in gradual rearrangement of the whole to form conditions typical of the resting cell. But careful and extensive study of the unicellular genera, and even renerred observation of different thread-forms such as the Rivularieæ, must be made before final conclusions can be reached.

The decided trend of eridence however is strongly in favor of the riew that a loose or open chromatin nucleus exists in the higher Cyanophycex; that this is deroid of a nuclear membrane, and has not yet aggregated centrally to form a nucleus; that, from behavior of the chromatin previous to division of each cell, it is becoming an initiative and directive substance that stimulates and guides the protoplasm during division; while from its staining reactions and behavior, in comparison with higher plants, it seems to be composed of like substance with the chromatin of the latter, and so in all probability is becoming the main hereditary center of each cell. But one important fact deserves to be emphasized. It is that in the simplest Acaryophyta, where no chromatin 
constituents have yet been described, hereditary peculiarities of chromotophore color, wall formation and thickness, glycogen formation, and other details, are all handed down by simple division.

Further, since these simple species have gradually varied, so as to give rise to new species that not only differ in structure but may inhabit different surroundings, it follows that chromatin mas not primitively, and need not be, the only bearer of heredity for protophytic types. So also chromatin formation and heredity were not ultimate and sudden developments of certain primitive cells, but have evidently resulted from slow modifications and condensations of energy, correlated with the building up by that energy-on the principle that we have called stereoenergics - of a greatly more complex colloid, the chromatin, that has become the highly sensitive or cognitic material of the cell.

But we may now pass to another and more complicated question that directly pertains to the present chapter. The studies of many observers have revealed that certain cells of several genera amongst Cyanophycece under definite seasonal conditions, such as changes in moisture, temperature, and light intensity, become more or less enlarged, their contents become granular, the wreathed chromatin increases in quantity, the wall becomes thick, often modified into several layers, and not infrequently is pigmented. These "perennating cells,", then, correspond in every respect to the so-called "akinetes" of higher algæ described later, except that they have no nucleus.

Again, in Hyella and other genera, some of the normal cells may increase in size, the contents become rounded off from the parent wall, rupture of the latter occurs, and the entire mass already enclosed in a new wall layer is set free. This exactly corresponds to "aplanospore" formation, as described below for the higher algæ.

Further, in the Chamrsiphonex and some Stigonemacex, at certain seasons or under definite conditions a few or most of the vegetative cells (p. 251) become enlarged, have their protoplasm divided and redivided, till four to many rounded masses have arisen inside each cell. These escape by rupture of the parent-wall, each secretes a new membrane around itself, and in time grows and divides to become a new individual. So far as known none of the cells thus set free is pro- 
vided in any species with motile processes or cilia. But though passive the entire process resembles, and is apparently the evolving forerunner of, swarmspore formation in higher algæ.

Our knowledge is still imperfect and fragmentary as to the exeiting causes that start formation of these different types of cell. But all indications decisively point to changing environal stimuli as the causes for starting varying energizing modifications - either increased or decreased in energy amountwhich by long continued action have slowly molded the organism.

We can now investigate another and more complicated question that directly pertains to the subject-matter of this chapter. Phillips (35:303) states that in an Oscillatoria studied by him, and which he repeatedly demonstrated to the writer, an apparent breaking down of adjacent cell walls and union of adjacent cell contents occurred, the diffused chromatin of each cell seeming to participate largely in the process. Phillips says regarding it: "Whether this fusion should or should not be looked upon as a form of sexuality is problematical, however, for the reason that spores are sometimes formed in Oscillaria, as in Cylindrospermum and other Cyanophyceæ, by the transformation of only one cell, but even here we find the same passing of chromatin into the single spore cell from the adjoining, or, as they might be termed, the nurse cells."

While we would not attach too great an importance to these results till they are carefully and repeatedly verified or disproved, they at least suggest that commencing sexuality is associated with forming or evolving chromatin substance, a position that is strongly suggested by all studies made on nucleate organisms.

So for the simple Cyanophyceæ it can be said that sexuality is absent or possibly rarely indicated, and that multiplication is effected by some mode of vegetative division of a cell or of a chain of cells. In the higher thread forms, where chromatin formation and aggregation can be traced, several modifications of asexual division are developed, that correspond to akinete, aplanospore, and endospore formation in higher algæ. These seem in all cases to be due to rather rapid and pronounced changes in environal conditions, that have gradually modified lines of energy distribution, and so have modified the protoplasmic substance. It is suggested that a possible primitive sexual fusion may occur in a few genera between adjacent cells, though as yet the question requires fuller verification. 
But the writer believes that extreme importance-attaches to the mode of division, followed by spore formation, in the Chamæsiphoneæ and allies as cited above. For this seems in every respect to represent the commencing phase of endospore formation, which plays so important a role in the higher or nucleate algæ, and is a usual preliminary to sex-cell formation.

It seems highly probable that, though the Chamæsiphoneæ is the group which mainly shows endospore formation now, members of the simple spherical genera may also have advanced to a like state. But either from the simpler and more roundcelled Chamæsiphoneæ, or from evolved types of the Chroococcaceæ, we would derive the typically unicellular but at times nucleate Pleurococcacece, Protococcacece, and Tetrasporacece. A study of sexual cell formation, and of evolving sex-cells in the higher or nucleate algæ, can therefore now be undertaken.

As amongst the simpler unicellular Cyanophyceæ, so in the above groups, direct division and rounding off of each cell may give rise to new individuals. Or again "akinetes" may result from thickening of the wall of a unicellular individual, or of a cell in the multicellular one, with occasional enlargement of it. The contents also seem often to undergo change or reformation, and then, or only after a rest period, do these germinate to form a new individual. In formation of "aplanospores" the contents of a cell contract and round off from the parent wall. The mass then secretes a new wall round itself, and in time is set free by dissolution of the parent membrane. This may undergo a longer or shorter rest period, and then germinates into a new plant.

The "palmelloid spore" results from division and redivision in successively different planes, at right angles to each other, until a number of spherical cells are formed. These may rest within the parent wall for a time, and then escape to produce a new plant, or they may instead give rise to the swarmspore soon to be studied.

All of the above types of new cell formation seen in pleurococcoid organisms are exactly shown, in some members of the Cyanophyceæ as already noted, and in the former as in the latter they have evidently resulted from periodic exposure of organisms to definite environal states, as subjoined evidence will indicate.

But another and highly important asexual spore form is that generally known as the endospore or swarmspore. Here the contents of a vegetative cell may have become rounded off, but more usually become divided and re-divided until four to many smaller ones have arisen inside the parent mem- 
brane. On rupture of the membrane, the several swarmspores are set free, each being provided with rarely one, usually two, at times four, seldom numerous, cilia. From their ciliary motility, these bodies have commonly been called zoospores, and the mother-cells in which they have been formed zoosporangia.

Such highly active and energized swarmspores may seem to differ very widely from any condition seen amongst the Blue-green Algæ. But morphologically connecting stages exist, we would consider. Thus amongst various of the Tetrasporaceæ the round or oval cells that are embedded in mucilage bear elongated plasmatic processes that project through the mucilage and into the surrounding medium. These have been called pseudocilia by Correns; they are apparently motionless or slowly vibratile, and seem to be mainly tactile in function. But end-cells in filaments of Oscillatoria described by Phillips, and which have been watched for hours by the writer, bear several slowly waving and rather blunt protoplasmic processes. So betreen the last and the active cilium the main difference may be that the cilium is connected with and largely energized by nuclear chromatin, as has definitely been shown for some algoid cells and for spermatozoid cells of the ferns and higher groups.

We would therefore claim that the morphological connection between blue-green and green algæ now living is a close one, when we compare certain groups of each. It is entirely appropriate then to consider that many types which would once have made the connection much more gradual and continuous have been obliterated during past geologic periods.

When we compare the behavior of the different kinds of spore described above, the at times rapid transformation of one into another is noteworthy. Thus in some genera of green algæ, such as Trentepohlia, Stigeoclonium, and others, the akinete cells may either grow into new individuals, or may form a palmelloid stage, or advance instead to formation of swarmspores. So from examples such as these, and others already indicated, we consider that the four modes of asexual spore formation above described, can all be traced to have evolved gradually from the Cyanophycex upward; and that, with completed evolution of the nuclear chromatin, originally passive non-ciliate (or possibly in forms now largely lost to us, 
but suggested in the sluggishly pseudo-ciliate Tetrasporaceæ) palmella spores became active ciliate swarmspores.

Unfortunately we do not as yet know the exact and minute nuclear or chromatin changes that go on in the above cells, and specially in swarmspores, when they fix down, or change into another and later stage. But again we would consider that these have all resulted from long-continued environal stimulatory action, that at one period of the year or season has differed much from the condition at another. Distinct evidence of this is got when the life history of many algæ is traced.

Thus in Ctenocladus of the Chætophoraceæ, that is largely intermediate in structure and aspect between the Chamæsiphoneæ and the higher algæ, autumnal akinetes with thick walls arise on the ordinary branches and these act as perennating winter spores. In late spring on lower branches of the plant, cell groups are formed that become summer akinetes.

Again in five genera of the same group (Chcetophora, Ctenocladus, Endoclonium, Stigeoclonium, and Trichopilus), that seem all to be transition types between the Cyanophyceæ and higher algæ, two sizes of swarmspores-large and small-arise at different times. Even in Trichopilus, these - as in the palmelloid spores of Choanophoraceæ-are destitute of cilia, though in the others two to four are formed. In Draparnaldia $(99,1$ : 232) two readily distinguished types of swarmspore arise, the so-called macro- and microzoospores. Both have four cilia, but the latter have a denser aspect, a more deeply placed eyespot, and can pass directly into the resting-cell stage. While the former can fix down and reproduce new plants directly, the microzoospores may become active and conjugate as sexcells according to Klebs.

Many other cases might be cited which demonstrate the frequent alternation or interrelation of akinetes, resting-spores and swarmspores, in the life history of single genera.

From evidence advanced by them, Chodat, Klebs, and other workers have considered that such varying structural states are due to varying environal factors. 
So it seems to the writer that the bulk of evidence is in favor of the position that the different types of asexual reproduction and spore-cell formation had already evolved amongst the Cyanophycer, and that as chromatin organization became perfected the swarmspores originated, that represent not merely the structural details and functional capabilities of a biotic energy, but the much more specialized phase of cognitic energy.

Now, as shedding a certain light on evolving sexuality, it is important to note that all of these asexually produced spore cells are capable of exactly reproducing the type or species of adult plant from which they have been derived. And this holds true, not only for the nucleate algæ that we are now considering, but equally for all of the non-nucleate or semi-nucleate blue-green genera. So every vegetative cell which becomes a potential spore-cell-and in many cases, as Radaisia and Hyella amongst Cyanophyceæ, or Stigeoclonium and Ctenocladus amongst green algæ, such make up the bulk of the plant at certain seasonshas the potentialities and energizing tendencies locked up in it which enable it to reproduce the adult form, and this in turn to reproduce the varied kinds of spore -akinete, aplanospore, or zoospore - characteristic of the species.

We shall now attempt to ascertain how sexual differentiation gradually arose amongst the lower nucleate algæ.

According to our present knowledge of their life histories, it can be said that many living genera amongst some of the simplest nucleate green algæ are invariably characterized by the formation of swarmspores, but these neither become sexual gametes to conjugate with each other, nor are sex-cells formed at any stage in the history of the genera. Thus the Pleurococcaceæ and Protococcaceæ that we would regard as most directly derived from unicellular Cyanophyceæ (p. 308) have hitherto failed to show sex-cells in the thirty or more genera that make up the groups, except in a few like Chlorochytrium and Endosphora. Many even of the Pleurococcaceæ fail to form swarmspores, and multiply only by such methods as are seen in the Cyanophycex. A considerable margin of doubt however must attach to these, till they have all been 
exhaustively studied by algologists. But, even granting such, we believe that the above pretty surely represents the state of the case.

If such be so, it can be said that differentiation of the complex chromatin substance, that is mainly centered within the nucleus, and which we have regarded as a delicately sensitive mass for storage and distribution of cognitic energy (p. 129), took place antecedently to the origin of sexuality. As will be pointed out below the same seems to be true for animals.

But it would be a mistake to suppose, as has often been done in recent years, that sexuality arose after formation of swarmspores, and in connection with their active movements. For, in the large but comparatively primitive and wholly freshwater group of the Desmideæ, the conjugating cells are ordinary vegetative cells, which at definite periods undergo a profound change in the chromatin, so as to start the conjugating process. The Conjugatæ generally and the Diatomaceæ also conform to this.

In nearly all the primitive unicellular and multicellular caryote algæ then, except those above named and others soon to be cited, the formation of swarmspores is a general phenomenon, though their production need not be followed by sexual differentiation in them. Each swarm spore may represent the entire rounded-off contents of a cell, as in Oedogonium, or the contents may divide again and again to produce several swarmspores. In nearly every case, it may be said that each stands in striking contrast to the cell that produced it, in being greatly more sensitive to external stimuli. This is shown first in the production of the cilia, which are oftenperhaps always - formed as prolongations of the chromatin or cognitic substance.

Further the cilia protrude from the clear and usually pointed end of the spore, the posterior rounded green end of which is evidently the more purely biotic or vegetative portion. The cilia and soft anterior end then are readily permeable by heat and light rays, by chemical agents, and by currents of electric or other energy, so that alike in character and in behavior it 
is highly energotactic. But each spore is, in higher examples, often provided with a red sensitive eye-spot, that is placed below or near the cilia, more rarely at the rounded end as in Leptosira, when the position indicates the rounded end as that for attraction and coming together of the swarm-cells, if these are to copulate as sex-cells.

As preliminary to the larger question of sex-cell origin, we may next inquire whether any pronounced cytological changes occur when vegetative or resting cells become changed into swarm cells or spores. The knowledge that would enable us to give a satisfactory reply is still extremely limited. But a few carefully described cases seem at least indicative. Thus Wille (100:62) in condensing Klebs's original account gives the following for Scotinosphera, one of the Protococcaceæ:

"In S. the swarmspores are formed from perennating (or winter) cells in May and June, as soon as they reach fresh water. The differentiation of the protoplasm appears then clearly, and the entire protoplasm is finely granular. The individual masses then separate gradually more and more from each other, while they become shrunken together, and between them appear small red areas. The masses condense increasingly under contraction, and a red coloring matter appears in ever greater quantity around. Finally a dark blue-green protoplasmic ball is formed which lies in the red granular mass. This now begins to split through successive cell-divisions, and the red granular mass is again absorbed. After 12-14 divisions the swarmspores ripen, and by swelling of the parent wall they become free."

What happens physico-chemically while these modifications proceed we cannot as yet say, though a profound rearrangement of the entire bio-cognitic substance is suggested.

Whether maturing sex-cells have been derived from ordinary vegetative and passive cells, as in desmids and higher Conjugatæ, or first developed as swarmspores, as is most widely true for groups of green algx, it invariably seems that some physico-chemical change so occurs in the maturing or matured gametes that they show marked attraction for each other. 
Not only so, it is true that, whether we deal with passive or with motile cells, these usually seek for and unite with some definite portion of each conjugating individual. This strongly suggests that powerful and very exact lines of energy act along the conjugating or gamete substance of each. Thus in swarmspores the pointed, ciliate, and at times "eye-spot" end is that where attraction-contact is first made. So, when the eye-spot or similar mass is lateral or sublateral, fusion in like position is made, while in Leptosira-already referred toattraction and union occur at the rounded posterior end, toward which the eye-spot is nearest. What relation such union bears to the position of the cell-nucleus has not yet been determined.

In the desmids a like exact line of attraction and union of the passive sex-cells is observed. But the multicellular conjugate genus Spirogyra is most informing. When the annual period for conjugation comes round-and this is often extremely exact according to environal conditions-some filaments become wholly giving filaments, others wholly receiving. Though numerous efforts have been made to establish a chemical difference between these, it is doubtful if any sure result has been reached; in fact we would interpret it in a different manner. But in such species as S. maxima and S. nitida, though not apparently in $S$. longata, striking nuclear interactions occur between the cells of sexually different threads. Thus the writer pointed out in 1884 (101) that, even in such filaments as have not yet conjugated, the commencing swellings of opposite cells are put out in a direct line with the nuclei of each, no matter whether these are exactly opposite, or at varying oblique positions to each other. Again, if a cell in one filament is slightly nearer to an opposite cell than to another in that filament, a stronger and feebler tube may be formed in the first; while, if it is exactly opposite the septum between two cells, it may form two equal but oblique tubes to meet one from each attracted cell opposite. Now all such tubes are developed in exact line with the cell nuclei, and are not mere haphazard growths of the wall under the molding influence of the protoplasm alone. Overton and others have since recorded like phenomena.

So here at least it can be said that the nuclear chromatin of the future conjugating cells is traversed by lines of force 
so far-reaching as to affect the protoplasms of each and cause definite growth of each cellulose tube, a considerable time before these tubes have even met.

The indicative evidence then at present for the simpler algæ is that either the nucleus or the eye-spot when present, or perhaps both, represents the specially attractive substance of the cell. But from the viewpoint of cell-structure already advocated (p. 130) this means that the chromatin is the special sex-related material.

If in a few sentences we pursue the same line of investigation further amongst plants and animals, and return later to its more detailed study, it is now accepted by most that as one isogamete cell becomes gradually smaller, through successive stages (as in Oedogonium, Sphcroplea, Volvox, Coleochocte, and Chara) up to the minute active spermatozoids of Bryophyta and Pteridophyta, decrease of the male or sperm body occurs, till it consists only of a small refractive mass mainly of nuclear chromatin. Even the cilia, that many have regarded as protoplasmic, seem from the researches of Ikeno, Shaw, Strasburger, and others, to be in large part at least chromatic in origin.

Again, as we similarly advance from the averagely small swarmspore to the egg of Bryophyta and Pteridophyta, a steady increase in relative bulk of nuclear material takes place, so that the nucleus assumes a striking aspect inside the egg. As in Spirogyra nitida so in each egg-cell a powerful attraction of the spermatozoid toward it is exerted, till fusion of both occurs. Yet that the sperm contributes nearly or quite as much molecular energy, and starts equal stereoenergetic flows of molecules as does the egg chromatin, is shown alike by the balanced embryo, and in time adult plant, that is produced. Moreover the writer's studies on plant hybrids (63) definitely demonstrate that every cell of a hybrid may show the balanced influence of both parents, though reduced each to about half the parental strength.

We accept it then as fully verified that lines of some kind of attractive energy are set up between the chromatin of com- 
plemental sex-cells, when these are ripe for conjugation. But in order to grasp the significance and connection of this it will now be necessary to review shortly the entire question of cell division as witnessed in nucleated plants.

In the preceding context we have developed the riew that the nucleated cell consists of two main hereditary factors, the protoplasm that is associated with biotic energy, and the chromatin that is associated with cognitic energy.

The chromatin is, in most cases, centered in the nucleolus, and from the latter threads radiate out into an area of the cell substance that forms a chromatin "sphere of influence," which we have commonly called the nucleus. These threads again fuse in part to form the nuclear membrane, while through this, and in part also from its surface, chromatin threads radiate out into the protoplasm, and in some cases at least-probably always in surface cells - end in many fine processes in the ectoplasm, though in tissue cells they seem to form intercellular connecting threads through pores in the common walls.

The above view, first reached by the writer in 1882, from study of dividing plant and animal cells, was again shortly set forth in his paper on hybrid structure in 1892 (63). In recent years the valuable and original studies of Hartog on "dual forces of the dividing cell" have shed confirmatory light on it, as the subjoined pages will bring out.

Of all ordinary tissue cells known to the writer none in his estimation approaches Spirogyra as yielding a vivid picture stage by stage of cell division, that, in the act, gives a key to other and more complicated types in the higher plants and animals. S. nitida and S. maxima are the most convenient, and the former is a widely distributed type, for study.

When cell-division commences the nucleolar substance first becomes the center of energizing changes that result in subdivision of its substance into many smaller masses or chromosomes, as set forth by Mitzkewitsch (102:85). Though too minute for detailed study hitherto, these bodies evidently correspond to the chromosomes of higher cells, and in breaking up into definite masses we doubtless have a halving or segregation of the constituent chromatin materials that are to confer definite characters on the daughter cells. When this has been 
effected the nucleolus becomes, as described by the writer (103: 207), the seat of two opposing forces or lines of energy, that cause separation of its substance into two daughter nucleoli. But that there is an intimate energizing and morphological connection between the nucleolus-even in its modification into chromosomes - and the peripheral protoplasm of the cell is demonstrated by the early appearance in the latter, and contemporaneously with the above nucleolar changes, of scattered microsomata that gradually come together to aid in formation of the future cell wall.

Meanwhile the daughter nucleoli retreat from each other, as if they had become the centers of opposing and radiating forces, which cause them as they retreat to drive apart the surrounding nuclear chromatin threads and associated nucleoplasm. The substance of the latter passes through clearly visible pores or areas in the nuclear membrane, and in time aggregates outside the nuclear membrane. Soon thereafter the chromatin of the membrane, that is in contact with these masses, begins to break down and fuses with them, at the same time that both masses seem to get driven apart by radial lines of repellent energy passing out from the daughter nucleoli. At length these last reach and embed themselves in the chromatin-nucleoplasmic masses, that then become astral radiant centers from which chromatin threads radiate out. The most distant sets of these represent the original radial chromatin threads of the cell, that pass from the nucleus to the pyrenoids of the peripheral protoplasm, but the inner and nearer to each other form at first threads in the nuclear spindle, that for a time connects the daughter nuclei.

A stage is thus reached when a balanced adjustment seems to be effected between this chromatin force or cognitic energy, and some neutralizing or counteracting force. Meanwhile a beautiful nuclear spindle, in part constructed of chromatinlinin threads, in part of protoplasmic substance, has resulted from the nuclear- and nucleolar-derived chromatin threads that radiated outwardly. By the activity of this spindle, and in part of the peripheral protoplasmic layer, microsomata aggregate which later aid in formation of the cross partition-wall.

In describing the above succession of events, the writer wrote (10.3: 207 ) that "the nucleolus is now the center of two opposing forces, acting along the long axis of the cell; in fact that from this point onward the nucleolus presides over, guides, and impels the movements of the nucleus," and again, refer- 
ring to division in relation to Chara and other plants, he said: "I regard the building up of cells to form a definite plant, or the parts of it, as the result of a force radiating from the cell center, stimulating to division; and either that the energy giving rise to this force is equal to producing only a certain amount of tissue, or that it is inhibited or resisted by some external force, which prevents it forming an excess of tissue."

No other interpretation seemed possible than that definite lines of energy were traversing, rearranging, and finally readjusting, the chromatin and protoplasmic substance of the cell. At that time however the existence of a cognitic energy peculiar to the chromatin substance, and of a biotic energy peculiar to the protoplasm, had not occurred to him.

But already the existence and possible relation of these energies had been worked out, when he became acquainted with the highly suggestive results of Gallardo and Hartog. The latter especially has pointed out that, alike in dividing cells as in electrically charged solids and "foam" mixtures, the behavior and appearances are only explicable if we suppose that "the cell centers are the seats of opposite charges," that the fibers of the spindle (and of asters when such are present) are differentiated in a "field of force" and transmit lines of force under action of a pair of "unlike poles." Not only has he succeeded in producing artificially bipolar figures by introduction of one or two terminals put to earth, tripolar and quadripolar spindles could be formed that exactly simulated those seen in various plant tissues.

Hartog further concludes, from comparison with magnetic models, that the chromosomes, the nuclear membrane, the spindle fibers, and the ectoplasmic zone must all be good conductors of, or of high permeability to, what he calls a metakinetic energy, that apparently corresponds to our cognitic: energy.

Fol in his study of animal eggs (104), so early as 1873 , also considered that radiant streams of energy, somewhat resembling electric or magnetic forces, could alone well explain the phenomena of division. 
But, while Spirogyra and some related simple conjugate algæe exhibit the above karyokinetic changes, most tissue cells show rather different division phases, that require consideration and comparison with Spirogyra. In these the chromatin substance, while still largely massed in the nucleolus as in Spirogyra, gradually becomes more and more richly distributed radially from the nucleolus so as to form a chromatin network within the nucleus, and so in this network, as much or more than in the nucleolus, the first division changes are observable. These changes consist in the chromatin of the network becoming increasingly prominent and massed. Since some of the threads are clearly continuous with the nucleolus or its membrane, and since the nucleolus becomes gradually more and more pale and emptied of its refractive contents as division proceeds, it seems that much of the thread skein or wreath material is derived from the nucleolus. After the aggregating chromatin wreath or so called "spireme-thread" has assumed a definite size, it becomes transversely divided into a number of chromosomes that in number vary for different, and at times even closely related, genera. From the studies of Strasburger, Flemming, and many more recent workers, we now recognize that each of these chromosomes is made up of definitely arranged granules or chromospheres, arranged along and embedded in the chromosome substance. These undergo division, some time before division lengthwise of each chromosome in which they are embedded, so that the latter becomes dotted lengthwise with pairs of minute chromospheres.

Soon after the chromosomes have been established, two centers of energy-flow usually appear within or immediately outside the nucleus. These start radiation figures as in Spirogyra and so the radiation centers were regarded as important and distinct morphological entities by some, that for a time were called centrosomes. They indicate areas of stereoenergizing activity round which the chromatin can gradually redistribute itself in formation of the daughter nuclei. But as yet we are ignorant as to the exact physico-chemical changes that start their formation. The chromosomes next aggregate more or less toward the nuclear center, as if attracted mutually by energizing currents, and they then collectively form what has been called the nuclear or equatorial plate.

Each chromosome next splits lengthwise, much as did the minute chromosomes of Spirogyra that made up in it the nucleolar substance. Then the daughter chromosomes seem to be driven apart till they reach a position where balance between them and the radiating threads, as well as the surrounding 
protoplasm, is reestablished. Here gradual redistribution of the chromatin material, along with nucleoplasmic substance, starts the formation of two daughter nuclei.

The earlier phases then in division of most cells, both plant and animal, that take place up to the point where splitting lengthwise of each chromosome occurs, and which has been designated the prophase and the metaphase, are scarcely if at all represented in Spirogyra, and constitute an added complexity.

It would be inappropriate here and now to follow many details of division that cytologists have discussed. But, in the light of what we already know of the process, cell division in nucleate plants represents a segregation and selective sorting out of molecular complexes of chromatin and protoplasm, under the action of cognitic energy, that here shows positive and negative polar effects, which exhibit themselves much as in electric fields of action. Why these effects should be regarded as due to discharge of cognitic and not of electric energy is discussed elsewhere (p. 130).

But, in relation to the disruptive discharge of cognitic energy from the chromatin substance, it may be that biotic energy, as traversing the protoplasm, exerts a certain inhibiting or restraining action, in relation to the centrifugal action of the cognitic energy.

Now in connection with cell division on the one hand, and sex-cell formation on the other, it should be observed that the more minutely the continuous life history of the simpler plants and animals is studied, the more exactly do we realize that the process of division proceeds, under normal environment, to a strictly limited extent. Even in highly complex plants and animals the same principle seems to hold, and is associated with the terms maturity, senility, decay, death. If we confine attention to the simpler organisms meanwhile, the species of Desmidex, of Zygnemex, of Oedogoniex, and of Coleochætex, all show very definite, even though varying, periods of cell division, and at equally definite but short periods sex-cell formation occurs.

As examples the writer need only refer to his own observations made on species of Spirogyra in the region round Phila- 
delphia. S. nitida divides its cells very regularly each night from late April till October or even November, and so forms about 230 divisions on the average. In late March and early April a few divisions occur, followed by conjugation, in the second to the third week of April. S. longata shows a similar rate of division, but conjugates a week to ten days earlier than the last. S. maxima after similar dividing activity conjugates in the first to the second week of September.

An enormously wide field of study is here opened in algoid phytophenology, and which has as yet been little cultivated. But not a few observations tend to show that division, and even more sex-cell formation, is largely dependent on environal conditions, just as is growth and fertilization amongst flowering plants. Thus Oltmanns states for Coleochcete pulvinata that formation of sex-cells takes place in the waters of low ground during July to August, while in mountain lakes it occurs during October to November.

If we may now quote an illustrative case from animals, it can be said that, though exact observations are still limited mainly to the Infusoria, it is true for some of these, and probably is for many, that, after a definite period of dividing activity, this is followed by differentiation of the cell contents into complemental gamete cells, with or without a previous rest period. Thus after 170 normal divisions in Paramocium (Joukowsky), after 175 in Stylonychia (Maupas), and after 935 in Oxytricha (Woodruff), a marked cessation of dividing activity takes place, followed soon after by death of the mass of organisms. In other words the supply of some kind of energy that behaved like a disruptive or expansive energizing discharge seems to become so reduced or enfeebled in amount as to fail further in stimulating to division. But Calkins and others since have shown that change in the diet, or supply of certain salts, or special stimulation by such a complex compound as extract of pancreas (105: 136) may reestablish what might be called a recharged state of the organism. Then division may be carried on to the 620th or even to the 742nd generation. 
In such cases it seems that a definite chemical agent can recoup the protoplasm, and through it the chromatin substance, in energy supply; that the chromatin or cognitic energy therefore becomes recharged in amount to a greater or less degree, according to the nature of the nutritive material. The phenomenon then is one, in which so much colloid substance holds a definite supply of some highly complex and perfect form of energy, as an intramolecular charge. This, in the cases above cited, and in many beside if we judge from known conditions of life, daily becomes so piled up in amount through supply of normal food constituents that it gradually rearranges and then drives asunder the inert but definitely linked molecules of chromatin, and in turn reacts on the protoplasm so as to rearrange and subdivide it into oppositely charged masses.

With such views on cell divisions before us we can now compare sexual differentiation and sex-cells in ascending stages of evolution.

Sexuality in the Algo-Immediately above the Blue-green Algx, and showing many affinities with them in the simpler genera, are several large groups of green algæ, that may be classed into two main series, each showing lines of advancing but diverging evolution. These can be distinguished as the Conjugatæ and the Zoogametæ. The former includes the desmids, the higher Conjugatæe and the diatoms, in all of which division, with or without subsequent separation of cells, is the only or most frequent means of vegetative multiplication, but in which, at very exact periods usually, the cell individuals or chains of cells cease to be vegetative, and conjugate union occurs between them.

The latter includes the Protococcacex, Pleurococcacex, Tetrasporaceæ, Ulothricaceæ, Chætophoracex and other groups (p. 308), in all of which division as well as other modes of cell multiplication may occur, but in which at very exact periods usually, and nearly always after formation of swarmspores or zoospores, the actively motile zoospores differentiate in time as gamete cells and conjugate.

Regarding these two groups, the tendency in recent years has been to ignore the former and to emphasize the latter 
as that which suggests and presents evolving sexuality. But both are equally graded, instructive, and fundamental, though they must early have diverged along two pathways. One of these - the Conjugatr - has ended in an organic cul-de-sac, while the other or Zoogametæ has led to the development of higher and more complex algæ, in turn also has given rise to the archegoniate plants. The latter in fact has become the dominant line, as generally accepted during the past quarter century.

We can only refer now to the thread-shaped Conjugatæ, in which varying degrees of cell affinity are shown in the conjugating cells. The cells throughout the greater part of the year resemble each other, and are purely vegetative. But at surprisingly definite periods the cells become denser and richer in reserve food. Then in Mougeotia either adjoining pieces of sister cells become cut off ( $M$. uleana) and the common partition between these breaks down with subsequent fusion of contents, or from adjacent cells of an individual thread two hump-like tubes are formed (M. mirabilis), which join and unite their contents into a fertilized conjugate cell.

But in Debarya and Staurospermum opposite cells of distinct filaments put out tubes which meet, the contents of each cell seem to be equally attracted to the other, and so, after absorption of the bridge partition, fusion of the contents occurs in the middle of the bridge. But in some species of Spirogyra and Zygnema the tubes that meet from opposite cells are rather irregularly directed, though the contents of one cell, or possibly its nucleus, so react on the other that the latter mass flows over to fuse with that opposite. So here we can now say that one cell is a giving or male, and the other is a receiving or female cell.

But, in the more modified species of Spirogyra already referred to (p. 257), distinct lines of stereoenergizing action are manifested between the opposite or sub-opposite cell nuclei, so that the molecules of each wall are molded into shape, and so placed that they form a tube, each of which exactly grows toward and joins its neighbor, with the result that the conjoint tube thus formed is in exact line with the cell nuclei. 'This action and reaction relation may be due to the giving off from each of a definite chemical substance, that starts chemotactic action and reaction; or to definite interaction of opposite kinds of energy, much as we might regard a + 
and - cognitic energy discharge to set up. But of this more anon. Frequently and possibly always, the above conjugation changes are ushered in by repeated divisions, in rather rapid succession, of the cells that make up the entire filament. Such is probably associated with "reduction division" changes that are referred to later.*

Turning next to the Zoogametæ, the component gamete cells are in nearly all cases exactly like the asexual swarmspores. In some cases even (Uronema, Ctenocladus, etc.) swarmspores are set free, and after a motile existence fix down, without showing any tendency to conjugate. In other cases (Trentepohlia, Leptosira) the same vegetative cells of the adult plant give rise to ciliate cells, some of which live through a motile stage and then fix down to form new adult plants, while others conjugate in pairs to form a zygospore from which new growths arise. Again, and very usually, all of the swarmspores, on being set free from vegetative cells, may live a motile existence and then unite in pairs to form zygospores.

The above and many similar facts strongly indicate that, in the simpler green algæ, ordinary cells of the vegetative thallus may become modified, may separate from it, and forming those types of asexual spore that have been distinguished as akinetes, aplanospores, palmellospores, or swarmspores may disseminate, and give origin to like individuals as the parent species. But by the most gradual stages of transition-advance they may become either passive conjugate cells (Conjugatæ), or motile conjugate cells (Zoosporeæ), which show increasing and increasingly exact stereoenergized attraction for each other by nuclear action and reaction. Such zygospores then differ fundamentally from the asexual spore cells, in that they combine the blended peculiarities of two distinct plants. But as the mature plants, produced by these zygospores, subsequently teach us, and as many facts from the history of higher plants amply verify, the blended parental characters are reduced by about half.

Another peculiarity of each zygospore cell is that, previous to conjugation, the future conjugate cells display definite

\footnotetext{
* Recent studies on Spirogyra and other of the simpler algae seem fully to confirm the above.
} 
lines of stereoenergizing attraction, which may cause cellulose walls to be molded in shape, or may cause ferments to be exuded that break down the walls at definite areas, or cause lines of morement in the entire cell-masses, so that these are equally attracted to each other (Desmidece, Staurospermum); or one of the cell-masses so preponderates in its stereoenergesis that it draws the other mass to it.

But a third and very important peculiarity hinted at above, and almost of necessity bound up with the first peculiarity, is much better known to us as yet amongst higher plants and most animals. This is the evident reduction, disappearance, or absorption, in each conjugating cell, of approximately half its hereditary characters, and this at some period anterior to the fusion of the two cells. For in no other manner can we explain the retention of parental characters typical of both parents, and yet the development of a new plant that is not double the size or vigor or structural detail compared with either parent, but that is a fair average between both. Such reduction has been widely and satisfactorily demonstrated, from some of the higher algæ up to the higher flowering plants. The strong probability then is that it will yet be demonstrated to occur in the above simple algæ.

Reference has already been made (p. 264) to the effect of environal agents in promoting continued cell division. But certain agents seem at times to promote, at times to inhibit sex differentiation. Oltmanns has admirably synopsized (99, 2: 249-262) the experimental results already obtained. He shows that changes in temperature, in light intensity, in chemical nature of the environal liquid, in stillness or motion of the water, may so influence many algæ as to quickly promote or completely retard the formation of sex-cells.

Another indicative suggestion that many of these plants are still in a state of wavering and evolving sex differentiation is yielded by the numerous recorded examples of parthenogenesis, as well as by the ease with which gamete cells may be modified by enrironal agents, so as to become parthenogenetic in their future history. Thus Oltmanns (99, 2:255) says: 
"Among all of these the gametes can sometimes conjugate, sometimes develop individually, according to the surroundings and relations; sexuality is found still undetermined as it were in unstable equilibrium. That can be understood, since most of the above-named algæ stand, as isogamete forms, at a still relatively low stage. In higher groups of the algoid kingdom where oogamy dominates, sexuality has become a quite stable arrangement."

Again Klebs's observations on Protosiphon (99, 2: 256) show that, if the temperature of the surrounding water be raised from $20^{\circ} \mathrm{C}$. - when abundant conjugation occurs-to $27^{\circ} \mathrm{C}$, gamete cells that are about to conjugate fail to do so, but instead form a wall around themselves, and in time develop into new plants. The same result is got in weak Knop's solution. And when the ripe gametes of Chlamydomonas are darkened they develop parthenogenetically. These and many similar facts suggest that slight changes in energizing stimuli, either thermic, chemic, or lumic, may faror or prevent sexcell maturation, but yet that the gametes retain the power to grow into new individuals.

Even in the same genus varying differences may be observed. Thus, in Spirogyra varians (99, 1:70-71) and others, commencing conjugate cells may, if placed in running water or salt solutions, become parthenospores that derelop new plants. On the other hand, in S. nitida, S. maxima, etc., when cells fail to conjugate in filaments where most of the cells do so, they soon become discolored and disintegrate.

So just as cell-division is a seasonal activity that depends on the cooperation of environal factors, and seems to be due to steady discharges or liberations from the center of the nucleus or the nucleolus of cognitic energy, that drive apart and rearrange the chromatin substance of each cell, in like manner conjugation seems to be due to the establishment of unlike or differently charged amounts of cognitic energy, and the resulting attraction of the differently charged gamete cells when a definite specific climax in such differently charged states is reached. 
Even if we accept it, as has generally been done in the past, since Pfeffer published his studies on the fertilization of fern eggs, that union of the gametes results from chemotactic attraction between substances given off from the egg and certain substances in the sperm, this seems only to be an accessory and minor step in the much more fundamental union of the entire gamete cells with each other, and is accomplished to ensure that at least one gamete shall be brought within more exact attraction-range of the other. Lillie's recently published studies on animal eggs not only confirm, but seem greatly to extend such a view (107:524).

But it may well be objected here, that gamete cells amongst the simpler algæ are, as a rule, indistinguishable from each other, and up to the point of union have been exposed to like environal states. This seems to be entirely true, but we shall shortly mention a possible phase of the case, to which all of the preceding studies have led up, and which will be further discussed after study of animal sex-cells.

It is that, in the gradual upbuilding phylogenetically of the highly complex substance chromatin, such is only able, under normal environal states, to grow, to divide, and to produce new individuals up to a definite limit. During this period of activity, the stored intra-molecular cognitic energy, that has built up and energized the chromatin molecules, becomes so expended and reduced in force that a stage is reached when further division and growth become enfeebled or even cease, unless some antithetic or complemental stores of cognitic energy become accumulated in distinct groups of cells. Now immediately preparatory to conjugation, in the simpler as in the higher plants and animals, two noticeable changes nearly always occur. First, the cell-protoplasm, under the activity of biotic energy, accumulates abundant supplies of reserve food in or near the future sex-cells; second, the change that has already been referred to as "reduction division" occurs in each sex-cell, or in some cell preliminary to its formation.

The former act, or storing of reserve food substances, need not now concern us further, except that it indicates that thus 
reserves of available energy are conveniently placed for the future sex-cells so as still later to nourish the succeeding embryo plant. "Reduction division" however seems biologically to be of prime importance in all interpretations of sex-history, and so will receive detailed attention later.

Sexuality in the Protozoa-We can pass next to studies in cell-history and sexuality amongst the Protozoa. The entire group, as now living, may conveniently be divided for our present purposes into three series, that seem to show advancing complexity in their cytological history. The first is made up of the Rhizopoda, the fresh-water Foraminifera, the Heliozoa, and the Sporozoa. The second consists of the marine Foraminifera, the Radiolaria, and the Hæmoflagellata; the third of the Infusoria.

It seems increasingly problematical whether such a morphological group as that of the Proteomyxa exists, of simpler constitution than the higher Rhizopoda or Lobosa. But in such genera as Leptophrys, Gymnophrys, and Protamceba no nucleus has been detected. In these and in most genera of the Proteomyxa also, no conjugation act has been observed. Here then, as amongst the Protophyta, the absence of a nucleus and of sexual differentiation of cells seem to be associated features. But the cell protoplasm is evidently so capable of rejuvenescence in its molecular substance that it grows, divides, and multiplies endogenously, as do many bacteria and Cyanophyceæ. The variety of species and genera also that have evolved in the past is again suggestion that nonnucleate or semi-nucleate animals are capable of variation, of hereditarily transmitting acquired variation characters, and of fixing these to a greater or less degree, and for a longer or shorter period, so as to constitute recognizable specific or generic characters.

The genera and species of the Lobosa vary in an interesting manner, alike in the structure and distribution of the protoplasm or biotic substance, and of the chromatin or cognitic substance. Thus in some cases the former may be soft, fluid, and loosely alveolar in structure, while the latter is a spherical 
alveolar sac, with chromatin masses irregularly scattered or massed toward the interior of the nuclear membrane (Pelomyxa, Lithamoba). Or the protoplasmic substance may be interpenetrated by a fine chromatin network that we would regard as homologous with the kinoplasm of the plant cell, and as radiating from the nuclear membrane, while the nucleus shows a more or less granular chromatin texture (Diffugia). Again, as in the plant cell with its plastids, some of these chromidial masses may be specially set aside for nutritive purposes, when they have been called trophochromidia, or they may represent detached parts of the nuclei that are specially concerned in irritable and reproductive action, when they have been called idiochromidia (Centropyxis). Or again a paranuclear mass of chromatin lies near or beside the nuclear membrane, and seems to take active part in conjugation as in Paramoba. Finally a distinct nucleolus or chromatin center to the cell may be formed as in Arcella.

In the fresh-water Foraminifera or Gromiadæ there is usually a well-defined nucleus, and this is also true of the Sporozoa, where the nucleus with a distinct nuclear membrane and enclosed nucleolus or nucleoli are evident.

From the above it will be seen that the amount and degree of differentiation of the chromatin varies from a condition where it exists as scattered chromidial granules in the protoplasm, through stages of evolution to where a complex chromatin network traverses the protoplasm, passes inward to the nuclear membrane, is continued inward as a nuclear network, and finally becomes centered in the nucleolus or nucleoli.

The second and more complex group shows even greater and more varied chromatin relations than the first. The striking difference between megalospheric marine Foraminifera with their single large nucleus, and microspheric forms with many small nuclei, is still so little understood in its exact biological significance that it would be superfluous to treat of such here. Amongst the Radiolaria there is a great diversity of detail. But in not a few, such as the Acantharia and Discoidea, fine threads from the nucleus radiate outward through 
the protoplasm to the periphery, while the nuclei are bounded by a definite nuclear membrane, and show within either scattered chromidia or an evident nucleolus, at times of elongate shape as in Tuscarora.

In the Hæmoflagellata, according to Schaudinn and successors, as development of a maturing trypanosome takes place, the parent nucleus divides (p. 223) into a larger half or trophonucleus that presides over the general regetative functions, and a smaller half that is largely hereditary and irrito-reproductive. This again divides into a kinetonucleus that directs movement, and an elongated portion that spreads into the flagellum and the membrane. During division each of these contributes daughter nuclei to the new cells. As will be noted below the trophonucleus and kinetonucleus fuse, previous to formation of gamete cells.

The third group includes only the Infusoria, but these, in the relation and distribution of the protoplasm and chromatin materials, are as suggestive as they are complex. The apparently homogeneous structure of the ectoplasm in some cases, or division of it into as many as three layers in other cases, the diverse functional nature of the latter, the extreme mobility and yet correlated action (42:44) of the cilia, the detectable existence at times of a radiating endoplasmic reticulum (Dendrocometes, etc.), the division of the chromatin into a micronuclear and meganuclear constituent, as well as the size and occasional branched nature of the latter in some genera (e. g., Stentor, Ephelota, Opalinopsis), all indicate that this group is not only the most specialized in protoplasmic and chromatin substance amongst Protozoa, but that though unicellular not a few of its members deserve to rank high alongside many Metazoa, in morphological differentiation for physiological work.

With such details of protoplasmic and chromatin or nuclear differentiation to hand, it may further be said that, as amongst the simpler Algæ, so also throughout the Protozoa, asexual multiplication by division, by budding, and by free cell for-mation or endogenous multiplication occur, while the redis- 
tribution of chromatin during these processes also indicates that repulsive lines of energy-flow traverse the chromatin substance. As amongst these algæ, free cell formation, with subsequent production of amoboid or ciliate swarmspores, is a frequent occurrence. Here also it has been demonstrated, in at least some instances, that such formation is due to environal changes either in food supply, in composition of the surrounding water, in exposure to dessication conditions, or in relation to some host. Further, as among the simpler algæ, such free cell-formation may only result in the setting free of numerous spore-cells, each capable of developing into a new individual. Amongst some Radiolaria even, as amongst algæ already cited, one crop of swarmspores-the so-called isospores-may directly give rise to new individuals, while another-the anisospores-require to conjugate for formation of new individuals.

As to the origin and occurrence of sexuality amongst the Protozoa, this question seems to be largely bound up with the possible origin of the entire group from some more primitive ancestral types. This involves again the question of the possible monophyletic or polyphyletic descent of the group. If, as advocated in a succeeding chapter (XII), we view some of the autotrophic or the saprophytic bacteria as ancestral forms, these are still regarded as having no definite nucleus and no sexual differentiation. It is by no means unlikely therefore that organisms which have been classed in the division Proteomyxa are alike anucleate and asexual, or show only evolving nuclear and sex differentiation. Thus in Gloidium, Protamoeba, etc., careful study has hitherto failed to reveal nuclei, in Tetramyxa only scattered chromidia occur, in Eudyonema the organism is devoid of "nuclear apparatus" during the regetative phase, but the aggregation of chromatin into a nucleus takes place when reproduction is about to occur, while in Pseudospora and Nuclearia there is a definite nucleus.

But, given the formation of a nucleus, sex-differentiation seems then gradually but invariably to appear. As already pointed out also (p. 264) there are definite proofs amongst 
at least Infusoria, as amongst Algæ, that after a certain period of dividing activity this is followed by differentiation of the cell-contents into complemental gamete cells, with or without previous encystment. For, when such a stage is not reached, senile decay sets in, unless indeed some new and different stimulant form of energy incites to continued division.

We can now examine phenomena of sexuality in the Protozoa. Amongst the Rhizopoda-Lobosa enough is known to indicate that, after a period of division-activity, a stage is reached when the nuclear chromatin undergoes reductiondivision by the extrusion of a certain amount of its substance, that then undergoes disintegration. During this process, cells that are nearly related to each other in origin (Endamoba), or that may belong to distinct broods, become gamete cells. These show such energizing reactions that pairs come together, fuse, and produce a zygospore from which a new individual develops.

In Centropyxis and Trichosphorium transitions from the above simple conditions to the complex details seen in Foraminifera are presented. For, during reduction-division in the former, two sizes of shell arise, the individuals in the smaller of which probably decay, while those of the larger continue to increase, and eventually produce large conjugating cells or megagametes, and also-by subdivision-four smaller cells or microgametes. One of the latter conjugates with the former to produce a new and fully rejuvenated individual.

In Trichosphorium one generation arises that seems to be mainly nutritive in function, and which forms spicules in the gelatinous outer coat. It shows numerous nuclei in its protoplasm, and multiplies by division. This stage in time gives place to another that is devoid of spicules, which after repeated divisions encysts, produces flagellate gametes, and in order to fertilization a gamete from one cyst must unite with a gamete from another cyst, so as to form a zygospore that starts again the first or trophic generation.

Amongst Foraminifera two types of shell are-at least in some genera-produced, the megalospheric and the micro- 
spheric. The latter seems to correspond to the trophic stage in Trichospharium while the former gives rise to broods of swarm-cells which escape, conjugate with each other, and develop a microspheric generation. The last may produce in turn either a megalospheric or microspheric crop of new forms.

In the Heliozoa conjugation occurs after encystment, and in such cases as Actinophrys the resemblance to what happens amongst desmids is considerable. Exact details are lacking in most genera as to the minor changes.

In the Radiolaria the formation of ciliated isospores and heterospores, as also of ciliated microspores and megaspores, has already been noted, but, though conjugation may occur between some of these, such has not yet been observed.

From the standpoint of sexuality the Sporozoa and Hæmoflagellata are semi-parasitic or parasitic groups, each of which has either had a polyphyletic ancestry as proposed by Woodward $(108,1: 246)$ for the latter, or if each group is monophyletic it has undergone varied and advancing modification in the sex-cells, that have given rise to an instructive series. It should however be borne in mind that our knowledge of both groups is still very limited and imperfect, in spite of the great advances made in the past quarter century.

The group Sporozoa presents the most varied advance, from simple conjugation of like gametes, to the union of sperm and egg constituents. In the subdivision of the Gregarinida, the genus Monocystis has been minutely observed. Here two adult individuals surround themselves by a common cyst. 'The nucleus of each becomes resolved into chromatin or chromidial portions, that seem at least in part to disintegrate. The remainder reforms as a new nucleus, that then divides into numerous small nuclei. These attract the surrounding protoplasm of each individual, so that it becomes divided up into many oval or irregular bodies, which surround a central residual mass. These bodies become isogametes, and through breaking down of the parent cyst-wall they mingle and conjugate. Whether two individuals, derived from the 
same adult, can fuse to constitute close fertilization, or whether individuals from distinct adults only fuse, is still undetermined. The conjugate mass then starts a spore-forming generation.

In the genus Stylorhynchus two adults also encyst, but one of them becomes divided up into male gamete cells, each of which forms a flagellate tail and pointed head. The other divides up into smaller cells that remain passive. Then the larger motile male gamete cells penetrate the adjoining cavity that contains the smaller gametes, and union in pairs takes place.

In Pterocephalus one of the two adults in a cyst breaks up into numerous small attenuate chromatin bodies or spermatozoids, each bearing a posterior flagellum. The other breaks up into large eggs with abundant stored food. The latter, remaining passive, are fertilized by the motile male cells.

The subdivision Coccideæ includes Coccidium in which the sexual stage has been fully traced. Here the adult cells, at an early period in sex differentiation, become recognizable as male and female cysts, since the former become filled with finely granular, the latter with coarsely granular, materials. The chromatin substance of the nucleus in the male cyst, derived mainly from the nucleolus, flows out along radiating threads into the peripheral part of the protoplasm, and forms small chromatin granules that unite, elongate, and along with the protoplasm form each an anterior and posterior flagellum. The spermatozoids or microgametes thus formed then escape. The probability further is-judging from like cases-that chromatin material is excreted before the above granules unite to form the male chromatin masses. The future female cysts produce each one egg, and during the process the nucleolar chromatin is largely or wholly extruded. The enucleolated nucleus then matures, shows chromatin granules in its substance, and at length travels to the surface of the now ripe egg. 'Here a 'cone of reception' is formed as a little prominence of clear hyaline protoplasm, from which a thin streak of similar substance extends as far as the nucleus. I microgamete touches the cone and adheres to it 'The cone of recep- 
tion is at once drawn in, and partly in this way, partly by its own movements, the microgamete penetrates the macrogamete, and its pronucleus reaches the female pronucleus.

"No sooner is the entry of the microgamete completed than a clear membrane, gradually increasing in distinctness, appears over the whole surface of the zygote, excluding the less fortunate microgametes, which die off and break up. The clear membrane very soon becomes an exceedingly tough protective envelope, the oocyst" (109: 219).

Highly interesting for our present purpose is the following, "Schaudinn has observed some curious points of considerable interest in this process. Before the macrogamete has expelled the fragments of its karyosome [nucleolus mihi] from the cytoplasm, it is not attractive to the microgametes, but no sooner has it done so than they are drawn to it as by a magnet. The attraction, which is evidently exerted by the substance of the karyosome [nucleolus mihi] itself, acts very suddenly, and reaches from $48 \mu$ to $130 \mu$. If exerted near to developing microgametes, it stimulates them to great activity; even still imperfect microgametes then develop flagella, and in their struggles to free themselves from the microgametocyte they carry away lumps of protoplasm with them. The substance of the karyosome [nucleolus] seems to be absorbed by the microgametes that swarm to it, and the remarkable fact was observed that the attractive power of the macrogamete was limited as regards the number of microgametes drawn to it, the usual number being about twelve or fourteen. When this number was made up, fresh microgametes approaching the macrogamete were no longer attracted. After the substance of the karyosome has dissolved up, a fresh attraction seems to be exerted by the female pronucleus, which travels to the surface.

"The fusion of the two pronuclei in the zygote takes place in a very remarkable manner. The female pronucleus passes back to the center of the zygote and becomes drawn out in the form of an elongated spindle, on which the chromatin granules are arranged in parallel rows running in a meridional 
direction. The male pronucleus, at first huddled up at one pole of this nuclear spindle, also breaks up into granules of chromatin, which mingle with those of the female pronucleus, and cause the spindle to increase in size until it finally stretches through the entire oocyst. One pole of the spindle marks the point of entry of the microgamete, where its disappointed companions are still to be seen outside the oocyst. The peculiar spindle-like arrangement of the nucleus in the zygote has nothing to do with nuclear division, but is simply a means of mixing intimately the chromatin derived from two different sources, and when this is effected the spindle contracts and rounds itself off, the final result being a spherical nucleus, the fusion-product of the two pronuclei."

The details for the Hæmosporidia in their main outlines resemble the last.

The large series of the Myxosporidia seems to be a group of organisms which, by parasitic degradation, has lost the sexual phase during their life-cycle, or this is reduced to simple conjugation between small and similar gametes.

In the important group of Hæmoflagellata, mature trypanosomes become transformed into an egg in one case and a cluster usually of eight sperms in another. In both instances, a certain amount of chromatin material undergoes disintegration, and is absorbed or extruded. The remainder, during differentiation of the egg-cell, becomes a large female nucleus that, after fertilization, produces the trophonuclear and kinetonuclear constituents of the adult. The remaining chromatin substance of the male cyst produces eight double nuclei, made up each of a tropho- and kinetonuclear constituent. Round these two constituents a motile sperm mass forms, and, on escape, one fertilizes a large egg.

The sexual condition met with in Infusoria is in the nature of a conjugation of similar individual cells, and is periodic in its occurrence. The apparently invariable presence of a meganucleus and a micronucleus, the active part which the former seems to play in the vegetative and even sensory activity of the adult, the active part which the latter plays in con- 
jugation, and the origin of the meganucleus from the latter, all suggest that here, as amongst the Hrmoflagellata, the chromatin or cognitic substance early separates after fertilization into a trophonuclear and a kinetonuclear portion.

As to details of the conjugation process Calkins (105: 156) says for Paramœcium aurelia: "At the outset the two organisms are more loosely attached, so that forceful ejection from a pipette is sufficient to separate them. After twelve hours union, however, the attachment is so firm that no amount of force will break them apart without killing one or both. The normal course of conjugation requires from eighteen to thirty hours, according to the temperature, and during the process the micronucleus of each cell divides twice; one of the four micronuclei then divides again into dimorphic nuclei. One of these nuclei is smaller than the other and acts as a spermatic or wandering nucleus, while the other remains in the parent cell. Each cell receives a wandering smaller micronucleus from the other organism; this fuses with the larger micronucleus to form the fertilization nucleus of the new individuals. Each fertilization nucleus then divides three times in quick succession, and eight micronuclei are formed. Four of these begin to swell and to metamorphose into four new macronuclei, while four remain as micronuclei. In the meantime the two conjugating cells separate, soon after the interchange of micronuclei, and the processes of reorganization are carried out independently. The old macronucleus begins to disintegrate by first forming a skein-like structure, and then breaking down into granules which are finally absorbed by the cell protoplasm. The process of reorganization requires from one to three days before the first division of the fertilized cell, which, as we have seen, contains four micronuclei and four macronuclei. Each daughter cell, after the first division, contains two macronuclei and two micronuclei, and the normal nuclear relations are not reestablished until after the second division, when the resultant four cells have each one macronucleus and one micronucleus."

Amongst the Infusoria the number of divisions previous to reduction in and extrusion of chromatin substance has 
in not a few cases been accurately observed. This, so far as number of extruded and retained nuclei may form a criterion, gives rise to seven degenerating and one persistent nucleus in Paramocium aurelia (82, 1:390), to five degenerate and one persistent in Dendrocometes, to three degenerate and one persistent in female Vorticella, to seven degenerate and one persistent in male Vorticella, and to about seventy degenerate and one persistent in Bursaria sp.

Reviewing the above, and related evidence that it would be impossible to treat in detail here, it may be said for the Protozoa, as for the main lines of Algæ, that multiplication may be effected by regular division, by budding, by rejuvenescence, or by encystation with subsequent free cell formation. In all of these cases the nucleo-nucleolar chromatin undergoes division into definite chromosomes that are distributed to each cell, and evidently bestow on it definite hereditary qualities.

Each division of a cell is apparently inaugurated by the gradual accumulation of a store of bio-cognitic energy of like sign or quality in the nuclear and nucleolar chromatin, up to the stage when steady disruptive discharge occurs, and in the process a redistribution of the plasmatic-chromatin material occurs. So long as the environal conditions remain the same, this process may be repeated up to a definite limit.

Under normal conditions it has been shown for various Infusoria and is probably true for many Protozoa, as it is for most Algæ, that after a limited number of divisions a senile state is established, unless by change of environal conditions some new stimulus incites to continued division, or unless sexual fusion of individuals, that had previously become sexually differentiated, occurs.

In the gradual evolution of sexuality moreover, this seems always to be associated with chromatin formation, and is absent or rudimentary in the simple bacteria and Blue-green Algæ, possibly also in the simplest animals.

Subsequent to formation of chromatin material and resulting sex differentiation, and after a definite period of active 
multiplication, regetative cells may directly become sex cells by extrusion of certain chromatin constituents, and so of definite amounts of some kind of energy. Such regetative cells, in becoming thus changed, may remain unaltered in size and in general aspect, but undergo some fundamental change in their store of chromatin and so of cognitic energy. These are then termed isogametes.

Such sexually different isogametes, when set free or brought within measurable distance of each other, usually show powerful and exact attraction for each other and specially for the chromatin substance, in a manner that can alone be explained as due to their being charged by opposite or complemental amounts of energy that we would regard as cognitic.

From such isogametes graded stages of evolving modification can be traced in the size of each complemental sex cell, till small forms are produced as sperm cells that consist almost wholly of chromatin, and large forms are produced as eggs that consist of a plasmatic and chromatin nucleus, also of abundant protoplasm. These two are termed heterogametes. Fusion of the relatively small chromatin sperm-mass with the nucleus of the relatively large egg results in fertilization and formation of a morphologically balanced individual, that combines half of the characters of both parents. The extruded material of each sex cell represents the remaining and superfluous half of the hereditary chromatin material.

But till the Hæmoflagellata and the Infusoria are reached there is no indication of cytological differentiation into substances, or separate chromatin and cell constituents, that might in any sense be called somatoplasmic and germoplasmic masses. In both of the above groups, however, there is strong suggestion of a somatochromatin and a germochromatin differentiation. Such is noteworthy from the standpoint of heredity.

The phenomena of sexuality amongst the nucleate Algæ and the Protozoa, from their simplest beginnings, run closely parallel, and suggest that the two groups of plants and animals early diverged from a primitive and probably colorless holo- 
biotic protoplasmic and specially bacterial ancestry. The line of green plant advance seems to have resulted from formation in these bacterial forms of autotrophic pigments, and later the evolution of chromatin, that started sex differentiation. The line of animal advance probably resulted from autotrophic bacterial organisms through formation of a saprophytic, a parasitic, or an epibiotic dependence on plants or other animals, and simultaneously or somewhat later through the evolution of chromatin as in plants, that gave rise to sex differentiation.

In the evolution of the above described stages of conjugation in Algæ as in Protozoa, it should be emphasized that the simplest types are made up of cell-gametes that in size, shape, and behavior to each other are apparently exactly alike. Both equally attract, and are equally attracted, so that it is impossible to speak of either cell as male or female gamete cell. From the standpoint of stereoenergetics therefore, it seems to be constantly suggested that equal amounts of cognitic energy of opposite sign or quality energize both, and so cause balanced union of isogamete cells.

So the highly important distinction has to be drawn, that sexuality as compared with maleness or femaleness are distinct even though usually correlated conditions. For, while any two complemental cells that fuse to form an organic unit are sex cells, it only is when one of these becomes recognizably different and behaves differently that we can speak of them as male and female cells. From the one condition to the other however, the most gradual advances are shown.

But in Zygnema, Spirogyra, and others that are passive, and which have conjugate cells exactly like each other, as well as in Algæ and Protozoa that form sperm and egg cells, the receiring cell is evidently so differently energized from the giving cell that it draws the contents of the latter toward it. In the case of motile gamete cells also, when even a slight difference is established in size, one becomes the attracting, the other the attracted, cell. The manner also in which, after fusion of the conjugating cells in Spirogyra, and in some Protozoa, rapid energizing changes spread through the conjugated sub- 
stance, indicates that porrerful and rapid currents of energy are set up, and that profound stereochemical changes are established immediately after fusion of the conjugate cells, and which render superfluous the need of more than the two cells.

Sexuality in the Fungi. Reproduction and sex history in the fungi-diversified in the extreme-can only shortly be considered here and now. Asexual multiplication by cell division, also the formation-as amongst Algæ and Protozoa -of akinetes, aplanospores, and swarmspores, reaches its climax of diversity in this group. The mode of formation and the structure of sex-cells closely parallel conditions already described for Algæ and the Protozoa.

Thus in Zygochytrium and Polyphagus of the lowly organized Chytridineæ, similar or nearly similar tubes are produced, which accurately grow toward each other, fuse, and then unite their contents as in Mesocarpus amongst Algæ. Again conjugation between like non-motile tubes in such conjugate fungi as Piptocephalus, Mucor, or Sporodinia resemble conditions seen amongst Algæ and Protozoa.

But amongst the Chytridinex the genus Reesia shows conjugation of similar motile gamete cells. In this case also, as amongst Algæ, the motile gametes are swarmspores, which in other and related genera give rise directly to asexual growths. But here instead they first undergo fusion, and thereafter become asexual resting spores, from which in time a new crop of swarmspores may arise.

In Zopf's genus Lagenidium studies by that observer indicate that conjugation may take place between non-motile cells of a thallus, which are neighbor cells of that thallus, or that opposite cells of two distinct thalli may unite. The sexual process also seems to occur only during June, while division and asexual spore formation go on during the other favorable months of the year.

Numerous graded transitions can be traced also from such isoconjugate tube-unions to other groups of the fungi, such as the Saprolegniex, where, in its simplest expression, a smaller 
and often adjacent tube grows out as an antheridium, and fuses with a larger spherical swelling, the oogonium. The numerous spermatozoids of the former are then discharged into the four to eight eggs formed inside the latter. But the careful studies and experiments of the past quarter century have demonstrated that not a few of the Saprolegnieæ which evidently had such sexual differentiation have gradually lost it, so that parthenogenetic development of the egg cells occurs.

In this connection, and also as shedding a striking light on the whole question of sexuality, are Klebs's exhaustive studies on Saprolegnia mixta (110:513). He has shown that, when cultivated on pure gelatine, the plant can give rise to an active vegetative growth, but forms no reproductive bodies. Change into pure water starts the formation of zoosporangia and setting free of zoospores. Abundant growth, as well as zoospore formation, occur when the fungus is cultirated on agar albumen in running water. In a rather strong solution of hæmoglobin $(1 / 20$ to $1 / 10 \%)$ or in leucin solution, growth with subsequent formation of sex cells occurs. On dead insects or flesh extract in water, growth with subsequent zoospore formation and succeeding sex cell formation take place. In water with fibrin or syntonin added, growth with simultaneous development of zoosporangia and sex cells is observed. Finally actively vegetating material, when brought into $\mathrm{a}^{1 / 100 \%}$ hæmoglobin solution forms sex cells and subsequently zoosporangia.

Amongst the higher divisions of the Fungi the sexual process is often so greatly reduced or condensed, in other cases apparently so entirely obliterated, as to render exact comparison with lower or higher groups difficult. But the view now frequently accepted, that such condensation or obliteration is largely due to the rich and complex organic material in which they often live, having furnished the needed energizing stimulus that ordinarily is yielded by gamete fusion, has much in its favor. It would be beyond the scope of this work however to follow the intricate ramifications of asexual and sexial modification in the Fungi. 
But for the Fungi, as for the Algæe and Protozoa, we nould assert that asexual cells, exactly comparable to akinetes, aplanospores, and swarmspores in their mode of formation and relation, can be set free, and that each accurately reproduces the structure and life role of the parent. In the production of sex cells on the other hand, each cell has been exposed in the parental cell that produced it, as well as in itself, to similar or dissimilar environment, that may produce similar or dissimilar results in formation of each sex cell. Since cross fertilization also is frequently between distinct individuals, a constant response to, adaptation to, and perpetuation of biotic and cognitic lines of energy flow, as well as of flows of simpler forms of energy, that place, build up, and energize definite material particles, must constantly be proceeding in the sex cells. So the sex cells on fusion must, unless all physical and physiological laws are inapplicable, transmit such exact lines of energy flow, and the exact grouping of particles that these effect.

Sexuality in Higher Plants. We pass now to the higher groups of plants. Without entering here into the long-drawn question of the origin and significance of alternating gametophyte or haploid and sporophyte or diploid generations, from the Algx and Hepaticæ upward to the Cycadaceæ and Ginkgoacex, it can with considerable safety be now said that for these, as for many of the previously treated simple plants and animals, a reduction division occurs during the stage of spore formation, so that the sex cells have an amount of chromatin that, in energizing capacity and molecular adjustment, represents for each about half that of a diploid cell.

Such might explain why, in slow process of evolution, the haploid or gametophyte generation has become gradually reduced in size and vigor, while the diploid has become increasingly enlarged till in the giant redwood tree each sporophyte individual may be at least 1500 years old and 300 feet in height. But it is possible to emphasize orermuch the mere number of chromosomes. For the numbers may vary, even in species of a genus, and very strikingly in genera of some families, amongst the higher flowering plants. 
Now, in all the groups from the Hepaticæ upward, sexuality as such does not differ fundamentally from what we encounter in the higher green algx. That is, two complemental cells unite, or apparently more exactly one chromatin nucleus of small size, but of like or nearly like morpho-physiological value with the other, approaches and fuses with the protoplasm and nucleus of the attracting cell, owing to lines of attractive physico-chemical energetics exerted by the egg and most powerfully by the egg nucleus, and reacted to by the sperm nucleus. If the two belong to parent plants that for long generations have been exposed to like environment, the resulting embryo will develop under the same environment into a like organism as the parents. If the two belong to parent plants that differ from each other varietally or specifically, the resulting embryo will develop into an organism that more or less exactly blends the characters of the diverse parents, if the environment and the degree of molecular blending and physico-chemical energy make for the formation of a medium between, or a divergence from, that typical for one or other of the parents. If the two nuclei originate as in the first instance, but the resulting embryo be exposed to different environal states than was either parent, and if such states be not prejudicial to its growth and continued life, the resulting organism will be so affected that it or its descendants under like environment will show changed morpho-physiological features.

But, whether the resultant organism be like or unlike a blend of the parents, all our knowledge tends to prove that the likenesses or unlikenesses are fundamentally due to lines of stereoenergetics that traverse molecules and place these in definite connection with each other. So whether we study the motile sperm nucleus and the archegonial egg of Bryophyta and Pteridophyta, or the motionless sperm nucleus and the embedded egg of flowering plants, the researches of Pfeffer, Shibata, and Miyoshi amongst others indicate that an attractive action and reaction in line with the egg and sperm nucleus occur, while as yet these are removed from each other by several diameters of the egg. Such attraction seems at least in part 
to be due to definite substances excreted by the egg, or by accessory cells cut off from it some time before fusion of both sex cells. But, no matter whether the excreted substance be sugar as is probably true for mosses, or malic acid for ferns, or cane sugar for flowers, or even other and varied products, the attraction is due not to the molecules of these bodies as physical particles, nor to the molecules of the egg and sperm as physical entities, but to definite discharges of chemic, electric, biotic, or other energies that traverse the particles, so as to start and continue in these the attractive relation.

But; when the sperm and the egg nuclei fuse, the extremely complex highly energized molecules that make up both become accurately distributed and minutely interpenetrated as organically balanced constituents, that start the egg on a definite course of evolution, and so there results an organism that in every cell may show the structural and physiological details of both parents in blended relation, though reduced about half in potential value. Now in the above sentence the words "fuse," "distributed," "interpenetrated," "balanced," "start," "results," "show," are all terms that denote energy. So the writer claims that the minutest details of structure are all expressive of accurate flows or "tubes of energy," that traverse the molecular substance.

The truth of the above was demonstrated by the writer in his study of the hybrids of flowering plants. For the size, shape, degree of thickening, protoplasmic activity, and character of the plastids, in different cells, furnished exact evidence that profoundly delicate and yet powerful lines of energy flow were constantly traversing the embryonic cells and stimulating these to behave in a manner that was an approximate or a balanced mean between those of the parents.

But abundant studies by many workers have revealed the fact that reduction by half of the nuclear chromatin, both in egg and sperm, takes place in Hepatica and higher plants in the maturing spore cells, and that this halved, reduced, or haploid state is continued till the eggs and sperms have been developed, matured, and attracted to each other. On fusion of 
both, the halved or haploid number is doubled and forms the resulting sporophytè plant.

The quality and condensation of energy implied in such complemental unions and subsequent unfoldings by the egg represent an amount and a kind of energy far more perfect than even electricity. And from the preponderating part played by the chromatin over the protoplasm, under very many conditions observed, we would conclude, for the higher as for the lower green plants, that sexuality is due to a modification or change in the sign or character of the cognitic energy in two cells, so that one becomes charged with negative or minus cognitic energy, the other with positive or plus cognitic energy. So, when set free in the mature state, each becomes attractive for the other.

Here however we have again to distinguish two distinct phenomena, as amongst the simplest plants and animals. First, we recognize sex differentiation as such, that is, the production of two sets of like cells that are complemental to and necessary for fusion with each other, in order to the production of a new plant, and in both of which cells the amount of plus and minus cognitic energy is exactly balanced. Such cells could then be called isogametes, and the phenomenon as isoamphimixis. But over and above in all of the higher plants and animals, as well as in many unicellular ones, the complemental sex cells have become unequal in size, different in bio-cognitic constitution, and so organized that one cell attracts and the other is attracted. Such secondary sexual modification might then be called anisoamphimixis, and the cells as anisogametes.

We do not as yet know accurately for plants how such anisoamphimixis is to be explained. But important studies of animal and specially of insect gametes, made in recent years by Henking, McClung, Morgan, and others, suggest that often in the male cell, more rarely in the female cell, one chromosome may have an increased amount of chromatin, and so may give rise to maleness or femaleness; or eren as in insects that a supplementary chromosome in each spermatozoid confers 
special male characters that are hereditarily transmitted. Ircording to Baltzer the converse is true for the sea-urchin, since it is the egg cell in it which forms the supplementary sex chromosome. For recent synoptic literature on this, reference might be made to the papers of Morgan, published during the past five years in "The American Naturalist" and cognate journals; to his work on "Heredity and Sex" (1913); and to Walters' "Genetics" of the same year.

But in a minute critical study of plant and animal hybrids as well as in the behavior of the male and female gametes in process of fusion, also during subsequent divisions in formation of the cell lineage, it becomes increasingly evident that the two parental constituents evidently become physically apposed in their colloid molecules, rather than chemically blended, since varying degrees of separation, or of swaying toward one or another parent, may occur in some one structure, or in the organism as a whole. So the writer concluded, in connection with the share that each sex-element might contribute, that one of three conditions may exist in hybrids, and present evidence generally indicates that the same is true for individuals of a species:

(1) Often "each sex element, after union with its complementary sex element, represents half its former individuality, or retains half of its former hereditary properties." In this case the resulting organism shows a minute blending of both parental characters, but reduced by about half.

(2) Where one or more physically or chemically diverse substances, and therefore characters, are "found only in one parent, and with no corresponding counterpart in the other," these "are handed down though reduced by half."

(3) Where one or more diverse physical or chemical chararcters appear in each parent that "do not readily blend, each may be reproduced by the hybrid but reduced by about half."

Reviewing then the observed facts on which the above results were founded, the writer wrote: "Van Beneden (Rech. sur lar Maturation de l'ouf. 1883) went further than most of his zoological coworkers were prepared to go when he as- 
serted that each cell in an organism is a hermaphrodite structure. To this thesis many subsequently took exception and with some show of reason perhaps, seeing that no direct proof in individual cell life was forthcoming. But one is forced to accept its absolute correctness from study eren of one hybrid. It is this hermaphroditism of the entire hybrid organism which not only impresses on it the structure that the naked eye and microscope reveal, but which causes it to have a life cycle whose successive steps are intermediate between the parent extremes. Thus sufficient facts are in our possession and will, we hope, be greatly supplemented ere long to prove that the period of bud-bursting, of leaf-expansion, of flowerproduction, fruit-ripening, and other vital phenomena in hybrids are all dependent to a wonderfully exact degree on hereditary inheritance."

This separation of the stereoenergized molecules that are distributed, in definite amounts and directions, during successive divisions of the chromosomes is suggested by the behavior of the male and female chromosome constituents, in various plants that have been studied, and whose chromosome constituents fuse by apposition of molecules, rather than blend chemically. But the most direct evidence is got from such studies as those of Haecker on the developing eggs of Cyclops. In these the apposition but continued distinctness of the male and female chromosome constituents could be traced through a great part of the history of each dereloping animal.

Again the cases recorded by Sanson (106) for hybrid "Nivernais" breeds of cattle and many like records in breeders' journals testify to the frequent separation of cell characters derired from one or other parent.

Since many phenomena of sexuality then are common alike to plants and animals, in the few succeeding pages of this chapter a comparative review of these phenomena will be attempted. So many details however still remain obscure or unknown to us that what follows must be regarded merely as a tentative and imperfect presentation of results.

First: In tracing the gradual evolution of sexuality amongst the simpler algæ and fungi it was noted that there is often a 
wavering between asexual swarmspore formation of new plants; formation of these by parthenogenesis, but in which the swarmspores show a tendency first to weak sexual differentiation; and formation of these by true sex fusion of like or unlike gamete cells. Studies of the past quarter century have equally shown that, amongst the higher plants and animals, wavering transitions from natural or artificial parthenogenesis to union of sex products may occur. Thus in Chara and Marsilea amongst cryptogams; in Thalictrum, Alchemilla, and Antennaria amongst flowering plants; in many rotifers, smaller crustaceans, and genera or even groups of insects, frequent and extensive parthenogenesis occurs alongside occasional sex fusions. Loeb's experiments also with echinoderms prove that artificial parthenogenesis may be ensured by treating the eggs with various kinds and strengths of solution.

The question then arises: what is the morphological and physiological state of the egg that causes it to return from the sexually mature to the more primitive parthenogenetic condition? This seems to be due amongst animals and probably also amongst plants to the relative amount of chromatin retained in or thrown out from an egg as one or more polar bodies. In eggs that are normally fertilized, two small chromatin masses or polar bodies are thrown out of the egg in succession, one of which remores half of the chromosomes, and so expels half of the cognitic or main hereditary substance of the egg. In parthenogenetic eggs this half is retained, only one polar body being extruded.

Second: If such be true some evolutionary cause must have existed that gave rise to the parthenogenetic state with its one polar body, in place of the usual prefertilization state with its two extruded polar bodies; and in place of the most primitive condition of all, as seen in simpler algæ, where asexual cells alone exist. Amongst the five factors of organic evolution already cited (p. 204) the only two which seem to be here concerned - either one alone or both combined - are environment and reproduction. Now, in the reproduction of such colloid masses as 'Traube's cells, the two factors which might 
cause variation in their formation are diverse environment or altered chemical nutrient materials. Both seem to be inciting conditions alike in asexual cell formation, as already discussed, and also in sexual cell formation.

It must frequently have happened during mid-archæan times that hosts of individuals of the same species of acaryote, and as yet therefore asexual, organisms became exposed to two or more different environal states. Thus one group may have reached or been carried to areas in which a rich and stimulating food supply existed around, while another may have been carried to areas poor in such food. Two such diverse regions at the present day might be a richly charged mineral basin and a rather stagnant lake between an inflowing and outflowing river. Were individuals of a species able to survive in two such regions, all known facts indicate that altered lines of energy flow would be established, which would cause one lot to diverge more or less from the other. The abundant experimental results of Klebs (110) are here exactly apropos.

Differences in energized tension alike of protoplasm and of evolving chromatin would establish stereoenergetic placing of molecules of diverse chemical nature. Were such action so to continue as to affect the chromatin granules of each lot, one of these might assume what we may call the positive state of cognitic energizing, the other the negative state. Then as on the physical principle of plus and minus electric attraction, of satisfied and unsatisfied chemical states, or of warm and cold bodies, when these have developed under appropriate environment they would show complemental stereoenergetic bio-cognitic attraction. If it be here objected that we have as yet no material basis for such riew, it can well be replied that, as in electric, chemic, and thermic positive and negative states, a satisfied or balanced condition results, when currents of energy between the plus and minus bodies are established.

In the daphnid or water flea Simocephalus Issakowitsch showed that rich nutrition, and also high temperature, which probably acts indirectly by influencing nutrition, caused the production of parthenogenetic females, while starvation and 
cold caused the appearance of males. Related results have been obtained by others, these differing chiefly in indicating that the changed conditions are only effective at certain times in the life cycle. Whitney and Shull have shown that pure spring water acts on the parthenogenetic eggs of the rotifer Hydatina during their growth in such a way that they develop into male producing females, while eggs of females grown in an infusion of horse-manure always give only female producers.

Third: Since attention was drawn to Mendel's forgotten studies an abundant literature has appeared, from the time of De Vries' and Correns' suggestive publications, on the results of crossing experiments. Unfortunately Mendel and nearly all of his followers have treated of naked-eye appearances, few indeed have examined cytological details. Now, as the writer's studies on plant hybrids demonstrate, one or more characters from one or both parents may be inherited separately, owing to the absence or incompatibility of inheritance of such characters from the other parent. Such phenomena he termed unisexual and bisexual hybridity. But in the varied cases that he studied microscopically each character inherited only from one parent and reproduced in the offsprings appeared only of half or nearly half the potentiality seen in that parent. In other words there is no dominance or recessiveness shown either in the invisible molecules or in their aggregate masses that make up the inherited characters, but there is either a halved inheritance or if there is incompatibility chemically in fusion of the complemental parental details chemical disintegration and disappearance of the character seems to result.

So the writer considers that most of the cases of so-called "Nendelian inheritance" in plants and animals will probably be found to conform to such conditions, when they have been studied microscopically, and still more microchemically. But even in the case of macroscopic details very loose statements have been made. Thus the writer was recently shown an ear of white and of black corn along with a so-called "black" ear, obtained by crossing of the two parents. When the three 
were placed side by side a white ear, a dark slate-colored ear, and a black ear were evident. So the writer would candidly state that he has little faith in much of "Mendelian" literature, though he gladly welcomes and profits by the valuable and suggestive nature of Mendel's experiments, as by those of the older hybridizers of last century, and of the present.

Fourth: Another and highly intricate question involved in sexuality is as to why and how sex-cells have slowly erolved from ordinary somatic cells; also why and how they have been able to acquire, to lock up or epitomize in their substance, and to transmit to succeeding progeny all of the characteristic somatic details shown in the individuals from which they are derived.

In study of this question, and as a result in part of what has gone before in this chapter, we accept it as demonstrated equally for plants and animals: (a) that sex cells are extremely stable units, which hand down hereditary details with marvelous exactness, and which are only slowly and indirectly modified by action of environal agents; (b) that, from the simplest fungi and algæ upward through the scale of plants and animals, the sex cells have been derived erolutionarily from somatic cells, which have gradually undergone cytological changes that make an adjoining cell of a filament, then distinct cells of distinct filaments or individuals equally complemental and attractive to each other; thereafter that increasing growth in protoplasm and nucleus in occasional cells of one plant or animal with decreasing growth or division in a complemental cell protoplasm and its nucleus of the same or of another plant or animal originates the unequally sized but complemental egg and sperm; (c) that the two gametes thus formed have developed equally or more or less unequally balanced states of plus and minus cognitic energy, so that they are equally attracted, or one attracts and the other is attracted; (d) that in all the simpler plants and in groups of simpler animals no indication of segregation into somatoplasm and germoplasm occurs, but in higher plants and animals such is gradually established. 
But of equal importance is the great fact that the varied types of "spore" or "bud" cells in acaryote as well as caryote plants and animals are hereditarily somatic cells which by cytological modification perpetuate and reproduce all of the parental details. Further with evolving chromatin formation, one race of "spore" cells shows varying wavering between ordinary spore or bud multiplication, parthenogenesis, and production of complemental sex cells. When the last is effected reduction by half of the chromatin substance takes place in each gamete cell preliminary to fusion of the two.

We conclude then that sexual cells have originated evolutionarily from spore cells, that spore cells were derived from and were preceded by somatic cells formed by simple division, and this mode is anticipated and doubtless initiated by that physical process of division or budding which proceeds in many inorganic colloid mixtures, and which causes multiplication and hereditary perpetuation of inorganic colloid cells.

How then has progressive extrusion of chromatin substance been effected, and what benefit biologically has been secured by formation of complemental sexual cells? If unicellular or multicellular nucleate plants and animals, or their common ancestors during archæan times, became temporarily exposed as individuals to new and diverse environal states, such might cause amongst one group of these an increase or plus condition in definite tubes of cognitic energy, and correspondingly equal decrease or minus condition in another group. Associated with, and possibly even originating such, might be a gradual breaking down and extrusion of definite products with which such increase and decrease were connected.

Given then a return of both groups to primitive arerage conditions, the unicellular groups or certain cells of each multicellular group, charged each with either a plus or a minus supply of cognitic energy, would undergo fusion. So as compared with long continued exposure of a group of organisms to one set of conditions, temporary exposure of two groups of these organisms to diverse conditions from each other, and from the past environment, and subsequent intermingling of the 
two groups amid their primitive environment, the commencing causes might be secured that started sexuality.

The resulting advantage of this we claim is, that thereby through constant crossing of what from the long drawn hereditary standpoint were primitively somatic cells, more recéntly asexual spore cells, and still later bisexual cells, a constant blending and so hereditary averaging and fixing of entironally derived or acquired characters was ensured. Therefore a steady and solid adrancing adaptation in organisms to their average environment would be effected, as steadily advancing modification in cosmic environal factors took place.

Fifth and finally we would note that, during the past quarter century or more, much has been written and spokenmainly from the zoological side-as to "somatoplasm and germplasm," "immortality of the germplasm," and kindred topics. As the writer has endearored to demonstrate, the protoplasm (or protoplasmatin if one desires similar terminations) is the most ancient and probably simplest constituent of each living cell. In other words primitive unicellular acaryotic cells, resembling types still existing, exhibited all the fundamental life functions of irritability, nutrition, respiration, growth, and reproduction by division. So to express such in Weismannian language, protoplasm is the most ancient and highly immortal living substance known.

For, from its first evolution as a complex and labile chemical body, which made up the entire living unit and that probably evolved during early mid-archæan age, on to its existence at the present day in the most complex nerve cells of the human brain, we are forced by the observational and experimental evidence to conclude that it has had unbroken biotic continuity. Otherwise we must accept it that in higher or caryotic plants and animals a sudden new formation of protoplasm took place, or that the chromatin of a cell nucleus developed and existed alone for a time, and from its substance reformed a new mass of surrounding protoplasm. Few, we judge, will venture to accept either position.

The next more complex substance chromatin seems gradually to have evolved from the protoplasm, conjointly with and 
as a result of that condensation phase or state of energy that we have called the cognitic. Originating amongst the higher autotrophic Bacteria, later and more perfectly amongst the derivative Blue-green Algæ and the primitive Protozoa, this constituent has since persisted as a hereditary portion of every higher cell up to man. It is therefore the next most ancient constituent of the living cell in succession to the protoplasm, and may have evolved during the later mid-archæan age.

While amongst the nucleate cells of such simple plants as the conjugate alga and fungi any or even every cell of an indiridual may become a sexual cell, in higher plants and in animals higher than the Acaryozoa, definite sexual cells are early separated off from others that in time decay. Such sexual cells, possibly by migration of hormones, of complex peptones, and other materials along profoundly complex but definitely localized lines of energy flow, lock up or condense within themselves the entire set of characters of two parental organisms. But alike in evolutionary origin and in historic continuity they appear to be less immortal than is the chromatin as such, and greatly less so than the protoplasm. For irritability and nutritional capacity must always precede reproductive effort.

The still more complex substance neuratin, that the writer would regard as typical of most invertebrate and of vertebrate ganglionic cells, seems to have segregated off from the protoplasm or chromatin, or even more likely as a conjoint product of both. We have suggested that its first beginnings seem to be traceable (p. 223) amongst higher Protozoa like the Hxmoflagellata and Infusoria; that it gradually became concentrated in special elements-the nerve cells-and that such appear in simplest conditions amongst the Coelenterata and Echinodermata. Evolutionarily and historically then it is the newest and highest of the cell elements, that probably segregated by slow degrees during late archran age, and has now reached its most complex amount and expression in superman.

The writer will not attempt in this chapter to discuss a possibly more complex energy and ether aggregation that in 
a succeeding chapter he has called the spiritic. It may possibly only represent the climax of densely aggregated neuratin elements that give rise to high cogitic activity in man.

But in closing this chapter we would anew emphasize that there seems to be not the slightest indication. of break or discontinuity along the entire chain of evolving plant and later of derived animal life. The whole chain of being suggests a great processional elaboration of increasingly organized physico-chemical material, that is due to the action of definite lines or tubes of energy in progressive degrees of condensation. Starting with minute colorless autotrophic organisms, simpler possibly than the unicellular spherical bacteria, the organic evolutionary procession has been continued in orderly manner alongside general cosmic evolutionary progression, till it has culminated in superman. 


\section{CHAPTER XI}

\section{THE EVOLUTION OF PLAN'TS}

Since plants are autotrophic, that is, are capable of building up their organic food from simple inorganic substances, and since animals are allotrophic (heterotrophic), or feed on the organic compounds that plants have elaborated, it follows that animals probably originated later than plants. We hope, in succeeding pages, to demonstrate this, and to show further that the simpler animals evolved as offshoots from colorless bacterial lines of plant organization, through the slow acquisition by the former of certain characters that now cause us to distinguish them as animals rather than plants.

If such be the biological relationship, it becomes necessary to determine, if possible, the environal surroundings of primitive plants and animals, the period approximately when they first appeared, how far traces of ancient organisms have been preserved in the fossilized state, whether very ancient types have surrived long and checkered changes so as to exist still in a condition little if at all altered from their primitive state, and what the main lines of evolutionary advance have been for those plants and animals that underwent progressive evolutionary modification. These questions will be considered in the succeeding chapters.

We believe all will agree that primitive organisms were soft micellular, or later multicellular, types which would leare no trare of their existence in the earth's crust. During the slow but gradual erolution therefore of the soft algoid, fungoid, and moss-like forms of plants, as well as of non-calcified or non-silicified animals, many species, genera, and even families may have arisen of which no record exists.

But, as already stated (p.53), in the most primitive stratified archacan or proterozoic rocks, extensive beds of limestone, 
of siliceous chert, of carbonaceous shales, or of colloid-iron rocks are not infrequent, and all suggest the activity of simple cellular organisms belonging to the groups of the bacteria or of the Blue-green Algæ. Elsewhere (13:953) the writer has advanced reasons for believing that the plant groups just named were the most primitive of which we have any exact knowledge at the present day There also he has tabulated or indicated the existence of nearly a hundred species-in part algoid, in part bacterial-which can flourish in hot waters of $45-75^{\circ} \mathrm{C}$. temperature.

Now, while there are clear indications that dry land and seas, denudation areas, and shore cliffs, much like those of the present day, then existed, there is equally clear eridence that extensive volcanic action, rock displacement, stratigraphic distortion, and often intense baking or destruction of rock masses prevailed over wide areas, during archæan times. The agents that effected such changes, and the raried changes in themselves, would all contribute to variation, modification, and evolution of organic types. So an increasing richness and diversity of species would slowly arise, at the same time that a gradual decrease in temperature occurred amongst many inland expanses of fresh or mineral water.

Such a view presupposes that organisms developed at first in warm fresh-water or mineral-water expanses that corresponded closely to the hot-spring regions of the present day, and such has been accepted above for reasons given elsewhere by the writer (13:253). If further it is found on investigation that a preponderating number of plant and animal species have evolved in land-locked water areas of greater or less extent, or upon dry land, added testimony is secured for such. a position. In the present and succeeding chapters the attempt is made to collect botanical data that may guide us. In later chapters a like attempt is made from the zoological side.

But, before studying such, a well-known and important fact should be taken account of that has far-reaching consequences. When the palxontological record is examined eren cursorily, one is vividly impressed by the apparent sudden 
advent of a rich and highly evolved animal life at the beginning of the cambrian epoch, and by the entire absence, even of traces, in the rocks of the archæan system. Thus graptolites, brachiopods, lamellibranch gasteropod and cephalopod molluscs, ostracods, and trilobites, that represent leading and often highly evolved groups of invertebrates, are all found to occur in the cambrian rocks.

Even in the absence of direct fossil evidence, therefore, it becomes a necessity of the case to assume that, throughout a considerable stretch of archæan time, numerous and abundant organic species and genera were evolving, but in part owing to their soft and perishable nature, in part to the extensive terrestrial changes proceeding, they have left no recognizable trace of their existence.

How far back into archæan times the evolutionary process for organisms may have been carried, and how long may have been the period during which organic forms were gradually elaborating from the simplest bacteria and algæ on to such high types as the cephalopods, are questions which will be considered in another place. But that the period was an immensely extended one is conceded alike by geologists (p. 11) and biologists, and judging from the thickness of mid-archæan rock strata may conservatively be estimated at between $10,000,000$ and $25,000,000$ of years. We are compelled therefore to accept it that during mid-and late-archæan times an abundant flora and fauna originated; also that on the plant side a degree of development had been reached that by the close of archrean days probably represented the moss or bryophytic, and even the simpler types of the fern or pteridophytic alliance, while on the animal side all of the main groups of invertebrate animals had been mapped out. The widespread rolcanic, metamorphic, and denudation changes proceeding, combined with the often soft perishable substance of the earlier plants and animals, would sufficiently explain their apparent absence.

As to the possible persistence of ancient species through intervening periods, say from the cambrian up to the present 
day, a number of well-known instances might be cited such as Lingula from the cambrian beds, Crania and Rhynchonella from the next higher or ordovician beds, all three being brachiopod genera; while the gasteropodous molluse Capulus and representatives of other groups might be cited. This is direct proof that organisms still living may afford fairly accurate data in some cases, regarding the main lines of evolutionary advance in the past. But alongside this we must ever place the great and demonstrable fact that wholesale destruction of many thousands of genera has been effected during the evolution process.

We will now bring together statistics and structural details regarding living plant species, and try to link these, so far as possible, with groups of the past.

The Blue-green Algæ or Cyanophyceæ, which we have already regarded as including many of the simplest plants, consist of 81 genera and 653 species. Of these, 47 genera are purely fresh-water, 26 genera consist mainly of fresh-water but in part of marine or brackish species, while 9 genera alone are marine. The total number of fresh-water species (accepting average results from the works of Gomoni, Kirchner, Tilden, and others) is 548, and of marine 105 . Of the last 31 species are unicellular or loosely joined into threads, and the remaining 74 belong to the nostocaceous, oscillarioid, and higher groups.

It thus appears that fully five times as many species are fresh-water-including under this hot-spring and related areas - as marine, and that of the latter the great majority are more evolved and so later in origin. The primitive development of the entire group therefore in land-locked fresh-water areas is strongly suggested. But, when about 41 species (13: 253) are met with in thermic waters at a temperature of $50-75^{\circ} \mathrm{C}$. , their primeval origin in thermic areas amid the volcanic activities of the mid-archæan epoch seems highly probable.

We need not suppose that even the simplest living thermic species absolutely resemble their primeval ancestors. It is by no means unlikely that the latter were of simpler structure 
and may have evolved-on the plan of Traube's cells-from complex colloid substances that were energized by combined thermic, lumic, electric, and evolving biotic energies amid the abundant thermal springs of the archæan age. As they spread into cooler areas during succeeding ages, new species must have arisen, many in turn must have become extinct, and some must have migrated into the sea. Such would exactly explain present-day conditions.

The only group of plants that seems closely related to the Cyanophyceæ is that of the Bacteriaceæ. For between some of the paler "bacteroid" unicellular Cyanophyceæ and the rod-shaped bacteria the morphological resemblance has often been insisted on; while between the paler, smaller, and multicellular thread species of Cyanophycer and some of the thiobacterial and "Beggiatoa" types of bacteria the same is true.

Of living bacteria about 31 genera and 600 species have been recorded as taxonomically distinct fresh-water or land examples-including saprophytic and parasitic types-while 4 genera and about 50 species are marine. The relative proportions, here again, strongly indicate a fresh-water origin for the group, while their morphological derivation, as allotrophic or heterotrophic feeders, from the autotrophic series of the Cyanophycer, seems strongly suggested. But the probable origin even of the Cyanophycex from colorless types resembling the autotrophic sulphur and iron bacteria should ever be kept in view, and has already been explained (p. 42). Even more markedly amongst the bacteria than the Cyanophycex, we note the occurrence of numerous "thermophilic" species, the existence of which has only been revealed fully during the past decade. The condensed account of them given by Ambroz (111:25\%) sets forth well their biological activities. Setchell $(3 \%: 934)$ has shown that certain of these can live at $89^{\circ} \mathrm{C}$, while we know that spores of some will survive to $100^{\circ} \mathrm{C}$. for a considerable period.

Many and often conflicting views have been expressed during recent years as to the phylogeny of the higher algr. Reference will be made to this below. But the subjoined table 
sets forth the distribution of the genera and species of the different families that now live in fresh and salt water respectively.

Distributional Table for Algae-Fungi

\begin{tabular}{|c|c|c|c|c|c|c|c|c|c|}
\hline \multirow[b]{2}{*}{ Group } & \multicolumn{2}{|c|}{$\begin{array}{l}\text { Fresh- } \\
\text { water }\end{array}$} & \multicolumn{2}{|c|}{ Marine } & \multirow[b]{2}{*}{ Group } & \multicolumn{2}{|c|}{$\begin{array}{l}\text { Fresh- } \\
\text { water }\end{array}$} & \multicolumn{2}{|c|}{ Marine } \\
\hline & & 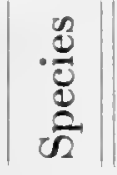 & & 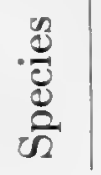 & & & 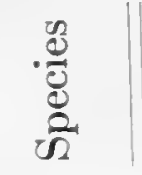 & 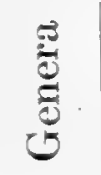 & 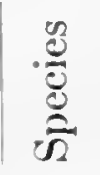 \\
\hline Cyanophyceæ.. & 73 & 548 & 8 & 105 & Valoniaceæ.. & & & 12 & 55 \\
\hline Desmidiaceæ. & 31 & 1029 & & & Diatomaceæ penn... & 38 & 1244 & 44 & 1287 \\
\hline Zygnemaceæ. & 4 & 108 & & & Diatomaceæ & & & & \\
\hline Mesocarpaceæ. . & 2 & 32 & & & centricæ. & 7 & 165 & 59 & 1046 \\
\hline Volvocaceæ..... & 20 & 27 & $\mathscr{2}$ & & Gymnodiniaceæ. & $\mathcal{Z}$ & 8 & 5 & 35 \\
\hline Tetrasporaceæ.... & 11 & 28 & 1 & & Prorocentraceæ. & & & 3 & 10 \\
\hline Pleurococсасеæ.. & 33 & 110 & 1 & 3 & Peridiniaceæ.. & 3 & 12 & 21 & 105 \\
\hline Protococcaceæ.... & 13 & 48 & 3 & & Phaeophycex & & & & \\
\hline Hydrodictyaceæ. & 4 & 36 & & & Litho'ae-Pleuro'æ. & 2 & 4 & 1 & 4 \\
\hline Tívaceæ......... & 5 & 29 & 6 & 49 & Phaеophyceæ & & & & \\
\hline Ulothricaceæ..... & 5 & 29 & 2 & 4 & marinæ.. & & & 145 & 759 \\
\hline Chaetophoraceæ. & 17 & 86 & 6 & 8 & Bangiacer. & 1 & 1 & 1 & 33 \\
\hline Mycoidaceæ..... & 5 & 10 & 1 & 1 & Lemaneacex. & 5 & 22 & & \\
\hline Oedo-Coleoch'æ. & 3 & 184 & & & Helminthocladiacere. & 4 & 46 & 7 & 18 \\
\hline Cladophoraceæ & 6 & 108 & 5 & 69 & Rhodophyсев...... & & & 291 & 1975 \\
\hline Vaucheriaceæ... & 2 & 26 & & & Bacteriaceæ....... & 31 & 600 & 4 & 50 \\
\hline Characeæ......... & 6 & 34 & & & Fungi....... & 2670 & 24000 & 10 & 200 \\
\hline & & & & & Total. & 3003 & 28574 & 658 & 6018 \\
\hline
\end{tabular}

The above reveals some suggestive peculiarities. If, excepting the Cyanophycex, account be taken of the next sixteen groups along with the group of the Pennato, or simpler diatoms, which together represent the lowest and most primitive algæ, 206 genera and 3168 species are fresh-water, while 71 genera and 1430 species are marine. So, if account be taken of those which show simplest individual structure, simplest cell structure, simplest modes of reproduction, and widest geographical distribution, the fresh-water species and genera greatly predominate. But when the highest and almost assuredly the four most recent divisions of the "Centric" diatoms, the brown and the red algæ, as well as the highest green algax are compared, it is found that there are 24 genera and 258 species in fresh water, and 565 genera with 4233 species that are marine. 
As will be again emphasized later on, we would view the above four large and preponderatingly marine groups as of primitively fresh-water origin, and as still including freshwater connecting links with the yellow, the brown, the browngreen, the pink, the red-green, and the green Cyanophycece. But, while we have abundant evidence to show that the above four groups, on passing from a fresh-water to a marine existence, branched out into many genera and species that contributed to the large existing total of 4233 species, few observers have ever regarded them as the originators and ancestors of the dominant lines of ascent that later gave rise to the bryophytic or moss series, the pteridophytic or fern series, and others still higher. These have generally been sought for, and we believe rightly, amongst some ancient representatives of the Chotophoracece and Coleochcetacex, both fresh-water genera.

Now we hope to show that the great processional change from fresh to salt water that the above four groups exhibit has been gone through again and again in the history of animal groups, which starting from more primitive fresh-water types have passed on to a salt-water existence, and have then evolved a wealth of marine genera and species, but have failed to evolve higher groups than themselves.

Morphologically and taxonomically then we would regard the fresh-water Cyanophyceæ as the ancestors of higher algoid organisms, while the conclusions of most workers in the past, that the green fresh-water alga have given rise to higher palustral and later to land forms, seem fully warranted by evidence from every side. The morphological evidence in favor of continuity from the Cyanophyceæ to the highest algæ can now be studied.

Each simple spherical cyanophyceous organism like Chroococcus or Gloocapsa shows within the mucilaginous wall a cylindrical zone, as Hegler has pointed out, of yellowish, yellow-brown, yellow-green, blue-green, or pink-green color-the chromatophore. This consists of a nearly homogeneous plasmatic ground-substance in which chlorophyll and phycocyanin bodies are closely distributed. The chlorophyll pigment evidently enables the plasmatic substance to elaborate glycogen, 
which appears as refractive masses within it. But alongside these in some genera, and embedded in the colorless central protoplasm, are often small refractive bodies that behave to stains, digestive agents, and chemical substances as does chromatin material. Such seems to be closely related to the chlorophyll bodies, and even united with them by fine threads, while they suggest the diffuse beginnings of nuclear chromatin, which, it should be emphasized, are early associated with the chromatophore on the one hand and with the central protoplasm on the other.

In the higher or thread forms of the Cyanophyceæ, glycogen, albumen masses, and protein granules may all be present in the chromatophore, or may be stored-often at opposite ends of each cell-in the central protoplasm. But they further show an increasing formation of chromatin granules in the central protoplasm, which, instead of being loosely connected by fine threads as at first, gradually unite into granular loops or wreaths, which occupy a considerable part of the central area in Oscillatoria and Cylindrospermum. Such represent, as most recent observers have accepted, a developing but still diffuse state of the cell nucleus in higher plants. And it should here be emphasized that the refractive chromatin granules of the chromatophore, and those of the evolving nucleus, seem always to be connected by delicate threads, that act as an elastic connecting substance between the protoplasm and chromatin.

As regards the pigment of the chromatophore, this may vary, even in one genus like Pleurocapsa from yellow to olive, then to blue-green and violet, in Dermocarpa from violet-pink to rose-red, in Plectonema from light green or yellow-green through blue-green and pale red to rose-colored. This pigment has been called phycocyan, and spectroscopically is allied to phycophæin that is typical of the brown seaweeds, and phycoerythrin that is typical of the red seaweeds. $\mathrm{By}$ transmitted light it is of a rich blue color, but in mass it exhibits a carmine-red fluorescence.

Now from the spectroscopic researches of Engelmann (112: 1) and Gaidukov (113:484,517) we learn that the color of blue-green and of other algæ varies according to the law of "complementary chromatic adaptation," and even in one genus like Oscillatoria threads may become green in red light, blue-green in yellow light, pink to red in green light, and brownyellow in blue light. Such results accord with the observations that red algæ occur in the deepest marine zones $(20-500 \mathrm{ft}$.) where mainly blue-green rays penetrate; that the brown and 
yellow algæ occur in shallower depths up to tide margin where yellow-green rays penetrate; that the green algæ occur at or near the surface where red light is averagely strongest; and that blue-green algæ would be best adapted to orange-yellow light.

But, even amongst the variously colored blue-green algæ, the rariation in species from blue-green to olive-green, from this to greenish yellow, then to greenish brown, and from this to brownish or violet-pink and thence to pink-red, is usually correlated with a like environal relation to light rays. Thus the green or the greenish yellow species are mostly met with in exposed situations, and they best resist sun-dessication when combined with appropriate fungi to form lichens as in the pleurococcaceous Verrucaria and Lecidium or in the nostocaceous lichen C'ollema. The blue-green or brown-green forms occur in moister and more shady positions, where they may give rise to such lichens as Peltigera and Ramalina; those which show a brownish red to rose-pink color are marine as in Dermocarpa violacea and rosea, Plectonema roseolum and $P$. gelenkinianum, which are found on rocks, on algæ, or on sea animals at varying depths.

So equally for the Cyanophyceæ as a group, and for their probable ancestral connection with the green, the brown, and the red algxe, that will be discussed later, the color relations indicate that the Cyanophyceæ have been a highly adaptable series.

Confining attention for a time now to the question of chromatophore evolution, we consider that it is possible to trace here three lines of advance, that represent the green, the brown, and the red algoid groups respectively.

In the simplest and probably most primitive green algæ that are included under the Pleurococcacex, Tetrasporaceæ, and Protococcacer, the chromatophore is nearly always single, and either occupies the entire circumference of the cell, as in Tetraspora, Oocardium, etc., or is an extensive lateral or posterior expanse as in Dimorphococcus, Chlorocystis, and Palmophyllum. Or it may be divided into two chlorophyll bands or chromatophores as in Chlorangium, or there may be further division into from five to twelve masses as in Oocystis and some stages of Pleurococcus.

But further, the minute chromatin granules in the chromatophore of the most evolved Cyanophycese appear now to unite and enlarge into one bright refractive "pyrenoid," and then instead of glycogen the elaborated carbohydrate is deposited in or round the pyrenoid as starch. Or several pyre- 
noids - two to twenty-are formed as in Stephanosphcera and Chlorochytrium, each of which becomes a starch-forming center.

On the other hand in such genera as Chlorella, Conferva, and Botrydium that make up the group Heterokontæ pyrenoids are not formed, and here oil instead of glycogen or starch appears. Alike from the chemical and nutritive standpoints this may be regarded as a transition phase in the history of the green algæ, since from the standpoint of molecular colloid organization the oil is intermediate in chemical complexity between glycogen and starch. In now forming a somewhat aberrant assemblage apart from other green or from higher algæ, it is by no means unlikely that the Heterokontæ represent a special advancing group from the Cyanophyceæ, that have become a morphological side-line.

From ancient and now extinct members of the above groups several diverging lines of evolution seem to have arisen, most of which have remained amid fresh-water surroundings. Living genera like Schizochlamys, Nephrocytium, and Scenedesmus indicate by their structure and life history transition connections from the Cyanophycer to the Desmider, and that extinct forms doubtless united in finely graded series. The group Desmidex now consists of 31 genera and 1029 wholly fresh-water species. Great diversity in advancing evolution of the chromatophore is seen in these. Thus some genera have a single greatly expanded peripheral mass; in others it is subcentrally or centrally placed with radiating bands or arms; or again two to seven peripheral or central or spirally placed chromatophores occur. Equally varied are the pyrenoids, which may be single and central in the mass, or two to many may be distributed through its substance.

From an ancestral desmidean stock again, the mesocarpous and zygnemaceous groups have evidently started. Though together they now include only six genera and 140 species of wholly fresh-water habitat, these may well be taken to represent small remnants of two once abundant families. They may show one to seren peripheral spiral chromatophores with numerous pyrenoids at intervals along them, or one to several central chromatophores may show a like number of pyrenoids.

The Desmidex and two last groups seem all to form a phylum that diverged into a separate pathway of variation from other alga, but which is still represented by many and world-wide species.

Another diverging line has probably passed from greenish unicellular cyanophyceous ancestors through protococcaceous forms, allied to Endosphera and Phyllobium to the simpler 
Tolvocacere like Sphcerella and Chlamydomonas, and thence through allies of Coccomonas and Chlorogonium to Stephanosphara and Folvox. As in Endosphora the chromatophore forms a complete postero-lateral and peripheral investment to the cell, in which one to several pyrenoids occur.

A striking morpho-physiological structure that becomes highly differentiated in this group is the red spot or eye-spot, that attains most perfect condition in some of the highest Volvocaceæ.

Twenty of the twenty-two genera are fresh-water. The living species number 31,27 of which are fresh-water, and some of these are world-wide in distribution.

Another diverging evolutionary line that seems early to have originated with the three above named, but rather from a greenish yellow or green-brown cyanophyceous ancestry like Synechococcus, includes the Gymnodiniaceæ, Prorocentracer, Peridiniaceæ, and Diatomaceæ. Of these the first is simplest, and includes genera like Amphidinium with green or greenish brown chromatophores, and so may have arisen with pleurococcaceous organisms from a common or nearly allied ancestral cyanophyceous group, through perfected evolution of a nuclear body. The greener species are still freshwater, but others are now yellow-brown and have migrated into a free marine environment where, though few in species, they now are often abundant in individuals. But still others like Gymnodinium and Cochlodinium show all transitions from green through yellow-brown to pale yellow and finally colorless conditions, while they pass from fresh-water to salt-water life. Such colorless flagellate free-swimming organisms have often been claimed as protozoic animals by the zoologist. They rather should be viewed as a small side-line of plant life, the species of which have passed from typical plant life to types that simulate animal form and relation.

But while in their more primitive stages of development, and while still retaining a single or later a divided brown chromatophore, types somewhat intermediate between Hemidinium and Pyrocystis of the Gymnodiniacer showed deepening of the longitudinal furrow and thickening of the cellulose membrane, at the same time that minute pore areas were left in the latter. Some of these, developing and perfecting one or two flagella, seem to have branched out into the Peridiniacex that includes three genera and twelve species of fresh-water and 21 genera with 105 species of marine distribution; also the Prorocentracex that is now wholly marine in its three genera and ten species. 
As would seem to be true from palæontological evidence, the specifically numerous and varied family of the Diatomaceæ originated at a comparatively recent date from organisms intermediate between the two last divisions. In the frequent retention of a single greenish yellow to yellow-brown chromatophore, with one or more pyrenoid centers, in the division of the chromatophore amongst some to form two or more variously expanded plates, in the gradual silicification of the cell-wall to a degree that is at times baffling in its minute complexity, and in its present geographical distribution, the group is highly suggestive.

It now includes 148 genera and about 3800 species. But a detailed examination of these, alike as regards their chromatophores, the shape and relative complexity of the wall structure, as well as their present distribution in fresh and salt water, reveals interesting features that appear to have an important bearing on their evolutionary history.

The entire group has been subdivided, in many recent treatises, into the Centricæ and the Pennatæ. Now, if we accept this as an approximately natural classification, the latter include 38 genera with 1244 species that are fresh-water and 44 genera with 1287 species that are marine. That is, distributionally, the genera and species are about equally divided between the two habitats. The Centricæ on the other hand include 7 genera with 165 species that are fresh-water and 59 genera with 1046 species that are marine. Now, while some have regarded the Centricæ as the more primitive group, the general structure and complexity of the shell, the number and distribution of the chromatophores, as well as auxospore formation methods, all indicate that the Pennatæ are the more primitive. Since they contain numerous existing fresh-water species, and since the more complex Centricæ are evidently evolving much more as a marine than as a fresh-water group, there seems considerable evidence for believing that the entire series has wholly or mainly been derived from a common freshwater ancestry, but during the process of increasing specialization they are becoming increasingly marine. The entire series constitutes another side-line of evolutionary progress, that both in its fresh-water and marine species has failed to evolve higher groups than itself.

The widely disseminated class Ulvacex has generally been viewed as related to Tetrasporacex, though it represents at great advance in cellular organization. But in both divisions each thallus cell contains a single large chromatophore, with one - rarely more-pyrenoid center. But, during evolution of 
the Ulvacex from simpler tetrasporaceous ancestry, the writer considers that the first striking evidence is got amongst the green alga of migration from fresh through brackish into salt water. For, while the Tetrasporaceæ include 11 genera and 28 species that are fresh-water, and 1 genus with 2 species that are marine, the Ulvaceæ include 5 genera and 29 species that are fresh-water, also 6 genera and 49 species that are brackish or marine. It might however be argued that all have been marine primitively, and have gradually sent motile gonidia into brackish estuaries, which there established their respective species, while later they again migrated into fresh water. So many and so grave objections can be offered at once to such a suggestion that we do not propose at this stage to linger over it further.

The Ulvaceæ then may well be designated a transition family, but one which traces fresh-water descent. It is one also which presents many transition conditions in some of its genera and species. Thus Monostroma bullosum has been regarded by Kützing rather as a Tetraspora and is wholly fresh-water, while related species, with more ample thallus, are brackish or marine. $\quad$ H. Grevillei-widely known also as Ulra Lactucais marine or subbrackish and of wide geographic range over the world. Ulca, in restricted sense, includes brackish and salt-rater species, but Enteromorpha-which like Ulva is world-ride-includes not a few fresh-water species. The genus Ilea is of interest, in that it closely resembles Enteromorpha, but has developed a brownish color. The two species that make up the genus Letterstedtia are the most highly modified members of the family, and are now purely marine. From their occurrence at Port Natal and in Australia they may have become fixed in their environal relations during cretaceous times, and spread to both areas when shallow seas united both regions.

The Ulothricaceæ, Hydrodictyaceæ, Mycoidaceæ, Oedogoniacex, Cladophoraceæ, and Vaucheriaceæ are all important green algoid families whose purely fresh-water, or preponderatingly fresh-water, origin and distribution seem unquestionable. I glance at the table (p. 305) makes this at once evident. But we can well afford to linger somewhat over the group Cladophoracese. It includes six genera and about 200 species, of which one genus Spongocladia with 3 species is marine, another Pithophora with 8 species is fresh-water, while the remaining ones like Cladophora and Rhizoclonium, that are world-ride in distribution, contain species which occur in fresh, in brackish, or in salt water. So this again may truly be designated a transition family. 
But the above six families, with some smaller related ones which cannot be individually considered here, show graded connection in the number of chromatophores, from one large expanse that almost encircles the cell, to another that is reticulate and may separate into parts as in Chotomorpha, or numerous small subdivided bodies that closely line the peripheral protoplasm as in Conferva. Simultaneously the single pyrenoid of the simpler genera multiplies by degrees into many that dot over the chromatophore substance, except in such a group as Vaucheriaceæ, where oil instead of starch formation takes place, and the pyrenoids are absent.

We now reach two families that seem to the writer of exceptional importance, as being probably related on the one hand to some of the higher Cyanophyceæ, and on the other to the simplest conceivable Bryophyta and Pteridophyta. We refer to the Chætophoraceæ and Coleochætaceæ, that will be treated separately. Amongst the Blue-green Algæ the family Chamæsiphonaceæ deserves special attention, in that most of the included genera produce conidiospores inside conidangia, that are some multiple of four. Each conidiospore is passive and non-ciliate. But as Phillips has shown, and as the writer has often observed, there is a strong tendency toward production of semi-ciliate waving processes in the end-cells of many Cyanophyceæ, as well as fine intercellular threads, either of which may well represent rudimentary ciliary formation. If then the conidial cells before being set free retained or developed such processes, swarm-cells would result.

Now in cytological aspect and structure of the entire plant, there is striking agreement betreen some chamæsiphoneous genera and those of the Chrtophoracex, the presence of a distinct nucleus in the latter being the main difference. In Microthamnion and Phoothamnion also the color is yellowgreen, blue-green, or yellow-brown. In this family then, we might look for a common point of divergence from the Cyanophyceæ to the higher green and the brown algæ. The diversified structure, aspect, and environal connection of the thallus in the family suggest that the living genera are scattered remnants of a greatly larger assemblage, which in remote past periods were much richer in connecting genera. The single or more or less subdivided chromatophore may readily in higher derived types have become distinct small chlorophyll bodies.

But a feature of special interest is the structure of the swarmspores, and their often exact similarity to sexual or gamete cells. 
While it is true that most of the genera and species have now adopted an epiphytic or subparasitic habit, this may merely indicate that by such artifice biologically they have been able to survive, while many related genera of freer habit may have succumbed. The family includes 17 fresh-water genera with 86 species, and 6 salt-water genera with 8 species, while 2 or 3 species are mainly brackish in habitat, a sufficient indication that they originated as a group in fresh-water areas.

They again exhibit close affinities with the Mycoidaceæ that consists of 5 genera and 10 species of fresh-water habitat, and one genus with a single species of marine habitat.

The Coleochætaceæ is a family that has excited attention during the past thirty years from its possible affinities with the green algæ on the one hand, and the bryophytic or even pteridophytic groups on the other. These affinities were connected with the mode of growth of the thallus, the structure of the green cells that compose it, the tendency in some to formation of flattened expanses, and not least in the origin and structure of the sex cells. In the structure of the thallus, however, the family closely agrees also with some types of the Chætophoraceæ and Mycoidaceæ. So forms that would connect all three, and that might at the same time act as a connecting bridge with bryophytic and pteridophytic groups, were greatly desired. Such seems to exist in the fresh-water genus Aphanochoete, that has been carefully studied by Huber (98: 264). Formerly united with the Chætophoraceæ, it is now placed by Oltmanns (99:240) in a separate family owing to its interesting reproductive methods. For, while the Chætophoraceæ mostly develop motile biciliate conjugating or gamete cells of like size and appearance, in Aphanochote they are motile, tetraciliate, and of unequal size, the male cell or spermatozoid being about a fourth to a sixth the size of the egg cell.

But it should be noted that Phoophila has been considered to have similar tetraciliate gamete cells. Accepting it-as can quite safely be done - that these and the other genera of Chætophoraceæ, as well as Coleochætaceæ, are a mere remnant of abundant and allied genera, that once flourished so far back as archæan times, they furnish collective evidence that from the condition of motionless conidiospores, produced in cells of the cyanophyceous Chamæsiphonacer, to the motile biciliate or tetraciliate swarm cells of some Chætophoraceæ, from these again to biciliate or tetraciliate similar gamete cells of most Chxtophoracex or of Phoophila, from such to biciliate or tetraciliate sperm cells and ciliate-as in Aphan- 
ochoete-or non-ciliate and fixed egg cells with small biciliate sperm cells as in Coleochote, the transition stages are graded and suggestive.

Along this advancing chain of types the plants show green branching cellular threads that at times tend to form flattened cell plates as in Ctenocladus, Endoclonium, and some species of Coleochcete. They all possess one chromatophore of considerable to large size, or which may divide into 5-8 separate masses. All moreover are fresh-water genera fundamentally, for the few species that are now brackish or salt-water are evidently derivatives from fresh-water ancestry.

From the above cited evidence we would suggest that two main lines of evolutionary advance started which may be termed (a) the biciliate and (b) the polyciliate spermatial lines. As will be discussed later, both persisted during their earlier advance as fresh-water organisms, but gradually a palustral and then a land stage was established, that started the biciliate spermatial branches of the Bryophyta and probably the Lycopodineæ; and the polyciliate spermatial branches of the Filicinex, Pteridospermex, and Metaspermeæ. Consideration of this will be taken up in later pages. Meanwhile we will continue the probable history of the algoid derivatives.

The Cladophoraceæ, Vaucheriaceæ, and Characeæ are either wholly or largely fresh-water groups. As stated by Wille (100:117) the first suggests decided affinity through Urospora and Rhizoclonium with Hormidium and other representatives of the Ulothricaceæ, while all are allied to the Chætophoraceæ. But, like the Ulvaceæ of very different structure and affinity, a decided tendency to migration into brackish and then into salt water is shown. So there seems early to have originated, probably from the Tetrasporaceæ and more advanced types, an abundant and common ancestral assemblage such as is feebly represented now by Gomontia, Botrydium, and Phyllosiphon, and from these on the one hand started the unicellular fresh-water Vaucheria and the marine series of the Caulerpaceæ and Codiaceæ, while on the other started the multicellular fresh-water Cladophora, Cylindrocapsa, Pithophora, Sphcroplea, and the Characeæ, as well as the salt-water Valoniaceæ and the Dasycladiaceæ.

During this process of evolution the single chromatophore of tetrasporaceous and ulothricaceous character became extended over the protoplasm as a uniform or banded or reticulate expanse that developed one or more pyrenoids; or, as in some species of Cladophora, sent reticulate extensions inward toward the center; or, as in other species and in the Characex, 
Valoniaceæ, and Dasycladiaceæ, it divided up into many small chromoplasts each with a pyrenoid body.

In a sentence now we may sum up the distributional value of the green alga-excluding diatoms and related organismsby stating that 167 genera and 1924 species are fresh-water, while 59 genera and 391 species are marine. The two latter numbers include also the great majority of the most complex and modified species.

The Brown Algæ or Phæophyceæ can next be examined. These are so thoroughly marine, and so advanced in structure, that the task of connecting them with ancient forms is confessedly a difficult one. But suggestive connecting links seem still obtainable. Tracing back origins for these, as for some of the Green Algæ, to the chamæsiphoneous Cyanophyceæ, most of the genera of the latter include species of a yellowish or brown color. Further, while the majority of the species are fresh-water and of bluish or violet-green tint, not a few are marine and yellow-brown.

But, amongst living members of the Phæophyceæ, such genera as Lithoderma and Heribaudiella (Engl. Nach., p. 174) resemble, in growth of the plant individual, in cell structure, and in the arrangement as well as in the formation of terminal conidangia with conidiospores, such cyanophyceous genera as Pleurocapsa, Chamcesiphon, and Hyella, except that the former are nucleate and give rise to sex cells, while the latter have only evolving or diffuse nuclei, and are, so far as we know, asexual. Phocococcus and Phocothamnion too are interesting fresh-water phrophyceous genera, that in many points of history recall the Cyanophyceæ. The origin of the gamete cells also in Phжососcus, as described and figured by Borzi $(99,1: 14)$, indicates that formation of gamete cells with lateral cilia, so typical of the Brown Algæ, may early have started even amongst unicellular species.

So we would regard the bulk of available evidence, even though slight, as favoring a primitive and very remote origin for the Brown Algx from fresh-water yellow or brown-green ancestors of a cyanophyceous nature, and that early migration into littoral margins of varying depth accentuated brown pigment production in the chlorophyll substance. Having reached the shore area with its ceaseless wave-motions, its changing mechanical strains, and its varying light intensities, 
wide dispersal and steady though varied modification must have occurred, till in the long-drawn interval between archæan and present times there evolved those abundant species that amount to 759 , included in 145 genera. But these, unlike some of the green algæ, failed to develop higher and strongly progressive descendants, so that they form a marine cul-de-sac like many Ulvaceæ, the Valoniaceæ, the Rhodophyceæ, and as we shall see later the Spongida, Echinodermata, and other animal groups.

In the restricted though steady evolution that they have undergone, graded advances are shown in chromatophore and in reproductive cell details, that are exactly paralleled by the green algæ. Thus, in the relatively simple Ectocarpaceæ, Encoliaceæ, Chordariaceæ, and allies, the chromatophores may vary from one large semi-cylindric of pale greenish yellow hue to a few oval or strap-shaped ones, or to many that are small lenticular and of deep orange color as in the highest groups of the Fucaceæ and Laminariaceæ.

Such advance in chromatophore condition is fairly well correlated with progress in sex cell differentiation, from union of like ciliated gamete cells as in the above three first-cited families, to the Cutleriacex where-as in the green group Aphanochætacex - both gamete cells are ciliate, but the egg cell is much larger than the sperm cell. From this to the condition seen in Fucaceæ where the egg cell is large and passive, while the sperm cell is minute and ciliate, transitions can still be traced.

The red seaweeds or Rhodophycex form the remaining great algoid division. This, like the last, is so preponderatingly marine that a fresh-water origin for it has seldom been claimed. Thus Oltmanns (99, 1:537) says: "The Floridere are found scattered through all the seas of all zones, and one can scarcely say whether they prefer any one region. Many of them have become restricted to very saline regions, and aroid seas that are less salt; others, however, inhabit these, and many species of Floridere have even migrated into fresh water. Batrachospermum, for example, lives in still as well 
as running water; species of Lemanea, Tuomeya, Chantransia, and Delesseria live in cold swiftly flowing brooks, etc. That such forms have migrated rather recently from the sea will be considered probable in all parts of this book; here it may only be hinted that Bostrychia Moritziana, through its occurrence in fresh as well as in salt water, makes such migrations plausible." But the entire evolutionary trend of the red algæ suggests such fresh-water origin. In tracing this we would again commence with the Cyanophycex. Amongst the living genera of the latter many species are of a bluish violet to violet color, a fact attested to by there being seven species belonging to as many genera that bear the specific name violacea. Some of these are fresh-water, others are marine. The marine species Dermocarpa violacea may vary in its cell-contents from violet to rose-red. Amongst some of the fresh-water and marine Rhodophyceæ the color varies from bluish to purple-pink and crimson. This is due to the pigment phycoerythrin, which Kützing considered to be identical with the pigment of many purple-red or red Oscillatorieæ.

Some species of the genera Radaisia, Chamcesiphon and Hyella of the Cyanophycer exhibit a cellular aspect, a color relation, and a production of non-motile conidia that might well cause them to rank as precuisors of the Rhodophyceæ. Some of these are fresh-water, some brackish, and some marine in habitat. Some are bluish, some purplish, and some purplepink to rose-colored. But such non-nucleate and asexual organisms seem to be connected with primitive Rhodophyceæ such as the Bangiaceæ and Helminthocladieæ by nucleated but asexual or doubtfully sexual genera like Bangiopsis, Goniotrichum, Phragmonema, Porphyridium, and Cyanoderma which have adapted themselves to most diverse habitats and surroundings. The resemblance of the first named three to Bangia, Porphyra, and Erythrotrichea, specially when the latter are in their youngest growth condition, also of Porphyridium to Erythropeltis, is, we would consider, more than a coincidence. It suggests rather that, from ancient organisms related to these, the Rhodophycex have slowly originated as a side-line of evolutionary modification, which, starting from bluish violet fresh-water and ultimately violet-red fresh- or saltwater cyanophyceous or semi-rhodophyceous ancestry, have 
culminated in the highest batrachospermoid, rhodomeloid, and ceramioid divisions.

If now we compare numerically the living species of Rhodophyceæ that show simplest cellular structure, simplest chromatophore conditions, and simplest asexual as well as sexual reproduction with those that are decidedly complex in all of these points, it is seen that the class naturally divides itself into a smaller section within which fall the Bangiaceæ in widest sense, the Helminthocladieæ, the Thoreaceæ, and related smaller families. The larger section includes the ordinary red marine algæ. Now in the former section are 10 genera and 69 species that are wholly or in great part fresh-water, and 8 genera with 51 species that are marine. Such would suggest that there is at least as much evidence for a freshwater origin of the whole group as for a marine.

In the latter and much larger section 291 genera and 1975 species are wholly marine, for, while Bostrychia Moritziana and a few Delesserias may at times occur in and show capacity for fresh-water life, all indications are that they are temporary or adaptive migrants from the seashore.

Examining the two sections more in detail as to individual structure, chromatophore formation, and methods of reproduction, added points of interest appear. Thus, from the short or elongated cellular threads that make up the plant body in some chamæsiphoneous algæ, transition is got to Asterocystis and Goniotrichum in which more abundant branching and expansion occur. From these the transition to Bangia and Erythrotrichia in their young state is easy, while the later subdivision of their threads into multicellular cords, or flat or branched expanses, leads up to Chantransia, Batrachospermum and species of Lemanea. It seems also to be generally true for the fresh-water species, as for the marine ones that grow between or slightly below the zone of battering tidal action, that the thallus remains filiform, slippery, and often much branched. In deeper marine species, where the plants grow amid less agitated surroundings, a more expanded and usually flattened thallus is often formed.

In chromatophore development the simplest and most primitive evidently is seen in Goniotrichum, where instead of being peripherally placed, as in Cyanophyceæ, the single chromatophore has assumed a central position, but sends out radiating arms toward the periphery. In Bangia and allies it is similarly placed, but the star-arms become more extended, and at the periphery of the cell form longitudinal bands which run in the peripheral protoplasm. In Chantransia, Nemalion, 
and allies a like disposition is seen, but the longitudinal bands may branch, as they run over the periphery of the cell, a condition well shown in Helminthocladia.

When we pass to the marine genera, the chromatophore becomes divided, and later on increased in number till many small rounded or stellate masses result. Thus in the genus Rhodochorton there may be two spirally wound chromatophores in some species, or from 6-12 stellate ones in others, each embedded in the peripheral protoplasm and bearing a large pyrenoid center. In other of the marine genera, e. g., Ceramium and Polysiphonia, the several chromatophores may assume diverse shape according to the size and position of the cell.

In the development of reproductive organs a like evolutionary advance is shown from simple fresh-water to the most specialized marine genera. Thus, if we start again with the Chamæsiphoneæ, the genera Chamoesiphon, Radaisia, and Hyella derelop non-ciliate monospores from enlarged cells of the thallus, as well as numerous and smaller conidiospores. In Phragmonema, Goniotrichum, and allies usually placed with the red algæ a similar origin of monospores occurs, but sexual cell formation has hitherto not been observed, though such may exist. In Bangia and allies monospores are formed in a variety of ways, though all resemble fundamentally those named above. But here sexual reproduction is also effected. In this case cells that differ little from the spores, except that they are much smaller, become physiologically differentiated as spermatia or motionless male cells, while other cells of the thallus that differ little from the vegetative cells, become richly protoplasmic and evidently attract a spermatium by energotactic action. The fertilized egg forms a 1-8 celled spore-fruit that soon is liberated.

In passing now through the simpler and mainly fresh-water genera to those that are marine, a progressive complexity alike in spermatial and egg production is observed, the main stages of which are figured in the accompanying illustrations. In Lemanea the spermatia vary from a few to a dense cluster of surface cells, while in addition to the egg cell there are $2-10$ accessory carpogone cells, the whole somewhat sunk in the thallus. The fertilized egg produces loosely clustered chains of carpospore or spore-fruit cells (Fig. 10).

In Batrachospermum and Chantransia there may, as in most of the above, be monospores formed, and often in clustered fashion. Or in some species of the latter genus tetraspores arise by subdivision of a primitive monospore cell. The sex cells consist of densely clustered spermatia and of clustered 
carpogonia that project amid cellular threads. The fertilized egg gives rise to dense clusters of carpospore cells.

In transition to the marine groups the formation of tetraspores amongst these is an almost invariable feature. The clusters of spermatia are produced as prominent terminal masses on special branches. The increasingly complex carpogone with subsequently formed cystocarp and enclosed carpospores have been so often described in text-books that the accompanying figures will suffice to illustrate them (Fig. 10).

A short summary may now be made of the main details of the present chapter. In connection with arguments advanced already here the position has been taken that living organisms originated during the archæan age, amid warm thermal waters, and by gradual colloid synthesis. The primitive organisms were probably colorless bacterial forms similar to the sulphur and thermal bacteria, and from these by slow evolution the unicellular Blue-green Algæ originated. The latter consisted of a cylindrical zone of pigmented protoplasmic substance, which surrounded a central mass of clear protoplasm or synthesizing substance. The pigment zone seems to have functioned equally as an absorber, a condenser, a transformer, and a storing area for energizing food material.

The cyanophyceous organisms probably originated from more primitive bacterial ancestors first in thermal fresh-water areas, and at temperatures of $75-50^{\circ} \mathrm{C}$. We have accepted it that 41 existing species which still live under such conditions are probable direct descendants of these. By acclimatization to cooling terrestrial conditions in fresh-water, on moist surfaces, or in shady moist places, and later in the sea, a wealth of species probably evolved during mid-archran times that became the ancestors of all green plants, while the preponderating wealth of species now living in fresh-water or moist land areas, as compared with the much smaller number of marine species, seems to be a correct index to their primitive environment.

From green or reddish green unicellular Cyanophyceæ we have traced what are probably the simplest nucleate green algæ like Pleurococcus and Tetraspora, while from olive-green 


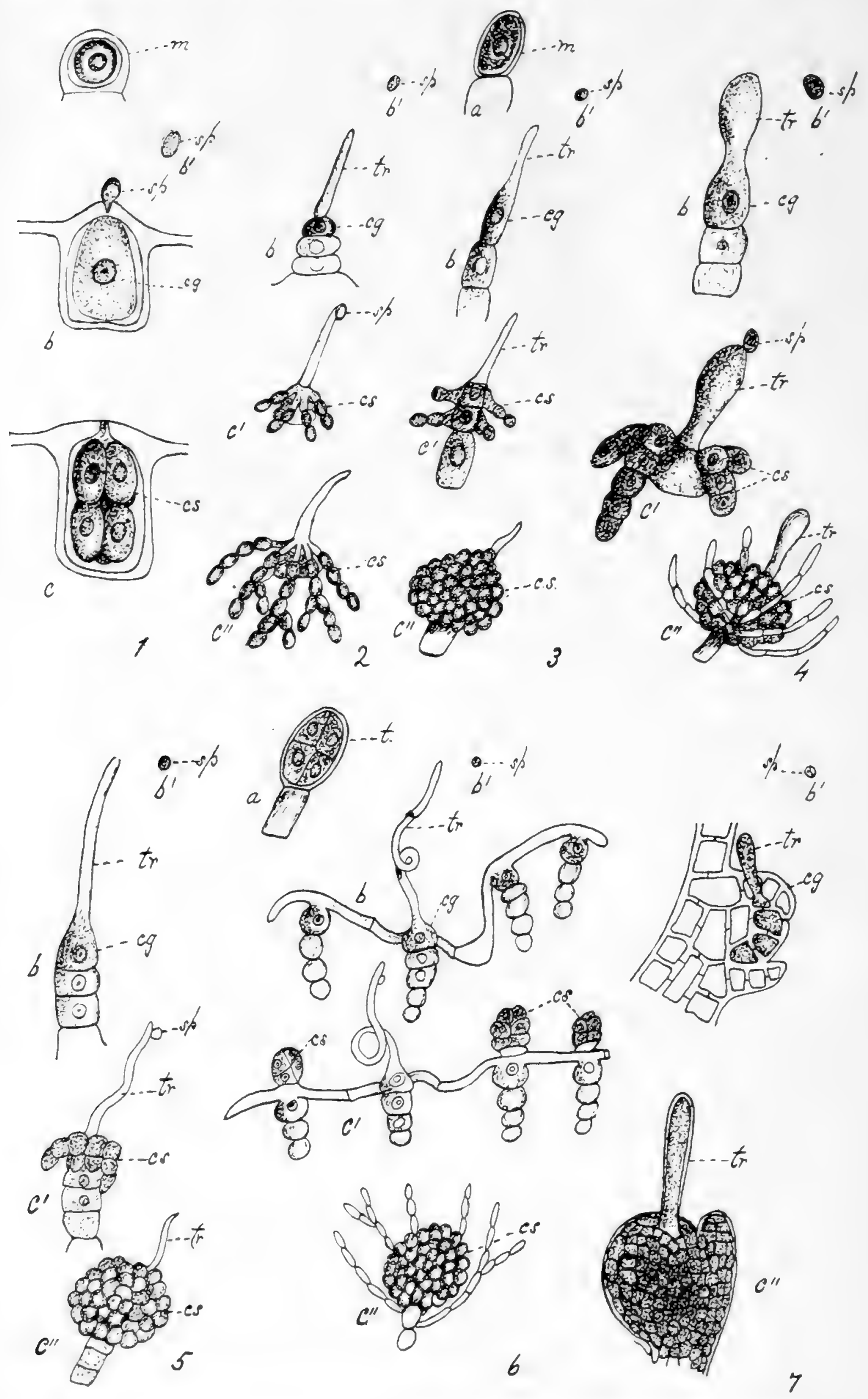

Fig. 10. - Stages in evolution of the asexual and sexual generations in Red Seaweeds. 1, Bangia atropurpurea; 2, Lemanea torulosa; 3, Chantransia sp., 4, Batrachospermum; 5, Nemalion, 6, Dudresnaya; 7, Rhodomela (after Falkenberg); $7 c^{\prime \prime}$ Chondria (after Bornet). $1 \mathrm{~m}$. 3m., monospore, 6, a, tetraspore; $b$, female apparatus with carpogone cell bearing trichogyne $(t r) ; b^{\prime}$, spermatium; $c^{\prime} c^{\prime \prime}$, carlier and later stages in formation of carpospores from fertilized carpogone cells. 
or bright green unicellular members of the same division the Desmideæ and higher green Conjugatæ probably sprang, and these have practically all retained a fresh-water environment. From protococcaceous ancestors we have derived the Volvocaceæ that are fresh-water and also represent the climax of unicellular specialization. From some simple unicellular greenish brown fresh-water genus like Amphidinium, derived in turn from pleurococcaceous ancestry, the four divisions from Gymnodiniaceæ to Diatomaceæ have apparently arisen. A large number of these, and the simplest genera of them, are still fresh-water, but many have permanently become marine livers. These last have given rise to many and raried marine species, but have failed to evolve higher organisms than themselves.

But, from fresh-water ulvaceous, cladophoraceous, and siphoneous divisions, migrants shoreward originated the abundant genera and species of green marine algæ, which failed however to originate higher organisms than themselves. On the other hand the Aphanochætaceæ and Coleochætaceæ amongst higher fresh- $\pi$ ater algæ have been regarded as related to ancestral organisms, from which the primitive Bryophyta and Pteridophyta have probably originated, whose morphological continuity we shall endeavor to trace in the next chapter.

The probable origin of the brown seaweeds has been traced from a yellow-brown chamæsiphoneous ancestry, through fresh-water genera like, or allied to, Phoococcus and Phoothamnion, on to marine derivatives of increasingly advancing structure, and that ramified into a wealth of species, but failed to evolve higher organisms than those of the class. From bluish, purplish, or pink Cyanophycex of fresh-water habitat, the red seaweeds have started, for commecting genera that are now alive serve well to link all into a continuous organic chain. But while most of the simplest groups are mainly fresh-water, or still retain some representatives there, diverging lines from some of these early took on a marine existence, assumed a fixed red or claret color, and produced a great wealth 


\section{Causes and Course of Organic Evolution}

of genera, but failed to evolve higher organisms than themselves.

In the diagram that forms Fig. 11 the phylogenetic affinities and derivations are set forth graphically. If now, in connection with the table already given (p. 305), the algæ be divided into a simpler and a more complex or advanced group, that we may term the Archæophycere and the Neophycere, we find that 221 genera and 2809 species that are freshwater, and 30 genera with 99 species that are marine, are included in the former group; while 62 genera and 1502 species that are fresh-water, and 623 genera with 5570 species that are marine, belong to the latter. Comment on such a fact is unnecessary.

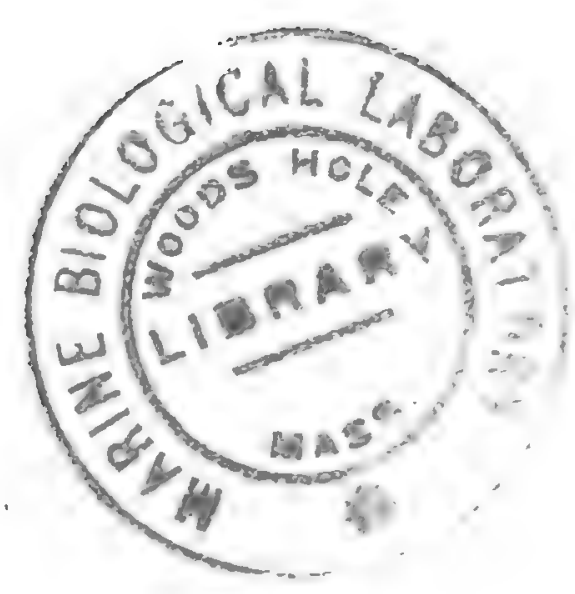


Frestl-ivater on Latro Forms

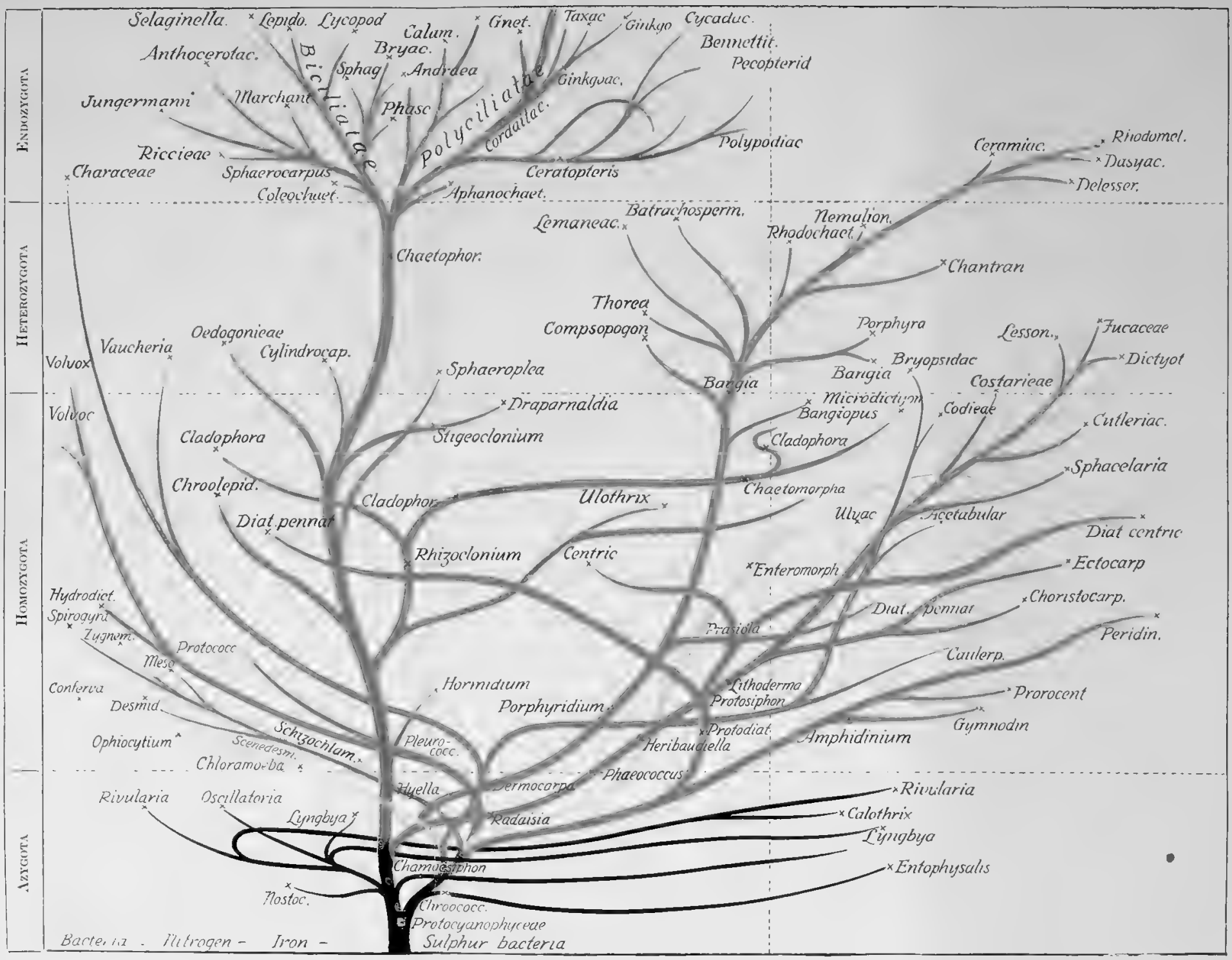

l'IG. 11.-Genealogical 'f'ree of Plant Genera and Groups as determinel by recent and fossil forms. Group names often contracted. 



\section{CHAPTER XII}

\section{THE EVOLUTION OF PLANTS (Continued)}

The oft-expressed view that the ocean has been the great center and originator of living bodies is almost wholly negatived when a study of the bacteria and fungi in general is made. For, since practically all of these depend on organic food for sustenance, it might be supposed that many marine plants and animals would habitually be associated with definite bacterial or fungoid plants. But, comparatively speaking, they are almost wholly absent from the sea. For, while at the present day 31 genera and about 600 species of bacteria live in fresh or brackish water, or saprophytically and parasitically on land plants and animals, only four genera and about 50 species are marine.

Again, while 2670 genera and about 24,000 species of fungi are distributed over land or swamp areas, the estimate is generous which would place the marine ones at ten genera and about 200 species. The recent recording and description of two new marine species from California indicates that a few may from time to time be added, but the number undoubtedly will always be small.

There seems therefore absolutely no reason for supposing that either the bacteria or fungi originated other than over land-locked areas. An attempt may therefore be now made to account for their primitive origin, in terms of what we can ascertain as to their life-history.

We have already postulated the origin of the cyanophyceous algæ amid warm thermal springs of mid-archæan age, and at a temperature in some cases of $60-75^{\circ} \mathrm{C}$. since living representatives still show such ancestral capacity. But in manyprobably it will be demonstrated in all-thermal waters of $60-80^{\circ}$ C. a variety of bacteria exist and multiply, the opti- 
mum for their growth and multiplication being at $60-70^{\circ} \mathrm{C}$. This physiological series of the "thermophile bacteria" includes also those that vegetate and multiply best at $50-70^{\circ} \mathrm{C}$. and which are abundant as well as world-wide in distribution amongst soils, decaying and fermenting regetable remains, animal manure, and other heated organic products. The studies of Globig (115: 294), of Miehe (116), of Kruyff (117: 65 ), and of Ambroz $(111: 257)$ in particular show that all of these have great capacity of adaptation to, and endurance under, varying environment; that types are included which belong to the genera Bacillus, Bacterium, Beggiatoa, Leptothrix, Actinomyces, etc.; and that the nutritive relation may vary from one that is autotrophic, as with the sulphur-iron bacteria, to one that is eminently heterotrophic and dependent on organic decay, as in the "hay bacillus" (B. calfactor).

Naturally we would consider that the former or autotrophic group is the more primitive. Now, in nearly every study that has been made of thermal springs, observers have noted a gradation of color variation in the organisms from pale colorless ones, that flourish nearest to the warmest water, to those furthest removed, that are of a bluish or an olive-green. Thus speaking of the western thermal springs Weed (14:619) says that the organisms "appear first in skeins of delicate white filaments which gradually change to a pale flesh-pink further down stream. As the water becomes cooler this pink becomes deeper, and a bright orange, and closely adherent fuzzy growth, rarely filamentous, appears at the border of the stream, and finally replaces the first-mentioned forms. 'This merges into yellowish green, which shades into a rich emerald farther down."

Again Rabinowitsch (118: 154) found that, of eight species examined by her, the color varied from white through grayyellow to brown, red, and gray-green. Others in different parts of the world have referred to like phenomena. In view of such evidence the question may well be asked: Are the Cyanophycex the simplest and also most primitive organisms; or are we to regard the pale and specially the colorless 
inhabitants of the warmest waters of thermal springs as conforming to such conditions? Much can be said in favor of the latter view. Thus so far as surely determined the cell of a sulphur, of an iron, or of a silicate bacterium consists only of a cell-wall that would correspond much in aspect and relation to the colloidly precipitated membrane of a Traube cell, and of a finely granular or at times vacuolated foam-like protoplasm within. In living examples the granules seem at times to be particles of food, at times as in sulphur bacteria pure sulphur masses. Some however, by staining and by chemical tests, seem to show chromatin granules that may represent evolving nuclear substance, as yet in diffuse state.

But the sulphur or Thiobacteria, when living amid free hydrogen sulphide, are able to utilize the latter for their life needs. For, as Fischer (39:67) well puts it, "seeing that a solution of $\mathrm{SH}_{2}$ in water is very easily decomposed by the oxygen of the air, and free sulphur deposited, the sulphur bacteria would be able to take advantage of this oxidation for their life processes, even if they merely had the power of existing in $\mathrm{H}_{2} \mathrm{~S}$ solution. The $\mathrm{H}_{2} \mathrm{~S}$, entering their cells, would be oxidized by absorbed atmospheric oxygen, and the sulphur set free would represent an abundant source of energy for further oxidation." Then "the oxidation of this sulphur represents a very abundant supply of energy, a supply that is probably far more than sufficient to cover the wants of the organism living under the conditions already described, and building up its protoplasm from the minute traces of fatty acids and ammonia contained in the water it lives in."

Autotrophic action evidently occurs also in the iron and the siliceous organisms. It seems therefore highly probable that these, even more perfectly than the Blue-green Algæ, represent the most ancient organisms now left to us.

But the pink and purple bacteria probably are derived from the colorless sulphur ones by slow elaboration of a pink pigment in the outer cell region that is sensitive to and capable of absorbing red sunlight or even infra-red or dark rays as a source of energy. So "the Erythrobacteria have therefore 
a double source of energy, the oxidation of sulphur and the absorption of sunlight or infra-red light, a great advantage to them in the struggle for existence, inasmuch as they would be able to subsist in places where, from want of sulphuretted hydrogen, the colorless Thiobacteria would perish."

Gradually then a yellowish or etiolin and later a greenish or chlorophyllin pigment may have been elaborated, as increasingly perfected screens for absorbing the sun's rays and transforming these into biotic energy. If such be true, the succession of color growths in many thermal springs would be a living picture still left to us of the graded steps in colorerolution from colorless autotrophic organisms in hot springs, through pink to pink-yellow, yellowish green or yellowish bromn, on to emerald-green in cooler waters as already stated.

So, from primitive colorless cells or cell-threads, cyanophyceous organisms with colored peripheral chromatophore may well have originated. During such changes the plant organisms would less and less depend on sulphur and iron compounds as sources of energy, and would more and more depend on absorbed and condensed sunlight as the source of energy. But the continued need still of iron, in order to the formation or activation of chlorophyll, or even in complex union as a conductor and condenser of energy, may possibly be explained as a hereditary chemical relation that is still retained.

Whatever the primitive origin and mode of sustenance of plants may have been, however, it seems undoubtedly true that they evolved in fresh-water, not in marine, areas. For, even if it be shown that Drew's marine chalk-precipitating bacteria ("Science" N. S. XXXV (1912) 441) derive energy or food-materials from the ocean, this could quite be a migrant marine derivative from earlier autotrophic fresh-mater organisms. The more recent studies of Taughan seem to emphasize this.

It might well be argued however that the Cyanophycex are the primitive plant organisms, and that the pink and colorless ones are degraded derivatives from these. From our fairly intimate knowledge now of parasitic plants that are 
derived all along the scale of plant life by degradation change from autotrophic plants, such a view has considerable in its favor. Then it might be claimed that from an emerald-colored chlorophyll slow degradation changes through greenish yellow to yellow-pink, pink-white, and finally to white took place. In the process also transition might conceivably have been made from the decidedly specialized mode of chlorophyll elaboration to the simpler one of sulphur or iron energizing action.

Against such a riew should be placed the fact that parasitism or saprophytism, even amongst fungi and higher plants, always leads to a dependence on increasingly rich sources of organic food, not on comparatively simple inorganic material. It seems quite probable therefore that the sulphur, iron, and siliceous bacterial organisms represent the most ancient and the simplest existing types of life, from which or from nearly related types others have been slowly derived.

But as plant life increased in quantity, and steadily underwent decay, derivative species and genera of colorless bacterial organisms may have evolved, which biochemically slowly adapted themselves to a saprophytic, next to a facultative epibiotic, and finally to a holoepibiotic life.* But even to judge from examples of Blue-green Algæ now living, such as species of Dichothrix and Calothrix, it seems fairly assured that not a few species passed from a green state to a colorless condition of saprophytism, and still later to a semiparasitic or a parasitic state.

We may therefore say for the bacteria that the simplest genera consist of a wall enclosing vacuolar or finely foam-like protoplasm in which there may not be, or there may be, distributed chromidia or chromatin granules. Some of these

* In discussing the possible origin of animals from a primitive plant ancestry, it is important to distinguish between attaching parasitism and the ver different relation where a free colorless organism moves actively in search of food. The former state seems always to be associated with advancing degeneration, and to it strictly the term "parasitic" should alone be applied. The term "epibiotic" may then be used in connection with colorless organisms that move freely in search of, and live directly on, fresh plant or animal tissues, and epibiotism as the condition. 
genera are capable of chemical auto-nutrition by utilization of sulphur or iron compounds as sources of energy, by which food material can be elaborated. Others show varying degrees of pigment formation through a pink or bacterio-purpurin phase to a yellow, a yellow-blue, a blue-emerald or emerald stage, in which latter three absorbed sunlight becomes increasingly utilized as a source of energy for formation of food compounds.

So it seems probable that the simpler blue-green and emerald-green cyanophyceous algæ, with their peripheral chromatophore, and frequently skene or wreath-arranged chromatin threads, represent a slow evolution from more primitive colorless types, in which chemico-energesis has been replaced by lumino-energesis. With increasing abundance of organisms and their subsequent decay, some of the colorless autotrophic bacteria assumed a saprophytic role, and still later through the stage of facultative parasitism many became purely parasitic, while others through a stage of facultative epibiotism became largely or wholly epibiotic, and so originated animal organisms.

The pleomorphism and enormous abundance of living fungi is one of many parallel proofs that fresh-water and more especially land organisms always tend to vary more, and to give rise to advancing and dominant lines, than do marine organisms. So the 2670 genera with 24,000 species of existing fungi far outnumber the entire assemblage of the algæ.

But the mode of origin of these has been variously accounted for. We accept fully the view, propounded by De Bary, Sachs, and many since, that the fungi are dechlorophyllized offshoots from the algæ, at different stages in the evolution of the latter. For, with advancing critical investigation of algoid and of colorless or fungoid forms, the more perfectly do we recognize the close continuity, structurally, that exists between both. Thus of the Pleurococcaceæ, Protococcaceæ, Oocystacex, and Volvocacex, that we have regarded as groups connecting the emerald Cyanophycese with the higher green algæ (pp. 254, 308), pale green or colorless genera are now known, like Eomyces, 
Harpochytrium, Prototheca, Polytoma, and Tetrablepharis, that live in fresh-water and on decaying organic material there. These again show decided affinity with some unicellular or mucilaginous multicellular fungi.

Not a few, however, of the Chætophoracex and Chroolepidaceæ are epiphytic or endophytic on other algæ, on Sphagnum moss, or on a variety of organic bodies, while a few like Cephaleuros seem to be largely parasitic. The close resemblance in structure and life history between some of the conjugate algæ and the conjugate fungi, or between such siphoneous fresh-water algæ as the Oogonieæ and corresponding fungi, has often been remarked.

But, as to the supposed near affinity, that some have claimed, of the red marine seaweeds with some ascomycetous fungi, we believe that several fresh-water lines of derivation can well be traced, any one of which may have originated the now very heterogeneous divisions of fungi that show spermatium and trichogyne union, or formation of ascospores. Thus in several genera of Chætophoraceæ elongated hair-processes are produced from zoosporangial or neighbor cells, and so in rather simpler types, intermediate between these and cyanophyceous organisms in which sexual differentiation was just developing, passive spermatia that united with such hairs, in order to reach or pass their contents to a central cell, may have been the starting points for such organisms as the Laboulbeniacex.

Again the elongated thread-like neck formed by the oogonium of Coleochcete may have remained closed, and have been associated with spermatia in some rather drier types that became largely land forms. But many now extinct genera allied to Bangia, Lemanea, Sterrocladia, Batrachospermum, and Chantransia must also have evolved and existed in fresh water at early periods and in great abundance, if present-day evidences are to form any key to the past. The growth of these at first epiphytically, and later parasitically, especially in their early growth-stages, may have started a wealth of fungoid organisms, before the dawn of the cambrian epoch.

By slow but sure proenvironal adaptation,"mainly of a chemotactic kind, to decaying organic material and alternately to a 
living host, or later to two alternating hosts; by formation of several kinds of asexual gonidial cells and of sex-cells; by exposure at one season of the year to one set or intensity of environal agents, and at another season to a different set; by variations in the host-plants, or in the organic nutritive medium, and reflex variation in the fungus; that remarkable wealth of species has evolved, to slight degree in fresh water, to a very small degree in salt water, and in exceptional degree on land, that are now omnipresent and preponderatingly rich in individuals.

So, from all the experimental and observational evidence to hand regarding the bacteria and fungi, we would conclude that these show abundant hereditary characters, but that they also show variation-effect owing to action and reaction between them and their environal surroundings; that, in constantly responding to the sum-total of most satisfying environal agents, they have projected proenvironal variations that have given rise to new species; and that during the process other types of the fungi, that were imperfectly suited to their environment, have died out. The last result would frequently occur when some species of plant or animal that acted as a host, and on which some fungoid species was holoparasitic, died out. But it is as true that, when plant and animal species increased and occupied every suitable area, the production of new bacterial and fungoid species would also be possible. This it is which explains the enormous number of species now alive, as compared with the algæ, from which the fungi were primitively derived according to the above accepted views.

The exact chemico-physical and biological correlation that exists between most bacteria and fungi on the one hand, and their hosts on the other, is well shown in the nitrifying, the ginger-beer, and the leprous bacteria, also in the potato fungus, the black knot fungus, and the oak Dodalea (Drdalea quercina) amongst many others.

But, before passing from the fungi, special attention should be given to the fact that, in spite of their ancient origin-as rare palxontological evidence sufficiently testifies - they have 
remained almost wholly a fresh-water or terrestrial group of organisms that have only rarely adapted themselves to marine life. Such we regard as one of many proofs that sea-water and a marine life are conserving and disseminating agents, but permit of limited variation and slow advance by any one group, and even of frequent extinction of whole genera or tribes, through such environal changes as rise or fall in temperature of sea water, volcanic submarine activity, discharge of submarine gaseous effluvia, and other causes. A freshwater or land origin for all of the dominant lines of advance is thus correlated with a steady increase in species of saprophytic and parasitic bacteria and fungi, that are closely associated with such lines.

We have already accepted it that, while the green, the brown, and the red algæ have all descended from a cyanophyceous fresh-water ancestry, the latter two early passed-possibly in archæan times-in large measure into a marine environment and have there slowly undergone subsequent variation, evolution, devolution, or extinction. The green algæ continued in fresh-water surroundings and in preponderating numbers, so that now there are 172 fresh-water to 59 marine genera, and 1924 fresh-water to 391 marine species, or there are about three times as many genera and five times as many species of green fresh-water as of marine algæ. So it is to fresh-water green algæ that botanists have turned in recent decades for the origin of higher plants.

An attempt will now be made to trace the main evolving lines, and to note morphological modifications that have accompanied these. Here it can be at once clearly and unhesitatingly postulated that rapid and advancing migration of plants took place from a shallow fresh-water to a marshy and later to a dry-land situation, and that this must have been effected largely during later archxan times, and accentuated during the cambrian epoch. For the appearance of scorpions and insect-life, along with highly organized plant remains, in the silurian rocks necessitates a long previous period of evolutionary activity on land. 
Examination of living plants higher than the Thallophyta, in all of their life phases, and consideration of this in the light of palæontological evidence, equally suggest that two probable series of organisms early started from a related green algoid ancestry, and progressed along diverging pathways of ascent. One of these probably originated the classes of the Hepatica or scale mosses, and the Musci or leafy mosses, also the Lycopodinex or club mosses and selaginellas. These will be spoken of as the Biciliatce. The other gave rise to the ferns and waterferns, the Cycadofilices and Gymnospermia, finally attaining a climax in the higher seed-plants or Angiospermia of our existing flora. These will be spoken of as the Polyciliato.

Statement was made earlier (p. 314) that Coleochote and A phanochcete might be viewed as nearest living representatives of organisms that started the above. In comparing them and the higher organisms that we consider they led up to, a point of great morphological interest claims attention. We refer to the graded evolution of alternating generations of sexual or gametophyte, and of asexual or sporophyte growths, in the life-cycle of each individual, and the number of chromatin masses or chromosomes that respectively characterize the cells of these two generations.

A more detailed study of this has been made in a previous chapter that deals with sex-evolution. But shortly it may be indicated here that, from the next higher class to the algæ, namely the Hepaticæ, upward to the highest plants, the cells of the plant body or gametophyte that culminate in production of sexual cells show, during each cell division, half the number of chromosomes up to the point when the egg and sperm fuse. Since each of these introduces as many chromosomes as were typical of the parent plants that bore them, the fertilized egg that is the climax of the sexual or egg-producing or gametophyte generation shows double the number that it or the cells that produced it had, up to the moment of fertilization.

But the fertilized egg on segmentation, while retaining the double number, gradually builds up a new structure that is known as the asexual or spore-producing or sporophyte gener- 
ation, which reaches its growth climax in the formation of spores, and it shows, up till then, the double number of chromosomes at each cell-division. Therefore we speak of the egg-producing and related cells as having a haploid number of chromosomes, the succeeding or spore-producing growth as having a diploid or doubled number.

As the spores mature, however, they throw off and break down so much chromatin material that on final maturation they show only half the number of chromosomes. So when each spore has matured and germinated it gives rise to a new sexual generation with haploid chromosomes, that in turn reaches its climax in the production again of male and female sexual cells, each with a like number.

In beginning now to trace what we would regard as the probable and approximate steps of advance from the algæ upward, it is a matter for regret that we still know too little as to the above haploid and diploid cytological details, for the different genera involved. But so far as accurately known they will be utilized.

While accepting. Coleochote, with Pringsheim and many successors, as the nearest living representative of those algæ that advanced from the Mycoidaceæ or Pleurococcaceæ to the degree of bryophytic or moss dignity, there is no need to view Coleochoete other than as a related organism to the main line of bryophytic advance. The nearest living group of higher grade than it seems to be the Ricciaceæ, a simple division of the Hepaticæ. This means however that many intervening types must have become extinct, the approximate structural characters of a few of which we may vaguely attempt to outline, and so one or two of these are suggested below.

But in this evolving advance two fundamental features are revealed. First it may be said that all future specialization and modification in plant-forms occurred to a diminishing degree in fresh water, and to an increasing degree on a moist or even fairly tenacious substratum, and ultimately on land. So a great and ever multiplying congeries of marsh and ultimately terrestrial genera originated which only in rare cases sent isolated outliers toward or into the seashore. 
Not only then is the eridence for a fresh-water origin of plants preponderatingly perfect, if we regard mere number of species and genera now existing, their entire evolutionary and palæontological history emphasizes this.

A second feature is that the sum-total of the forces that represent an average land environment constitutes a greatly more stimulating, molding, and proenvironing medium than does sea rater. For, though a few botanists have suggested the derivation of sex cells, or even of important plant families of dry land habitat, from the brown or red algæ, nearly all are agreed that a fresh-water ancestry in every respect suits the case.

The transition from a fresh-water to a marshy and at length terrestrial environment must have been accompanied by changes in the relation of the plants to environal conditions, that could not fail to fundamentally influence, and stamp themselves on, the organisms. The development of cuticular protective tissue, of indurated strengthening strands or bands or even sheets of fiber cells, the formation of transpiration pores, the restriction largely or wholly of water absorption to the lower or geotropic part of the plant, and of sap transpiration to the upper or heliotropic part, are only a few of many changes that must gradually have been effected.

But seasonal and environal rariations during the annual growth-period are much more pronounced in their action on fresh-water alga than they could possibly be on marine ones; while the crowding of the former on to a drier substratum, or their proenvironal tendency to accommodate themselves to this, would still further accentuate the forces of variation in their action on fresh-water algæ. Such suggest an explanation, as several writers have pointed out, for the origin of that striking phenomenon, in classes of plants higher than the algx, that is now known as "alternation of generations." For the phase in the complete life-history of members of the moss and fern alliances, that we now call the sexual or gametophytic (characterized also by a reduced or haploid number of chromosomes) generation, suggests throughout adaptation 
to an aquatic life, while that known as the asexual or sporophytic (characterized by a full or diploid number of chromosomes) generation as clearly suggests advancing adaptation to a land life.

In returning now to a study of the relationship between the green algæ and the Ricciacer, we would accept it that, while Coleochote indicates valuable transition characters, large groups of connecting genera, that would have enabled closer connection to be traced, are now extinct.

In all of these the germinating spore probably formed first a swarmspore tube of passive character (p. 340), that became in the higher examples the "protonema." This in turn gave rise, by repeated divisions, to a somewhat flattened thallus, that either grew radially into a condensed dorsiventral mass, or by elongation produced a thread-shaped body that lengthened dorsiventrally. In all of them simple cellular downgrowths from the mass became attaching organs or rhizoids, while apex cells by continued division produced dichotomous or false dichotomous branches. Each vegetative cell thus produced retained a single chromatophore with pyrenoid center in Coleochocte, and this is retained in the Anthocerotex group of the Hepaticæ, that for many reasons should be regarded as an early derivative from protohepatic organisms, and that evolved separately from the Riccieæ. But in the last the originally single chromatophore of each cell divided - as already shown to have happened in higher green algæ -into several separate masses.

The sexual plant body in Coleochoete is usually of one layer, but a tendency toward two layers is seen in types like $C$. scutata. But in Thallocarpus and Sphococarpus, both simple genera of Hepaticæ, the thallus consists of a single cell layer, except along the middle line, where it is subdivided into several zones. Extinct forms, in which two to many cell layers developed, must have connected both of the above with the Ricciex, whose thallus now consists of six to ten similar or more often dissimilar zones, the lower being made up usually of closely united pale cells, from which attaching rhizoids and cellular scales arise, while the upper is generally spongy, and contains abundant chloroplasts in the cells.

In all of them outgrowths from the upper surface of the thallus appear as fine delicate hairs, that are probably sensory in function. 
In all, multiplication by gonidial or bud cells has been a typical condition. In the aquatic genus Coleochoete most of the vegetative cells of the thallus can become modified under certain conditions for this purpose, when the entire contents of each cell become rearranged, and a pair of cilia is formed. The mass escapes by rupture of the parent wall, and, after a free-swimming stage, fixes down and divides to form a new plant. In connecting types between this and the Ricciexthat became in large part palustral or sub-terrestrial-the production of two cilia ceased, so that mainly from the lower layers, more rarely from the rhizoids or from cells of the plant body, bud cells or groups of bud cells formed, that detached from the parent plant and reproduced it.

Study of the sexual organs shows that numerous connecting types have entirely disappeared. But the accompanying diagrams suggest a graded series that very probably existed, and which may have united genera related to Coleochcete with others allied to the Riccieæ. In $C$. scutata each antheridial mother cell has its contents rounded off into a spherical or oval mass, the spermatozoid, which develops two cilia, and by rupture of the parent wall this escapes into the watér. In hypothetical forms now extinct, a stage doubtless existed where each antheridial cell gave rise -as in some other living green algæ-to 8, 16, 32, or more spermatozoids. Each of these spermatozoids seems also to have assumed a more elongated shape for passage through the water, and even more for penetration of the egg-cell and its accessory growths.

But in gradual elaboration of the antheridium, and through modification of what were once peripherally placed sperm cells probably, the peripheral cells in such an antheridial body ceased to mature as spermatozoids, and became protective strengthening or bounding cells (fig. 12). This persists throughout the whole of the Bryophyta and higher groups as the multicellular antheridial wall (fig. 12, 3a-6a). The contained spermatozoids became elongated and even curved in shape, at the same time that they retained their cilia. From such a condition to that seen in the Ricciese the transition is an easy and progressive one, and is correlated with reduction in size, and conversion into a thin coiled body, for the spermatozoid.

The origin of the archegonium in the Hepaticx can be traced with tolerable certainty from the simple oogonium of Coleo- 
chœte. The latter arises as the end cell of a cell row, and is at first conical. Gradually the apex of the cone elongates greatly into a narrow neck, while the basal part swells out as an oogonial cavity, that encloses a rounded egg. Rupture of the tip of the neck and subsequent passage of a spermatozoid down the neck
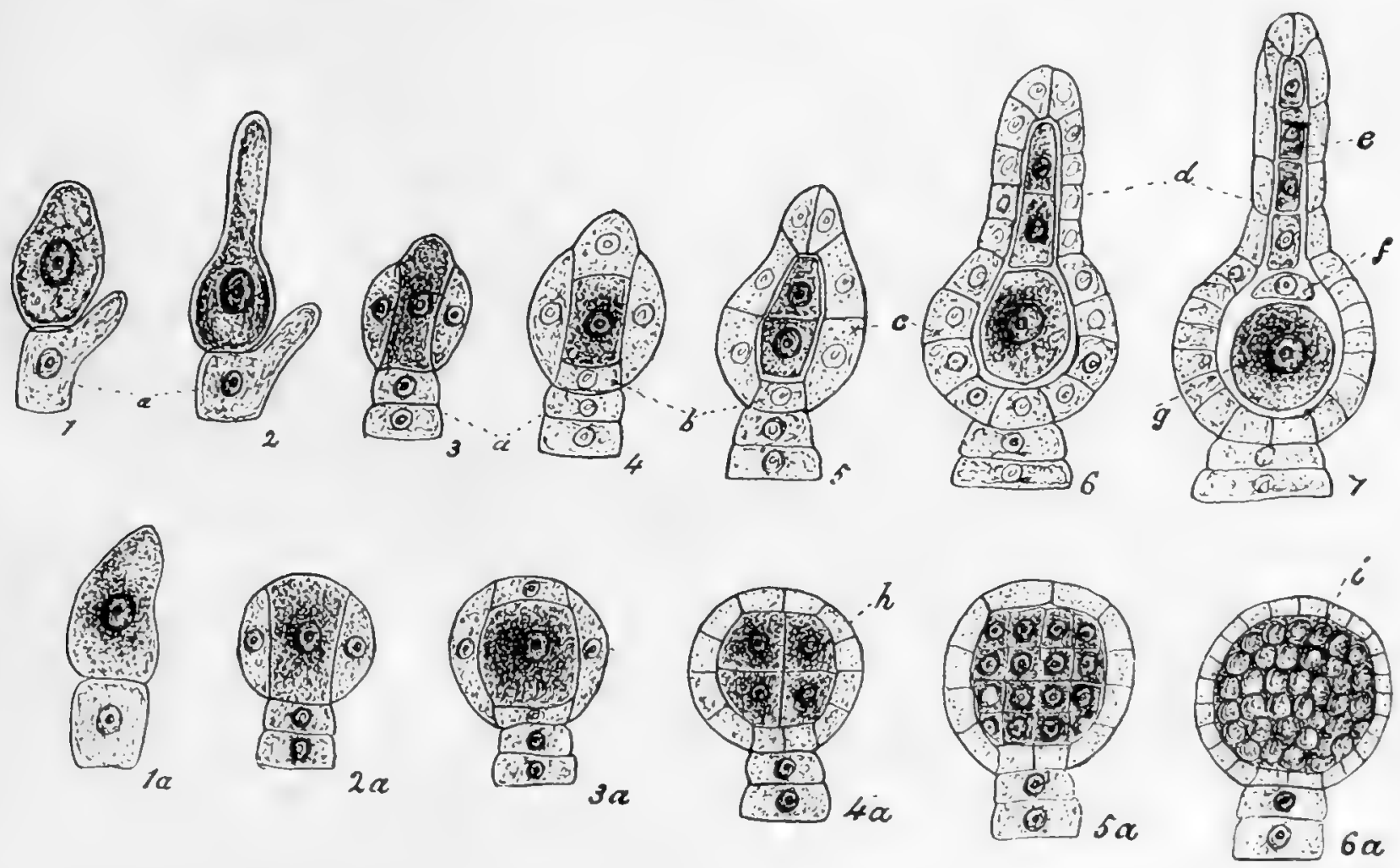

FIG. 12-Diagrams of probable stages in evolution of archegonium and antheridium from coleochætoid to sphæroriccioid and ceratopterid types. 1 young, 2 maturing oogonium of Coleochate. 3-7 stages from last to archegonium of primitive Hepaticæ. 1a maturing antheridium of Coleochoete. $2 a-$ 6a transition stages to Hepaticæ. a stalk cell, b ventral cells, c wall cells, d neck cells, e neck-canal cells, $f$ ventral canal cell, $g$ egg, $h, i$ maturation of spermatozoids.

effects fertilization. In hypothetically intermediate and extinct examples, peripheral cell segments must have been cut off as in the antheridia, to form a wall layer; while the oosphere, by cutting off accessory cells in line with itself, gave rise to a set of conducting and chemotactically secreting cells, that could guide the spermatozoid down the now multicellular neck to the egg (Fig. 12, 4-7). Finally the perfected archegonium of the Ricciex and other Hepaticx has become a stable and practically uniform structure throughout the entire series of the Bryophyta. 
The changes subsequent to fertilization, and that lead up to formation of the alternating or sporophyte generation, are at present by no means so easily explicable. Pringsheim and Oltmanns have shorn that in Coleochote the fertilized egg divides and redivides till 8 "octant cells" are formed. But, if the chromosomic cell details of $C$. scutata have been correctly interpreted, such might suggest that a very short-lived sporophyte generation in it, that closes when division into octants is about to occur, becomes extended in other species-as Lotzy $(119,1: 193)$ has suggested-to include the group of 8 or in some cases even 16 cells.

If such be correct, and considerable evidence can be advanced in favor of it, a more complex advance next toward Riccia mould be the formation from and around these 8-16 cells of an enclosing wall-layer, the sporogonial wall. For it should be noted that the zone of pseudoparenchyma formed round the fertilized egg of $C$. pultinata is a purely accessory structure, and even suggests, by its formation, the view that Coleochote is a surviving type along a lateral line of green algoid advance, rather than along the direct pathway.

In Riccia the fertilized egg divides and redivides to form an octant mass, which by continued division in time gives rise to a wall-layer and numerous enclosed spore cells, the whole forming a subspherical mass inside the archegonium.

Each of the 8-16 zygospores of Coleochcte, when mature, bursts and sets free a biciliate swarmspore, that after a time fixes down to form a tube or cylindric cell. This then produces a sexual or gametophytic plant at its extremity. But, in passing through protoriccioid forms that frequented drier surroundings, the germinating spore seems to have gradually changed from a ciliate swarmspore condition to that of a nonciliate cylindric cell, that early fixed to a substratum by rhizoids, and which constitutes the protonema of the hepatics and mosses. This has remained as a simple cell in nearly all of the Ilepaticæ, and even in Riccia the colorless rhizoids that grow out from it do not become septated off by walls. But the swarmspore or-as we would view it - the correspond- 
ing protonemal stage, that intervenes between zygospore germination and the development of a sexual plant, is a very constant feature in the Coleochætaceæ, Hepaticæ, Musci, and even higher organisms; while the formation of the sexual plant on it takes place either as a terminal or lateral outgrowth from it.

We would conclude then, from the above comparisons, that the Hepatica have directly originated from ancestral forms that approach nearest to the Coleochætaceæ of living freshwater algæ. Lotzy has proposed the name Sphceroriccia for a hypothetical intermediate type $(119,2: 88)$ that may have combined characters of the Coleochætaceæ, the Ricciaceæ, and the Sphærocarpaceæ. The term "sphæroriccioid ancestor" would be preferable, for, while Sphorocarpus shows nearer approach in some details, Riccia shows points of closer affinity in others. An exact generic term also for a hypothetical form is inadvisable.

It would be superfluous here to trace the genealogical ramifications of the Hepaticæ further, but, in spite of their almost complete absence as fossil remains, all evidence points to their early origin, possibly during the middle period of the upper archæan epoch. But increasing adaptation to a land life, and continued action of subaerial stimuli, with proenvironal response in varied ways by the organisms to such stimuli, has resulted in evolution of the more complex and specialized groups, such as the Metzgeriaceæ, the Anthocerotaceæ, the Marchantiaceæ, and the highest or Jungermanniaceæ, though none of these has advanced to the degree of complexity shown by the leafy mosses.

But in making such a statement it is well to bear in mind that ancient and even greatly more complex types may have existed but may have become extinct, owing to the relative softness of their tissues. For, had not the giant Calamites and Lepidodendra of the coal period possessed hard and resisting tissues, we might have inclined to the riew that the humble Equisetums and Lycopods of our existing flora represented the highest and most complex types of their classes, a view that would have been greatly wide of the mark. 
In passing from the genealogy of the Hepaticæ to that of the Musci or leafy mosses, a question presents itself that has been considerable debated, specially by Wettstein (120). This bears on the fact that in the Hepaticx from Riccia and Sphcerocarpus upward the growth of the sexual plant is dorsiventral, and so each forms an expanded flattened thallus, as in Riccia, or an inclined axis with dorsiventral bilaterally placed leaves, as in the scale mosses. In the true or leafy mosses on the other hand the growth is typically radial, and so upright axes bearing spirally arranged leaves are characteristic of the group.

But in referring the mosses as well as the hepatics back to a coleochxtoid ancestry ample explanation is got. For the living species of Coleochcete evidently represent survivors not merely of a group of species, but even of genera that varied considerably amongst themselves in general structure and lifehistory. Now the surviving species of Coleochcete may be said to fall under three heads as to their mode of vegetative growth. First, C. divergens forms elongated cellular threads that branch at varying angles, while many of the branches grow upward as green processes. This seems to be the most primitive type that approaches most nearly to forms like Chetophora. Second, in C. pulvinata the protonemal tube of the swarmspore produces branches that grow out in one plane as a dorsiventral expanse, but from these branches others arise that grow upward and branch repeatedly in radial manner. From this or some related type of organism we would consider that the mosses originated. Third, in C. soluta and specially in $C$. scutata, that we regard as nearest in protoriccioid characters, the protonemal tube early gives rise to a dorsiventral type of growth, and to branches that arise by equal or unequal dichotomy, as in Hepaticæ.

So even the living species of Coleochocte exhibit that degree of variability and adaptability to environal substrate and subaerial stimuli that would enable us to account satisfactorily for evolution of the Hepaticx and Musci from them, or from a related extinct group.

A greatly wider morphologic and taxonomic gap now exists between Coleochote and the simplest mosses, than between it 
and the hepatics. All existing mosses likewise advance to a higher degree of specialization. So far as we can judge from existing examples, three distinct groups - the Sphagnaceæ or bog mosses, the Andreæaceæ or mountain mosses, and the Bryaceæ or common mosses-early branched off from an ancestral type with radial branching that was intermediate between Coleochoete and protobryaceous organisms.

Here the germinating oospore has produced, instead of a swarmspore cell, a protonemal cell that has elongated and undergone septation. On it as on the swarmspore tube of Coleochoete, an outgrowth has become enlarged to form a multicellular gametophyte, that has branched radially as in $C$. pulvinata, but has advanced on the latter in that the main axis has become a multicellular radial structure, while the lateral filaments have subdivided and broadened out into spirally disposed leaf-like growths. Similar advance in antheridial and oogonial structure has taken place as in primitive hepatics, but the fertilized egg has divided into a sporophytic mass that in complexity and irregular apical rupture resembles types like Marchantia, and which is still retained in Phascum or related simple genera.

Before leaving the Hepaticæ and Musci it may be pointed out that no single representative of either seems ever to have adopted a marine life. Riella Paulsenii is a submerged brackish-water species of interior Asia, that belongs to a fresh-water genus of hepatics, while such moss genera as Fontinalis and Hypnum aduncum var. gracilescens seem in structural detail to indicate that they have been migrants from a terrestrial to a fresh-water environment. The usually soft, slippery, and elongated growth, the brownish green color, and the rapid dessication that occurs on exposure to the air for a few hours or days, in such aquatic genera as the above, are combined proof of environal action and proenvironal response, in the return of what had become a land type back to an aquatic existence.

In spite of persistent efforts put forth by many workers, during the past half century, to bridge over the great mor- 
phological gap between the simpler cellular groups, like those already studied, and the rascular plants, it must be accepted that still the gap is an enormous one. The problem is also further complicated by our knowing that there existed, throughout the geologic period that extends from the upper devonian to the lower permian, extensive groups of vascular plants that attained to huge size, as compared with living representatives. Their extreme abundance, complicated structure, and capacity for reproduction were all so pronounced that alongside them their representatives now existing are truly starved remnants. So true is this that the devonian and carboniferous epochs together have been spoken of as "the age of vascular cryptogams."

We are compelled therefore to accept that during the cambrian and silurian epochs numerous transition genera between algoid and vascular plants erolved, gave rise in some cases to higher forms than themselves, and in turn were blotted out. In the process, some of the higher types for a time surrived, and originated still higher organisms, thereafter disappearing like their progenitors.

Though the position may be a mistaken one, we incline to accept the biciliate and polyciliate structure of the mature spermatozoid as a useful guide in determining affinities. Accordingly, in line with the biciliate coleochætoid and bryophytic groups reviewed above, we now pass to the vascular series of the Lycopodinex, or club mosses, and the Psilotinex of minor importance at the present day. Thereafter starting with the polyciliate type Aphanochcete we shall advance to the fern, the horsetail, the fern fycad, and higher alliances.

The group Lycopodinex, that includes the living genera Lycopodium and Selaginella with a considerable number of species, and Phylloglossum with one species, will mainly concern us.

When the spore of Lycopodium cernum bursts it produces a cylindric tube, which we would again regard as the homologue of the swarmspore tube of Coleochate. 'This divides into three to six cells by transverse walls, and so forms a protonema that is of green color. At its extremity a subterminai 
or lateral expanse of cells forms, that is the rudiment of the sexual or gametophyte plant, and so corresponds to that of Coleochrete scutata, of Riccia glauca, of Sphcerocarpus or the leafy rudiment of a Phascum. As in Coleochcete or Riccia this soon may transform surface cells into antheridia and archegonia, which scarcely differ even in minor details from those of the latter genus except that condensation in the neckcanal cells is observed. But the fertilized egg gives rise to a very different and greatly more complicated structure than even the most elaborate sporophyte of the Hepaticæ or Musci. And it is in the absence, at the present day, and as yet also from the palæontological record, of connecting stages in the evolution of the sporophyte body or plant that the great gap exists.

It would lead us too far here to give even an abstract of the varied views that have been advanced to account for the course of morphological development taken. These have been reviewed by Lotzy (119, 2:388) and others comparatively recently. So a brief outline will alone be given of what seems to the author to have been the probable course of events.

Alike in the Hepaticæ and Musci the sporophyte is a structure that grows into and absorbs all of its nourishment from the sexual plants. Its entire function is to develop spores within a more or less simple or complicated wall-cavity, the sporangium. But, through cooperation of agencies, most of which have already been named as environal factors, it assumed an increasingly dominant size and complexity, so as mechanically to press on and reduce in size the sexual generation, till this even in its early phases arose close to the ground. Now through inability of the sexual generation to keep pace with the nutritive needs of the sporophyte, in line however with the growth-continuity relation of one to the other, as well as unceasing environal action and proenvironal reaction between each organism and its surroundings, absorptive and elaborating structures, that each more or less closely simulated those on the nutritive body of the sexual generation, grew out of the sporophyte body.

So the green cellular lobes, filaments, or flattened expanses -that we now know various prothallia amongst species of 
Lycopodium to possess as a response to varied environal stimuli -would tend to reappear on the sporophyte generation as it increased in size. On the principle also of growth compensation, this regetative advance might occasionally though rarely be effected in the later history of each individual, through conversion of spore-forming tissue into regetative tissue.

There ultimately originated then from the fertilized egg, as an alternating or sporophyte generation, a leafy body that slowly but surely attained increased size and greater capacity for resistance to subaerial dessicating influences, during the later cambrian, the silurian, and the early devonian periods. The diverse species, genera, and families that branched off from each other in the process culminated in part in the arborescent Lepidodendra, Sigillarias, and Ulodendra of the devonian and carboniferous periods. These represent ages of exposure - as their microscopic structure is gradually revealing-to alternating hotter and colder, moister and dryer, brighter and duller, still and windy, atmospheric states, that all constituted environal stimuli to which the plants made steady and ever more elaborate proenvironal responses.

Throughout the lycopodineous group, as in the bryophytic, the leaves tend to be simple, numerous, closely aggregate along the axis; while the axis is usually elongate, resisting, and fibrous. But with our present very limited knowledge of extinct types it is impossible to say whether the lycopods arose from a primitive but common stock with the true mosses, or whether their ancestry is to be sought for in even older and more generalized types. But this in no way militates against their derivation from representatives of green fresh-water algæ.

The small living group of the Psilotaceæ, that includes only the genera Psilotum and Tmesipteris, suggests by its structure and habits that it is a greatly reduced and condensed off shoot from the main line of the lycopods. The finding of Psilotum over a large part of the tropics and subtropics in epiphytic or saprophytic connection, and of Tmesipteris over a wide area of the southern hemisphere, are indications that they 
are surviving ancient types, representative of a large assembly of now extinct forms, but which once were spread over a great part of the world.

While some of the Lycopodineæ remained of low herbaceous or semi-shrubby habit, and started lines of growth that have culminated in the club mosses and selaginellas of our existing flora, others attained to giant stature and bulky habit, as in Lepidodendron, Sigillaria, and allied genera On the principle of steady evolutionary progression, the origin of these must be looked for well back into silurian times, for during the devonian period they were already abundant. They reached a remarkable climax, however, alike in size, in number of individuals, and in widespread distribution, during the later carboniferous epoch, since it is to their softened and pulpified remains that we mainly owe our large beds of coal.

Several noteworthy points in their structure deserve special mention. First, in the evolution of their large stems, a close similarity or parallelism and yet a varying diversity were established, that caused them to simulate, and also to differ from, those of polyciliate Cycadofilices, Cycadaceæ, Pinaceæ, Gnetaceæ, and also dicotyledons, while far removed apparently from these in systematic affinity. Second, in their leaf structure a close agreement is shown between them and existing lycopodineous plants. Third, in sporangial formation they all evolved elongated "strobili" that closely resembled those of living lycopods.

But here, as in the great polyciliate groups above cited, advance from a homosporous state, such as is retained in Lycopodium still, to a heterosporous condition occurred, so that microspores and megaspores were produced as in Lepidodendron. Fourth, in those carboniferous types that produced the fruits known as Lepidocarpon, a further evolutionary advance took place, so that structures fundamentally resembling seeds were built up. In this respect again a parallelism with the above polyciliate groups is shown.

The genus Selaginella, that like Lycopodium is now worldwide as a terrestrial group, is represented by about five hundred 
species. Though greatly more advanced than Lycopodium in their life history, all so clearly suggest a common origin, and have so many common structural details, that they constitute collectively the class Lycopodineæ.

Palæontological evidence shows that our existing humble selaginellas are a very ancient group, which were fairly abundant during carboniferous times, and which originated from a greatly more ancient stock in common with the lycopods. But, early branching off from the latter, they in common with the Lepidodendrex advanced to a heterosporous state and produced ligulate leaves. Though somewhat imperfectly known, the genus Miadesmia even indicates that carboniferous representatives of this group advanced to the stage of seedproduction as in Lepidocarpon.

The ramifications therefore of the Biciliatæ might be set forth as in Figure 13 facing p. 356. 


\section{CHAPTER XIII}

\section{THE EVOLUTION OF PLANTS (CONTINued)}

The polyciliate vascular plants and their derivative higher allies can now be considered. In looking to Aphanochoete or possibly to some related genus of the Chætophoraceæ, rather than to the Coleochætaceæ, as their starting point, the writer is only influenced by the consideration that in such genera as Aphanochoete, Draparnaldia, and Stigeoclonium the microzoospores which reform as gametes have four cilia, while in the first named the large egg cell has four cilia. Such would strongly suggest that they represent the initial types for origin of polyciliate groups. The varied configuration also of the vegetative body, from an expanded green thallus to a branched creeping filiform green mass, is correlated amongst higher plants in the flat prothallus of Ceratopteris, or the branched filiform prothallus of Schizcea.

But, as many observers have pointed out, a wide gap now exists between the most advanced algæ and the simplest of the ferns, that proclaims the obliteration of numerous preexisting types. As in so many other lines of advance this morphologically represents a gap that chronologically extended in all likelihood from the ordovician to the early silurian age, when the primitive ferns probably first appeared.

In attempting to bridge over this gap, the existing organism which seems to the writer most nearly to conform to required details is the semi-aquatic fern Ceratopteris. Its abundance and world-wide dissemination in tropics and subtropics is evidence of its antiquity. The sexual or prothallial generation is, as in riccioid examples of the Biciliatre, a flat cellular expanse that bears marginal antheridia on smaller male prothallia.

'The young sporophyte stem that springs from the fertilized egg forms a very short and simple axis that has the primitive 
character of being for a time monostelic (121:95). That is, in the midst of the differentiating stem tissue a central core of rascular elements matures into wood or xylem, and bast or phloem, constituents. With advancing age and growth of the stem, the axial core divides, so that a circle of several bundles results.

The leaves are at first simple lateral lobes of the axis, but become increasingly enlarged as new ones arise, till expanded simple or lobed floating leaves are formed. In the variety fluitans, that the writer has kept under observation for years in tanks at the Botanic Garden of the University of Pennsylvania, such leaves or only deeply pinnatifid leaves are produced. Abundant buds, that propagate the plant asexually, arise in the sinuses of these.

But, in the normal form that advances to a semi-aquatic state, greatly more complex and finely divided leaves arise, that bear simple scattered subglobular sporangia. The simple but variable annulus with its abundant closely-placed cells, the short broad stalk, and the few spores produced withinusually 16-are all suggestive of a more primitive type than is seen in any other existing fern. We accordingly place it at the base of the fern series, for, though it may have undergone a degree of simplification by exposure from changing subaquatic to aquatic conditions, the steady advance shown in the life history of each individual militates fairly against carrying such a view too far.

But between a Ceratopteris type and the highest chætophoraceous algxe a wide gap exists, that at present we can only fill in by hypothetical examples based on combined views obtained from living forms. Thus by reference to Figure 13 (p. 356) it will be seen that between the Chætophoraceæ and Ceratopteris a wide stretch of life evolution must have proceeded during which the great equisetal, cordaital, and possibly the cycado-filicineal alliances may have become somewhat established.

Now these all still clearly indicate that they had a thalloid or algoid origin, in that they show in common a purely cellular 
prothallus on which the sexual organs arise, and which still is, or once was, an independent plant. All however develop antheridia and archegonia, these arise in a fundamentally similar manner in all, and they all have polyciliate sperms, that in number of cilia show considerable advance on the four cilia of Chætophoraceæ.

If now we attempt to trace the probable origin of the Equisetales or great horsetail alliance, by combining palæontological and morphological evidence in an effort to fill the gap between them and the green algæ, it may be considered that a group early evolved in which the fertilized egg was borne on a semi-aquatic to subaerial prothallus. Such prothallia were probably produced during a moist period of the year and may then have become exposed to a rather suddenly changing subaerial xerophytic state, that caused retardation in growth of the first two or three leaves of the young embryo and arrest of the associated internodes, with resulting reduction in size and whorled placing of the leaves. So the formation of two to three leaves from a common foliar ring on the embryo plantlet, followed by whorls of leaves of increasing number, must have occurred. It seems not unlikely that such may have arisen over some land area exposed to hot suns, and in which periods of alternate flooding and drying of low flat lands took place.

Thus must have originated, during the late silurian or early devonian period, types like the Sphenophylleæ, that are the most ancient and most primitive in structure of the great horsetail alliance or Equisetales.

In succession to these the Archæocalamitex and the Calamiteæ appeared, from the middle devonian onward, and attained maximum development during the later carboniferous period, alike in size, in structure, in abundance, and in wide distribution. The highest members of the series like Calamostachys advanced to the stage of bearing microspores and megaspores in adjoining sporangial sacs, while all of the Calamitex had a capacity for indefinite stem increase, that is not shown in their nearest existing representatives, the horsetails. Mean- 
while the more humble types that constitute the Equiseter or true horsetails began to evolve, possibly as a sideshoot from herbaceous carboniferous representatives, and this from the jurassic period to the present day has alone survived, as representing the once abundant group of palæozoic age.

But coeval with them in origin seem to have been three great group-serjes, that for convenience we may designate respectively the Archæocordaiteæ, the Archæofilicineæ, and the Archeocycadeæ, which were already established during the upper deronian, and whose earliest evolution from primitive cellular thalloid forms must have dated back to the cambrian period at least.

The material is still entirely lacking that would enable us to follow or reconstruct the early pathway taken by these in branching off from a common ancestry. But, piecing together such details as living examples and fossil specimens furnish, we would consider that such lines of advance as are indicated in the diagram facing p. 356 (Fig. 13) were pursued.

All three, from an originally common kind of green cellular thalloid expanse or prothallus, seem to have produced, from the fertilized egg borne on it, an alternating sporophyte generation that gave rise to successive stem and leaf parts, that were joined to or reared on each other as several phyta that made up a regetative system. In the floating aquatic form of Ceratopteris we get some notion of what such a generalized type may have been. Here on short successive stem segments simple floating thalloid leaves are formed, that are traversed by delicate vascular bundles. Along the edges of the leaves abundant buds are produced that separate and form new plants. But equally might swellings have formed on these that could have matured into sporangial sacs, and so probably arose that still aquatic group-the Hydropteridexthat in the long-drawn ages since has branched out into four distinct representative genera still living.

But, with increasing development of the sporophyte stem and roots, with increasing assumption of a land existence by the sporophyte, and with evolved tissue elements that were 
suited to such existence, the sporophyte growth assumed increasingly bulky proportions. In the process, the above named three groups diverged apart from a common ancestry, probably during late silurian time.

Of the three, the group that seems most closely and directly to have survived till now in a comparatively lowly organized condition is that of the Archæofilicineæ, that has produced derivative evolving fern families and genera, from the devonian to the present day. The usual retention of the concentric stem and leaf bundles, the tendency to formation of a root or roots at each node, the wide expanse of the mature leaves, and the structure of the sporangia, have all remained fairly constant. But very early in their history two divisions were produced, whose characters depend on the origin of the sporangia. These, that are often designated as the leptosporangiate and the eusporangiate divisions, are both recognized in carboniferous strata, and have persisted till now. Of the two, we would regard the former as the simpler, and so probably the more ancient. For in it we can still trace simple root, stem, and leaf conditions, as exemplified in Ceratopteris, and the sporangia all arise as outgrowths each of a single epidermal cell. In this they resemble the origin of the vegetative buds formed on the leaf edges of Ceratopteris, as elucidated by Hofmeister. Such also is their origin in that fern.

In the eusporangiate ferms the sporangiferous tissue includes not merely the epidermis, but one or more layers of subepidermal tissue, from the latter of which the spores are formed. These however almost certainly originated as two distinct and derivative series from distinct members of the Leptosporangiatæ, and are now recognized as the Ophioglossacex and Marattiacex. The two have few points in common, except that the sori and their sporangia are greatly more complex than in the Leptosporangiatæ.

Of all examples of Filicinex known to us, whether fossil or recent, Ceratopteris conforms to the simplest conditions, as has been indicated above. So the group Parkeriacex, made for its reception, we would regard as representing a family of 
ferns of lowly organization, all of which except Ceratopteris are now extinct.

Somewhat distantly related to the last is the carboniferous group of the Botryopteridex, which in its simple monostelic stem, its spirally placed leaves-that like Ceratopteris were at least dimorphic-, in the reduction of the sporophyll lamina, in the often spherical sporangia with extensive annulus and short broad stalk suggests affinity to the Parkeriaceæ. But the Botryopteridere again have been regarded by most recent observers as showing marked affinities with the Osmundaceæ, or the royal and cinnamon fern types. The origin of other subdivisions of the Filicineæ it would be inadvisable to trace here in any detail.

The term Archæocycadeæ we use to designate that assemblage of palæozoic plants which-as set forth in the tablegradually gave origin to distinct families like the Pecopterideæ, the Lyginodendreæ, the Medulloseæ, and Bennetiteæ, that are now wholly extinct, as well as to the Cycadeæ, that are represented by living genera. As set forth in the diagram, these all probably had an ancient common origin with the Archæofilicineæ, and especially with Protomarattiaceæ.

So much is this the case that many lower carboniferous plant organisms, whose leafy impressions the writer has often laid bare in countless number, appear exactly fern-like in aspect and veining. But studies made of the stem, the branches, and the reproductive organs, as well as the leaves, during the past quarter century, have clearly demonstrated that these belonged to plants which had evolved, even during the lower carboniferous period, to the stage of being seed-bearing plants or spermatophytes.

The mode of growth of their stem showed a striking parallelism to that of pinaceous and even dicotyledonous stems, as well as to that already noted for the group Lepidodendrea. But we would regard it purely as a case of morphological parallelism. On the other hand much can be said in favor of our viewing the four extinct groups and the single persisting group as having all been derived from one or a few common and very ancient ancestors, of at least lower devonian if not silurian age.

The Lyginodendrex, Aneimitex, Pecopteridex, and Medullosex attained their growth climax and greatest wealth of individuals, during lower to upper carboniferous times, but they in turn had largely or wholly died out when the Bennetitex were attaining to their developmental climax in the juras- 
sic period. They in turn were being pushed aside by the allied Cycadeæ of common derivative stock with the Bennetitex. These cycads evidently became a highly abundant factor alongside the Bennetiteæ during the jurassic and early cretaceous period. But, while the latter group developed striking and highly modified lateral hermaphrodite flowers-as the researches of Wieland (122: 139) have abundantly and beautifully demonstrated-and then gradually died out, the cycads have evolved larger and terminal diøecious flowers with correspondingly large fruits. Whether the huge polyciliate spermatozoids that now characterize cycads were equally typical for all of the above is a point that has not yet been determined.

As regards the flowers and fruiting organs of these groups, sufficient naked eye and microscopic studies have been made to prove that they had evolved to the stage of naked-seed production, or the gymnospermic habit. Thus the staminate flowers and "Lagenostoma" fruits of Lyginodendron, the staminate flowers and "Neuropteris" fruits of the Medulloseæ, and Grand d'Eury's "Pecopteris" fruits from the coal measures, the Bennetiteæ with their "Cycadoidea" flowers and fruits of jurassic and cretaceous age, as $\pi$ ell as fossil and recent cycadaceous flowers and fruits, all verify the true gymnospermic character of these families.

It is therefore of considerable morphological interest that these have all evolved staminate and ovulate structures in diverging from a more primitive spore-bearing state, just as Lepidocarpon of the biciliate lycopodineous alliance has attained to the same dignity, though from a totally different ancestry. This added example of parallelism in evolution is therefore of value, as suggesting that, when similar environal agencies act as stimuli, they give rise to closely parallel lines of growth and resulting morphological details, at the same time that other and diverse environal agencies or stimulation intensities act, so as to originate divergent types, such as the Lepidocarpeæ and Lyginodendreæ.

The primitively monostelic stem-bundles that sometimes became broken up in higher types, the capacity for secondary increase of the stem by cambial activity, the formation of barred tracheids in the secondary wood, the relatively ample size of the vegetative leaves, the gradual evolution of a heterosporous condition, the differentiation of the heterosporophylls into stamens and carpels, the predominant development of one megasporangium as an ovule of considerable size, the anemophilous pollination of this, the ultimate formation of the gymnospermous seed that enclosed an embryo with two 
cotyledons, are all characters in common that distinguish the six groups from other phyla.

Did space permit, detailed reference might be made to the structure of the elements that constitute the stem of all of the above. It will suffice now to say that alike in the cellular, the sieve, the tracheidal, and other elements a surprising similarity exists, alongside certain important differences in grouping, that constitute family distinctions.

Reference to the phylogenetic diagram (Fig. 13) will set before the reader the ramification of another, and according to the writer the specially dominant, line of evolutionary advance, that started from a nearly related source with the two great polyciliate lines already considered. This protocordaital stirp has had an equally ancient origin with the other two. For in devonian rocks of America, and in the lower carboniferous or "calciferous" rocks of Scotland, abundant remains of the leaves and twigs, or at times giant trunks which floated some distance before being fossilized, occur.

When we consider their extremely high organization, and attempt to correlate this with form-evolution in connection with time-duration, we are compelled to consider that they had already developed as primitive vascular plants during the silurian age. But in this case, as for the two great phyla already studied, the softer and more ancient remains that might have guided us as to their primitive origins are entirely unknown. Again, therefore, we must endeavor to piece together information derived by comparison of fossil and living examples.

As compared with the equisetineous, the archæofilicineous, and the archæocycadineous groups, the archæocordaital ancestors must very early have matured the stem segment of the embryo sporophyte as the prominent and steadily-growing member, at least in some genera. The leaves on the other hand remained simple or only lobed, were usually lanceolate, and had a few to many parallel or sometimes branching veins. The primitive monostele of the evolving vascular sporophyte stem must very early have divided into a set of four or more meristeles, that were placed in a circle round a quantity of cellular pith tissue. 


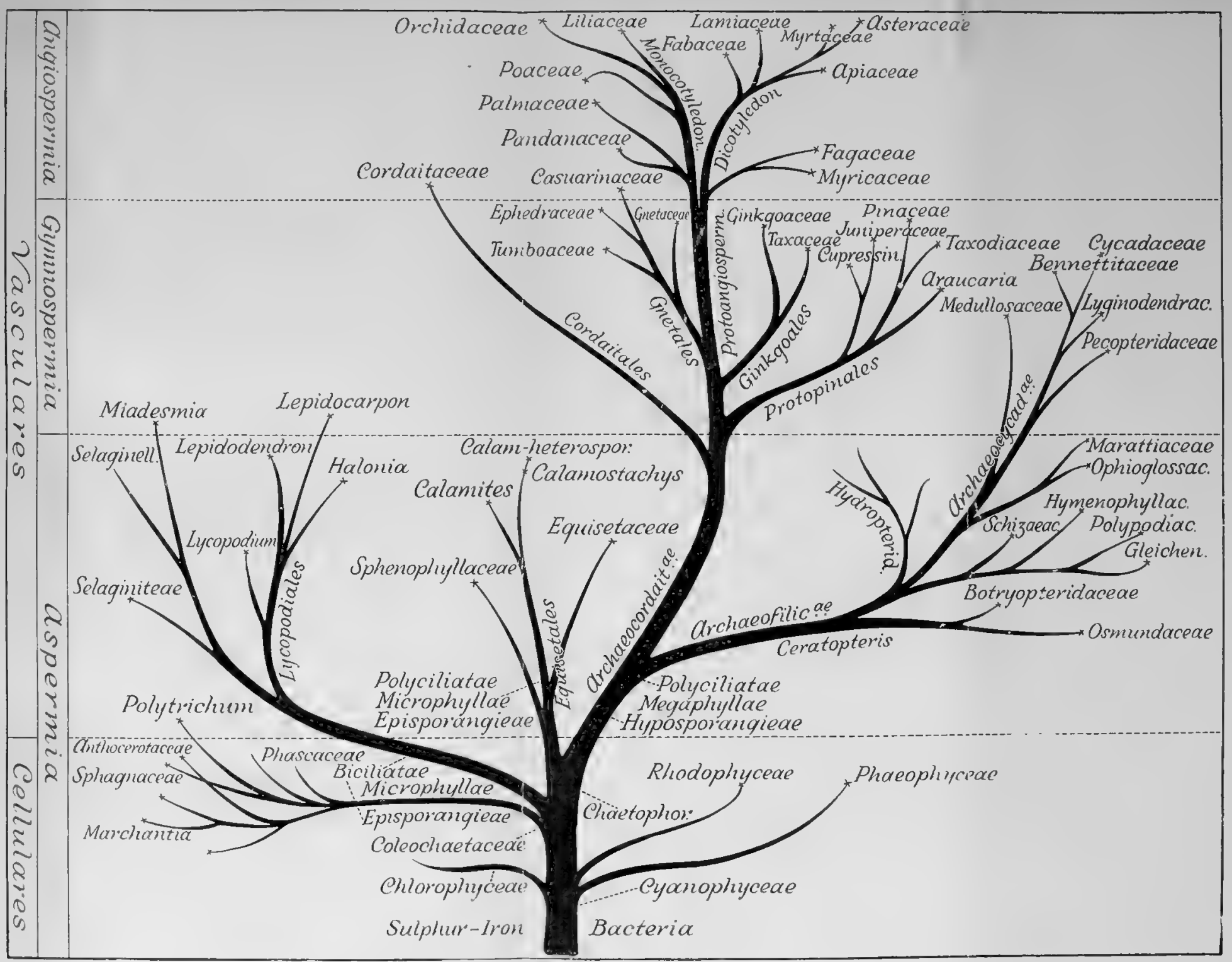

Fisi, 13,-1'rohaldre crolutionary velations in lending groups of Cellular and Vascular l'lants.

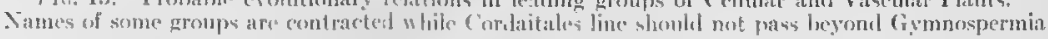



But a marked histological difference was early established in the secondary wood of these, which contrasts with that seen in the other vascular groups already considered. For, while in the latter abundant scalariform or barred tracheids made up the bulk of the secondary nood or xylem tissue, in the group now under consideration narrower, more elongated, and much lignified elements, with pore markings along their walls, became a frequent, often a sole, feature of the wood.

The precociously rapid evolution of floral or anthophytic conditions through the heterosporous stage, up to that of staminal and ovular production, is even more marked than in the other two groups. Our knowledge also of these is now fairly helpful, alike from the macroscopic and the microscopic standpoints.

The "Cordaianthus" flowers were staminate and ovulate. The staminate flowers had characters that foreshadowred those seen at the present day in Ginkgo, in part also in the Taxacex, and in part in the genera Ephedra and Gnetum. The carpellary flowers are decidedly variable, and viewed comparatively suggest several evolving stages, that became more pronounced in later and higher groups. Thus in some genera numerous ovules in close relation to accessory leaves were arranged along an elongated axis and so foreshadowed the cone of pinaceous and related alliances, as well as the apocarpous polycarpellary pistil of angiosperms. Others with few ovules and accessory leaves resembled thus some of the Cupressinex, and the fiveto three-carpelled pistil of many primitive angiosperms, while others bore a solitary ovule that suggests resemblances to Ginkgo and the Taxaceæ.

From the variety of seeds found in the carboniferous rocks, that seem to have cordaital affinity, it is almost assured that numerous genera will in time be identified in their regetative parts. But, as transition is made from the carboniferous to the permian period, genera like Walchia, Noggerathia, Baiera, Glossopteris, Ullmania, and others became increasingly abumdant, and indicate a splitting up of the cordaital stock into one line of deviation that led, as set forth in the diagram (Fig. 13) to the heterogeneous gymnospermic genera commonly known as the Gnetales, and to two other gradually separating groups that are there designated the Ginkgoales and P'rotopinales.

The former or Gnetales is the one that might claim our deepest interest, since from it we would trace the origin of the 
highest flowering plants or Angiospermia. Varied connecting links, therefore, between the higher carboniferous Cordaitales and evolving gnetal and angiospermic branches might well be expected to occur in the fossil state throughout the permian formation. But it is a striking coincidence that the permian rocks, which we would expect to yield varied and important connecting links between the Amphibia and Mammalia as well as other higher vertebrates, are as poor relatively in these as they are in the plant links now desiderated.

Dependence must therefore be placed largely on the three very different and yet interrelated genera, Ephedra, Gnetum, and Welwitschia that may well stand as types of three great gnetal families. The stem of these shows, as in Ephedra, close agreement in growth with that of the Casuarineæ or Shea oaks; or, as in Gnetum, a combination of cordaital details with those now seen in curvembryonal dicotyledons like Chenopodiaceæ and Nyctaginaceæ; or, as in Welwitschia, a parting of the cordaital way that might lead either to monocotyledons or dicotyledons.

Histologically all three are of great interest, for the elements that make up the secondary wood consist in largest part of porous fibers or tracheids as in the Cordaiteæ; but the pores become fewer, less prominent, and smaller, thus indicating gradual approach to the fibers of angiosperms. In smaller part the wood is made up of pitted tubes or vessels, that in aspect and in disposition amongst the fibers strikingly recall the pitted ressels of angiosperms. The reduced opposite scale leaves of Ephedra, the ample net-veined opposite leaves of Gnetum, and the long leathery parallel-veined leaves of Welwitschia suggest transition stages from Cordaitales to the Angiospermia, such as no other series present.

In floral structure these three genera show striking advance on cordaital and even on average gymnospermic flowers, at the same time that they present very exact angiospermic details. For, in addition to showing transitions from bracteolar to what can appropriately only be called floral leaves, they exhibit in their sub-hermaphrodite (Welwitschia) or diclinous 
flowers (Ephedra, Gnetum) a staminal and ovular structure that is greatly more specialized than in Cordaitales, and even than in other gymnosperms. For each stamen is now divided into filament and anther, while the stamens surround the pistil and mature with it, in sub-hermaphrodite flowers of Welwitschia, after an exactly angiospermic manner.

While too much stress need not be placed on the condition, Pearson's demonstration of insect pollination in flowers of Welwitschia is indicative that this genus is probably the oldest now surviving in which such a biological connection had developed.

The formation of secondary floral leaves round the ovules as in Gnetum, and the great prolongation of the ovular integuments beyond the ovular mass, so as physiologically to simulate a style, in all three genera, are adumbrations of higher angiospermic characters. The varying modes of development of prothallial tissue, of egg-cells, and of embryo, which have been elucidated during the past two decades, cannot be treated in detail here, though some consideration will be given to them later (p. 365). All however emphasize the transition value of the three genera.

But in all of these, as in the higher Gymnospermia and in all of the Angiospermia, the production of large motile spermatozoid nuclei-a hereditary derivative by descent from plants with free vegetative prothallia-has entirely ceased, and instead only motionless sperm-nuclei, that are guided -to the egg-cells by chemotactic affinity, are produced.

At this point now, instead of advancing to immediate consideration of the angiosperms, we may turn back to trace two important pathways of advance up to the gymmospermic stage, both of which evidently have been derived from a more ancient cordaital ancestry. These in the diagram we have designated the Ginkgoales and the Protopinales, or in common language the maidenhair trees and the primitive pines.

Though by no means abundant, and only imperfectly known to us as yet in the fossil state, not a few genera of plants have been described that strongly indicate a gradual splitting up 
of the Cordaitales into two additional branches beside the Gnetales, and which became increasingly dominant through jurassic and cretaceous times, while as a coeval event the stock genera like Cordaites, Dorycordaites, and allies were dying out. They constitute the two groups already named, the former of which or Ginkgoales we will now consider. In doing so we may be aided if we consider living material, and then work back to types that seem to connect with the parent stock.

Ginkgo biloba, the maidenhair tree of China and Japan, is the sole surviving representative of a genus that can be traced through the tertiary rocks into the jurassic strata of the mesozoic age, or even according to Saporta back into the permian. The greatest number of species apparently flourished during the late cretaceous or early tertiary times. Like the more ancient Cordaitales the stem grows indefinitely, and shows similar porous fiber elements. The leaves, in shape and veining, can well be connected by genera like Psygmophyllum from the permian and Dicranophyllum from the carboniferous rocks with cordaital forms. But in the small number of prothallial cells in the pollen grain, and in the solitary succulent seeds, the Ginkgoales show specialized details, at the same time that they retain the evidently ancient peculiarity of producing large polyciliate spermatozoids.

The genera Ginkgophyllum, Baiera, and Noeggerathia, in part from the structure of their leaves, and, when known, from that of their flowers, were apparently related genera of the family that occur in permian rocks, reach their climax of development during cretaceous times, and are wholly or largely extinct by early tertiary times.

But a third family now to be considered, and with which Ginkgo was formerly united, is that of the Taxacex or yew alliance. Fron what we know of fossil remains it may be said that of the eight living genera (Saxegothea, Microcachrys, Dacrydium, Phyllocladus, Podocarpus, Cephalotaxus, Torreya, and Taxus) some are unknown in the fossil state, others like Podocarpus and Torreya can be traced back to the eocene or even cretaceous age, while some that have been described as belonging to Taxus are even more ancient.

In all of these except Phyllocladus, there is a tendency for the leaves to show condensed, simplified, and more leathery or resistant texture than in the Ginkgoales, the leaves being often lanceolate to linear or even tooth-like. Unfortunately we still know little regarding the flowers and fruits of the fossil 
examples, or their still more primitive ancestors. But, while the stem structure, the flowers, and the fruit of the Ginkgoales and Taxales show many marked points of affinity, the condensed leafage of the latter, and the tertiary spiral thickening that is so marked a feature of the fibers in the yew alliance, indicate that during the early permian, in all probability, a more or less marked separation of genera from the Ginkgoales took place, and from such derivative genera in turn evolved the taxaceous organisms of today.

During the process the gradual absorption of the circlet of cilia round the spermatozoids, that is still present in Ginkgo, must have taken place, and reduction of the resulting spermnuclei to rounded masses that pass along the pollen-tube and toward the egg-cells by chemotactic action and osmosis was simultaneously effected.

The Protopinales or primitive Coniferales form a division that can recognizably be traced back as such to jurassic times. It seems highly probable also that their ancestral connections are to be met with in such cordaital genera of the coal period as Cordaianthus, in which staminate and pistillate flowers form close spicate masses, that are largely made up of imbricated sporophyll leaves.

The most ancient group of them now living, and the group also which appears to have made closest contact with cordaital predecessors, is the Araucarieæ. In truth so strikingly does the wood in the often large stems of carboniferous cordaitals resemble that of existing Araucarias that it was called Araucarioxylon by the earlier palæophytologists of fifty years ago. The leaves also of Agathis, and of some species of Araucaria now living, conform closely to cordaital affinities.

But in discussion of the phylogeny of Coniferales it may be well to refer here to the extreme diversity of foliage in the one genus Araucaria. Thus the leaves on vigorous shoots of $A$. imbricata recall greatly those on seedling shoots of Agathis (Dammara), while histologically they show not a few points in common. Those of $A$. brazilinesis and $A$. Bidwillii accord more nearly with the genus Cunninghamia; $A$. Cookii resembles Abies or Picea types; while A. excelsa in general morphology and histology approaches to cupressinoid characters. In other words this ancient genus, through long ages of adaptive variation in part in the northern but mainly in the southern hemisphere, has undergone modification, that greatly duplicates the variations shown by leading divisions of the entire group Coniferales.

The genus Voltzia that extends upward from permian to the Keuper beds, and Walchia that shows close affinities with 
it, are transitional in leaf and also-so far as accurately known -in floral parts between cordaitals, araucarias, and the genus ('unninghamia. From the jurassic or lower cretaceous upward, progenitors or representatives of the araucarioid, the spruce, the pine, the redwood, and other pinaceous genera become increasingly common, various cupressineous genera succeed during the cretaceous and early tertiary, while Cupressus, ('hamocyparis, and Juniperus seem to have appeared last in the early- and mid-tertiary periods. In stem and leaf modifications, as in floral and fruit structure, the three last are the most condensed, specialized, and evolved genera of the series. Accordingly we have placed Cupressus and Juniperus at the top of the coniferal line of ascent.

That the more evolved coniferal genera such as Abies, Picea, Pinus, Taxodium, Cupressus, and Juniperus formed a dominant evergreen group in northern temperate regions within even historic times becomes evident, if we reflect on the enormous areas in North America, Europe, and Asia that are still covered by such forests, or that were covered within the past 10,000 years, and have been denuded by man. The manner also in which they cover the ground, to the almost total exclusion of other and especially herbaceous species, is a biologic and ecologic feature of prime importance.

There is left for consideration now the highest and most completely dominant line of plant ascent, namely, that indicated on the diagram (Fig. 13) as Protoangiospermia, which leads up to and branches out into the great dicotyledonous and monocotyledonous branches.

But, before treating of these, the peculiar group of the Casuarinales requires study, owing to its intimate relation, as the writer believes, to Ephedra of the Gnetales, a view already suggested by Engler (124: 18). As subsequently treated (p. $365)$ and as set forth in the diagram, the writer would view this small unigeneric family as an offshoot and evolved product from the Ephedracex, and so as comparatively remotely related to the main angiospermic line. The reasons for such treatment are numerous and fundamental. Thus the bundle structure and mode of growth of the stem are closely alike; the functioning of the stem and branches in place of the leaves is similar; many and most suggestive resemblances are revealed in the histology of the bundle tissues; the structure of the 
stomata as set forth by Porsch (123: 41) forms a highly important similarity; the reduced, whorled, scale-like leaves are also suggestive. But it is in the flowers and fruit that even nearer affinities are shown.

In both, the flowers are diclinous often diøcious, and of condensed but simple type. In both they are arranged in sessile decussate or whorled manner, though Gnetum gnemon in this respect more closely suggests Casuarina than does Ephedra. In both, the staminate flowers are surrounded by 4-2 decussate perianth scales; in both, a single staminate pillar ends in an antherine mass, that in Ephedra bears 8-2 anthers, in Casuarina 2 only; in both, the anthers are bilocular and contain oval pollen grains. The pistillate flowers in both form condensed oval spikes, and each flower has been considered to be devoid of a perianth. Each flower however consists of parts that have excited much discussion. The writer believes that for these an exact homology can be established between them.

In Ephedra helvetica the careful studies of Jaccard fortify the somewhat diverse and yet related views of Van Tieghem and Strasburger, that each flower is surrounded by a pair of fused leaves. These we would regard with Jaccard as a rudimentary perianth. In Casuarina suberosa, according to Treub, two leaves also arise right and left of the developing pistil, which we would also interpret as corresponding to the perianth of Ephedra. But in Ephedra trifurca according to Land four perianth leaves are formed; and this again is duplicated in Casuarina equisetifolia, which bears a like number.

In Ephedra trifurca, Land has shown that the so-called integumen arises as two swellings which elongate greatly after fusing. This has usually been regarded as an ovular or megasporangial integument, but the writer would regard these as two carpels which early fuse and elongate to form a long open stylar canal. Such then presents a close approximation to an angiospermic state. In Casuarina Treub's embryological studies show similarly that two carpellary swellings are formed which early fuse and rapidly elongate to form the long style. 
Within this is the "stylar cylinder" of Treub, which exactly agrees with the open stylar canal of Ephedra, except that it is more intimately fused and reduced in Casuarina.

In Ephedra a single orthotropous ovule is formed, and this, according to the above interpretation of the "integument" and descriptions hitherto given, would seem to be devoid of special ovular integuments. The two orthotropal ovules in C'asuarina arise in succession and develop each two ovular integuments. So unless detailed study of other species of Ephedra than those already examined reveal one or two ovular integuments, it would seem as if these had been absorbed, or had failed to develop in that genus.

It will thus be seen that a dimerous and decussate floral symmetry is typical for both groups, as for Welwitschia and Gnetum.

Passing to the ovular derelopment, the studies of Land for Ephedra and of Treub as well as Frye for Casuarina prove that in both a patch of megaspore mother cells arises in the megasporangial or orular tissue. If the condition in $E$. trifurca is typical for the genus, it would appear as if considerable reduction had taken place as compared with Casuarina, for, while four is the number in the former, an extensive patch or plate is met with in the latter. It is worthy of note in this connection that Gnetum gnemon as described by Lotzy conforms to Casuarina.

An ordinal difference however is now observed. For, while in Ephedra one megaspore-the innermost - enlarges to form a single daughter megaspore cavity, whose nucleus divides repeatedly to form several hundred megaspore daughter nuclei, round which protoplasm accumulates and walls are formed to constitute a gymnospermic prothallus, in Casuarina division into numerous-15-20-complete cells takes place, each of which becomes a megaspore or embryo-sac, while disorganization of others around takes place. This would indicate that in the evolution of the angiospermic Casuarina from the gymnospermic Ephedra the prothallial tissue of the former has divided up into the several prothallial constituents or daughter mega- 
spore cavities. This is further emphasized by future behavior of the cells. For in Ephedra the outer prothallial cells become structures that push out into the megasporangial tissue and there absorb nourishment; in Casuarina the resulting cellgroups to the number of 10-20 grow downward as mature megaspores or embryo sacs into the megasporangial tissue, and there act in part as absorbers.

The next stages are of special interest, as probably affording a direct connection in the relation of Ephedra and other gymnosperms as to formation of their egg-cells, to the condition as it occurs in angiosperms.

In Ephedra the prothallial tissue, as it forms within the megaspore, shows rough division into an inner or antipodal mass, an outer generative or micropylar mass, and a loose central tissue. On the micropylar summit of the generative mass, one or two typical archegonia arise. In Casuarina each of the ultimate megaspore or embryo-sac cells divides by free cell formation into an antipodal set of four cells and an outer or micropylar set of four. From their future relation and behavior the writer would consider that each set of four represents a divided up prothallial mass, which bears one rudjmentary archegonium that forms sex-cells. This view however he sets forth with much reserve, and would await future comparative studies of the species in both genera.

In some species of Ephedra, and also in Casuarina, the seed is surrounded by firm bract leaves that form a protection to the "fruit."

The above remarkable points of fundamental agreement between Ephedra and Casuarina seem amply to warrant the conclusion that the latter has evolved through an ancestry that was closely related to Ephedra, though partaking of some characters that lean toward Gnetum. Further we would regard Ephedra as semi-angiospermic, in that the terminal lobes of the carpels do not fuse to form stigmas, and that this fusion in Casuarina constitutes it angiospermic, though the stylar cylinder in it points to a more primitive semi-angiospermic state. Casuarina then would stand as a unique genus 
in a unique cohort Casuarinales, that has evolved directly from a gnetal ancestry, and so separate from the main angiospermic line.

The above conclusion the writer hopes to elaborate elsewhere, but as a necessary corollary it involves the view that the Angiospermia had at least a diphyletic origin.*

The highest and remaining division of the Protoangiospermia can now concern us. Up to the close of the jurassic period the predominant regetation was gymnospermic, and to a less degree of a fern character. Such was largely true also during the earlier cretaceous age. But towards the close of the jurassic age, or very early in the cretaceous, primitive angiosperms must have been evolving. As already set forth above, the only living group that combines in its genera most structural details of angiosperms is that of the Gnetales. But neither of it nor of undoubted angiosperms have we as yet traces, till the early cretaceous rocks are reached. It is however a necessity of the case to consider that during triassic times numerous and diversified gnetal genera evolved, and that these in turn gave rise along the ephedral line to Casuarinales, along another and slowly diverging line to progenitors of the Angiospermia proper, that we may term the Protoangiospermia.

In attempting to trace the evolution of this group, one is confronted by the fact that, so far as fossiliferous rocks have hitherto yielded evidence, the two great divisions of dicotyledons and monocotyledons appear practically simultaneously, and in rocks of lower cretaceous age. The question then at once occurs as to how these two very natural divisions arose, and whether one preceded the other.

On the whole the writer believes he is correct in saying that, till within the past decade, the great balance of opinion leaned toward the view that the monocotyledons were the more ancient and in structure the more primitive group, and either arose separately from, or gave origin later to the dicotyledons.

\footnotetext{
* Since the above was written, now nearly five years ago, the valuable and suggestive paper by W. P. Thompson has appeared (Amer. Jour. of Bot. III (1916) 135). His views form a welcome confirmation to the above statements.
} 
But Henslow's able advocacy $(125: 485 ; 126: 717)$ of the view that monocotyledons have dereloped as an offshoot from the protoangiospermic line, and that they have become simplified by the adoption of a semi-aquatic or aquatic habit, caused a restatement of the older position by several more recent workers. Thus Dr. Sargent's paper on "Primitive Angiosperms" (127: 121) still further expands and fortifies the view that dicotyledons retain a more protoangiospermic set of characters than do the monocotyledons.

The above view also is somewhat strengthened when we compare lists of the earlier angiospermic plants in their two constituents. Thus in Lester Ward's enumeration (U. S. Geol. Surv. 15th Ann. Rep. (1893-94) 313) of plants from beds of cretaceous age, that characterize the Potomac formation of North America, 330 species, or 45 per cent. of the entire recorded flora, are dicotyledonous and few are monocotyledonous, while the remainder belong to gymnosperms or ferns. The above result may be explained in part at least by the usually firmer nature of shrubby or arborescent dicotyledonous than of monocotyledonous leaves. But, granted such, we may next try to ascertain whether any morphological details shown by the primitive ancestors of both can guide us to a correct estimate of their origin. If the monocotyledons did not arise from and in common with the dicotyledons, then we must accept it that we have not a fragment of the ancestors of monocotyledons in a fossil state.

But, if we accept it that they all sprang from the great cordaital stock, then we must believe that all had originally two seed-leaves to the embryo, a stem with bundle-ring that increased indefinitely, and leaves that were more nearly parallel than net-veined. In other words characters that are now largely separate, and typical of each division, were then conjoined. This also is what we might expect on morphological grounds. So, while the Gnetales were evolving and reaching a probable climax of generic diversity and abundance during permojurassic times, coeval with them there evidently arose a group of forms that had for a time mainly dicotyledonous tendencies, 
but which later separated into two distinct and diverging series. In later jurassic times then, the forerunners of some of the dicotyledonous and monocotyledonous families must have become fairly abundant, and by combined structural adaptations to environal conditions must have begun to press on and excel the preexisting filicineous and gymnospermic; floras.

The occurrence of an evident and fairly rich angiospermic flora in the Potomac beds of America, and in the Aptian beds of Europe, brings up the necessary corollary that their progenitors must have been evolving into dicotyledons and monocotyledons during jurassic times. As yet however no remains from these rocks have been obtained, so that we must rely on morphologic and taxonomic details presented by recent plants, as well as determinations of scant fossils from more recent strata.

But, as regards the identifications of many fossils from cretaceous and tertiary strata, the writer is compelled to claim a very conservative attitude. Many of these identifications have been made from leaves alone; in a few cases only have flowers or fruits been secured. But the state of preservation of the last is often very imperfect. Where specimens are found that evidently and frequently show a typical parallel or reticulate venation, there seems a fair likelihood that these are monocotyledons or dicotyledons respectively. But the attempt, from study of the leaves alone, to refer many remains, even from the cretaceous rocks, to such genera as the oleander, holly, and guelder-rose; can only give rise to false hopes and mistaken conclusions.

The arguments for and against a more primitive origin of angiosperms from plants with preponderatingly dicotyledonous details have been ably presented by Henslow and Sargent. We can only attempt now to add suggestions that may fortify that position. And such largely center round three questions.

First: If the cordaital and protoangiospermic stocks possessed embryos with two cotyledons-as we know the former at least to have had-what causes operated to effect absorption of one cotyledon, and bring the other into a terminal axial position? Second: How was the vascular bundle-ring of cordaital and protoangiospermic ancestors redistributed and 
reduced to scattered closed strands? Third: How did the trimerous flower of monocotyledons and the dimerous or pentamerous flower of dicotyledons arise?

As regards the first of these questions, it can be said that throughout their entire history, whether amongst recent plants or within the seeds of extinct cordaital plants, the cotyledons clearly functioned as organs of intermediation between the stored food passed in from the parent, and the succeeding embryo plant, during its growth into a seedling. So the two periods of their energizing activities are: first, that period when they are forming on the young embryonic axis up to the stage of seed maturation; second, that period when, under the stimulus of heat, moisture, and oxygen supply, the cotyledons start to absorb the stored food, to pass it into the growing axis, and often during the process to themselves grow considerably in size.

Now Henslow's fundamental thesis is, in regard to the origin of monocotyledons from a dicotyledonous ancestry, that transfer to an increasingly moist environment brought about the change. In connection therewith, and speaking of the probable absorption of one of the cotyledons, he says "that the energy of growth is arrested in one and not in the other appears to be the whole interpretation of a monocotyledonous embryo."

But, if lines of growth energy have caused the change, such change should have been effected gradually during the specially active growth-period. Now in nearly all monocotyledons the single seed-leaf is small up to the time of germination, while it increases enormously in size during the germination period. Thus that of a leek or onion seedling is easily one hundred times the size that it was while inside the seed; that of a coconut is several hundred times larger.

The increased size however is mainly due to great growth in length, to a much less extent and only later, to growth in width of the cotyledon. Now a broad fundamental difference at the present day between dicotyledons and monocotyledons, in root, stem, and leaf, is the steady and diffuse intercalary 
growth that soon prevails orer apical growth in the former, as compared with rapid and continued apical growth, followed by growth cessation, in the latter. This growth tendency longitudinally, especially in leaves, is to be explained and became accentuated, as Henslow has pointed out, by increased adaptation to a moist environment. We would further suggest also shade surroundings as an added factor.

Now in such rapid elongation from a growth apex, along with frequent bending downward of the radicle when the seed fell on its side, a slight but appreciable difference in length of one cotyledon over another would almost inevitably occur. The longer cotyledon, by its absorptive apex, would reach and digest more abundant reserve food. Through long continued action thus, a slow but sure absorption of one cotyledon, or possibly displacement of it through longitudinal growth between it and the cotyledon retained, would be effected, while the remaining one, or the lower one of the two, would gradually encroach on the stem apex, as being increasingly in direct line with the lengthening radicle. The diffuse intercalary growth and frequent absorption-activity over both of the external faces of the cotyledons, in cordaital, in gnetal, and in primitive dicotyledonous embryos, would ensure a more balanced cotyledonary growth, and thus a retention of both seed-leaves.

The comparative stem structure of dicotyledons and monocotyledons is characteristic. But, as Henslow and Sargent have pointed out, various exceptions in the growth of both types are met with. Thus amongst the former the nymphraceous, the piperaceous, and the curvembryonal alliances show marked resemblances in their stems to those of monocotyledons. Conversely not a few of the simpler monocotyledons show one or more tolerably well-marked bundle rings.

In the latter also marked apical and longitudinal growth of the foliage leaves, in relation to shade and to moist environment, have had much to do, in the writer's estimation, with the breaking up of the bundle-ring, with apical increase in the stem, with irregular scattering of the bundles, and with increase in number of these bundles. For the extensive wind or water strains caused through the leaves on the stem would 
tend to stimulate the formation of widened leaf-base, and would start longitudinal straining of the bundles on each other lengthwise. This would equally break up the ring-continuity, and would prevent steady cambial formation and cambial additions. Apical growth would thus steadily tend to be established, and to be selected along the line of organisms so varying.

The geophilous theory of stem origin, it seems to the writer, has much less in its favor than the cooperating factors of shade and moisture.

The usually trimerous floral symmetry of monocotyledons stands out in marked contrast to the pentamerous or tetramerous symmetry of dicotyledons. Even where, as amongst Graminaceæ, the foliar arrangement is $1 / 2$, the floral order is seen to be $1 / 3$. As we already have had occasion to remark for the gnetal and casuarinal alliances, a 1/2 floral symmetry is evidently an ancient one for that phylum. Was this then the common primitive foliar order for other angiosperms? There seems little if any good evidence in favor of such a view.

But that the $1 / 2,1 / 3$, or $2 / 5$ foliar order may arise readily is proved by their not infrequent occurrence in one family or even genus. In such cases too, adaptation to environal conditions seems at times clearly indicated, as the originating cause of the leaf order. Thus, while the foliar order in Nepenthes is typically $2 / 5$ or $3 / 8, N$. Veitchii shows a $1 / 2$ or alternate system, which so enables the leaves to grow in clasping fashion to tree branches on which this species is epiphytic that the plant is held and balanced thereby. But that the floral symmetry persists as a $1 / 3$ system amongst Graminaceæ, Musacex, Zingiberaceæ, Orchidaceæ, etc., while the foliar arrangement is often $1 / 2$, seems to suggest that the $1 / 3$ system was once typical of monocotyledons throughout, as is still true of most Cyperaceæ, some Liliacex, Amaryllidaceæ, and other families in whole or in part.

As Henslow (p. 526) has pointed out, however, in this connection, not a few genera of monocotyledons show a tetramerous symmetry. It is equally true also that some important and probably rather primitive dicotyledonous families, such as Anonaceæ, Magnoliaceæ, and Berberidaceæ, show trimerous 
symmetry. But until more exhaustive and extensive studies have been made from the ecological, the experimental, and the taxonomic standpoints, as to foliar and floral variations in symmetry, it seems superfluous to advance any explanation.

From all the morphological evidence now to hand, we would consider that monocotyledons branched off, probably during early jurassic times, from a parent stock that showed pronounced dicotylar, and to a less degree gnetal structural details in root, stem, and seed-leaves, but occasional incipient monocotylar details in foliage-leaves. Largely in line with Henslow's position, we would further consider that the group erolved through exposure to moist or in some cases aquatic environment and shade conditions. But such probably alternated in many cases with exposure to hot dry atmospheric states. The former may thus have evolved the geophilous and soft-foliage types of monocotyledon, the latter the semixerophilous or xerophilous types.

All morphological and palæontological evidence however would lead us to consider that the monocotyledonous stock did not arise as a monophyletic, but at least as a diphyletic series. For the presence in older cretaceous rocks of pandani and palms, and in these rocks or in late cretaceous rocks of gramineous, araceous, and other herbaceous types, would indicate at least two, if not three to four, important though diverging lines of evolution. But here and now it would be inappropriate to go further.

The main line of adrance that culminated in the dicotyledons of existing world floras must have been fully differentiated from monocotyledons, and must have subdivided into minor lines of evolution during later jurassic times. For in this way only can we explain the varied remains that have been met with in early cretaceous rocks of America and Europe. But we would consider that the remains now known represent a mere fraction of the genera and particularly herbaceous ones that had then appeared. For if we attempt to group together the few localities of present-day age, or of pleistocene date, where considerable plant remains have been found fossilized, the localities are few indeed, and the remains are scant 
in the extreme. This would even more apply to plants of cretaceous age, which since fossilization have been subjected to upheaval, depression, denudation changes, and other terrene alterations, along with the rocks that enclose them.

Revieming the evidence for dicotyledons now to hand, the writer would consider that at least three, possibly four or five, diverging groups early arose, that may be designated by the existing cohort names of the Fagales, the Laurales, the Chenopodiales, and the Ranales. Fairly recognizable and probably correctly identified remains of the three first named are included amongst the oldest dicotyledonous fossils. Of the fourth it seems fairly assured that magnoliaceous remains coexist with the other three.

Given the existence then of a few primitive representatives, both herbaceous and arborescent, of each of the above great morphological upbuildings, it seems by no means difficult to understand how the existing dicotyledonous flora may have been evolved therefrom. In this connection the probable appearance of the Hymenoptera and Lepidoptera during the cretaceous period, and their undoubted specialization during oligocene times into divisions like those now existing, doubtless contributed not a little to rapid variation and specific selection amongst both dicotyledons and monocotyledons, from early tertiary times onward.

The origin and widespread distribution of a northern and simultaneously of a southern monocotyledonous and dicotyledonous flora, each largely distinct from the other, seems to have occurred during the cenomanian and senonian periods of cretaceous age, and on into eocene times, when a gradual separation into the present continental masses seems to have been effected. Throughout these times the genera and species that now exist gradually spread. But only after an exhaustive investigation has been made of palrentological remains from the early cretaceous up to our day, and when botanical comparison is then instituted between these and the geographic localities of living genera, as well as the relative morphological dignity of these genera, will we be able to trace successfully the history of angiospermic development. 


\section{CHAPTER XIV}

\section{THE EVOLUTION OF ANIMALS}

Alike as demonstrating the unity of Natural Science, and the all-embracing nature of evolutionary processes, every one now concedes the close and interlinked relations of chemistry and physics, as explaining to us the fundamental actions and reactions of inorganic bodies. With advent also of biochemistry and of biophysics, it is being gradually accepted that the lans of physical chemistry are equally those which govern and determine changes in organisms. It has been a main aim of the present volume to try to demonstrate the continuity relation that thus exists, and to remove the idea that there is a mysterious and impassable gap between the living and the non-living, or that another planet furnished the beginnings of life for us.

But the past quarter century has brought into increasing prominence the intimate continuity relation that exists between plants and animals. The botanists and zoologists of thirty to seventy years ago, recoiling perhaps from the strained and artificial views of their predecessors of a previous century, constantly strove to demarcate the two realms of life by supposed hard and fast lines, alike morphological and physiological. The impossibility as well as the scientific inaccuracy of such a proceeding has caused us now to realize that between botany and zoology an equally intimate and interrelated connection exists as between chemistry and physics. It is now generally accepted that organisms exist at the bottom of the biological scale that even the skilled specialist finds it hard or impossible to relegate to one or another division of life. So the statement made in a recent leading zoological text-book holds true regarding them "the formal distinctions which are commonly drawn between the animal and the vegetable kingdoms vanish" and 
"could only be made by deliberately ignoring obvious genetic relationships."

While such is true in actual evolutionary continuity, it is we believe exactly correct to say that from these common beginnings plant and animal life evolved in diverging lines to a high degree of organization, but the sedentary and autotrophic habits of plants have prevented, while the motile and heterotrophic or epibiotic habits of animals have stimulated to, the attainment of the highest possible phase of such organization. But it should be constantly emphasized that none of the fundamental morphological and physiological inheritances slowly acquired and steadily absorbed into the organismal constitution during the long-drawn later archæan age has been cast overboard during the subsequent voyage of life over the ocean of time. Added complexities of energizing and molecular nature have resulted on the way as an outcome of the evolutionary unfolding of the world as a whole in every relation. But the great morpho-physiological inheritances have been carried forward as a progressive whole.

So, though in part a recapitulation of facts already presented, we consider it well now to set out in detail the fundamental resemblances in plants and animals, and to follow this with the differences that they generally show. The resemblances are:

(1) In both, the lowest or primitive forms are nonnucleate and asexual, while they propagate by division of cells.

(2) In both, some of the sub-primitive forms show evolving chromatin threads.

(3) In both, the same fundamental protoplasm forms the basis of life action, and shows the same phases of synthesis of molecules, through activity of biotic energy associated with and helped by the inorganic energies.

(4) In both, the same physiological functions of irritability, nutrition, respiration, growth, and reproduction occur.

(5) In both, like hereditary, variational, environal, proenvironal, and selective capacities are shown. 
(6) In both, the same irritable phenomena of environal stimulation, excitation period, response, propagation of stimulus effect, (summation of stimuli), neutral period, and frequent reexpansion are seen.

(7) In both, the evolving chromatin ultimately forms (Caryota) a nuclear and nucleolar structure for rapid perception of environal relations through activity of cognitic associated with biotic and lower energies.

(8) In both, sexuality with its most primitive expression conjugation, evolves pari passu with nuclear-nucleolar evolution.

(9) In both, the protoplasm or some constituent of it elaborates like products as carbohydrates, fats, amides, proteids, ferments, and additions to the protoplasm; and also produces like secondary products as glucosides, alkaloids, volatile oils, mineral excretions, etc.

(10) In both, absorption of oxygen and liberation of carbon dioxide occur during the respiratory act.

(11) In both, evolving sexuality proceeds from the production of asexual reproductive cells, and later conjugation of like cells, on to phases where one becomes smaller and motile as a spermatozoid, and the other relatively large and richly protoplasmic as the egg.

(12) In both, the fundamental phenomena during cell division of the chromatin, and during chromatin rearrangement for fertilization, are the same.

(13) In both, the egg and sperm structure (of Caryota) agree, except in minor details of number and distribution of cilia, relative size, etc.

(14) In both, the egg on fertilization undergoes segmentation into equal or unequal cells.

(15) In both, the entire ontogeny of each organism becomes condensed and locked up in one cell the egg, with its needed and complimentary cell the sperm.

(16) In both, each new organism reproduces the blended characters of both parents to a greater or less, usually to a balanced, degree. 
(1i) In both, asexual multiplication persists from the Acaryota far up into the caryotic stage of evolution, and frequently the entire historic structure of the organism becomes locked up in one spore cell. Such spore cells usually inherit from their acaryotic ancestry a much greater degree of environal resistance than do the egg cells.

(18) In both, capacity is shown for hybridization, and in correspondingly narrow and wide degree up to a definite species- or at most genus-limit.

(19) In both, as advancing evolution of tissues proceeds, like protective, conducting, connecting, elaborating, digestive, glandular, hair-forming, strengthening, and irritocontractile tracts or areas arise.

(20) In both, like substances or functions may often appear and again disappear, or be absorbed according as environal stimuli act or cease to act.

(21) In both, physiological disuse of elaborating tissues is gradually followed by morphological degeneration, and often by death of the individual and of the group.

The differences are:

(1) Plants have gradually evolved, from primitive colorless ancestors, etiolin and chlorophyllin, so they have become autotrophic, in that they build up complex organic from inorganic products.

Animals seem to have evolved from a plant line in which non-formation of the chlorophyll caused them to depend on the plant world for elaboration of primary organic food products.

(2) Plants have usually a gelatinous or cellulose wall, rarely one in which nitrogenous compounds are present.

Animals have a nitrogenous cell wall when such is present.

(3) Plants are sedentary, except in some simple groups. Animals are rarely sedentary, usually actively motile.

(4) Plants respond very or rather sluggishly to environal changes. 
Animals as a rule respond quickly.

(5) Plants accordingly have not developed rapidly contractile tissues except in some simple motile types, or in sensitive and in related forms; nor have they developed special sense centers nor nerres, except the sensory conducting areas in root tips, stem tips, and leaves, be so viewed as in rudimentary form.

Animals have developed rapidly contractile tissues, and nerves. In connection with these and through energizing activity of cogitic associated with cognitic and biotic as well as the lower energies, nerve or ganglionic cells have been evolved.

If now we pursue a similar mode of treatment for animals as has already been done for plants, in an effort to follow their evolutionary trend, it may be well to trace their ecological origin and gradual distribution over the world. A remarkable similarity with plants will be found to unfold itself, but one that is widely at variance from many recently accepted views.

Though it must be conceded that the Proteomyxa form a highly heterogeneous and even doubtful assemblage, it seems generally true of at least some of them that they are devoid of, or possess only a rudimentary nucleus; that they are rather sluggishly responsive to environal stimuli; that there is no sex differentiation, or only a commencing indication of it, where the gametes are similar to each other; and that multiplication is by division, or by its modified method endogenous division.

When we combine the accounts of Zopf, Rhumbler, Buitschli, and Calkins with the classified list given by Hickson (82: 6), it may be said that 30 genera, which include about 178 species, are known. Of these 16-18 genera are fresh-water or parasitic on land organisms, and they include about 120 species; 5 genera are mainly found in like places, but include a few species that are marine; 7 genera are marine, but include only $20-25$ species.

Study of the unicellular Caryozoa gives striking confirmatory results. We will review these in their probable evolutionary relationship, and accordingly will treat of the Lobosa, the Mycetozoa, the Heliozoa, the Foraminifera, and the Radiolaria first, since all of these are naked pseudopodial organisms. Next the Mastigophora, the Sporozoa, the Hæmoflagellata, and the Infusoria which generally or always develop 
cilia or flagella, have a definite outline, and often a highly specialized body structure. Here it may be remarked however that we nould not regard the former or pseudopodial group as necessarily the older or more primitive.

The 17 genera of Lobosa include 14 that are fresh-water, 1 that is adaptable to fresh or salt water, and 2 that are marine. In structure also they show graded transitions from types that closely resemble the higher Proteomyxa to others that become testiferous and exhibit easy passage to the fresh-water Foraminifera.

The genera of the Mycetozoa are wholly land types, and are 48 in number. The species according to Lister amount to about 280. The Heliozoa include about 30 genera of which 15 are fresh-water; 5 contain species that are mainly so, but are in part marine; 8-10 genera are wholly marine. As some genera of the Lobosa show graded passage to the Foraminifera, so do some members of the present group show suggestive transitions to the Radiolaria.

The Foraminifera can best be treated numerically, as they seem to have evolved morphologically, namely, as composed of two groups, a more primitive one that includes simple forms with mostly chitinous or adventitiously derived tests, like Diplophrys, Lieberkühnia, and Pseudodiffugia; and a more advanced group with simple or more often complex test incrusted with carbonate of lime, as in most of the marine species. The former group includes 12 genera that are fresh-water, 2 that are fresh- or brackish-water, 2 that are mainly freshwater but in part marine, and 5 that are marine. But migration of some of the ancient and primitive representatives from fresh water into brackish and eventually salt water, has here resulted-we would consider, as in nearly every animal genus that so migrates - in production of a great wealth of foraminiferous genera and even more of species, that are as complex and beautiful in their shell structure as they are in their nuclear structure and mode of conjugation.

The Radiolaria run fairly parallel to the last group in numerical and morphological value. But as often restricted and defined in text-books, the group includes a great and diverse assemblage of genera that are purely marine, are extremely rich in species, and are highly pleiomorphic. As explained later (p. 382) it would seem a preferable treatment were such genera as Acanthocystis, Pinacocystis, Lithocolla, etc., which are fresh-water, removed from the Heliozoa, also Euglypha, Trinema, various of the Astrorhizidea and Lituolidea, removed from the simpler Foraminifera, and placed as generalized 
representatives of primitive Radiolaria. For the stiff pseudopodia and central capsule are more or less foreshadowed in these apparently transition groups. All of the above are fresh-water, or include species that pass from fresh-water to marine life.

The Sporozoa, that might well be viewed as a group intermediate between the Lobosa and the next group the Mastigophora, is at the present day exceptionally rich in genera and species. This is in part probably due to their being often free at one time and parasitic at another, and both under a great variety of circumstances. It is very difficult at the present stage of our knowledge of these organisms to reach an exact statistical position, but from comparison of the monographs of leading writers on these we would consider that there are about 60 genera of land parasites, 5 neutral, and 40 genera of marine parasites.

The Hæmoflagellata includes 8 or 9 genera, of which 6 are wholly or chiefly land or fresh-water parasites, two of the genera are either marine parasites or include a fair number of marine forms.

The Mastigophora or flagellate Infusoria is a group that is exceptionally rich in genera and species, though some of these may prove to be doubtful or valueless when the complete life cycles are known. Since the fresh-water forms have received so much attention by microscopists, and the marine ones have been less minutely examined, it is likely that the number of genera and species in the latter will be considerably increased. As delimited at present however there are 94 fresh-water genera, 13 that are common to fresh and salt water, and 36 that are purely marine. Bütschli (93) considered that the group included from 450 to 500 species. In our calculation of the genera, the Chlamydomonidre have been excluded as having been already reckoned amongst the algæ.

The ciliate Infusoria show, like the last, similar diversity of structure, variety of genera, and richness in species. Ninetyfive genera are fresh-water or rarely are land parasites, 31 are common to fresh and brackish water, while 24 are purely marine.

The above results are tabulated on the opposite page.

In view of the above statistics the question may well be asked for the simpler animals, as we already have for plants, whether these originally evolved over a lacustrine and more or less landlocked, or over a marine, area. The latter view 


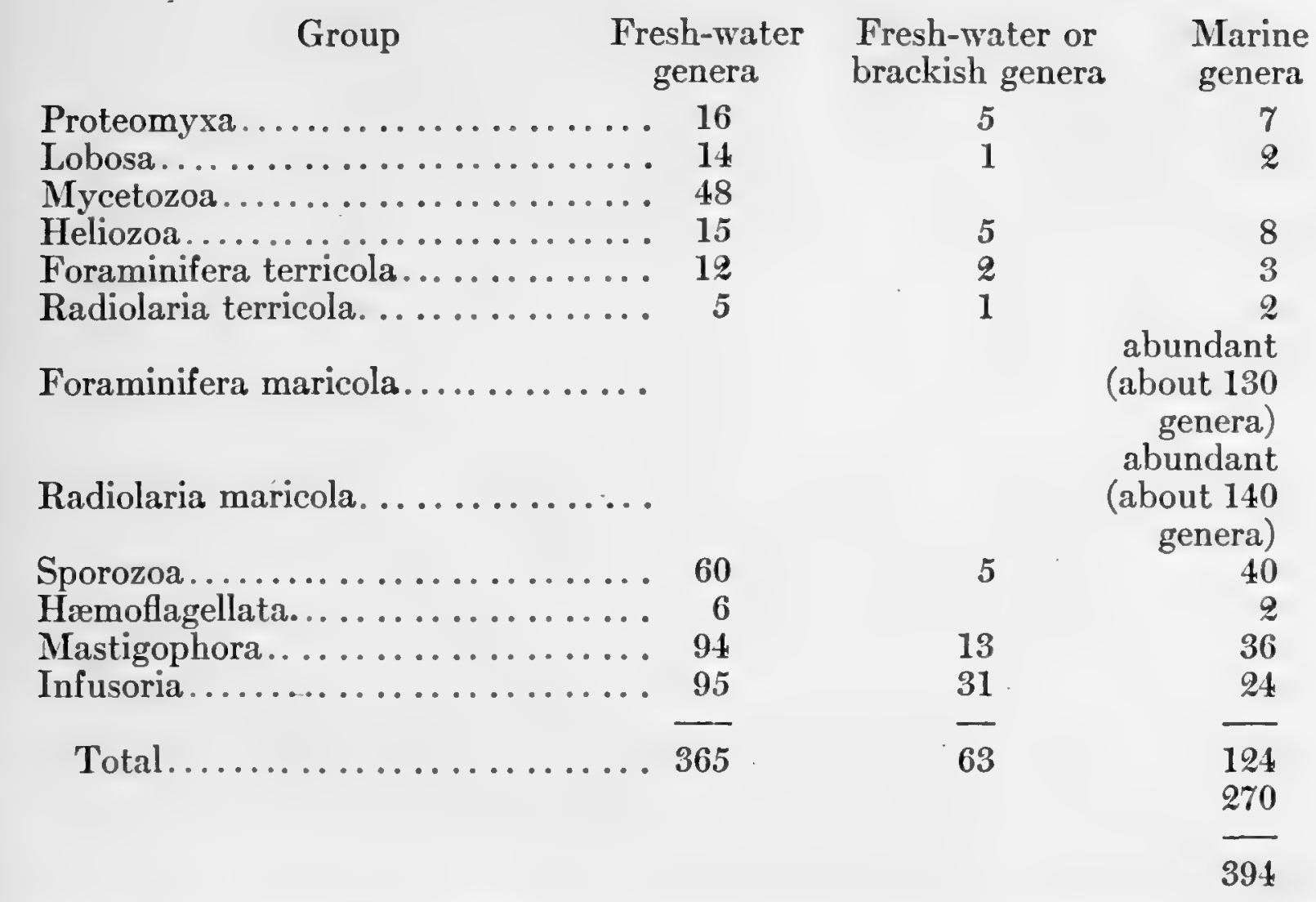

has been uniformly accepted for practically all animals. Thus Sollas's highly suggestive paper (128:87) and Semper's volume equally emphasize this. Sollas says:

"That the sea is the fertile mother of all life was a poetic fancy which has now become a fair deduction from admitted facts. Indeed, all naturalists are now agreed that all freshwater animals have descended, directly or indirectly, from marine ancestors; so that the adaption in question must have occurred at some period in the past history of all fresh-water races." And after giving statistical lists of fresh-water and marine forms he proceeds (p. 95): "It will be seen that, as the analysis proceeds, each group furnishes a large number of exclusively fresh-water divisions, while the mixed fresh-water and marine genera, omitting admittedly exceptional cases, are very few indeed." He then advances various weighty evidences in favor of the view that animal life originated in the sea and migrated landward.

In order to reach a correct conclusion it is necessary that each group should be viewed alike from the phylogenetic, the structural, the taxonomic, and the distributional standpoints. But in the present work only a few observations can be offered. 
The Proteomyxa, while including some doubtful types, contain several species that find their nearest allies of a more advanced type, amongst the fresh-water forms of the three succeeding groups. The Lobosa and Mycetozoa seem equally to have been derived along with members of the Proteomyxa, from primitive myxobacterial forms, that gradually evolved a nucleated and sexual condition. Most of these it is true can resist drying, and so might be carried in dust-winds or as spores from seashore to inland situations, or be left stranded from the sea in lagoons. But we should expect to find as rich or even richer a variety still living in the sea or along its shores, had the inland forms been of sea-derivation. The plastogamy or multiple fusion of young cell-individuals, so strikingly seen in the Mycetozoa, is observed outside the group almost wholly in fresh-water types like Difflugia and Centropyxis.

The simpler forms of Heliozoa show decided affinities with the fresh-water Proteomyxa and Lobosa, while such specialized fresh-water types as Acanthocystis and Pinaciophora approach very nearly to the marine Radiolaria, and include a few species that may be marine. We believe that many will concede a probable fresh-water ancestry alike for the marine groups Foraminifera and Radiolaria. For the genera Diffugia, Arcella, and Phryganella are all fresh-water forms, or brackish-water transition forms, from the Lobosa to foraminiferal types like Pseudodiffugia, Gromia, and Lagena, that are fresh-water, brackish, or marine. In like manner Trinema, Acanthocystis, and Euglypha, though showing little if any indication of an internal capsule, approach very near to the simplest Radiolaria, like the group Physematiidæ. On the other hand no known simple marine types lead up by graded steps to either of the large polymorphic marine groups, Foraminifera and Radiolaria.

But, if we assume that ancestors of both were derived from a fresh-water source, the open free swimming life and chemically active sea water that they eventually reached would act as a double stimulus in addition to those of light, gravity, and other energies, so as to evolve them into that wealth of species, variety of pelagic contrivance, and complexity of structure that are now so characteristic of most of them. But we would by no means consider, as some have done, that such changes took place mainly at great ocean depths. The sea surface or the comparatively shallow littoral zone are the marine regions where actions and reactions would always be most expressed, and so where species would evolve most plentifully. 
No one we believe will deny, on review of the ciliate and flagellate Infusoria, that the entire present-day evidence is in favor of their fresh-water origin. For, whether we regard their richness in genera and species, their comparative structure, and their morphological relation to physiological stimuli, the fresh-water forms fulfill all the requirements for such an origin. Furthermore, they possess an added interest, since Phalansterium, Codonodesmus, and Proterospongia are at once fresh-water and are the only living types that readily connect the Protozoa with that aberrant and lowest group of the Metazoa, namely, the Spongida, Porifera, or Parazoa, while other genera are regarded by the writer as progenitors of the metazoan Rotifera (p. 415).

It would be inappropriate in the present volume to expand at length on the possible geographical origin and present geographical distribution of the invertebrate Metazoa. Sollas has given (loc. cit.) a full list of the groups and has ably argued for a marine origin. A more detailed comparison however of these groups not only weakens his contention; it seems to be entirely negatived. But it should be observed, in following out the inquiry, that where any group becomes preponderatingly marine, and specially semi-pelagic or pelagic, it may, as in the cases of the Foraminifera and Radiolaria, branch out into many generic and specific modifications, that impress the mind more than do the steadily advancing, though possibly less striking, forms that originated on land.

As cases in evidence we might cite the Spongida or Porifera, the Coelenterata, the Echinodermata, the Brachiopoda, the Mollusca, and Cephalopoda.

Amongst the Spongida or Porifera, the division Spongillinæ includes eleven genera, all of the species of which are fresh-water or rarely marine as in Lessepsia. Though referred to the "heterogeneous" sub-group Demospongieæ, they are relatively simple in structure, while their distribution is most suggestive. The fresh-water and the river sponges (S. lacustris and $S$. fluviatilis) are the only living representatives of the entire group that are cosmopolitan, there being scarcely a region of the world where such do not appear, if conditions during recent geologic time have been fairly favorable. But the 
other genera though starved in species at the present day, and though greatly restricted in range, together show a distribution that much resembles Spongilla. For Europe and South America, Asia, and Africa make up their continental spread.

When we consider that the Flagellata are preponderatingly fresh-water, also that Phalansterium and Proterospongia likewise are, a lacustrine origin for the entire group seems quite likely. It would be difficult to explain the distribution of Spongilla, in any other way, even if ne grant that the gemmules or pseudonavicellæe are often distributed by other animals or even by wind.

But it must be acknowledged that on first view it seems wholly unlikely that a large group like the sponges, which includes 350 genera, nearly all of which are marine, should have originated elsewhere than in the sea. So zoologists have consistently and tacitly accepted such origin. But if this be true it means that Spongilla has repeatedly adopted the habit of migrating from the sea into inland waters over different regions of the earth; or has very early passed from a marine life in one or a few localities, and in the extensive changes of the earth's surface has spread by eggs or by gemmules into the world-wide areas where it is now found. But its comparatively simple skeletal structure, the continuity of the surface and connective-tissue spongin fibers, the varying and apparently diverse development of the embryo as traced by Evans, as well as the origin and disposition of the tissue cells, are all structural details that might favor the view of its being one of many ancient fresh-water types, some of which have died out, some have persisted in the other 10 or 11 genera of Spongillinse, while others have originated the complex, heterogenous, and abundant genera now found in sea waters. When like evidence for the possible origin of other largely marine groups is presented, it will be seen that much can be advanced in favor of a lacustrine origin. About 350 living genera are now recognized, of which 12 out of the 13 genera of Spongillinæe are alone fresh-water. 
The large division of the Coelenterata or Enterocœla presents a most suggestive study. Unquestionably arising during mid- or late-archæan times, and early branching out into a multiplicity of lime-crusted as well probably as soft-bodied species, it is at the present day represented by several thousand species, that are littoral, marine, or pelagic, with the exception of six genera that are fresh-water. These, however, deserve special notice, for they belong to the most primitive living types, and have a geographical distribution that greatly recalls that of the Porifera. Thus the genus Hydra includes 3, possibly 4-5, species that are world-wide, for equally in Europe, North America, tropical South America, Africa, Australia, and New Zealand it has been met with. Microhydra, even more simple than the last, is found in eastern North America, while Polypodium is found in the Volga River. No marine forms approach these in simplicity of structure. Protohydra and Haleremita, though marine, seem closely related, and may well be migrants seaward.

But the rather more specialized freshwater genus Cordylophora is a type that perfectly unites the Hydroida division or Eleutheroblastex with the littoral or Gymnoblastex.

The two unique fresh-water medusoid genera Limnocodium and Limnocnida are found, the one probably in the Amazon waters, amid the Victoria lily, the other certainly in Lake Tanganyika and Victoria Nyanza, also in the river Niger of central Africa. The theory which explained the presence of the latter as an imported marine type-when Tanganyika was a supposed arm of a jurassic sea-has been rejected by Boulenger. The production of a medusoid stage by Microhydra is equally interesting. On the other hand the occurrence at times of sea anemones "in brackish or almost fresh water of river estuaries" is fair proof of partial migration from a marine to a fresh-water life, since the entire group of the Anthozoa is littoral or marine at the present day.

So unless it can be shown that one or more genera, as simple as Microhydra and Hydra, also about as abundant, exist in the sea, it seems as logical a position to assert that the group 
had a fresh-water origin in probably mid- or late-proterozoic or archæan times, developed simple forms like Microhydra, like the fixed stages of Limnocodium and Limnocnida or the tentacular Hydra, and that from these in latest proterozoic and early cambrian times marine types originated; while the above fresh-water forms may have spread widely, and may even, between the silurian and jurassic epochs, have given rise to many hydroid types now entirely lost.

Included in the three great classes of the Colenterata are about 300 genera of Hydrozoa, a nearly equal number of Anthozoa, and 25 genera of Ctenophora. From this one can readily estimate how overpoweringly abundant the group is in marine distribution at the present day.

The Echinodermata seem very early to have assumed and retained a marine existence, possibly from their early extensive utilization of skeletal lime salts, so that from the time of the cambrian rock-deposits up to the present day their remains or living representatives are abundant. But the fact that they seem to be derived as a group from a primitive Dipleurula larva with bilateral symmetry might carry them back to a common ancestry with the fresh-water Enterocœla, while the fact that some can live in brackish water, in spite of the great majority being marine, indicates the possibility either of primitive fresh- or brackish-water origin, or the capacity for adaptation to such environment. But, before passing from them in the present connection, it might be pertinent to ask why they have not frequently and in different parts of the world produced migrants from sea to fresh water, that might persist for a time at least. Such seem to be as entirely unknown as are like examples of the more highly modified genera of Foraminifera, Radiolaria, and Porifera.

The group is made up of 8 genera referable to the Crinoids, 58 genera to the Holothuroids, about 94 genera to the Asteroids, 98 to the Ophiuroids, and 220 to the Echinoids, or a total of 478 genera; all which are marine, seldom indeed found in brackish water.

Regarding the Rotifera it has been said (130:200) that they "are of cosmopolitan distribution. Most of the species 
inhabit fresh water, whilst some are brackish and a few marine; 84 genera and about 700 species have been described." Of these and more recently discovered types some 73 genera are fresh-water and 11 or 12 are brackish-or marine. The embryology and the mature structure both indicate that the group probably sprang from a common ancestry with the turbellarian and nemertean worms as well as with the primitive brachiopods and molluses. For all possess in common a more or less extensively ciliated trochosphere larva in which the alimentary, the nervous and sense organs, the circumoral disk, and other structural details agree (p. 507).

But, while nearly all of the above-named higher groups have greatly advanced in structural complexity and in size, the Rotifera have remained comparatively retarded and may be said to represent permanently the post-trochosphere or subveliger stage of turbellarians, molluses, and other groups.

So far as the writer is aware a marine origin-even a littoral marine origin-has never been claimed for them. The fact also that about 20 living species have a marked capacity of adaptibility to fresh-water, to brackish, or to marine life suggests that the marine types have slowly become adapted from an originally fresh-water environment. The small size and soft nature of the animals explain their absence from geologic records.

The large and world-wide group of the flatworms or Platyelminthes presents many interesting problems in structure and distribution. The evident transitions shown from the free-swimming turbellarians to the usually parasitic trematodes, and the highly modified parasitic habits of the cestodes, all involve suggestive questions of phylogeny that cannot now be considered. No one, we believe, will question the land origin of at least two of these.

Numerically the polyclad turbellarians include 28 to 29 genera, all of which are marine; the triclad division consists of 27 genera that are either fresh-water or terrestrial and 8 that are marine; while the rhabdocole division consists of 38 genera that are marine and 24 that are fresh-water. The 
total of the marine genera, therefore, is 75 , and of the land or fresh-water 51. But thanks to the detailed treatment in Von Graff's splendid work (131) we learn that, of the simplest, and as we believe originally lacustrine, group Rhabdocola proper, 167 species are fresh-water, and 153 are marine.

A point worthy of at least passing notice is that in the otherwise marine genus Plagiostoma the species $P$. lemani is found in Lake Geneva.

Of the above groups then the rhabdocol turbellarians are evidently most primitive and typical in structure, while the sub-group Rhabdocola, that includes fresh-water, terrestrial, and littoral marine species, may well have been the originating one for other and more modified types, some of which like the triclad and polyclad groups have greatly evolved, while others like the Acoela have become degenerate types.

The Trematoda is made up of 45 genera of fresh-water or land parasites and 10 that are marine, but, when one finds genera like Distomum and Gasterostomum, that parasitize on land or on fresh-water animals, to include one or two species in each genus that inhabit marine fishes, the question may well be asked whether the entire group has not been of land origin.

In the third group the members of the Cestodes or tapeworms are so evidently adapted in the great majority of cases to a parasitic life in land or lacustrine animals that we would regard the few marine ones as derivative and adapted types. Sixty to sixty-five genera may be taken as making up the group.

Van Beneden's interesting though restricted group of the Mesozoa is so far as known wholly marine and parasitic, but it may well be that they represent very ancient forms remotely descended from simple land ancestors allied to the Turbellaria and Trematoda, and which have remained alive through assuming the parasitic habit.

The small group Gastrotricha includes about 50 species that are fresh-water in habit.

The present-day forms of the Nemertinea are largely marine, but it is highly significant that two genera of the more com- 
plex types are not only land or fresh-nater, they exhibit a geographical distribution that is practically norld-wide. Thus the genus Stichostemma includes seven species that are found in Europe, Asia, North America, and East Africa; while Geonemertes consists of eight species that are met with in New Guinea, Australia, New Zealand, the Mascarenes, and Bermuda.

As stated later we would regard the group as one of exceptional phylogenetic value (p. 418). It becomes therefore a matter of some importance to determine whether the terrestrial or swamp species have been derived from a marine ancestry or vice versa. According to Bürger's beautiful monograph (132), supplemented by a few recent papers, the total number of species is about 450. Of these 15 species are terrestrial or fresh-water, while the remainder are more or less marine. But when we find the genus Stichostemma widely distributed over fresh-water areas, and Geonemertes to inhabit land areas at varying elevation, even though the larger number is now marine, there seems no inherent reason why the group should not have had a terrestrial or lacustrine origin, as has in part been suggested by Montgomery.

The large and diversified division of the polychæte worms may truly be called marine, for of the $\mathbf{1 4 5}$ genera, that include a much larger number of species, all are littoral and marine (or pelagic) except two genera that may well have been passed from the sea into a fluviatile life, while the genus $N$ ereis that is largely marine includes two fresh-water species. Though most of them are soft-bodied, and have therefore left few traces of their remains fossilized, the genus Spirorbis with its calcareous test has been traced back into the silurian rocks, so that the group is an ancient one.

In contrast to the last, but probably having a primitive common origin with them as forms that all originated in landlocked areas, are the earth and river worms or Oligochieta, also the leeches, or Hirudinea. For, if the Polychxta may well be regarded as an ancient marine group, equally may the two last-named be regarded as terrestrial. 
The earth worms or Oligochata consist of 118 genera or thereby, and include many hundreds of species. Most of them are in the truest sense earth-livers, but genera like Nais, Tubifex, and Branchiura are fresh-water and may bear gills. Their soft bodies have prevented fossilization, but this need in no way militate against our regarding them as equal in age to, or even older than, the oldest polychrtes.

The group of the leeches or Hirudinea is made up of about 35 genera, only two or three of which are marine and parasitic in rays and sharks, the remainder being fresh-water parasites. Their wide distribution, like the last group, over practically the whole world, is a strong indication that they are parasitic derivatives from free-swimming fresh-water ancestors, which have undergone slow but extensive modification that has suited them to a parasitic life.

The purely marine group of the Gephyrea is made up of 19 genera, the species of which are world-wide in distribution.

The Kinorhyncha is a small derivative group of about 20 species that are all marine, and may be derivatives from primitive Turbellaria or Gastrotricha.

The Nematoda that are mostly parasitic include about 62 genera. There is no indication that they have originated otherwise than in shallow pools, ponds, lakes, or amid moist shaded earth, even though some are now marine. Their high vitality under dessicating conditions, their frequent infestment of the roots of land plants, and their occurrence as parasites in land invertebrates, all favor a land or fresh-water origin. Fifty-four of the genera are fresh-water or parasitic, and eight genera are marine. The allied group Chætosomatida includes 3 marine genera with a few species. The Archiannelida agrees with the last in habit and genera (3-4).

The Acanthocephala is made up of four genera, most of the species of which are parasites in fresh-water or in land animals, while a few only are marine. Even one genus like Echinorhynchus includes forms that are parasitic in freshwater fish, in a species of whale, in the mouse, the toad, in salt-water fish, or in seals. 
The Polyzoa form a group that may be regarded as preeminently marine in the living species, of which there are 1700 included under 47 genera. The fresh-water species are relatively few, and are included under 8 genera that are purely fresh-water and 39 genera that are mainly marine but contain fresh-water species. That the group is a very ancient one is shown by the abundant remains of marine flustroid species amongst the oldest palæozoic rocks, and often side by side with brachiopod or molluscan shells. These have been preserved on account of their tough membranaceous or more often calcareous colonial shell or test covering. But it is unquestionably true that all of these must have been preceded by soft-bodied types. Now at the present day such are found in divisions that contain, or consist of, fresh-water species.

Thus Urnatella is a fresh-water genus of the subgroup Entoprocta; Paludicella, Bowerbankia, Alcyonidium, and Victorella are fresh- or brackish-water members of the Ctenostomata; while the Phylactolæmata belonging to the Ectoprocta contain only fresh-water species, though these seem to form the most primitive group.

But a persistently free-swimming habit in any of the Polyzoa, along with soft-bodied conditions, might indicate retention of two ancestral and primitive states. This is still shown to a limited degree by the genera Cristatella, Lophopus, and Pectinatella belonging to the fresh-water group Phylactolæmata.

Structurally the Entoprocta have often been regarded as the more primitive of the two main groups. But the retention of the anus outside the lophophore circlet, the persistence of a distinct body cavity that remains continuous with the zooids, the retention of a muscular body-wall outside a mesodermic layer, as well as the continuity of the body cavity with the circular canal and thereby with the tentacular cavities, seem all to point to the Phylactolæmata as probably most directly descended from the most primitive division. On this view the other groups and the genera of them-though now mainly marine - may all have had a fresh-water ancestry, though in extremely remote periods of the past. 
The Brachiopoda are so primeral a group, stand so severely alone amongst other invertebrates, and are, as well as have been, so thoroughly marine as far as we have any present evidence, that to propose a possible fresh-water origin for them in archran times may seem an utterly useless and chimerical suggestion. But, when we find a variety of species belonging to the unarticulate and the articulate divisions in the cambrian rocks, this is absolute proof that these must long before have descended from types destitute of shell, of greatly simpler structure, and also of much smaller size. Such seem to be represented embryologically by Beecher's typembryo and phylembryo, that suggest highly modified trochosphere derivation as indicated by the studies of Kovalevsky and Morse. But even then we have no evidence that the group had not become marine. So the Brachiopoda, like the Echinodermata, many Polyzoa, and three groups of the Mollusca seem all to have acquired their group characters in littoral or marine surroundings, during some period in the late archæan epoch. But such in no wise militates against their earlier trochosphere stage having originated in and been derived from species originating in land-locked areas of the mid- or late-archæan age. The question is more fully discussed in later pages (pp. 518, 520, 528).

The group is now represented by 15 genera, a porerty in numbers that forms a striking contrast to their wealth of genera, and particularly of species, during the silurian and devonian periods of the earth's history.

The large cosmospolitan group of the Mollusca, like that of the Echinodermata and of the Brachiopoda, seems eminently to faror the view of a marine origin for life. So Cooke in the opening seatence of his volume on "Mollusca" (133:1) says: "It is the generally accepted opinion among men of science that all life originated in the sea." Throughout his work therefore this view forms the leading thesis for an explanation of the distribution of living molluscan forms. If an appeal be macie to the palxontological record, one is struck by the fact that all of the main molluscan groups can be traced 
back to the period of the upper cambrian, or the ordovician. So there must certainly have been a period long antecedent to the later cambrian when molluscs existed as adult animals in the "veliger" stage, but were modifying the cement or shell gland that hereditarily was common to them, to the Rotifera, and to the Brachiopoda into a shell secreting organ. For long ages however the group must have existed as soft-bodied animals only, and the geologic period between the origin of veliger cephalopods and the origin of orthoceratite genera with shells a foot to six feet long, as encountered in cambrian and silurian rocks, must have been an enormously extended one.

So one is compelled to assume that not merely the common ancestral types of the group, but these, along with other organisms that linked such ancestral forms with other phyla of the animal kingdom, had developed during the early cambrian or more likely even well back into late-archæan times. Now, if successive embryological stages, and the principle of developmental recapitulation, are of any value, both indicate that in the trochosphere, and later in the veliger stages of molluscs, direct contact is made with the Rotifera, as well as with turbellarian and nemertean ancestors, as several zoologists have already emphasized. But the body-substance alike of a rotifer, a turbellarian, a nemertean and of a veliger molluse is extremely soft. It is therefore not surprising that their fossilized remains are unrecorded in rocks of the early cambrian or of precambrian age. But proofs have been adduced in favor of the view (pp. 38\%, 389) that the Rotifera and the Nemertinea both originated in lacustrine areas.

So, in late-archæan times, ancestors of the rotifers, the turbellarians, the nemertines, and post-riliger ancestors of the molluscs probably all lived side by side in such waters. The frequently saline character of some lacustrine areas, into which dissolved salts were carried, formed a ready natural source from which to draw calcareous shell-constituents as well as to accustom such organisms to a subsequent marine life. Now at the present day a considerable number of molluscan genera and species show marked capacity of adapta- 
bility to fresh water, brackish, and salt water, and, unless it can be shown that this is a recently acquired capacity, it should rather be accepted that this indicates a variation tendency slowly dereloped in primitive life to suit varying environal changes that the group became exposed to during the process of evolution.

Accepting the estimates and classificatory values of several recent conchologists, the entire group of living Mollusca is made up of 813 genera, 483 of which belong to Gastropoda, 997 to the Lamellibranchiata, 61 to the Amphineura, 11 to the Scaphopoda, and 59 to the Cephalopoda. But of the above total about 260 genera are either fresh-water or terrestrial or include species that are such as well as others that are brackish or marine. In view of the statements that have generally been made this seemed a.rather startling as it was an unexpected result, when the writer first compared the genera statistically and geographically.

But, in trying to unravel the phylogeny of the above five groups of the molluscs, zoologists have often accepted it that not merely the fresh-water forms are derived from marine ones, but even that the Gastropoda-pulmonata have also had such an origin, and later formed a pulmonary sac accessory to the branchial sac, and which ultimately replaced it. It is necessary therefore that we try to reach a correct estimate as to the possible origin of each of the five groups. It will generally be accepted that all possess a free veliger stage, or have descended from forms that possessed such. Now in passing from this to the adult stage many reasons could be adduced for regarding the Gastropoda as the most primitive group, while several genera belonging to it are found in cambrian rocks. But of these the soft-bodied genus Vaginula seems to represent a simple and ancient line in which diffuse tegumentary respiration was effected, in which the animal and its interior organs were straight, and the hepatic as well as reproductive systems are like those of many worms. A second line of origin seems to have led to the testaceous pulmonates in which pallial breathing was also retained, but in 
restricted form by an aquatic involution, or by an aerial lung. Both groups are lacustrine or terrestrial, and include about one-third of living forms of such habitats.

From a common ancestry with the aquatic pulmonates the patellid, nudibranch, and related marine forms seem to have been derived, and while often retaining a soft body, or one with spicules, in others there was developed a shell from the shell gland that has hereditarily been derived in common with the cement gland of rotifers, and the egg-cement gland of some annelids.

The gradual development of ctenidia for breathing, as an advance on simpler mantle respiration, has occurred most extensively in marine forms, and this, along with various important details of alimentary, nervous, renal, and reproductive structure, suggests that they are derivative types from lacustrine or river inhabitants. Such however need not militate against the view that some of these last may have migrated back into rivers, lakes, and other inland localities. Varied evidence favors such a conclusion, but it by no means indicates that molluscan life mainly originated even along littoral areas.

The Scaphopoda are all marine, but, though showing affinities with the Gastropoda, their simplified structure makes it difficult to determine their relationship and primitive geographic area. Their first appearance and rarity in the midsilurian, and their climax of abundance in the cretaceous age, help little in the elucidation of the group. Further observations on them are made in a later chapter (p. 527).

The Lamellibranchiata as a living group seems to favor strongly a marine origin, while the greater number of the genera are marine, only about thirty, or one-seventh of the entire number being estuarine or fresh-water. It seems to be a more modified and recent one than the two last, even though concentration of the nervous ganglia does not reach to so marked a degree. Many, probably all, of the freshwater genera have been derived from a marine ancestry, while the most primitive division - the Protobranchia - are all marine so far as known, alike in the fossil and the living species. But 
a long interval of time existed between the early cambrian when the first gastropods appeared, and the silurian when lamellibranchs seem to have originated. Whether primitive soft species with rudimentary bivalves may then have evolved from a fresh-water ancestry is only a conjecture for which we have no fundamental evidence. This is further discussed on p. 523.

The Imphineura and Cephalopoda seem to resemble the last in their marine ancestry, and equally in the entire absence of any types that would connect them primitively with a freshwater origin.

In reviewing the molluses now it might well be emphasized that, considering the abundance of genera of living freshwater and land gastropods, in view of the undoubted early origin of these in cambrian and almost certainly in the latearchran period, in view of the extensive changes and denudation action going on then in the land areas, and in view of the wide possible range for life of aquatic forms when they reached the sea, we would suggest strongly a primitive freshwater origin for the entire group in the late-archæan, an early separation of the Gastropoda into several fresh-water and land areas of evolution as well as into several marine lines, and a later origin of the now exclusively marine groups of Scaphopoda, Amphineura, and Cephalopoda, as well as of the mixed lamellibranchs, from primitively fresh-water ancestors of simple structure.

The Arthropoda include the four solid groups of the Crustarea, the Arachnida, the Myriapoda, and the Insecta. The first of these alone deserves to be regarded as marine, the others are in truest sense land or fresh-water organisms. But, when the comparative structure, the embryology, and the taxonomy of the Crustacea are examined in detail, one finds that the genera and even species of the acknowledgedly primitive group, the Lntomostraca, are nearly all fresh-, rarely brackish-water, still more rarely marine. Here again, however, as in previous examples, when members became thoroughly marine, they 1. ranched out into several phyla and many genera, that show 
considerable variations and adaptations to marine life. No one would probably doubt, however, that the crayfish and allied fresh-water or brackish species are migrants from seashores. * Ecologically they resemble the salmon, the lamprey, and other vertebrates in showing considerable adaptability to changed environment within a comparatively short period. The total genera of the Crustacea according to the authors in Bronn's Tierreich are about 1092, of which at the present day 213 are fresh-water and 879 are marine. But, when it is remembered that, even amongst the divisions of the simplest and most primitive group Entomostraca, the Branchiopoda are fresh-water in the division Phyllopoda, largely so in the division Cladocera; that the Copepoda and Ostracoda are in part fresh-water, in part marine; and that the Cirripedia, which represent the most modified degenerate and sedentary group, are wholly marine, a key seems to be suggested to the probable origin of the Crustacea as a whole.

A comparison of living representatives of the group with the fossil forms may also aid in a proper determination of the origin and present distribution. The resemblance of many annelidan larvæ to the simpler nauplioid larvæ of Crustacea has often been commented on, and so the latter may have represented the diverging point of the Crustacea and the Annelida from the Rotifera. But, like most living and extinct Entomostraca, the primitive types doubtless had a soft even delicate body, and so have left few fossilized remains. During the long geologic ages of the later archæan formation however, they must have become very abundant, since in the early cambrian rocks, and from these into the base of the silurian, representatives of most entomostracan groups have been found.

Now the phyllopod or branchiopod and the cladoceran Entomostraca are both largely fresh-water or lake dwellers, while some species of Branchipus and Artemia can even live in very saline inland lakes that seem to have resembled like areas of the archæan age.

\footnotetext{
* This entire question needs revisal, for with Ortmann's paper on Patagonian Crustacea before us it seems possible that the crayfishes are fresh-water progenitors of the marine lobsters.
} 
But many of the Ostracoda and Copepoda are of like habit now. It seems therefore superfluous to argue that the Crustacea primitively had a marine origin. Rather we should view all or most of the marine types of the two latter groups as derivatives from the originally lacustrine and even it may have been cooler hot-spring areas, in which as now an abundant saline constituent was dissolved. The appearance of the purely marine group of the Cirripedia in cambrian rocks, however, would indicate that by that time at least littoral forms had developed, probably by derivation from some ostracoid type like Cypris that had migrated to the shore, and there undergone modification.

Amongst the Malacostraca numerous species are freshwater, while a good many of them are so nearly related to purely marine types that their origin from marine forms which more anciently were lacustrine seems likely. But even here it is well to keep in mind the wide geographic range of not a few of these lacustrine or land forms, their unquestioned ancient land distribution, and their occurrence in lakes at high elevation. Now fossil remains of Decapoda only begin to appear in earlier mesozoic strata, and so even here the possibility exists of lines of erolutionary divergence having taken place amongst these, from lacustrine or brackish areas to freshwater or even land areas on the one hand, and to littoral or in time deep sea situations on the other. Even amongst the divisions of Macrura, Brachyura, Cumacea, Isopoda, and Amphipoda, species, genera, or even whole families are either terrestrial, fresh-water, amphibious, or marine at all levels from the upper shore limit to miles of ocean depths, so we begin more fully to realize that the entire group is one that may have wavered since early cambrian times down to the present as a fresh-water series that has in one case given off lines of descent that have reached high on mountains, and others that have descended to depths of ocean, while many have kept along the sea margin.

So, while we estimate that 213 genera of living Crustacea are land, lacustrine, or brackish-water, in their environment, 
and 879 are littoral or marine, such statistics do not give a correct estimate of the origin of the group, nor of the evolution of most decapodous marine forms since mesozoic times. For the primitive Crustacea an origin in land-locked areas of fresh or slightly brackish water seems practically certain.

Palæontologically the Arachnida can be traced back into some of the oldest palæozoic rocks. The silurian genus of scorpions call Palcoophonus shows close affinity with existing genera, and affords definite proof that such specialized types must have been preceded by numerous related, though more primitive, groups that passed into the cambrian or even into a more ancient period. Fossil scorpions, pedipalps, and spiders have also been discovered in carboniferous rocks. We may therefore safely conclude that convergent ancestors of these three groups were land forms that were derived in turn from a fresh-nater ancestry. But in recent years the very ancient and wholly extinct groups of the Trilobita and Eurypterida have not only been included with the king-crabs - of which there are living examples-amongst the Arachnida, these three have been viewed by eminent authorities as primitive and marine types from which fresh-water or land forms have descended. This view is favored by the occurrence of the eurypterid genus Strabops in cambrian rocks. But against this it should be said that remains of giant eurypterids are found in fresh-water rocks of carboniferous age.

But these three groups all exhibit decided affinities with the phyllopod Crustacea that are and have been fresh-water inhabitants. So a much more likely theory of descent for the Crustacea and Arachnida would be from some common fresh-water or estuarine form of late-archæan age, that gave rise to the main lines of fresh-water phyllopods and related Entomostraca, that produced at first small estuarine or brackish-water ancestors of the Trilobita and Eurypterida, and which early started air-breathing or dry land groups that became the progenitors of the scorpions, pedipalps, and spiders. Except for the king-crabs-some ancient forms of which at least were fresh-water-that have lingered on, the marine 
forms-after attaining at times gigantic size-perished soon after the close of the palæozoic epoch. The fresh-water entomostracan Crustacea and the land or air-breathing Arachnida persisted and have branched out into numerous phyletic ramifications.

So, on reviewing the living arachnidan groups, it can be said that, while one to three genera of king-crabs are recognized and are marine, at least forty-six genera of Scorpionida, twentytwo of Pedipalpi, six hundred and fifty-one genera of Araneida or spiders, one hundred and seventy genera of Solipugæ, Chelifers, Acarids, and related orders, and nine genera of Tardigrada or Pentastomida are land or rarely fresh-water. The Pycnogonida alone, that include about 42 genera, have branched off into a marine life. Thus, out of 886 arachnid genera, at most 45 can be regarded as marine, and these seem evidently to have originated from fresh-water or land ancestors.

The Centipedes and Millipedes, that together formed the older group of the Myriapoda, include 67 genera, all of which are land animals and air-breathers, though some live at times by the shore. Fossil forms have been traced back to the devonian age, but with no suggestion even then of a marine origin. All existing evidence favors the view that they, along with the Insecta, were probably derived from an annelidan ancestry, the genus Peripatus being one that connects the present group with the Annelida. But weighty reasons might be advanced for regarding both as derivatives from the entomostracan line, and so still more primitively from Rotifera.

The enormous group of the Insecta, that includes at least 350,000 living species, alike in its entire structure, history, and present distribution is so typically terrestrial that only vague suggestions have been made as to its possible derivation from some marine ancestry. The order Aptera is now generally regarded as the most ancient and primitive, while of it and of the orthopterid order fossil remains have been found in deronian, silurian, and even in ordovician rocks, while Palcoblattina has been regarded as a primitive type of the cockroaches. In common with the millipedes, the arachnids, 
and the crustacea, they were almost surely derived from an ancient cambrian or late archæan ancestry, of rotiferan structure and lacustrine habit. The writer has not attempted to estimate the number of living genera, which after all, in the present group, is of minor import when one has regard to their varied structural ramifications. For sake of comparison he has roughly included 3500 genera, most of which are truly terrestrial, a relatively small number are now freshwater, while the very few littoral or semimarine types may well be neglected.

The Hemichordata is a small group that we would view as marine derivatives from a form intermediate between higher marine Nemertinea and the Cephalochordata. The almost world-wide distribution of Balanoglossus suggests an ancient ancestry for the group, that now consists of 11 genera and about 32 species.

The Ascidiacea or Urochordata is also a marine series, which during the past quarter century has been shown to be rich in genera and species. In all 130 genera at least are recognized. The above two groups are further referred to in a succeeding chapter (pp. 537-540).

The simplest living chordate or vertebrate genus Amphioxus or Branchiostoma inhabits sandy seashores, where it burrows readily. But as stated later we would regard it as a divergent, and rather widely divergent, type from the main line of invertebrate-vertebrate ascent. As now known the group includes 4 marine genera.

The Cyclostomata or Marsipobranchii may be said to consist of 5 well marked genera, Myxine, Bdellostoma, Petromyzon, Mordacia, and Geotria. These for the writer have a special interest, as being, according to his views, in almost direct continuity with the main vertebrate line of evolution (p. 418). Bearing in mind that such genera of the Nemertinea as Geonemertes, Tetrastemma, and Stichostemma are wholly or chiefly land or fresh-water forms, even though the large majority are marine, if we build up a compounded type of nemertean from these, the resulting structure would very largely conform 
to a primitive cyclostome form, as traced in the next chapter. It becomes then a question of some importance to ascertain whether any or considerable evidence exists for viewing the Cyclostomata as primitively marine or fresh-water. Of the above 5 genera of the group, the species of lamprey or Petromyzon are now most abundant. Several species of these are purely fresh-water, all of them whether living a part of their life in the sea or at the mouth of estuaries run up rivers to sparn. As now known the larval lamprey or Ammoccetes spends several years of its life in fresh water, even though the adult may be frequent in brackish or marine waters.

Now in most features of organization Petromyzon is the most primitive genus of the group, and we are amply justified in concluding that the Cyclostomata may as well be claimed for fresh-water as for marine environment. But further the semi-parasitic habit shown in adult life indicates that Myxine and the other genera which are now wholly marine derive their environal relation and often parasitic habit from having been carried to sea by fish hosts like the salmon that are migrant between fresh and salt water. Therefore of the living species of Petromyzon we would claim all as fresh-water; the species of the four remaining genera may now be ranked as marine.

Next to the molluses the true fishes of the present day, that include 12,000 species at least, present a serious puzzle to the geographer. When we find 700 or more species that are often modified for abysmal depths of the ocean, almost as many that are inhabitants of high land regions, others that are now land-locked fresh-water forms, and some that can live for months in a dried up dormant or hibernating state, a slight idea is obtained of the difficulties that surround the problem of determining as to their probable environal origin and generic distribution.

But the fact that at least 90 per cent. of the entire group have become preeminently adapted for aquatic existence, and thus that marine facilities are enormously greater than river, lake, or swamp facilities, must be kept in view. It is 
not surprising therefore that of the 12,000 to 13,000 species already recorded about 8000 species or more are marine, fully 4000 are fresh-water, and a few hundred like the salmon, the blenny, and the stickleback show surprising adaptability to salt-, brackish-, and fresh-water conditions. As with the lamprey so with many of the adaptable forms like the salmon, their present-day instincts and irrito-environal responses cause them to seek the sea or even the ocean as a great and rich feeding ground, then to return to rivers where their inherited instincts cause them not merely to spawn, but to excavate special cavities amid stones or gravel for this purpose.

The entire question of the relation of the spawning migrational or anadromous fishes, to their phylogeny on the one hand and their inherited or acquired habits on the other, is one of intense interest, but is one also that has yet to be thoroughly investigated in light of all the facts of palæontology, of embryology, of structure, and of taxonomy. That the "homing" instinct is one which may be lost, or more or less retained, or again acquired seems to be indicated in the case of the sea lampreys that return to the rivers, in those fishes which now seem to breed in deep sea recesses, and in the salmon that waver between the brook trout on the one hand and the marine or Quinnat salmon on the other.

We would shortly sum up our conclusions by saying that the Cyclostomata were probably, as they still are in part, of fresh-water origin; that the Selachii are and have been through long epochs nearly all evolved as marine forms; that the Polypteridæ are and probably have been fresh-water in habit and history; that the Dipneustei are similar to the last; that the Chondrostei or spoonbill and sturgeon series agree with the two last except that some of the sturgeons pass into the sea to feed and return inland to spawn; that the Holostei are now wholly fresh-water; that of the 13 suborders of the Teleostei given by Boulenger the first or Malacopterygii are almost wholly fresh-water, the third or Symbranchii are mainly fresh-, more rarely brackish-water inhabitants, the fourth or Apodes-that includes the eels-are rarely fresh-water, 
usually marine and breeding there at considerable depths, the fifth or Haplomi-that includes the common pike-are largely fresh-water though a fair number live and even breed in the sea, and the remaining nine (Heteromi, Catosteomi, etc.) are almost wholly marine with rarely fresh-water inclusive genera.

But a comparative review of the number of fresh-water and marine genera of the present day, in the light of their known first appearance in geologic time, brings out some rather remarkable results. Thus, of the entire group of the Gnathostomata or toothed fishes, 453 living genera are purely fresh-water in their life history, and 902 genera are marine, with occasional fresh-water representatives. But when such statistics are compared with the geological record, another and different relation is revealed. The oldest known groups of the true fishes are the Crossopterygii, the Dipnoi, and the Chondrostei, representatives of all of which have been found in the lower devonian. All of these are fresh-water forms at the present day, and many if not all of the fossil forms likewise were. But as these attained to the greatest climax of their derelopment in inland seas, in lacustrine and in fluviatile situations, the group of the Selachii probably evolved from the primitive acanthodeans, took to a marine life, and soon became an increasingly powerful type of fish up to the period of the carboniferous epoch or even later. Their aggressive and roracious tendencies, their often lithe movements, and their inclination to feed on dead as well as living animal matter seem to have enabled them to contend successfully with the giant representatives of the cephalopod molluses that at that time largely held the seas. But the highest or teleostome fishes gradually evolved from the permian period onward in ever increasing numbers. It is a striking fact however that, if we compare the number of living genera of fishes whose ancestry dates from the period of the cretaceous or further back, 320 living genera are fresh-water, and 155 are marine. Only during the period from the cretaceous and especially from the beginning of the eocene onward do the teleostean 
fishes attain that enormous marine development which now characterizes them; so that, while 133 genera of recently evolved living teleosteans are fresh-water, $7 \pm 7$ genera are marine. This remarkable development seems to have been correlated with a gradual dying out alike of the dominant groups of cephalopod molluscs and of selachean fishes. So, when we sum up all of the living genera of gnathostome fishes and find that 453 are fresh-water and 902 are marine, this by no means represents the primitive distributional relation.

We would therefore consider that all palæontological, structural, and geographical evidence strongly favors the view that the primitive fishes have had a fresh-water origin and that derivative marine forms have evolved from these, which, in the case of the anciently derived selacheans and of the recent teleosteans, reached each to a high climax of species diversity - the former during the cretaceous period, the latter in our own day.

The Batrachia or Amphibia are and have been so evidently inhabitants of rivers, ponds, or land areas that an enumeration of the genera might seem to suffice for our present purpose. Boulenger concludes "that the geographical distribution of the Amphibia agrees in general with that of the fresh-water fishes" (Camb. Nat. Hist. VIII (1909) 69). But the haunting notion of a marine - at least a shore origin - for the main vertebrate lines-seems to pervade so much the minds and the volumes of most students that it may be well here to emphasize - what is expanded on succeeding pages-namely that from a fresh-water nemertinean ancestry we would derive the main vertebrate line through a fresh-water cyclostomatous type to a coecilian and thence to a urodelan stage. On the other hand the derivation of the varied carboniferous and permian cuirassed batrachians from primitive fresh-water ganoid progenitors, or from more ancient early silurian or cambrian forms ancestral to both, seems at least possible, but the question is open to discussion. Of the nearly 2200 species that fall under 171 genera, that now make up the Batrachia, all may truly be reckoned as land or fresh-water, 
and as having been similarly derived in their past environal history.

The Reptilia are acknowledged by all zoologists to have originated from fresh-water or terrestrial ancestors, for whether we consider the flipper-limbed plesiosaurians and ichthyosaurians of the Lias, or the phases of paddle-limb formation seen in the marine chelonians from the cretaceous period onward, both are accepted as gradual modifications on a land ancestry. The 528 genera therefore, and fully 5000 species fall under that head.

The 440 genera and at least 12,000 species of birds, as well as the 494 genera and 5000 species of mammals, have equally clearly originated as land forms. A synoptic table therefore of genera that make up the Hemichordata, Urochordata, and Chordata would show the following results:

\begin{tabular}{|c|c|c|}
\hline Group & $\begin{array}{l}\text { resh-water } \\
\text { genera }\end{array}$ & $\begin{array}{r}\text { Marine } \\
\text { genera }\end{array}$ \\
\hline Hemichordata................ & & 32 \\
\hline Urochordata................. & & 130 \\
\hline 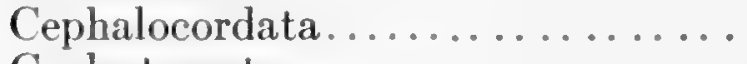 & & 4 \\
\hline Cyclostomata................. & 1 & 5 \\
\hline Gnathostomata... & 453 & 902 \\
\hline Amphibia.... & 171 & \\
\hline Reptilia.......... & 528 & 11 \\
\hline Ares............ & - 440 & \\
\hline 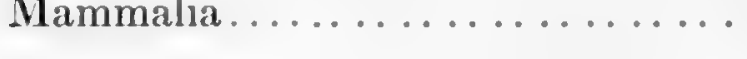 & - 455 & 39 \\
\hline secent & 0000 & 11200 \\
\hline
\end{tabular}

While mere statistics apart from historical evidence can proverbially prove anything, we have further attempted briefly to show the developmental trend that most have taken, where this is fairly accurately known. The evidence indicates clearly that some groups like the Spongida, the Enterocoela, the Echinodermata, a considerable number of the Annelida and of the Crustacea, a good majority of the Mollusca, all of the Brachiopoda, and most of the Polyzoa amongst Invertebrata are marine. But of these the morphological, the embryological, and the geographical evidence, as well as in some cases the palsontological, very strongly suggests that the Spongida, the Enterococla, the Annelida, and the Crustacea very early originated as fresh-water, not as marine, organisms. 
In this connection however it is well to remember that owing to active volcanic agency, and the activity of hot springs and other thermal influences from the archæan to the silurian epochs, many land-locked water areas, and probably even streams, must have been charged with free acids, salts, and other constituents much as are some of the siliceous, carbonated, iron, and other areas of streams at the present day. The variety of these, their comparative frequency, and the adaptation of many organisms to such waters, would either give an elastic adaptability to the organisms, as is still shown by many species or genera of the present day, or it would accustom some organisms by slow but sure stages to a marine existence.

If we attempt to estimate the number and variety of environal agents that fresh-water, marine, and land animals are relatively exposed to, it may safely be said, we believe, that fresh-water species are most exposed throughout the yearthough not perhaps during each successive day or week-to varying environal changes. The inhabitants of shore lines and estuaries have frequently sharp daily changes, but the more extended periodic changes are averagely less marked probably than for the occupants of lakes and rivers.

In this connection Cooke says in his discussion of molluscan distribution (133: 1): "It is the generally accepted opinion among men of science that all life originated in the sea. Not that all parts of the sea are equally favorable to the development of forms of life. The ocean surface with its entire absence of shelter or resting place, and the deep sea, whose abysses are always dark and cold and changeless, offer little encouragement to plant or animal life, as an original starting point." And again: "It was no doubt the littoral region and the shallow waters immediately below it, a region of changeable currents, of light and shade, of variation, within definite limits, of temperature and tide effects, which became the scene of the original development of plant life, in other words, of the food supply which rendered possible its colonization by higher animals. But the littoral region, besides the advantages of tenancy which it offers to animal life, has also its drawbacks. The violence 
of the surf may beat its inhabitants in pieces, the retreat of the tide exposes them, not merely to innumerable enemies in the shape of predatory birds and beasts, but also to a change in the atmospheric medium by which they are surrounded."

Later (p. 11) he reaches the conclusion: "The ultimate derivation of the whole of the land and fresh-water molluscan fauna must, as has already been remarked, be looked for in the sea." These arguments have strong weight, at least for large groups of the Mollusca. But we must place against such the fact that eren the highly developed scorpions and insects were already evolved during the carboniferous period. Further the enormous development in species and genera which the "worms" and the land arthropods have undergone is proof that a fresh-water or land environment is prolific of organic change, even though the strain on life-the struggle for existence-there is greater than in the sea. Accordingly proenvironal response and selective survival act more sharply to evolve new types.

The extensive and often rapid transfer of marine forms by sea currents, or by attachment to other sea animals, are unquestionably important factors in spreading these abroad. Where groups therefore like the Spongida, the Echinodermata, or the Mollusca become preponderatingly marine, they branch out into a great variety of types. But such are not the groups which seem to have steadily evolved into higher and specially alert organisms, that by quick nervous movement could equally escape from enemies or untoward physical conditions, and also secure food supplies most quickly and extensively.

No one we believe would now doubt that the Sirenia and Cetacea are greatly modified marine forms that have originated from a terrestrial ancestry amongst the mammals. And yet they are as greatly modified morphologically from what must have been the originating land type as many of the marine crustaceans and molluses are and have been from fresh-water types that we would regard as closely resembling their ancestral originators. But the close fundamental agreement between larval forms of many coelenterates, echinoderms, worms, and 
molluses, of which examples from the first and last seem clearly to have been of fresh-water origin, is an added argument for the primitive development of all such groups in fresh water, though possibly often in fresh water of a kind that may have been highly charged with dissolved salts.

In view then of the statistical, phylogenetic, structural, and other details, brought together in this chapter, in view

Statistical Details Regarding the Animal Kingdom

\begin{tabular}{|c|c|c|c|c|c|}
\hline & \multicolumn{2}{|c|}{ Genera } & & \multicolumn{2}{|c|}{ Genera } \\
\hline & 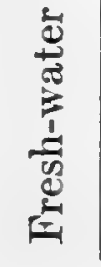 & $\stackrel{\stackrel{\mathscr{E}}{\tilde{E}}}{\stackrel{\mathscr{E}}{\Sigma}}$ & & 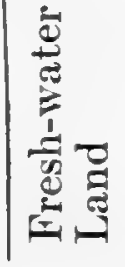 & 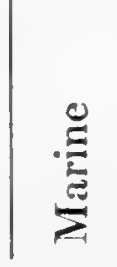 \\
\hline Protozoa & & & Nematoda. . & 54 & \\
\hline Proteomyxa. & 23 & 7 & Oligochæta. & 118 & \\
\hline Lobosa... & 15 & 2 & Hirudinea. . & 33 & \\
\hline Mycetozoa. & 48 & & Polychæta... & 3 & 145 \\
\hline Heliozoa.... & 20 & 8 & Gephy'a-Kinorh'a......... & & 20 \\
\hline Foraminifera & & & Acanthocephala........... & 4 & \\
\hline (a) terricola... & 14 & 3 & Gastrotrichea............ & 6 & \\
\hline $\begin{array}{l}\text { Foraminifera } \\
\text { (b) maricola.... }\end{array}$ & & 130 & $\begin{array}{l}\text { Chætosomatida........... } \\
\text { Nemertinea............ }\end{array}$ & 2 & $\begin{array}{r}3 \\
39\end{array}$ \\
\hline Radiolaria & & 100 & Polyzoa............... & 8 & 39 \\
\hline (a) terricola.. & 6 & 2 & Brachiopoda............ & & 15 \\
\hline Radiolaria & & & Mollusca........ & 257 & 560 \\
\hline (b) maricola & & 142 & Crustacea. & 213 & 879 \\
\hline Sporozoa............... & 65 & 40 & Arachnida............. & 841 & 45 \\
\hline Hæmoflagellata.......... & 6 & 2 & 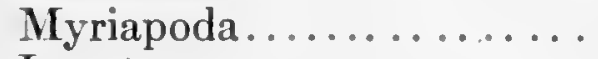 & 67 & \\
\hline Mastigophora........... & 107 & 36 & 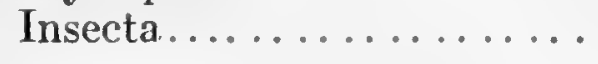 & 3500 & \\
\hline Infusoria.............. & 126 & 24 & & 7881 & (20\% \\
\hline Metazoa & & & $10 \mathrm{ta}$ & 8101 & 0081 \\
\hline (a) achordata & & & Metazoa & & \\
\hline Porifera...... & 10 & 340 & (b) chordata & & \\
\hline Coelenterata. & 6 & 619 & Hemichordata....... & & 11 \\
\hline Echinodermata.. & & 478 & Urochordata. & & 130 \\
\hline Rotifera.............. & 73 & 11 & Cephalochordata........... & & \\
\hline Mesozoa.. & & 6 & 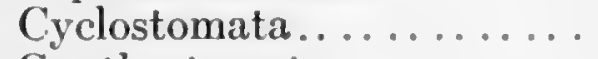 & 1 & \\
\hline Turbellaria & & & Gnathostomata... & 453 & $90 \stackrel{\mathcal{Q}}{2}$ \\
\hline (a) rhabdocœela. & 24 & 38 & 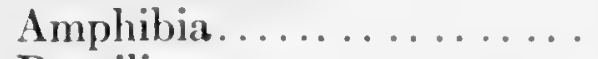 & 171 & \\
\hline Turbellaria & & & Reptilia.... & 528 & 11 \\
\hline (b) tricladida. & 27 & 8 & Ares.............. & 440 & \\
\hline $\begin{array}{l}\text { Turbellaria } \\
\text { (c) polycladida. }\end{array}$ & & 29 & Mammalia............... & 455 & 39 \\
\hline Trematoda. & 45 & 10 & Total & $20+8$ & 1102 \\
\hline Cestoda.. & 60 & 3 & Protozoa-Metazoa achordata & 5781 & 3697 \\
\hline & & 4 & Grand Total. & 7829 & 4799 \\
\hline
\end{tabular}


also of the acknowledged land or fresh-water origin of nearly all vertebrates other than the more recent teleost fishes, and in riew finally of the considerations to be presented in the next chapter, the writer is forced to the conclusion that the main and dominant lines of animal evolution have all originated in fresh water or on land, and that only side lines have assumed a marine life, though these have often branched out profusely into species, and even have given off again groups that have in rare cases returned to a fresh-water or a land life. 


\section{CHAPTER XV}

\section{THE EVOLUTION OF ANIMALS (CONTINUEd)}

In a study of the palæontological record of animals, and of the facts it reveals as to the periods when different species, genera, and families appeared, one is often impressed by the apparent suddenness with which many of the leading groups are ushered in. This applies not merely to invertebrates, but even to vertebrates, as in the case of fishes, which appear apparently suddenly in such biologically ancient rocks as the silurian. But it is permissible to estimate the comparative evolution of the species, and the length of time needed for such evolution, by the thickness of the strata in which their remains occur. Then the appearance of arthropods and of gasteropodous molluscs in the cambrian, as well as of fishes in the lower silurian, rocks is appropriate. For the frequent thickness of these rocks, in various parts of the earth, indicates long ages of biochemical activity.

A variety of genealogical tables and of classifications has been proposed, each one of which sets forth the supposed main line of evolutionary advance from a unicellular type to man, as well as the important side branches that have diverged from it. Most of these in passing upward from the Protozoa early reach some of the annelidan sea types, that conduct thence by rather sudden and discontinuous morphological variations to the Hemichordata or series that includes Balanoglossus, thence to a primitive free-swimming member of the Ascidia or Urochordata and again to the Cephalochordata that has as sole representative Amphioxus (Branchiostoma), while from it a saltatory advance is made to the scaled fishes. Therefore, from the early "vermes" or worm ancestors, on to the fishes, a marine environment is claimed for the vertebrate phylum. 
While not denying a somewhat distant affinity in the main phylum with such a series of marine types, the writer hopes to show that a more condensed and much more direct continuity can be traced at the present day, through small, simple, but multicellular forms, intermediate between some of the ciliate Infusoria and living forms of Rotifera, thence through primitive and later through advanced members of the Turbellaria to the fresh-water Nemertinea. From the last we hope to show that the continuity is wonderfully perfect through a semi-cyclostomatous to a proto-cyclostomatous fresh-water ancestry. From the latter through a cæcilian to a semi-urodele type, the gradation is equally marked, while by some fossil forms we seem to pass onward to a marsupial and later to a high mammalian stage.

We are fully aware that such a proposed plan upsets many cherished classificatory tables of past years, but if the present substitution is truly based on sound morphological principles and on phylogenetic continuity it will doubtless be accepted. If it is unable to stand the test of time, it can be set aside as another earnest even if misdirected groping after the truth.

But contrary to usually accepted views, and in line with the evidence presented in the last chapter, we would emphasize the statement that all of this evolution has probably-almost certainly-proceeded, either in relatively shallow fresh-water areas (using this designation in its widest sense) or even at times on land, not in salt-water expanses and least of all in ocean depths. Accordingly in speaking of some structural details amongst the Rotifera, the Nemertinea, and the Cyclostomata, that may now be best traced in brackish or marine forms, we accept it that in not a few cases similar structures may have been-probably even were-developed amongst fresh-water or land species that may either be alive or are now extinct. Our reasons for such a position are as follows: while for example amongst the Nemertinea the fresh-water and land types are now decidedly in the minority and do not belong to the highest orders of the class, they are so evidently and fundamentally in line with the higher marine forms, and 
exhibit so many features of diversity in themselves, that the likelihood of land forms having been evolved of equal or even of greater complexity than the marine types is extremely likely, specially if we can connect these with higher fresh-water illustrations. In other words, we do not hesitate to attempt the construction of a composite morphological photograph from the species of any order, if such dovetails into and elucidates a gioup of species from an order below or above.

In starting on such a task with man, the anthropoid apes, the lemurs, the lower mammals, and some still more primitive forms, as increasingly advancing types from the last- to the first-named, what we might expect to deal with throughout the entire scale of progress would be graded examples, that showed in common a soft body of rounded or somewhat dorsiventrally flattened outline and with bilateral symmetry; that had a skin devoid of scales, scutes, or feathers, but which was rich in mucous or sebaceous skin glands, and bore cilia bristles or hairs; that showed a somewhat enlarged head with dorsally placed brain which connected with olfactory, optic, and auditory sense-centers; that had some active, energized, and adaptable structure or structures that would bring it into constant and varied contact with its environment; that possessed a dorsal nerve cord and lateral nerves connected by transverse threads; that exhibited no distinct "somites," but had an internal, more or less segmental, arrangement of the myotomes, the nerves, the blood vessels, the excretory and the reproductive organs; that had a more or less discoid sucking mouth and simple alimentary canal; that showed a dorsal notochord or evident stages originating it and explaining its ontogeny; in which the egg segmented holoblastically, and from which an embryo was derived, that was wholly or in part ciliate.

Since practically all metazoan organisms start with an egg, the last phase in the above group of requirements represents the one that we may consider first, as it was doubtless the one that originated the metazoan group.

In the holotrichous ciliate Infusoria there are still preserved to us the primitive unicellular nucleate organisms from which 
most of the higher groups sprang. The tendency of some of these to form not merely fine cilia of complicated relation to the cell and its nucleus, but also strong tactile bristles disposed at definite points, deserves emphasis. The next step therefore in the main line of ascent is to ascertain how and by what stages the ciliate infusorian organism became multicellular, and what are now the simplest representatives of that multicellular state.

But for the entire group of the Spongida we evidently must look not to the Infusoria ciliata, rather to the flagellate infusorians or Mastigophora. And in such semicolonial, semimulticellular genera as Proterospongia and Phalansterium we have examples of what may have been during the mid- or late archæan epoch abundant evolving types. The unicellular embryo of these, early segmenting and attaching itself, started a zoological cul de sac that has persisted to this day.

As regards the main line of evolutionary progress through ciliate infusorians, the first morphological advance we believe is in the enlargement of each cell organism or bio-cognitic mass, and the formation of several nuclei that become biocognitic centers in it.

Next in the intimate relation of each organism to its environment this is beautifully assured, first by the usually extremely flexible and adaptable body mass; second, by the ciliary investment; third, by the frequent presence of special centralized cirrhi near or round the mouth or head end, along the body, or posteriorly; and, fourth, by the formation in some genera like Trachelius, Ileonema, and Dileptus of a preoral process of a tactile proenvironal nature.

Again the great majority of the Infusoria are markedly bipolar and have a definitely restricted sucking mouth, an endoplasmic biotic mass for digestive and general vegetative activities, and occasionally even a restricted anal region.

The abundance and uniform character of the cilia over the cells of the embryo or even the adult animal, in many divisions of invertebrata, and their retention over soft protected surfaces of the vertebrata would lead us to suppose that possibly 
these may have originated from ciliate infusorians, through repeated division of the nucleus, and succeeding separation of the cell protoplasm into nucleated areas or cytoplasmic masses. On the principle of stereoenergics also, if definite and characteristic lines of energy-flow have caused the morphological origin of definite layers, structures, or masses of matter in the Infusoria, that have severally become associated with definite functions, such might be continued upward from the unicellular to the multicellular organism, and little altered as to position or relation.

Now in not a few infusorian genera, such as Lacrymaria and Oxytricha, division of the nucleus and subsequent diffuse distribution of the resulting nuclei occur. Further in the higher Ciliata, exact differentiation of the cell-substance, first into a surface zone with sensory cilia and often trichocysts, second into a muscular and excretory zone, third into an alimentary zone with mouth, canal, and anus, is shown. Can such a condition then be correlated and connected with any of the simpler Metazoa?

Of the latter, we regard the Rotifera for many reasons as the foundational group. In the accompanying diagrams (Fig. 14, a, b) are set forth the structural details of a composite ciliate infusor and of a rotifer. Alike in the relation of ventral and dorsal surface during feeding, as in the opening of mouth and anus on these two surfaces; in the possession of an anterior ciliated wreath; in the differentiation of the external or surface layer into a sensory tissue and the formation therefrom of cilia; in the formation of myonemes or myotomes and of excretory organs from the middle-body substance; in the origin of a mouth, at times of a protrusible pharynx, of an alimentary canal, and of an anus; in the polar actions and reactions shown by both; as well as minuter details that cannot here be examined, both show an exact fundamental agreement.

As the embryological studies by Zelinka and the mature studies of the Rotifera by many observers show, the demarcation of the individual cells of the Rotifera is faint, and becomes increasingly so as one passes from the early to the late embryo, 
and then to the adult. But the lines of stereoenergesis in the Rotifera remain fundamentally as in ciliate Infusoria. While such is true, however, the writer is deeply sensible that no single organism now exists which would readily bridge over the highest existing infusor with the simplest rotifer. But this is not surprising when we bear in mind the wide and deep organic gaps which exist all along the biologic scale.

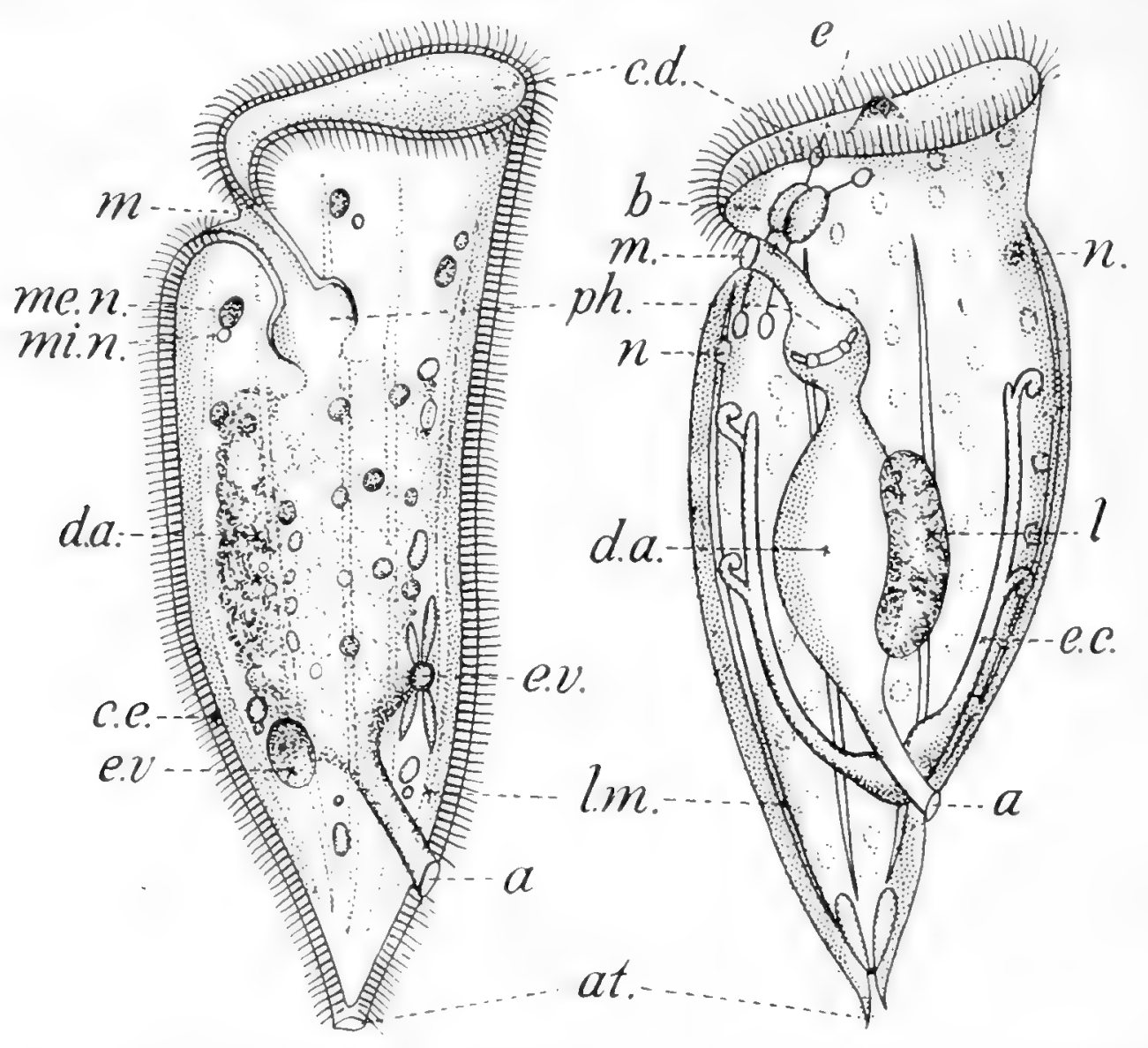

FIG. 14.- $a$, ciliate infusor; $b$, rotifer; $c . d$. , ciliated disc; $m$, mouth; me.n., meganuclei; mi.n., micronuclei; $n$. , nuclei; $p h$. , pharynx; d.a., digestive area; $l$., liver; $e . v$. excretory vacuoles; $e . c$., excretory canals; $a$, anus; l.m., longitudinal muscles; $b$, brain; $e$, eyes.

So, we would accept it as likely that continuous morphological progress was made from higher ciliate infusorians to simple rotifers. During the process, in addition to the establishment of a soft and faintly differentiated system of ecto-, meso-, and endodermal cell layers, as traced by Zelinka, there further originated that double swelling and ingrowth of ectodermal cells in the head segment that formed the commencing nervous system.

As regards its origin and value, though the following is only in the nature of a vague surmise, it might be recalled that 
in the Hæmoflagellata and in the Infusoria the nuclear chromatin early separated after fertilization into a trophonuclear (for the former) and meganuclear (for the latter) constituent, also into a kinetonuclear (for the former) and micronuclear (for the latter) mass. The trophonuclear and meganuclear bodies seem to correspond largely in energizing and organization action to the cognitic nuclear substance of plant and simpler animal cells. The kinetonuclear and micronuclear bodies seem to be greatly more complex, energized, and hereditarily important, as if commencing centers or substances for cogitic energizing action and reaction.

It would be of special interest therefore to learn whether, in the formation of reproductive cells and of ganglion cells in rotiferan embryos, there is not given off to or accumulated in these a more complex super-chromatin Nissl substance, or kinetonuclear substance, that becomes associated with flows and condensations of cogitic energy. The future will alone determine whether such a possibility exists.

So while we would view the coelenterate and echinoderm stocks as side linés of evolutionary advance, which both develop a nervous system that is typical for them, it is to the Rotifera and progressively derived groups therefrom that we would look for the direct line of invertebrate-vertebrate ascent. This then is our immediate quest.

But, in comparing these and in considering the possible origin of higher invertebrates and of vertebrates, we are brought face to face with several well known circumstances. First: If we regard the cambrian epoch as that during which life can be traced directly in its earliest fossil remains, and glance backward to the archæan as well as forward through all succeeding epochs, it seems as if life had comparatively suddenly appeared in telescoped complexity and variety. For, apart from readily obliterated plant life and from simple soft animal organisms that may equally have been obliterated, we are suddenly confronted with elaborate representatives of nearly every invertebrate group, while, in the ordovician formation above, remains of insects, scorpions, and fishes tell us that 
the fresh-water or terrestrial invertebrate half of animal life had already reached its highest platform, and that the vertebrate half was already well under way. So between the midarchrean and the late cambrian we unquestionably have to look for the beginnings and the full development of the invertebrate line, as well as for the established foundation of vertebrate organisms; while from the close of the cambrian till our day both invertebrate and vertebrate groups have been some of them erolving, some stagnating, some devolving, many, rery many, disappearing, in the great struggle for existence.

Second: During the evolution of the invertebrates into the rertebrates the writer would suggest that two main lines have arisen, which show their first divergences from a common stock in the group Rotifera above considered. These are the soft-bodied or scaleless, and the firm-bodied or scaled, protovertebrates, that are first shadowed forth in members of the above group.

Third: In comparisons of the Vertebrata hitherto made, it has not generally been recognized that two such lines can be traced, one that includes the Cyclostomata, Cæciliada, Lrodela, Marsupialia, and higher mammals; the other that includes the scaled or plated fishes, the ancient or scaled and plated amphibians, the reptiles, and the feathered birds.

Fourth: That in spite of their soft bodies and therefore perishable characters, sufficient living and fossil evidence can be secured that enables us to trace the main line of ascent from the rotifers through turbellarians, nemerteans, cyclostomes, and urodeles to higher mammals, and so that Huxley's contention is correct when he connected the Amphibia (Batrachia) and Mammalia in a continuous ascending series.

With the above principles to guide us we may first review general features of resemblance from rotifers to the mammals, and thereafter, in the remainder of this chapter and in the next, treat with minute detail the structural characters of the Nemertinea, the Cyclostomata, and higher groups.

The body surface in the Rotifera, in rhabdocœel Turbellaria, in Nemertinea, in the ammocœete cyclostome larva, in 
the apodous and the urodele batrachians, also in the mammalian embryo, is wholly or largely, or in reduced and restricted degree only, covered with cilia. In the Batrachia the cilia become either partially or wholly shed in later life from formation of an external cuticular covering. Owing doubtless to the relation of the embryo to surrounding food, to a passive viviparous embryonic stage, and the formation of a cuticular layer as in Batrachia, the mammalian embryo wants cilia. But that it is derived from one that possessed such is evidenced by the different regions or sensitive cavities which show such, and which are derived directly or indirectly from the epidermis. The restricted and transitory state seen in the Batrachia has therefore become more circumscribed in Mammalia.

In the Rotifera, the rhabdocoel Turbellaria, and in most of the Nemertinea the skin is provided with one or more sets of secreting cells, that exude definite surface excretions. In most of the Nemertinea simple subepidermal goblet cells that secrete mucus may be mixed with rod cells (Drepanophorus), or deeply sunk clustered cells may pour out a surface exudate by long ducts (Carinella), or again nematocysts, that remind one of the thread cavities in some Infusoria, may be encountered. Usually therefore an abundant mucous secretion is poured out that may become an enveloping mucous tube in some nemertean land forms.

In Cyclostomata goblet mucous-secreting cells and nematocysts are both developed. The quantity of mucus excreted may be moderate (Petromyzon) or great (Myxine). In the Batrachia apoda deeply sunk mucilage glands occur, along with squirt gland cells that exude an irritant poisonous juice which at least physiologically may be compared with the liquid of the nematocysts above. In the urodele batrachians abundant mucus or slime glands are intermixed with poison glands. As newt-like forms became increasingly terrestrial the slime glands would tend somewhat to dry up unless under special or supraoptimum stimulus. Thus transition would be effected to the mammalian skin where sweat glands, sebaceous follicles, and milk glands are all present, and excrete under appropriate conditions.

In all of the groups now under consideration the mouth is anterior and mostly subterminal, the simple alimentary canal is continued backward and ends in a subterminal anus in most, though in some rotifers and in turbellarians the canal ends blindly. The body surface exhibits no definite segmentation, though annular constrictions of the muscles and connective tissue, as well as the internal disposition of nerves, 
vessels, and of segmental organs, suggest embryonic somites.

Lateral sensory lines are scarcely traceable in rotifers or in turbellarians, since the ciliated disk of the former and the ciliate surface of the latter are highly sensory. But the socalled "marginal groove" of some turbellarians may yet be homologized with that now to be noted.

In nemerteans and all higher forms such as the cyclostomes and urodeles two highly sensitive lateral lines are connected with the lateral nerves, and only in mammals - with their hair covering -is the sensitive relation and the recognized morphological aspect of it largely reduced. But even in man lateral tracts are more sensitive than are the dorsiventral regions.

The circumoral area in all is circular to elliptic and is surrounded by swollen ridges or lips except in simpler rotifers and in turbellarians. In the latter groups the mouth leads into a buccal or pharyngeal region that is chitinous and protrusible as a short proboscis, and which can be retracted into the top of the gullet or œsophagus. This pharynx develops rods, teeth, or hooks of a horny nature that are used when the proboscis is protruded. In the Nemertinea the proboscis may vary from a short to a greatly elongated highly sensitive body that can be rapidly shot out or retracted again into a special cavity-the proboscis sheath or rhynchocoel. As this structure assumes special importance in connection with the phylogeny and morphology of the entire vertebrate series, it deserves detailed study.

The proboscis and proboscis sheath, from all present embryological evidence, seem to arise as correlated structures. The former according to Hubrecht and Salensky is formed as a tubular ectodermal invagination of preœsophageal origin, which becomes surrounded by the sheath that has become constricted off dorsally from the mesoderm or more probably from the endoderm, though exact evidence on this important point is still lacking. Now, in many of the 'Turbellaria that show decided affinity to the Rotifera though on more advanced and derived lines of their own (p.421), there are formed above or behind the mouth a cœecal œesophageal invagination and a central proboscidiform swelling, both of which may attain relatively great size and lie at right angles to or above the mouth, while the inner walls of the former become strengthened by muscular fibers that may aid in evertion and retraction of the proboscidiform part. Now were such a cœeal sheath extended backward parallel to the alimentary canal, and were its orifice to open along with the œesophagus by a common 
anterior pore, the condition would be as seen in the Metanemertinea, but were the pharangeal orifice to be carried forward outside the oral area the typical proboscis sheath or rhyncocœel and associated proboscis of other Nemertinea would result. Various stages in this process can be traced that are all illustrated and described by Buirger.

Phylogenetically then, we would regard the simple protrusible pharynx of some Rotifera as the primitive rudiment of the proboscis. In the simpler fresh-water Turbellaria or Rhabdocola, that show in many details characters intermediate between Rotifera and Nemertinea, the deep dorsal pharyngeal invagination with muscular wall and evertible proboscis would represent the next stage (Plate Fig. 15a). By backward prolongation of the invagination or coecum, and forward traveling of the orifice till it lay outside of and in dorsal front position to the mouth, the typical sheath and proboscis of some nemerteans would result. This would be stimulated to development on the principle of action and reactionstimulation flows of energy and stimulatory response-through increasing sensitivity and elongation of the proboscis, and its increased use as a tactile, an offensive, and an absorptive organ. In the Turbellaria all of these functions, specially the tactile and absorptive, are clearly shown. But in the Nemertinea the proboscis became more and more an elongate sensitive rapidly expanding and contracting structure, that has brought the organism into constant contact with environal conditions. So it acquired a very fundamental association with, and became richly innervated by processes of, the brain between which it lies in all higher nemerteans. Through constant muscular activity also, in relation to nerve supply, a steady increase in its investing sheath took place, till this became the long hollow tube or rhyncocœl that stretches backward and is a powerful support for the body parts.

But its possible future evolutionary history gives to the combined proboscis and proboscis sheath a surpassing interest. In the years 1883 and 1885 the distinguished Dutch zoologist Hubrecht promulgated the view that the latter is the precursor and first foundation of the vertebrate notochord.

His hypothesis was thus expressed: "According to my opinion the proboscis of the nemerteans, which arises as an invaginable structure (entirely derived, both phylo- and ontogenetically, from the epiblast), and which passes through a part of the cerebral ganglion, is homologous with the rudimentary organ which is found in the whole series of vertebrates without exception-the hypophysis cerebri. The proboscidean sheath 
of the nemerteans is comparable in situation (and development?) with the chorda dorsalis of vertebrates."

The arguments he adduced $(134: 349 ; 135: 470 ; 136: 121)$ were alike weighty and suggestive, but they have hitherto stimulated to no marked advance. Somewhat strangely also Hubrecht, though greatly improving his original position by additional embryological and histological evidence, does not seem to have followed out his fruitful suggestions by study either of the lower or of appropriate higher animals. This we believe was probably due largely to the predominating attention given by other zoologists at that time to the Enteropneusta, the Urochordata or ascidians, and the Cephalochordata or Amphioxus group, all of which seem to be decidedly lateral or even degraded offshoots from the main ascending line.

To the writer, Hubrecht's position forms one of the most far-reaching and brilliant steps ever taken in zoological science. F. M. Balfour had advocated a nemertean ancestry for vertebrates, while Lankester decidedly favored Hubrecht's view. But the past quarter century has witnessed no further advance. The writer will therefore try to advance the position further, in hope that zoologists may take it up in its many fruitful ramifications.

From the standpoint of advantageous organization in the struggle for existence, the formation of the proboscis and proboscis sheath has two high recommendations in the abstract. From early dorsal invagination of the whole structure, in some now lost type of the rhabdocœel turbellarians, it came to lie directly between and against the dorsal nerve-mass and to be increasingly stimulated by the latter; it thus placed the organism in intimate and delicate relation to its environment. In other words, between the brain as the evolving cognito-cogitic center and the environment of the organism, the most direct and sensitive pathway possible was established. This, we consider, explains why the nemerteans gave off phyla that in time progressed by selective survival to higher and successful stages of organization, alongside armored animals of the arthropod and molluscan types that have largely succumbed.

Second, the increasingly perfect protrusion and retraction of the proboscis into a muscular wall or sheath, such as we see in its primitive form amongst turbellarians, started a nucleus or center of resistance for the whole body, such as no other parallel group possesses. Thus the sheath, originally a dorsal mesodermal or more likely endodermally derived tubular investment to the proboscis, became a cylindrical 
elongated tube that was gradually separated from the alimentary canal or blastocoel, through strains exerted on its walls by the extensor and retractor muscles of the proboscis, and by sliding action of the proboscis itself. Favoring this view-apart from confirmatory details furnished by turbellarians - is the occurrence in Drepanophorus of lateral lobes that correspond in position and verticality with coeca of the alimentary tract. The structure of the sheath equally suggests its embryonic origin, for this consists of an investing cell layer similar to that lining the alimentary canal, that is surrounded by an inner longitudinal and outer circular muscular wall. In most genera the sheath contains loose irregular semistellate cells, but Hubrecht draws attention to the fact that in Cerebratulus its posterior end, that is not now occupied by the proboscis, becomes filled with soft continuous nucleated cellular tissue, much resembling the central cells of the vertebrate notochord. This is a suggestive partial proof that, where the proboscis has come to fill its sheath imperfectly, the latter instead of being obliterated has so fully assumed another function-that of strengtbening in embryonic and later life - that it increases its cellular continuity, instead of becoming absorbed.

As Hubrecht, Bürger (132), and others have also emphasized, the sheath extends lengthwise anteriorly to "the level of the nervous commissure, through which the proboscis passes." Further in its disposition amongst typical vertebrates "the anterior end of the notochord terminates immediately behind the attachment of the pharyngeal membrane" (134: 436). This is typically the line of insertion of the proboscis into the anterior end of the sheath in nemerteans, so that in both details given the two structures accurately agree.

Hubrecht viewed the proboscis in its entirety as "homologous with the rudimentary organ which is found in the whole series of the vertebrates without exception-the hypophysis cerebri" (pituitary body). This brilliant explanation might have obtained rapid and wide acceptance had its author somewhat limited or modified its scope, and at the same time pushed his comparisons further.

That the proboscis for some Nemertinea and the hypophysis for vertebrates are both primarily developments from the roof of the oral sinus are now accepted facts. Thus Hertwig says regarding the latter (op. cit. 436): "It first arises at a very early stage, when the oral sinus has just arisen, and is still separated from the cavity of the head gut by the pharyngeal membrane. It is therefore a product of the outer 
germinal layer and not a growth from the cavity of the head gut as earlier had been maintained.:" Also "it remains a long time in connection with the oral cavity by a narrow duct."

The proboscis in the fresh-water and land forms as well as in such nemertean genera as Amphiporus results from dorsal uppitting and backward growth of the pharyngeal area, in the front part of the gullet, the whole extending backward above the alimentary canal and beneath the dorsal nerve cord. Capacity for protrusion of the eversible anterior part gives rise to the proboscis. Now it is in the genera indicated above that primary and accessory rods or stilets - the importance of which phylogenetically we hope soon to emphasize- originate; that the proboscis is often richly loaded with nematocysts; and in which the mouth is in front of the brain.

We would regard the shifting of the proboscis upward anteriorly, and its coming to open by a distinct orifice in line with the sheath but in front of the mouth, as a derived arrangement evolved because it then did not interfere with the direct use of the mouth as an absorptive structure. Such genera therefore as Eupolia and Valencinia are modified in this respect from a more primitive stock.

In order to facilitate further description we would now draw attention to the accompanying diagram, of some nemerteans and simpler vertebrates, as favoring Hubrecht's view and extending it in various directions (Plate Fig. 15).

In emphasizing the direct homology of the hypophysis of vertebrates with the proboscis of nemerteans, Hubrecht does not seem to have considered the possibility hereditarily of only a part of it being thus utilized in transition from nemerteans to primitive cyclostome vertebrates, and of a remainder being utilized in other ways. We may best ascertain if such is likely after examination of the proboscis when at rest, and what might result if, by gradual filling up of the sheath as we see proceeding in Cerebratulus, it thus becomes converted secondarily into a dorsal sensitive and strengthening organ, thus causing the entire proboscis to be extruded and again united with the oral cavity.

Plate Fig. 15, $\mathrm{f}$ is a diagram of the proboscis extruded in front of the now solid notochord, and separating into the posterior glandular portion that is pushing upward from the roof of the mouth, and underneath the infundibulum. This transformation seems a necessary step in evolution from the nemerteans to a group somewhat distantly related to the cyclostomes and which in the figure we have termed a paracyclostome type. The anterior and originally inverted part of the proboscis 

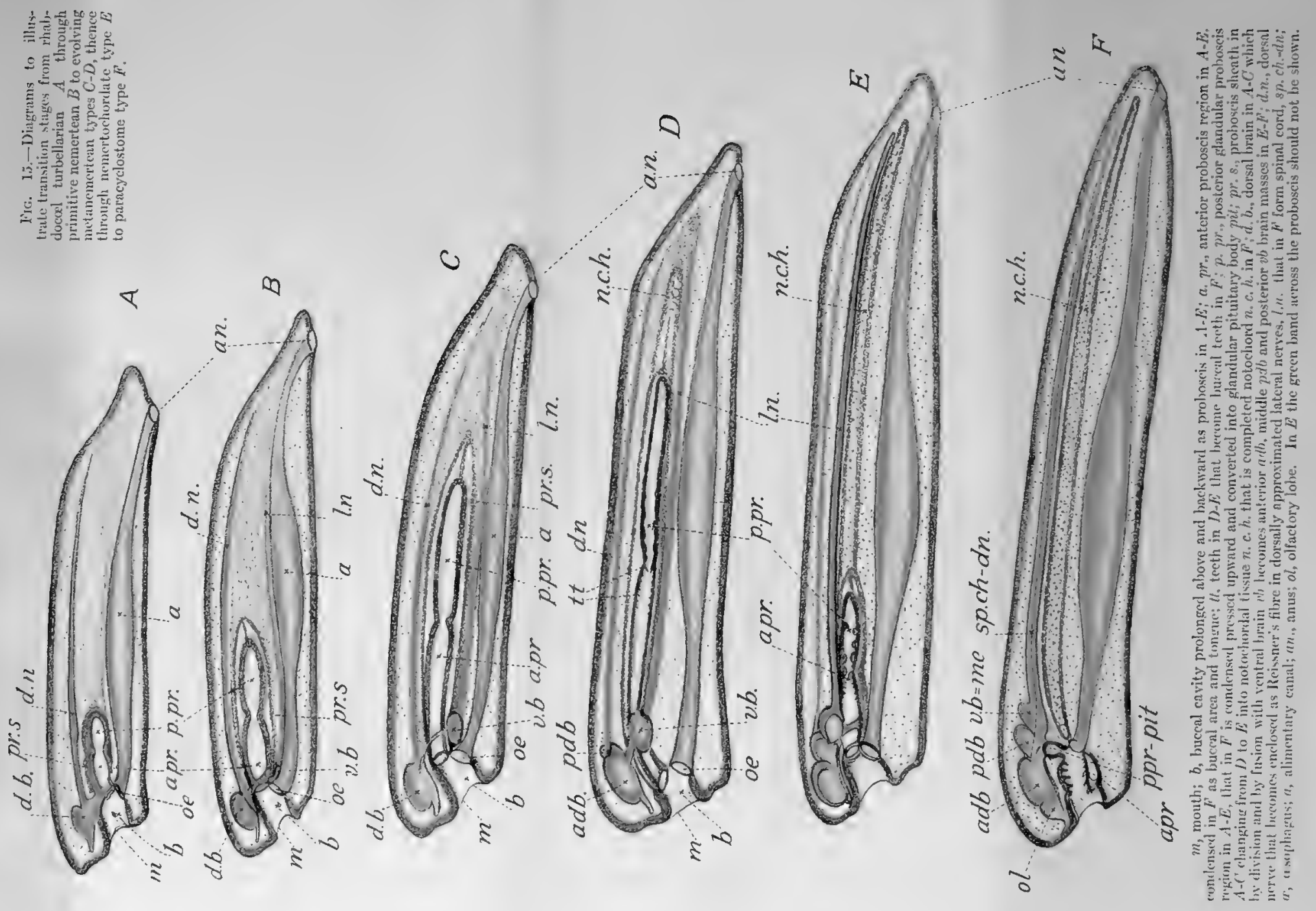

becomes now the lining of the oral cavity, and as in some nemerteans retains the capacity to develop strong muscles and tactile papillæ, also glands for excretion of the juices into the oral cavity. The mid region of the proboscis, the so-called tongue in Myxine, retains the capacity for development of pharyngeal teeth, either a median horny tooth as in Myxine, or a series spread over the entire area as in some nemerteans and in Petromyzon, or vomerine and other accessory buccal teeth as in Batrachia.

Further the sunken glands of this posterior buccal region, where it joins on to the oesophagus in nemerteans, may well represent the rudiments of the thyroid and of the thymus glands of vertebrates. A critical and detailed study of these in relation to vertebrate cephalic glands is highly desirable.

That the notochord of vertebrates has somehow originated as a structure that primarily had a different function has often been expressed. Thus Wiedersheim (138:34) says: "The notochord or chorda dorsalis, lying in the longitudinal axis of the embryo between the neural and visceral tubes, is the first of the endoskeleton to be formed, and is the forerunner of the vertebral column. It is developed as a ridge of the hypoblast from which it becomes constricted off, and is therefore of epithelial origin [writer's italics]. The large parenchyma-like cells of which it is composed consequently do not give rise to any intercellular substance; vacuoles, however, soon appear within the cells, the protoplasm of which undergoes modification, and thus a retrogressive metamorphosis sets in. The fact that this occurs at such early stages of development shows that the notochord must long ago have begun to lose its primary function, whatever that function may have been" [writer's italics].

We can now sum up our studies of the relation of the proboscis and its sheath in nemerteans as related to vertebrates by saying that with Hubrecht we regard the sheath as a mesodermic but in all probability primarily an endodermally derived tube for retraction of the proboscis. As to its present origin from mesoblast or from invagination of the primary epithelium, both such might well be adaptive possibilities. By gradual filling up of the cavity from behind forward the sheath has been converted into the notochord of rertebrates, where it still retains the same loose cellular structure, investing epithelium, and strong double wall-sheath. The longitudinal and transverse muscular coats of the sheath have gradually 
been changed into the stronger but more resisting double "elastica" or primary, and "fibrillar" or secondary sheath.

As extruded by degrees from the sheath the nemertean proboscis has largely retained its former functional relations. The inner non-eversible glandular part has separated as an upgrowing pit that has fused with the infundibulum of the brain to form the rertebrate pituitary body. So it has been described as "a coecal diverticulum from the roof of the mouth" (139:370) while its continued secretion as a glandular structure in higher vertebrates is noteworthy. But its striking union with the nasal sac in lampreys indicates how the proboscis has distributed its tissue in evolving vertebrates.

The middle highly muscular and glandular zone may have originated on its dorsal side the urula, while along its laterorentral sides the glands may be possible homologues of the thymus or thyroids. The more muscular protrusible and papillar portion might well suggest origin for the vertebrate tongue which Jordan states to be extensible in Myxinidæ, while, as above indicated, the stylet or tooth-plate cells seem to be the forerunners of the horny or calcareous accessory teeth of vertebrates.

The writer might here add, to the above very imperfect comparisons, that he was guided to a study of the proboscis and notochord relations several years ago, and only after he had noted the many points of structural resemblance between the groups concerned. These points will be taken up below.

The surface of the body, in several genera or even groups of Rotifera, evidently secretes a mucous substance, which often acts in the cementing together of extraneous particles. We do not as yet know whether this is due to excretions from all of the cells, or whether from special gland-cells that are prophetic of the mucus and other glands of rhabdocoels and nemerteans.

In Rotifera, the important external sense-centers are massed in the cephalic area. So far as has yet been traced, these centers are the circumoral disk, which from its mode of use, and the general behavior of the animal when feeding, seems to be a diffuse chemotactic center, which receives nerves from the brain. No indication of an undoubted olfactory organ 
has been traced, but it may be that chemotactic and olfactory functions are conjoined in the disk area, and only become separated as recognizable sense-organs in higher groups. But Zelinka has described $(140: 424)$ a median anterior mass of four cells in front of the developing brain in embryonic Callidina, that becomes in the adult animal a median sensory depression with sensory bristles, and which he terms the "taster." It receives a median anterior nerve from the brain, and is the first sense organ to differentiate. So alike in time of origin, in position, structure, and nerve supply it agrees with a median olfactory organ.

When we compare this with the median ciliated center in some turbellarians and nemerteans, with the median ciliated olfactory sac or sacs in cyclostome fishes, and with the minute studies of Herrick and Johnston $(162,163)$ on the origin and relation to the evolving cerebrum of the olfactory apparatus in vertebrates, a direct morphological and physiological continuity is suggested that may yield valuable results when exhaustively pursued.

The eyes may be 2,3 , or 4 in number, may either remain separate or be fused in pairs, and they receive paired nerves from the brain. In some genera, two minute openings lead into sacs containing refractive particles, and these, in position, nerve-supply, and connections, suggest the geotactic portion of the vertebrate ear. One to two pairs of antennæ that may remain distinct, or that may fuse more or less, are referred to in another chapter (p. 531). Except in Vorticeros, these organs seem to be absorbed in rhabdocœlids, as the evolving tactile proboscis of that group takes their place with increasing efficiency.

In rhabdocœl turbellarians certain of the epidermal cells produce varied secretions. Such glandular cells are grouped as mucus, pigment, and hyaloid, according to the substance secreted, or the aspect of the contents.

The special sense-organs are rarely tentacular as in Vorticeros, since the progressive formation of a proboscis evidently causes a concentration of the tactile function in the latter.

In several fresh-water rhabdocœel genera a median orifice in front of the mouth, and close to the cephalic ganglia, leads into a cavity that may become bilobed, is richly ciliate over its anterior area, and has a nerve-ending in it. This we would regard as the rudiment of the olfactory organ, though more advanced than in Rotifera, that leads up to the corresponding structure seen in nemerteans, and is there called the frontal organ. In other wholly or mainly fresh-water genera, paired 
organs of even more elaborate structure open at the anterior or antero-lateral part of the head, and lie in front of, or lateral to, the cerebral mass. As described and figured by Von Graff, these seem to be paired olfactory organs, of more elaborate detail than in the former type.

Eyes occur in the majority of the rhabdocols, though not a few species want them. Usually two, sometimes four, occasionally more true pigment eyes are developed. In position they may be lateral and paired, or two may remain so, while the other pair may fuse in the middle line, or one of the two in a pair may be placed in front of the other. Such relations exactly recall conditions seen in some nemerteans, and explain the commencing origin of the paired and the median eyes of cyclostomes and higher vertebrates. It would be impossible here to deal with the refractive non-pigmented organs described by Von Graff, though their possible phylogenetic relation is most suggestive.

The statocyst or probable geotactic organ is slightly advanced on that of Rotifera, but in the size of the statocyst and statolith, as rell as in the nerve-supply, it is intermediate between those of Rotifera and Nemertinea.

In a few of the genera-and these mainly advanced in structure and marine in habit-two lateral ciliate furrows appear below and behind the brain, and by their upper ends extend at times near to the statocyst. They are ciliate cavities of greater or less depth and extent. The opinion might be ventured that these represent the common beginnings of a first or branchial furrow and auditory sac, such as in the nemerteans become the "head furrows." Reference is made to these later.

The frontal organs of nemerteans consist either of one (Eupolia) or three (Cerebratulus) slightly elevated but at times retractile papillæ into whose apex open the ducts of gland cells. Sensory nerves also pass directly to them from the brain. They have been variously regarded as tactile organs or taste papillæ. From their position, nerve supply, and glandular supply they seem almost certainly to represent a rudimentary olfactory system, a view further strengthened by their becoming retracted or depressed into a slight pit. As described and figured by Buirger also, the epithelial cells of each depression consist of ciliated sensory or olfactory cells, that alternate with interstitial or supporting cells, an arrangement exactly similar to that seen in the vertebrate olfactory organ.

In cyclostomes alone, amongst vertebrates, the nostril and olfactory organ are single as in metanemerteans, but, since 
it is supplied by two olfactory nerves from the brain, it might equally be suggested that two of the three areas in Cerebratulus have temporarily fused, or that a single original olfactory nerve is splitting, preliminary to separation of the olfactory organ into two structures seen in other vertebrates. Minute comparative study will alone determine the question. But from the studies of Brookover (141:49) and of C. J. Herrick (162: 413) it seems highly probable that one median mass in front of the brain originally constituted the olfactory body. Division of the sensory olfactory sac into halves, as seen proceeding in transition from Myxine to Petromyzon, has evidently resulted in splitting of the mass into halves, so that a primitive common "placode" is difficultly traceable.

In the Nemertinea eyes may either be absent (by absorption?), or they consist of a simple pigment spot, or show advancing stages up to an organ with retinal, choroid, and lens constituents. That they vary in number from 20 down to 2 , but tend in most cases to be paired on either side of the head, suggests that we are here dealing with diffuse evolving sense centers for heliotropic perception such as still occur in many Turbeliaria, but that become reduced to two paired highly developed organs in vertebrates, and to a median parietal pair still largely functional in Petromyzon and lizards, and probably retained along the evolving vertebrate line, though functionally absorbed before the mammals had developed.

The "cerebral organs" of nemerteans have been much discussed, though unanimity of opinion as to their function has not yet been reached. These however seem, like most other parts of nemerteans, to be the simple rudiments of more evolved structures in vertebrates. They are paired organs developed along the postero-lateral sides of the head. Each in its simplest state is "a mere groove in the epidermis not extending deeper than the basement membrane; it is lined by ciliated cells, and at the bottom are large gland cells; while the organ is supplied by nerves from the dorsal ganglion of the brain. In Carinella rubicunda and others the groove becomes an oblique canal, the blind end of which is surrounded by a mass of ganglion cells, lying outside the cutis. In the higher forms the canal penetrates deeper into the body as far as the brain. The gland cells and the associated nerve tissue increase in amount, and the canal becomes differentiated into two regions - an extra-ganglionic 'lateral canal' and an intra-ganglionic 'cerebral canal' (c) which frequently terminates in an enlarged sac. In Drepanophorus the cerebral canal is quite exceptional, in that it bifurcates, one branch terminating in a sac with sen- 
sory epithelium, the other being glandular; this in $D$. crassus extends backwards beyond the brain as a free tube. In several genera of this order the cerebral organ lies in front of the brain (Tetrastemma, sp. of Eunemertes, and of Amphiporus); in others it lies at the side, and in still others behind the brain-in which case it attains a great size. In all cases the organ is separate from the dorsal brain mass, from which it receives nerves." (Benham in Lank. Zool. IV, p. 185).

A comparison with the embryology and structure of the vertebrate ear leads us to believe that here one has to deal with evolving stages leading toward that organ. The accompanying diagram, copied from Bürger's beautiful work, is suggestive, and can be compared with a figure of the embryonic ear in a mammal.

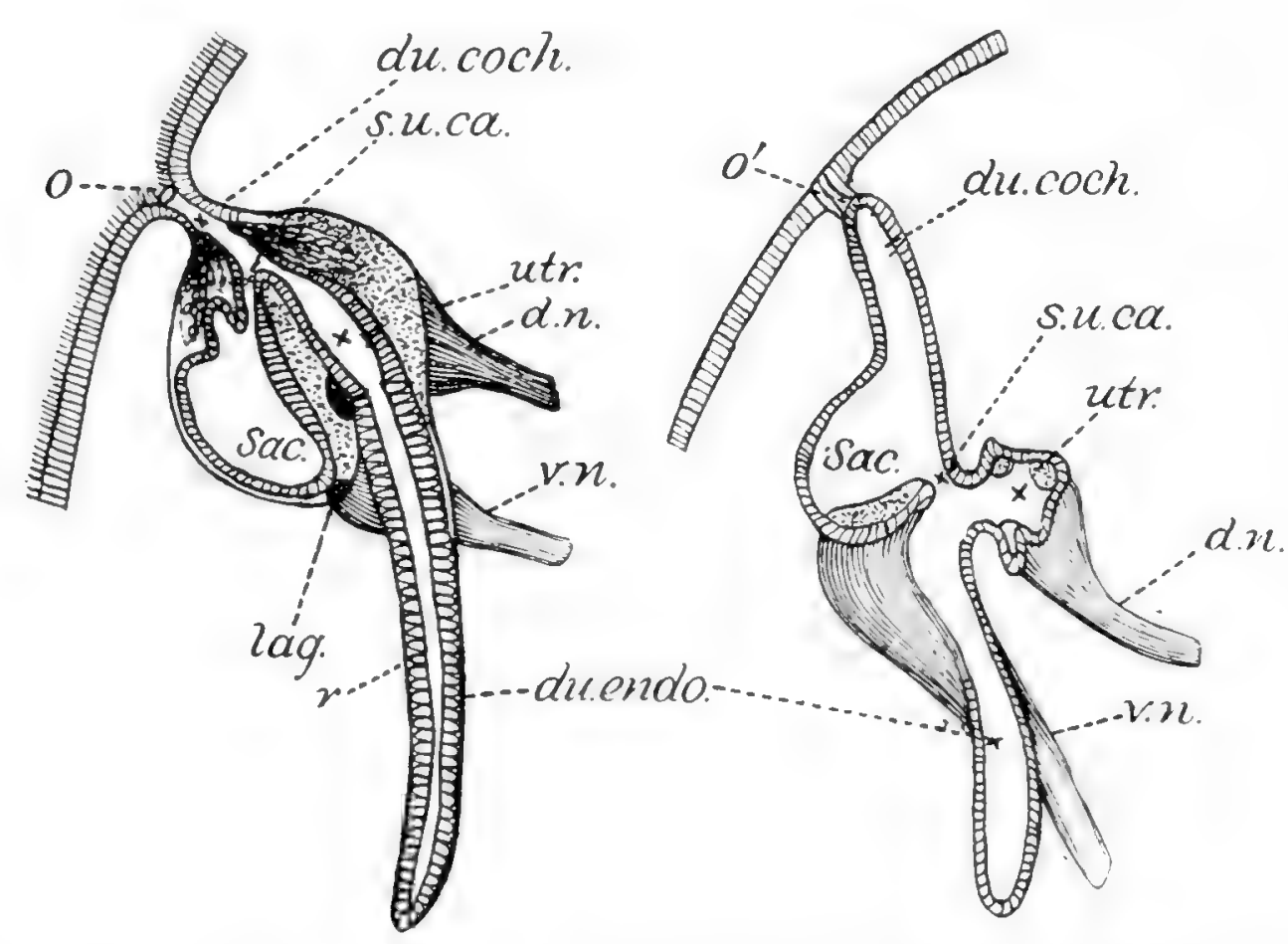

FIG. 16-a (to left) cerebral organ of Drepanophorus compared as an auditory organ with $b$ (to right), the young auditory organ of mammal, $o, o^{\prime}$ open or closed external orifice; $d u$. coch. ductus cochlearis; $s, u$. ca. sacculo-utricular canal; utr. utriculus; sac. sacculus; $d . n ., v, n$. dorsal and ventral auditory nerves; du. endo. ductus endolymphaticus.

It is now well recognized that the ear is innervated by two distinct branches of the auditory nerve, and that it performs a double function. The cochlear branch, distributed to the cochlea and ampulla of the ear, enables the latter to perceive sounds; the restibular branch that passes to the semicircular canals has for function the maintaining of equilibrium, or, as we would suggest, is geoperceptive or geotactic. 
In Cerebratulus, as described and figured by Bürger, the external orifice, the ciliated canal, the two diverticula, and the distinct nerve branches seem well to correspond to the ear orifice, the ciliated lymph duct, the sacculus and utriculus, and the cochleo-vestibular nerves of the ear. That of Drepanophorus is even more exact. Two to three auditory nerves here are inserted between the two organs, the sacculus and the utriculus, as in vertebrates (Fig. 16), the maculæ extend along their base, while the elongated process seems from position, relation, and shape to represent the ductus endolymphaticus.

If the comparison made above be correct it follows that the ductus cochlearis of vertebrates, and not the ductus endolymphaticus, represents the primitive invagination tube of the auditory organ. Further the elongated process shown for Drepanophorus in Fig. 16 suggests exact homology with the ductus endolymphaticus of vertebrates; while Bürger's statement, that it passes backward and becomes embedded in the cellular tissue of the body, recalls the often extensive ramifications of it in many fresh-water teleosts. The opening of its upper end on the dorsal surface of the head in elasmobranchs would then represent a special new formation. The histological description of the organ given by Devoletzky suggests that ciliated sensory epithelium and supporting cells are here often surrounded by a mass of nerves as in the vertebrate ear; while the secretion of mucus from surrounding gland cells and the presence often of refractive "körnchen"that are possible rudiments of otoliths-is most instructive.

A minute comparative study of living nemerteans may yet reveal more exact relationship, while the group seems to present us with suggestive stages from a comparatively simple type like Carinella up to a high degree of organization. It will be noted, however, that the auditory function seems largely to predominate over the equilibrating one, since no distinct semicircular canals are traceable. But when we remember that only one exists in Myxine, and that two are alone formed in Petromyzon, we need not wonder if the equilibrating function here is diffused along the sides or bases of the sac attachments.

The suctorial or semisuctorial nature of the mouth, along the main line of animal evolution now outlined, deserves notice. The swollen lips that bound the oral margin in most nemerteans have great powers of distension, so that in feeding "the nemertine dilates its mouth to a large extent, and the anterior end of the œsophagus is thrust out and engulphs the animal." 
In cyclostomes the suctorial character has become, so to say, typical of the group. We would even consider that this in large part has started the semi-parasitic habits in fresh-water ancestors of some ancient type, that is now more pronounced in the fresh-water or temporarily salt-water Petromyzon, as well as the almost complete parasitism along with the marine life of Myxine.

Apart from the protrusible gullet, the alimentary system in the Rotifera, and in some rhabdocœl Turbellaria, shows a straight alimentary canal made up of muscular pharynx, glandular stomach, short intestine, and in most females of the former group a posterior anus. A varying degree of advancing complexity is seen in the nemerteans from a simple tube (Malacobdella) to one with slight lateral enlargements at intervals as in Carinina, and from this to types in which the coeca are expanded and even subdivided. This is combined in many cases with similar annular disposition of the transverse blood vessels, nerve threads, and reproductive sacs, as worked out by McIntosh and Bürger with great care. This also clearly prepares for or anticipates the like segmentation observed throughout the vertebrates, while some of the alimentary cœca may have been the starting points for the pairs of secreting accessory glands of it.

But the nervous system, next to the proboscidial system, seems exceptionally suggestive; in fact it is even more confirmatory of the vertebrate relation. For it shows homological details throughout, such as neither Balanoglossus, the ascidians, nor Amphioxus show.

In the Rotifera a relatively large paired cerebral ganglion is the center of origin for nerves to the supposed olfactory organ, to the tactile organs, to the eyes, and other cephalic sense-centers. A commissural ring connects this with a ventral pair of ganglia. But from the cerebral mass according to Zelinka two strong nerves arise that branch into feeble dorsal, into strong lateral, and into strong ventral threads which pass to the foot, the muscles, etc.

In many fresh-water rhabdocoel turbellarians, there is a pair of fused and well-developed cerebral ganglia from which two strong ventral, two rather strong lateral, and two weaker paired dorsal nerve-threads are given off. But we would regard the last of these as of extreme importance. For by gradual approximation and ultimate fusion in higher rhabdocols and in nemerteans, as their function in relation to the eye on the one hand and the anterior external muscles on the other became increasingly accentuated, they furnish 
the natural and evolutionarily continuous origin for Reissner's fiber that is present in vertebrates generally.

In practically all cases the nemerteans show a relatively large dorsal brain that consists of paired lobes. Each of these lies directly above or somewhat behind the mouth in forms where the proboscis opens in front of and separately from the mouth. But in some land nemerteans the main mass of the brain lies in front of the common orifice of the œesophagus and proboscis-sheath. The dorsal lobes also, as in the verte-
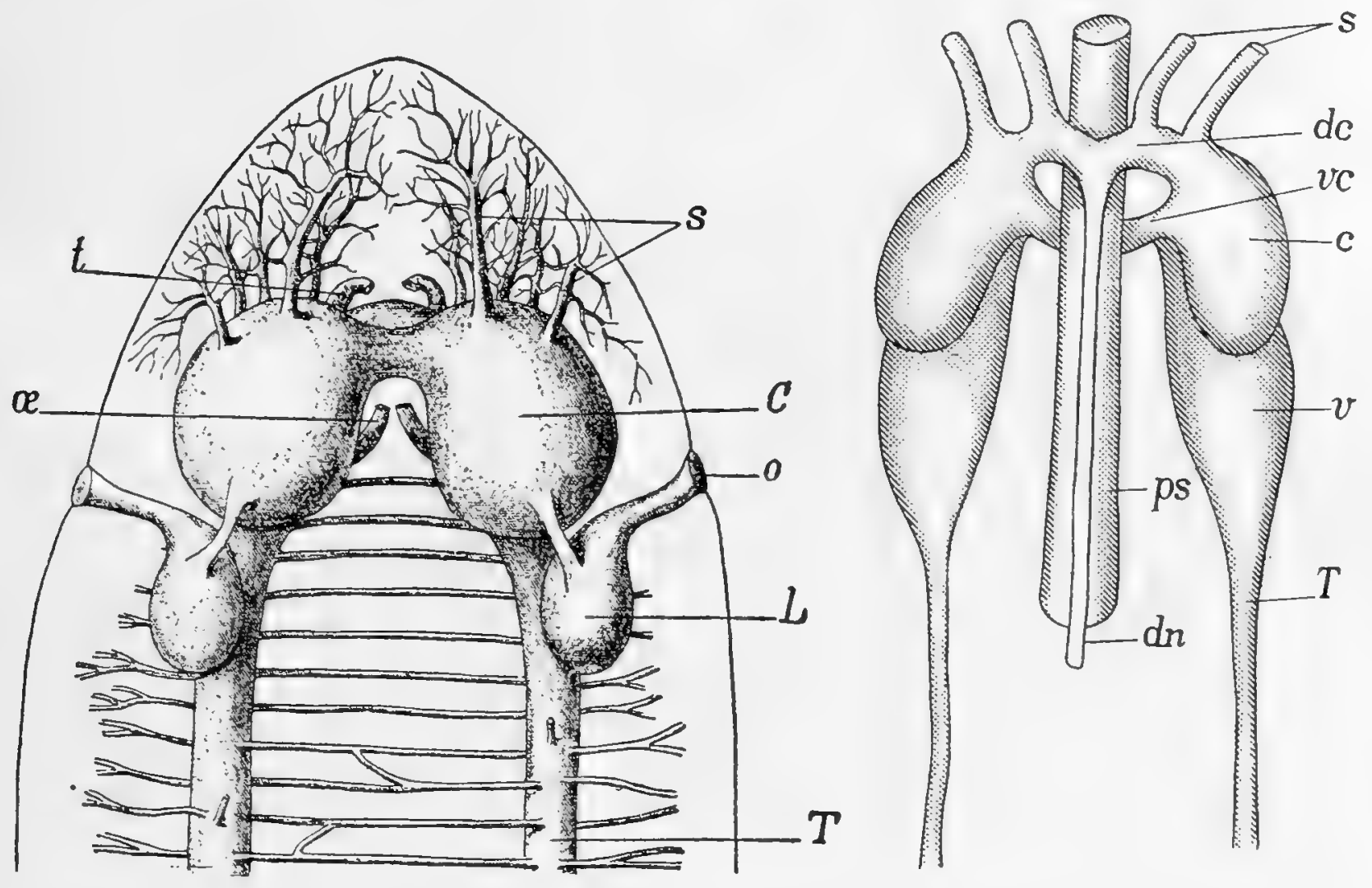

Fig. 17.-a. Nervous system, to left, of Drepanophorus (after Hubrecht). C dorsal brain; $s$, anterior sensory nerves; $t$, nerves to proboscis; $\not 2$ nerves to digestive tract; $L$, probable auditory organ and orifice $o ; T$, lateral nerve trunks with their branches. b. Nervous system to right of Hoplonemertean (after McIntosh). $c$, dorsal brain; $s$, probable olfactory and optic nerves; de, dorsal commissure with junction of dorsal nerve $d n$; $v c$, ventral commissure passing beneath proboscis sheath $p s ; v$, ventral ganglion; $T$, lateral nerve trunks.

brates, may in higher types undergo subdivision into an anterior lobe or thalamencephalon, and a posterior or mesencephalon as in Eupolia. The former are not only anterior to, they largely overlap, the posterior lobes (Fig. 18 a). Anteriorly in some genera of nemerteans, as in vertebrates, these lobes give off branches that might appropriately be termed in the former group, did we know their function exactly, the olfactory, the 
optic, the trigeminal, and the auditory nerves. Below the dorsal brain masses and often closely apposed to them are the two ventral ganglia, that are like the dorsals united by a ventral commissure. Through the circle thus formed the proboscis-tube and proboscis pass. The mouth and alimentary tract however lie rentral to them. The nervous system then in all nemerteans resembles the other systems in showing progressive advance from a comparatively simple to a complex condition. It might truly be said further that, from the simplest to the most complex nemertean brain, the advance is almost as great as is the simplest vertebrate brain-that of Myxine-an advance on the nemertean. But all show an evident homological continuity. We shall here concern ourselves only with the most advanced nemertean types.

In Drepanophorus (Fig. 17 a) the brain consists of two relatively huge dorsal ganglionic lobes that are united by a dorsal commissure with each other, and also with two smaller ventral or infraproboscidial lobes. From the dorsal lobes three main sets of sensory nerves arise. The olfactory and optic are given off anteriorly; the pair to the cerebral organs arises posteriorly. The two last enlarge into special sacs, and for reasons already given we would regard them as "auditory" nerves with double audito-geotactic function.

Such a dorsal brain system might therefore be regarded as the primitive and evolving type of vertebrate swelling, from which the fore and mid brain are to be evolved.

In making such a comparison we readily grant that an olfactory function has not been demonstrated for the ciliated pits, nor an auditory function for the cerebral lobes, but, if structure, position, and relation are to count for anything, then such a view is warranted.

The ventral lobes can now be examined. In most nemerteans they are distinct from the dorsals, and are united with them and each other by a commissural thread below the proboscis. In some genera, howerer, all are closely joined above the gullet and round the aperture of the proboscis sheath. From their nerve distribution to the proboscis, osophagus, and surrounding as well as posterior parts, the suggestion is warranted that these ventral ganglia may gradually join with the dorsals to form a medullary element, from which nerves corresponding in value to those of the glossopharyngeal and vagus are given off. In such a case the ventral ganglia may then represent the hind brain or myelencephalon of vertebrates, while the dorsal divisions may evolve into the thalamencephalon 
and mesencephalon. Only detailed comparative study can again accurately determine. In distribution and function two pairs of lateral nerves that arise from the ventral ganglia correspond well with the 9 th and 10th or the glossopharyngeal and vagus nerves of vertebrates.

The point of origin, invariable superficial position, and median dorsal course of the dorsal nerve cord are all highly instructive in relation to the vertebrata. Arising from the middle part of the dorsal commissure, it would follow that, were the dorsal ganglia completely fused, this dorsal nerve cord might become the leading tract for conveyance of stimuli lengthwise through the body of the animal.

But we would consider that the earlier position taken up by Hubrecht, and by his predecessor Harting, namely that the lateral nerves of nemerteans, and their anterior or ventral cephalic ganglionic expansions, have gradually converged and enlarged dorsally to form medullary and spinal cord constituents, has greatly more in favor of it than Hubrecht's later view $(136: 131)$ that the dorsal nerves, by gradual growth and backward extension, gave rise to the medullary-spinal system.

For it is to be observed that the two divergent groups of the annelid-arthropod series and of the nemertean-chordate series start in common amongst primitive rhabdocœl forms with a strong ventral nerve pair, a feebler lateral pair, and a delicate dorsal pair. This becomes a very strong ventral pair, a small lateral pair, and an attenuate or absorbed dorsal system in annelid-arthropods. In Nemertinea on the other hand the ventral pair becomes small, the lateral pair specially strong, and the dorsal system reduced to a small median medullary or dorsal nerve. With gradual absorption of the proboscis into the oral cavity, and increasing solidification of the sheath-notochord, the ventral ganglia and their nerves would undergo condensation behind and above the front of the evolving notochord, to form the medulla and spinal cord.

So, if we compare the diagram of the brain of Eupolia with that which we would regard as a protochordate type (Figs. 18a, 18c), an exact progressive similarity can be traced. For by conversion of the ventral commissure into the Pons Varolii, as the proboscis sheath became closed anteriorly and functioned as a notochord, the ventral lobes would rise upward and backward, so as to be below and behind the dorsal centers, and thus constitute the medulla; the formation dorsally of a correlating commissure above the notochord would give rise to the cerebellum, that is still a rudimentary crest in Myxine; 
convergence above the notochord of the ventral lobes would leave between them the fourth ventricle, while the lateral nerves springing from these would be, as Hubrecht originally contended, largely of a vagus and a glossopharyngeal character.

So the fundamental conception in evolution of the main invertebrate-vertebrate line of animal life as here sketched is the manifold reception, the concentration, and the correlation of every form of energy that may environally affect the organism, and the most satisfying response of the organism to this environment.

Now, if such a dorsal uprising and backward extension of the ventral ganglia in nemerteans gradually took place, we might expect to find in cyclostomes and even higher vertebrates all or some of the following structural relations: (1) the dorsal mid area between the dorsal ganglia would be open or covered only by a thin investing membrane; (2) the dorsal mid area between the uprisen ventral ganglia would be even more open; (3) the functions performed by the dorsal brain masses as receptors and correlators of olfactory, optic, geotactic, and auditory stimuli, also by the ventral masses as receptors, correlators, and distributors of thermo-, thigmo-, and chemotactic stimuli would be continued and even more perfectly correlated; (4) as the uprisen ventral ganglia became more closely applied to each other the lateral nerve cords would become increasingly approximated, and on account of the frequent transverse connecting fibers that joined each would become in time symmetrical halves of a bilobed cord, in which the connecting fibers remained as a bridge between the more or less united halves; (5) receptor currents to, as well as motor and inhibitory currents from, the cephalic ganglia would increasingly traverse this bilobed cord, which would thus increasingly distribute the motor and inhibitory impulses throughout the body; (6) some rudiment might be left of the dorsal nemertean nerve, which starts from the dorsal commissure that joins the olfacto-optic areas of the dorsal ganglia, and running backward distributes to the skin muscles according to Bürger (p. 108).

All of the above conditions are fulfilled in Petromyzon, in the Apoda, and upward through higher types in increasingly perfected manner as one rises throughout the series. In this process the areas between the ganglionic masses become the brain ventricles, or the "iter" connections between these. But a remarkable confirmation of the writer's position, as well as of the earlier views of Hubrecht and of Harting, is 


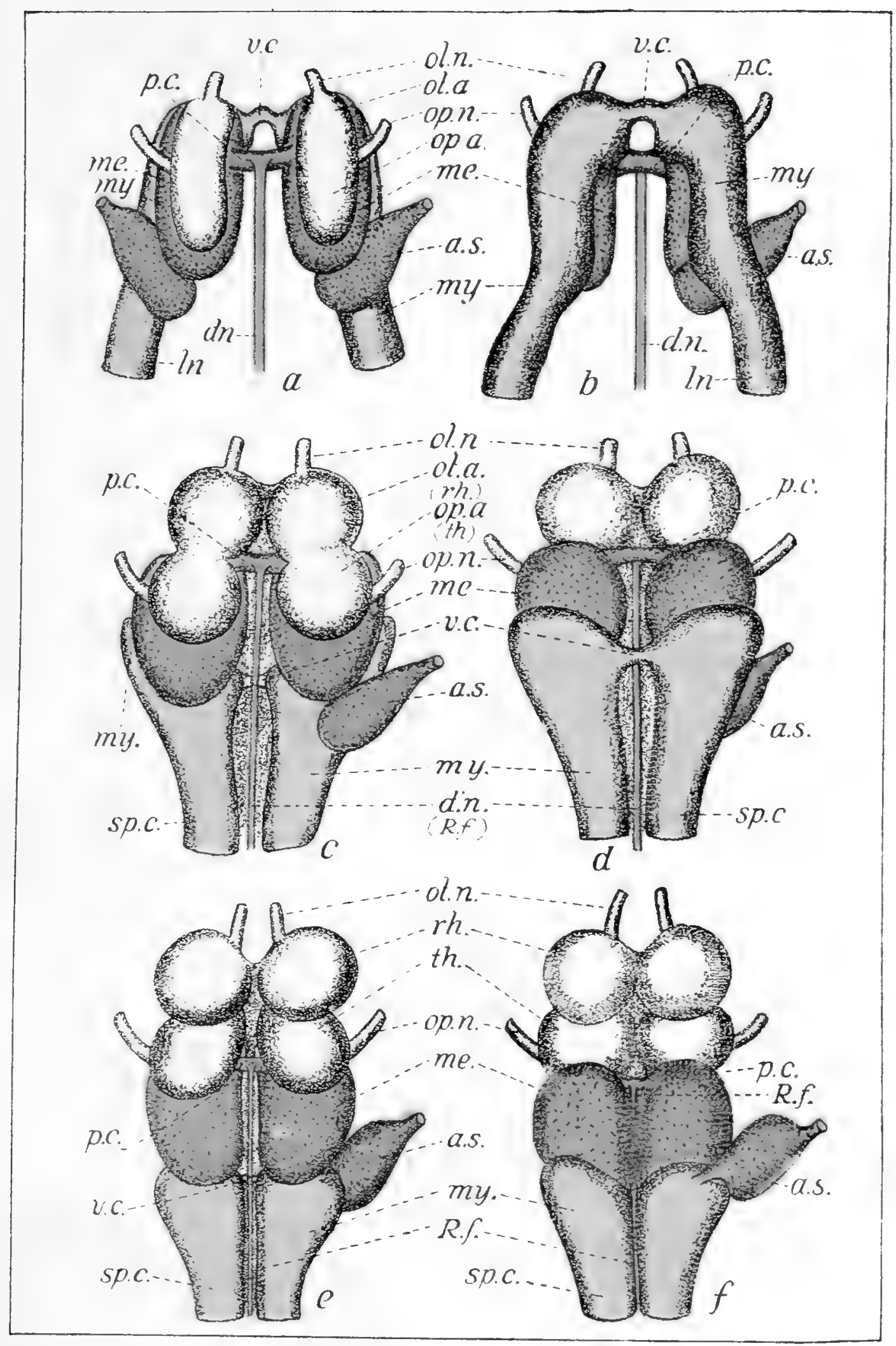

FIG. 18- $a$ dorsal, $b$ ventral view, brain of Eupotia Giardii (after Hubrecht); $c, d$, of hypothetical protochordate type; $e, f$, of cyclostome type; ol.n., ol.a., rh., olfactory nerves and area or rhinencephalon; op.n., op. a., optic nerves (which should be yellow in all) and area; th., the last evolved as thalamencephalon; $p . c$. , posterior commissure; d. $n$., dorsal nerve becoming Reissner's fibre in $c-f ; v$. $c$, ventral commissure; me., mid lobe or mesencephalon; $a . s_{0}$, auditory sac; $m . y$, ventral ganglia becoming myelencephalon or medulla in $c-f ; \ln$, lateral nerves becoming sp. $c$. or spinal cord in $c-f$. 

got under the sixth caption, and this in the following manner. In connection with captions 2 and 4 , as gradual apposition and more or less perfect union of the ganglionic or brain masses and nerve cords took place, one of three "fates" might also have happened to the median dorsal nerve. Either it might have risen above the apposing masses to occupy a free position dorsal to them, or it might have become included in the cavity that resulted from their apposition and union, or it might have transferred its functions to another nerve and become obliterated. The second is clearly the result that has been worked out, and as now existing in vertebrates the dorsal nerve is known as Reissner's fiber, though retaining its primitive origin, distribution, and function (Fig. 18, c-e).

Had Hubrecht's attention been turned to this structure of the vertebrate brain, such would at once have confirmed him in his and in Harting's original contention, and furnished his most powerful argument for derivation of the vertebrates from nemerteans. For, discovered by Reissner in 1860, within the neural canal of Petromyzon, it was subsequently observed by Kutschin, Stieda, Sanders, and Studnicka in Petromyzon, selachians, teleosts, and by the last even in amphibians and mammals. But the most extensive and accurate investigation of it has been made by Sargent (142:129), who also gives a full bibliography. He has further added descriptive details that strengthen some of the above six captions.

Sargent shows (p. 139) that, while cavities or ventricles of the central nervous system are of small size in higher vertebrates, in the lower and specially in Petromyzon and Myxine the cavities or rentricles are large. Such would be appropriate evolutionarily, if the vertebrate brain has resulted from convergence of nemertean ganglionic lobes.

Next, the origin of the dorsal nemertean nerve and of Reissner's fiber from the connecting commissure of the opticoolfactory areas is identical. Reference to Figure $17 \mathrm{~b}$ shows the origin of the dorsal nerve in nemerteans midway between the pairs of optic and olfactory nerves. In this connection Sargent (p. 162) says: "It is evident that in the cyclostomes, where the eyes are poorly developed, Reissner's fiber serves as a short circuit for the transmission of reflexes arising from olfactory as well as optic stimuli."

In histological detail as given respectively by Bürger (132: 108) and Sargent (p. 145) the resemblance is marked in both groups of animals. That it is a structure also that has become overshadowed by a more powerfully developed system, and rather readily tends to disappear when its function as a lumino- 
muscular correlator becomes reduced or superfluous, is shown by Sargent's study of such blind fishes as Amblyopsis and allies, in which it may suffer varying degrees of degeneration to the point of ultimate disappearance.

The quite unique position and course of Reissner's fiber can only be explained by its having become surrounded and included by two longitudinal cordal structures, that primitively were more widely apart. Its continued presence and structural similarity, even up to the mammals, are probably explained by its having retained its functional activity throughout the entire scale from turbellarians and through the nemerteans upward to mammals. As in some rhabdocoels, and apparently also in nemerteans, to judge from illustrations, so in vertebrates, Reissner's fiber arises from two roots, but these remain separate only in rhabdocols.

The writer would therefore regard the structural, the anatomical, and the physiological evidence above cited as convincing proof that a direct connection exists between the nemertean and cyclostome brain systems, and in epitome he would now outline the probable evolution of the vertebrate brain and associated parts from that of nemerteans as follows.

In primitively fresh-water types allied to the existing Geonemertes, a pair of dorsal ganglia, hereditarily related to those of rhabdocoels and rotifers, was formed by dorsal involution of ectoderm. These were connected by a dorsal commissure that became the posterior commissure of the vertebrate brain, and from this arose a dorsal nerve that represented the fused dorsal nerves of rhabdocœels, and which in nemerto-vertebrates became Reissner's fiber, that still arises by double roots. The antero-inferior parts of the dorsal ganglia gave off olfactory nerves to a single olfactory body that remains such in Myxine, and probably was also in primitive cyclostomes. But in Petromyzon and higher vertebrates it has divided into two olfactory sacs. The antero-superior parts of the ganglia gave off branches to the two paired eyes, and to the two unpaired antero-posterior eves seen in many metanemertines. Commissural threads, alike from the olfactory and optic nerves, crossed the dorsal commissure, and passing into the dorsal nerve enabled the latter to send correlated olfactomotor and opticomotor stimuli to the anterior skin muscles. This function according to Sargent, is continued in what the writer would regard as its hereditary derivative, Reissner's fiber.

A tendency to division of the two dorsal ganglia or brain masses into an anterior and posterior pair of lobes, as in Eupolia, occurred, and then from the latter pair nerve branches sprang 
that passed to the cerebral organs, which the writer would view as in part an auditory apparatus.

Two ventral ganglia, closely placed below and behind the dorsal in many nemerteans, are united with each other by a strong ventral commissure, that in nemerto-vertebrates apparently became the Pons Varolii. In front of these ganglia the proboscis-tube and proboscis opened into the oral or buccal cavity. But as the proboscis-tube became a strengthening rod of tissue, and filled its cavity from behind forward with loose cells to thus constitute the notochord, the posterior or basal glandular part of the proboscis grew upward above the buccal cavity as the pituitary body, while its glandular middle zone probably gave origin to the thyroid and thymus glands. In regard to these latter glands, however, it may well be that they originated from the mass of cesophageal gland-tissue that Bürger appropriately suggests might be named (p. 194) the "drusendarm." A comparison of the structure of these in existing fresh-water and land metanemerteans with the andostylar or thyroid organs of vertebrates would constitute a valuable and welcome study.

During the above changes the ventral ganglia rose upward through the region once occupied by the front part of the proboscis, and became apposed behind and somewhat below the bilobed dorsal ganglia, to constitute the medulla oblongata. The cavity between the increasingly convergent dorsal anterior lobes became the third ventricle, that between the dorsal posterior lobes the iter, and that between the now uprisen and converged ventral ganglia the fourth ventricle. The ventral nerve ganglia, therefore, which we consider to have fused dorsad to form the medulla oblongata, are largely concerned with correlation of the simpler or biotic functions. In this connection C. J. Herrick (143:668) says: "While much remains to be explained in the comparative anatomy of the medulla oblongata, the underlying morphological pattern has been exposed, and is found to be surprisingly constant in all vertebrates. This constancy of type grows out of the fact that this part of the brain uniformly serves the simpler vital functions, such as feeding, respiration, etc., whose peripheral mechanisms are broadly similar in vertebrates." In contrast to this the dorsal ganglia are largely cognito-cogitic in function, since they rapidly bring each organism into contact with light, sound, gravity, and the more delicate chemic stimuli, and correlate these so as to give rise to cogitic responses. Figs. 18a-18f illustrate the above changes. 
The originally strong lateral or dorso-lateral nerves, that are a continuation backward of the ventral ganglia, by gradual upward approximation, as seen proceeding in the nemertean genus Langia, ultimately united to constitute the spinal cord of vertebrates.

The rather strong paired nerves described by Bürger as starting from the rentral ganglia, and named by him "mundschlund nerven" and "ruissel nerven," seem well to correspond, in their origin and distribution, with two important systems of nerves in vertebrates. The "mund-schlund" system, from its origin along the lower posterior edges of the ventral brainmasses, and its abundant distribution round the mouth, the pharynx, the alimentary canal, and the skin, would correspond with the ventral nerve threads of turbellarians. This seems gradually to have evolved into what might be termed the ventro-sympathetic in protochordates, and later into the sympathetic system of cyclostomes and higher vertebrates. The latter or "ruissel" system specially and richly innervates the entire proboscis-tube area and the anterior part of the alimentary canal. Unfortunately, little seems to be known of its complete and ultimate ramifications, specially toward the posterior part of the nemertean body. The entire system however suggests homology with the glossopharyngeal and the vagus nerves.

A detailed morphological and physiological investigation of both systems in some of the larger land nemerteans is highly desirable. More careful study also of the ventral ganglia of the Metanemertinea, alike morphological and physiological, may yet reveal evolving but exact rudiments of the fifth, sixth, and seventh nerves.

The numerous points of morphological continuity thus indicated furnish strong proof that the nemertean brain passed by progressive evolution through a nemerto-vertebrate stage to that seen in cyclostome or allied but now extinct types.

It may correctly be said then that the vertebrate brain, as foreshadowed in that of the higher nemerteans, consists of an anterior olfacto-sensory lobe, the prosencephalon; of a median optico- (or helio-) sensory lobe, the mesencephalon; and of a conjoint audito-geotactic-sensory portion, derived in part from the last and in part from the myelencephalon. Further, that the diffuse mechanotactic, thermotactic, hydrotactic, and chemotactic (gustatory, etc.) sense perceptions were concentrated in the latero-ventral lobes that became the myelencephalon. Or, in other words, of the eight most important animal senses or perception centers, four became localized 
in the upper brain lobes as the important cognito-cogitic centers, and four in the lower brain lobes as the important biotic centers. So the correlating cognito-cogitic center or brain, in the main line of invertebrate-vertebrate ascent, represents the result neither of a segmentation nor cephalization process, but of a sensorization process, or is a center for increasingly rapid condensation and correlation of all external sensory impressions.

We would pass beyond the scope of the present work were we to deal with the circular nerves of nemerteans that recall interesting homologies with vertebrates. But we would merely add that the abundant and serially arranged pairs of nerve threads that connect the lateral nerve cords with each other and with the ventral pairs (Fig. 17a) cannot but call up a close parallel with the spinal nerves and nerves of the lateral line, as well as the spinal-sympathetic nerves, in vertebrates.

In proceeding to a study of the nervous system in Myxine, the writer should state that, while the existing cyclostomes are regarded by him as nearly direct types in the line of vertebrate evolution, forms must have existed that we might view as leading up from the nemerteans to an ancestor that was common to the living cyclostomes which are now lateral and somewhat degraded offshoots, and to a direct series that led upward through paracyclostomatous types toward the Apoda or Cæciliada of the Batrachia. But the Cyclostomata represent living material that alone is to hand.

Reference has already been made to the median nasal sac on which much work needs to be expended.

The eye of cyclostomes is not greatly advanced over. that of the most complex nemerteans. But the evolutionary connection between the pineal and parapineal eyes, alike with nemerteans on the one hand and higher vertebrates on the other, deserves notice. As Bürger points out for the former, the eyes may not only at times be somewhat sunk below the skin, they may tend to show an uneven position or number, or a clustering into two or more. Now in fresh-water or land genera like Geonemertes and Tetrastemma, that usually have four eyes, we have an exact morphological basis for origin 
of the tro lateral eyes of cyclostomes, and equally for the rudimentary pineal and parapineal eyes of this group. In this connection comparison might be made of the figure in Buirger's work (Pl. 10) for two of the multiocellate organs of Drepanophorus, with that from Studnicka of the pineal and parapineal eyes of Petromyzon. As regards the region of the brain from which the optic nerves spring, Bürger states (p. 171) that in nemerteans they arise from "the anterior circumference of the brain." How far this might be correlated more exactly with the habenular and posterior commissure of the brain in Petromyzon yet remains to be determined.

But we would trace a direct continuity from the four-eyed state, typical of various land and fresh-water nemerteans, in which two of the eyes form one paired series and two form another unpaired series and often also in reduced state, to the paired eyes and the rudimentary pineal and parapineal eyes of Petromyzon, and from this again to the restigial pineal eyes of amphibians and mammals.

While the auditory sac is closed off in its endolymph canal from the exterior in vertebrates generally, it is worth noting that in elasmobranchs an orifice exists, the possible origin of which has been already explained (p. 431). The simple ear of Myxine is an advance on what we regard as the nemertean ear, in that a semicircular canal has dereloped along with two sacs. The presence of two semicircular canals in Petromyzon, along with other details that are typical for higher vertebrates, leads us to the ear of the latter, with its three semicircular canals.

A blood-rascular system seems to be wholly undereloped in the Rotifera. In the Rhabdocoela, it is also absent as such. But in view of the extensive and varied ramifications of the excretory system, as described and figured by Von Graff (131: 2137-2151), in view also of what is noted below regarding the joint blood-rascular and excretory systems of nemerteans, it seems not unfair to suggest that, in transition from the Rhabdocola to the Nemertinea, a portion, and specially certain of the main trunks of the excretory system, in the former, 
may gradually have been set aside for the double function of tissue-aeration and of waste-removal, while the finer branches and capillaries may have remained as excretory vessels purely. The distribution of the blood-vascular system in the Nemertinea has been carefully studied during the past sixty years, though we still desiderate fuller physiological details. While the simplest system (e. g., Cephalothrix) shows only two longitudinal vessels in close proximity to the lateral nerves, in higher forms there are two lateral and a dorsal vessel, which with accessory vessels to the proboscis sheath, and transverse ones connecting all, show a marked anticipation of the vertebrate vascular system.

In considering this system further, alike in its own distribution and in its aerating and excreting connections, we believe it is correct to consider that proximity to the nervous system for metabolic renewal of the nervous substance, and the periodic transfer of its blood to some aerating region or regions, are of prime importance. The former is effected by the frequent formation of expansions of the bloodvascular system into two special longitudinal sinuses near the brain, and the passage of blood-vessels parallel to the nerve trunks. The latter is effected by those expansions that run parallel to the proboscis sheath, or near to the cephalic groove, whose vessels seem to correspond to the posterior cardinals of Cyclostomata and higher forms, since in all extensive connections are made by these vessels with the renal or excretory, and with the reproductive systems. In the forward course of these vessels to the heart, in the nemerteans as in the cyclostomes, the posterior cardinals run directly beneath the sheath or notochord.

In many genera these two vessels unite anteriorly into a common cavity that seems to correspond to the venous sinus in lower vertebrates, while two anterior veins that often form a loop system in the front part of the head and that also unite with the sinus would equally correspond to the anterior cardinals. A single vessel in the higher nemerteans, starting posteriorly from the anal commissure, corresponds in origin, course, and position to the dorsal aorta of most vertebrates, and it, along with the anterior cardinals, gives rise to the above mentioned vascular loop-system round the front part of the head, that agrees with the circulus cephalicus of vertebrates. 
But further, in many Heteronemertinea, an oral-cervical pair of vessels (Schlundgefäss-system of Bürger) starts from a median ventral vein given off from the cardiac ring or commissure, and which agrees in relation with the ventral aorta of vertebrate types. These oral-cervical vessels branch repeatedly after the manner of afferent branchial arteries, are in direct connection with the -as we shall term them tentatively-anterior cardinals, and ramifying round the cardiac organ and cephalic groove agree well with the internal jugulars. The anterior ending of the median ventral vein, from which these spring, and the side vessels given off from it in front of the mouth (Bürger, p. 254), agree well with the hyoidean sinus and mandibular veins of lower vertebrates. The posterior fusion again of the oral-cervical vessels with the sinus venosus-like enlargements of the lateral vessels constitutes another agreement.

Vessels which Bürger has called the proboscis-tube vessels arise at their anterior end in vascular swellings, and posteriorly join the lateral (cardinal?) veins. These might therefore be the beginnings of the lateral veins of cyclostomes.

As regards the transverse vessels the description that has been given by authors for the cyclostomes could accurately apply for the nemertean segmental somatic arteries, that are regularly supplied to the myotomes from the dorsal aorta. A corresponding series of somatic veins also empty into the cardinals (laterals).

As to the methods and direction of blood circulation our knowledge is still vague. Bürger considers that the blood flows along the dorsal aorta which is the most powerful pulsating one. It then flows into the transverse vessels, thence into the lateral vessels, from which it is propelled forward. This if fully proved to be correct would agree fairly well with the cyclostome circulation. Interesting also is Böhmig's discovery in nemerteans of unicellular valvular swellings that occur at irregular intervals along the interior of the ressels, and there act as valves for the course of the blood flow.

The nucleated and often pigmented blood corpuscles contain true hæmoglobin in not a few cases, both of which are characters that lead up to the vertebrates.

Before learing the blood vascular system, a feature that has considerably impressed the writer may be here mentioned, whether much morphological value may attach to it or not. 
The primitive mode of origin of gills has been much investigated and debated. In nemerteans all stages of development (Bürger, 132-137) of two rather similar structures can be traced. These have been called the "head furrows" and the "head grooves"; and in some cases they seem almost to merge into each other. The "furrows" usually surround the neck as a pair of dorso-lateral and vertical or oblique grooves. The "grooves" are rarely oblique, usually longitudinal, and run forward from the neck to the front part of the head. Each furrow may be a shallow or rather deep simple but ciliate depression, or richly ciliated ridges may project from its cavity as pointed out by Hubrecht (145:1) fully thirty years ago. He then advanced the view that these are a rudimentary respiratory apparatus. In some genera a second pair may appear in front of the normal ones. The latter or grooves are longitudinal ciliated depressions, which in the region of the brain may penetrate almost to the brain substance. Their lining cells are devoid of a cuticle. Both structures are usually placed close to extensive blood sinuses, as well as to the brain substance. But, while the furrows seem to have no nerve endings, the grooves show these abundantly.

A highly interesting and we trust exact morphological comparison can be suggested between these structures and the gill-pouches of cyclostomes. The latter have always been viewed by zoologists as diverging widely from the typical piscine type. But from the ciliated furrows of nemerteans to the "gill pouches" of Amphibians the transition can be gradually traced. Further, however, the writer would suggest that the "Wimperrinnen" and "Ringfürchen" of turbellarians described by Von Graff (131:2201-2204) seem in position, structure, and probable function to correspond to the "head grooves" and "head furrows" of nemerteans, and so to represent possibly the first origin of gill structures.

The somewhat diagrammatic Figs. 19 a, b, c, d and e will aid descriptions. The slight or deep paired grooves with enlarged ciliated cells in various Rhabdocoela, and the paired depressions of the nemerteans, with their internal projecting ciliated lamel- 
lae, end blindly against or in the mesoderm substance. In the Cyclostomata the depressions are continuous by their inner faces with the pharyngeal cavity, while the branchial lamellæ are represented by a series of vascular horizontal and parallel ridges, radiating outwards along the roof, floor, and lateral walls of each gill sac, and invested by an epithelium that is partially ciliated. In lower Amphibia the "gill pouches" or depressions are also continuous from without into the pharynx (Fig. 19e). In those genera that develop external gills first, these arise as dorsal lamellæ, which instead of merely projecting

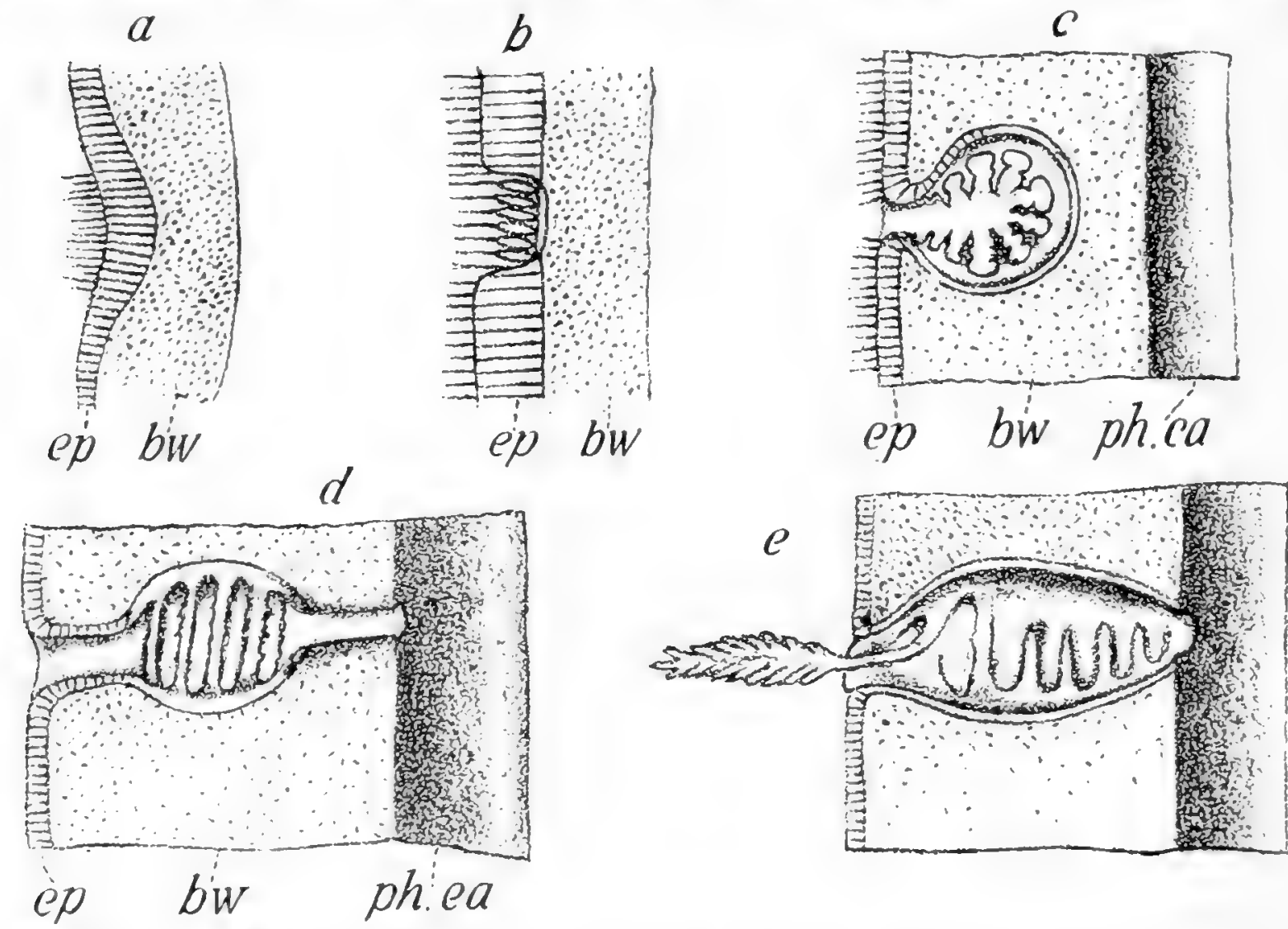

FIG. 19.- $a, b$, section of ciliated head-grooves in Turbellaria rhabdocoela; $c$, section of ciliated furrow of Nemertean; $d$, section of branchial sac of Cyclostome; $e$, do. of caecilian Amphibian.

into the pouch, as in nemerteans and cyclostomes, push outward and may attain great length as in Ichthyophis of the Apoda or Caxciliada so carefully studied by Sarrasin. The internal gills are lamella that arise on the inner ventral surface of each pouch, later as a rule than do the external, and which become greatly more branched and complex than in the two more primitive groups of the cyclostomes and the nemerteans. 
As previously explained the "cerebral organ," that we have viewed as a probable auditory structure, arises from some part-usually the cervical or precervical portion - of the groove or furrow. But, from the simplest vertebrate up to man, the auditory organ arises from the first gill cleft, visceral cleft, spiracular cleft, or hyomandibular cleft, as it has variously been called. Such a coincidence alone, between some turbellarians, nemerteans, and vertebrates, seems more than a homoplastic resemblance. It may rather afford us valuable glimpses into the history of vertebrate ontogeny and organogeny.

All evidence therefore indicates that these furrows or clefts of nemerteans are the primitive beginnings of the first pair of vertebrate gill-clefts, in which the epiblastic depression often becomes quite deep, but in which also no hitherto traceable outgrowth from the pharyngeal region has arisen to meet it. These probable rudimentary gills, then, are still epiblastic depressions in nemerteans into which currents of water are driven, but in connection with which auditory organs have already arisen.

When we consider further the abundant formation of vascular girdles or loops, in the cervical region of some nemertean genera, and their immediate proximity to the lateral furrows on the one side and the osophageal region on the other, we have here the necessary fundamental requirements for formation of at least 10-20 gill clefts with alternating arches. But no such nemertean, nor type intermediate between that group and the Cyclostomata, seems to exist, if we except $A m$ phioxus, which however we would regard as an abberant side type, organized somewhat along the line now desiderated. But a rich series, abundant in species, may well have lived during ordovician or early cambrian times, and yet have left not a trace of their soft bodies in the fossil state.

If however we may shortly try to piece together the now fragmentary evidence, it seems as if, with gradual disuse of the proboscis sheath, its conversion into a notochord, and the establishment of currents of water into the pharyngeal 
region that now could freely conduct such, not only were the furrows converted into a first pair of pouches that came to open internally; gradually additional posterior pairs developed till possibly 15-20 pairs may have existed. Of this variable number Bdellostoma of the Cyclostomata may still have from 14 to 11 pairs in one species (B. Stouti), 10 in another (B. Dombeyi), or 7 to 6 in the Cape species which thus closely resembles Petromyzon and Myxine. Amphioxus seems also to have had from 11 to 9 primary pairs. In the branchiate Batrachia, the sacs gradually become absorbed, so that from 5 to ultimately none are left, unless we continue to consider the spiracular or hyomandibular area as the last modified remnant.

It would be impossible here and now to enter into many other striking morphological details, as for example the disappearance of cilia from the outer faces of the gill pouch walls or bars, in Amphioxus, and even from the whole body of the adult, due evidently to the long-continued burrowing habits of the animal.

The excretory system in Rotifera and Rhabdocoela consists of a pair of coiled tubes which run forward on either side of the stomach and which each sends a coiled process forward toward the neck. Each tube opens externally by a minute rentral pore, and then branches copiously in the mesoderm substance. The branches all end internally in "flame cells." But in the higher or "alloiocoel" Rhabdocœlida-a few of which such as Bothrioplana are fresh-water-the strong cephalic and abdominal loops and transverse branches formed between the main longitudinal canals (as seen in Fig. 5 p. 20 of Lank. Zool. IV) might be the commencing basis for a bloodrascular system, as already suggested (p. 442). The finer branches then of the excretory system would become more and more separated off from the evolving vascular system, though retaining intimate relations with it, alike in order to draw off excretory products from the vascular fluid and to be itself aerated. Such fine coiled branches then, ending in flame cells, would constitute a primitive pronephros, as outlined for nemerteans below.

The excretory system in nemerteans shows a gradually evolving complexity from a simple pair of anteriorly placed tubes, 
each with an external pore as in the last, to a segmentally arranged and unpaired or paired system, in which many'sets of nephridial or excretory glomeruli are in intimate contact with special branches of the vascular system, as in the highly complex arrangement described by Montgomery for Stichostemma Eilhardi (95:1). It would be impossible with our present knowledge to say whether we have here a graded advance from a simple pronephros of two tubules to a highly complex pronephros composed of many sets of tubules, or whether rather the nemerteans may not present us with all stages in formation of a pro-, meso-, and metanephridial system of still diffuse type in Tetrastemma.

But, as indicated in a preceding paragraph, it seems extremely probable that the primary and specially the posterior main excretory tubes of Rhabdocola became closed externally, in transition from that group to the Nemertinea, and so formed a rudimentary vascular system. The anterior ends of the tubes then, along with their branches, made special, usually paired, antero-lateral external openings for themselves. In the earlier stages of the process, as exemplified by most nemerteans, only two paired anterior openings were formed. But, in types that must once have connected these with Stichostemma, the primary nephridial tubes became broken up into separate constituents, that gradually ranged themselves along the length of the body. Some of these remained closed, but most made external openings along the sides of the animal. Internally, they gave origin to groups of flame cells of excretory character, that ramified alongside the vascular tubes.

Since the tubes are of unequal length in Stichostemma, alike in themselves, and on the two sides of the body, and since the external openings, real or potential, vary from 16 to 19 on each side, there is strongly suggested here an archinephric system such as Lankester has postulated. Further the very irregular lengths of the tubes, and the irregular disposition of the openings, might indicate that, in some still more evolved fresh-water form that once existed, the archinephridial system consisted of at least 20 pairs of archinephridia, that opened symmetrically by as many pores, and which internally formed as many sets of coiled tubules. Still later, a common internal archinephric duct may have joined the outer ends of the nephridia, and so the external openings would become closed up. In this connection Goodrich remarks (146:85): "In the cyclostomes there is evidence of the whole duct being really formed by the fusion of segmental rudiments representing tubules, 
much reduced in Petromyzon, but reaching fuller development in Bdellostoma."

The excretory system of cyclostomes is of exceptional interest in the above relation. For, while the anterior nephridial portion or pronephros appears in embryonic or larval stages of these as of other craniates, it persists unaltered and in segmental relation only in Myxine, though the pronephric tube has largely degenerated even in these. The position occupied however by the pronephros in Myxine in front of the gill pouches is exactly like that seen in most nemerteans. In Petromyzon the pronephric tubules are absorbed, though the duct more or less persists; while the solid kidney of this genus is composed of numerous closely coiled tubules that have lost all trace of segmental disposition, though they show a similar histological structure to that already described.

That a gradual transition occurs again to the Batrachia is indicated by the following statement of the distinguished worker Goodrich $(146: 86)$ : "There is no fundamental distinction between the pro- and the mesonephros; in the Myxinoids and Gymnophiona the transition from one to the other is gradual. Such differences as are found in the development appear to be chiefly due to the fact that, as the mesonephros arises later, the mesoblastic somites are by that time more completely differentiated." The carrying backward of the excretory pore has probably proceeded pari passu with backward development of the nephritic system and of its canals.

The eggs in Turbellaria, Nemertinea, Cyclostomata, and Batrachia undergo holoblastic segmentation, and the resulting embryo in all is completely or partially ciliated. All stages from direct and continuous development in land, fresh-water, and a few marine forms to an extensive metamorphosis and shedding of embryonic tissue in many marine types of nemertean can be traced. In spite of our still limited knowledge of immature cyclostomes it is now known for the larva or Ammocœes of Petromyzon that it undergoes a considerable degree of metamorphosis in reaching the adult stage. In the Apoda or cxcilians a slight degree of metamorphosis occurs, that becomes even less marked in urodele batrachians.

Nany other characters - alike embryonic and adult-might be cited to demonstrate that striking morphological continuity can be traced from the Rotifera and Rhabdocoela through 
the Nemertinea to the Cyclostomata and Batrachia. All such evidence tends to show that the above is in all probability the rapid and condensed line of evolution pursued from a simpler invertebrate to a fairly high vertebrate ancestry. For greater completeness, intermediate forms are specially desirable between the higher nemerteans and precyclostome forms. But even though these may never be found in the fossil state in virtue of their soft-bodied condition, and probably are not now alive, we can fairly well picture the transition phases that would be passed through.

Reduced size of the proboscis, and shifting of its functions to the steadily elaborating sense-centers and increasingly more muscular head; resulting filling up of the sheath, as Hubrecht has so far traced, by loose notochordal tissue; conversion of the muscular walls of the latter into firmer but highly elastic notochordal sheath tissue; added strength and pliability given thereby to the body, which would result in increased growth and rigor of movement; more perfect and rapid respiratory interchange with resulting formation of gill pouches behind the cervical furrows; increased concentration of the brain lobes, and dorsal convergence of the lateral nerves to form the spinal cord, as well as the specialization of the brain as a sensory correlating center; these are a few of the modifications that must have been passed through as nemerteans slowly became modified in some of their genera to a precyclostome condition. There seems no reason either to consider that any of this took place other than in fresh-water or subterrestrial surroundings. But in the process numerous offshoots may well have arisen that migrated seaward, and from some of these we would derive the Hemichordata, the Urochordata, and the Cephalochordata.

An important histological modification that is often regarded as of craniate origin is the production of protective or strengthening patches or plates of connective tissue and of cartilage, as well as their later replacement by bone. One or two closing suggestions on this may end the chapter. 


\section{Causes and Course of Organic Evolution}

In some nemerteans Bürger describes a connective tissue (Bundegerrebe, p. 87) that forms an enclosing box to the brain masses. If we may judge from its structural appearance and staining capacity it seems to be related to cartilage. Further histological and microchemical study might well be made of this tissue.

The possible relation of the Cyclostomata, the Batrachia, and the Mammalia to each other will next be considered. 


\section{CHAPTER XVI}

\section{THE EVOLUTION OF ANIMALS, Part III}

A consideration of the evolution of craniate animals has engaged many naturalists since Darwin's "Origin of Species" and some of Haeckel's earlier works appeared. It would be superfluous here to attempt a review of the various classifications proposed, though in connection with some often valuable treatises vague and even random statements have been made, that might prejudice a fair consideration of the facts before one. Thus when within a few pages of each other in one volume it is said, "if degeneracy consists in the loss of parts without complimentary addition of other parts, then the batrachian line is a degenerate line," and "the Reptilia of the permian present us with types with fish-like vertebræ, from which the class Mammalia may be distinctly traced," and again "the later reptiles diverged farther and farther from the mammalian type with the advance of geological time," we have generalized assertions made that-unless backed by detailed facts-may produce impressions wide of phylogenetic value.

In the present chapter we shall endeavor to compare structural details of the Craniata-alike living and fossil-and see how far these may contribute to some helpful positions. In doing this we shall dwell rarely on the Urochordata or Tunicata, since the consensus of opinion now is that they are very primitive chordate forms that have become much degraded and modified even from the larval state owing to their assuming a sessile life. Amphioxus, though greatly higher in structure, we shall view as a much modified and in not a few respects degraded type from the main vertebrate line (p. 543). The burrowing habit has conferred both modified and degraded conditions of structure. While greatly 
nearer to the cyclostomes than to higher nemerteans, it has become diverted from the main line in structural modification.

But, as already indicated, while we start with living cyclostomes as existing types of Craniata, we regard even these as somewhat lateral derivatives from a set of stem-types that connected the nemerteans with the Gymnophiona (Cæciliada) or a nearly allied group. Amongst fishes, then, the Cyclostomata alone will receive our immediate attention. For, as will be explained somewhat fully later, we would regard the scaled fishes or Gnathostomata, i. e., all except the cyclostomes, as being derived very early from a separate ancestry than that which directly originated the cyclostome-cæcilian series.

That the cyclostomes differ very fundamentally from the other groups of fishes is a fact which has often been stated by naturalists. 'The glandular scaleless skin, the soft circular oral area, the branchial sacs, the absence of lower jaw and of limbs, the numerous segmental areas and the mode of origin of the spinal nerves, the rudimentary character of the excretory system, and the history of development are all diagnostic features. The fire great remaining orders of fishes diverge so much from the above, and agree so well amongst themselves, in broad details, that they and the cyclostomes must early have diverged as two separate phyla. One group of the fishes howerer, the Dipnoi, includes species which show so many features suggestive of amphibian or even reptilian structure that they have often been regarded as likely ancestors of the highest vertebrates. When minutely compared, however, the inference does not seem warranted. Rather the resemblances are to be viewed as cases of parallelism or homoplasy in evolutionary history.

In line with evidence advanced in a previous chapter, the strong probability is that the entire group of cyclostomes as well as protocyclostomes were fresh-water in origin. The almost world-wide distribution of the few living species suggests also, apart from structure or affinities, that the group is an ancient one. But, from the softness of all parts except the horny teeth, one need not wonder that fossil specimens 
are unknown. We need not here speculate as to the nature of horny tubercles found in some of the oldest rocks, and viewed at times as the horny teeth of primitive cyclostomes.

While the Amphibia as a group has been accepted by most evolutionists as contributing to the direct line of vertebrate ascent, this has more been because it included attractive fossil forms, often of large size, from some one phylum of which reptiles may have been evolved. But, in conducting toward animals like reptiles that formed scales or bony scutes, that developed only one occipital condyle, that had a mandible of five or more distinct pieces, that formed procolous vertebræ, and that remain oviparous, we seem wholly to have left the natural pathway of advance.

We would propose then that a more likely line of advance would be from the Cyclostomata to the Amphibia apoda (Gymnophiona or Cæciliada), from these again to the Urodela, and - even though the present-day gap be a serious one-from the last to the simpler mammals. Considerable known remains in the carboniferous rocks of Ohio, Ireland, and Bohemia enable us to assert that batrachians of the group Aistopodes flourished then, and showed decided affinities with living Apoda. But the Aistopodes probably had devonian ancestors, that could well unite them with more primitive cyclostomatous progenitors.

If we consider then the characters of living Apoda in the above light, the view which has often been cursorily accepted, that they are degraded urodele batrachians, is in no way justified. A detailed comparison can now be made of structural characters that might favor such ancestral connections as above suggested.

The olfactory organ may first be dealt with from nemerteans upward.

In the latter two closely related structures arise on the anterior or antero-dorsal part of the snout, the cephalic glands and frontal organ or organs. The former are saccular paired glands that surround and often open into or beside the orifice of the latter. The latter or frontal organ is usually single, but in several rather primitive nemertean genera (Bürger, 
pp. 175-76), one median and two paired organs may arise, of which the median persists in higher types. The paired glands, in relation to the frontal organ or nasal sac, seem to correspond with the accessory nasal glands met with in vertebrates from the Amphibia upwards. The frontal organ in nemerteans is at times prolonged downward and backward as a richly glandular structure, whose posterior part is on a level with the nerve ganglia of the brain, also with the front part of the proboscis sheath and of the pharynx. The relation of these structures in Prosadenoporus suggests that the posterior glandular part of the frontal organ might readily in course of evolution open into the pharyngeal region, and so though still unpaired seems to correspond exactly in position, relation, and development to the organs of Jacobson soon to be mentioned.

In Amphioxus the median ciliated "olfactory pit" evidently corresponds to the frontal organ just described, though it shows secondary changes of relation.

In Cyclostomata the nasal organ is, like the frontal organ of nemerteans, a single median dorsal involution on the anterior end of the snout, and like it consists of an anterior orifice, a canal, a nasal sac or pouch, and a down-growing process that in the lamprey as in nemerteans ends blindly, but in Myxine is prolonged downward to open "into the oral cavity, perforating the skull from above, instead of from below as in other vertebrates" (138: 198). In cyclostomes the single nasal sac becomes either partially or completely divided by a median partition; and the ciliated lining tissue becomes raised into radial ridges. On either side of the nasal sac patches of glandular tissue arise, that very probably represent the head glands of nemerteans. But in Bdellostoma, according to Dean, the internal division of the nasal sac seen in Petromyzon and $M y x$ ine becomes complete, since in it the olfactory organ arises as a pair of ingrowths.

In Batrachia Apoda the nasal organ has now become paired throughout, but still occupies the anterior dorsal region of the snout. Each passage opens posteriorly into the oral cavity as in Myxine, and so a possible respiratory relation is opened for these, specially if they become land forms in the adult state, as most now are. Lateral glandular outgrowths are also formed ventrally here, that in some Apoda communicate with the nasal sacs, in others become shut off from the nasal sac, though innervated by the olfactory nerve. These form the organ of Jacobson, that seems to have a chemotactic function, and that persists even up to man. As in cyclostomes 
"the mucous membrane" of the sacs "is raised into radial folds" (138: 200).

Much speculation might be indulged in as to the pseudonares of many Apoda. Shortly it might be suggested that these are the paired frontal organs of some nemerteans still persisting in reduced state, or even are the paired functional organ of Bdellostoma. With good show of reason it might thus be claimed that they become the paired nasal sacs of higher craniates. Carefully prepared embryological and adult histological material can alone throw proper light on the question.

As to the histology of the organ in the three groups, Bürger's description of the frontal organ in nemerteans is unfortunately scant. His figures strongly suggest that it is lined by cells of two kinds, in one of which the cells are elongate and bear a fine tactile process. These two kinds seem to correspond to Schultze's olfactory cells and epithelial cells, to the former of which olfactory nerve-threads pass according to Blanc. In the Cyclostomata and Apoda the structural details are similar to the last, but in Mammalia the epithelial cells have been crowded out by the olfactories which now wholly line the cavity (137: 513).

The eye and ear need not concern us further than as already discussed (p. 429).

The alimentary canal presents points of comparative interest.

Wiedersheim says (138: 250): "The glands of the mouth, like those of the orbit and integument, appear first in terrestrial vertebrates, that is, from amphibians onwards. They have the function of keeping moist the mucous membrane which comes into contact with the outer air. From being at first almost entirely unspecialized and giving rise simply to a slimy fluid, they become differentiated later into structures the secretions of which take on a very important function in relation to digestion."

In contrast to the above, Lönnberg (14\%:316) says of the lower epithelial layer of the oral cavity in Myxine: "The basal cells are small-polygonal, between them appear larger mucilage cells which are quite similar to the mucilage cells" in the upper epidermis. (See also Retzius, 148.) But further Haack for Petromyzon has carefully described paired salivary glands which lie directly under the tongue and open by special papillæ. Of particular interest is it to note that these are 
absent in the larval Petromyzon, and only appear during the transformation stage as solid cell rows, which later form a lumen. They seem to be absent in Myxine. Mucous and salivary glands are both present therefore in some cyclostomes. The possible relation of these to the buccal glands described and figured by Bürger may be noted.

In the Apoda "oral glands are abundant," these being gobletshaped with large nuclei. Similar glands occur in Urodela, but beside these a median intermaxillary gland occurs in the area of the nasal septum.

While the tongue in ordinary fishes is merely a slight fold of mucous membrane, in the cyclostomes it is a large ventral mass of oral tissue, broadly attached to the floor of the mouth, and which in Myxine is covered with teeth so as to form a rasping apparatus of great efficiency, that is worked by strong muscles. Amongst the simpler urodeles the tongue is also well developed and similar in shape and wide attachment. In most of them it is nearly smooth (Proteidæ, Amphiumidæ). But in the Amblystomidæ the tongue may be broader than long, and anteriorly is covered at times with coarse papillæ. In the higher urodeles the tongue varies from oval to much elongate, and may be highly protrusible as in Spelerpes.

Reasons for regarding certain preœsophageal glands in nemerteans as homologous with the thymus and thyroids have already been given (p. 425). Wiedersheim synopsizes well as follows their origin and relation amongst craniates: "The thyroid arises primarily as a median ventral diverticulum of the pharynx in the region of the first four or five visceral clefts, and in the course of development may become subdivided into two lobes. In addition to this unpaired diverticulum, paired portions, situated more posteriorly, are developed in mammals.

"In the Ammocoete the single diverticulum, which is lined by a ciliated epithelium, opens into the pharynx between the third and fourth clefts, but in the adult Petromyzon the organ, as in all vertebrates, loses its connection with the pharynx, undergoes a modification, and gives rise to numerous closed glandular vesicles enclosing the albuminous substance.

"In the Urodela and Anura the thyroid gives rise to numerous vesicles situated close to the anterior end of the pericardium, 
posteriorly to the second ceratobranchials in the former, and on the ventral side of the posterior cornua of the hyoid in the latter."

The œesophagus in cyclostomes is continued by a rudimentary cardia into the straight intestine. But, though a definitely enlarged stomach is absent, the researches of Alcock and Haack prove that functionally it is represented by a considerable part of the cesophagus and intestine, whose gland cells secrete pepsin. The morphological resemblance of the above to the condition in nemerteans is more than striking, while functionally the remark of Shipley for nemerteans (149: 366) is equally so: "There is some evidence that in this group the ectoderm of the oesophagus is chiefly concerned with digestion, whereas the endoderm of the intestine is limited to the absorption of the soluble products."

In the simpler urodeles the stomach may be little larger than the osophagus as in Siren, or may be decidedly swollen as in Amblystoma, while the intestine is slightly or only moderately coiled on itself. In the higher urodeles the stomach is usually more enlarged and the intestine is mostly much coiled. Liver, gall bladder, and pancreas are a common heritage with all the craniates. From the much elongated liver of the Cyclostomata and Apoda to the at times elongate one of simpler Urodela, easy transition is made to the usually short compact liver of higher urodeles, and of mammals.

The purely cartilaginous brain-box of cyclostomes, to which no lower jaw is attached, represents the simplest craniate condition, though the opinion often expressed that the lower jaw is here atrophied has only a little evidence in its faror.

The advance shown in Apoda is most marked, and would indicate that between the higher cyclostomes, that almost certainly must have flourished in the early devonian or even in the silurian, and the aistopodous or related batrachians of the carboniferous great specialization had taken place.

In this connection the permian genus Lysorophus, as recently expanded in description by Case $(150, n .146: 141)$, seems in its known characters to connect cyclostome and apodous types. Thus there "is no distinct atlas or axis, and the vertebræ of 
the column are all similar. The centrum is perforate, and the cavity is widely open, so that the notochord was very little constricted. . . . The neural arch is free from the centrum, and the two halves are separate. . . There is no trace of intercentra. The ribs are single-headed." The relation of the bones in the skull, also, suggests ossifications amid the cartilages typical of cyclostomes. The above are all characters that would unite cyclostomatous with existing apodous genera, and in this connection Case adds: "The apparently limbless condition and the probable position of the eyes far forward in the orbital spaces suggest some affinity with the limbless amphibians."

The time and place of origin of the cartilaginous areas in the cyclostome brain-box correspond very well with similar areas in Apoda and Urodela where afterwards bone is deposited. But it should be said that it equally well corresponds with what one finds for various groups of fishes, of reptiles, and even of birds. As we shall shortly explain later, this may be one of many cases of homoplasy or developmental parallelism, that run through the entire organic world. Possibly however it may have a closer and more genetic explanation than the writer is at present prepared to accept.

The formation of two articular condyles for the skull is typical of recent Apoda, is equally so of Urodela, and persists up to the highest mammals. The ossification of the mandible from three centers only - dentary, splenial, and angular-is true alike of Apoda and Urodela, and is much simpler than that of many fishes and specially of reptiles. The presence of the dentary alone in mammals will later be dealt with.

The numerous true teeth in Apoda are small, hard, conical, pointed, and resemble each other, but show similar mode of growth to that of higher craniates. Here a wide gap exists between the cyclostomes that have only horny epidermal structures, and such perfected developments. Fossil remains may yet bridge the gap however. The similar or homodont teeth of Apoda and Urodela as compared with the usually varied or heterodont teeth of Mammalia is another morphological gap in the record. But Wiedersheim's remarks (138: 245) are apropos: "The heterodont dentition characteristic of the Mammalia as a class must have arisen by a modification of a simple homodont condition in which the teeth were all conical, and of similar size and shape."

The absence of limb-girdles and of limbs, alike in Cyclostomata and in Apoda, at times even in some Urodela, is a 
morphological condition that has been variously interpreted. Owing to the burrowing habit of many Apoda, and the burrowing or cave-dwelling habit of some Urodela, a certain degeneration has undoubtedly taken place, usually in the eyes, to some extent in the color and consistency of the skin. There seems however small evidence that the degeneration has become fundamental or deep-seated. But the often tacit, though in some cases expressed, opinion has gained ground that these groups are decidedly degraded, rather than types that link up for us an evolving line. We can now examine the evidence. But before doing so we would express the opinion that the very burrowing or retreating habits of these animals are probably the factors that have caused them to be left as connecting types of high interest at the present day.

The general primitive character of the endoskeleton and of other parts in cyclostomes agrees well with the idea that these have not yet evolved limb-girdles or limbs. The active gliding habits and slippery skin, also, scarcely serve to set up the needed irritable stimuli that would start paired limbs as a response result. The constant balancing efforts amid marsh vegetation, or progression on land, have called forth and established this by degrees, as we shall trace soon.

The presence in carboniferous strata of early apodous batrachians, that resembled in many points those of recent date, at once proves that the type is a very ancient one, and that an ample period has elapsed for the evolution of more complex forms from them, at the same time that some, from habit and environment, have retained primitive conditions largely unchanged up to the present day. We would therefore, and for many other reasons, regard the existing apodous condition as truly ancestral, and as being retained owing to continued preservation of a like balance between structure and environal relation. But the finding by Brauer (96:500) of "a pair of little swellings behind the last gill cleft, and an unpaired swelling (corresponding with a double one in Ichthyophis) in front of the vent," is of great interest. For "not unreasonably he sees in these swellings the last, very transitional vestiges of 
the paired limbs" (155:93). But why Brauer should be credited with regarding these as disappearing vestiges rather than evolving organs the writer fails to understand. Strasser's discovery then "of small cartilages in the position of the inferior" elements of the scapular arch" (152: 222) in Amphiuma we would take to be not a sign of degenerate limb remnants, but rather of the first rudiments of evolving limbs.

The existing members of the Urodela present great diversity in limb development, but we hope to show that this represents in most cases advancing specialization on a definite evolutionary line, where increasing mechanical friction and strains acted as stimuli that caused increasing complexity of structural response. The view has been strongly urged by eminent authorities, and has been almost unreflectingly accepted, that many of the simpler Urodela are degraded in their simplicity. We shall try to bring the facts together so that they may speak for themselves.

For reasons that will appear later we regard the Sirenidæ as most nearly serving to bridge over the Apoda with the Proteidæ, and these again with the Amphiumidæ, that in turn lead to the highest urodeles. Cope (152: 223) considered the Sirenidx to have become simplified from higher ancestral types "due to a long process of degeneracy." While showing slight degeneracy in some details the group seems rather to be a valuable one as demonstrating progressive evolution from apodous origin, and this is shown not least in limb relation.

Regarding the general question Wiedersheim (138: 107) remarks that in Amphibia "the pectoral arch shows no direct connection with that of fishes, but is similar in plan to that of all the higher vertebrates." The pectoral girdle in Sirenidx is alone present, and is largely cartilaginous; the fore limbs are short and the bones are partly cartilaginous; the carpus is purely cartilaginous (152: 224, Pl. 43), and bears four digits. The pelvic girdle and its limbs are wholly wanting.

As regards the gradual origin of limbs and their girdles, this has been treated in many works on vertebrate morphology, to which the reader may refer $(138: 102 ; 146: 73$, etc.). Limbs, amongst Batrachia and higher groups, evidently pushed out first as simple processes of the body, the fore limbs being the first to appear, and these were succeeded later by the hind limbs. Regarding the embryological origin of the digits more- 
over Cope says (152: 32): "The feet are first simple at the extremity, but soon become bifurcate, or with two digits. This was first observed by Rusconi in 1821 in the European Hemisalamandra cristata. Professor Baird first observed this in the Amblystoma punctatum in larvæ of $1.4 \mathrm{~mm}$. in length. The same has since been observed by myself in 1869, and later by Götte in his essay on the development and regeneration of the foot-skeleton in the triton; also by Strasser and by Hoy. In Amblystoma punctatum the number of digits succeed each other as follows: First stage, 2 before, hind limb none; second, 3 before, hind limb none; third, 3 before, hind limb 2; fourth, 3 before, hind limb 3. Sometimes the anterior digits are completed in number before the posterior foot appears, and we have combinations of numbers from 4-0 to $4-3$, and 4-4 to the full number, 4-5, which is found in all specimens of $2.5 \mathrm{~mm}$. and upwards."

Now in connection with the above numerical advance it may be well to inquire whether, in Sirenidæ and other groups of Urodela, a corresponding advance is not shown in the cartilaginous and bony internal parts. Here again we are met by the degeneracy theory, which must be balanced with all the facts of the case.

In Proteus, which has feeble fore and hind limbs, the three digits of the former are united to the radius and ulna by three carpals, of which two are in line with the ulna. In the later formed hind limb there are two digits similarly placed in line with the tibia and fibula. In Siren (152: Plate XLVI) there are six carpal cartilage bones joining the limbs. In Amphiuma the number of carpals and tarsals varies from three, placed as in the last, to $4,5,6$, or 7 . In higher urodeles the number may be nine to ten. Does such then represent a reducing series along a degeneration line, or an increasing series along a progressive line? Later additional facts, drawn from other systems, may enable us to decide.

Having accepted it, as strongly confirmed by the evidence in last chapter, that the notochord represents the now modified proboscis sheath of nemerteans, we find it rumning almost to the anterior end of the head in Amphioxus, thereby strongly suggesting that this genus is derived from one of the nemerteans in which the proboscis and its sheath opened at the front of the head, whereas in land nemerteans generally it opens within the oral cavity as in Plate $15 \mathrm{~b}-\mathrm{d}$. This is seen also in 
marine Malacobdella and Akrostomum still living. In the latter cases, and in types related that may once have been abundant, we hare to look, it seems, for the more typical notochordal extension seen in craniates. For in such a case when the sheath filled up with central occluding tissue its anterior extension would reach only to the mouth and the hinder part of the brain. Round this as a mechanically flexible but yet resistant rod, added strengthening substance would naturally center, as new and varied stimuli became added.

So we have a continuous notochord in the cyclostomes, that bears only cartilaginous neural arch processes, and occasionally ventral processes as well. In the Apoda however these arches are not cartilaginous but bony, and become continuous round the notochord to form bony amphicœelous rings or vertebræ. The biconcave or pseudocœelous vertebræ thus formed are the simplest type in construction, though they are a marked advance on the conditions in cyclostomes. But further in Apoda cartilaginous material is laid down round the fibrous notochord sheath between each osseous ring, and even grows inward within each biconcave ring, so as to absorb there the whole notochordal substance. Thus the originally continuous notochord with its fibrous sheath and cartilaginous hoops, as seen in cyclostomes, becomes by degrees an alternating set of cartilaginous rings and osseous biconcave rings with hollow centers, that surround the notochord; while ultimately as maturity is reached the cartilage, having grown into the hollow centers of the osseous vertebræ, where only notochordal substance was, replaces that substance entirely.

A transition is made from the Apoda through the simpler urodeles like Siren and Amphiuma to more advanced types like Desmognathus and from it to Diemyctylus and Salamandra, in that the pseudocoelous (amphicolous) osseous rings become more and more lengthened, the cartilage between more and more narrowed, and the substance of the notochord more and more completely replaced by bone, till in groups that are represented by the three last named genera the most complex condition is reached. Here each osseous vertebra has its 
anterior facet or articular area enlarged into a cup, and its posterior facet hollowed into a socket, so that the vertebræ now are firmly but most flexibly locked together. In the last condition the entire notochord has been replaced mainly by bone, there being only cartilaginous patches over the articular surfaces.

In the present connection it may be noted-though possibly the point may be of minor importance - that just as Hubrecht has shown that filling up and progressive differentiation of the proboscis sheath in nemerteans starts posteriorly, so in the formation of completed dorsal arches in cyclostomes, and later in the appearance of ventral arches in these, the process starts posteriorly.

In cyclostomes the cartilaginous skull is attached by muscles to the continuous notochord. In apodous types owing to high ossification of the skull-probably as suggested through action and reaction in the burrowing habit-two occipital condyles are formed that fit into sockets of the first vertebra. This vertebra however is a mere ring. But in the urodeles a part of the first vertebra grows forward as a hanging peg for the skull, and is the odontoid process.

The heart and blood-vascular system in cyclostomes have already been compared with those of nemerteans, but can now be compared with those of Apoda and Urodela. In cyclostomes the heart is placed-in contrast to the condition in other fishes-some distance back from the head, and is threechambered. The venous sinus passes from the dorsal to the ventral side, there opens into a thin-walled auricle, and from it by a two-valved aperture into a thick-walled rentricle. This is continued forward-at least in Bdellostoma-into an elongated conus arteriosus, that is separated by two valves from the ventricle. From the conus the ventral aorta is continued forward to the level of the gill pouches that are the fourth pair from the front, and here it breaks up into four pairs of efferent vessels distributing venous blood to the pouches.

The heart in Apoda, alone amongst Batrachia, is placed some distance back as in the last, and shows also the same divisions and valvular parts in one row. The conus also is elongated, straight, simple, and is continued into the ventral aorta, from which four similar pairs of afferent branches spring, 
to distribute blood to the gill pouches. But a decided advance is the division of the auricle into two chambers by a septum. The septum, however, is still imperfect, being perforated by apertures.

In some of the simpler Urodela (Proteus, Necturus, etc.) the structure remains as in Apoda, except that the heart has advanced slightly nearer the head. But in other of the simpler genera (Siren, Amphiuma), and in the salamandroid forms, the conus shows a decided spiral twist; internally it has valves at its fore and hind ends also, as well as a spiral flap projecting into it. The entire heart further in the last assumes a more condensed and rounded aspect. All of these details constitute increasingly perfected mechanical devices for forcing the blood flow, and for ensuring increasingly perfected separation of the impure and the oxygenated blood.

An equally noteworthy advance and condensation can be traced in the blood vascular system.

From our knowledge of the cyclostome Bdellostoma Stouti, and to a minor degree of Amphioxus, it may safely be said that in types between the nemerteans and cyclostomes-now evidently lost to us - a series of twelve or more pairs of gill pouches was formed. Into these as many afferent vessels passed; and as many efferent ones departed. Though slight rearrangements have taken place, we can still in the above named species trace twelve to fourteen pairs of these (p. 448), which in other Bdellostomata become reduced to six, the typical number for higher craniate embryos, and also for Myxine. In Petromyzon, after four pairs of afferent vessels have spread out from the ventral aorta, it splits and each branch gives off four lateral vessels. These latter, in process of condensing specialization, and substitution of aerial for aquatic respiration, would tend to disappear.

In some of the Apoda distinct indications of five sets of gill pouches occur, but in others only three are functional in the young. Brauer has shown (154: 496) that Hypogeophis and Ichthyophis form three pairs, but only the anterior two of these later develop expanded external gills, the third set of gills being rudimentary.

The gill pouches produce the external gills, which in origin represent lobe-like expansions of the membrane formed from the outer edge of each pouch that originates them. The anterior three pairs of afferent ventral vessels supply them, while the fourth or posterior pair proceeds to and joins with the 
pulmonary artery. Essentially the same arrangement persists in the Urodela.

But mention of the pulmonary artery leads to a study of the lungs and lung circulation. Amongst the cyclostomes no swim-bladder nor related structure apart from the gills occurs. But in seeking for possible first beginnings of lungs "that arise at the hinder border of the branchial region of the pharynx" a structure - the osophagœo-cutaneous ductdeserves mention. Regarding this Goodrich (146:46) says as to the myxinoids: "There is always on the left side a simple tube leading from the pharynx to the exterior, and opening in common with the last gill pouch." This he views as "probably a modified gill slit." With passage from an aquatic to a semi-terrestrial and at length terrestrial existence and coeval reduction of gill tissue, this might gradually have enlarged into a simple sac below the pharynx at first, that later might close off internally and dividing give rise to paired lungs. Interesting in this connection also is Cope's observation (73: 363): "The habit of holding in the osophagus large quantities of air while engaged in seeking food in foul water, or on land, on the part of vertebrates which normally oxygenated the blood by means of gills, was probably the mechanical cause of the development of a pouch, and afterwards of a diverticulum of the œesophagus, which became ultimately a swimbladder or a lung. . . . The development of a lung at once produced a change in the uses to which the various branchial arches were put. The posterior, which supply the lung, would be subjected to greater pressure owing to the increased blood supply demanded by the lung, and a correspondingly diminished pressure would be experienced by the now unused branchial portions of the bows."

In living Apoda, so far as observed, the lung is in the form of a bilobed sac, of which the right is well developed, the left is minute. This may be due to the habit that many, and specially the females, show of curling circularly round the eggs during the breeding season, but doubtless also is due as in snakes to a certain unilateral tendency in which one side comes more into play than another, as during rest and hibernation.

In the simpler Urodela like the Sirenidx and Proteidx, the same inequality is shown, though the two lobes tend to become increasingly near each other in size, till in Amblystoma they may be said to be paired. In the Salamandridx they are equal and of considerable size. Internally they show varying transitions from thin-walled sacs (Proteus) to sacs with coarse 
vascular meshwork (Siren) and again to others with "a complicated trabecular network" (Apoda) or a finely meshed lung system (Menopoma).

The urino-genital organs afford valuable comfirmatory evidence of the affinities now under discussion.

The relation of the nemerteans to the cyclostomes has already been dealt with. The clearly metameric character of the tubules in the former is reproduced exactly in the myxinoids, and in these alone the primitive or larval pronephros persists, while the opening of the nephrostomes into the pericardial cavity may well represent the nemertean and still earlier rhabdocol condition of distinct skin openings in which the pore has now closed, and the renal products are shed internally. In the Ammocœetes larva of Petromyzon the pronephros is present, but in time it becomes small and is replaced by the mesonephros or kidney proper. In the adult Petromyzon the permanent mesonephros or functional kidney is represented by a single longitudinal duct on each side, into which open a number of tubules leading from closed renal capsules with glomeruli. These capsules are much more numerous than the segments of the body they occupy. The above conditions are in marked contrast to what holds for ordinary fishes.

In the Apoda "the pronephros is well developed in the larva," which may have "as many as 12 or 13 nephrostomes" (138). In the adult, however, the pronephros is replaced as in Petromyzon by a long mesonephros or kidney, composed in the larva of metameric bodies which in the adult may lose metameric number-relation, and by gradual development may at length amount to hundreds. Who will say whether this is a progressive or degenerate change? But so far as phylogenetic morphology goes "there is no fundamental distinction between the pro- and the mesonephros; in the myxinoids and Gymnophiona (Apoda) the transition from the one to the other is gradual. Such differences as are found in the development appear to be chiefly due to the fact that, as the mesonephros arises later, the mesoblastic somites are by that time more completely differentiated" (146:86).

In the Urodela the condition is fundamentally as in the last, but there is a strong tendency for the posterior part of the mesonephros to enlarge, and become the active functional part, the narrower anterior portion being connected with the reproductive organs. This state of affairs may have foreshadowed, and even stimulated to, what probably occurred in the amphibio-mammalian origin of a metanephros. 
We may profitably pursue the history of the reproductive organs from the Nemertinea. In genera like Tetrastemma and Geonemertes, that include species which are mainly freshwater or terrestrial, the species may be hermaphrodite, but the great majority of the Nemertinea are diœcious. Amphioxus is dioecious, but, while the majority of the Cyclostomata are so, it has been shown evidently beyond doubt that Myxine is protandrously hermaphrodite. Much weight need not be given to this, though there is no sound reason why such may not be a lingering ancestral inheritance. The Urodela are entirely dicecious.

In Nemertinea the reproductive organs are simple sacs which lie at intervals alongside, and on either side of, the alimentary canal, or between the coeca of it when such are formed. They arise as swellings of cells in the body cavity, which by multiplication form a lining membrane and an enclosed packet of eggs and sperms. When ripe each sac becomes applied to and forms a pore in the body-wall, slightly above the line of each lateral nerve cord. The genital pores are therefore temporary openings, extending as a double row from the anterior-mid to the hind region of the body.

In Amphioxus the sacs occupy a similar position along the animal as paired, rarely unpaired, organs. Each consists of an investing epithelium enclosing the eggs or sperms. But when each sac is ripe, instead of opening outwardly by a separate pore as in the last, the contents are shed internally into the atrium and escape by its pore.

In the Cyclostomata the sacs appear as unpaired organs "extending nearly the whole length of the cœlom" (139:402). In structure they agree with both above. When ripe their contents are shed into the body cavity and from there are carried through a pair of "genital pores" into the common urino-genital sinus, and thence to the exterior.

EIn the Apoda a marked advance on the above generally similar condition is observed. The sacs-egg or sperm-are disposed in lines along the middle part of the body. They are united by a common tube in the case of the testis, which in turn communicates by transverse tubes with another longitudinally placed tube, that ultimately sheds its contents through the urinary tubules and the urino-genital duct to the exterior.

In the Urodela a distinctly segmental disposition is traceable in the ovaries and also in the oviducts, but the testes are reduced and condensed into an oval mass from which a few (2-4) efferent canals convey the products as in Apoda. 
From the foregoing comparative studies we are compelled to draw the conclusion that the cyclostomes are not only in almost direct line with nemerteans, they are equally removed from the true fishes. Further they show so many graded morphological connections with the Apoda, and these again with many living Urodela, that all of them, or some not distantly removed ancestral types, form a common and graded line of ascent of surprising completeness.

But, further, amongst the Urodela a progressive relationship in the living types seems fairly well traceable, while evidence for any extensive degeneracy of genera or families seems practically negligible. For, while a certain morphological overlapping or crossing of genera and families is at times observable, most structural details point to the Sirenidæ as the most ancient and primitive structurally of those now living, and as the group that most nearly approaches to the Apoda. The taxonomic and phylogenetic connections even seem in a general way to be indicated by the number of vertebræ in the entire vertebral column. Thus in Apoda 200 to 300 may be counted, in Siren 90-100, in Amphiuma 100-150, in Proteus 60-70, in Cryptobranchus 50-60, in Amblystoma 45-50, in Salamandra 45-50, in Triton 40-50, in Spelerpes 35-40. The numbers unquestionably vary - at times considerably - in different species or genera, even in different individuals as to tail vertebræ especially, but the average seems to agree with other characters that may now be quickly reviewed.

In the Sirenidx the gills are perennibranchiate; hind limbs are absent; the fore limbs have four digits; the eyes are devoid of eyelids; the jaws are toothless, but horny teeth and horny jaws may occur in larve (138:243); the skeleton is largely cartilaginous and the vertebre are pseudocentrous; there are no supra- or basi-occipitals, no intercalary, maxillary, vomeropalatine, prefrontal, or pterygoid bones; there are four epibranchial cartilages and of the four larval gill clefts the ond, 3rd, and 4th remain open; the carpal cartilages are few; the alimentary canal is simple. These are all characters that are absent in cyclostomes, because of their greatly lower posi- 
tion in the line of direct evolution. But we may compare them with living Apoda as follows:

In Apoda the gills are caducibranchiate; fore and hind limbs are absent; the eyes are rudimentary and without eyelids; the jaws bear teeth; the skeleton is largely cartilaginous and the vertebræ are pseudocentrous (amphicœlous); "the hyoid and branchial apparatus is more primitive than in any other recent Amphibia"; there are no supra-occipital, basi-occipital, supra-temporal, or intercalary bones, but the others are strongly and well developed; the alimentary canal, except for the liver, is simple. The outstanding feature here is the well formed skull, evolved doubtless in relation to the burrowing land habit. This also in all probability had to do with the rather precocious formation of lungs, and so with absorption of the gills.

The Proteidæ seem to be somewhat higher than the Sirenidæ, but present the following characters. The gills are perennibranchiate; the fore and hind limbs are both developed but often feeble; the digits are 3-2, or 4-4, or 4-5; eyelids are absent; upper and lower jaws bear teeth; the skeleton is largely cartilaginous, and the vertebræ pseudocentrous; there are no supra- or basi-occipitals, supra-temporal, maxillary, nasal, or prefrontal bones; there are three epibranchial cartilages but of the four gill clefts of the larva two remain, the first and the fourth closing; the carpal and tarsal cartilages are few; the alimentary canal is simple.

The Amphiumidæ are caducibranchiate; the fore and hind limbs are developed though feeble, owing probably to degeneracy, the digits are 3-3; the eyelids are absent; upper and lower jaws bear teeth; the skeleton is in part cartilaginous but to a less degree than in the above, while the vertebræ are still pseudocentrous; there is no median sternal cartilage; the skull is well formed and osseous but there are no supra- or basi-occipitals or intercalary bones; there are three epibranchial cartilages but of the four larval clefts only one remains open; the carpal and tarsal cartilages are few; the alimentary canal is clearly divided into areas and the intestine is convolute. This group is an advance on the two former, but the numerous 
vertebræ, feeble limbs, poorly ossified pectoral and pelvic girdles seem to be in part primitive, in part-specially as regards the limb girdles-degenerate conditions.

The Cryptobranchidæ are caducibranchiate; the fore and hind limbs are well developed; the digits are $4-5$; eyelids are absent; upper and lower jaw bear teeth; the skeleton is in part cartilaginous but is condensed and strong, the vertebræ however are still pseudocoelous; there is a sternal cartilage; the skull is well-formed and osseous, but there are no supraor basi-occipital or intercalary bones; there are three epibranchial cartilages and one mature gill cleft as in last; the carpal and tarsal elements are still cartilaginous, but are numerous ( 8 to 9 ); the alimentary canal is extensive, divided into distinct areas, and the intestine is long and greatly convoluted.

The Amblystomidæ are caducibranchiate; the fore and hind limbs are well formed; the digits are 4-5; eyelids are absent; teeth are as above; the skeleton is only slightly cartilaginous but the vertebræ are pseudocœlous; the skull is closely as in the succeeding; there is one epibranchial cartilage; the carpal and tarsal elements are now ossified and numerous (8-9); the alimentary canal is as in the last. Reference may here be made to the gradual formation of the digits in fore and hind limb as synopsizing the phylogenetic history of the group. "In Amblystoma punctatum the number of digits succeed each other as follows: First stage, 2 before, hind limb none; second, 3 before, hind limb none; third, 3 before, hind limb 2 ; fourth 3 before, hind limb 3 . Sometimes the anterior digits are complete in number before the posterior foot appears, and we have combinations of number from 4-0 to 4-3, and 4-4 to the full number $4-5$, which is found in all specimens of $2.5 \mathrm{~mm}$. and upwards" (152:32).

The two last groups show decided transition to the next and last one Salamandridxe, the genera Desmognathus and Thorius serving in many respects to unite all three.

The Salamandridxe are caducibranchiate; fore and hind limbs are well developed and relative to the size of the body are set well down from it, so as to raise the animal on them; 
the digits are typically 4-5; there are movable eyelids except in Typhlotriton; teeth are as above; the skeleton throughout is well ossified and the vertebræ are now opisthocolous in most genera, though Plethodon, Spelerpes, and other genera have pseudocolous; there is one epibranchial cartilage, and all of the four larval clefts close up; the carpal and tarsal elements are ossified and numerous (8-9); the alimentary canal is much as in the last.

In setting out the above characters the writer would by no means assert that all of the included genera as well as their groups represent a continuous and rigid line of advance. For the extensive organic denudation that must have occurred during past geologic periods since the permian epoch has left us these only as island-groups of scattered organic continuity. But the above assemblage of characters, that could be increased by many additional minor ones, seem to indicate for us the main pathway of urodele advance, and the sign posts, so to say, that point the way. But if more detailed comparisons be carried out, as, for example, along the lines of comparative pelvic structure, as figured by Wiedersheim (138: 112), or of pectoral structure, the correctness of the writer's views will probably be granted.

One point here deserves emphasis. If the main trend of evolutionary progress toward the mammals is as has been sketched, then we must look for the first beginnings of true tetrapodous limbs amongst either the Apoda, or the sirenid and the proteid series. The careful embryological studies already made on these by Götte, Strasser, and others should be carried to all species of the component genera of the groups.

But the objection may, at least on first glance, be pertinently urged, that the Apoda are scaled animals while the main groups emphasized above have been scaleless. To this it can be replied that a smaller half of the living genera of Apoda are scaleless, while even the scaled genera have the scales formed deeper in the body substance and in a quite different manner embryologically from true scales. They might well, therefore, be designated the "Pseudolepidapoda." 
Finally, while we have stated that the true fishes, as a great group, form a side line of development that has not now concerned us, it should be conceded that living Dipnoi show many resemblances to the Batrachia, and not least to the Apoda. This point along with other phylogenetic details will be considered later. The "family tree" facing this page may represent for us the supposed relations existing between the groups studied up to the present.

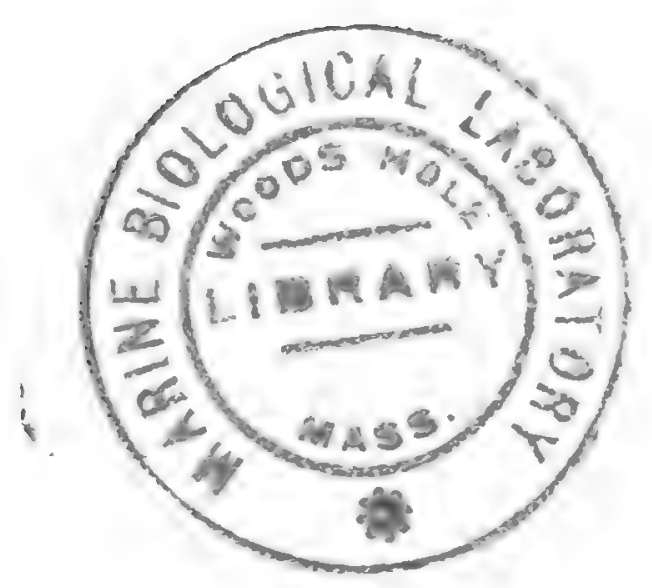




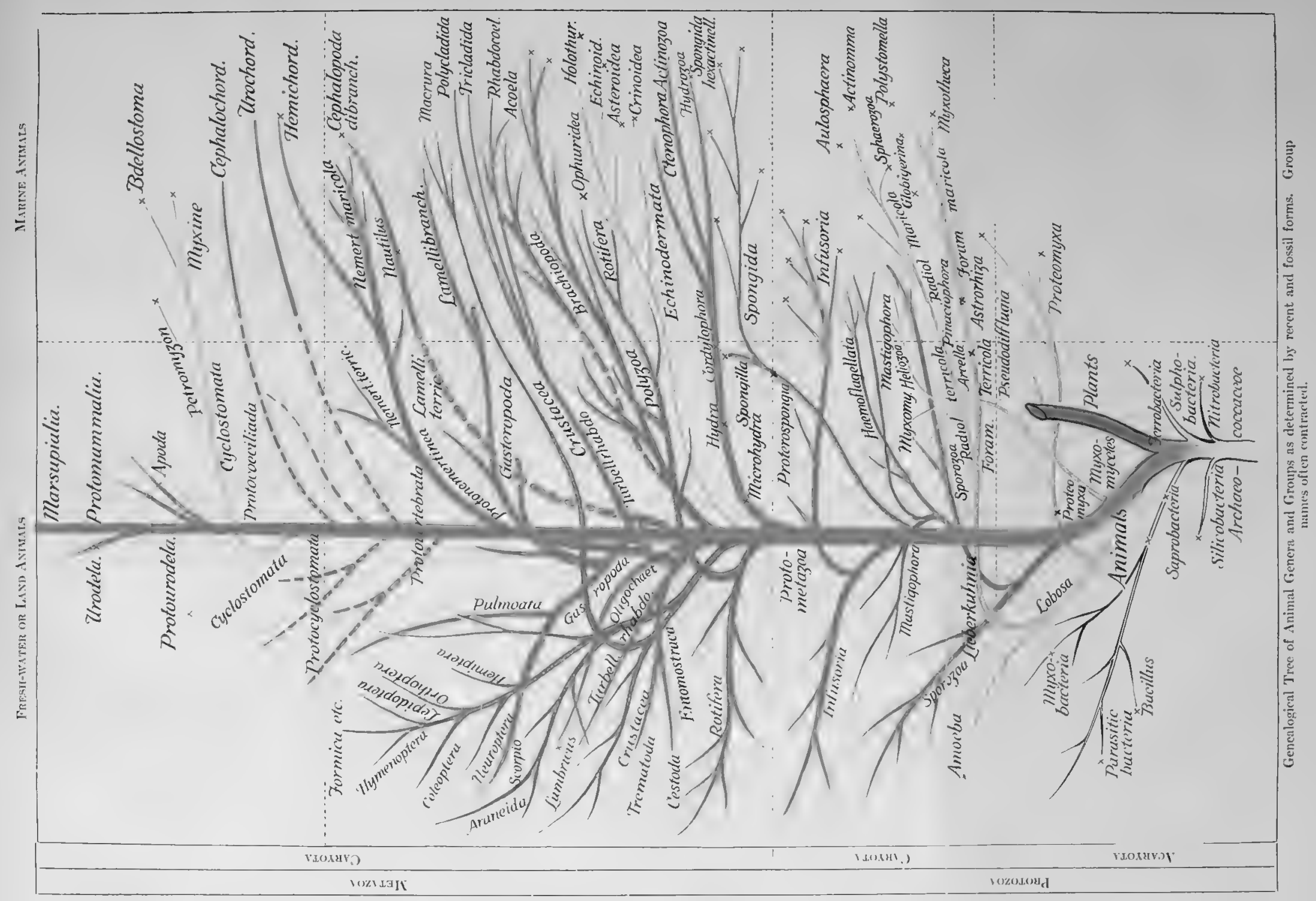





\section{CHAPTER XVII}

\section{THE EVOLUTION OF ANIMALS (Continued)}

There remains for consideration in this chapter the possible origin of mammals from simpler types. This inquiry narrows itself essentially to the batrachian (amphibian) origin that Huxley advocated, or to the reptilian origin that most recent naturalists have favored. The writer can not refrain from expressing the opinion that in the past discussion of this question too much emphasis seems frequently to have been laid on minor bones of the osseous system, that often appear and disappear in mammalian groups. Further the question of dentition has been so emphasized as a safe guide that other important evidence is often ignored. Again the fragmentary parts of fossil remains have at times had large generalizations tacked on to them-in some cases very appropriately, in other cases with doubtful results. Finally the lack of soft parts in fossil forms often cuts off from us important means of reaching valid conclusions.

A highly valuable and necessary matter to determine here is the environment that obtained when primitive mammals were evolving into those of recent times, and the environal relation of these animals to that environment. The preponderating number of living batrachians and Amnionta consists of land or fresh-water animals, while those that are marine have evidently become adapted from a land life. Thus the sea turtles, the seals, whales, etc., are conceded to have sprung from fresh-water and more primitively from land ancestors. Now our knowledge of land forms is largely confined to three geologic periods, the carboniferous, the liassic, and the eocene, we know much less as to the permian and the cretaceous.

But, owing to constant and extensive denudation changes, chances for the preservation in fossil state of land animals 
were relatively-small. Swamp-loving animals, like many of the batrachians, reptiles, and early mammals, stood greater chance of being more quickly buried from disintegrating change.

Every known fact points to the conclusion that the predecessors of mammals were essentially land animals. Though the remains must represent a mere fraction of those that at one time inhabited the earth, we already know of many and diverse types of fossil batrachians, reptiles, and mammals, which, while serving at times to link together isolated groups now living, in the majority of cases rather suggest that great cohorts of families have been swept out of existence. The greatest destruction too must undoubtedly have come to land species. This therefore complicates and makes difficult the task of bridging over the gaps now existing amongst living species.

But we may be helped toward a right estimate of phylogenetic lines of descent if we pass in review the leading characters of the three great classes, Batrachia,* Reptilia, and Mammalia, that alone now concern us. These can be set out in correlated series as below:

In Batrachia (higher Urodela).*

(1) The skin is smooth, scaleless, richly glandular, with abundant sensory nerve endings and sensory hairs; pigment cells are also frequent.

(2) The limbs are tetrapodous and four- to five-dactyl. When digital reduction occurs, digit I always disappears first, and later digit V (159:1).

(3) Larval gills and gill clefts are formed, but these are replaced functionally by lungs in the adult.

(4) There is a lateral line connected with abundant sense organs.

(5) The skull articulates with the first vertebra by two exoccipital condyles.

(6) An auditory columellar apparatus fits into the fenestra ovalis.

* For reasons that will appear later we give the characters here of the higher Urodela, and neglect the simpler groups. 
(7) The mandible consists of three pieces, and articulation is with the quadrate bone.

(8) The vertebræ are pseudo- or in highest urodeles opisthocœlous.

(9) There are abdominal ribs, or these fuse to form a sternum that joins with the coracoid. No distinct coracoids however are formed.

(10) The heart consists of a ventricle, two auricles, and conus with one row of valves.

(11) The aortic arches are strictly symmetrical.

(12) The red blood corpuscles are nucleated, oval, and biconvex.

(13) The lungs are simple and paired, at times with trachea and cartilages.

(14) The vagus is the last cranial nerve, and the hypoglossal is a true spinal nerve.

(15) There are kidneys with persistent nephrostomes.

(16) Most are oviparous, a few only are viviparous.

(17) There is no amnion, but the allantois is feebly represented by a swelling of the cloaca that is a urinary bladder.

\section{In Reptilia.}

* (1) The skin develops scales, shields, warts, or scutes, never hairs; it is non-glandular; the sensory nerve endings are embedded; pigment cells are occasional.

(2) The limbs are tetrapodous and four- to five-dactyl, or have been absorbed. When digital reduction occurs, digit $V$ always disappears first, followed by digit $I$.

(3) Temporary gill clefts are formed, but lungs alone aerate the blood.

(4) Sense organs of the lateral lines are rare or absent.

* (5) The skull articulates with the first vertebra by one basi-occipital condyle.

(6) An auditory collumelar apparatus fits into the fenestra ovalis.

* (7) The mandible consists of six pieces, and articulation is with the quadrate bone. 
(8) The vertebre are procolous and gastrocentrous.

(9) The ribs form a true sternum that is calcified and that joins the coracoid.

(10) The heart consists of two ventricles with incomplete or complete septum, two auricles, and conus absorbed into the ventricle that has a row of ventricular valves.

* (11) The right aortic arch only persists and is tripartite.

(12) The red blood corpuscles are nucleated, oval, and biconvex.

(13) The lungs are paired, and have trachea and cartilages, also bronchus.

(14) The hypoglossal nerve becomes intracranial, and so posterior to the vagus.

(15) The kidneys have no nephrostome, but each kidney has one ureter.

* (16) All are strictly oviparous.

(17) There is an amnion and allantois.

In Mammalia.

(1) The skin is smooth, hair-covered, richly glandular, and has abundant nerve endings; rarely has scales or ossified plates; pigment cells are usually developed.

(2) The limbs are tetrapodous and four- to five-dactyl, rarely they become rudimentary or absorbed. When digital reduction occurs, digit I disappears first, and later digit $\mathrm{V}$.

(3) There are embryonic gill clefts, but lungs alone aerate the blood.

(4) Lateral line sense organs are developed.

(5) The skull articulates by two exoccipital condyles.

(6) The ear bones consist of a chain of three or four separate bonelets.

(7) The mandible consists of one piece, the dentary, that articulates with the squamosal.

(8) The veretbræ are flat and gastrocentrous.

(9) The ribs form a true sternum that is ossified and is free from the coracoid, except in the Monotremata. 
(10) The heart consists of two ventricles complete, and of two auricles with one row of ventricular valves.

(11) The left aortic arch persists.

(12) The red blood corpuscles are nucleated in the marrow, spleen, etc., in other regions they lose the nuclei; they are oval in the camel, circular in others, and biconcave.

(13) The lungs are paired, with trachea and cartilages, also with bronchi.

(14) The hypoglossal nerve is intracranial.

(15) The kidneys are the metanephros and each has one ureter.

(16) All are viviparous except the Monotremata.

(17) There is an amnion and an allantois.

When one compares the above characters it appears that between the Amphibia and Mammalia all are either common and fundamentally alike, or that certain characters in Amphibia could by progressive evolution be gradually changed into mammalian ones, except for the formation of the amnion. Comparing the Reptilia and Mammalia the last named character is one of fundamental agreement along with others, while five most important characters disagree. These are indicated in Reptilia by an asterisk.

Such would yield at least slight presumptive evidence in favor of the Amphibia being the ancestral mammalian stock. Further the tailed forms or urodela - not the mature tailless Anura that have branched off along a line of condensing specialization-particularly claim our attention. If one think of a salamander or an axolotl, one marked external difference, as compared with ordinary mammals, is the real or apparent absence of hair. But more careful study reveals the abundant presence especially at certain stages in the life history - of many fine sense organs or side organs, with delicate hair endings in each, that are disposed around the mouth and in longitudinal lines along the body. These are in contact with nerve endings from the vagus or hypoglossal, and correspond to similar structures met with in cyclostomes, in a simple state 
amongst nemerteans, and even in Rhabdocola and in Rotifera. Amongst apodous and urodele batrachians these tactile hairs are most persistent, and as a rule most abundant, around the anterior head region and the lips, where they are innerrated by fine branches of the trigeminal nerve. But they are often abundant along the entire length of the animal, mostly arranged in rows. They are supplied by the vagus and by the hypoglossal nerves. Like the hairs of mammals, these sensory hairs of cyclostomes and batrachians are purely epidermal formations, and though greatly simpler in structure result from invagination of the epidermis, which forms a follicle round a central cell mass from which the clear hair springs. Were more abundant cell-divisions to take place in the follicle, and specially in the hair, a structure would result that would closely approximate, in origin, in relation, in morphology, and in function, to the stronger and more persistent hairs of mammals. Like mammalian hair-cells also these are often pigmented or contain fatty products. Such an origin has already been suggested by Maurer (156:717). A possible connection may exist also between two conditions observed by Schultze and Meijerle respectively. The former found amongst Batrachia that each hair-area might develop as few as one, as many as eight straight stiff hairs (15\%:370). The latter has shown that amongst mammals the hairs, instead of being scattered, irregular, and so-to-say unsystematized, may arise in groups of $3,5,6,12$, etc., which may either be equal or unequal in size $(158: 312)$.

In this connection it is significant to observe that the first mammalian hairs to appear during development, and the last to disappear by specialization or degeneracy, are the sensitive tactile hairs round the mouth, ears, eyes, along the lateral line, and at times also along the fore limb, when this is used in a specially sensitive manner. But these are the hairs which are most primitive and persistent along the entire nemertovertebrate line.

In view of the above evidence an origin for the haired mammals from the urodele amphibians seems strongly suggested. 
If we next select for comparison the skin-glands, these form an equally important feature in common for Batrachia and Mammalia, while their absence in reptiles is in striking contrast. The abundant and diversified glands in salamanders and other urodeles, which often secrete several diverse substances, only find an evolved parallel in the sweat, the sebaceous, the meibomian, the mammary, and other glands of mammals.

Much stress has been laid on the similarity of the teeth and tooth groupings in some fossil reptiles to the mono- or diphyodont and diversely grouped teeth of mammals. But many facts go to show that this is probably a case of parallel development or homoplasy. We may therefore review here the entire question of tooth formation amongst craniate animals.

We have already learned that horny teeth are a frequent characteristic of nemerteans. 'These persist as the median horny tooth of Myxine or as the numerous scattered teeth of Petromyzon. Such seem unquestionably to have been the primitive tooth type, and are reproduced as the only functional teeth in many fish genera, and over considerable areas of the buccal cavity. But these again correspond to the dermal plates or denticles, that have constantly tended to appear in several lines of animal development. In this connection Wiedersheim (138: 30) says: "The first and most primitive hard structures in Vertebrates are . . . in the form of small pointed denticles (placoid organs) in the skin; these consist of enamel and dentine, resting on a basal plate of bone, thus resembling in structure ordinary oral teeth" [author's italics]. Again Bridge (139: 248) says, "True calcified teeth first make their appearance in fishes, where they assume the form of modifications of exoskeletal structures. The teeth of elasmobranchs are identical in essential structure, as well as in the manner of their development, with the ordinary dermal spines of the skin, and in the embryo the dermal spines form a continuous series with those which invest the jaws and erentually become teeth. It is only later, when lips become apparent, that the continuity of the teeth and dermal spines is interrupted, and the two structures assume their distinctive characters."

Now alike amongst fishes and batrachians such teeth constantly appear and disappear over definite areas, and funda- 
mentally are only distinguished from the horny teeth of Petromyzon in that they fail to form odontoblasts and so form no calcified tissue. Thus romerine, palatine, mandibular, maxillary and premaxillary teeth may be formed, or may again disappear. Amongst Batrachia the writer inclines to consider that a suctorial mouth with horny teeth originated first in the group, and that these were afterwards shed without replacement by calcified teeth as is still seen in Siren and in many Anura, where in change from a vegetable to an animal diet the tongue and the lips are used to seize and hold the prey. But in the Apoda and simpler Urodela one to several sets of teeth are formed, of which one row along the premaxilla and maxilla and another along the dentary have survived through the higher Apoda and Urodela to the Mammalia.

So amongst urodeles the originally numerous and diffuse teeth on the vomero-palatine and other bones tend to become more and more restricted till they form a row along upper and lower jaw. But in Spelerpes, Plethodon, Desmognathus, and others there is a distinct tendency toward heterodont tooth localization, in that there is a subdivision of the crown of some teeth into two equal or subequal cusps. Such formations, taken in conjunction with Cope's observations (73: 318 ) on the mechanical origin of incisor, canine, premolar, and molar teeth, suggest that with increasing tendency to life on land, with greater use then on hard objects and at different angles, also with specialization in the nerves and muscles of the upper and lower jaw, the heterodont mammalian type might well have evolved from the simpler and mainly homodont batrachian.

But study of such mammalian groups as the marsupials, the toothed whales, the rodents, and others show that even amongst these the teeth may be homodont or some that are usually present may never be formed, as in the canines that are entirely absent in rodents.

The cervical vertebræ in batrachians and mammals suggest a rather profound difference in number and relations, that at the present day would diagnose the two groups more easily than any other. In all existing batrachians the number is two, of which the most anterior unites with the occipital region of the skull, while the second is recognizably absent in Apoda but forms the odontoid process in urodeles. The succeeding vertebræ bear ribs, but, if one define as cervicals all in front 
of the articular line of the humeri, then 3 to 5 cervicals can be distinguished. In mammals there are rarely 6 , usually 7 , seldom 8 or 9 . But even this degree of variability renders permissible the view that land forms may have appeared between the carboniferous and recent epoch, in which the usual number of seven became fairly steadily established; this resulted from two changes, the absorption of the anterior vertebral ribs, and the shifting backward of the pectoral girdle and line of insertion into the humeri. But the shifting backward would be a morphological one, rather than in actual distance relation. For, while the ribbed cervical vertebræ in front of a line joining the humeri in Cryptobranchus, Chondrotus, and others are as long as the dorsals, in mammals they are nearly always much shorter. A suite of genera of amphibio-mammals from the close of the permian or early part of the triassic age is here greatly to be desired, as we feel fairly confident of their former existence. Some of the recently described Aistopoda from Ireland and this country seem to be the desiderated types.

In the relation of the skull to the lower jaw, and in the structure of the latter, the urodele Batrachia approach much more nearly to mammals than do the reptiles. For in the former the squamosal closely corers the quadrate, and lies directly above and outside of the articular surface. The quadrate however still acts as the articulating bone for the lower jaw. Now according to one view the quadrate and articular in mammals slip inward to form the malleus and incus bones of the ear, while the squamosal directly articulates then with the dentary. The retention of the quadrate as a very evident bone in reptiles, and its broad fusion with the articular, as one of six bones in the lower jaw, form a grave objection to the reptilian view of mammalian ancestry.

Lull (159:1) has pointed out that "the laws which govern digital reduction among vertebrates lead to an interesting grouping of the Mammalia with the Amphibia, in which the order of reduction is first digit 1 , then digit 5, as contrasted with Sauropsida-reptiles and birds--in which the fifth digit is invariably the first to disappear, followed by digit 1." 
The absence of an amnion and well-developed allantois in Batrachia and their presence in reptiles and mammals seem highly favorable for an alliance of the two last. But the undoubted developing rudiment of the allantois as a cloacal outgrowth exists in Batrachia, while in what we would regard as the most primitive group of mammals, namely the Marsupialia, an allantois is not seen in most, and only attains to the condition of an allantoic placenta in Perameles. The amnion as such is entirely unrepresented in the last, but when we consider how striking and fundamental a modification occurs in Salamandra maculosa and S. atra (155:117) so as to make these viviparous, while the group is oviparous, such is proof of a degree of adaptability and modification that might readily have stimulated the further marked enlargement of the allantoic rudiment, and equally have started new formation of an amnion during the mid- or late carboniferous, or at latest in the early permian, epoch of the earth's history.

Mention of the above formation of a viviparous habit in some of the higher urodeles forms a point of contact between Batrachia and Mammalia that we cannot afford to overlook. That the habit should appear amongst such Apoda as Dermophis and Typhlonectes, and that it should reassert itself in Spelerpes and Salamandra amongst the Urodela, is proof that here at least a typically mammalian habit has been fairly well established. But attention might also be drawn to the post-embryonic and yet premature nourishment effected by members of the Marsupialia, a group that we would look on as the basement series of the mammals. For, when the freed fotus or embryo is transferred by the mother marsupial to its pouch, it continues-unwittingly doubtless by chemotactic action as with milk for a sucking child-to absorb food from a free source, much as do the fotal salamanders, when they at one stage absorb the products of surrounding and arrested eggs alongside them.

Mention of the marsupials can now serve for introduction of the question as to which of the mammalian groups the Batrachia most nearly approach. It must freely be confessed that palæontological knowledge is here so scant, and the present gap that separates Batrachia and Mammalia is so wide, that only an approximate and suggested answer can be given. But many reasons tend strongly in favor of the marsupials as at least the commencing members of the main line. For it includes such diverse types as the edentate-like Myrmecobius, 
the ungulate-like kangaroos, the carnivorous Dasyurus, the rodent-like wombat and opossum, the insectivore-like marsupial mole (Notoryctes), the lemuroid tree kangaroos (Dendrolagus).

The subjoined is a tabulated comparison between urodele Batrachia and Mammalia in which the frequent contact points with the marsupials in particular will appear. But it should be said that in arranging the table it has seemed entirely permissible to quote morphological details of similarity even in one or two genera, though otherwise they may be of minor value. Thus, if it can be shown that one or more species of reptile has a lower jaw that articulates with the squamosal rather than the quadrate, such would prove that the at least potential capacity for such morphological change existed amongst the reptiles, and thereby made a possible alliance with the mammals.

\section{Batrachia (Urodela)}

(1) Specially sensitive hairs round areas of the head and over the body.

(2) Eyelids rudimentary to well developed.

(3) Lips bounding a shallow oval vestibule.

(4) Fourth toe usually longest.

(5) Digital nails in higher urodeles (Onychodactylus, 152: 119).

(6) Teeth numerous, alike or nearly so, sometimes slightly heterodont.

(7) A double set of teeth developed.

(8) Slight to firm union of the rami.

(9) Mandible inflected.

(10) Horny teeth or horny pads or both may be formed.
Mammala (excepting Monotremata)

(1) Sensitive hairs abundant.

(2) Eyelids well developed.

(3) Lips and oval vestibule well developed.

(4) Fourth toe longest in marsupials, often also in other mammals.

(5) Nails usually well developed.

(6) Teeth numerous to definite, rarely alike, usually heterodont.

(7) A double set in all typical mammals.

(8) Slight (Cetacea) to firm union of rami.

(9) Mandible inflected in marsupials and Otocyon, straight in other mammals.

(10) Horny teeth and pads occasional (whales, etc.). 


\section{Batrachia (Urodela)}

(11) Vertebral segments having cartilage formed from connective tissue, also cartilaginous intervertebræ, which in time fuse with vertebræ.

(12) Axis vertebra has ribs.

(13) Ribs are double-headed.

(14) Epipubic cartilages in most. Carpal and tarsal bones of nine pieces in higher genera and that are usually distinct.

(15) Cerebellum fully exposed, and corpus callosum rudimentary.

(16) Cloaca well developed.

(17) Lungs long and simple to condensed and complex, lobulate.

(18) An allantoic placenta as a saccular outgrowth of ventral cloacal wall that functions as urinary bladder.

(19) Eggs holoblastic approaching to meroblastic in segmentation, with unequal cleavage.

\section{Mammalia (excepting \\ Monotremata)}

(11) Vertebral segments with cartilage formed directly in the vertebræ, later intervertebral masses remain as distinct bony epiphyses.

(12) Axis ribs in Perameles and Phascologale, but absorbed in other mammals higher.

(13) Ribs are double-headed (single in Prototheria).

(14) Epipubic cartilages in $T h y-$ lacinus, but becoming bones in other marsupials, possibly also in dog. Carpal and tarsal bones of nine pieces that are distinct or may somewhat fuse with each other.

(15) Cerebellum fully exposed, and corpus callosum rudimentary in marsupials and lower mammals.

(16) Cloaca rudimentary in marsupials only.

(17) Lungs rarely long and simple, usually complex and lobulate.

(18) Allantoic placenta present in most higher mammals, and in marsupial Perameles; absent in other marsupials.

(19) Eggs holoblastic rarely approaching to meroblastic (Marsupialia), with equal segmentation.

But in addition to the above characters the pelvic arch forms a feature of contact between the two groups that was strongly and deservedly emphasized by Huxley. For, apart from the epipubic cartilages or bones of higher urodeles and their homology (Duges, Cuvier, Huxley) with the marsupial bones of mammals, the relative development and position of the ilium, ischium, and pubis in Salamandra as compared with marsupials, caused Huxley to say (160:404): "These facts appear to me to point to the conclusion that the Mammalia have been connected with the Amphibia by some unknown promammalian group, and not by any of the known forms of Sauropsida." 
With the above characters now before us, and bearing in mind other structural points of the Amphibia and Mammalia, it would not be difficult to construct an imaginary form that might be temporarily called the amphibio-mammal. If such existed we should expect to find some confirmatory eridence from fossil remains. Unfortunately urodelan and primitive mammalian remains are still rare from their early occurrence in the triassic up to tertiary times when they become more abundant.

In suggesting a probable arboreal ancestry for the Mammalia, Matthew (161:811) considers that a primitive central mammalian type would show the following characters:

1. Size very small, skull of moderate length, brain case completely enclosed in bone, brain of high type compared with that of Reptilia although lower than in the modern mammals.

2. Molar teeth "bunodont," i. e., low crowned, the crowns composed of few low broad rounded cones, heavily enamelcovered.

3. Neck rather short, slender, and flexible, permitting quick and easy turning of the head in all directions. Trunk slender, flexible, ribs rather short and few in number, lumbar region long and comparatively flexible. These features characterize all early tertiary mammals without exception.

4. Tail very long and flexible with strong muscles toward the base and probably prehensile.

5. Shoulder girdle of scapula and clavicle. No indications of a separate coracoid have been observed in early tertiary mammals.

6. Ilia narrow and rod-like, gluteal muscles long and slender.

7. Upper members of limbs comparatively long and loosely jointed to the trunk, permitting great freedom of motion. The great relative length of the humerus and femur is a striking feature in the limbs of early tertiary mammals.

8. Ulna and radius separate, equal in size, radius with round head, permitting free supination and pronation.

9. Wrist and ankle very flexible, all the carpals separate and a centrale present.

10. Five digits on each foot, the joints permitting of very free motion of fingers and toes, which are tipped by small claws. pes.

11. First digit more or less apposable in both manus and 
But if we assume it as safe that batrachians had already attained to urodelan level of organization in the lower carboniferous age and the Aistopoda practically assure such a possibility-, also that primitive mammals appeared in the early triassic - as all palæontological evidence tends to show-, then the extended period from the upper carboniferous through the permian would represent the amphibio-mammalian and promammalian age.

During this permian period the higher urodeles would have become thoroughly land animals. So the sparse sensitive hair growths would spread abundantly over the body in part for sense perception, in part for aerial temperature protection; the ear membrane would develop round its cartilage an external pinna for collection of the air waves; the quadrate bone would slip in to form a bone of the ear, and the articular be replaced by the squamosal; the three bones of the lower jaw would become, with increased masticatory work on firmer plants or animals, condensed into one; the originally abundant similar vertebræ of Apoda would become from the changed condition in urodeles condensed from 270 in some Apoda, through the 100 or more of Siren allies and the 70-50 of higher urodeles, to the average number of about 50 for typical mammals. With varied running, climbing, and swimming habits on land and in water, these vertebræ would become from specialized muscular action sharply divided into cervical, dorsal, lumbar, sacral, and caudal; with increasingly rapid observation-motion of the head, increase in the cervical vertebra and associated muscles would occur; greater and more rapid freedom of movement in the air would result in more perfect innervation between muscles and nerves. Surrounded by a medium that would show more rapid and pronounced stimulation changes than did water, the entire brain would increase in proportion. The anterior or olfactory lobes, that from Rotifera and Turbellaria upward seem to represent the first formed and most anterior sense center, would increase rapidly in size through increased action and reaction. All of these environal changes and results would be gathered up in and 
would affect slowly the main brain vesicles. But, as the important researches of Johnston and Herrick have brought out, it is largely through increase in the olfactory region of the first vesicle, and later the development of this as a center of higher sensory or cognitic, and mental or cogitic, correlation, that commencing growth and steady increase in the cerebral lobes took place. Thus Herrick, speaking mainly of urodele amphibians (162:499) says: "Primitively the evaginated cerebral hemisphere was simply a primary and secondary olfactory center. In very early phylogenetic stages ascending fibers entered this secondary olfactory center from the pars dorsalis thalami for olfacto-tactile correlation, etc., and from the hypothalamus for olfacto-visceral correlations.

"Since the dorso-medial part of the hemisphere is to a less extent under the direct domination of any single one of the functional systems which enter into the cerebral hemispheres, in it the higher correlation tissue was first developed. The preponderating element at first in this pallial correlating apparatus was undoubtedly olfaction. Nevertheless cerebral cortex is not developed under the influence of any single sensory system, no matter how elaborately organized, and it is probable that the primordium hippocampi, even in selachians and amphibians, is concerned with the correlation of all the various types of afferent impulses which reach the cerebral hemispheres in these animals."

Johnston again expresses (163:457) a similar view.

Further, as has been strikingly and perfectly pointed out by Cope, Ryder, Osborn, and others, the whole osseous system-teeth, skull, vertebræ, limbs-would show increasingly perfect modification to suit perfecting response to varied environal stimuli that were passed into the brain centers.

When we examine the very fragmentary record of premammalian fossil remains, and attempt to link these with living types, certain broad results seem to the writer to be indicated. Of existing mammals the marsupials, as already hinted, seem to approach most nearly to an amphibio-mammalian origin. For, from the triassic to the eocene period or even later, pre- 
marsupials and marsupials became increasingly abundant, enlarged in size, and varied in generic detail, till in mid-tertiary times they had spread over North and South America, Europe, and possibly southern Asia and East Africa.

Now, though the very name of the group is derived from the presence in some of a marsupium or pouch for sheltering the young, all morphological and palæontological evidence indicates that such is an acquired, not a primitive, character of the group.- For of the two subdivisions of the family now living, viz., the Polyprotodontia and Diprotodontia, the former is the more ancient and structurally is the most primitive. It is also the group that still extends from North and South America to Australia. Thus in the jurassic age Spalacotherium, Amphilestes, and other genera represent a series of small creatures varying from a mouse to a rat in size, and which from the structure of the jaw and teeth conform to the above group. In more recent strata additional genera occur along with examples of Diprotodontia that seem to have evolved contemporaneously with groups of carnivorous and insectivorous mammals to which reference will later be made.

But, in thinking of a possible connection between such jurassic or even older forms and the urodele Batrachia, the most fundamental question that can concern us is the development of the embryo and its nutritive connection with the parent. While the great majority of the urodeles and all of the reptiles are oviparous, mention has already been made of viviparous salamanders. We may therefore compare the maternal and embryonic relations in the last with the conditions seen in marsupials. In the simplest urodeles like Siren and Proteus the allantoic bladder (urinary bladder) is a narrow elongated sac that opens on the ventral side of the cloaca, but in higher groups it becomes swollen above, or even the swelling may become bilobed. In marsupials the allantoic bladder is comparatively small as in the last, and never expands in any part to form a nutritive and respiratory sac for the embryo, except in the genus Perameles where a true allantoic placenta is formed. This, amongst other characters that will later be referred to, indicates that it is from one of the primitive polyprotodonts that most, though not all, characters have evolved. 
Alike in urodeles and marsupials the rectal and urino-genital canals open into a definite cloaca, though in the latter, as in embryonic higher mammals, it may be shallow or reduced to a common circular fold of integument. In the urodeles and marsupials the oviducts and uteri are paired structures, which in the former open into the cloaca. Now in Salamandra maculosa the eggs are fertilized in the upper region of the oviducts, but then descend into the uterus and there undergo development. The resulting embryos feed on the egg-yolk, but later absorb nourishment through the wall of the uterus, and may undergo gestation for about ten months. The egg capsules with enclosed embryos are then ejected, when the perfect viviparous animal in each at once escapes. In S. atra an even more intimate relation exists between egg, embryo, and the parent uterus, for the several eggs passed from the oviducts into the uterus are gradually absorbed by one or at most two that survive. These develop rich red vascular gills in time, that grow closely against the red richly vascular uterus of the parent. Nutritive and respiratory interchange are thus effected between parent and embryo, till the latter has become fully developed. The former species then might be said to have a uterine placenta, the latter a branchial placenta.

But in marsupials the usually solitary egg passes down from an oviduct into the uterus. Here "the outer covering of the ovum or false chorion is free from persistent villi, and not adherent to the epithelium of the uterine walls; for, although fitting into the folds of the latter, it is perfectly and readily separable in its entire extent from them. The umbilical vesicle or yolk sac is large, vascular, and adherent to a considerable portion of the false chorion or subzonal membrane, while the allantois is relatively small, and, although the usual blood vessels can be traced into it, it does not appear to contract any connection with the false chorion, and therefore much less with the walls of the uterus, of such a nature as to constitute a placenta. In other forms however, such as the opossums, the umbilical vesicle or yolk sac develops temporary villi, which unite with the subzonal membrane, or false chorion, to form a disk-like area closely attached to the cells covering the utricular glands of the uterine epithelium and thus forming a so-called yolk sac placenta. The function of this organ is considered to be the transmission of the secretions of the utricular glands to the embryo by means of the umbilical vescicle; the function of the allantois being either respiratory or the absorption of the fluid secreted in the uterine carity by the utricular glands" (164: 78). 
But "in Perameles obesula a further approach towards the formation of a true allantoic placenta is seen, the allantois giving rise to small vascular villi. In most marsupials the allantois serves merely as a urinary reservoir, and in none of them does it possess any important function as an organ of nutrition, the young being born at a relatively early stage" (138: 338).

It will thus be seen that graded stages in viviparous embryology can be traced from a simple collateral uterine and egg wall-absorptive relation (uterine placenta), through an at first similar but later collateral uterine and branchial wall connection (branchial placenta), on to a collateral utricular-gland -iterine epithelium and yolk-sac union (yolk-sac placenta) and finally reaching a more or less intimate villary allantoic union between parent and embryo (allantoic placenta), from which the transition to the most advanced condition of this, as seen in higher mammals, is easy. So vivipary and easy transition, from simple uterine development of many or two or one embryo, to vivipary and uterine allantoic development of an embryo in mammals, can be clearly traced in our suggested line of ascent. Finally that the allantoic blood vessels are branches of the pelvic arteries that ramify over the batrachian bladder, and from it over the developing allantois, seems an added anatomical feature of some value.

But the amnion that is so precocious and striking a feature of reptiles, birds, and mammals is wholly absent from urodeles and other batrachians. This however is an embryological character that may have arisen in early permian times, as various means of attaching and feeding the embryo were evolved. Stages in its evolving history are as yet entirely unknown.

Still another mammalian structure that we can only vaguely trace or suggest the origin of is the mammary gland. But it should be remembered that the urodele skin is very richly supplied with sunken epidermal glands. Now, if forms derived from these produced more and more feeble and dependent viviparous embryos, these might have nestled against the venter of the parent which may have curled round them. The coiling of the parent round the eggs, up to hatching period, in Ichthyophis and IIypogeophis amongst the Apoda, is suggestive. By constant sucking on the part of the embryo and stimulation then of the adjoining ventral surface of the mother by the imperfect but viviparous young, some of the abundant 
sweat and mucous glands along either ventro-lateral side may have deepened, enlarged, and changed more and more the secretion from a mucous to a lacteal condition. It may even be that the large "Körnchendrusen" of Engelmann which occur along the center and sides of higher urodeles, which contain a milky-like secretion, and which in living forms seem to be a poisonous excretory and defensive substance, may have had its secretion primitively different or gradually changed, so as to utilize it for nourishment of the young. Such structures, alike morphologically and physiologically, furnish a basis and starting point amongst urodeles for mammary glands, such as we have no trace or suggestion of amongst reptiles.

In spite therefore of many apparent points of morphological contact between reptiles and mammals, we would strongly second Huxley's position, that it is amongst the urodele Batrachia that we have to look for progenitors of the primitive mammals. The latter we will now study somewhat in detail.

As Cope, Osborn, and others have emphasized, the primitive marsupials were on the whole generalized types, from which higher and specializing groups have eridently started. In addition to features already observed while comparing the above groups, and which eminently favor the riew that the marsupials are the starting point for at least most of the mammals, the following comparative details deserve special attention:

When the still immature embryos are transferred to the maternal teats they develop a sucking mouth that at once reminds one of the like organ in many larval stages of batrachians, of the larval mouth pads of Amblystoma, the oral attaching cirri in Triton, Spelerpes, and other genera, the suctorial disk of cyclostomes, and the labial expanse of many nemerteans.

As regards the mamma, the following seems a likely riew as to their number and distribution. If in ancient viviparous types, that conformed somewhat to Salamandra maculosa, several eggs matured in the uterus up to a certain stage, and were then set free to be retained along the curled-together abdomen of the mother, the sucking action of these might 
have started a double series of mammary glands, as response to the right-and-left-side coiled condition of the parent at different hours. Fifteen to twenty pairs of these may have formed, according to the nursing vigor of the parent, and natural selection acting in connection therewith.

Now at the present day one of the ancient marsupial groups, alike as judged by distribution and structure, is that of the opossums, which may have from 13 to 4 pairs of teats. The teeth are numerous, usually 50 to 52. There may be no pouch, or it may be imperfectly suggested by two lateral folds of skin parallel to the teats, or as in the Virginian opossum it may be a true pouch. The genus Myrmecobius has 4 to 2 pairs of teats, the teeth are 50 to 54 in number, and there is no pouch though "a tract of skin shows indications of a pouchlike structure" (166:154). Both of the above belong to the Polyprotodonta, or more primitive and ancient group (p. 490) as we would view it.

In Sminthopsis, Peragale, Perameles, and Chœropus belonging to the same group there are four to five, rarely three, pairs of teats, the teeth vary from 46 to 48 , and the pouch is developed but opens backwardly. These however seem to be derivative from forms like Phascologale, in which there may be three to five pairs of teats, a like number of teeth as above, but in which the only beginning of a pouch is a mere skin-fold.

In Thylacinus - a highly modified carnivorous polyprotodont - the teats are in 2 pairs, the teeth are 46 , and there is a backwardly opening pouch. Of other polyprotodonts Dasyuroides and one or two other genera have either no pouch or it is represented by slight lateral folds.

From evidence of fossils it can correctly be said that the polyprotodonts were the world-wide and dominant marsupials of late cretaceous or early eocene time, while the second group of the diprotodonts were largely, perhaps wholly, australopatagonian, of later appearance, and of more specialized structure in nearly every important detail. Of these it may be said that the teats are nearly always two pairs, in Phascolartos even one pair. The teeth are on the average 38 if we take account of species, but they vary from 40 through 36 to 24 in Phascolomys and even 20 in the nectar- and insect-feeding genus Tarsius. A complete pouch in these is nearly always formed.

From the above and similar abundant evidence that might be adduced, the writer would consider that the polyprotodonts 
formed the primitive group of small mammals most of which, like some of the present day, varied in size from a small mouse to a rat. In line with such evidence as Matthew has brought forward (161: 811), some of these took to an arboreal existence, but as indicated later they modified along many lines of response to varied environment. The entire group however seems to have represented amphibian derivatives of permian age, whose remains are almost wholly unknown to us, at least as yet.

During late permian or early triassic times an extensive land connection seems to have formed from Central and South America across the southern hemisphere to Australia, and which permitted eastward migration of many plant genera like Araucaria, Myrsiphyllum, and others. Simultaneously also probably, specializing polyprotodonts, some of which were developing diprotodont habits, spread into Australia and there became an isolated zoological island from the time of the later cretaceous and early eocene. But connections of the southern land, probably by Central America with northern Africa and southern Europe, permitted Conolestes and the opossums to work north and again east, so that we have fossil remains of a myrmecobius-like form in southwestern Europe, and the living representative in western Australia; while the eocene and lower miocene Peratherium or Didelphys of Europe tells how widespread were and are the opossums.

The marsupial migrants from America and outside parts to Australia were evidently cut off from contact with the extraaustralian area in late cretaceous or early eocene times, and so alongside more primitive polyprotodonts that have largely retained primitive characters, like Myrmecobins, Peragale, and Phascologale, others have evolved by condensation and specialization into diprotodont groups. For apart from ('onolestes of Equador, and the extinct Epanorthida of Patagonia that represent a group intermediate between the polyprotodonts and diprotodonts, the latter seem largely, if not wholly, to have evolved in the australian area.

In addition to specializing characters already giren for the latter, it might also shortly be indicated that the syndactyly 
of the digits, and their tendency to condensation, as well as the relatively more convolute brains, are all evolving features along a definite line.

We might sum up our position at this stage then by considering that higher urodele amphibians, during the permian period, probably evolved into purely land animals of small size and promarsupial structure; that during the triassic period these increased in size, in number, and in distribution, until by the close of the cretaceous they had spread over an extensive southern continent, and northeastward through America into Europe. Their main line of advance however was into the australian area that received many polyprotodont as well as evolving diprotodont types, and after separation of that area during late eocene or early miocene times they there attained highest development along both lines.

A morphological and phylogenetic question can now be studied that has long appeared to the writer as one of the most remarkable and suggestive in the entire range of vertebrate history, though paralleled largely by the connection indicated in this work between the Rotifera and several other invertebrate groups. We have already shortly alluded to it, and it may now be presented as follows. If the marsupials are the oldest of the true mammals - and all palæontological evidence fortifies this-, if further they gave origin to succeeding groups-and facts seem clearly to point in this direction, then in process of evolution they gave rise to two ascending but divergent evolutionary lines. These are: First, a typically marsupial line, which while retaining inflected lower jaw, epipubic bones, double uteri and vagina, rudimentary cloaca, immature foetus, etc., has still branched out in eight or ten variational directions that have given us an edentate, an ungulate, a carnivorous, a creodont, a rodent, an insectivore, a dermopterous, and a lemuroid simulation in many structural details and equally in environal relations. Second, an apomarsupial line also arose, which gradually departed from marsupial characteristics, and assumed new peculiarities of structure, such as a non-inflected lower jaw, rudimentary 
or no epipubic bones, single uterus and vagina, functional allantoic placenta, well-formed young, etc. This line however split up into exactly similar forms in morphological detail and environal relation to those of the marsupial line, except that one of these, passing through and including the true lemurs, culminated in the anthropoid apes and in man.

Now some extremely exacting taxonomic zoologist who regards the presence or absence of a rib process, a muscle, a nerve, a skull bone, as a matter of fundamental importance may object to these two morphological lines as involving any feature of cardinal importance. But we would view the whole as proof that, amongst the small primitive marsupials of triassic age, there were focussed up all the past hereditary tendencies of ancestral forms, as well as certain lines of easiest energy flow, food distribution, respiratory repair, reproductive modification, that gave surprisingly similar organization when varied and more or less parallel environal stimuli acted, at the same time that certain wide features underwent slow change, in evolution of the higher mammals; while they remained largely unobliterated in the segregated group of the australian area that includes the bulk of existing marsupials.

With such a morphological conception to guide us, some otherwise perplexing structural details are readily explained. For it is extremely difficult otherwise, even by the most liberal use of the homoplastic principle, to explain why the jerboa, hyrax, and shrew, representing three distinct groups, have the typical marsupial character of the dentinal tubules of the teeth being prolonged into the enamel; and why the wombat of the marsupial group has evolved in this detail to the stage of the groups to which the above three types belong.

Again the inflection of the lower jaw, that is typical of marsupials but wanting in Tarsipes, is retained in Otocyon and other higher mammals. The gastric glandular patch of the wombat and koala is accurately continued in the bearer. 'The patterns of the teeth, that are typical of the varied morphological groups of the marsupials - the carnivorous, the rodent, the herbivorous - are often strikingly reproduced in the ecologically corresponding groups higher. 
It is not surprising, therefore, that, over a large part of the world during the jurassic, and on through the cretaceous period, the remains of forms are found that have retained or possibly reverted to some marsupial details in assuming new morphological adaptations, and alongside them others that have largely lost the former details through dominant reaction of the latter adaptations on the life relations of the animal. According to this view therefore, while in one sense living marsupials are a decadent race that are descended from once greatly more abundant, larger, and widely distributed ancestors, in another sense the marsupial group has been a dominant race whose evolving and adaptable mammalian progeny now stocks the earth.

In attempting to follow the phylogenetic history of the higher mammals it seems difficult to trace successfully the progress of the Cetacea, of whose earlier origin even we are still largely ignorant. These and some other groups will therefore be set aside for the present, as unnecessary for our immediate purpose.

While the later jurassic and cretaceous rocks were being deposited, ample time and opportunity were given for the evolution of some polyprotodont mammals of America and of Europe into those rather small essentially carnivorous mammals that have collectively been called the Creodonta, from which again the later typical Carnivora probably sprang. While they have unquestionably lost many of the marsupial characteristics, such statements as follow clearly indicate the drift of opinion. Thus "the curious resemblance of the molars of many of these forms to those of the marsupials may indicate a genetic relationship between the two groups, but on the other hand the presence of a full set of milk teeth, and the absence of palatal vacuities, or of an inflection of the angle of the mandible, sharply distinguishes them from that order" (164: 608). But the discovery of an additional set of rudimentary teeth which never cut the gum in marsupials does away with one of the supposed differences.

Again regarding the creodont Hyanodon Scott has remarked of its skull that it is "somewhat like that of an opossum," 
while "their likeness to the carnivorous marsupials has also been insisted on" (166:455).

While then there seems a direct traceable relation between the Marsupialia and the Carnivora, it is equally true that in the cretaceous and eocene periods like continuity can be traced for the Rodentia, the Insectivora, and the Ungulata. Thus while in Australia the pure and primitive marsupials were branching into physiologically and ecologically distinct groups that retained many marsupial characters, in America and western Europe-probably also over a much wider southern area now submerged - the primitive marsupials became dissolved into physiologically and ecologically dissimilar groups, which however lost the marked marsupial characters in assuming more perfect or more adapted morphological details, that we now associate with the higher mammals.

But, for our immediate purpose, one line of early and apparently direct modification from marsupial ancestry was evidently the lemuroid, representatives of which group have been traced back to the earliest eocene, possibly even to the late cretaceous epoch. Thus Indrodon, Notharctus, and Tomitherium, of the western states, Palcolemur and Hapalemur of western Europe, and Canopithecus of central Europe, all conform to the lemuroid type.

The living phalangerids of Australia most nearly connect marsupial with lemuroid structure. Below is a table setting forth their points of agreement:

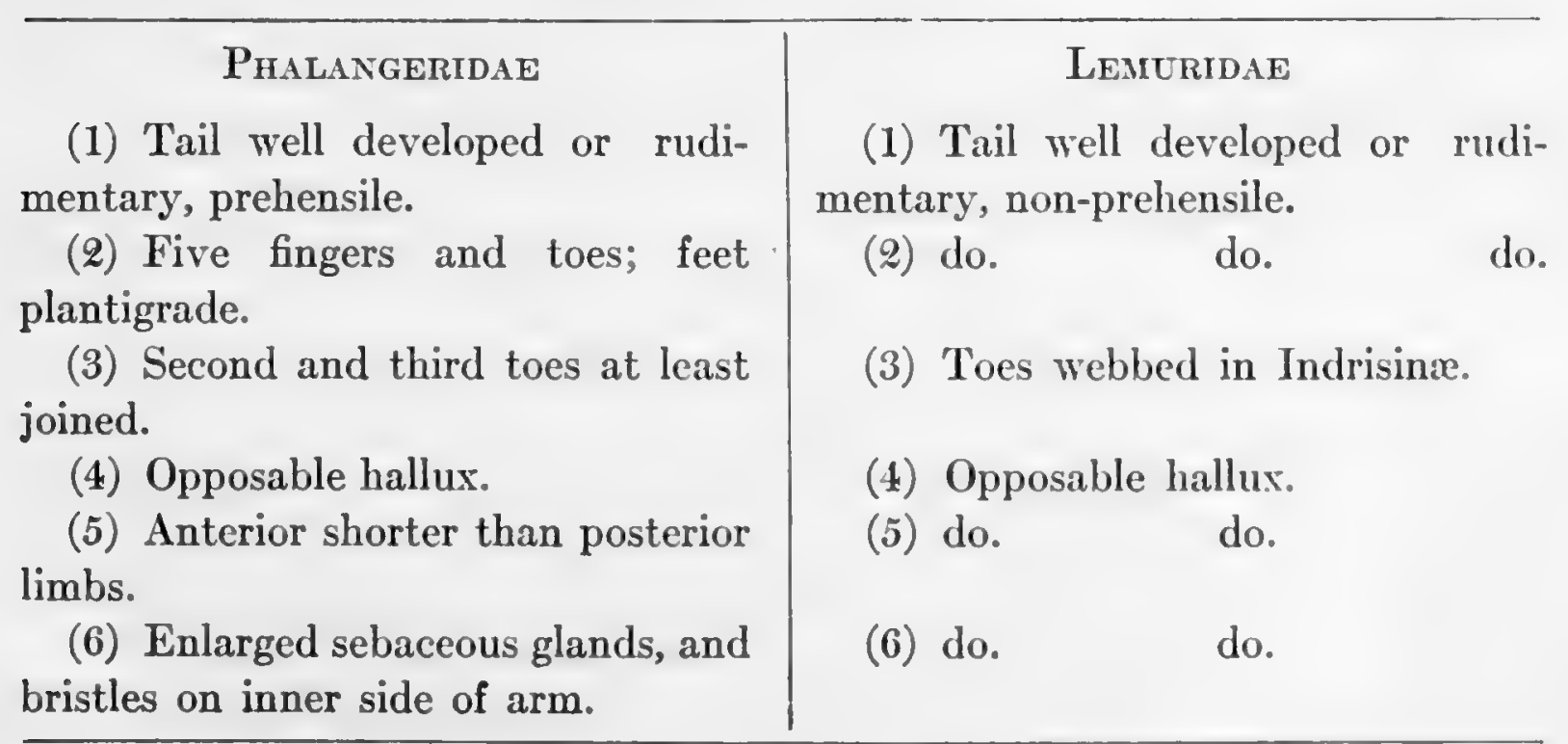




\section{Phalangeridae}

(7) Check pouches rudimentary or developed.

(8) Mammæ typically 4, abdominal.

(9) Teeth $\frac{3}{2}$ or $\frac{3}{3} ; \frac{1}{0} ; \frac{2}{3}$ or $\frac{3}{2} ; \frac{4}{4}$ or $\frac{3}{3}$.

(10) Incisors and canines similar and projecting forward.

(11) Molar teeth tuberculate, with 3-4 cusps.

(12) Palate with posterior fenestræ.

(13) Stomach simple, cœcum often large.

(14) Brain with cerebellum exposed, cerebrum slightly convolute.

\section{LEMURIDAE}

(7) Check pouches absent, developed in apes.

(8) Mammæ 4 to 2, abdominal to pectoral in position. $\frac{3}{3}$.

(9) Teeth $\frac{2}{2}$ or $\frac{2}{3} ; \frac{1}{1}$ or $\frac{1}{0} ; \frac{2}{3}$ or $\frac{3}{2}$;

(10) do.

do.

do.

(11) do.

do. with 3-5 cusps.

(12) do.

do.

do.

(13) do.

do.

do.

(14) do. do. but cerebrum with simian fissure.

If in addition to other general mammalian characters the above existing marsupials and lemurs show so many contact structural details, it might be expected that the above-named fossil genera would even more exactly bridge over the gap. Such they effect in various ways. So, from higher urodeles to the primitive polyprotodont marsupials, from these to the phalangerid diprotodonts, and from these again to protolemuroids, the transition seems fairly continuous and suggestive.

But here we would not insist too closely on direct descent of the lemuroids from phalangerid ancestors. For given certain polyprotodont ancestral characteristics as a common starting point for further evolutionary advance, fairly similar groups of environal factors may have combined to evolve from such common ancestry an arboreal lemuroid line, and a phalangerid line of ascent that simultaneously diverged from each other in certain fundamental structural features.

But Haeckel and Cope have both adrocated the view that the lemurs are very directly related to the higher monkeys. Thus Cope says (73:154): "I have advanced the . . . hypothesis that the Anthropomorpha (which include man and the anthropoid apes) have been derived directly from the 
lemurs, without passing through the monkeys proper. This close association of man with the apes is based on various considerations. One of them is that the skeleton of the anthropoid apes more nearly resembles that of man in the most important respects than it does that of the monkeys. This is especially true of the vertebral column, where the anapophyses are wanting in the Anthropomorpha (insignificant rudiments remaining on one or two vertebræ, as pointed out by Mirart), while they are well developed in the monkeys and lemurs. The molar teeth of the apes and man resemble each other more than do those of the monkeys, since they lack the crests which connect the cusps, which are general in the latter.

"The frequent presence of the tritubercular molar in man suggests the superior claim of the lemurs over the monkeys to the position of ancestor. Another significant fact pointing in the same direction is the existence of large-brained lemurs with a very anthropoid dentition (Anaptomorphidæ) in our eocene beds, which have the dental formula of man and the Old World monkeys and apes." And later (p. 156) he adds: "I now maintain as a working hypothesis that all the Anthropomorpha were descended from the eocene lemuroids."

It is unnecessary in this chapter to do more than refer to the general opinion of naturalists that the genus Homo is not directly derived from any one of the highest living apes, but is descended rather from a form that combined various characters of these in more or less intimate blending, at the same time that steadily evolving characters were superadded to these, which gradually gave him his dominant position.

Summing up then the conclusions indicated in the preceding chapters it may be said that, from a unicellular ciliate infusorian, the main line of progressive evolution has been pursued through a loosely multicellular ciliate type somewhat intermediate between infusorian and rotiferan organisms. From this direct advance has been made through the Rotifera and Rhabdocøela to the Nemertinea, the latter of which, in the fresh-water forms especially, more and more closely foreshadowed and advanced toward future vertebrate organization as strongly advocated by Hubrecht. While such Hemichor- 
data as Balanoglossus and such Cephalochordata as Amphioxus are side derivatives from these of greatly higher organization, the ciliated nemerteans exhibit so many and so detailed points of morphological and physiological contact with the cyclostomes that one can readily construct what has been termed in this work a protocyclostome form. This would serve to connect higher nemerteans with a type pretty closely related to the now scarce-generically-and somewhat degenerate examples of cyclostomes at present alive. The organic gap now existing however is a wide one. From allies of the cyclostomes, that are extensively ciliate in the larval state, to allies of the Apoda, the morphological continuity is again pronounced, and from the latter to simpler urodeles, thence to higher urodeles with ciliate larvæ, the connecting chain is surprisingly clear. (See "family tree," facing p. 474.)

A wide organic gap in the evolving process, that is represented in geological time by the upper carboniferous and permian epochs, again separates the higher urodeles from the primitive marsupials. For the writer decidedly follows Huxley in considering that the true pathway of advance to the primates is to be sought for in batracho-marsupial and marsupio-mammalian affinities. Evidences therefore have been advanced for connecting the marsupials as a primitive but plastic group with the Lemuridx, at the same time that the marsupials served as the starting point for most of the mammalian families. The opinions of Haeckel and of Cope have been accepted, which advocated that extinct types of Lemuridæ made connection with the anthropoid apes, while the latter have been accepted as being in a nearly direct line of ascent to man.

such a trend of evolutionary progress does away entirely with the need for a marine ancestry at any stage in the process. For, even though, in the case of some nemertean and cyclostome features, our descriptions have directly or tacitly pertained to semi-marine or marine forms, such strong evidences exist of the original fresh-water origin of these, and of their probable great abundance in such waters in past geologic time, that we have used the existing marine types as accessory collateral evidence only. 
Another advantage that can be claimed for the above pedigree is that it is at once condensed and fairly continuous in morphological advance and specialization. Balanoglossus, Megalocercus of the tunicates, Amphioxus, and the Reptilia are all of high interest and show us how life modifications have often occurred. But all of them seem to be side developments of minor value alongside the more direct, condensed, and now dominant line.

It follows however from this that, were a diagram made of the zoological tree which would include living and fossil species, while the main trunk would be relatively condensed and low, the lateral branches would be enormously extended and ramified. Next chapter will be devoted to consideration of the connection of the main branches with the stem, and therefrom the zoological tree as above suggested will be constructed.

A striking and far-reaching principle is illustrated in the above history, which may be synopsized in the classic and trite sentence "the race is not to the swift, nor the battle to the strong"; but it is to those who become the most adaptable, the most responsive, the most plastic; in a sentence the most highly, abundantly, and perfectly innervated. So the elongated soft-bodied, ciliated, proboscis-stimulated, or cephalicbrained and delicately skin-nerve-covered organisms have not only survived; they have become the progressive and dominant types. For through constant action of the environment, and reaction of each succeeding organism that formed a link in the long chain of animal life, slow but sure proenvironal outreaching unceasingly occurred, that ensured steady if slow organization and advance in the chain of life.

On the other hand, as not a few biologists of recent decades have emphasized, the mailed, the shelled, the tusked, the carnivorous, the fighting groups have survived for a season, but one after another they have been exterminated amongst themselves, or have failed to adapt themselves to changed physical or biological environment. Softness, plasticity, correlated nervous organization, and adaptability are and have been leading characters in determining organic survival and advance. 


\section{CHAPTER XVIII}

\section{THE PHYLOGENY OF ANIMALS}

In this chapter the writer will endeavor to trace broadly the phylogeny of the animal kingdom, in conformity with riens expressed so far in the foregoing pages. This phylogeny will illustrate four principles that have already been emphasized: first, that animal life, like vegetable life, primarily originated in fresh-water areas; second, that the main line, as well as many lateral lines of animal advance, have long remained amid such surroundings, but gradually adopted a terrestrial life; third, that successive migrations from fresh to salt water have taken place along the entire evolving animal scale, and that the marine immigrants usually developed or further strengthened either a hard, often heavy, calcareous shell or a chitinous investment along with creeping or swimming habit; or became soft, pelagic, and much modified in structural detail; fourth, that all the great invertebrate groups had already been fully established and broadly distributed by the close of the archæan epoch.

Distributional and structural evidence has already been advanced to prove that all of the primitive protozoan groups are now mainly fresh-water in habit, while we have also accepted it that an easy transition from colonial flagellate infusors like Proterospongia to the multicellular or metazoan sponges is clearly indicated. For in sponges a colonial group of cell individuals, formed by repeated division from a single cell individual as in young stages of Proterospongia, has merged into a multicellular organism often of complicated structure. Now Proterospongia and some of the simplest as well as worldwide sponges are fresh-water, and give no indication of ever having had a marine existence. But, while Spongilla-of world-wide distribution - and allies of it are still fresh-water, 
members of the Spongida must, in the early history of the group, have assumed a marine life, so as to leave their remains in the earliest cambrian strata that are of marine origin. In the process the group became an evolutionary side line, and in persisting to the present day skeletal frame works of horn or lime or silica, often of great intricacy, have been built up. But the group failed to evolve higher organisms than those composing it. Here and subsequently reference should be made to the "family tree" facing p. 474 .

A second great phylum - that of the Cœelenterata or Enterocola-includes as its simplest representatives fresh-water genera like Microhydra and Hydra, the latter being world-wide in distribution. The comparatively simple cells that build up both of these genera, and that result from segmentation of one egg-cell as in Spongida, indicate that the beginnings of the group resembled colonial ciliate infusorian cells, in which a colonial protozoan life was replaced by a metazoan individual life. We can best explain the history of the entire group if we consider that descendants of the simple fresh-water genera - which may have existed abundantly during mid-archæan times-migrated seaward, and by a process of continuous budding developed the often large colonies of individuals that now mainly characterize the group. These deposited, in or round themselves, chitinous or calcareous secretions as in many Hydrozoa and Actinozoa, or single individuals became much enlarged and succulent as in some Actinozoa, or evolved reproductive pelagic buds or individuals of watery texture, as in medusiform and lucernarioid examples.

But, for reasons that cannot be enlarged on now, we accept it that these, like the Spongida, became a side line of evolution, and in persisting from the cambrian to the present period have slowly built up reefs and island masses, but have failed to evolve higher organisms than themselves.

A somewhat similar history attaches to the Echinodermata, except that the earliest ancestral members seem to be entirely unknown, and so our existing ones are all derived secondarily from ancient marine organisms, but whether these were in 
turn early evolved from fresh-water types is a question on which no light has yet been shed.

A point is now reached where the palæontological and the zoological records are equally defective. For granting the existence of archran organisms that formed from the single egg cell a morula, or cellular embryonic mass, as in young phases of the Spongida, Colenterata, and Echinodermata, few organisms seem to exist now which connect such with

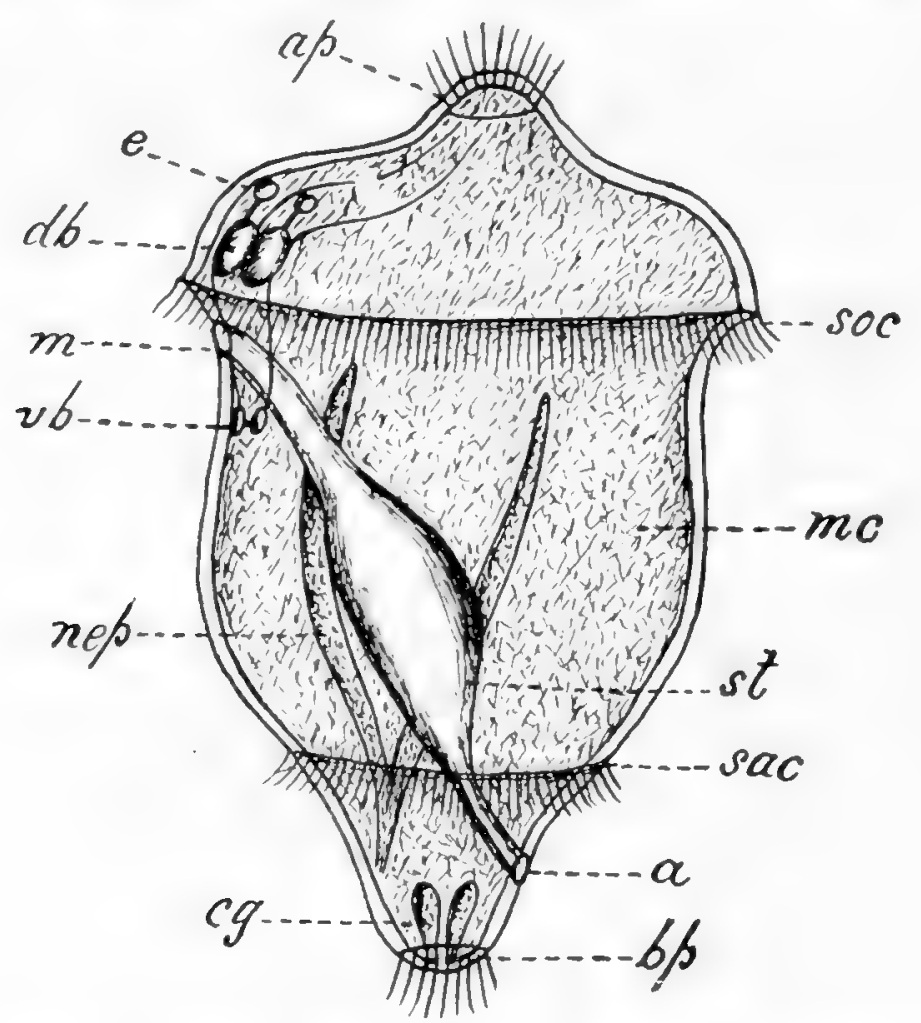

FIG. 20-Diagram of Rotifer after Zelinka: ap apical plate or area; $b p$ basal do.; soc anterior ciliated ring; sac posterior do.; $m$ mouth; $a$ anus of alimentary canal; $a b$ dorsal brain or cerebral ganglion; $i b$ ventral do.; $e$ eyes; $n c p$ nephridia; $c g$ cement glands.

those higher and more complex alliances that evidently represent the main line, as well as many collateral or diverging lines of ascent, like the Rotifera, the Turbellaria, and the Polyzoa, not to name higher classes.

But larval or developmental stages of the higher classes seem clearly to point the way backward toward the Rotifera as a great basic group, from which have sprung most of the dominant classes now existing. As leading up to this it may be said that zoologists are now largely agreed in regarding the so-called trochophore stage of many large phyla or branches of invertebrate animals as representative temporarily of what 
once must have been a widespread set of organisms. A generalized illustration of such is given in Fig. 20. This probably was primitively a holociliate organism whose entire surface was stimulotactic. But, through repeated and continued stimulation in relation to the earth below, to the light above, and to the surrounding medium from which food and other materials were derived, the sensory cilia became largely or wholly restricted to a lower or geotactic patch, an upper or heliotactic patch, and one or two circlets above and below the lines of the alimentary and excretory apparatus. Such is the example shown in the figure, and which is typical of many adult Rotifera, of larval stages of Annelida, Brachiopoda, Mollusca, etc.

Mention in last sentence of "adult Rotifera" as closely resembling larval trochophores of more highly developed invertebrates might suggest the view that the class Rotifera ranks amongst the most primitive, if it is not the most primitive, of the great classes from which the higher invertebrates and the vertebrates have sprung. This view we propose now to open up.

Many zoologists, during the past quarter century, such as Hatschek, Zelinka, Korschelt, and others, have remarked on the striking structural resemblance of many rotifers to the trochophore or the still more advanced veliger stage of molluscs, to the trochophore of annelidan worms, or to the like stage in the history of Polyzoa. But we believe that an even closer and also wider phylogenetic connection exists than has hitherto been suspected, between different genera of rotifers and the higher classes of invertebrates. In now attempting to trace this a helpful explanation may be furnished of many distributional problems that have hitherto seemed inexplicable.

As already explained (p. 415) the Rotifera are, and seem to have been, of fresh-water origin, derived probably from a colonial ciliate infusorian ancestry. Their eggs after fertilization probably gave origin to a multicellular organism, whose cells either by epiboly or invagination assumed different positions and took on different functions. Such a history is recapitulated in the development of each adult rotifer. The great majority as already shown (p. 387) are fresh-water, a few show considerable adaptability to fresh-water and marine life, relatively few are purely marine.

A generalized picture of an individual shows, as in a trochophore larva, three areas that make up the body mass, viz.: an apical or cephalic, a somatic, and a basal. But tendency to constriction into minor areas, especially of the two latter regions, may occur. The apical area may be uniformly cili- 
ated, or more commonly there is a central or terminal patch or circlet of cilia, or cilia and bristles, that form the so-called trochal disc; while around the widened margin is a circlet of cilia. The region thus demarcated may produce two pairs of sensitive antennæ, a dorsally and a subventrally or ventrally placed pair, or one pair may be absent or absorbed, or the members of each pair may show all stages of fusion. They are often jointed, they usually bear one or more bristles of a tactile nature, and they may be capable of protrusion and retraction. Between and usually near these, one to two patches of surface cells early sink in from the surface to form cerebral ganglia, while two, three, or four, rarely more, eye-spots are formed above or on either side of the ganglia, and receive separate or fused nerves from them.

The mouth opens beside the marginal circlet of cilia, and toward the ventral surface of the organism, which very often swims or explores the muddy bottom of its territory by keeping the oral side toward it, so that the opposite side becomes a dorsal region. The mouth passes into a gullet or pharynx that is protrusible at times, and in this are chitinous teeth arranged in definite fashion, as will be emphasized afterwards. The oesophagus often has paired salivary glands opening into it, and then enlarges below into the stomach. The alimentary canal next receives in some forms secretions from two large glands that may be biliary in function, and then passing backward bends slightly upward to open as an anus, or it may, in tube-forming genera like Melicerta, bend into a dorsal loop which ends in an antero-dorsal anus.

The ventral area below and in front of the anus usually becomes a jointed attachable foot, or may have a ciliate rim, or be expanded into a disc, while paired "cement glands" open at its extremity. These glands can secrete an adhesive cement, or a glutinous exudation that is gradually poured out as an investment which enables many species to resist drying, heating, or cooling for months or years. Primitive kidneys take the form of two longitudinal tubes with or without branches, and that open into a cloaca or bladder posteriorly, but in their inner end openings are provided with "flame-cells" of excretory nature. The diøecious reproductive organs consist of ovary, yolk gland, and oviduct in the female, and of testis, seminal vesicle, and penis in the male. A seemingly degraded and derived condition in the adult is the semi-obliteration of division walls between the cells throughout, but this probably represents a loose separation of the cell protoplasms, derived hereditarily from an infusorian ancestry. 
We have thus recapitulated details such as can be got in any zoological manual, in order later the more readily to refer intelligently to minuter details that genera or species show, and which may indicate points of contact with other, higher, and, as we would view it, derived groups.

With such details before us we believe that evolutionary progression and descent can be readily traced to the Turbellaria, the Nemertinea, the Annelida, the Polyzoa, the Brachiopoda, and the Mollusca, while we will refer finally to clear affinities with the Arthropoda, such as Gosse claimed fully a quarter century ago. In other words, the Rotifera present us with a highly generalized and primitive group from which all of the above may be derived, and which indicate their ancestry by the temporary larval rotifer-like trochophore or veliger stage that they still pass through.

But of the above large groups the one that, in its smallest and simplest members, shows close affinity to the Rotifera, though in its largest and most complicated genera advances far beyond these, is that of the Turbellaria. Comparison of the structure of the smaller rhabdocœl turbellarians with the Rotifera and with the trochophore of higher classes suggests to us what may have been a common and simpler type from which all have arisen, or in other words, a primitive rotifer. This was in all probability holociliate, faintly divisible into an apical, a median, and a basal region, had a simple blind alimentary canal (as have many male rotifers still, though owing apparently to degeneration), without or to a slight extent with accessory glands, with simple eye-spots that were formed on or near epidermal invaginations that became at first separate and later fused ganglia, while outgrowths formed from the last that became nerve threads.

Every detail of this composite picture can even now be followed in rotifers or in fresh-water rhabdocœl turbellarians. But all known analogy suggests that during early epochs, and most abundantly probably during later mid-archaean times, organisms swarmed in the warm stimulating fresh waters of that period that were similar to the above, and which contributed gradually to the evolution of higher types than they. 
But by retention of the cilia over the body surface, by continued elongation of the body, and correlatively by elongation of the nerve fibres in it, also by formation of an increasingly evident body cavity, organisms must have evolved that differed little from typical rhabdocœl turbellarians; while, by restriction of the cilia to localized areas, by condensation of the body substance into an oval active mass, and by formation mainly of muscular body tissue, other organisms evolved into typical rotifers.

In subsequent history the turbellarians in large measure branched off into a side line of development, in which some offshoots by migration on to land or into the sea produced there the increasingly complicated and enlarged triclad and polyclad divisions of the series; while others, adopting a parasitic life mainly in fresh water or within land animals, have evolved into the trematode and cestode series of organisms. But most of the fresh-water as well as the marine and parasitic derivatives from the primitive stock seem all to have failed in the evolution of higher groups than themselves.

One division of the Turbellaria however-the typical Rhabdocola-deserves special mention, for even living fresh-water representatives of it like Dalyellia, Mesostoma, and Prorhynchus conduct us, from small forms allied in structure to the Rotifera, upward to organisms with close affinity to the Nemertinea. This last group has already been treated in detail as being in the direct line of evolution with the Chordata (p. 450).

A considerable number of the living Rhabdocœela are freshwater, and these we would regard as still inhabiting ancestral environment. Others have assumed a marine habitat, and have there ramified into diverse genera. So we would give a totally different interpretation, than have some naturalists, to the occurrence of species of Plagiostoma. The genus is made up mainly of marine representatives, but the species $P$. lemani occurs at varying depths in the Lake of Geneva, as well as northward into Russia, along with species of allied genera. These we would regard as very ancient and primitively fresh-water species, from which the marine species have been derived.

As to the affinities then of the Rhabdocœla, we would regard them as intermediate in structure between the more elongate Rotifera like Notommata and Taphrocampa on the one hand, and the Nemertinea on the other. Thus, in the oval to linear shape, in the dorso-ventral orientation, in the secretion of mucus from ventral cement glands, in the simple alimentary canal, in the sucking protrusible pharynx, in the presence of 
longitudinal muscles amid a colomic fluid, in the formation of cerebral and smaller subœsophageal ganglia as well as longitudinal nerves, in the origin of simple eyes adjacent to the cerebral ganglia, in the structure of the primitive kidneys with their excretory tubes, and in the constituent parts of the reproductive system, points of exact morphological contact are made between all of the above named three divisions of invertebrates. We cannot share the opinions of those writers therefore who trace the Turbellaria back to a ctenophoran and so coelenterate ancestry.

Now all of the organisms hitherto reviewed as belonging to the Rotifera, Turbellaria, and Nemertinea are soft-bodied, and so easily perishable. Therefore, even though they may have swarmed in fresh-water or marine areas of archæan age, no trace would be left of them. So during evolution of the entire protozoan assemblage we would expect no fossil remains till rhizopodan derivatives like the Foraminifera and Radiolaria had elaborated mineral tests; nor of the lower metazoa would we expect remains till simple siliceous-more rarely calcareous-sponges began to deposit spicules in their tissues; nor of the lower Coelenterata would we expect remains till chitinous and calcareous investments or deposits had begun to form as in marine derivatives from fresh-water hydroids, like the graptolites and corals of cambrian deposits; nor of the Rotifera, Turbellaria, and Nemertinea could other than the minute chitinous teeth have a chance of survival in fossil form.

For millions of years, therefore, organismal activity and evolution may well have proceeded without the possibility or likelihood of even faint indication from the palæontological side. Yet during this period biotic, cognitic, and even cogitic energy may have combined to elaborate organic structures, that became the wide foundations on which an enduring superstructure could be reared.

But we shall now attempt to trace how the primitive Rotifera became prolific originators of numerous higher groups than themselves.

From rotiferan ancestry the Archiannelida and Annelida evidently originated, as Hatschek, Zelinka, and Korschelt amongst others have indicated. Though Dinophilus is a marine coastal genus, it so perfectly unites structural details of both groups that we may well believe it to have been derived from a more primitive fresh-water rotiferan type, in common with primitive microdriloid worms, or it even may have become modified from an ancient marine rotifer. Now an outstanding 
feature of the Annelida (Chætophora) is the presence of chætæ or bristles, on some or most of the segments of the body. So, if the Annelida have had a rotiferan ancestry, we should probably be able to trace in some of the latter both annulation and suggested segmentation of the body, as well as the rudiments of chætæ. As to the former we need only cite Notommata pilarius and aurita, Taphrocampa annulosa and Albertia intrusor or naidis to prove that alike annulation and commencing segmentation are exhibited in several genera of rotifers (fig. 21). Setæ, typical of the cephalic tentacles of many rotifers, are often elongated and aggregated into patches of two to five. But further, in Copeus and the Triarthrida, spines or groups of spines, some of which greatly recall those of the Annelida, are set into the body wall as genuine chætæ, and are moved by appropriate muscles.

Again in the evolution of the Annelida from the Rotifera, we might expect still to find rather advanced larval annelidan stages that would aid us in bridging over the gap which may seem now to separate the two. One such from amongst many is presented in the larva of Eupomatus which we compare in the adjoining page (Fig. $21 \mathrm{a}, \mathrm{b}, \mathrm{c}$ ) with a compounded diagram of Pterodina from the Rotifera, and with Polygordius from the Annelida. These speak for themselves. But an important change passed through in this evolution process must have been the retention of rows of cilia, left from a primitively holotrichous condition, at the same time that these were retained across areas of advancing segmentation. Alike in the mature animal Dinophilus, and in the larva of the annelidan Ophryotrocha puerilis figured (Fig. $21 \mathrm{e}$ ), the so-called telotrochal larva, typical of Mysostoma and of the adult rotifer compounded above, has become a polytrochal type by retention of the ciliary rows and commencing segmentation. But neither in Dinophilus nor in the larva are setæ as yet observed. Segmentation from before backward is then gradually established, as pointed out by Korschelt.

Thus there arises a polysegmental condition, which in many points still largely retains rotiferan characters. Such exists now in that simple group of fresh-water annelids, the Aphaneura, which A. Sedgwick has suggested should be placed amongst the Archiannelida. It along with Dinophilus suggests that many genera once existed which formed graded and perfect transitions from primitive rotiferan and trochophore types to planarian worms on the one hand, and to highly segmented annelidans on the other. 

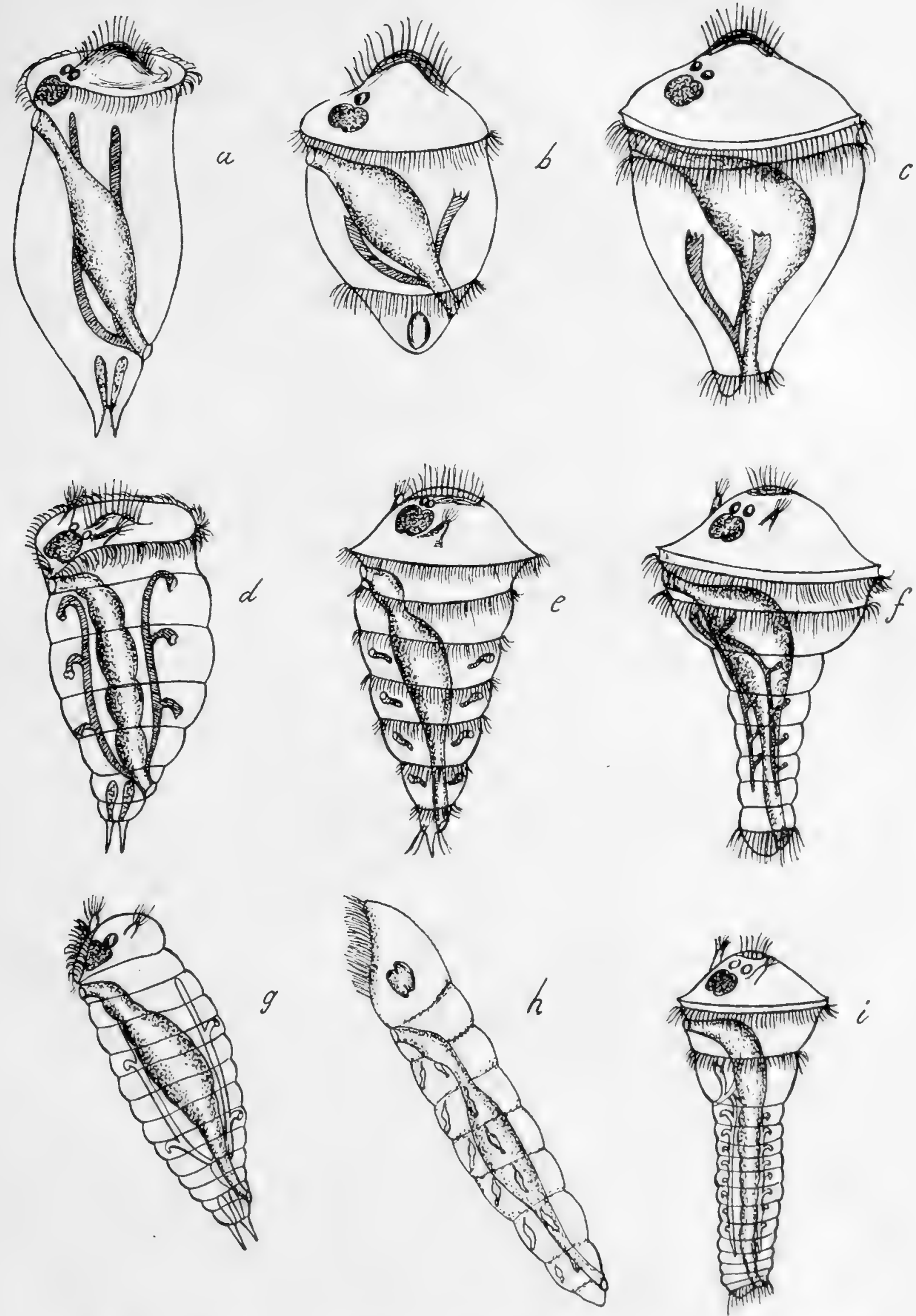

Fig. 21.-Relation of Rotifera to Annelida. a, simple Rotifer; b, young or trochophore stage of Annelid; c, trochophore stage of Polygordius: d, segmented Rotifer Nolommata; e, polytrochal larva of the annelid Ophryotrocha; $f$, polytrochal stage of Polygordius; $g$, compounded rotiferan diagram of AlhertiaTaphrocampa; $h$, embryonic stage of Acolosoma; $i$, third stage of Polyjordius. 
The remarkable organism Stylochota fusiformis that was first described as "a new Rotifer" under the name Polyarthra fusiformis (167:59) is a type that strikingly combines rotiferan, gastrotrichan and chætophoran characteristics; though most nearly conforming to the second of these groups, as Hlava has shown (168: 247$)$.

The seven fresh-water species of Eolosoma belonging to the Aphaneura show a ciliated prostomium that corresponds to the trochal disc in Rotifera (Fig. $21 \mathrm{~g}, \mathrm{~h}$ ); a delicate chitinous layer that suggests the similar layer of the latter; setæ that are delicate and embedded in epidermal pouches as in the rotifer Copeus, though here they have become numerous and segmentally disposed; a secretion of copious mucus at definite periods in the life history; an alimentary canal that is complete and simple; perfect segmentation indicated only in the anterior area as in some rotifers; nerve ganglia of equally primitive state as in rotifers; simple excretory tubes that show structural similarity to those of Dinophilus and to Rotifera; while as in many of the latter the secreted mucus can be fashioned into a conserving coccoon, inside which the animal can survive adverse environal changes.

But, apart from Dinophilus, other of the Archiannelida such as Polygordius suggest remarkable agreement with some rotifers. In Figs. $21 \mathrm{~g}$ and $21 \mathrm{i}$ are shown the proto-annelidan stage of Polygordius, and a compounded view of AlbertiaTaphrocampa prepared from Hudson and Gosse's beautiful illustrations.

The Annelida or Chrtophora then we would regard as common descendants with the Platyelmia from fresh-water rotifers. Very early they seem to have split up into a series that remained fresh-water or in time became land dwellers, such as the aquatic or terrestrial oligochætes or "earthworms"; also into a group that migrated shorewards and there became the polychæe or marine worms. Such migration may have taken place while the migrants were still more nearly rotiferan than annelidan in structural detail. But the few connecting types now left to us make more exact decision impossible.

That even the highly modified and greatly more complex polychrte Nereis is largely molded on a rotiferan basis is abundantly shown by comparison. For in both we note the soft flexible dorsiventral body, the prooral region that in the larval 
or adult state is ciliate, the presence of four antennæ two of which in Nereis (as in some rotifers) have become palps, the accessory peristomal setæ, the ventral mouth and posterior subdorsal or dorsal anus, a protrusible chitinous pharynx which in rotifers bears the paired plates or mastax teeth that seem to have become the jaws in Nereis, the accessory masticatory plates of rotifers that seem to correspond to the paragnaths of Nereis and other polychæts, an œesophagus with paired salivary glands, a brain in the præoral region that gives off nerve threads to the antennæ and the eyes, the four eyes that in some rotifers and in Nereis occupy similar positions and are developed in fundamentally similar manner, an excretory system that in both shows like structure to start with, but which in adult polychæts like Nereis is greatly advanced on, cement glands of rotifers that suggest exact correlation with the parapodial glands of Nereis, and the formation in Nereis, as in many rotifers at certain seasons, of gelatinous investments from the cement glands inside which both animals survive.

Fundamentally the Annelida have advanced beyond the Rotifera, in increased development of the body tissues, in formation of body segments and of added setæ for these, in the evolution of a blood-vascular system, and in increased complexity of the excretory system. But the evolutionary continuity of members of both groups seems evident.

Affinities of the Polyzoa with the Rotifera have been repeatedly suggested by zoologists, since the polyzoan larva passes through a distinct trochophore stage. Some are soft-bodied, and so would leave no fossil remains. These at the present day are mainly fresh-water, others are calcified, and so have left abundant remains from the base of the silurian rocks up)ward. The latter are wholly marine. A few from both divisions are solitary animals, but the great majority have, by continuous budding, gradually evolved an extensive colonial system. For the solitary state is naturally the more primitive, the colonial is more evolved.

The changes which result, on fixation of the previously free-swimming larva, as set forth diagrammatically in figs. 20, a-e, are in all cases so fundamental, and are further complicated in most by extensive budding even in the larval state, that the relation of the Polyzoa to the Rotifera has often been 
overlooked. The class has generally been divided into the Entoprocta that has been regarded as the more primitive, and the Ectoprocta that is greatly modified in the mature state and somewhat even in the trochophore larva. As already stated (p. 391) we incline to reverse this view. The former includes three existing genera, of which one Urnatella is fresh-water, while Loxosoma and Pedicellina are marine.
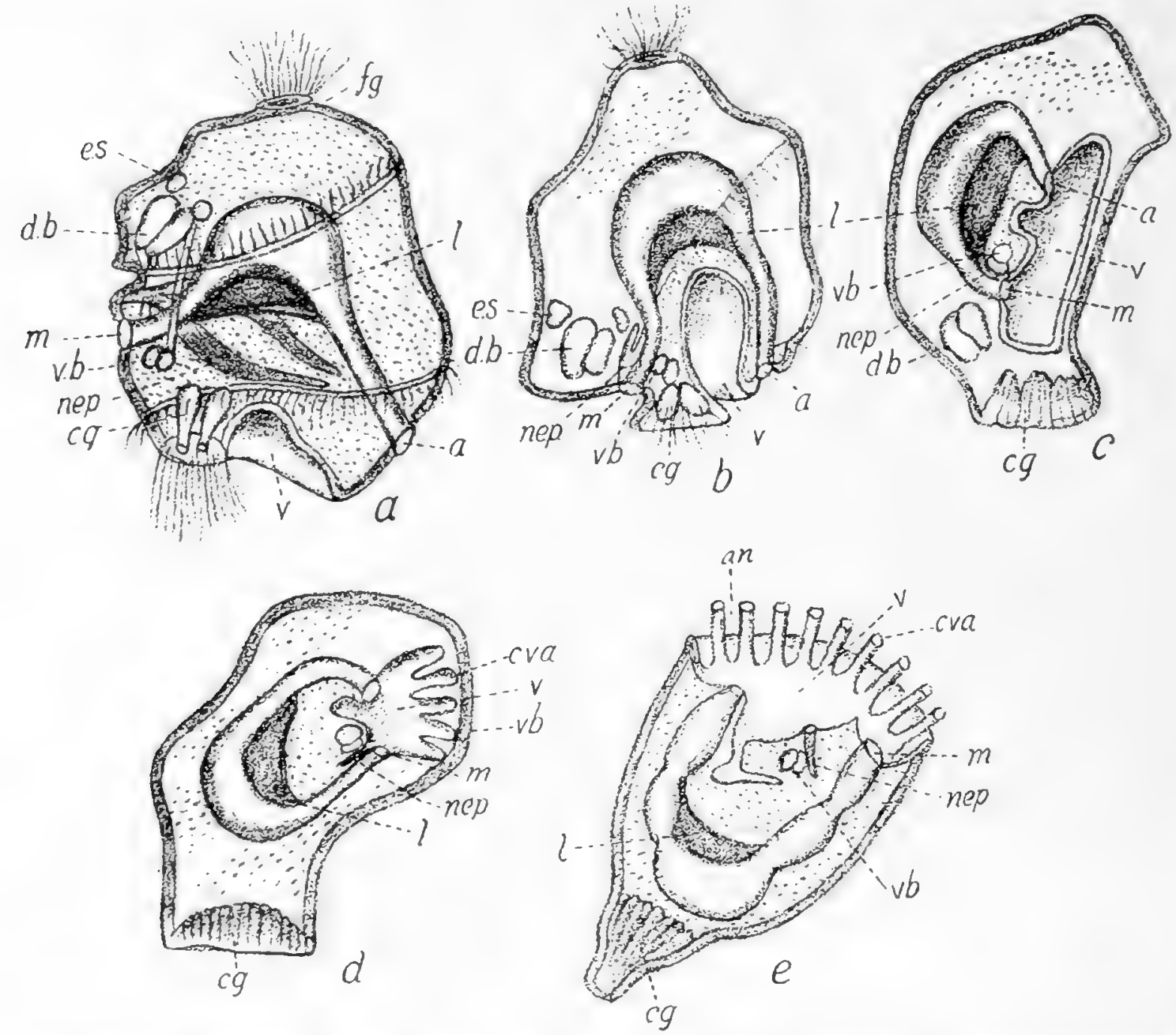

Fig. 22.-a, trochophore stage of Pedicellina; $b-e$, stages in development of marine polypide of Polyzoa; e.s., eyes; d.b., dorsal brain; v.b., ventral brain; $m$, mouth; $a$, anus; $v$, ventral area; nep, nephridia; $c g$, cement glands; $c v a$, circle of tentacles.

In Fig. 22a is shown in normal position the somewhat advanced trochophore stage of Pedicellina as given by Hatschek, which unfortunately in many text-books is represented as standing or floating on its head. The area in front of the præoral ciliary ring corresponds to the trochal disc of Rotifera, and here has been called the "ciliated disc." As in rotifers "the larva of Pedicellina, when swimming, always carries this organ directed forward." In front of it, and similarly derived as in rotifers according to Harmer, is the_dorsal brain- 
mass; above this, in Loxosoma as in some rotifers, a pair of simple, but in this instance temporary, eyes arise. Below the ciliated ring, and ventrally placed, is the mouth. The ventral area between it and the foot (v) is greatly shortened; the alimentary canal has become bent and opens well forward on the restricted dorsal surface; while the supposed excretory tubes of Verworn and Cori occupy appropriate position for such, though greatly reduced in size. This however is to be expected, when the capacity for excretion alike by the arms and the atrium, as well as the sluggish character of the adult, are considered.

The so-called Cyphonautes embryo of many marine genera undergoes striking change owing to enlarged forward growth of the supra- or epi-oral region, by ingrowth and then deep invagination of the atrium, and by transfer of the alimentary canal in the process, while the mouth and anus both open into the atrium (Fig. 22 $b-e$.)

A study of the forms of polyzoan embryo therefore shows that all evidently started from a common ancestry with the Rotifera, and that gradual but striking modification has been effected by slow evolutionary processes. Such simple and soft-bodied forms as branched off from older rotiferan ancestors continued mainly if not wholly amid fresh-water surroundings. Remnants of these are seen in Irnatella amongst Entoprocta, and in the phylactolæmids amongst Ectoprocta. By degrees adopting the colonial habit, they seem to have migrated or been carried seaward, probably during late archæan times. There by chitinous growth, or by secretion of lime salts from the ocean, as with the seaward migrating Spongida and Coelenterata, they had by silurian times established a varied and rich assemblage of species that are in part known to us in the fossil state.

In frequent past systems of classification the Polyzoa and Brachiopoda have been united as the Molluscoidea. But, whether such be accepted or not, many zoologists regard both as fairly well related. So far as we are aware, however, no attempt has hitherto been made to trace the Brachiopoda back to a rotiferan ancestry, though the trochophore character of the larva has usually been recognized. 
In tracing the class backward from the present time, zoologists have recognized that gradual simplification can be noted from living and also extinct members of the division Testicardines to species and genera of the much simpler Ecardines, the latter of which are found fossil in the most ancient cambrian rocks. Throughout this entire history also, all were marine, and secreted at first a chitinous or semicalcareous shell that eventually became modified into the huge heavy calcareous shells of examples like Productus giganteus that the writer as a boy laid bare abundantly in rocks of the carboniferous age. So, in any attempt that may be made now to trace their phylogeny, we must trust to embryonic resemblances, or to picturing their developmental trend during archæan times by interpreting more primitive stages in light of the changes passed through from cambrian times till now. The latter seems to avail little, the former therefore must be the mode of attack.

Though our knowledge of brachiopod embryology is still very imperfect, enough has been published to act as a fair guide. As in adult rotifers the young embryo of Cistula or Argiope shows division into three regions, the head, the body, and the foot. Similarly the head region, which in structure and relation can exactly be likened to the trochal disc, is ciliated and bears a circlet of cilia round the margin - the præoral ring. In exact position as amongst some rotifers, four simple red or brownish eyes are formed. From the position of these in relation to the ganglia of the adult, the ganglia seem to arise between the eyes which are doubtless connected with them. The body region is deeply constricted off from the head, much after the manner of Megalotrocha amongst rotifers. Further the upper circular rim of the body grows outward and downward as a skirt around the body, and this bears four patches of setæ. Alike the outgrowth and its setæ correspond well to the setigerous lobes of Pedalion amongst rotifers, while in both the setæ are movable and can be expanded. The simple alimentary canal opens by a mouth under the præoral ciliated ring. The stalk or foot is as in rotifers a tapered cone, the apex of which is naturally the future attaching or pedal center. From its relation to the adult animal it seems probable that pedal cement glands are present here as in rotifers and polyzoans, for "after swimming about for a few hours, the larva fixes itself finally, apparently adhering by some secretion produced by the stalk" (or foot) "segment" (170:482).

The free-swimming brachiopod larva moves exactly as does the rotiferan, for it keeps the ciliated highly sensitive head 
segment in front, and at times directed downward, "as if investigating the nature of the rocks on which it may settle," at the same time often rotating it.

When the larva fixes by its pedal end, changes occur and structures can be traced that can best be interpreted if we keep in view a possible rotiferan ancestry.

When the larva fixes down, the skirt, at least in some cases, becomes slipped forward so as to encircle the head, and gives rise to the adult mantle lobes, a development that does not seem to be reached by any rotifer, and then gradual degeneration of the eyes takes place. In relation to rotifers the formation of the first or embryonic shells is highly suggestive. In that group is included a large and varied division known as the "loricata," or shelled rotifers. In these, two distinct chitinous plates or shells are formed, one on the dorsal, the other on the ventral, surface of the animal, and are well exemplified in Monostyla, Diplax, and Cathypna; or instead and more rarely two latero-ventral plates may be formed. In various ancient and recent brachiopods similar shells occur, the dorsal plate being termed the prodeltidium, the ventral the deltidium. These undergo various modifications as the adult shell is deposited alound them in the form of dorsal and ventral secretions. Such a feature might suggest that the Brachiopoda sprang from a loricate member of the Rotifera, and this is possibly further strengthened by the fact that most of the marine rotifers now living are included in the more evolved loricate section.

As the fixed and maturing larva passes into the adult, the brain, or supra-œesophageal ganglionic mass, grows in from the antero-dorsal epidermis as in rotifers, and similarly retains connection with the epidermis. Sub-œesophageal ganglia and longitudinal nerves complete the resemblance.

But the most striking structures of adult brachiopods are the two long and coiled "arms." These arise as lobular expansions on either side of the mouth, and alike in origin, position, function, and relative size correspond to the huge trochal discs seen in such a loricate rotifer as Brachiomus, or the even larger ones of Floscularia, Melicerta, and Limnias.

A possibly trivial feature, but one nevertheless that may indicate parallelisms in evolution, is the blind ending to the intestine in Asplanchna and Paraseison among rotifers, and in the higher group or Testicardines of the Brachiopoda.

The primitive kidneys in both are elongated tubules that run forward to the oral and trochal region, and in both groups these end in flame- or funnel-shaped inner endings. 
The above study indicates that, if the existing preponderating abundance of rotifers in fresh water is a true key to past conditions, it seems likely that the brachiopods sprang from a primitively lacustrine rotiferan ancestry, and that through loricate members of the Rotifera, which migrated seaward during later archæan times, loricate and afterward shelled brachiopods evolved, that even in early cambrian times were abundant marine organisms. Such a view furnishes at least a working hypothesis that further embryological study may strengthen or weaken.

The phylogenetic origin of the Mollusca may next be examined. For these as for some preceding groups, not a few zoologists have claimed an origin common with the Polyzoa and Annelida, more rarely with the Rotifera. The last seems perfectly to furnish the fundamental starting point, and, as Korschelt-Heider $(171, I: 260)$ have emphasized, some adult rotiferan groups supply strong points of contact with molluscan larvæ. But, as the Mollusca now exist, we are at once confronted with widely different larval types that doubtless represent equally divergent and early diverging adaptational modifications to environal relations.

As in other of the rotifer-derived groups already studied the ecto- and endoderm of the trochophore larva may arise either by epiboly or emboly. Except in the most highly modified group of the Cephalopoda an embryonic stage is reached by molluses that succeeds to the simple trochophore, ${ }^{*}$ and has been called the veliger stage. It is in the earlier or in the mid period of veliger development that perfect homologies to the rotifers are best observed; while, from this period on, localized growth activities are then ushered in-much as with the Polyzoa and Brachiopoda already reviewed-that can best be interpreted in terms of continued rotifer-molluscan evolution.

\footnotetext{
* In the above connection J. 'T. Cunningham has recently written "most remarkable is" the trochophore's "resemblance to the adult form of the Wheel Animalcules or Rotifera, which retain the preoral ciliated band as their chief organ of locomotion and prehension throughout life."
} 
Fig. 23 is a somewhat composite diagram that illustrates details common to some rotifers and to gasteropodous, pelecypodous (lamellibranch), pteropodous, and chitonoid organisms. One feature alone first deserves consideration, viz.: the primitive or cuticular molluscan shell. This at once suggests correlation with the dorsal cuticular lorica of shelled rotifers, and equally with the prodeltidium of brachiopods. Its dorsal position, even in lamellibranchs, as a median body is noteworthy, while the fact that in some existing loricate rotifers there is an expanded dorsal plate only, or a dorsal and ventral plate, or two lateral plates, might indicate that
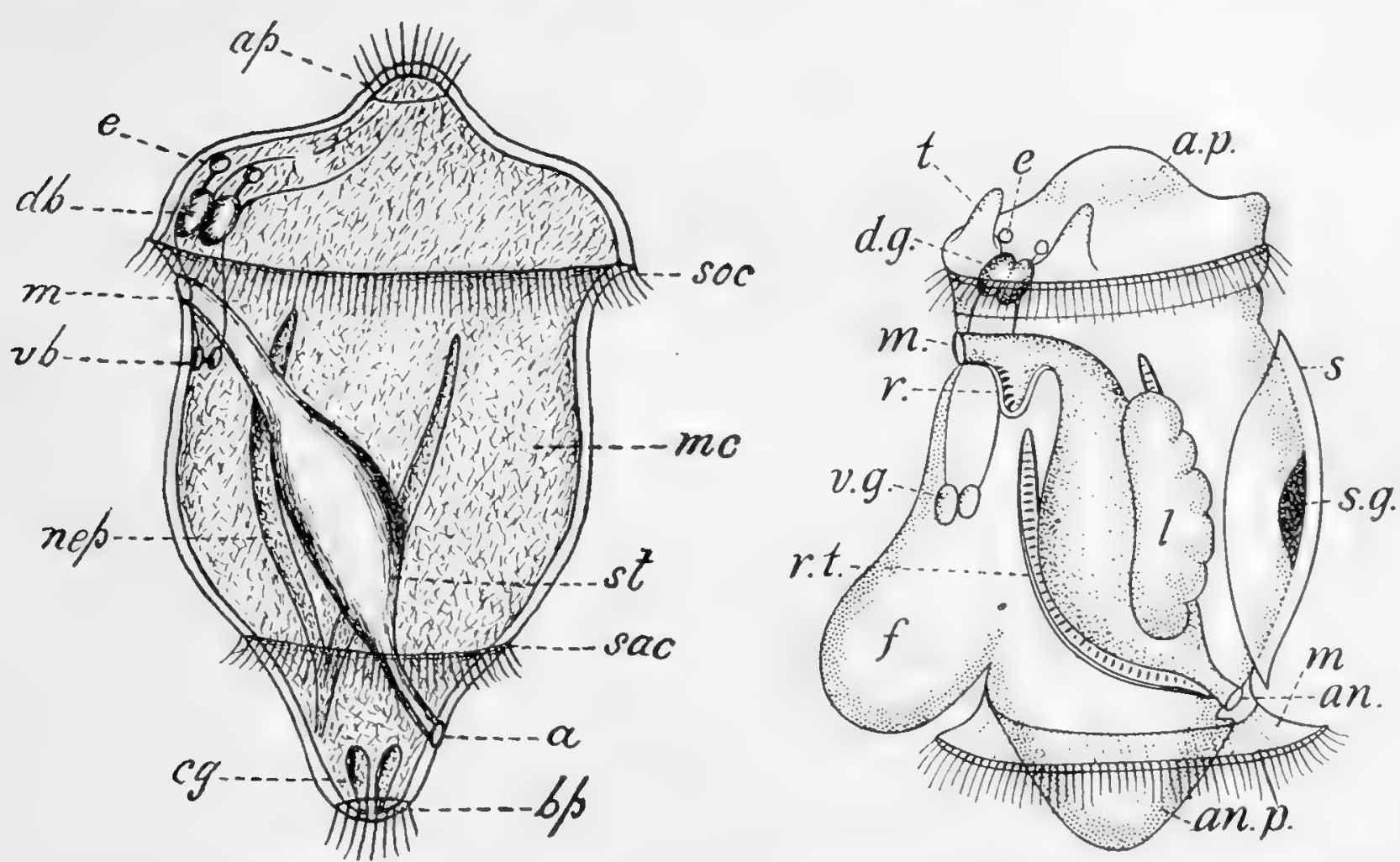

FIG. 23.-Diagram of Rotifer to left, of gasteropodous embryo to right. a.p., apical area; b.p., an.p., basal area; $m$, mouth; $a_{.}$, an., anus; d.b., d.g., dorsal brain; v.b., v.g., ventral ganglia; $e$, eyes; nep., r.t., nephridia; $l$, liver; $t$, tentacles; $s$, shell; s.g., shell gland. Compare also with Fig. 25 a., p. 534.

some ancient rotifers formed a dorsal, later also a ventral, and still later two lateral plates that fused as the tubular lorica of various genera. Of these four, the dorsal and ventral have been retained and expanded in Brachiopoda, the dorsal may be the cuticular shell, and the two laterals the future calcified shells of Lamellibranchiata, while the dorsal shell alone has persisted in Gasteropoda. Such theoretical points suggest the need for a careful reexamination of lorica formation in rotifers, and particularly a more detailed study of the character and cytological nature of the cells that secrete the lorica.

As the free-swimming veliger matures and then passes into the creeping or sedentary or free-swimming (as in pteropods 
and heteropods) or fixed animal that is characteristic of the above named divisions of molluses, developments proceed that can often alone be phylogenetically explained in terms of a rotiferan ancestry. We shall therefore shortly consider the groups in succession.

The Gasteropoda seem in many points to continue rotiferan features more directly than other molluscan divisions. But, in order to conduce to a better tracing of subsequent changes, it may here be suggested as likely that from a fresh-water proto-gasteropodous ancestry three lines of evolving and diverging specialization proceeded. First: a series that retained the straight body and alimentary canal with posterior anus, that retained two simple excretory tubes, four to two antennæ or tentacles, and that retained a diœcious state, gave rise to genera that remained in fresh water as the Helicinida, Hydrobiida, etc., while extensive migration into the sea originated the Patellida, Pleurotomarida, etc., that early formed a heavy calcareous shell. Here, owing to weight of shell, progression by right- or by left-handed motion, relation of sex and oviposition, increasing unilateral muscular strains caused gradual twisting of the alimentary canal, even in otherwise axial types like the limpet. These constitute the group Aspidobranchia. In them antero-dorsal respiratory gills evolved with an evolving heart.

Second, and perhaps simultaneously, a series that retained straight alimentary canal, that retained 4 or only 2 tentacles and a well-developed pair of excretory tubes but that became monœcious, split up into one set which evidently assumed an increasingly land habit, evolved an anterodorsal pulmonary sac, formed no operculum, and either added to the chitinous primitive shell a light calcareous one, or absorbed the latter and became shell-less in the adult. These constituted the Pulmonata. Another set that seems to have migrated seaward developed in many cases a heavy calcareous shell, formed a pre-anal and post-dorsal branchial expanse, and so constituted the Opisthobranchiata.

Third, and apparently derived from both divisions of the first by later and condensing modification, arose two parallel 
series, in which twisting of the body and alimentary canal became most pronounced. With this, condensation into one unilateral excretory tube occurred, and into one branchia or gill that fused with the dorsal mantle expanse. The freshwater representatives became, we would consider, the pectinibranchiate Paludinida and Melaniida, the marine forms gave rise to an abundance of genera often with heary shells.

The Pteropoda and Amphineura will be dealt with subsequently.

A more detailed examination of some developmental points in the above can now be made. The preoral area in most gasteropods forms two eyes and four to two antennæ or tentacles as in many rotifers. The eye in the latter class may be a simple pigmented depressed cell, or in front of the depression a clear lens may be formed $(169, I: 91 ; I I: 133)$. In gasteropods both of these primitive conditions may occur, but alike the pigmented or retinal cell and the lens cell undergo numerous divisions. Advance to greatly more specialized eyes is also noted. Four, or three by fusion of two, or two antennæ only may be met with in Rotifera, each also provided with special nerve, and motile as well as retractile, according to the graphic account of Hudson-Gosse. Further while usually provided with terminal cirrhi or chætæ, some genera have soft rounded tentacular antennæ. Like conditions exist in various Gasteropoda.

The very characteristic mastax or pharyngeal sac of rotifers with its associated teeth has already been referred to in correlation with the jaws and paragnaths of the Annelida. But a most interesting correlation and evolution can be studied in passage to the gasteropods. The masticatory system in Rotifera consists typically of a median often bilobed plate, the incus, each half of which is a ramus. This, by slipping forward, may be the homologue amongst pulmonate molluses of the single scraping jaw seen in Helix, Limax, etc., which, as Wiegmann has shown, is composed of two pieces in the young that fuse in the adult. On either side of the incus are paired plates, at times with serrated edges, in the Rotifera, each of which is known as an uncus. These are found in all pulmonates, and at times equal in size to each ramal half of the incus as in Limnoa. External to and attached to the margins of the two unci are oblique or longitudinal pieces, the manubria. These seem to be represented in Limnca by the oblique outer pieces of the unci with which they may have become fused. In prosobranch molluses there are paired 
jaws, which from their shape, position, and function probably represent the incus completely separated into its halves. Alike in rotifers and molluses these plates are protrusible from the mouth, and act as triturating organs.
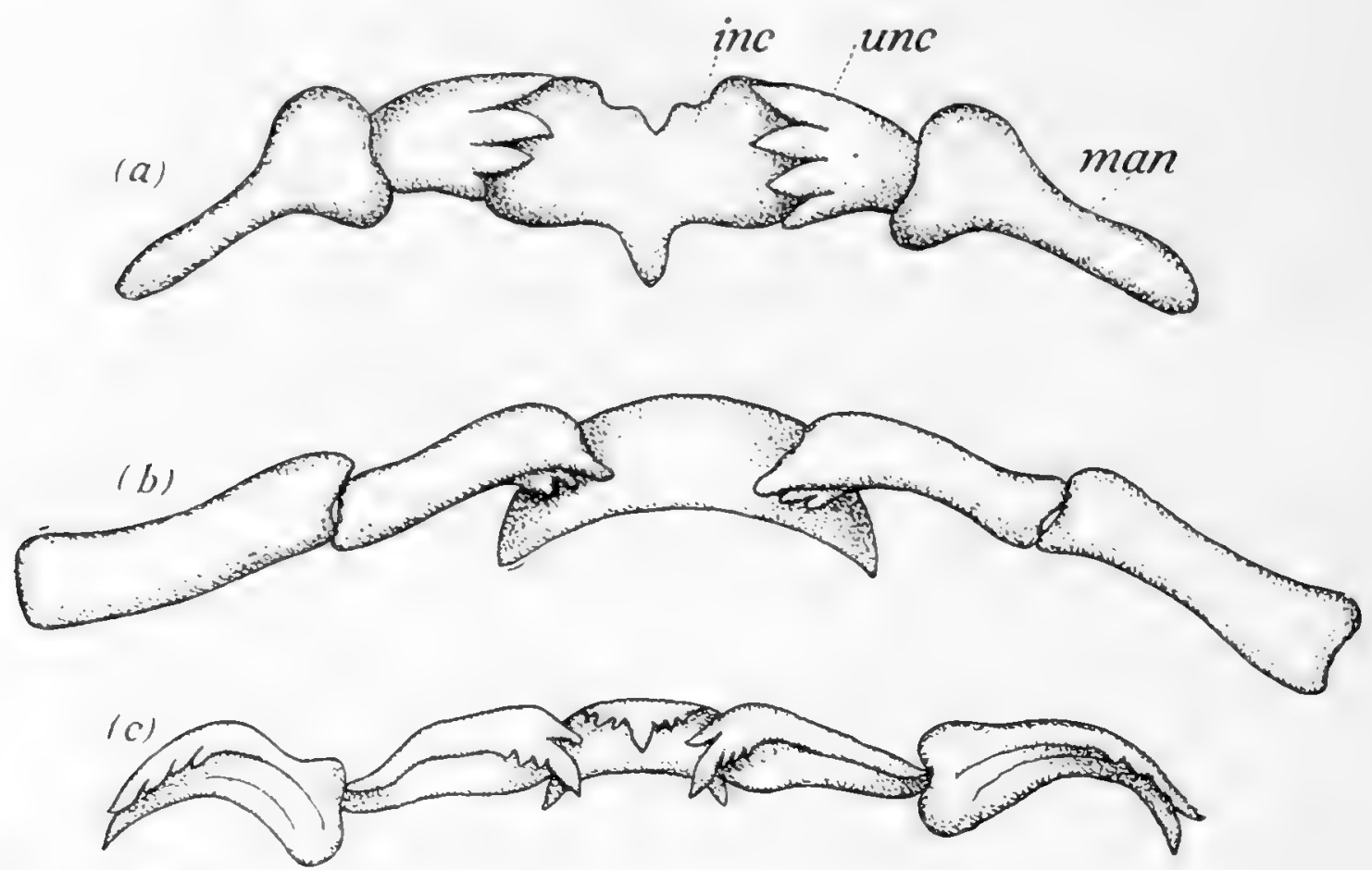

Fig. 24-Comparative view of masticatory plates from (a) a rotifer, (b) a mollusc Dentalium and (c) a gasteropod mollusc Vermetus.

But further in some Rotifera, e. g., Philodina, "there is a multitude of minute ridges parallel to the teeth." These we would suggest are the first beginnings of the elaborate gasteropodous radula or tooth-ribbon. Now in the radula of many molluses it can truly be said that each radular row of teeth is a vegetative repetition, in aspect and position, of the masticatory teeth. For comparing Fig. 24 a from a rotifer, with a row of teeth in Fig. $24 \mathrm{~b}$ from the mollusc Dentalium, and that of Fig. 24 c from Vermetus it will be observed that the central radular teeth correspond to the halves of the incus, the lateral teeth to the unci, and the marginal teeth to the manubria. But again, where the teeth are small, uniform, and harrow-like, these may represent a umiform development of tubercles from the "multitude of minute ridges" spoken of above. We incline therefore to regard the mandibulo-radular apparatus of gasteropods as a direct evolutionary advance on the rotiferan mastax.

IVe cannot here trace all of the changes in size and growth of parts of the head, body, and foot, in the subdivisions of gasteropods, as the larva changes into the more sluggish adult. But it is of interest to note that the bilobed cement or pedal 
gland of rotifers is continued in development, and becomes the glandular mass on the front region of the foot in many genera of molluscs, alike fresh-water and marine. This exudes a viscous mucilage, which has been shown by Cunningham to be used in formation of the tough egg-cases. The foot in most rotifers ends in a bilobed process, and it is at least of interest to observe that in fresh-water molluscs like Limncea and Succinea, as well as in the marine limpet, it arises as a paired structure.

The shell-secreting dorsal gland of gasteropods is a structure that early appears and becomes very conspicuous in the larva. Below (p. 526) we refer to its possible homologue in rotifers, when discussing lamellibranch structure.

The arrangement and finer histology of the excretory tubes in both classes may be said to be identical, while the similarity of the sexual organs and their accessory parts need merely be stated.

If now inquiry be made as to whether any molluscan group permanently exhibits only slight advance on rotiferan structure, such is unquestionably furnished by the Scaphopoda or tuskshells. These, while exhibiting both molluscan and rotiferan characters, agree with the latter in having no heart or distinct blood vessels, as well as no gills. The corpusculated bloodfluid is retained in hæmal sacs at different regions of the body. There is no indication that such negative characters, in what is ordinarily classed as a molluscan animal, are connected with degradation processes, for progressive modification throughout is observed in it from the trochophore stage onward.

The trochophore and early veliger stages of marine lamellibranchs or Pelecypoda agree exactly with rotiferan conditions above noted. But all distributional, developmental, and palæontological evidence would tend to show that while still in the early veliger stage of evolution the bivalved molluses branched off from a fresh-water-derived but marine rotiferprotomolluscan ancestry, and long evolved in the sea, only later, and probably during the devonian epoch remigrating into fresh waters to produce there the "fresh-water bivalves."

Perhaps the first feature here deserving of comparison is the origin and relation of the lateral shells. The lorica of rotifers, as already indicated, seems most commonly to consist of a larger arcuate dorsal and of a smaller-often flat-ventral chitinous plate. But such genera as Colurus, Monura, and Mytilia show instead two lateral plates often hinged together along the dorsal edge. Again in Metopidia a dorsal and two lateral elements seem to fuse to form the lorica, while 
in Pompholyx four elements - a dorsal, a ventral, and two laterals - together make up the lorica. But in all of HudsonGosse's accounts the dorsal shell seems to be the largest and first formed. Now in lamellibranchs, as in several classes already cited, a dorsal median shell is first formed, while in lamellibranchs only the lateral shells arise as dorso-lateral calcareous formations in connection with the highly typical molluscan shell-gland. A phylogenetic indication of this gland in rotifers would therefore be of interest.

Now Gosse $(169, I I: 102)$ speaks as follows regarding Colurus deflexus: "In the middle of the back, just under the lorica, are two curious organs, each apparently an agglomeration of minute clear vesicles, perhaps of air, perhaps of oil, observed long ago by Ehrenberg. He declared them inexplicable; and I cannot supply the explanation." Again (p. 104) Gosse says for $C$. amblytelus, "one oil globule (sometimes two) occupies in general the middle of the back, and is conspicuous." And again he says for Metopidia lepadella (p. 106): "No organization was visible within, save two conspicuous clear vesicles, side by side in the middle of the body, not at all like oil globules, being irregularly oblong; nor accidental, being found in each of a large number of individuals seen at different times."

Though meager in detail, this account may next be compared with those given for the shell-gland, and its relation to the shell in lamellibranchs. "The shell, as in the Gasteropoda, is unpaired in its origin, and is formed by a secretion of the epithelium of the shell-gland. This embryonic cuticular shell is retained, and passes over into the periostracum (epidermis) of the adult shell. The latter arises through a secretion of granular calcareous substance; this at first accumulates in two complexes lying symmetrically at the two sides of the body beneath the cuticular shell, which, by further increase, yield the shell-valves. These grow out dorsally until they meet. The dorsal part of the cuticular shell, that lies between the two calcified shell valves, which are growing towards one another, yields the ligament of the shells. The larval shell which thus arises, and which has a very simple structure, is retained in Ostrcea, the Unionidx, and, as has recently been proved, in a number of other Iamellibranchs" (171, $I: 60)$.

Further Ziegler has shown that "an accumulation of small calcareous concretions can be seen on each side of the dorsal middle line, beneath the umpaired cuticular integument. These masses increase and soon take the form of the definitive shell. There is now a shell-plate on each side; the two, however, are not in contact, but are somewhat far removed from the middle 
line." The above accounts for rotifers and lamellibranchs seem to agree so well that additional evidence either of negative or confirmatory kind is highly desirable.

Though the resemblances may be only structural parallelisms and trivial, it might further be pointed out that a striking similarity seems to exist between the circlets of - in part ciliated-plates in the primitive genera Yoldia and Nucula, and the tesselated plates of the rotifers Anurcea and Metopidium.

So the dorsal shell or prodeltidium of brachiopods, the single larval shell of gasteropods, and the dorsal larval shell of lamellibranchs, seem all traceable, as primitive structures, back to the dorsal lorica of rotifers, while in the last the first faint beginnings of the molluscan shell-gland seem strongly suggested by Gosse's accounts for the rotifers.

The trochal or velar area in larval lamellibranchs gives rise to the two pairs of highly sensitive labial palps. These have been regarded by Loven $(1 \% 1: 46)$ as mere expansions of the velum. But their position in relation to the head and larval eyes, as well as their innervation, would suggest rather that they are the four rotiferan antennæ in soft expanded and somewhat modified form.

The musculature of bivalve shells is so conspicuous a feature that some commencing indication of it might be looked for in rotifers. An observation of Hudson regarding Pterodina valvata is worth quoting. He says: "While watching some of the new Pterodinae, I was surprised to see one of them sailing by, with its lorica folded down like the flaps of a Pembroke table; its outline was so altered that it scarcely seemed the same animal. This curious infolding of the lorica is due to the contraction of two conspicuous transverse muscles, which do not necessarily act together; as a friend, who was watching with me, saw some specimens with only one side folded at a time" (169, II: 113).

As to the main longitudinal muscular bundles, the striking resemblance in disposition, insertion, number, and function of those seen in Plate XI, fig. 3, of the above work, for $A s$ planchna, or the description on page 61 for Stephanoceros with those of Teredo larva as figured from Hatschek, may be a case of parallel coincidence. But in view of the many other fundamental points of agreement, we can scarcely view such otherwise than as a case of adult rotiferan and larval molluscan continuity.

The foot exhibits equally great variation in size and function in both groups. Thus it may be elongated, tapered, and attaching quickly at will, or greatly elongated, muscular, and 
saltatory, as in Adineta raga amongst rotifers, and Cardium amongst lamellibranchs, or discoid and creeping, or reduced in size and function, or absorbed as in Triarthrida of the lower, and Ostroea of the higher group.

The cement gland evolutionarily is even more suggestive here than for Gasteropoda. Thus (173: 42): "A pit-like depression of the ectoderm is found on each side of the middle line, lying exactly above the mass of cells from which the pedal ganglion develops. This is the paired rudiment of the byssal gland. These two depressions together with the whole of the ectoderm lying between them soon sink in deeper, having then only one common aperture; their epithelium becomes modified into glandular cells which secrete the well known byssal threads that serve for the attachment of the embryo or young lamellibranch. As the body grows further the paired rudiment of the byssal gland is drawn further and further inward, and finally opens out through a long duct with a narrow aperture. This gland degenerates later (in Cyclas), and in the adult is a mere sac-like vestige. In other lamellibranchs, on the contrary, as is well known, it functions throughout life and is very highly developed."

The secretion of this gland functions also in molluses for attachment to foreign objects as in some rotifers, but similarly the secretion may be poured out round the mollusc, as in Modiola, Dacrydium, and others, to form a viscuous envelope in which extraneous particles may be lodged $(174, I I: 913)$. So far as the writer is aware no faint trace even of a pedal ganglion has been observed in most rotifers, but there seems a possibility that such may exist in several genera. For the detailed description given by Zelinka in the case of Discopus (175: 353), as well as the accompanying illustrations, alike demonstrate the existence of a pedal ganglionic system, the close similarity in the nervous system of that genus and of lamellibranchs, and an exact relation between the pedal ganglion in Discopus and the adhesive gland. The origin of the gland also, as a pair of ventral swellings, is noteworthy.

The absorption of the mastax in development of the lamellibranchs from a rotiferan type seems wholly correlated with the soft nature of their food. The close similarity of the alimentary canal, of the larval eyes, of the nerve distribution, and not least of the excretory organs in larval stages, need merely be indicated.

As regards Cephalopoda the remarkably early and abundant development of this group palæontologically has often been commented on. It would therefore have been of great in- 
terest, could the embryology of such a primitive organism of the class as Nautilus have been before us. In want of such the recent members of this great class have evidently become so highly modified, even in the early larval stages, that it would be inopportune now to attempt the tracing of their phylogeny.

The Polyplacophora or Amphineura seem to have a history that constantly wavers in its earlier phases between the annelidan and molluscan lines, but eventually ends toward the latter. The later larval stage, described and figured by Kowalewsky, shows marked agreement in detail with the rotifer Dinocharis, and specially D. Collinsii. In both the organism is dorsally arched and ventrally somewhat flattened, in both transverse areas are early indicated that suggest six to eight transverse plate segments, in both an anterior head plate arises; setæ that project through the cuticle or lorica are arranged transversely, the test early shows fine spots or markings, the details of which we are still ignorant of for Dinocharis, but which develop the æsthetes and possibly eyes in Chiton. A minute examination and comparison here is very desirable.

Further in both the alimentary canal is somewhat arched but straight, the eyes are situated rather far back, while many other points of agreement with typical rotifers are revealed.

We may sum up then by saying that the various molluscan divisions show, from the trochophore and veliger stages onward, so many fundamental points of agreement with definite living genera of rotifers, that these can all be exactly accounted for if we bear in mind that living rotifers are long-drawn descendants doubtless from once greatly more abundant and varied genera, forms of which would equally have united the living with the extinct, and would as perfectly have furnished connecting types between themselves and the Mollusca, as well as other great divisions.

The Arthropoda now remain for consideration. By many zoologists it is now recognized that the Rotifera, Chotophora, and Articulata form divisions that are fundamentally interrelated, and all distinguished by the tendency to produce bristles on the surface of the body, or on special expansions of the body. But, though Gosse has very clearly stated that he regards the rotifers and crustaceans as near allies, many have rejected such a view. So the remarkable genus of roti- 
fers Pedalion has by them been regarded as an example of parallel or convergent development between groups that are distinct. Such a view is entirely tenable, if no more helpful one can be advanced. We would however decidedly favor the view that the many structural and developmental resemblances shown by some rotifers and specially Pedalion to primitive and mainly fresh-water entomostracan genera, as well as to larval stages of higher arthropods, indicate a real and close phylogenetic affinity. So, instead of tracing the Arthropoda from an annelidan ancestry, we would derive it directly from the Rotifera.

The early larval or nauplius body of Crustacea showslike that of all previous groups which we have regarded as descended from the Rotifera-division into a preoral or cephalic region, a body or postoral or thoracic region, and a tail or caudal or abdominal region. Though it is still difficult to correlate the exact organs or appendages that each of these may bear, every new investigation is adding to our ability in effecting this. Such a division as that now given is fully recognized by Korschelt-Heider (172, II: 191, footnote) who say: "Although there is no external segmentation in the nauplius body, we can nevertheless recognize in it the following segments: an anterior preoral or primary cephalic segment, a posterior terminal or anal segment at the posterior end of the body, and the true trunk segments which lie between these."

A striking difference in the surface aspect of the crustacean larva, as compared with those already treated of, is the absence of cilia or ciliated areas, so that one has largely to be guided by the average position and historical relation of parts in correlation efforts. Reasons for this ciliary absorption seem to be, increased thickness of the egg wall, retention of the young larra within it till it has formed swimming organs, precocious formation and functioning of the latter, and abundant storage of rich yolk food in many cases-Entomostraca exceptedthat enables precocious larval development to be effected.

In what follows we would premise that the Crustacea of entomostracan affinity are the most primitive arthropods, from which by increased segmentation and specialization, most of the other arthropod groups are derived.

Probably the most fundamental point that might first be considered is the origin and value of the paired, jointed setaceous limbs that are so characteristic of nearly all larval crus- 
taceans, and of many adult ones. We would suggest that the complete evolutionary history of these starts with some of the Rotifera, and is carried up unbroken through the Crustacea to still higher arthropods. As is now well recognized the larval nauplius and zoæa stages of Crustacea show sometimes two, usually three, occasionally four pairs of setaceous appendages, the antennæ, antennules, mandibles, and maxillæ. These act at once as feelers, propellors, and accessory mouth appendages. All of them, in varying degrees of development, or of dorsal and ventral paired fusion, can be traced in Rotifera. Unfortunately, where almost certainly fusion occurs, we as yet have little exact knowledge as to double nerve supply, or indications of nerve fusion.

In not a few genera of Rotifera the trochal disk, with its cilia or ciliary circlets or tufts, constitutes the only motile and directive structure. Many of the loricate genera exhibit this, also Lacinularia, Megalotrocha, and others. But in Scaridium two dorsal outgrowths of the disk that have been described as "rocket-headed antennæ" appear as slight swellings. These again, as Floscularia mentioned below indicates, may fuse to form a single short dorsal movable process, usually with setæ, as in Brachionus, or a long flexible appendage with terminal setæ as in Cephalosiphon and Rotifer.

But below and in front of, or more ventrally than the above, two other appendages may arise as setigerous papillæ or knobs or elongated and highly flexible organs. In this connection Ecistes is noteworthy. In Ecistes serpentinus the dorsal organs appear only as two hooked processes adnate at the base, the ventral organs as two minute papillæ. But in other species the latter may become elongated and conspicuous, while"they move independently of each other but not spasmodically."

In Floscularia the species $F$. Hoodii has two evident dorsal appendages, and two small setaceous latero-rentrals, while in other species the dorsal ones are evidently fused into a long median dorsal process. In Melicerta the latero-rentrals are long supple setigerous appendages, while the two dorsals seem to have fused into a small dorsal setigerous papilla. In $1.5-$ planchna Ebbesbornii "there is a rectangular nerrous ganglion just under the corona," and a nerve thread passes from each corner of the ganglion to two dorsal setaceous swellings, and two lower latero-ventrals. But, further, just below the latter and above the mouth two added appendages appear with nerve supply from the latero-ventrals. 'The same is seen in 1 . priodonta. 
In Synchota pectinata four pairs of setigerous appendages can be traced. "At the summit of the dorsal hump meet two rocket-headed antennæ, each bearing a tuft of setæ; and the two tufts issue together from the same opening. A nerve thread passes from each rocket head to a nervous ganglion in the head" $(169, I: 125)$. In front of these are two lateroventral antennæ, each being a small tube that bears a swelling into which one or more styles are set. A third pair, corresponding to the buccal pair in Asplanchna probably, is placed more ventrally. But further, slightly behind the level of the mouth, and on either side of the front body region, are two "tongue-shaped fleshy protuberances, edged with powerful cilia, and as they can be set by special muscles at various angles to the body, the creature can dart, wheel, and stop, with the greatest ease." In Polyarthra four pairs of appendages seem also to be represented.

The above comparative details show that gradual evolution of two to four pairs of setigerous appendages may arise, and that even in the same genus each pair may be distinct, or may fuse into a median dorsal or sub-ventral appendage. All accounts agree in stating also that special muscles pass to and cause rapid motion of these, when they reach a fair size. Nerves also are as exactly distributed to them as to the eyes. And, as in Philodina, each may be simple or two to three-jointed.

Such conditions lead us by stages to the unique genus $\mathrm{Pe}$ dalion, in which are a small median dorsal antenna, that probably represents a fused pair, and two latero-ventrals below and on either side of the ciliated disc. Behind these are two pairs of gigantic lateral setigerous appendages, a very large median ventral one that may result from paired fusion, and a like though smaller dorsal one. In this case then six pairs of setigerous appendages are suggested, four pairs of which are clearly expansions of the body substance, and are moved by powerful muscles. In addition a pair of elongated setigerous appendages grows backward in telson-like fashion from near the anus.

The two pairs present in most non-loricate rotifers, as dorsal or lateral processes, may be correlated with the first and second pair of antennæe in Crustacea, the supra-oral pair seen in Asplanchna as the mandibles, and the slightly more posteroventrals as a pair of maxillæ. Possibly some of the large appendages in Pedalion may represent the first and second maxillæ, and others the ambulatory appendages. The two anal appendages seem entirely to agree with the furcal processes of the Crustacean larva. All of the above is in complete 
agreement with the principle that Korschelt-Heider lay down for Crustacea.

In relation to the Crustacea the eyes of rotifers are worthy of study. In the latter four paired eyes, or two paired and a median eye, or two paired eyes, or as in Anurcea and Notholea one median eye may appear, or as in Noteus they may be wanting. But Hudson observes regarding Notholea longispina "the adult had a single red eye, at the lowest part of the occiput, near the dorsal surface. Mr. Levick says that many of the first specimens he found had two eyes; and that he thinks these animals were young ones. It would be very curious should it prove to be the case that two eyes in the young approach with age, and coalesce in the adult, especially as Brachioni have an eye which has every appearance of being a coalesced pair" (169, II: 126-27).

Now in larval Crustacea there is a single median eye that is retained in the simple group of the Copepoda. Each is made up of two lateral and a median eye fused together, since there are three hollow masses of pigment tissue behind retinal tissue, and often three distinct nerves pass to these from the anterior ganglionic region. So it might well be considered that four primitive eyes evolved in successive pairs in at least some rotifers, that two slowly fused in the middle line, and that later fusion of this mass with the laterals occurred to form an apparently single but truly complex eye, with complex nerve supply. The compound eyes of many Crustacea have evidently developed as a character of the group, that is an advance on rotiferan organization.

The entire body or a large part of it is, in many crustaceans, surrounded by a common shell, that can only be understood phylogenetically and morphologically by reference to the Rotifera. This may be "a flat triangular or somewhat oval shell" or "a triangular dorsal shield to start with," or a circular or oval deeply convex lorica. But in the primitive group of branchiopodan Entomostraca the dorsal shell does not appear till after the body and some of its appendages are formed. So in Crustacea as in Rotifera we have a non-loricate and later a loricate stage of advance. In such Entomostraca as Moina a patch of cells forming the nuchal gland appears in similar position to the single clear vesicle already referred to in rotifers. Though various views have been expressed regarding it, it seems probable that both represent dorsal shell glands. Therefore the embryonic dorsal shell of Crustacea would be homologous to the dorsal shell of loricate rotifers, of brachiopods, and of molluses. 
But as Fr. Miiller and Grobben have suggested, this nuchal gland may give origin to the "dorsal organ" that is so marked a feature in many crustaceans. Now Gosse observed two dorsal bodies in some rotifers that closely resemble the one already referred to, while the dorsal organ in some Crustacea (Amphipoda-Isopoda) is a median dorsal ingrowth, in others a paired disk, or as in Mysis the paired bodies may be wide apart according to Nusbaum $(171, I: 153)$. This history is of special value in connection with shell origin. For, after the dorsal shell has formed in some larvæ, it is gradually replaced by a right and left bivalve shell as in lamellibranchs.
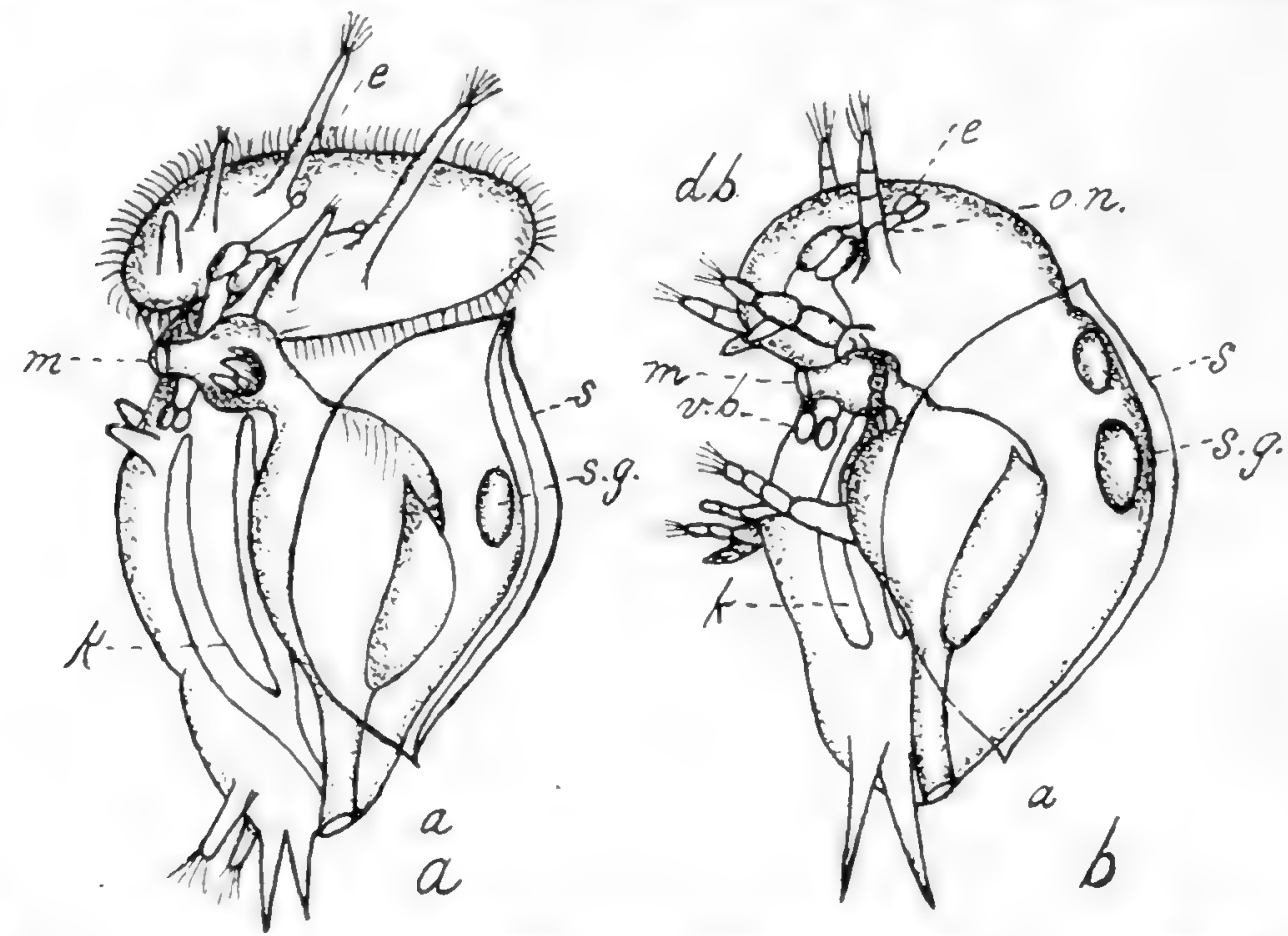

FIG. 25.- $a$, compounded diagram of loricate Rotifer; $b$, of larval Entomostraca; $m$, month; $a$, anus; $k$, cxcretory tubes; $d . b$., dorsal brain: $v . b$., ventral brain or ganglia; $e$, eyes; $s$, lorica or shell; s.g., shell gland. The liver in both is outlined on the dorsal side of the stomach.

Such is well seen in the Ostracoda, etc. But the connecting stage between these two phases that completely harmonizes with rotiferan conditions is seen in the cirripedes, where, in the early larva, "the lateral parts of the dorsal shield now alrearly begin to bend downwards, and, covering the body laterally, foreshadow in position the valves of the shell in the ('ypris stage." These "two shell-valves, which can be brought together by an adductor muscle, are directly continuous dorsally" however.

Korschelt-IIeider incline to regard even the lateral valves as extensions of the dorsal, for they say, speaking of Limnadia and Estheria, "although here, as in Cladocera, the two halves of the shell at first develop separately, we must, nevertheless, 
trace them back to an originally single dorsal shield, like that of Apus." Much may be said for both views, but till accurate study of the center or centers of origin for the rotiferan dorsal, ventral, and lateral plates of the lorica has been made it would be vain to say more. But the relation to lorica-formation in rotifers is most suggestive, except that from their usually active habits Crustacea do not appear to form a ventral shell, though even this may yet be recognized in the "sternal" element of crustacean calcification.

In passing to the internal organization attention may first be drawn to the comparative details of Figs. 25 a and b; that represent an adult rotiferan and a larval entomostracan phase. But, in the succeeding account, adult points of structure in the divisions of both classes will be referred to.

The mastax of rotifers is reproduced more or less perfectly throughout the whole crustacean series, and the teeth or spines of this "gastric mill" show surprising agreement as to structure and function in both. In Bairdia and some of the Phyllopoda, the chitinous plates are either styliform, bristle-like, or toothed and triturating, but in the highest division that includes the crabs and lobsters the entire system consists of a median plate resembling the incus of rotifers, and sets of lateral plates that work on it after the manner of the unci and manubria of rotifers.

The sections and the glands of the alimentary canal agree at least in their connections in both. The brain or dorsal ganglion is of like origin and position, and bears a like relation to the eyes, and the antennules or dorsal antennæ. But, till greater detail is secured for Rotifera, it seems impossible to correlate the suboesophageal ganglia and nerves in both classes.

A character that may be said to be largely diagnostic of the rotifers is the median anal spine, and even more constantly of the paired spines borne on each side of the median one. They may vary in size, length, and degree of flexible movement. They seem to be the precursors and homologues of the telson and anal spine of Crustacea. But we have already noted that two cement glands are developed within and open on these pedal processes, and further that, as this pedal portion expands in Brachiopoda, Polyzoa, and Mollusca, the glands are very often developed, while their viscous secretion is used in sereral ways. If then the processes of the telescopic foot in Rotifera correspond to the telson segments in crustaceans, one might hope even expect - to find cement glands in these.

The writer has searched in vain for any record of them. Frequent mention is made of viscous excretions in the Copepoda and Ostracoda, while the cementing of larval acorn shells 
and barnacles to their substratum has usually, and probably rightly, been attributed to antennary secretions. But it seems not unlikely, in view of Korschelt's statements (172, II: 215) and of the remarkable flexure in the body of cirripedes, that Darwin's assertion as to the part by means of which "the attachment of the larva at first takes place, does not correspond to the frontal extremity of the body, but to the most anterior portion of the ventral surface," may point to a similar secretion and fixation as already indicated for endoproctous polyzoans (p. 516). In any case further search deserves to be made for pedal cement glands, or their rudiments, in Crustacea.

As to the excretory apparatus, suggested homologies can alone be made, for our knowledge is still too incomplete for definite statement. Beside the longitudinal canals with their lateral branches that end in flame cells, several descriptions and figures of Hudson-Gosse would suggest that some rotifers develop an added system in the head region, just under the trochal disk. These are of two kinds: first, "in Pterodina the tubules ramify and spread into broad fan-shaped plexuses of flat laminæ, filling the wide triangular areas on each side of the mastax"; second, the so-called gastric glands. "These organs take the form of large reservoirs of delicate texture and wrinkled surface, joined to the œesophagus by long ducts, and affixed by threads to the lateral canals or to the lorica" $(169, I I: 138)$. Whether either of the above two structures may in any way be the forerunners of and homologous with the green or antennal glands and the shell gland of Crustacea remains to be decided.

It is worthy of note that, as amongst the scaphopod molluscs, a distinct heart and circulatory system seem to be undeveloped in several entomostracan as in rotiferan groups.

Without entering here into the relation of the primitive layers of the body to the alimentary canal, or into the structure of the reproductive organs, where interesting homologies could be traced, it may suffice to say that we would consider the Crustacea to have developed continuously and directly from certain evolving lines of higher Rotifera already named. Further that this progression has taken place through the simpler entomostracans like the Ostracoda, Copepoda, etc., which for eons of ages have been and still are largely of freshwater habits. By gradual multiplication of body-segments, the multisegmented bodies of the Phyllopoda were built up, 
and in the process successive migrations of evolving families or genera steadily took place seaward, there to originate types many of which became greatly calcified.

Thereafter specializing condensation toward the decapod series, and degrading condensation toward the sedentary and the parasitic groups took place, in part in fresh water, but more largely in the sea, that constitute great side lines of steady evolutionary change, but which have failed to originate forms higher than themselves.

We venture to make the above statements with full cognizance of two important positions reached during the past quarter century. The first of these is that so admirably synopsized by Korschelt-Heider, in which the view is advanced that the Crustacea have developed from an annelidan ancestry (172, II: 192). The second, advanced by Gaskell (176) and much more elaborately by Patten (178), is that the fishes and still higher vertebrates started from a crustacean basis. Both of these positions seem to the writer alike strained and morphologically unnecessary.

In the scope of this work it is impossible to do more than indicate that the three essentially land divisions of arthropods, namely the Myriapoda, the Arachnida, and the Insecta, seem all to have started from primitive entomostracan ancestry, and in assuming a terrestrial life have gradually adapted themselves to the action of environal agents by reactions of varied and in many cases increasingly perfected kind.

All palæontological evidence tends to prove that the more primitive divisions of the Crustacea must have been frequent in the fresh waters and seas of later archxan time, for the abundant remains of Ostracoda and Trilobita in oldest cambrian rocks, and of other Entomostraca in silurian to carboniferous rocks, often formed too in fresh water, could only have resulted by descent from greatly older ancestors. But the soft bodies or frail shells of many archæan organisms militated against preservation, while the quantity of oily compounds that these contained, combined with the baking by rolcanic agency that was then frequent, would conspire to disintegrate their remains. 
In summary at this stage it may be said that four great metazoan stocks or phyla have been recognized by the writer, from some one of which the higher groups have evolved. These are the Porifera, the Colenterata, the Echinodermata, and the Rotifera. Of these the first-named three failed to give rise to polyphyletic and dominant descendants higher than the most evolved members of their own division. The fourth, by formation of specialized and concentrated sense-centers, of active neuro-muscular tissue that was intimately related to these centers, of complete alimentary canal with associated glands that ensured high and continuous metabolic activity, of ramified excretory organs that prevented accumulation of waste products, and of specialized reproductive cells that ensured dissemination and perpetuation of the species, became a highly plastic, adaptable, and responsive group.

In virtue of such high and varied environal relations, correspondingly high and varied proenvironal responses were made. So originated the diverse structural details and adaptations that together constituted the Rotifera a polyphyletic ancestral group, from which the Turbellaria (and through them the Gastrotricha and Nemertinea), the Annelida, the Polyzoa, the Brachiopoda, the Mollusca, and the Arthropoda have all sprung.

But of these the two groups that most perfectly retained the qualities of rapid and plastic environal perception and proenvironal response were the Turbellaria and the Annelida. For, while the Arthropoda retained this to a large degree, the tendency to secretion of a chitinous or calcareous exoskeleton has unquestionably proved a serious obstacle to plastic adaptation, even though often aiding powerfully as a means of defense. So it is to one of the above two groups that we should look for an active and steady line of evolutionary ascent, that would lead us to the higher or vertebrate phylum. But a parting of the way occurred as they erolved from a common or related rotiferan ancestry. For in simple Turbellaria the bdelloid rotiferan dorso-lateral and ventral nerve cords were retained; in simple Annelids, on the other hand, the ventral system became the dominant and in time the only important longi- 
tudinal system. The simple or rhabdocoel Turbellaria however soon split up into a series that not only retained the dorsolateral nerres; these became approximated dorsally and increasingly strong.

The latter condition seen in the Nemertinea we have already emphasized as a perfect transition phase, that conducts from the Rotifera through the rhabdocol turbellarians upward to the simplest chordates or vertebrates. So when the flexible and tactile protrusible oral area of some rotifers became the dorsal protrusible proboscis of some turbellarians, and later became modified into the dorso-cephalic proboscis of early nemerteans, increasingly abundant, varied, and powerful nerve stimuli passed centripetally to it, and centrifugally from it. Such action and reaction stimulated the dorsal and the lateral at the expense of the rentral nerve cords. When therefore the steadily strengthened proboscis-sheath became a strengthening tube or rudimentary notochord, as claimed by Hubrecht, the attenuate dorsal nerve cord became the precursor of Reissner's fiber, while the laterals became the dominant transmitters of stimuli, and later by dorsal approximation and union formed the vertebrate spinal cord.

But during the past half century much attention has been given to three aggregates of marine organism, that show more or less markedly chordate characters. These are the Hemichordata or Balanoglossus series, the Urochordata or ascidian series, and the Cephalochordata or lancelet series. The first of these seems for many reasons to be a greatly modified marine derivative from marine nemertean ancestry, in which the larva has at times become distinctly pelagic, and the adult animal has undergone highly divergent modification from an advanced and specialized nemertean ancestor. In line with such a view, we would regard the proboscis as the permanently unfliped or evaginated proboscis of a nemertean, into the interior of which the nemertean proboscis-sheath has been pushed forward as a condensed and collapsed notochord, and is surrounded internally by the masses of muscle that in earlier history gave it its flexible character. The basal 
part of the proboscis attachment, and the entire area round the proboscis-sheath, have here become the collar. The anterior expansion in front of the notochord and inside the pro-

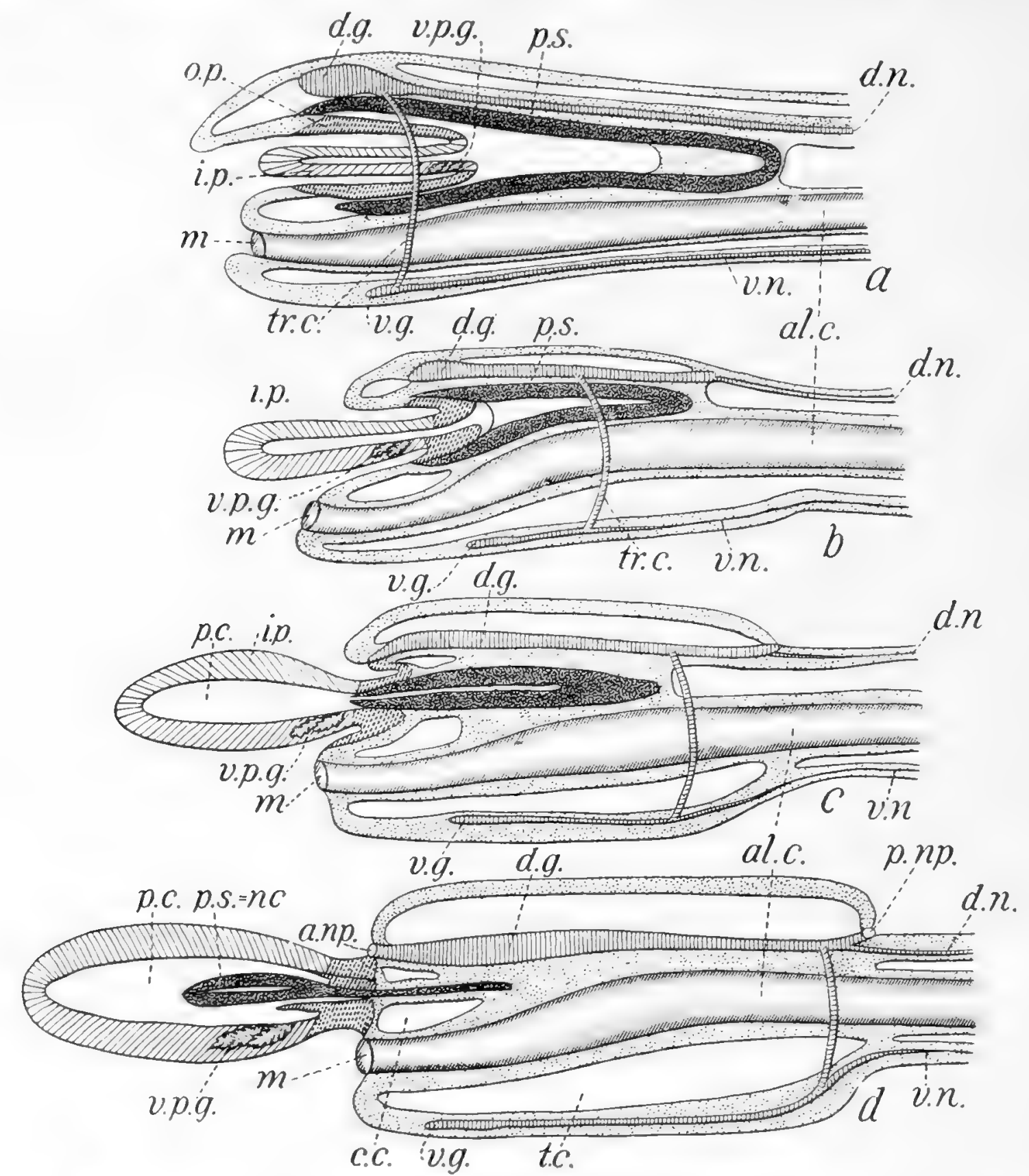

FIG. 26-Comparative diagrammatic views of cephalic region of, $a$ nemertean, $b$ nemerto-hemichord type, $c$ protohemichord type, $d$ Balanoglossus. $m$. mouth, $i$. $p$. everting inner glandular proboscis part in $a, b$, that becomes $p . c$. or proboscis in $c-d, o . p$. outer or muscular part of proboscis, $v, p . g$. ventral proboscis glands, $p . s$. proboscis sheath becoming notochord $n c$ in $d, d . g, v, g$ dorsal and ventral brain masses connected by transverse commissure $t r_{0}, c_{\text {. }} d$. $n ., v . n$. dorsal and ventral nerves, $p_{\text {. }}$ c. proboscis cavity, $c$. c. collar cavities, $t_{\text {. }} c$. trunk cavities, $a . n p ., p . n p$. anterior and posterior neuropores.

boscis known as the "proboscis colom" or "the anterior body cavity" would then represent a part of the nemertean bodycavity, that by joint extrusion of the proboscis and forward 
growth of the notochord in the cephalic region, has become abstricted off between the outer and inner muscular layers. The paired "second body-cavities" or "collar cavities" would similarly represent lateral coelomic enlargements behind the region of the proboscis attachment, that resulted from passage outward permanently of the entire proboscis and enlargement for absorption of water, as well as muscular strengthening of the collar area, as a mechanical basis for the proboscis. The paired posterior body cavities would again represent abstrictions round the area behind which originally the retracted proboscis lay. The accompanying diagrams illustrate such a graded transition series as would be involved in the above description (Fig. 26 a-d).

The hemichordal brain indicates alike derivation from an advanced nemertean ancestry, and degradation of the main nerve centers. For it is largely located on the dorsal surface, and there also gives off a strong median dorsal branch. But its diffuse flattened aspect, its loss of definite brain lobes, and its continued communication by neuropores with the epidermis, from infolding of which it is derived, all indicate simplifying and diffuse departure from a primitively higher type of organization.

The gradual expansion and diffusion of the dorsal and ventral ganglionic centers of nemertean type over the enlarging collar, and the shifting backward, during the process, of the ganglionic ring, till it occupies a position in the posterior part of the collar, in Balanoglossus, are evidently correlated with the increased use of the permanently exserted proboscis as a burrowing organ, and the utilization of the collar as a mechanical, muscular, sensory, and respiratory basis for the proboscis. The gradual shortening of the invaginated part of the proboscis, the conversion of the muscular and nervous ring of it into the strengthening fibrous layer of the proboscis neck in Balanoglossus, and the great development of the muscular coat of the proboscis round the proboscis colom, are all modifications that seem to have proceeded from a nemertean to a balanoglossoid type, on the plan indicated in Figs. 26 b and c. 
Its derivation as a group from an advanced nemertean and protochordate ancestry is suggested by the well-developed gill system with gill-pores and branchial sacs, by the bloodrascular system and other details that cannot here be discussed. So, as the illustrations suggest, it can best be viewed as a derivative from a decidedly specialized marine nemertean and protochordate offshoot of the originally fresh-water nemerteans, that shows structural tendencies of an evolving and a devolving kind, which together now typify the class.

The members of the Urochordata that we know as freeswimming ascidians represent a greatly more advanced type of organization than the last, and also are derivatives from a more direct nemertean-chordate stock than are the last. Thus the embryological stages in formation of the notochord, of the neural canal, of the body cavity, and other organs are exactly and typically of a "chordate" character. But the group as such furnishes no key as to the causes for origin of the notochord, for massing of the nervous centers and main nerve-trunk on the anterior dorsal region, or for the origin and position of the branchial sacs. All of these become clear when we refer them back to a nemertean ancestry, and to the relation there of proboscis-sheath and proboscis with the future notochord.

But the free-swimming ascidians are exceptionally interesting as being a group that fairly perfectly connects the nemerteans with Amphioxus and the cyclostomes. As to their derivation, the view seems most consistent with all the details of their structure, which would link them as derivatives from a marine protochordate ancestry that itself originated from a fresh-water type intermediate between the fresh-water nemerteans and a primitive ancestor of the cyclostomes. The degeneration that has taken place through fixation or sluggish response to environal stimuli, even in colonial groups, has caused the entire class to become a striking biological cul-desac. Secretion of defensive materials round the body has prerented extinction, and even contributed to increase and geographical distribution. The position of the class, as now estimated, is set forth in the chart. 
The Cephalochordata or group of the lancelets finally claims attention. These still more clearly than the last, and in all stages of their life-history, proclaim evident chordate characters. But the elongate notochord, the simplified spinal cord, the simple saccular liver, and other structural details, are all correlated with the burrowing habits, and indicate simplification by degradation.

In summary then we would conclude that fresh-water nemerteans, derived from a rhabdocoel turbellarian ancestry, became in increasing number marine, in part remained fresh-water, in part assumed life on land but amid moist surroundings. From the second of the three the Protochordata evolved, by conversion of the nemertean proboscis-tube, into a notochord, by modification of the proboscis into highly sensitive accessory pharyngeal parts, and by resulting development, specialization, and condensation of the brain-masses along the anterior dorsal region, where nerve action and reaction were centered round the proboscis, mouth, and dorsal sense organs.

Migration seaward of protochordate members originated the urochord and later cephalochord series, while the main protochordal stem gradually evolved into a protocyclostome and later a paracyclostome and cyclostome series. Some of these remained in fresh water like species of lamprey that are now world-wide therein, others became sea migrants but homing species in spawning and early larval years, as is true of the sea lamprey, still others like Bdellostoma and Myxine became littoral or deep sea genera. From the first we would trace the origin of the Protoamphibia and later the Apoda, that tended increasingly to pass from a fresh-water to a moist land environment, at least during part of their life history. The last in turn evolved the urodele Amphibia, from which, by transformation changes already indicated, the marsupial mammals evolved. 


\section{CHAPTER XIX}

\section{THE RELATION OF HIGHER ANIMALS TO MAN}

In zoological studies when the term "higher animal" is used, this is generally understood to be synonymous with one of the higher mammals, or at least with one of the higher vertebrates. Yet were we to study attentively a rabbit, a pig, a sheep, even a cat, alongside a lobster, a spider, a bee, or an ant, as to their movements, life relations, and successful survival as species or groups, a decided hesitancy might be felt in determining whether the former or the latter four deserved better the appellation "higher animal."

In the present chapter we propose to analyze and compare the structural details and life phenomena of groups of animals which, while apart-often widely so-in the zoological scale, all deserve to rank in a certain biological sense as "higher animals."

In immediately preceding chapters an effort has been made to trace the main line of animal advance that has culminated in man. But, while man represents the climax of organic evolution, it can truly be said that at least four, possibly six, other lines of marked advance are made from widely apart centers of origin. These all show an advancing bio-cognitic complexity and alertness that run parallel with a rapidly evolving cogitic perceptive response that has been superadded to the other two.

But, in trying to reach just conclusions here, human prejudice and hereditary bias are apt to sway the mind powerfully. For unless a living object conforms to certain preconceived notions of what we regard as a "higher animal" the evidence may be somewhat scantily weighed. In this connection Gaskell in weighing such evidence has truly written: "The law of progress is this: The race is not to the swift, nor to the strong, but to 
the wise" (176:19). And this was the summing up also reached by the writer on review of the apparent main line of zoological progress (p. 503).

It will generally be conceded that a highly evolved animal economy would be one in which the skin or environally irritable system, the protective or defensive system, the skeletal or strengthening system, the muscular or motor system, the alimentary or digestive system, the respiratory or aerating system, the nervous or sensory correlàting system, and the reproductive system are all sufficiently evolved and balanced as to ensure ample perpetuation and continued steady evolution of the species alongside destructive agents - either inorganic or organic. In a sentence it might be said that the ensuring of continued and even progressive synthesis in the midst of agents that might promote analysis represents true biological success.

Viewed from such a standpoint it can be said that at least five pronounced lines of evolutionary advance can be traced, that have branched off from the main line. Each of them, while showing sedentary or even degenerate species, contains others that have advanced to a high degree of specialization. These five in the apparent order of time and of evolutionary complexity are: the Cephalopoda, the higher Arachnida, the higher insects, the higher birds, and the higher mammals that are inferior to man. When therefore in this chapter we use the term "higher," we refer to those of the above five groups that have decidedly evolved along highest lines beyond the average of their kith and kin, and greatly higher than those of their kith and kin that have become degraded.

We incline much to add a sixth probably less important group, the Crustacea, did we know more, and more minutely, of their sense responses and modes of life. For the present we place it aside.

Now when one compares all of these with man, and with an average type of each group, it becomes evident that the most important somatic system is the nervous, since alike in its complex molecular substance and in its functional comnections it is the governing and correlating system. Spencer, Darwin, 
Lauder Lindsay, Romanes, and others in recent years have all emphasized the striking mental advance shown by members of the above groups over their fellows, or even over related groups that have shown little advance or even retrogression.

But our present aim is to demonstrate further the intimate bond existing between advancing nerve complexity and the exhibition of biotic, cognitic, and cogitic energies in connection therewith. Here it will be observed that the convenient and also morphologically correct hard and fast division into invertebrates and vertebrates, or into the more important minor groups of these, is set aside, for, while alike convenient and natural as an aid and index to general morphological and taxonomic distinctions, it entirely breaks down when we view animals from the biological or, to express it more exactly, the most energized and nerve-responsive standpoint.

The principles now to be dwelt on and expanded may first in part be illustrated by the annexed diagram in which the solid black lines set forth the taxonomic as well as ancestral dignity of the groups, while the broken lines indicate the degree of cognitic and of cogitic advance to which the highest representatives of the groups have attained (Fig. 27).

In reference to this diagram it may at first glance seem strange and even false to regard ants as greatly higher than fishes, batrachians, or even reptiles and many birds, even though Lubbock half a century since wrote: "If we judge animals by their intelligence as evinced in their actions, it is not the gorilla and the chimpanzee, but the bee, and above all the ant, which approach nearest to man" (17\%: 65.) But, if the evidences advanced below prove anything, they clearly demonstrate that some genera or groups, owing to certain combined biological factors, have run far ahead, in certain evolutionary characteristics, of the arerage members of their group. It is of prime importance then to learn what these factors have been, and how they have operated.

In the embryological development of every animal from the lower nemerteans upward, the first fundamental change noted in the multicellular embryo is the formation from the epi- 
dermis of a longitudinal tract that sinks inward to become the central nervous system. Next, three epidermic involutions of tissue sink inward round the head region to form in almost

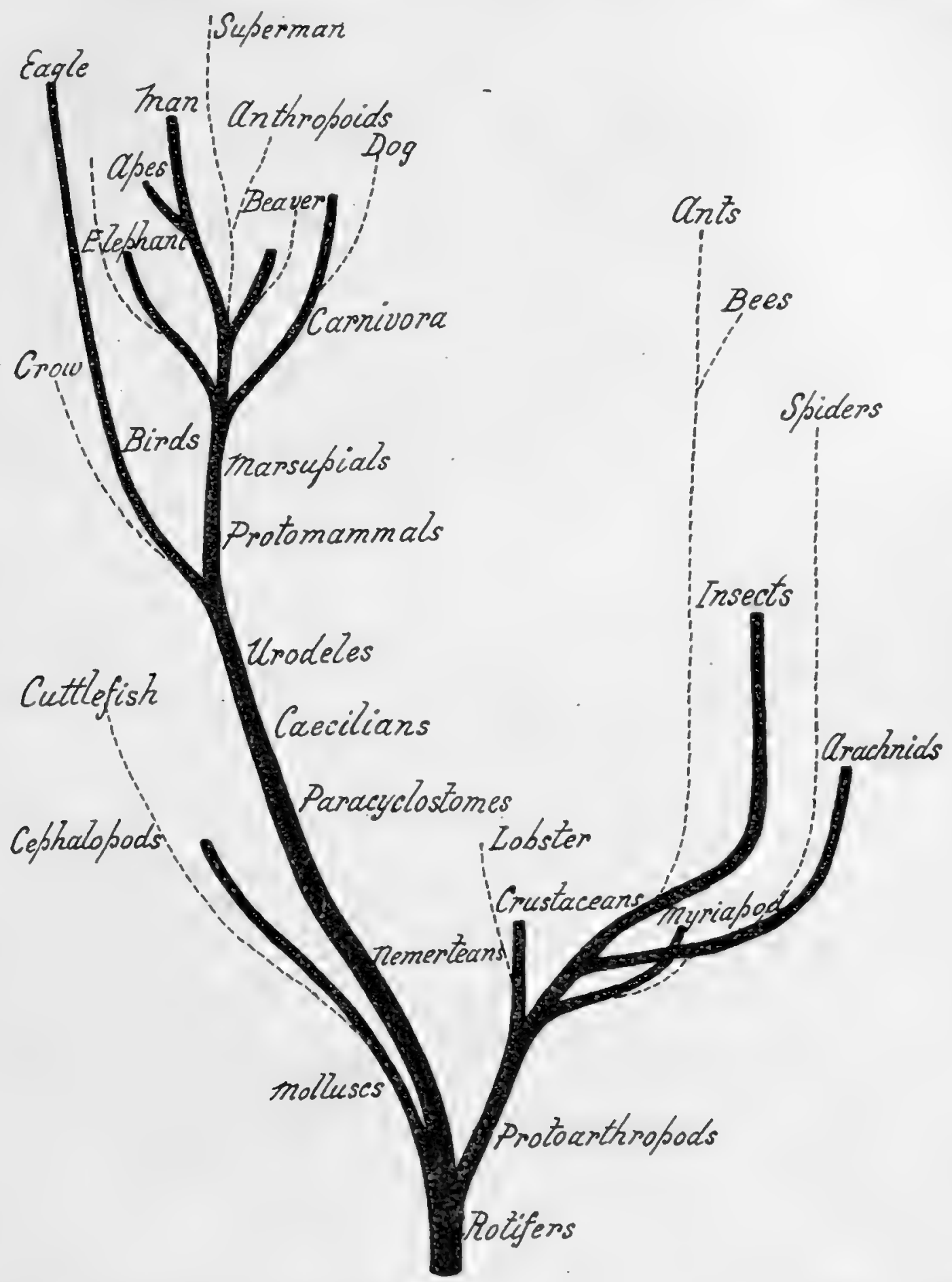

FIG. 27-Diagram to show comparative taxonomic and biologic relation of higher animals.

invariable order of appearance (1) the olfactory tissue or special chemotactic center, (2) the optic tissue, or special heliotactic center, (3) the auditory tissue or special tonotactic and geotactic tissue. Later other epidermal modifications, already re- 
ferred to under Nemertinea (p. 420), Cyclostomata (p. 429), etc., arise over various parts of the skin, that form electrotactic, surface chemotactic, thermotactic, hydrotactic, mechanotactic, and thigmotactic tissues. These all correspond functionally to certain tissues in higher plants, if we except the tonotactic, while in the animal, as in the plant, the response of the animal when these tissues are stimulated is a positive or negative chemotropic, heliotropic, paraheliotropic, thermotropic, geotropic, electrotropic, mechanotropic, or hydrotropic one.

The writer is unaware of any stimulation action in animals which differs from that of plants, except it be the tonotactic, and this, as several writers have suggested, is probably only a highly sensitized and condensed expression of mechanotactic stimulus. The epidermal system then, or some direct derivative of it, is that which receives, conveys, condenses, links up, and finally responds to, all stimuli in the animal kingdom. But, as compared with the diffuse condition in plants, definite groups or areas of cells are set apart to receive definite stimuli. It should however be kept in view that the very limited irritoperceptive area for geotropism in plants, and equally some heliotropic areas, are as condensed as many animal centers of impression. Similarly, the irrito-contractile centers or regions in leaves and leaflets of Dioncea, Drosera, Mimosa pudica, etc., are highly sensitive to several types of energy.

It follows from the above that the comparative size, weight, complexity, and tissue resistance of the nervous system may furnish a fair index to the comparative biological dignity of any individual, genus, or group. To a large extent it is the recognition of this relation that has caused $J$. Loeb to give us the remarkable and suggestive work "Comparative Physiology of the Brain and Comparative Psychology." But further evidence will be adduced to show that with the appearance of the nerve cell, the first recognizable beginning of organ formation for cogitic energy starts, and that, as the nerve cells become increasingly multiplied, linked up, and concentrated, in corresponding ratio does the animal show increased intelligence, memory, thought, reason, and complex volitional acts generally. 
From such a standpoint it will clearly appear that each of the five above-mentioned lines of animal progression approach - though by varying degree - much more nearly to man, than do others that started at the same level with them in the animal scale, but which, from environal action and proenvironal response, have lagged behind in the sweep of evolutionary advance.

Since in all of the bilaterally arranged animals the brain is placed anteriorly, those organs or appendages which can come in contact with environal stimuli most richly can most economically be aggregated round the head. Furthermore, if any structures or appendages, by proenvironal action, become increasingly utilized for conveying a variety of stimuli to the brain, or for conveying motor impulses from it, on the dynamical law of "action and reaction" the brain and accessory head appendages whether they be antennæ and mandibles, or tentacles, or forelimbs as a whole, or hands, will reciprocally affect, and be affected by stimuli from, these appendages.

If such principles be true, they should receive marked confirmation from study of the five or six above-named series of animals that have run ahead of most genera of their natural groups. These will now be examined for that end.

The Cephalopoda represents a very ancient group of molluscs, abundant representatives of which can be traced from the ordovician and silurian systems up to our day. The genus Nautilus, that is still alive, is closely related to forms that show such geologic range. In all of these the arms or tentacles result from forward growth of the muscular foot round the head, and subdivision of this into lobes. These lobes in Nautilus may be 90 or more, are smooth, and are devoid of hooks or suckers. Their function is - as in the feelers of the snail-tactile, apparently chemo- and mechanotactic for food material, while the strong shell has served as a protective covering.

But gradually, in genera of the group, the arms have become equipped with hooks or suckers or both, and have become reduced in number till now 10 or 8 are alone developed in other surviving genera. But these have become highly specialized 
structures for bringing the organism into most direct, rapid, and varied contact with its environment. The arms therefore of Loligo or Octopus are now tactile, prehensile, fighting, locomotory to a slight degree, and food selective. In corresponding degree then to perfectioning of the arms, the originally diffuse ganglia of molluses have become condensed in Nautilus to a broad ring round the gullet, as well as distinct buccal, pharyngeal, and visceral masses. In Loligo even these ganglia have become condensed into a huge ganglionic swelling that is almost on a level with the insertion of the arms, the eyes, the probable olfactory, the auditory, and the geotropic organs. Here is strongly suggested a direct action and reaction relation between the environal organs - especially the arms with varied functions - and the "greatly enlarged "brain" of the comparatively recent genus Loligo.

One result here, and biologically a very striking one, has been that the external protective shell has gradually been discarded, and the now soft-bodied animal trusts to less cumbrous but more intelligent means for offense and defense. This method has largely been adopted and has largely proved successful amongst many groups of animals in the struggle for existence. The shelled and mailed ones have often disappeared - like the cumbrously cuirassed knights of old-while the softbodied but resourceful ones have usually "pulled through."

That Loligo and related higher cephalopods have an extreme agility, resourcefulness, and caution is already fully recognized by naturalists, though abundant observations and experiments are still much needed. But the fact that the extensive and striking pigment-cell system is directly connected with the optic nerve (133: 380), and that the color of the animal can be changed almost instantly from gray to steel, to bluish, to purplish, to crimson-brown, and back again to gray, indicates capacity for rapid and delicate response to nerve stimuli. Again Romanes (50: 29), quoting first from Schneider, says: "The Cephalopoda show unmistakable evidence of consciousness and intelligence. This observer had an opportunity of watching them for a long time, in the zoological station at 
Naples; and he says that they appeared to recognize their keeper after they had for some time received their food from him. Hollmann narrates that an octopus, which had had a struggle with a lobster, followed the latter into an adjacent tank, to which it had been removed for safety, and there destroyed it. In order to do this the octopus had to climb up a vertical partition above the surface of the water and descend the other side. According to Schneider the Cephalopoda have an abstract idea of water [hydrotactic rather he should probably have said: author], seeking to return to it when removed even though they do not see it."

The writer once secured a Loligo, in a rock pool of some size, and experimenting with it for fully an hour, learned how alert, rapid, cunning, and determined it was, while the almost continuous play of color over its body suggested a highly excited state. Contrary also to the oft-repeated idea, it did not eject its inky fluid till all other means of escape from its tormentor had been exhausted. Then after many attempts to hide amongst the seaweeds, to escape by a shallow passage, to simulate the surrounding stones and weeds, it only as a final resource squirted out jets of brown-black "ink" that effectually hid it in the somewhat extensive but shallow pool.

One type, as well as one advantage and undoubtedly a most powerful one of environal stimulus, the Cephalopoda have not developed, viz.: the social or cooperative. They are solitary, combative, essentially analytic animals, that live for themselves.

The next series of highly evolved animals is that of the Spiders, amongst the Arachnida. That the latter is a very ancient group of land animals is proved by the discovery of forms near the living scorpions and spiders in beds of the carboniferous system. Whether their nervous complexity approached that of recent ones it is impossible to say.

Shipley speaks as follows: "The Arachnida, together with the Crustacea, Insecta, Myriapoda, and Peripatus, make up the great phylum Arthropoda, a phylum which, from the point of view of numbers of species and individuals, is the dominant 
one on this planet, and from the point of view of intelligence and power of cooperating in the formation of social communities is surpassed but by the Vertebrata" (179: 255). Some families of the Arachnida, like the ticks, the mites, and the gall-mites, may be more or less degraded offshoots from higher orders of the Class, but the scorpions and spiders specially concern us at present.

The viviparous habit, and the relation of mother to offspring, when these are hatched, indicate a degree of parental regard that is the basis for some of the finer mental qualities. The chemotactic and mechanotactic functions are highly developed, and it would be an interesting inquiry to ascertain to what extent the large dorsal neural mass is olfactory or chemotactic in function. Fabre's account of the scorpioid genus Buthus at once suggests a decided atrophy of some senses, and a high development of others, specially the thigmo- or mechanotactic. But the studies of McCook, Fabre, and the Peckhams, amongst others, show that the spiders in spite of their solitary lives have evolved high instinctive relations, that verge in not a few cases on simpler reasoning powers. For in making often distant but secure ties for the webs, in methods of swathing captured prey, in evolving and continuing mimetic resemblances between nest and environal objects, in cleaning their persons, and many other acts, there is such an interlinking of instinctive and reasoning qualities that man himself has to learn greatly more about them, and has to interpret more keenly and analytically, before final conclusions can be reached. But the want of cooperative effort in the entire group has undoubtedly prevented the development of those associative mental conditions which have so largely contributed to the elevation of other and higher groups like those next to be mentioned.

The third series of highly evolved animals is that of the bees and ants amongst the Insecta. In many families of insects the limbs and accessory mouth organs are used largely or wholly for progression and mechanotactic action. The eyes are usually well, or even very highly developed, but doubt exists as to the location of olfactory and auditory organs. The 
body, though usually encased in a hard chitinous exoskeleton, is brought fully and delicately into tactile-perhaps even other stimulation-relations by abundant nerve hairs that pass through holes in the chitinous layer.

The nervous system, in the more generalized forms, is an elongated double cord, with supra- and sub-œsophageal ganglia, also added ganglia that are scattered along the ventral cords. With increasing specialization and diversity of environal contact the first two of these ganglia steadily enlarge till they greatly exceed the others in size and complexity. It is therefore quite exact in such a case to speak of the mass as a brain. This brain even in high insect individuals of the same species may vary according to use. Thus Brandt finds that "the absolute size is not a criterion for the amount of intelligence, and we must rather look to the complication of the structure and to the development of certain parts for an index of this nature. The drone in the honey-bee has, correlatively with the superior development of its eyes, a larger brain than the worker, but the size of the hemispheres and the development of the gyri cerebrales are superior in the latter. In other words the mass of those great lobes of the brain that are directly connected with the faceted eyes must not be taken into account in consideration of the relation of the size and development of the brain to the intelligence of the individual" (181: 119).

As regards brain-weight in relation to body-weight Lowne (176: 120) has found that in insects it may vary from $\frac{1}{25000}$ to as much as $\frac{1}{150}$. In man the proportion is $\frac{1}{35}$ to $\frac{1}{40}$ while in the higher apes it varies from $\frac{1}{160}$ to $\frac{1}{180}$. The brain of the ant fills a large part of the head, and as figured and described by Leydig, is of highly complicated structure. The muscular system is also powerfully developed, and the striped muscle fibers of some insects - not least the ants-are amongst the most perfect in the animal kingdom. Thus have resulted the powerful tugging, lifting, shouldering, carrying, and other movements that one often witnesses in watching ants on their hills, or along their pathways.

Now if we concentrate attention on the brown or allied ant, every movement of the body indicates that it is constantly 
making environal contact by the antennæ or by the mandibles with each object passed, both of these sets of appendages being close to and ancillary to the head. By steady and continued use of these, in immediate connection with the brain, action and reaction have caused increased perfection of both. So, as compared with average insects, ants exhibit a range of activities that is only equaled by the more highly civilized human races, and even by educated individuals of these only. Thus they show extremely fine sense of touch, while the prehensile, carrying, cutting, building, digging, storing, feeding, harvesting, fighting, slave-making, and other activities are practised.

In connection also with questions taken up in later chapters, it should be emphasized that many of the acts which fall roughly under some one of the above categories are capable of a more extended mental analysis. Thus the housing and the feeding of Aphides by some ants, in order that they may be milked for their excretions; the nibbling and destruction of the radicle in grains that are to be stored; and numerous like acts, reveal a proenvironal analysis, synthesis, and response which is superior to that shown by savage races of men.

Now if one takes account in the above list of acts performed in environal relation, these result from many and often complicated stimuli passed into the central nervous system, through one or more of the circum-cephalic appendages, and which are aided or guided by the optic, olfactory, or other primary sense. But, while the more primitive insects have also these primary senses, brain increase has proceeded but little, since the active use of the accessory appendages has not been practised to at all so varied a degree. Strong evidence is therefore again suggested that brain increase and brain complexity proceed pari passu, with increasingly varied and active stimuli from without, that are passed in through actively movable appendages accessory to the brain.

But even stronger evidence is got when we compare the brain of those ants which have evolved three grades of indiridual, namely the most active or worker ants, the more passive and sedentary or female ants, and the least intelligent or 
males. The brain, according to Forel (180), is largest in the first, smaller in the second, and least in the third. Brandt and Kenyon have since shown that a similar condition obtains for bees and other of the social Hymenoptera.

In such cases therefore, primitively monomorphic species of insects, belonging to different groups, have slowly evolved a physiologically and morphologically distinct trimorphism. The type that used its accessory head parts in most active, varied, and continuous manner has correspondingly built up the largest and most complicated brain mass; that which used these least shows corresponding retardation.

But in the life-history of ants, as in that of bees, many wasps, and other insects, an important difference is observed in their individual and specific economies, as compared with those of the arachnids and cephalopods. This is the often highly evolved and mentally specialized exhibition in ants of social union for the general good, not merely of a colony, or of a group of them, but at times for the good of entire species.

Thus if we follow the exquisite and lucid descriptions of Huber for the ant, supplemented by the later studies of McCook, Lubbock, Forel, Wheeler, Janet, and others, as they have described the road-clearing, nest-rearing, tunneling, brick-making, plastering, door-construction, formation of granaries, drying of grain, conveyance of food, rearing of young, etc., that are engaged in, every candid and honest mind will acknowledge that this is not only equal to average human mentality, but that it greatly excels conditions shown by races of men now alive. It is a poor and paltry as it is an unscientific apology to make in trying to belittle their efforts, when one says that in returning to their nests they are utterly lost, if they miss their scent trail. Ninety at least out of every hundred civilized human beings would perish, were they to lose their eyes suddenly and be prevented crying when five hundred yards from their home and in the midst of a forest.

The fourth species that includes highly specialized animals morphologically is that of the higher birds. The group is specially noteworthy, for, as has often been remarked by zo- 
ologists, the body of most birds is constructed on a more perfect plan than even that of man. But a specialized body plan is one thing, a means for stimulating brain activity and brain increase is a very different one. This will be shown to apply strikingly in the present instance.

For the great majority of birds environal contact is effected mainly by the primary sense organs, specially those of sight, sound, and in a few doubtful cases of smell. But the bill also in relation to the actively moving head is often a most efficient conductor of tactile and possibly also of chemotactic stimuli. The accessory appendages here however, in the shape of forelimbs, have become wholly modified for the one function of air motion. But this one mode of stimulation of the brain, and reflex action from it, is of very limited range in variety. In the most mentally alert however, like the parrot, the crow, the jackdaw, and others, one foot is often used as a manual organ to grasp, to tear, to weave, to roll, to climb, and in conjunction with the bill to carry on varied acts, specally those connected with nest-building. This is in marked contrast to the other group of Sauropsida-the Reptilia-in which the limbs are purely organs of locomotion or are atrophied. The above-named birds, then, rank as the most intelligent and resourceful of their class.

But in birds the exceptional powers of flight have acted so as markedly to enlarge and complicate the cerebellum over what we find in reptiles. This has doubtless been aided, on the principle of action and reaction, by the increasingly quick alert movements, when they alight on the ground, and which shelter them from the attack of other animals. But though birds have evolved along an entirely distinct line from the mammals, and in some respects along a higher line, their brain has remained decidedly smaller relatively; it is also simpler, smooth, and less subdivided into cerebral areas. This result we would interpret to be largely if not wholly due to the modification of the forelimbs into wings, and the absence of any good accessory appendage, except in some cases one foot, for aiding in the rapid conveyance of varied stimuli to the brain. 
We thus consider that the increasingly high specialization of the anterior limbs for one particular and circumscribed function may become associated with a correspondingly high degree of specialization in the tissues, and may also reflexly confer alert and energetic movement for most parts of the body, as well as safety in connection with the organism's ability to secure food quickly and widely. But the size, complexity, and weight of the brain may not advance in corresponding ratio, owing to modification of the forelimbs into organs that have one limited function, and which thus cause a limited and uniform set of stimuli to pass to the brain, and so a limited degree of reaction to be exhibited by the organism as a whole.

The decidedly gregarious or social habits of the crow, the penguin, and not a few other birds doubtless have exerted a stimulating action on the brain. But until a minute comparative study has been made of the weight of the different centers of the brain in solitary as compared with gregarious species it will be impossible to reach any exact conclusion.

We have already accepted that the marsupials represent the most primitive group of viviparous mammals, and that strong evidence exists, alike palæontological, anatomical, and taxonomic, for considering that several families have evolved from them, of which the Carnivora, the Insectivora, the Rodentia, the Lemurida, and the Anthropomorpha are directly derived descendants from them. In the cretaceous and early tertiary beds all of these groups are well represented, and have assumed anatomic features by which they are classified. One group of mammals, however, that will receive attention in this chapter is that of the Proboscidea or elephant and mammoth series.

The evolutionary origin of the group is still very imperfectly known, though several palæontologists have furnished evidence of remarkable semi-primitive forms, mainly from the eocene of Egypt. Since that time to the present day the proboscis has doubtless been slowly evolving, and, as we shall try to indicate below, on the principle of action and reaction.

When comparison is made between the brains of the early mammalian groups cited above, and their existing tribes or 
representatives, a marked increase is noted in brain capacity and complexity. But this is more marked in some genera, tribes, or classes than in others, and reaches its climax in the higher Primates. We will now endeavor to trace the cause or causes which have operated to bring about this result.

If we start with primitive pentadactyl marsupials like the existing opossums, we are led by fairly easy transition typeseither existing or extinct-to the Phalangeridæ and Phascolarctinidx, that in turn exhibit decided resemblances to some of the ancient forms of Lemuridx. Now in all of these the forelimbs are more and more used for a variety of purposes other than progression, and not least for actions that aid, and are directly centered round, the important sense organs of the head. For this end the animals often rear themselves upward; grasp food and convey it to the nostrils and mouth; tear it to pieces; excavate nests for themselves, or grubs and other food from rotting trees. In short, the anterior limbs are increasingly used as arms and hands, that reach out in correlation with the eyes and nose, to environal objects and convey therefrom varied stimuli to the brain, through the different sense-centers of the head.

These therefore stand out in more and more marked contrast to the kangaroos and allies, in which the forelimbs, even though raised in the air with the often semi-erect body, are almost wholly organs of progression, accessory to the highly evolved hindlimbs, that are almost purely cursorial.

From the upper cretaceous rocks on to recent deposits a succession of advancing and related types such as Indrodon, Anaptomorphus, Adapis, and others, all indicate advancing affinities to the higher apes or Primates. But again the ancient American genus Mixodectes shows characters that place it somewhat intermediate between the Rodentia and Lemuroidea, while we have already suggested the origin of the Rodentia from more ancient gnawing marsupials, thus indicating that even the Rodentia, as we now understand it, may have had a somewhat composite origin. The evolution of the Carnivora also from carnivorous marsupial ancestors has already been considered ( $\dot{\mathrm{p}} .498)$. 
We have here then three diverging lines of placental mammals, that might be compared in some of their genera from the standpoint of mental evolution in relation to the utilization of appendages as conductors or originators of stimuli. Many of the Rodentia are purely cursorial animals, and receive environal stimuli from without only by the eyes, nose, ears, or skin. But in the squirrels, marmots, and others frequent use is made of the forelimbs for climbing, prehension, holding, and scooping. These are amongst the most alert, active, and mentally highly evolved of the group. But even they are excelled by the beaver that utilizes forelimbs and teeth as accessory structures for environal contact. The discernment and capacity for gnawing trees into suitable length, for guiding them in the water, for hauling them to their settlements, for rearing lake dwellings therefrom, for placing, plastering, and uniting them in so doing, for cooperating with each other in the common colonial work, and for defending the whole, indicate at once the passage inward to the brain of many and raried stimuli, as well as efferent impulses correlated with these. The beaver, therefore, exhibits a surprisingly high cognitic as well as cogitic capacity, the latter having enabled the group to elaborate and adopt a fairly perfect system of colonial or social living. Morgan's apparently exhaustive description suggests that more extensive observations and especially experiments might yield an added harvest of results.

Now the beaver is allied to the rabbit, guinea-pig, and other familiar rodents, that display an intelligence little if at all higher than that of the most simple mammal. But a rery fair index is got to their relative mental development, if we note the degree to which the forelimbs are used for progression only, or for added and more diversified environal acts. So, while the guinea-pig, the rabbit, the capybara, the squirrel, the marmot, and the beaver show close anatomical affinity, in active cogitic response they possess an increasing intelligence in the order given, to a degree that raises the last greatly above the first.

The remarks of Romanes therefore are so far true (50: 353): "In no other group of animals do we meet with nearly so strik- 
ing an exemplification of the truth that zoological or structural affinity is only related in a most loose and general way to psychological or mental similarity. Up to a certain point, however, even here we meet with an exemplification of what I may call a complementary truth, namely, that similarity of organization and environment is in a general way related to similarity of instincts (though not necessarily of intelligence). This is obviously the case with the habit from which the order takes its name; for, whether the instinct of gnawing is here the cause or the result of peculiar organization, the instinct is unquestionably correlated with the peculiarity. And similarly, though less obviously, is this the case with the instinct of storing food for winter consumption which is more prevalent among the rodents than in any other order of mammals-rats, mice, squirrels, harvesters, beavers, etc., all manifesting it with remarkable vigor and persistency. Here we probably have a case of similar organization and environment determining the same instinct; for the latter is not of sufficiently general occurrence among all species of rodents to allow us to suppose that the species in which it does occur have derived it from a common ancestry."

As will be explained later, we would attribute practically all of the above "instinct" and "intelligence" to environal action and proenvironal response. This starts, by long continued repetition, definite stereoenergetic currents, along certain tissues, that effect a stereo-chemical placing of definite molecules. There thus arises an acquired and inherited act, through lines of energy flow proceeding more readily along one pathway than another, and so determining the nervous, the muscular, the osseous, and other modifications that have generally taken place.

But we would take exception to the first clause of the above quotation from Romanes: "In no other group of animals," etc. For between a $N^{\prime}$ untilus and a Loligo, between a hemipterid and an ant, between an Apteryx and a parrot, the cogitic differences are at least as great, we should incline to say much greater, than between the least and the most advanced rodent mentally. 
The sagacity and high mentality of the elephant have evoked comment since the days of the early Greek naturalists. The large and highly convoluted cerebrum, though not greatly expanded posteriorly, is in keeping with the high mental development. Though our knowledge is still fragmentary, as to its earliest ancestors, it has evidently been derived from some form like Moritherium found fossil in the mid-eocene of Egypt. Starting with some such type the entire naso-maxillary region has steadily elongated and become flexible. Were the question asked: What would the elephant be without its trunk? all probably will concede that it would be a very sluggish and unintelligent animal. With small eyes, heavy neck, rather slowly moving head, awkward gait, digitigrade progression on heary feet, and thick tough skin, it is the opposite of an animal that might be expected to show high brain capacity. The evolution of the trunk explains the entire problem we believe, and this on the principle of action and reaction along proenvironal lines. For the proboscis is one of the most sensitive and plastic structures in the entire mammalian world, excelling in some respects the human arm.

By it the animal feels delicately, coils round objects, reaches to heights above its head and to distances otherwise beyond its range, grasps even the smallest objects, places and balances loads on its tusks, absorbs and distributes water over its body or to its mouth, conveys food to the mouth, absorbs and shoots out sand, projects objects that are out of reach by airjets, so as on rebound to seize them, constructs conduroy paths through swamps, and not least uses its trunk as an organ for showing caressing endearment toward members of its own herd.

Now, in the works of Houzeau (182), Lauder-Lindsay (49 passim), Romanes (50: 386), and other authors, numerous examples are cited of high mentality, memory, and reasoning power, that place the elephant, according to some of these authors, almost on a level with the dog and higher apes. Such mentality is an effect of high brain organization. 'The stimulating cause for the mentality has to be looked for and explained. No part or parts of the body, taken separately or 
together, compare even in feeble degree to the trunk, as a medium for conveying afferent and efferent impulses. In fact it may truly be said that the proboscis for the nemertean and a like organ for the elephant have been the fundamental organs for evolving the mentality of both groups. Composed largely as it is in the elephant of muscle and nerves, these are in intimate association and almost constant action, while they are innervated by, and are sending constant environal stimulatory currents to, the brain, that is placed exactly in line with the base of the proboscis. So if one watch the huge impassive mass of a caged elephant when confronted by a throng of admirers and food-contributors, except for an occasional shifting of the eyes, the trunk is the centre of constantly changing motion. Even when the animal is undisturbed, or is asleep, the twitchings and movements of the trunk indicate its extreme sensitivity.

Is the trunk then a developmental result of brain stimuli passing to the snout region, or has contact stimulus of the trunk with surrounding objects gradually stimulated the nerve substance to cell multiplication and increase, or have trunk development and brain growth proceeded in corresponding ratio? Alike on physical and physiological principles, the last is the only alternative. For the chemic, thermic, - possibly also electric-energy derived from food supplies is here converted into biotic energy, that is passed into and energizes the trunk. This organ is constantly reaching out proenvironally to new stimulatory objects, aided more or less by the eyes, the ears, or the nose-tissue. These objects affect it chemically, thermally, luminously, and so start cognitic stimuli that travel to the brain, there to be stored and linked up with other impressions, or from the brain efferent responsive impulses proceed. By incessant action, continued in ever-increasing ratio from the times of the mid-eocene onward-let us say conservatively for two millions of years - these raried cognitic stimuli and responses have been changed into higher and greatly more complex cogitic acts, that constitute the "high mentality" of the existing species. 
As in the case of the beaver, of ants, and of bees, the pronounced social habits that elephants have evolved unquestionably have aided much in accentuating and fixing many of the higher cogitic features now typical of the group. The many accurately recorded cases of social sympathy, fear, hatred, anger, effort, and accomplishment are proof of such; while the cogitic acts shown by "rogue elephants" confirm this in a striking manner. A flexible and richly nervous protrusion of the naso-maxillary region here functions in similar manner, and as an equally efficient organ, to the forelimbs and the gnawing teeth in the beaver.

Amongst the Carnivora, and even amongst the entire series of mammals lower than the anthropoid apes, it is usually conceded that the dog holds first place for intelligence and alert response. For our present purpose also it is an exceptionally interesting type, since it illustrates in striking manner what education by a higher organism - in this case man - can do for a lower one, that in the wild state shows rather brutal and even ferocious instincts. But, from our combined knowledge of the wolf, the fox, and the dog in the wild condition, it can definitely be accepted that the dog naturally exhibits decidedly high cogitic or mental qualities. On the principle of environal action and proenvironal response, we would consider that the dog has evolved in the feral state through increasing and varied use of the olfactory, optic, and auditory senses, combined to a minor degree with that of the forelimbs. The last of these are used for digging, for grasping, for pawing, and for cleaning itself. The olfactory sense is exceptionally keen, and has evidently had much to do with the erolution of the species. For thus it can follow and run down its prey, it can distinguish tracks of different animals, it can quickly recognize one of the same pack, or any animals that have proved friendly, it can scent danger in the lurking presence of some enemy, it can even scent out its prey when such is as yet unscen. This extreme olfactory sensitivity-doubtless gradually accquiredcombined with keen sense of hearing and usually of sight, have cooperated with use of the forelimbs to send many environal 
stimuli to the brain, which in return has sent many outward efferent impulses that have tended to quicken speed, to select those with keenest sense organs, and to perfect by natural selection other parts of the body that may be subsidiary to the important centers.

But, when man began, certainly 6000 to 8000 years ago as in Egypt, possibly 20,000 to 50,000 years ago or more, as in the case of the cave men of West Europe, to domesticate the dog, new possibilities began to appear. As in many similar cases a start was doubtless made by some hunter saving one or two cubs of a specimen he had destroyed in the chase, and bringing these into his rude family cabin, where they were petted by and grew up with the children. It should also be borne in mind that the dog is unlike the "wise" fox in being more or less gregarious, and therefore in having the social habits more or less developed even before being domesticated. The following observations of Romanes (50:437) then become apropos: "The intelligence of the dog is of special, and indeed of unique, interest from an evolutionary point of view, in that from time out of record this animal has been domesticated on account of its high level of natural intelligence; and by persistent contact with man, coupled with training and breeding, its natural intelligence has been greatly changed. In the result we see not only a general modification in the way of dependent companionship and docility, so unlike the fierce and self-reliant disposition of all wild species of the genus, but also a number of special modifications, peculiar to certain breeds, which all have obvious reference to the requirements of man. The whole psychological character of the dog may therefore be said to have been moulded by human agency with reference to human requirements, so that now it is not more true that man has in a sense created the structure of the bulldog and grayhound than that he has implanted the instincts of the watch-dog and pointer. The definite proof which we thus have afforded of the transforming and creating influence exerted upon the mental character and instincts of the species by long and persistent training, coupled with artificial selection, furnishes 
the strongest possible corroboration of the theory which assigns psychological development in general to the joint operation of individual experience coupled with natural selection. For thousands of years man has been virtually, though unconsciously, performing what evolutionists may regard as a gigantic experiment upon the potency of individual experience accumulated by heredity; and now there stands before us this most wonderful monument of his labors-the culmination of his experiment in the transformed psychology of the dog."

It would be superfluous here to enlarge on the manifold ways in which the dog is now utilized by man. We would merely affirm that all of its actions represent a hereditary feral capacity, due to exercise of the four centers of environal contact above mentioned, all of which have been, in one breed or another, added to and made more complex by man. These environal stimuli have all acted on the brain substance to cause increasing growth and complexity in storing sense-impressions, while proenvironal responses have started that constitute collectively what might be called the canine character.

There remain for study in this chapter the anthropoid apes, some of which deservedly rank next to man in intelligence and mental capacity. It has been accepted here that they seem to be derived anatomically by direct descent from the lemurs. This accordingly condenses the evolutionary line in one sense, though it gives increased time-duration for organic changes to proceed in the component species and genera.

In regard to them Romanes wrote: "These from an evolutionary point of view are the most interesting. Unfortunately, however, the intelligence of apes, monkeys, and baboons has not presented material for nearly so many observations as that of other intelligent mammals. Useless for all purposes of labor or aid, mischievous as domestic pets, and in all cases troublesome to keep, these animals have never enjoyed the improving influence of hereditary domestication, while for the same reasons observation of the intelligence of captured individuals has been comparatively scant. Still more unfortunately, these remarks apply to the most man-like of the group and the nearest 
existing prototypes of the human race. Our knowledge of the psychology of the anthropoid apes is less than our knowledge of the psychology of any other animal. But, notwithstanding the scarcity of the material which I have to present, I think there is enough to show that the mental life of the Simiadæ is of a distinctly different type from any that we have hitherto considered, and that, in their psychology as in their anatomy, these animals approach most nearly to Homo sapiens."

The desiderated information has to a slight degree been forthcoming during the past twenty years, but greatly extended observation and experiment are still needed.

If one starts with Romanes' account (50:484) of the doings of the Brown Capuchin (Cebus fatuellus), he is at once arrested not by descriptions given for movements of the eye, the mouth, the skin, the nose, or even of the body generally, though these all cooperate appropriately but to minor degree. The outstanding feature is hand-arm environal action that starts cognitic and cogitic stimuli, which result in proenvironal response.

Thus to abstract so far in the order given he struck nuts with a dish; he covered himself at night with warm shawls; he dashed to the floor and broke a wine-glass in mischief; after throwing down an egg-cup without breaking, he smashed it against an iron bedpost; he broke a stick between a heavy object and the wall; he carefully destroyed a dress by pulling out the threads; he used a hammer appropriately to break walnuts; he threw a nut, a hammer, a coffee-pot, and his shawls at a lady who had laughed at his disappointments; he caught fiercely at a domestic's hand; he carefully pulled over, balanced, then let crash repeatedly a marble-top washstand; he secured distant nuts that were outside his tether's area by pulling them in with a stick, or by whipping them in with a shawl; he spun round apples and oranges, then put his long thin finger deep into the fruit, and withdrawing it sucked from the hole; he pushed an arm between a heary washstand and the wall, then levered out the former and dropped behind it; he pulled the glazed leather cover off a trunk; he hammered at a chain-ring for hours in an effort to break it; he angrily beat servants who supposedly had 
offended him; he unscrewed a hearth-brush handle, and after considerable manipulation of both ends again screwed it on; he beat people with a cane, or failing beat the floor by proxy; he inserted a trunk key into a lock and turned it; he shook hands with family friends; he poked sticks into the fire, withdrew them and smelt the smoking end; he opened and shut folding window shutters. So the author concludes with an encomium on its "tireless spirit of investigation," by saying "the hours and hours of patient industry which this poor monkey has spent in ascertaining all that his monkey intelligence could of the sundry unfamiliar objects that fell into his hands [ital. writer], might well read a lesson in carefulness to many a hasty observer." Holmes' chapter on "The Mental Life of Apes and Monkeys" amplifies the above description (183: 260).

But since Romanes wrote this the chimpanzee has been "humanized" in some historic specimens. Thus "Edgar," "Coco," "Esau," "Sally," "Master Link," and "Peter" have been placed freely amongst men, have walked with them, lived in most cases with them, and conformed to their customs in the street, at home, at table, and during sleep. Master Link, brought by a ship captain from Africa to New York, and sold after three years to a gentleman there, was reared by him as one of the family. After six months he behaved perfectly, and never once put his hand to the ground. Were one to trace and catalog his actions, they would greatly excel those given for the Capuchin, and in contrast were of an agreeable and kindly turn.

Now, if it be asked by what cause has the brain of the Capuchin excelled that of the lemur, or the brain of the chimpanzee that of the Capuchin, one answer seems alone satisfactory and explanatory. In each case environal contact has been effected to an exceptional degree through the hand-arms, and particularly so for the two last. These, guided mainly by the eyes as sense-correlators, have converted kinetic into proenvironal energy that has brought each species into contact with an increasing variety of objects. Stimulated by these objects, afferent impulses have passed into the brain as stereoenergetic 
stimuli, that have very slowly but all the more surely affected its substance. From the brain efferent impulses have proceeded, that have resulted in the diversified "life movements."

Continued proenvironal seeking out toward new and more varied objects has reflexly stimulated the brain cells and fibers to growth and multiplication. But as many, we might even say most, of the acts in the capuchins and chimpanzees inrolved rapid correlation and resultant selective choice of one amongst sereral stimuli or groups of stimuli-a position more fully set forth in a succeeding chapter-groups of cognitic stimuli have been linked together in the gray matter of the brain, and have there developed cogitic resultants of a compounded character, that have either been stored up as groups of molecular aggregates that constitute memory power, or have in part been expended in the form of active cogitic energy or intelligent acts.

From our foregoing review of what are biologically the "highest" animals, we therefore conclude that, for increasing tissue complexity, for advancing organization, for more and more elaborate capacity of receiving and responding to environal stimuli, rapid and dominant advance is only effected when the brain and accessory sense centers constantly receive stimulating flows of impressions from without, through mobile and sensitive appendages like the foot-arms of cephalopods; the mandibles, antennæ, and forelimbs of ants or bees; the bill and foot of birds; the proboscis of the elephant; the forelimbs - and head sense-centers more or less - of the beaver, dog, and ape.

So, as regards their biological dignity, these six or seven groups would be related, as set forth in the diagram, in the ascending ratio of cephalopods, arachnids, birds, beavers, dogs, elephants, ants, and chimpanzees. But many of the ants, as well as to a less degree dogs and beavers, are higher even than some of the apes, in the complexity of their constructive and general biotic-cognitic acts. Such we would attribute to the greatly higher social organization of ants, and to the more primitive though yet advanced social organization of beavers and dogs. 
All of the above shows that morphologico-taxonomic organization and relationship, along one main ascending phylogenetic line, by no means corresponds to, or is an infallible index to, the true morpho-biological, or-if we prefer to so call it-the morpho-physiologic advance. Rather we find that, wherever an animal is brought to or reaches a more and more varied or complex environment, then there pass stimuli to the brain, which there give rise to proenvironal responses, that for the time lead to a "satisfied state," till in turn new environal stimuli are presented. Such energizing stimuli in turn excite the biotic system, the muscular, respiratory, alimentary, osseous, etc., to new growth-change or adaptation. These changes also affect slowly the nerves and brain or cogitic cells, we would consider, so as to cause a gradual readjustment, or increase, or much more slowly a chemical alteration of their substance, all of which are more or less shared by and influence the generative cells, which in turn affect the succeeding organisms hereditarily. Or, to repeat once more our fundamental position: flows of energy, often and steadily repeated from sense-collecting centers, start stereo-energetic stimulation-acts, that inevitably affect the brain cells, and these by expenditure of cogitic energy give rise to proenvironal responses that constantly tend to place the organism for the time being in "satisfied" relations to its environment. 


\section{CHAPTER XX}

\section{THE PHYSICAL EVOLUTION OF MAN}

Emerson in his essay on Plato says (p. 21): "If the tongue had not been framed for articulation, man would still be a beast in the forest." Like many of Emerson's sayings the passage is striking, but we hope to show in this chapter that it is only very partially true. Nay more, we trust to demonstrate by the close of the chapter that change in two words of the above quotation would make it a correct and telling statement.

Much also has been said and written on the "noble upright posture" of man, and "the human face divine," not a little of which is as ludicrous as by strict standards of the older theology it should have been denounced as impious. Darwin's words in this connection are ever true: "It is our natural prejudice, and that arrogance which made our forefathers declare that they were descended from demigods," that causes us to foster many a priori or baseless convictions regarding man.

Lamarck, Spencer, Darwin, Hæckel, and many succeeding have minutely compared man's physical frame with that of the higher mammals below him. Their conclusions, that there is a direct continuity in structural detail, which even impresses itself on the mental and moral acts, have been so fully verified that few now reject their conclusions, and a recapitulation of their results here would be superfluous.

But all are agreed that the outstanding characteristics of man are his elaborate and complex brain structure, and his immensely superior mental powers in connection therewith. In the present chapter we desire to ascertain whether, on the principle of cause and effect, of action and reaction, of environal stimulus and proenvironal response, a definite explanation can be given for the origin and increasing derelopment of this superiority, of the causes which have effected such, and whether stages in the process cannot at present be traced. 
In the preceding chapter we have tried to demonstrate, and we trust successfully, that the more complex brain structure, and the higher mental qualities of diverse groups of animals, over others closely allied to them taxonomically, have resulted from an increasing proenvironal use of parts around and accessory to the brain. The stimuli thus sent in increasing number and variety to the brain have stimulated it to increase in its cells and fibers, and these in turn have sent an increased number and variety of efferent stimuli outward. We shall now endeavor to learn how far this principle can be made applicable to man.

Along the entire mammalian line that leads from the marsupials to man, we have seen that varied use of the forelimbs, and a rearing of the animal more and more into a semi-erect and ultimately erect posture, have proceeded pari passu with added complexity in brain structure and action response. In man, as in the lower animals, increase in quantity and complexity of the brain matter - a purely material though highly complex chemical mass - can only proceed through increasing quantity and complexity of the energizing currents that traverse it.

That the erect posture is an effect and not a cause is clearly proven by study of the mantis, of penguins and many other birds, none of which possesses an exceptional degree of nervous complexity. Again human cerebral increase has evidently not resulted from special stimulating inflows of afferent energy, through the special sense-organs as the following show.

Had olfactory stimuli, entering and affecting the nasal regions, been an important cause, we should have expected to find, as we do in the skate, the buzzard, the wolf, and the dog, a considerable enlargement in the olfactory lobes, and an increase in the cerebral tissue associated with these lobes. Such, however, is not the case.

Again, had heliotropic or light stimuli been of special stimulating value, we should have expected to find the optic lobes in man smaller than in birds and many mammals whose power of vision at the present day greatly excels that of man. But 
even the penetrating and far reaching vision of an Indian or an Arab has not started exceptional brain development in the associated centers for these races, nor for the human race as a whole.

The auditory function, and its related brain center, though often well-formed, by no means excels and often even is inferior to that of many lower mammals.

The high quality of the vocal cords, and the resulting capacity for articulation, undoubtedly aid largely in conveying afferent and efferent stimuli, as the efforts of every orator and vocalist demonstrate. But we trust to prove that these organs of Emerson's fancy have been greatly inferior, as primary agents, to another source of stimulation.

The skin as a chemotactic and mechanotactic recipient of stimuli is highly important, but the stimuli thus received are neither sufficiently varied nor elaborate as to constitute a prime factor.

Man then, like most animals, comes into intimate sense contact with his outer world by six, possibly seven senses. These are the smell and taste senses, or the chemotactic; the sight sense or heliotactic (phototactic if preferred); the touch sense or mechanotactic; the sound sense, or tonotactic; the pressure sense or geotactic; and probably the thermotactic sense or heatperceptive. All of these except the tonotactic are common to plants as well as animals. Further alike in plants and animals some of the above senses, like the mechano-, the chemo-, and the thermotactic are diffuse, that is, many areas or surfaces over the body generally can act as receivers of such energy. In both also sense-perception and response may be restricted to limited areas, as the geotactic in root-tips of plants and in one part of the auditory organ in animals; or the heliotactic sense that is often highly diffuse in plants, but may be restricted to optic centers in animals.

Man then and the higher animals have evidently not attained to their present high physical and mental organizations by the addition of any special sense or senses of high quality or importance, but rather by having their physical organiza- 
tion so adapted to a high degree as to bring the sense organs into most rapid and perfect contact with environal stimuli. In some turbellarians and in nemerteans this is effected by the proboscis, whose substance, attachment-area, and orifice of protrusion are very richly nervous, and are situated in the midst of the sense centers; in cephalopods, spiders, ants, and other invertebrates that are highly evolved mentally as well as physically, we have seen that other structures surrounding the brain and the various sense centers act as rapid and varied environal perceptive organs, while in mammals the most perfect, persistent, and dominant organization, physically and mentally, is associated with increasing specialization of the forelimbs as environal contact organs.*

The highest exhibition of this is seen in the chimpanzee and orang as outlined in last chapter. Now, if man in his physical organization has evolved by degrees from ancestral forms that had a common ancestry with the gorilla, chimpanzee, and orang, the new details that he has undoubtedly acquired during the process were a permanently upright habit, and all that this would involve in straightening of the long bones of arms and legs; in perfect plantigrade motion; in total cessation in use of the arms for progression; in condensation and almost vertical placing of the pelvis; in condensation and contoured balancing of the spinal column as related mechanically to the head, arms, and legs; in a more vertical facial angle and larger brain capacity; in greater prominence of the brow and reduced hairiness of the body.

We believe that it is possible to show that steadily perfected adaptability of the forelimbs for increasingly varied contact of these with environal objects directly or indirectly effected such changes. This gives, therefore, to the fore limbs a predominant importance in man's physical evolution, but we trust

\footnotetext{
* Did space permit an interesting study could be made, and comparison instituted, regarding the highly specialized limbs of Artio- and Perisso-dactyli, as connected with race dominance on the one hand, and brain-stagnation on the other, the whole resulting in an evolutionary cul-de-sac. This has in part been referred to by Osborn and others.
} 
further to show in next chapter that it has stimulated the development of his highest qualities.*

Since the appearance of Charles Bell's well-known work on the hand, emphasis has often been laid on its immense value as a part of man's physical organization. Darwin has not failed to note the molding and modifying results of the functional activity of the hands. For in "The Descent of Man" he says (p. 67): "If it be an advantage to man to stand firmly on his feet and to have his hands and arms free, of which, from his preeminent success in the battle of life, there can be no doubt, then I can see no reason why it should not have been advantageous to the progenitors of man to have become more and more erect or bipedal. They would thus have been better able to defend themselves with stones or clubs, to attack their prey, or otherwise to obtain food." And again, "the free use of the arms and hands, partly the cause and partly the result of man's erect position, appears to have led in an indirect manner to other modifications of structure."

Possibly no more graphic method could be devised for bringing before us the enormous - the wholly preponderating-importance of the forelimbs than by asking ourselves the question: What would man be without his arms? By these he secures, cooks, and eats his food; cleans his body; grows, collects,

\footnotetext{
* From a reference in Parmelee's work (53: 271), the writer's attention was recently called to Cunningham's valuable address before the British Association (Brit. Assoc. Rep. 61 (1901) 539) which bears strikingly on the above position. Speaking of the high development of the frontal and parietal lobes in man as compared with anthropoid apes, he says: "The skilled movements of the hands, as shown in the use of tools, in writing, and so on, have not been acquired without an increase in the brain mechanism by which they are guided. So important, indeed, is the part played, by the human hand as an agent of the mind, and so perfectly is it adjusted with reference to this office, that there are many who think that the first great start which man obtained on the path which has led to his higher development was given by the setting of the upper limb free from the duty of acting as an organ of support and locomotion. It is an old saying 'that man is the wisest of animals because of his hands.' Without endorsing to its full extent this view, I think it cannot be a matter for surprise that the district of the cerebral cortex in man, in which the arm centers reside, shows a manifest increase in its extent."

Still more recently on perusal of Dr. Munro's valuable work on "The Antiquity of Man" and his article in Proc. Roy. Soc. Edin. (1917) the writer finds that Munro deserves fullest credit for the above view.
} 
weaves, shapes, sews, and puts on his clothes; digs, cuts, or hews down material for his home; prepares it often elaborately, pieces it together, and roofs in the whole; fashions and places lights for its illumination. He internally decorates it with materials at times of the most delicate and elaborate hand workmanship; he prepares the soil, sows, waters, reaps, garners, and distributes his grain, straw, fruits, etc.; he fashions instruments of musical harmony, of mechanical skill, of space-penetrating power, of microscopic exploration, of wholesale destruction. He so operates on the body of his neighbor--surgically taking it apart, and placing it together again-as to convert that body truly into a living machine; he catches, tames, pens up, and uses the animals below him as he wills, to ride on them, to be drawn by them, and to use them in many other ways for his purposes. Like the beaver he builds dams, digs canals, and drains or floods countries. He puts together machines that chain and store heat, chemical energy, mechanical energy, and electricity. He expands even on all of the above in endless manner, so that he can correctly proclaim himself "Lord of creation."

How then has he succeeded in this, compared even with the sagacious dog or elephant? Simply and solely because his forelimbs in relation to the brain, and on the principle of action and reaction, have gradually become highly perfected for all of the above work. But it may be objected that some deep and mysteriously acting agency caused increase of the brain substance, and that the latter, sending efferent impulses to the limbs, caused these to become molded with increasing delicacy to wider and wider uses. This might seem plausible, did we only trace one evolving line of animal life which showed such, and that one culminating in man.

But, as already demonstrated, the cephalopods, spiders, ants, beavers, elephants, and dogs, as well as monkeys, all exhibit along diverse lines the same specializing modifications that develop pari passu with diverse activity in circum-cephalic organs and with increasing and condensing brain substance. Such would strongly suggest that cooperative action and reac- 
tion went on between the plastic body organs and the brain substance to which these organs sent afferent impulses, and from which they received efferent impulses.

But in order to estimate aright how evolutionary progress can here be traced in man's historic progression from the apes to superman-so-called-let us follow in historic sequence the successive phases of forelimb activity.

A start might be made with human warfare. We have cited Emerson in the opening part of this chapter. In his article on Napoleon he aptly quotes the warrior as saying, in connection with one of his most decisive victories: "My hand of iron was not at the extremity of my arm; it was immediately connected with my head." The cold-blooded and unscrupulous but marrelously far-sighted general had here seized on and expressed a great biological fact, that can be clearly traced through anthropoid evolution, and onward through every stage of human progress.

As Romanes describes for his Capuchin monkey, and as every traveler in tropical forests testifies, the most primitive mode of human warfare was missile-slinging. And each missile meant a hand back of it, that by determined steady use-practice we call it-became increasingly adept. When the opponents came to closer quarters, a tree branch that the hand had broken could be utilized. As the branches of different trees with harder and softer wood were utilized, the brain registered and in time stimulated the hand in conjunction with the eyes, to select the hardest sort available, which for the early Irishman became the "tight little sprig o' shillelagh." So, as the history of rude nations still informs us, the stone and the club were the primitive offensive and defensive weapons.

But by slow, slight, often scarcely perceptible changes, due to hand and eye experiment that acted as a stimulus-action to start a brain-reaction, the rude club became enlarged at the end, then somewhat flattened on one face of the end, then sharp and splitting along that face. So by slow degrees, specimens of which every good ethnologic museum collection can show, the first battle-ax was fashioned that in time became the dreaded iron battle-ax of a half to two millennia ago. 
Again the rebound actions that the Capuchin monkey was not shown but evolved for itself, when often practised, became the initial stages with primitive man in the formation and use by hand of the boomerang, the arrow with its bow, the sling, and related primitive weapons. The use of the club as a handthrusting instrument, by hand-sharpening of its end, started the Pacific Islanders' weapon that in other lands gave rise to the bayonet. The arrow and the thrown stone started the more and more skillfully and artistically fashioned darts, spears, and javelins. Thus the wood, the stone, the bronze, and the iron age succeeded each other, during which the hand was increasing its cunning, and reflexly the brain its mental stimuli received. So registers were recorded, and proenvironal responses were made in steadily progressive complexity.

With the discovery of explosive materials and the mixing of these by the hand, a new era in relation to iron began. Now the hand, accustomed in relation to the eye and the thermotactic sense to chain and to use fire, melted, fashioned, welded, loaded, and fired the gun that struck wonder into the mind, by projecting a missile at rapid rate and with impressive accuracy to a great distance. We need only to visit a modern arsenal factory to learn how hundreds of human hands, by labor and by stored brain energy, are fashioning a breeched and rifled gun that will project a huge mass of metal eight to twelve miles.

The brain of the Neanderthal man who used his stones and clubs has evolved into the brain of the arsenal factory director or his assistants, who condense so to speak the efforts and potentialities of hundreds of hands into the formation of a modern gun. Nore truly, more scientifically even, it might be said, that the simpler action and reaction motions that began with stone-throwing have branched out into hundreds of distinct hand actions, that the highly educated brain uses and correlates into one complex compounded resultant in the finished gun.

Again we might trace the history of man's use of farinaceous foods. The primitive savage, like the monkey still, gathered 
and at once ate small cereal grains as these matured on the wild plants. The rery act of picking these stimulated the frame to an upright posture. The storing of these through seasons of drought or of cold stimulated him-like the antsto construction of granaries. This was gradually followed-as in the agricultural ants-by the preparation of selected ground, the sowing of the grains, weeding of the cultivated areas, and harvesting of the produce. More intensive cultivation in order to secure larger crops for an increasing population stimulated the brain and hand, by environal action and proenvironal response, to fashion more complex implements, to sow more carefully, to reap more quickly, to clean and store more perfectly, till all have culminated in the huge wheat-farms and elevators of Western States.

Alongside even this, however, the hand and brain have acted and reacted to rid the grain of its husk-slowly performed by the teeth alone originally - first by stone pounding or grinding, that are a hand imitation of teeth action; next by assisted animal labor, then by machines, till at length the complicated milling apparatus represents the mentally compounded and correlated action of hundreds of human hands that once were kept busy in a more primitive state, but which the evolving brain of recent man has condensed into hand-saving machinery, that a few hands can guide.

The supply of clothing in the fullest sense is even more suggestive. Exposed to marked drop in temperature toward night, during several months of the year, when they went to rest, primitive sylvan or arboreal men doubtless often constructed temporary nests or shelters out of leafy boughs and by hand action, like the orang of Selenka and of Moebius. These they would often line with leaves, and in colder nights nestling amongst these and beside each other, as monkeys almost habitually do in zoological gardens, would appreciate the added warmth. Palm or other leaves, grasses, or the fibrous hases of palm-stalks might slowly be substituted. The semiwoven aspect of the fibrous palm material would readily lend itself as a covering during dull cold days, and in turn might 
readily have suggested the weaving or plaiting of palm leaf segments, of grasses, and other leafy or stalked fibrous material into protective covers.

But noting the warmth of animal skins, that had been taken in the chase, and removed by hand action aided by rude stone knives, these skins in colder regions were gradually adopted and shaped into garments. This device, or the previous one of fashioning in warmer climes fibrous coverings, enabled man to adapt himself successfully to sudden changes of temperature in a manner that other animals were wholly incapable of. Such important results came, we claim, entirely from hand experimentation and stimulus of a cognitic nature, that, gathered up in the brain into more complex cogitic resultants, gave response in the form of dress.

A highly important feature, however, should now be considered in man's evolution. Descended from a hair-covered ape, what causes operated to decrease the hair covering to its present sparse extent? One or two environal conditions, either separatedly or at times perhaps cooperating, conspired to this result. Primitive man, like most monkeys and many rude tribes still, was mainly arboreal in habit. A shade or arboreal life, alike amongst higher plants and animals, invariably prevents stimulatory formation of hairs, or causes gradual absorption of these if change be slowly made to the environment next noted; sun-exposed and wind-exposed life often causes in higher plants and in the higher or mammalian animals hair formation. So long, therefore, as the urodeles sheltered under stones in shady places or amongst leaves they remained apparently smooth, with only fine tactile skin endings embedded in, or slightly rising as soft processes above, the surface-level. As we would consider that they became, during the later carboniferous and the permian epochs, animals of the semi-shade and of the open, the tactile endings projected increasingly as hairs, that conserved the animal heat toward night, and diffused or distributed it during day.

As we see in any good collection, over considerable areas of the body in arboreal monkeys, the hair covering often becomes 
reduced in amount and fine in texture. When primitive man took to dress, he thereby adopted a means of substitution and adaptability, that, combined with the frequent arboreal habit, ensured gradual absorption of hair owing to withdrawal of the stimuli that called it forth.

Another and alternate cause, however, seems in part to have operated. A few of the living species of monkey make nests in trees, or shelters in caves. That the latter habit was not unfrecuently adopted by primitive man is demonstrated by the care-dwellings and their human remains already known to us. This coupled with the clothing habit would equally tend to hair absorption.

If we again continue the study of dress, it can be said that appreciation of color blendings and contrasts, individual rivalry, sex selection, and other causes, would all conspire to the gradual production of more and more striking woven fabrics or dyed skins, such as many comparatively primitive nations still prepare and appreciate.

But the hand that first shaped a rude, even a huge, needle gave a great stimulus for the future. For more and more closely and delicately interwoven fabrics then engaged the eye, the hand, the brain; while it reciprocally stimulated currents of afferent and efferent energy along all of these organs. Such rough simple needles, that the hand and eye guided, may long have held sway. But a primitive loom, like that of some savage races now living, became a constantly moving needle with abundant reserve of thread that the hand, the eye, and even the foot guided in its active course. The climax came when the hand of man, working in rhythmic energizing activity with his increasingly complicated brain, fashioned steam engines and greatly more intricate machines, that enabled a pair of hands and an alert brain to accomplish in a day what once required a month or even a year to achieve.

The history of the shoe as an article of protective and defensive clothing equally demonstrates that hand, eye, and brain act and react on each other. But it should here be emphasized that the eye acts largely, almost wholly, as a sensitive con- 
ductor between the hand or arm and the brain in all or most of the above acts. It only becomes specially stimulated to highest action when it and the brain, apart from other organs, are acting and reacting directly in relation to each other.

In the gradual evolution of the nest, the leafy shelter, the hut, the simple home, the embellished house, the palace, and the cathedral, the cooperating and reciprocally stimulating action of hand and brain, with the eye to guide the stimuli passing to and from both, receives striking illustration. As with the orang, the chimpanzee, the Ainu, and some other neolithic human races, man's primitive shelter consisted of leafy boughs or piled branches and leaves. The twisting and bending of branches into shape and strengthened connection suggested and gradually evolved a more permanent framework or shed, as with ants and beavers. In time the hands were further exercised, so as to ornament, carve, fit together, and trace sculptures or drawings on the walls, like the primitive cave drawings of Central France or the rock and cave drawings of the South African Bushmen, which started "Art in the home."

The use of brick, plaster, and stone in succession to wood was evolved according to locality conditions, but their use drew out, more and more, the skill and faculty of the hand. This phase in human advance seems first to have been started 10,000 to possibly 12,000 years ago, and in the Irano-Assyrian region, where Sumerian and Semite both strove to excel and to rival each other. The first keyed arch to a door or window was a trophy of manual effort in brick, and opened up new possibilities. It probably originated in the Assyrian region, or in the Iranian region northeastward, 6000 to 7000 years ago.

The climax in combined artistic effort and strength of material may be said to have been reached in the late medieval cathedrals. In these the detailed hand effort and brain stimulation that devised and fashioned the massive walls, the gothic windows with their ornate carvings, the huge pillars with widely arched connections, the elaborately tiled floors, the upreared spires and minarets, the paintings and sculptures of 
the interior, the organ that rolls its melodies to each furthest recess, are all so blended in one great harmony of effect that the onlooker often fails to realize that thousands of pairs of hands and arms performed their allotted task and by correlated resultant effort-practised patiently and hereditarily through long millenia of effort and action-combined the whole into a noble and enduring edifice.

As already indicated (p. 207) the architect of it, by highly complex compounded resultant proenvironal response to many and raried previous environal stimuli, evolved by hand action and brain stimulation, the plan of the whole in every detail. Each constructive manager working under him proenvironed his own section of work so that he could guide intelligently the scores of hands working under and for him.

The enormously strong and interrelated nerves of the brachial plexus that innervate the arm, specially the $6 \mathrm{th}$, 7 th, and 8th cervical branches, are proof of the complicated communications that exist between the brain and the arm. So the hand-arms of each individual, by mechanotactic and to a much less degree thermotactic relation, gradually convey to the brain stimuli that influence its molecular substance, and which there are combined into resultant responses that, in the early nest- or hut-building activities of man, sufficed as efferent impulses to enable him to construct the whole. But, from that stage of house-building civilization to the one in which the cathedral is reared, added stimuli and groups of stimuli were combined more and more intricately by the Nissl or nerve-cell or neuratin substance, which started complex or compounded resultant responses of an increasingly high order.

We have, in the foregoing text, repeatedly referred to the contributory action of the eye and naturally the optic center, as a conducting sense-pathway from the brain. But, given the arm with its tactile fingers, the eye can at times be largely dispensed with. Thus the skill in reading, sewing, wearing, playing, and even writing, shown by the educated blind, is familiar to all, while a visit to an "Institution for the Blind" reveals many surprises in manual effort and brain stimulation. 
It can appropriately be said, therefore, that, when man is viewed from the standpoint of his physical frame, he is "the hand animal." His increasingly erect posture was at first contributory to hand action, and eventually determined by it; his plantar progression gradually resulted as an equilibrating adjustment, when he not only grasped objects, but carried them to a distance with him by aid of one or both arms; his readjustment of muscles, of bone-surfaces for attachment of these, of nerves and other tissues of the body, were, we would consider, mechanically effected to preserve the upright attitude, and to act as contributory helps to efficient working of the arms and hands. So the extremely slow but nevertheless steady increase in size of the brain and in its weight has, we consider, proceeded with, and been stimulated to growth by, activity of hand and arm as collectors and transmitters of environal stimuli to the brain.

This acquisition it is, also, which has enabled him more and more surely to become "the dominant animal." For in fashioning, by hand and brain action, rude gins and snares, by digging and skillfully covering pitfalls, by devising more and more destructive weapons, by utilizing poisons that his hand had concocted, by taming the horse and then organizing game drives, by excavating or building canoes or ships, and following prey into every recess of land and water, he has made other animals accessory to him, or has exterminated such as he desired.

And in recent decades the hand and brain have combined reciprocally to recognize, separate, cultivate, and apply antidotes, antitoxins, and antagonists, that lessen or destroy the action of those organisms, the bacteria and "microzoa," that once marched through the ranks of humanity as mysterious wholesale destroyers; or that spread as "blight," canker, or mildew, to blast the plants that he looked to, in the wild or in the cultivated state, for his support.

Even the international means now adopted by telegraph, cable, telephone, and wireless telegraphy, to locate disease areas, are a triumph of hand-arm action, reciprocally working with brain energy, toward a common end. 
But a striking expansion and corollary to all of the above is seen, when we study the "masters" of warfare, of industry, and of exploitation. In these, and in not a few others doubtless who by force of environment remained each a "mute inglorious Milton" or "a Cromwell guiltless of his country's blood," the brain capacity and activity so outran and gathered up in a proenvironal whole the hundreds of hands they looked to for aid in their gigantic schemes, that their plans drew in and engaged them all. So when Napoleon exclaimed that "there were no Alps for him," he truly meant that a million ordinary potential hands, either in the form of human hands, or of these guiding horses, or mechanical devices of a hand-condensing kind, were at his disposal to effect results that otherwise would only have been reached had no Alps existed. And when he declared that there was no defeat for him, he meant that his brain was big enough and active enough to keep a million hands fighting, in order to command success.

We arrive now at an important stage in our study, where the purely physical side of man becomes linked more intimately with his mental, and even moral, development. We refer to the formation and perfecting of Language.

No one at the present day will assert other than in a figurative sense, that language as a physical acquirement is "a hearen-born gift to man." The capacity for clear, varied, and expressive articulation by parrots is superior to that of some human beings, while the jackdaw and other birds all show linguistic ability of greater or less merit.

That the importance of the tongue, vocal chords, and associated parts, as cooperative physical or physiological agents in man's evolution, has been fully appreciated, is at once evidenced by the quotation from Emerson with which this chapter opened. We would object, however, to his sweeping conclusion. The grounds for this we shall now try to present.

As Darwin, Mivart, Lauder-Lindsay, Romanes, and other writers have stated, definite though primitive linguistic faculties are shown by various birds, dogs, monkeys, etc. Thus every one is familiar with the exultant notes of the hen after 
laying; of its low determined guttural notes when fighting; of its "purring" expressions when quieting its young beneath its wings; of its sharp inquisitive utterances when stalking in search of food; of its shrill sudden shriek when suddenly set upon and chased by an adversary; of its long-drawn warning exclamation or drawl when a supposed bird of prey flies across the sky. Regarding these Houzeau writes (182: II 348): "Now since the hen does not utter its warning voice after laying, nor its purring notes when in search of food, but each appropriate to a definite occasion and as definitely understood by others of its species even when not within sight, it is strongly suggested that in each case there is an exact concept formed in the brain of the bird that stimulates to an exact expression. Were we dealing with a human being, the writer believes that no one would doubt or criticize this, unless very strong proof were advanced."

Hobhouse (184: 281), in contrasting the intelligence of the highest animals other than man, with man himself, says: "We may suitably attack this problem, by inquiring first, what precisely the use of language adds to the practical intelligence which we have described An intelligent dog or ape can, if the evidence which we have quoted is sound, use experience intelligently, and even plan an adjustment of means to ends with a certain measure of inventiveness. But it cannot describe what it does to us, nor so far as we know to another individual of its own species." Here the writer accepts it that these animals can originate a concept or plan of action, though they cannot describe it. But when a cur, in danger of being whipped or kicked, flies and simultaneously utters its well known cry, such is a definite though simple descriptive warning to others of its kind that may be near. Similarly when the hen purrs broodingly over its young, simultaneously spreads its feathers even more expansively than before, and with its beak pushes gently inward the protruding head of a wakeful young one, it is describing alike to "another individual of its own species" as well as to each human listener, the fairly complex line of action mapped out in its brain. 
In relation to Emerson's statement, then, it seems not unreasonable to consider that at least for some kinds of "beast of the forest" language had become a developed possibility. According to the somewhat recent statements also of Garman and others who have closely watched and educated, as children are educated, some of the higher monkeys, these express, or can be taught to express, some definite concepts. But it must freely be conceded that, so far as direct evidence at present goes, a considerable, but by no means an enormous, gap exists between the linguistic performances of the highest monkeys, and that of uncivilized man as now living. Darwin expresses it fairly correctly when he says, "The lower animals differ from man solely in his almost infinitely larger power of associating together the most diverse sounds and ideas; and this obviously depends on the high development of his mental powers." Furthermore we would distinctly assert, from all known palæontologic, anatomic, intellectual, and utilitarian characters of man, that he represents an added period in evolutionary advance of at least $1,000,000$ to $2,000,000$ years over the chimpanzee, a still longer period over the orang, and a still more extended one over the gorilla. In other words that while man is a more or less compounded, and also advanced type out of the other three, the gorilla has passed into a progressive line that, measured by time relation, causes him greatly to lag behind the orang, while it and the chimpanzee have also relatively "lingered by the way."

If such be the case some cause or causes must have existed for the advance of man, and for the origin with him, in richest measure, of language. Most, we believe, will grant that many animals, invertebrate as well as vertebrate, show by their intelligent action that numerous "percepts" (Romanes 185: 184) originate in their brain, also that many of these animals are mute, or can feebly express their percepts by sound. Examples are seen in the spiders, ants, beavers, and elephants. Actions or gestures therefore may, as Romanes has pointed out for Indians, become a recognized though mute form of language. Now, as several writers describe, this gesture- 
language is wholly effected by action of the hand and arm. Such an initial or primitive mode of communication then would not have been possible till man had become fairly erect, and actively used his arms for the many purposes of which we have already sketched a few.

But, in view of the studies of Forel, Wheeler, Field, and others, few will now deny that ants have what might be called a mute antennal language, while many other animals express their ideas by some gesture or simple contact action. Similarly deaf-mutes or blind persons can converse* by the fingers almost as quickly as speakers beside them.

Clearly, therefore, if the brain has so evolved as to form percepts, these can, and may, be expressed or explained by a variety of organs, not necessarily by the mouth parts. Further Romanes (185: 123) says: "The higher animals unquestionably do understand the meanings of words; idiots too low in the scale themselves to speak are in the same position; and infants learn the significance of many articulate sounds long before they begin themselves to utter them." His succeeding illustrative statements are also apt, particularly the following: "The most remarkable display of the power to understand the meaning of words on the part of a brute, which has happened to fall under my own observation, is that which many other English naturalists must have noticed in the case of the chimpanzee now in the Zoological Gardens. This ape has learned from her keeper the meanings of so many words and phrases that in this respect she resembles a child shortly before it begins to speak. Moreover, it is not only particular words and phrases which she has thus learned to understand; she understands to a large extent, the combination of these words and phrases in sentences, so that the keeper is able to explain to the animal what it is that he requests her to do."

Not only, therefore, may animals, near to man in physical organization, originate concepts, they can understand these

* May it not be that the very word converse, "a turning together," may have originated from the turning or twisting in company of the finger's amongst rude primitive nations, who handed down the idea to more recent language builders? 
when articulated by man. In order to ascertain then how the simple articulation shown by some birds, mammals, and specially by some apes, evolved into the simplest language now spoken by man, we ought to consider the primitive or root words, and the root ideas or actions back of these, and that are most naturally associated with them. Here again Romanes well suggests that "although gesture language is not in my opinion so efficient a means of developing abstract ideation as is spoken language, it must nevertheless have been of much service in laying the foundation of the whole mental fabric which has been constructed by the faculty of speech." Also "where a vocabulary is scanty or imperfect, gesture is sure to be employed as the natural means of supplementing speech." Here we would again emphasize the undoubted fact that such gesture-language is practically wholly conducted through the arm and hand.

According to eminent philologists the root words of the three great types of human language amount to from 120 to about 500. Thus Max Müller reduced all Sanskrit words to 121, and regarding these Romanes truly remarks, a "most interesting feature of a general kind which the list presents is that it is composed exclusively of verbs." This peculiarity also of the ultimate known roots of all languages, which shows them to have been "expressive of actions and states, as distinguished from objects and qualities" is important. But a most striking ('ircumstance is that of the total number about 68 to 70 , or more than 60 per cent., are wholly connected with motion of the hand or arm. Thus to abstract the first twenty-five, of which such is true, we have "dig, weave (or sew), crush (or pound), sharpen, smear, scratch, divide (or share), cut, gather, stretch, mix, scatter, sprinkle, shoot (throw at), pierce (or split), join (or fight), tear, smash, measure, kindle, milk, pour, separate, glean, and cook."

We need not follow the discussion into which Romanes enters with Max Müller, as to whether these were truly primitive, or rather the selected survivors of a still more primitive language in which action and objects were both named. The 
important point for us now is that, whether primitive or selective survivals, Sanskrit roots are largely rerbs that denote hand-arm action. Of the remaining fifty or thereby, these like bite, shake (or tremble), fall, blow, and choose are mainly expressive of frequent acts that are common to all or most of the mammals.

Evidently, therefore, the increasing perfection, importance, and preponderating activity of the hand-arm was correlated with, and even stimulated to, word-formation and expression.

Various views have been propounded by philologists as to the possible origin of such simple root-words. That which unquestionably explains their derivations to a greater or less extent is the principle of onomatopoia, or the imitation by articulate names of sounds obviously distinctive of the objects or actions named. Thus the Anglo-Saxon creak, quack, thwack, crawl, croak, etc., evidently had such an origin. But, if we take as our guide what we may term the root-calls or rootwords of the lower animals, the conclusion seems fairly well established that these originated as rocal expressions, that in tone, intensity, and inflexion are the mental equivalent or response - on the principle of action and reaction-to the external stimulus.

It seems to the writer that Max Müller's objection to the onomatopœic origin of languages $(186: 181)$, is due to his perceiving, but perceiving too strongly, that many words may have originated as psychologic "appropriates," if we may so say, that in tone inflection and quality were suited to or psychologically appropriate to the action, though the one had no exact sound relation to the other.

But so appropriate in many cases are the sounds and the human word-expression for these that many verbs may be accepted as having originated in onomatopœic manner. 'Thus the "purring" of the hen and some related sounds in other animals that indicate restful satisfaction are exactly appropriate, and have no relation to other actions of these animals. It however is somewhat allied to the "crooning" cradle lullaby of a mother over her child. Again the wild exultant 
expression of some dogs when beating up prey in a brake cover is as appropriate psychologically as is the rather low warbling or prattling set of notes given forth when two or three are at play. Even more striking, varied, and appropriate are the sounds given forth by some species of monkey, when one watches them for a few hours, but all of which seem in harmony with the psychologic state of the animals at the time.

Waitz (187: I, 272), Max Müller and others have suggested that "the unit of language is not the word but the sentence." We would regard alike this and Romanes' conclusion therefrom "that historically the sentence precedes the word" as being unwarranted by all the facts known to us. Many substantives could well be represented by a gesture of the hand, and such when united to one simple root verb would form an expressive sentence. We would therefore conclude that primitive man developed a root-verb articulative language, along with a substantive gesture language. Partial evidence in favor of this is the fact that Sumerian and other primitive uritten languages have the substantive itself pictorially represented, and this, gradually simplified, became in time the written sign-word for the article itself.

But it may here be objected that while rocal or sound expressions, such as "rumble" for thunder-sounds, like "crackle" for wood-fire noise, and other like words could readily be explained, this could not be done, or done at least easily and naturally, for hand-arm action. A little reflection and investigation, however, will show that this, instead of being an objection, is farored by all we know of many words and their handarm connection.

Thus if we think of the early cave man of France and his pictorial representation of animal life on a mammoth tusk, the word "scratch" could well signify his hand-arm action, and the brain response to it. So after he had laboriously used a hand shell or stone splinter perhaps, to etch out an animal form on the tusk, when he again wandered with a companion and alit on some etching object that would be suited to the 
purpose, a hand-gesture with the new found object held appropriately in it, and the emission of the single sound "scratch," would be equivalent to saying "I have found a good shell for use in scratching figures on my mammoth tusk."

Again the verb "hew" doubtless has a primitive and apt sound-significance. For many laborers, when cutting down a tree or driving a wedge between stone blocks, habitually utter with each blow a semi-guttural sound that phonetically might be put as a "heough." The word "weave" could well signify the softly sliding noise of the shuttle as it slid through the fibers of a cloth from hand to hand. Smear, scatter, tear, pour, and many other of the above cited verbs may well have originated as sound words that the hand-arm called forth when in contact with and using soft fats, hard grains or pebbles, shredding leaves of palms, or in pouring out water or oil respectively.

The comparatively rapid origin of spoken language amongst men seems to have been due to a corresponding rapid development of the hand as a plastic environal contact-organ that rapidly and delicately brought the brain into cognito-cogitic relation with that environment, and so enabled it to proenviron a plan of response that, when put in action, constituted a sufficient or satisfied response. Further we would exactly follow J. Loeb $(60$ passim $)$ in considering that all of this involves fundamentally a series of continuous changes in definite colloid molecules due to lines of energy that temporarily act on and alter them. Therefore also the supposedly scathing, but truly superficial, criticism of a recent writer (189: 603d), when trying to follow Loeb's results, seems to us as smug and unscientific as his knowledge evidently of scientific facts is scant. We can never reach an intelligent psychology till we understand protoplasm, nor understand protoplasm till we have grappled physico-chemically with colloids.

But the above origin of language postulates, as a fundamental condition, that primitive man lived in communities or colonies, and hereditarily derived his social instincts from the anthropoid apes. Some writers, influenced possibly by the semisolitary or at most restricted social life of the gorilla and orang, 
have inclined to view primitive man as a solitary or family species only. But, except for the gorilla and a few lower types, the apes and monkeys evidently enjoy optimum existence when forming part of a communal life. Every zoological garden is witness to this.

Given such a communal life, when a few primitive men paddled a log or dug out canoe to a fishing station, the paddling at one time, the spearing of fish at another, the baiting of a rude bone hook, the throwing of the line that held it, the watching by eye and delicate feeling by hand for a nibble, the rapid inpulling of the captured prey, and the securing of it in triumph meant constantly changing and transferred stimuli between hand and brain. All such acts, as every civilized and even more uncivilized group show, tend to changing conditions of mental stimulation and excitement, along with ejaculations suited to the moment. But no parts of the body except the hand-arm, in conjunction with the eye, give rise to such diverse stimuli.

Even in the milder and more systematized efforts attendant on hut-building, land culture, garment fashioning, and other employments, communal or at least family aid and cooperation seem nearly always to have prevailed. Thus at the present day scarcely one rude tribe exists in which hand labor and simultaneous active talk do not go hand in hand, for a considerable period of each day. Advancing adaptation of the hand to an erer widening range of pursuits stimulated the brain to increased flows of energy, and so to increased complexity and growth. Simultaneously also it stimulated to an expression of riews on these pursuits, that even yet in "isolating" languages are general, simple, and primitive, but which in "agglutinative" ones are advancing to greater and greater complexity and analytic exactness, as skill of hand and advancing social organization conduce to more complex conditions of life.

In all such adrance by environal stimulation-action and brain reaction, followed by proenvironal outreaching and succeeding response, the great law of proenvironment is constantly at work. Thus given that two primitive tribes had 
come to occupy adjacent areas of country; that one had a more perfect, the other a less perfect manual dexterity in fashioning weapons of war; that the one also had a less rudimentary, the other a more rudimentary language. The former, in measuring themselves by cognitic and cogitic action against the latter, would plan or proenviron new and more perfect weapons than their less dexterous neighbors, and in battle-as in more recent football games-would agree to shout the most perfect words for command or team action. The others, poorer alike in planning, in executing, and in linguistic stimulation, would be crushed, unless other and compensating advantages aided them. Environal stimulation and proenvironal aspiration or outreaching to secure best results-as yet unattained-would combine to ensure victory.

Such was the biological history of Roman conquest over the tribes or nations of Europe and Asia. Such still is the history of wars and conquests, where the action of millions of erstwhile fighting hands are concentrated in death-dealing cannon, in explosive bombs, and in repeating guns, aided by the farreaching linguistic capacity of the telegraph wire, the telephone, or wireless signaling.

Equally true is it, however, that in spite of disintegrating agencies at work, hand, eye, and brain, as parts of the physical frame, have acted and reacted to map out and struggle toward a proenvironal position and result that lifts man ever higher in physical well-being, in mental superiority to his primitive ancestors, and in enjoyment of progressive social and national peace and prosperity. This has involved synthesis, growth, continued life, and that on an ever higher plane.

Speech, as Emerson claimed, has had not a little to do with raising man from the state of "a beast in the forest," but we would emphatically affirm that had man remained a four-footed beast, instead of gradually becoming a hand-and-foot beast, his brain would have remained relatively simple, his speech would have continued on a level with that of the dog or the cat, his aspirations would have remained limited, and his thought would still have been "chained to the dust." 
There remains, however, to be considered another hand-eye activity and achievement that has still more recently been evolved in human progress than language, and which has given to the latest stage of that progress the appropriate title of "the period of written history." The researches in Babylonia, in Assyria, in Egypt, in Crete, and in China of the past two decades have revealed that writing of modern character is of comparatively recent date, not going further back probably than $6000 \mathrm{~B}$. C. The three main types, the Chinese, the Sumerian, and the Egyptian, all show an evolutionary progression from direct pictorial representation of slow delineation and limited mental expression to word signs that can quickly be transferred from the brain to paper, and that in a sentence may convey abundant suggestion.

But much older even than these, and possibly representing a highly primitive type, is that revealed by Piette (190: 384) from Mas d'Azil in France. Since this belongs apparently to the oldest period of the neolithic age of man's history, it is of special interest. The type is set forth on numerous oval or flattened pebbles that were found scattered promiscuously in a gravel bed amongst perforated deer teeth, animal bones, and various fruit stones. Each pebble bears a sign mark that may be $\mathbf{U}$ or $\mathbf{V}$ or cross-shaped, or of a more complicated outline. Each may have represented a definite idea, or part of such, and in character may have corresponded to the incised or carved sticks still used amongst various uncivilized nations of this day and sent as messages to absent ones. Piette considered that they resembled letters of much more recent date, but it might even be that they were stones used in some game, much as dice pieces are used now.

The art of expressing one's thoughts in writing would almost inevitably result from the increasing skill with which neolithic man drew figures of plants, animals, and scenery around him. The wonderful accuracy with which many objects, found in the central and southern French bone caves, were delineated is a testimony to the growing artistic skill; at the same time it demonstrates how intimately hand-arm and eye were gathering 
and transmitting stimuli to the brain which were proenvironed into the mental images that took shape on the tusks.

So from the simian with extremely limited powers of expression, but with nearly upright habit, to the most primitive man in whom speech was fairly evolved owing to upright habit and skilled use of hand-arm; from this again to the neolithic man with fair vocabulary, free upright gait, skill and rapid use of hand-arm in many employments, as well as simple primitive sign-writing; then to the early Chinese, Sumerian, and Egyptian inhabitants, with good vocabulary, skillful use of handarm in bold and equally in delicate manner, also with striking pictographic writing; later to the advanced Medo-Persians, Egyptians, Babylonians, Cretans, and Greeks with elaborate and grammatically technical vocabulary, highly skilled use of hand-arm, and condensed character writing, the stages of advance as already accurately known to us are graded and easy in series. Man's recent combinations of speaking, writing, and hand-arm action, in the elaboration of the telephone, the phonograph, the grammophone, the Marconi machine, and like instruments, is a high testimony to the marrelous progress made by him during the past century.

But it must be conceded that writing, which is the highest art of the individual man, has been unrivaled in stirring the brain to rapid action, and in drawing from it mental responses that have become plans of proenvironal activity, for changing the trend of national histories, the pathways of rivers, the biological connections of nature, and often even the relation of the inorganic to the organic world.

Noiré (191), Geiger (192: 74), Sayce (193: II, 302), Darwin (194), Max Muiller (186), and Romanes (185) have all propounded views as to the stage at which language became worthy of the name, in the higher anthropoid apes or in primitive man. According to the physical basis on which the writer has proceeded, the strong likelihood seems to be that language became distinctly articulative only after, and probably a long period after, the simian stage had been passed, and when continuous upright progression had evolved, conjointly with a 
manifold and skillful use of the hand-arms in the manner already indicated. By this time also the brain, reflexly stimulated, increased, and specialized from reception of abundant and long-continued hand-arm stimuli, had doubtless attained to a fairly high conceptual stage.

But in stating this one must bear in mind how rapidly and strikingly the chimpanzee Master Link forsook all attempt at quadrupedal progression, and behaved "humanly." Against this, however, is the important principle that it had comparatively suddenly been projected into an environment that was stimulating to aspiration and imitation, whereas amid a crude natural environment the higher anthropoids had slowly and laboriously to proenviron, to practise, and to select a progressively nobler type of living, that is now a heritage of the race.

Therefore, while the writers named above have traced the evolutionary origin of language in a surprisingly perfect manner, the main instrument in stimulating to this origin was overlooked in its commanding importance. One highly important result however of their studies, has been the clear and scientific demonstration that man has gradually advanced from use of a simple language poor in roots and in inflectional expressions, to highly complicated spoken and written languages, like the Greek and Latin. But as already emphasized in this chapter such advance suggests increasing brain complexity, due to increased intricacy and complicated character of the hand actions involved. So we reach the position, already briefly outlined in Chapter IV, where cogitic energy is considered to have attained its maximal activity.

The study of this advancing brain complexity in "the higher animals" and in man, its relation to cogitic energy, and all the phenomena of mind, will concern us in the next chapter.

But in closing this chapter we might remind the reader that, in some systems of classification, man has been separated from the monkeys, that were all fittingly called Primates, and has been placed in a separate family the Bimanes, than which no better name could have been given. For his two hands have been the collectors of his most complicated environal stimuli; 
they have been the conductors of these stimuli to the brain; they have been the most important and delicate distributors of summated and resultant proenvironal plans; they also have mainly executed these plans, which then became proenvironed accomplishments. 


\section{CHAP'TER XXI}

\section{THE MENTAL OR COGITIC EVOLUTION OF MAN IN RELATION TO 'THAT OF THE HIGHER ANIMALS}

Regarding mental states Hume has said (195): "All our perceptions are dependent on our organs, and the disposition of our nerves and animal spirits." In accordance with such a century-old dictum, it will be our endeavor in the present chapter to ascertain how perfectly even the most complex mental processes can be traced back to simpler beginnings. For Romanes has well remarked: "It belongs to the very essence of erolution, considered as a process, that, when one order of existence passes on to higher grades of excellence, it does so upon the foundation already laid by the previous course of its progress."

In Chapter IV it was suggested that, amongst simplest nonnucleate organisms or Acaryota, a more perfect or condensed form of energy than electricity traversed and energized the protoplasm. This we named biotic energy. Further we adduced evidence for the persistence of this in all cell protoplasm, but accepted it that, with the gradual evolution of nucleoprotein and a nuclear mass, a still more evolved energy was increasingly associated with the biotic, and which we termed the cognitic. We considered that biotic energy exhibited itself amongst the Acaryota in simple direct responses to such environal stimuli as light, gravity, moisture, etc., so that a rather indefinite or rarying type of growth resulted, since a degree of direct response at one time to one stimulus might be altered or reduced in amount by another action at a later time.

In contrast to the last and in more evolved connection, we considered that cognitic energy gave to caryotic or nucleated plants and simpler animals the capacity to receive a variety of environal stimuli, to combine these often within the tissues, 
and to produce therefrom a resultant stimulus, that by proenvironal action started in the tissues a line of growth or motion reaction, that was a satisfying resultant of all the incident stimuli. The growth of Fungi and Algæ; the movements of roots, of stems, of leaves, of Paramœcia and other nucleated Protozoa, of sponges, and the simpler colenterates, would be accounted for on such a basis, and can clearly be demonstrated experimentally.

One important result to be here noticed, in connection with the studies of the present chapter, is that, within a single tissue, even within a small area of it, such as the root-tip, the stemtip, the leaf-node of higher plants, or one end of protozoan animals, when several stimuli have reached it from without these are absorbed, compounded, and intrinsically affect the living substance, so that one efferent stimulus, or possibly a correlated set of stimuli, give rise to a motion that is a resultant, in direction, in strength, and in duration, of all the stimuli combined. We have seen that "sensitive plants," so-called, exactly and quickly illustrate this, while protozoans and hydroids also agree.

But with the appearance of ganglionic cells or nerve cells in animals a new and more complicated situation presents itself, that we have considered to be connected with evolving mentality, though at first the manifestations are of a very primitive kind.

The fundamental feature of mind activity or mentality is that property which we call consciousness. As the word literally signifies, this is a knowing or comparing together of several distinct resultant stimuli. This then leads to a combining of two or more of these resultant impressions into a new compound resultant, that becomes the line of action taken, while not infrequently one or more of the primary resultants is inhibited, counteracted, or annulled in production of the new compound resultant.

Though somewhat anticipatory, therefore, of the illustrative cases to be cited in this chapter, we may shortly set forth the relation of biotic, cognitic, and cogitic acts as follows: 
Each biotic act is typical of protoplasm, and of the biotic energizing currents that traverse it. It represents a simple afferent stimulus or flow of energy, that temporarily alters some constituent or constituents of each cell, and sets free thereby a definite amount of biotic energy, which exhibits itself in a definite and simple response.

Each cognitic act is typical of protoplasm and of chromatin, and so of the bio-cognitic energizing currents that traverse both. It represents the passage into certain appropriate cells of one, or it may be of two or more, afferent stimuli, each of which temporarily alters some constituent or constituents of each cell. If two or more stimuli act these form a resultant material or energized combination which sets free a definite amount of bio-cognitic energy. This exhibits itself in a definite and complex resultant response.

Each cogitic act is typical of protoplasm, of chromatin, and of neuratin, so of the bio-cognito-cogitic energizing currents that traverse these. It represents the passage into certain appropriate cells - the nerve cells - of two or more afferent stimuli, that have by the chromatin been combined into resultants, and these again, fortified or altered or reduced by stored memorystates of the cogitic or nerve cells, become combined into a compounded or grand resultant cogitic impression in the nervecell substance, which then exhibits itself in a definite and compounded set of resultant responses.

Romanes, in delimiting mental conditions, expresses the following: "The distinctive element of mind is consciousness, the test of consciousness is the presence of choice, and evidence of choice is the antecedent uncertainty of adjustive action between two or more alternatives" (185: 18).

As in the relation of the cognitic to the biotic state, where a very gradual-practically imperceptible-transition seems to exist, so in passage from the cognitic to the cogitic the same gradual transition is observed. Overlooking this largely, mental philosophers have attempted, till within recent years, to assert that mentality, memory, choice, and reasoning are mainly peculiar to and characteristic of man. They have 
studied the highest and most erolved product; they have failed to trace step by step the manner in which that product was evolved.

The newer and more scientific attitude is well expressed by S. J. Holmes thus (183: 9): "Instinct, memory, volition, and reason are all parts of that general process of adjustment of the organism to its environment, in which life in all its stages essentially exists. As we pass from lower to higher forms we have an increase in the complexity and perfection of the adjustments; the corresponding increase in space and in time, in definiteness and in generality, but everywhere it is 'the adjustment of internal relations to external relations." "

If mentality represents the flow and activity of a special cogitic energy, that is more perfect, labile, and condensed than cognitic energy, then, just as we have given reasons for regarding protoplasm as the physical conductor for and storer of biotic energy, and chromatin of the cell nuclei as the conductor for cognitic energy, so we may inquire and try to ascertain how far cogitic energy is associated with, stored by, and conducted by ganglion cells, or their associated dendrites.

Deferring for a little any proof of such it is now conceded by all workers in animal psychology that if we start with primitive and simple members of such groups as we have studied in Chapter XIX, like the Cephalopoda, the Arachnida, the Insecta, the Rodentia, etc., the increasing abundance and complexity of nerve cells, and particularly of brain masses, are constantly correlated with advancing specialization in acts that denote mentality of a steadily progressive kind. So in some groups of ants that even excel some races of man in mento-social organization the brain in size, and in comparison to head and body weight, compares favorably with that of the highest apes (p. 553).

To study, therefore, the profoundly complex structure of the brain, and the equally complicated mental acts shown by man and the more mentally-evolved animals, such as ants, beavers, and dogs, is alike logically and morphologically misleading. Loeb has often and rightly emphasized that it is only by gradual 
tracing of commencing and adrancing mentality amongst the lower animals, and an interpretation at the same time of the acts of the latter, as they pass from simpler to more intricate morements and responses, that a possible explanation may ultimately be got of even the most involved mental acts of the highest animals. This method, already successfully pursued in part by Eimer, Lauder-Lindsay, Romanes, and subsequent workers, has yielded results of lasting ralue. But, as Washburn (196: 4) has well observed, the anecdotal method alone has always to be used with care.

Now, in explanation of even the most complex resultant cognitic movements, observational evidence has been adduced to show that such can be explained on chemico-physical grounds. If most facts of the case point to a like explanation for cogitic activity even on the highest plane, there is no need to call in an occult or hidden mental agency.

All higher mental effort is now recognized as involving great expenditures of energy. The lecturer, the author, the financier, the diplomat, the man of wide business affairs, the president of corporations or of nations, after four or five hours of mental effort, experiences a more fagged and depleted bodily state than does the manual worker after three times as many hours of labor. The results accomplished also by brain-workers, within such time, have correspondingly wide influence, and are an approximate index to the high quality of energy expended.

Such being necessarily true, it follows physically that either very large supplies of the more simple organic food compounds, or moderate supplies of some highly complex organic body, should be stored in or around the ganglion cells, and should gradually be used up during mental acts. After prolonged and intense mental activity, therefore, such a material would reach its lowest ebb. Now in rested ganglion cells an abundant reserve of fine aggregated granules-- the Nissl bodies or neuratin as we have named such-either crowd the cell substance irregularly as amongst many invertebrates cited, or are arranged in concentric layers or in patterns as seems frequently true of vertebrate nerve-cells. The granules become more cloudy, 
break down, and largely disappear during mental activity and resulting fatigue. After rest and repair of the tissues, the granules gradually increase in quantity and sharpness (p. 235). Illustrations of such cells are given below.
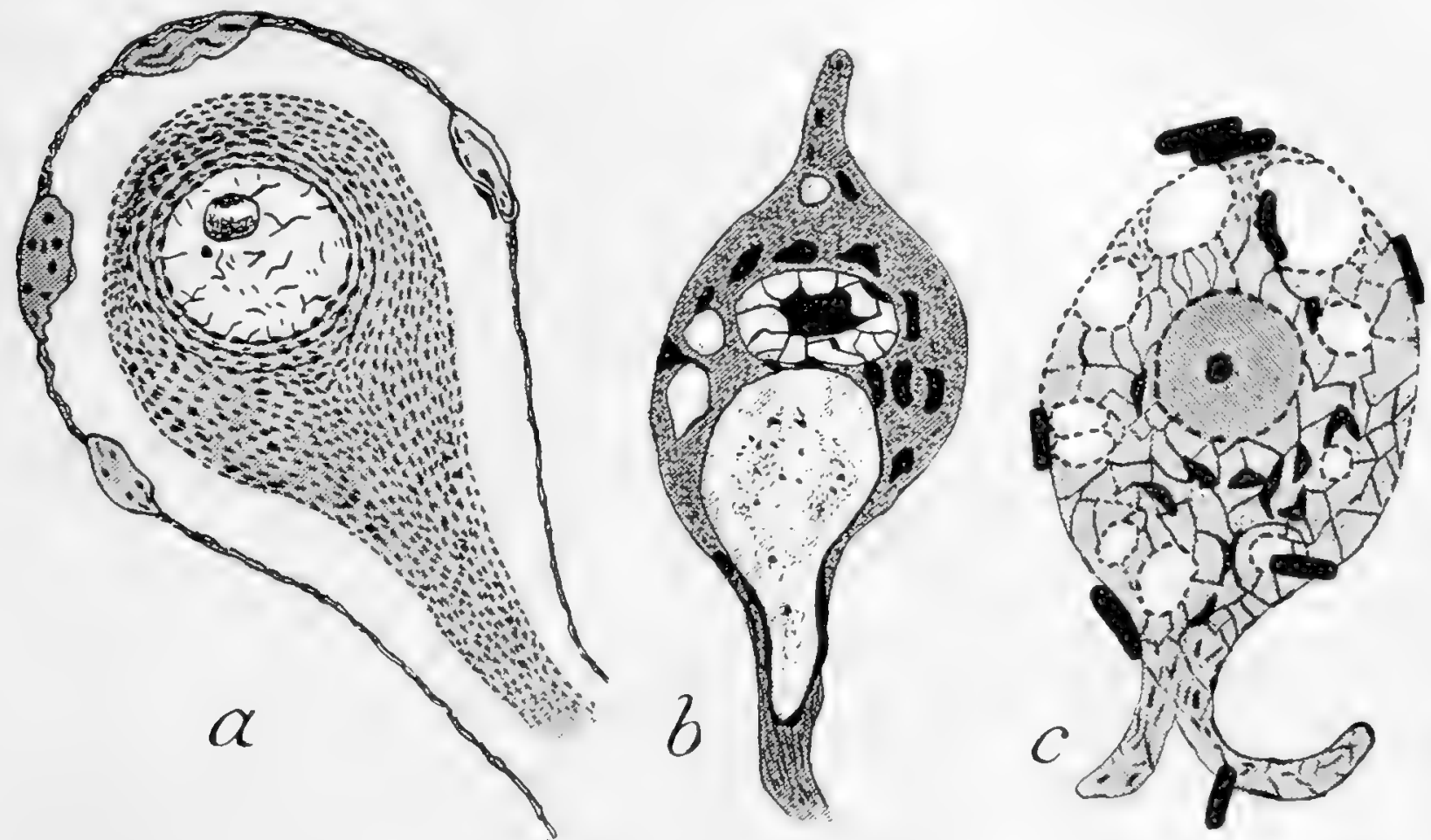

FIG. 28.- $a$, ganglion cell from Crayfish (after Pfücke) showing numerous fine neuratin granules intimately associated with the nerve fibrils: $b$, stained ganglion cell from infant (after Fwing) showing large dark neuratin granules; $c$, stained ganglion cell of Rabbit with neuratin granules as in $b$.

Here is definitely suggested, then, a substance that becomes used up as mental energy is expended, and that gradually accumulates again as this energy is recuperated. In all probability this energy, that we have called cogitic, is itself derived from, and is a greatly more condensed exhibition than is, the cognitic, that seems to energize and traverse the chromatin substance. For it is noticed that, during activity and exhaustion of the ganglion cells, the chromatin of the nucleus, and specially of the nucleolus, becomes reduced at the same time that the neuratin becomes first broken up and then largely absorbed. When rest ensues the nucleolar chromatin, as well as the neuratin, accumulate in quantity and assume a normal aspect $(86,18: 101 ; 19: 125)$.

Again between the cogitic and cognitic energizing material on the one hand, and the biotic on the other, there eridently 
is an intimate relation, when one considers the various connections of the living cell, and the trophic continuity that exists between the constituent parts of it.

In the latter part of Chapter IV the writer has shortly summed up evidence that constantly and clearly demonstrates the existence of three ascending phases of life-energy in man. Thus in the maturation of the newly born child there arealike morphologically and physiologically-the biotic, the cognitic, and the cogitic superposed and elaborated on each other up to at least the fourteenth year. Conversely in gradual bodily decay, "second childishness, and mere oblivion," or biotic life mainly exists before death. In sudden nerve-shocks, as in the case described, all three phases may be distinguished, first in descending and again in ascending order; while, in alcoholism and related drug poisoning, the descending phases are also seen.

Now in all of the above cases we seem to have to do with three groups of chemical compounds of increasing complexity, that exhibit an increasing degree of molecular instability in like ratio, and which also show a degree of heat and cold resistance that is greatest in the simplest, less extended in the more complex, and least in the most complex. Thus we have already pointed out (p. 57) that protoplasmic and related biotic material may live through a temperature range of $-200^{\circ}$ to $+100^{\circ} \mathrm{C}$; nucleated cognitic cells are active only through a range of $2^{\circ}$ to $40^{\circ} \mathrm{C}$, but show living response between $0^{\circ}$ and $75^{\circ} \mathrm{C}$. Naked human organisms again, in virtue of the presence of neuratin substance, have an optimum temperature range from $20^{\circ}$ to $35^{\circ} \mathrm{C}$.

In the history of individuals or even of nations, it is a fact of general observation that whole races of savages, and equally not a few members of civilized society, lose all effort toward aspiring proenvironal response, and, for reasons that we need not enlarge on here, sink into a condition where mere eating and drinking are the dominant life-activities. Their cognitic or chromatin, and even more their cogitic or neuratin substance and the energies for which these stand become clogged, 
atrophied, and buried beneath the load of biotic energy substance. Such are soon marked out for extinction by disease, by oversurfeiting, by want of active response-capacity in the struggle for existence. The higher and keener cognitic and cogitic life of other competitors overwhelms them.

But, as the history of man has emphatically shown, races or individuals who have observed to "keep under the body," and who have cultivated active cognitic or sense perception occupations, have yet failed to reach out to the more active mental improvements that have been introduced into their midst, or that have been discovered and adopted in other regions. So though quickly responding to cognitic stimuli they have lagged back mentally, and in time fall out of the race.

It never was more true than at the present day that the healthy "successful" human organism is that which combines in balanced proportion the biotic, the cognitic, and the cogitic energizing currents, and the capacity of responding to like stimuli.

Sleep is another act, common to man and many-perhaps most-animals, that shows certain well-marked stages. Through lowering of the blood-pressure in and round the brain, possibly also as a concomitant cause through accumulation of oxidizable substances in the brain, the mental organization or cogitic substance becomes largely passive; next the sensory-motor or cognitic follows; while the vegetative or biotic alone remains active during the sleep period. But the at times continuous and more or less active or irregular movements of limbs, specially of the hind limbs, indicates that there is a sub-conscious flow of cognitic energy releasing itself, while dreams and semi-conscious mental states suggest that the cogitic flow is for the time semiminimal to minimal.

In line with this Maudsley says (19S: 10): "Certainly it is true that volition, in its highest sense of control over the mental operations, is abolished in dreaming, as a moment's reflection will show must needs be the case. For such volition is neither more nor less than the expression of the fullest coordinate activity of the mental functions." And again he says: "For 
the same reason the sense of personal identity, the unity of individual character, is confused and seemingly lost." And again, "How can there be a clear sense of the unity of the ego, how any conscience, when there is an entire abeyance of that coordination of mental function, the self-consciousness of which is the feeling of personal identity."

Many of the transition stages or variation phenomena of sleep might well be interpreted, if we consider that the normal or waking passage of cognitic and cogitic energy becomes reduced in amount or in intensity, through gradual exhaustion of the supplies of biotic energy from the protoplasm as compared with the amount used up. But the sleep-period is the time when biotic activity continues in practically undiminished vigor and distribution of abundant biotic energy, in the form of digested, absorbed, and assimilated food, is proceeding. So a gradual recuperation of sense-perceptive or chromatin, and also of mental or neuratin substance proceed, till this very process results in reaccumulated cognitic and cogitic energy that stimulates to waking. Maudsley has advocated the view that waking does represent a gradual reaccumulation up to an optimum stage, of constituents that by diurnal activity became reduced to a minimum.

From the observations of Durham also (199), confirmed by several subsequent workers, the sleep process is attended by reduced flow of blood to the brain, and passage of it to the regetative organs. So, though the relative activity of digestion may then be less than during day, its calm and uninterrupted procedure, followed by absorption of the food, supplies material for the biotic flow that in turn is in part converted into cognitic and ultimately into the highest or cogitic phase. Waking seems therefore, physiologically, to be an optimal reestablishment of stores of cognitic and cogitic energy from the biotic system, succeeding to a state of more or less marked reduction of such stores, when the state supervenes that we term sleep. D) uring the latter state the biotic system alone functions steadily, the cognitic and cogitic are in a state of minimal activity. 
We can now deal with another phase of human and general animal history. If it be true that even the highest exhibitions of mentality are due to a definite energy traversing a highly complex energy-conductor and store-house, the process of starvation and its attendant phenomena deserve study. Here, the nutritive or biotic system being first checked in its food and therefore in its energy supply, pain becomes felt in the stomach, the digestive secretions and specially the saliva become thick and scanty, increasing feeling of thirst next supervenes, then pale facial emaciation. But meanwhile steady loss of fat content, next of blood, spleen, pancreatic, and intestinal content proceed. All of these changes up to this point indicate fundamental disturbance and check of the biotic energizing flow.

Then the eyes become unsettled, the gait becomes increasingly a totter, the voice becomes faint, the hearing often impaired. All such represent a collapsing condition of the cognitic substance. Next the mental sensibility becomes painfully delicate, this is succeeded by advancing imbecility, and often at length idiocy. By this time the body is in the last stages of emaciation, and soon thereafter death ensues.

In this case the remarkable and interesting circumstance is that, up to a late stage, the biotic energizing currents are recuperated from every bodily source, to minister to the cognitic and the cogitic; next the biotic and cognitic centers exhaust themselves in supporting the cogitic, that finally is depleted. Then sudden collapse of all remaining biotically conducting tissue accompanies death. But the post-mortem condition is equally noteworthy, for decomposition rapidly sets in, due it is considered to accumulation of effete material in the body tissues. But it may well be suggested also that such rapid tissue change is due to sudden breaking down of all the complex chromatin and neuratin constituents, through extensive withdrawal from them of their stores of intra-molecular encrgy, in the final effort to sustain life energy.

Slight reference was made in Chapter IV to the action of drugs. We believe that the view there and now set forth may yet form a basis for a better understanding of them. Regard- 
ing one of these Maudsley (198:5) says: "The stages in the gradually deepening unconsciousness which is produced by opium illustrate very well the gradations in the process of going to sleep: there is first a drowsy feeling which becomes soon an irresistible inclination to sleep; the person then falls into a slumber from which he may be roused sufficiently to make a reply to a question put to him in a loud voice, thereupon sinking back immediately into sleep, which deepens rapidly into a comatose unconsciousness from which the severest pinching, slapping, and irritation of all kinds hardly avail to elicit more than the least sign of feeling or the briefest responsive movement; finally he sinks into so deep a coma that he is insensible to anything that may be done to him; all the tortures which savage ever devised and inflicted upon his enemy, or Christian upon his fellow-believer of a minutely different shade of faith, would not touch him-he is in the unconsciousness of death before death. One sense goes to sleep after another, each sinking gradually into a deeper slumber, then the spinal cord and, last of all, the respiratory center in the medulla oblongata cease activity, when the man dies. In the production of insensibility by the inhalation of chloroform or of ether we observe evidence that the person hears after he can no longer see, and that the senses of taste and smell are lost before those of hearing and touch; and in natural sleep it is obvious that there are similar gradations of unconsciousness, one sense being sometimes more deeply asleep than another, or the spinal cord being awake when the special sensory centers are fast asleep."

While some poisons like oxalic and prussic acid, bichromate of potash, digitalin, and others are definite simple bodies, others such as snake poisons, curare, and various ptomaine poisons consist of two or more compounds, either alkaloids, glucosides, or unstable proteids. But physiologically some act at once to irritate and then destroy the protoplasmic substance, and so in the human subject these rapidly change the structure of the blood corpuscle, cause irritation and then disintegration in such vegetative parts as the alimentary canal and its associ- 
ated glands. Here then the sensory or cognitic centers and their nerves, such as the eye, the ear, the skin, and their nerve endings remain intact for hours or days it may be, while all mental acts are equally continued. In time these only collapse through gradual destruction of biotic flows of energy, that thus starve and then exhaust the two higher forms. Examples are found in the mineral and various organic acids, in snake poisons, and in some ptomaines.

The possible explanation in all of these cases may be that such poisons can act chemically on the molecules of the protoplasm or some food constituent of it, so as to rapidly disintegrate its complex molecular or energized machinery, and thereby retard alike the continued flow and the storing of biotic energy. The more complex cognitic and cogitic cell substances may from their greater molecular complexity and so higher energized condition be able to resist these more perfectly, till broken down by failure of biotic material to recoup the waste going on.

Again, the action of the alkaloid curarine seems to be specially on the sensory or cognitic nerves, so that the voluntary muscles presided over by these nerves become paralyzed, the eyes and other sense centers become dull and unresponsive, even though the involuntary muscles and associated organs continue their function, and an excited state of the higher nervous or cogitic centers may be observed at least at first. Here then the curarine seems so to alter the chromatin or cognitic substance in particular, and so retards the flow of cognitic energy, that paralysis of the appropriate main centers for its expression is speedily effected.

Ether and chloroform, in their action on plants and animals, are liquids that retard cognitic and cogitic flow, but in man and higher animals each first and quickly acts on the nerve centers for the latter, and later for the former. So correlated sensation and mental balance are first interfered with, memory next is lost, then the sense centers become paralyzed, and eventually the biotic or vegetative system alone remains active.

Accepting at least for the moment the foregoing conclusions as aiding toward the explanation, on a purely chemico-physical 
basis, of complicated cogitic as well as simpler life conditions, we can now consider various animal acts which by analysis may yield a key to mental processes. Here we would again accept it that, starting with plants and their irritotactic relations, viz., heliotaxy, geotaxy, hydrotaxy, thigmotaxy, paraheliotaxy, chemotaxy, thermotaxy, and electrotaxy, the same sense relations - no more and no less-characterize animals, if we except that to animals the auditory or tonotactic sense is superadded. If, however, as already noted (p. 118), we regard the tonotactic as a highly evolved state of the thigmotactic sense, plant and animal senses in environal relation would agree.

But in animals some of these become greatly concentrated into condensed and highly sensitive absorbers, as, for example, the eyes for the heliotactic sense, the nose for the chemotactic, and so on. Such animals as Hydra (200: 413), Eudendrium (60: 179), and others, however, exhibit a more or less diffuse heliotropic and paraheliotropic response.

From all the facts now before us the writer would conclude that all sense-impressions wholly and primarily arise for each organism from external stimuli, that are passed in through some one or more of the sense areas. In the case of man we can at least approximately picture to ourselves what a human infant would become if at time of birth all the sense areasthe eyes, nose, ears, touch-centers, etc.-over the body were destroyed, and the infant were yet to live. Compared even with the long recorded cases of infants reared in the silence of solitary cells, its condition would be miserable, and it could only grow up as an inert passive mass, even though living, that absorbed no impressions, and that propagated only uncorrelated movements.

Most naturalists and students of animal behavior now incline-and we believe rightly-to put the beginnings of mentality low down in the invertebrate scale, and we have already suggested at or somewhat below the stage where ganglion cells are first recognized. But in attempting to learn how cogitic energy or mentality is exhibited in the lower animals we may begin by selecting a case from amongst the worms. 
Tennent, in his "Natural History of Ceylon" (201: 481), gives the following account of an attack by land leeches on himself and party. "In moving, the land leeches have the power of planting one extremity on the earth and raising the other perpendicularly to watch for their victim. Such is their vigilance and instinct that, on the approach of a passer-by to a spot which they infest, they may be seen amongst the grass and fallen leaves on the edge of a native path, poised erect, and preparing for their attack on man and horse. On descrying their prey they advance rapidly by semicircular strides, fixing one end firmly and arching the other forwards, till by successive advances they can lay hold of the traveler's foot, when they disengage themselves from the ground and ascend his dress in search of an aperture to enter. In these encounters the individuals in the rear of a party of travelers in the jungle invariably fare worst, as the leeches, once warned of their approach, congregate with singular celerity."

Now here, from the sense-stimulation and sense-response standpoints, we would analyze and then summarize the complete action as follows. Chemotactic search for food, heliotactic light action, and geotactic stimuli that might correlate their movements would all be combined into a resultant motion-response that caused them to frequent the native's pathways. But, on appearance of a man or other animal, the moving object started successively changing heliotactic stimuli and heliotropic responses, simultaneously the sound of the moving party started tonotactic stimulus, ${ }^{*}$ while the poised erect attitude was an apogeotropic response due to the other stimuli causing an overcoming of geotropic or diageotropic position. These latter, combined into a resultant that linked up into the ganglion cells of the ventral nerve-centers with the immediately previously formed resultant, together constituted a compounded cogitic resultant that manifested itself as an "advance rapidly by semicircular strides."

\footnotetext{
* As in the case of the Nemertinea we would regard the slightly depressed sense-cells as a rudimentary ear.
} 
This again became compounded or linked with a thigmotactic stimulus when the animal fastened on the body of its victim, while finally the taste and absorption of the blood formed a chemotactic stimulus that doubtless greatly excelled, though it linked on with, those already compounded. The reaching of the traveler's leg therefore is a compounded resultant response, made up of at least two, possibly three, resultants each built up of several distinct stimuli.

The intrinsic capacity to link up these compounded resultants is evidently the function of the ganglionic cells, as experiment and observation will demonstrate from cases of a more and more complicated kind cited below. But at this point it may not be amiss to inquire as to the use of ganglion cells, and to ascertain how they differ in action from root-tip, stem-tip, leafstalk, and other plant cells, or the cells of some simple nucleate animal like Amoba.

Jacques Loeb has well remarked as to the tropisms in relation to ganglia, "The mystery with which the ganglion cell has been surrounded has led not only to no definite insight into these processes, but has proved rather a hindrance in the attempt to find the explanation of them." We have already seen that in many plant responses each such is a resultant of several stimuli. Each separate stimulus, whether geo-, hydro-, or apoheliotactic for a root-tip, causes definite chemical and so energizing changes in the perceptive root-tip region. These are propagated backward to the responsive part. But here the separate stimuli are combined into a resultant or proenvironal force, that causes the root to move in a resultant direction. This need not be in line with any one of the several separate stimuli, though in nature such often happens, specially if one stimulus be, in strength and direction, more powerful than the others separately or combined.

Now what occurs in the responsive part under such circumstances? We can only consider that the chemical molecules, severally affected by each separate stimulus, act and react on each other there, so as to produce new and definitely compounded molecular combinations whose energies distribute 
themselves in such stereoenergetic lines through the responsive cells that these elongate or grow more in one direction line than in other directions. This line of greatest growth or of energizing activity becomes the response line.

But a considerably more complex condition is introduced with the ganglion cell, and is witnessed in such movements as those of the leeches above described. As Loeb has insisted however, complexity need not cause us to regard a condition as one of insoluble mystery. One fundamental requirement for a satisfactory explanation, as Romanes and more recently Loeb have shown, is the gradual development of the physicochemical relation that has been called associative memory. This has too often in the past been regarded as a semi-metaphysical abstraction, without material basis.

To trace its rise as a condition that is bound up with the ganglion cell, and with cogitic acts, we must try to learn what intermediate states arose in transition from the higher unicellular and the simpler multicellular organisms devoid of ganglia, like the Spongida and the Mesozoa, to the simpler gangliate types. In these, as in still lower animals and in most plants, the tendency for separate energizing stimuli to combine their molecular actions and reactions into a resultant molecular combination becomes so mechanical and routine in relation that even in absence of the stimulus a definite motion may occur.

Thus, to cite a single case from plant life, the so-called sleep or nyctitropic state in which leaflets fold together is primarily due to withdrawal of light, but at the same time apogeotropism or diageotropism is acting on the sensitive pulvini or cushions to keep each leaflet in definite position in relation to gravity. The actual position assumed then during night is a resultant of these two actions, possibly even of a third or chemotropic. On the approach of morning light, the leaflets again expand fully. But if a plant be kept in darkness for two or more days it is found that the rhythmic periodicity of plant-movement is such that about the time of normal "awaking" the leaflets will expand more or less perfectly even in absence of light. Here we would consider is a beginning indication of associative mem- 
ory of primitive kind. A rhythmic energy distribution connected with one of several stimulatory acts becomes so adjusted to the molecular mechanism as to start and to effect movement, even in absence of the stimulus. The plant, in other words, has formed a habit.

But as already traced out in Chapter IX (p. 226) we would consider that early separation of a cogitic or neuratin constituent of the cell took place amongst the higher Protozoa. By steady increase in amount and complexity, this gave rise to the evolving nervous system of invertebrates and still later of vertebrates, till it reached a climax of amount and complexity in man. During this process myriads of proenvironal acts were performed in the history of each evolving individual and species. All such resulted in a temporarily satisfied state, that either conduced to advance, or stagnation, or even degeneration of the organism. But the combined states, in relation to definite environments, that proved most beneficial to some animal organisms, became by oft-repeated acts instinctive tendencies of a hereditary kind, through establishment of definite stereoenergizing currents along definite molecular pathways of stimulation.

These probably mainly altered the protoplassmic and nuclear or bio-cognitic material at first, but, gradually affecting and altering neuratin constituents of the nerve cells, produced in these such stereochemical placing of its highly complex Nissl or neuratin molecules that repetition of an act would tend to be started, even when an incomplete or partial stimulation act only affected the neuratin substance.

So we would associate manifold stored nervous impressions or compounded resultant sensory states with the neuratin substance of nerve cells, while the stereoenergetic tendencies that have become associated with the stereochemical molecules of these cells together constitute what in the past have been called instincts. But increase in the number, variety, and rapid correlation with each other of these instincts has gradually established that exhibition of animal activity that is known as intelligence. For this is the view that is more and more being accepted by physiologists and psychologists. 
Thus Holmes (183: 164) well says: "Psychologists nowadays with comparatively few exceptions agree in regarding intelligence not as a faculty standing in sharp contrast to instinct, as was formerly taught, but as one resting on a foundation of instinct and growing out of behavior of the purely instinctive type. The term intelligence is used here in the wider sense as embracing all those forms of profiting by experience through the formation of associations. It therefore includes psychic activity ranging from simple associative memory to complex trains of reasoning. What distinguishes intelligence from instinct is that in the latter the connections between acts are based upon hereditary organization, whereas in the former they are established through experience. The apparently new thing involved in intelligent behavior is the power of forming associations. So far as we can judge of the psychic states of an animal from its behavior, animal intelligence in its first manifestations consists in repeating acts which bring pleasure and in avoiding things which cause pain, and a discussion of the transition from instinct to intelligence naturally involves a consideration of the role of pleasure and pain as agents of accommodation."

The present writer would entirely agree with the above position, except that he would substitute for the word "pleasure" the expression "satisfied state." For pleasure, as applied in human and evidently also in mammalian psychology, would represent a combination of several satisfied states of the organism, and in which combination no-or at most entirely minorunsatisfied or discordant elements entered. For as with chemical actions and reactions, as with movements of root stem or leaf parts of plants, as with the motions of Amoba or Paramocium, so with higher animals; the result of every proenvironal change is to establish for a time a satisfied state.

Now in connection with H. Spencer's and Holmes' (183: 170) position as to why "animals which took pleasure in following acts that brought them benefit were preserved and those that did not behave in this manner were eliminated," Iolmes asks - "But why does an animal tend to repeat an act that hrings it pleasure and avoid one that produces pain?" "It seems so 
natural for creatures to behave in this way that the existence of any problem here is usually unsuspected; but this is the problem which confronts us when we endeavor to obtain a clear understanding of the way in which intelligence develops out of instinct."

In next paragraph he then says: "In the pleasure-pain response we have two problems of a quite different nature - (1) the problem of how behavior is modified by its results, and (2) the problem of. why pleasure is associated with certain physiological activities, such as securing movements; and pain with others, such as avoiding movements. The latter problem is one whose solution appears hopeless. [Ital. writer.]

"Why then should pleasure be connected with one kind of activity and pain with another? Why not just the reverse? This problem is, I believe, insoluble, because it is a question of the physical and the psychical."

To all of the above, the writer would say that every cogiticlike every cognitic and biotic-act represents a microchemical change due to a definite expenditure of cogitic energy on a definite micro-chemical substance. When such change works in harmonious and rhythmic physical or chemical relation with most or all of the other microchemical constituents of the animal, satisfaction - as a result of most component stimuli being in rhythmic harmony - or even pleasure - as a result of complete harmonic rhythm-is the result. So pleasure might be defined as: the harmonious and rhythmic agreement, in their energized motion, of all molecular compounds of an animal, when these compounds are acted on by one or more stimuli." Pain then is: the inharmonious relation in the energized motion of definite molecular compounds, that compose the tissues of any organism.

Numerous illustrative examples of both from the human organism might be given. Thus when a ripe pear or plum is eaten the sweet taste to most is chemically satisfying to the molecules of the taste sense endings, the soft mucilaginous substance starts another satisfying response, the contained sugar, acid, and mucilage are all chemically satisfying to the fer- 
ments and also to the absorbent cells of the alimentary canal, the substances as assimilated by these cells are satisfying from the nutritive standpoint to the general cells of the body and nourish these. So from the first acts of taste-stimulation till ultimate nutritive absorption has taken place a completely satisfied resultant or cumulation of sensations, or in other words a sensation of pleasure, is experienced.

In marked contrast to the above is the resultant action of honey on different human organisms. For most individuals a highly pleasing and satisfying food, it is for others a highly disagreeable and disturbing chemical substance.

Let us now cite, however, a somewhat opposite, but yet highly interesting case. Many can eat with satisfaction raw or fried onions, others on eating a piece no larger than a bean become nauseated within three to five hours, later on pained in the head, then flatulent, and for twenty to twenty-six hours highly unsatisfied or disturbed throughout the organism. Such seems to be entirely due to the presence in the onion of oil of garlic $\left(\mathrm{C}_{6} \mathrm{H}_{12} \mathrm{~S}_{2}\right)$, a definite chemical body which to many acts as a completely satisfying, i. e., pleasant compound, but to others develops a highly pained condition.

Many substances like alcohol, morphine, nicotine, acetic and citric acids, or annotto, are either at first or always unsatisfying, i. e., unpleasant, but are or may become highly satisfying and so agreeable to others. Some of them also may be nutritive and synthetic, others innutritious and analytic. The lower animals instinctively perceive these differences, owing to the usually limited range of substances from which they draw their food supply, and the usually high sensitivity of the olfactory organs. The higher animals, and especially man, with as a rule a wider range of food substances and more extensive change of environment, respond to a large degree intelligently.

Intelligence then is fundamentally a rapid as well as extended capacity for distinguishing between cumulated resultants of distinct stimuli that are satisfying and so helpful or pleasing to an organism, and like resultants that are unsatisfying and so retarding or destructive to it. Thus the intelligence of a 
brown ant as compared with a hemipterid insect is shown not only in the careful selection by the former of such food supplies as seeds, but even more in the removal to a nest underground, in the destruction of the radicle, in the storing of the grain, and the utilization of it as food at an appropriate time. Such extended provision for the future is, therefore, correlated with complex brain development.

A steadily cumulated mass of satisfied experiences of like nature, gathered along the nemertean-vertebrate line of evolution, has reached a present-day climax in superman, whose resultant stimuli are often gathered from a world-wide or even universe-wide area, and whose proenvironal responses already may have world-wide consequences.

so, in comparing instincts and intelligence, the writer would consider that the so-called instincts of many animals, even high in the zoological scale, represent the result of oft-repeated lines of stimulation or energy flow toward nerve cells, and the placing there of complex molecular groups which combine these lines of energy into a resultant state. Agreeing with this, though less fundamental in explanation, is Parmelee's definition of instinct (53: 226) as: "An inherited combination of reflexes which have been integrated by the central nervous system so as to cause an external activity of the organism which usually characterizes a whole species and is usually adaptive." Again, on page 266, he more briefly defines it as "an integration and correlation of reflexes by the central nervous system," and then adds "this kind of integration and correlation is inherited and is performed by the sensory and motor parts of the central nerrous system, which are specialized for those purposes. When a certain number of these instincts which are readily modifiable have evolved, and when the central nervous system has developed parts which are not specialized at birth, so that they can serve as association areas, then intelligence may make its appearance."

But, as the researches and experiments of Romanes, Uexkull, Lloyd Morgan, Thorndike, Yerkes, Cole, Davis, and others have demonstrated, instinctive acts may be performed by a 
small group of nerve cells as in medusæ or echinoids, or by localized ganglionic enlargements as in the earthworm, or by special areas of the spinal cord or lobes of the highly differentiated brain as in dogs, guinea pigs, etc. But it is when the ganglionic masses become more and more concentrated in, and correlated or summated by, the enlarging brain that instincts merge into intelligence. So, while in the groups Cephalopoda, Arachnida, Insecta, Aves, and in the lower Mammalia (p. 545) the more primitive members of each group show rather feeble instincts, as one rises through evolving genera of each group a stage is reached when the instincts become so condensed and correlated into summated resultants that such deserve fully the appellation intelligence.

We have already stated that such advance is correlated with increased condensation of the nerve centers, while both are dependent on and are an outcome of rapid transfers of afferent and efferent stimuli through highly specialized organs accessory to and around the brain, such as antennæ, trunk, or arms. In this connection we need merely draw attention once more to Edinger's diagrams of brain increase, and specially of cerebral increase, in transition from fishes to man (p. 240). As to the degree of intelligence shown by higher invertebrates and the lower vertebrates, Holmes in Chapters $\mathrm{X}$ and XI of his work (183) has ably discussed the question, though the writer inclines to consider that he has-possibly with commendable caution at the present stage of our knowledge - rather underestimated intelligence, and overestimated instinct in some groups, notable the higher insects.

But in the process of neural growth and condensation an important feature is that the sense-perceptors develop, from the Rotifera and Turbellaria upward, nearly always in definite order, and in certain ratios of sense importance. Thus even in Rotifera, but particularly from these upward along the turbellarian, nemertean, cyclostome, and apodous line up to man, after the first foundations of the nerrous system have been laid down the olfactory, the optic, and the audito-geotactic sense receptive centers appear in named succession. The 
brain areas connected with these also constitute the steadily enlarging masses in the above groups, while the tactile, the thermotactic, and the diffuse chemotactic sense receptive centers differentiate later and are mainly centered in the smaller rentral brain lobes of turbellarians and nemerteans, which we have considered (p. 435) to become the medulla in protovertebrates, and in still higher groups the medulla-cerebellum.

Now if we reflect on the enormously greater number of sense impressions that are of prime importance to each organism, which are received by the former or dorsal set, as compared with the fewer and less important ones received by the latter or ventral set, further how abundant are the cumulated resultant responses developed in the brain from summation of the former set as compared with those of the latter, it is not surprising that the dorsal brain mass of turbellarians and nemerteans has gradually led up to the enlarging fore- and midbrains of cyclostomes, urodeles, and mammals. For the increasingly abundant use of the anterior snout part of the head, specially of the olfactory area and of the eyes, in guiding each animal toward its food, in enabling it to ward off enemies, in aiding it toward suitable depositing of its eggs and caring for its young, have all conjoined in stimulating the brain to steady growth in size, and increase in correlated nervous responses.

Transition from a fresh-water to a land existence, and afterwards to an arboreal life, must have stimulated further the above centers to increasing complexity and correlated nervous activity. For in all cases along this ascending line of vertebrate evolution when side lines arise that reassume or retain an aquatic life these are nearly always less intelligent than the terrestrial ones. Thus the land as compared with the freshwater Apoda and Urodela, the land carnivora as compared with the seals and walruses, the ungulates as compared with the sirenians and cetaceans, are examples.

But again the development and steady perfectioning of four ambulatory limbs during the above transitions to a land and arboreal existence would powerfully cooperate with enlargement of the cerebral and optic centers in stimulating to nervous 
action. For all are aware how perfectly the nostrils, the eyes, and the limbs are linked up in their correlated connections with each other and with the brain. But, since the neuro-muscular innervation of the limbs seems in considerable part to be associated with nerves that spring from the ventrally derived brain lobes that became the medulla and later also the cerebellum, more and more complex correlation between the cerebrum, the optic lobes, the medulla, and the cerebellum would ensue.

Hence the highly intelligent actions that are characteristic of many carnivores like the dog, the fox, the cat, and the racoon; of some rodents like the mouse, the rat, and the beaver; of many monkeys like those already referred to (p. 565). But as traced in Chapter XX, when the forelimbs became increasingly used as prehensile, tactile, selective, fighting, and building organs, through increasingly upright posture amongst the higher monkeys, in corresponding ratio would increase take place in the main brain centers. Thus, in all of the above-named animals, multitudes of greatly varied sense impressions would be passed in daily to the brain, and while not a few might at once be summated and responded to by proenvironal effort, very many would become, if we may so express it, stored stimulant impressions that increased the neuratin substance of the brain, but to which no recognizable response would be made. Such however would tend, we consider, to start the rudiments of concepts, owing to the abundance, the variety, the complexity, and the correlation of the percepts poured in on the brain during the active hours of each day.

So the dawnings of concepts or evolving reason seem clearly to be traceable in all of the above groups, specially in the higher Carnivora, Rodentia, Proboscidea, and Primates. But in connection with the valuable comparative studies of Edinger, Herrick, and Johnston on the forebrain of vertebrates, which functioned primitively as an olfactory sense center, but which later in fishes and higher vertebrates gave origin to the cerebral lobes, it might well be objected that the upbuilding of this most important pair of lobes could more appropriately have been originated or effected from the midbrain or optic lobes. 
But when one watches the movements of any mammal up to the monkeys a helpful interpretation seems forthcoming. For, when a fruit or other food is presented, the eyes in connection with the optic lobes form a rapid and cumulated response activity. But the discriminating-one might almost say the intelligent-handling of the fruit in front of the nose, before any effort is made to eat it, is proof that the earliest formed of the animal sense centers is also that which retains the most fundamental importance. All again who have noted, as has the writer, the rapid and usually unerring sweep of a pack of hounds, over four or five miles of rough land, when led only by an invisible and faint trail of animal skin, can realize how important the olfactory center has been in the upward evolution of mammals. Though man has often allowed his primary sense function to lapse somewhat, owing to varied use of the hand, of the eye, and of taste, this merely indicates a transfer of sense-activity and response from one center to another.

Reason, as the term is ordinarily used, represents a steady advance on Intelligence, as the latter is on Instinct. It is a phenomenon of nerve cells in which these depend more and more on inherited associative tendencies, instincts, and acquired intellectual percepts; less and less on direct, and individual, peripheral stimulation of the sense organs. Holmes says (p. 274 ): "If we define reason as the derivation of conclusions through the comparison of concepts, it is not improbable that no animal below man employs this faculty. But this is far from implying that animals cannot perform mental operations, which are essentially inferential in their nature. Reason is not a faculty which stands sharply marked off from other forms of mental activity. Between simple perception on the one hand and abstract ratiocination on the other there is a fundamental kinship, and the latter process may be connected with the first by numerous intermediate stages."

So between percepts that have been gradually linked up into complex resultant percepts that start an intelligent response, but which at the same time so mold the nerve cells as to cause these, even on future faint stimulation, to evolve like 
intelligent response, and that state in which the evolving nerve cells link together several intelligent response-impulses, and in the process start a concept relation, the transition seems to be graded and clearly traceable. But here the "cross-questioning" of animals has only been practised to a moderate degree during the past twenty years. The comparatively scant results, however, clearly indicate the ever-widening avenue of research and reward that the remaining years of this century will bring.

But as a measure of caution, if we select only results from the higher mammals, the experiments of Cole (234: 211) and of H. B. Davis (235: 479) with racoons indicate the formation not merely of percepts regarding the position, connections, and motions of the various fastenings the racoon had to unloose, but also definite even though perhaps simple concepts as to how the position, connections, and motions of these were related to a definite end. The fact that this end was a satisfying one for them, in that they thus achieved greater liberty in some cases, and still better were rewarded by food in others, is proof that the satisfied state is the constant ultimate, eren though temporary, result that molecular combinations work toward, from inorganic particles upward in graded manner to highly complex neuratin interlinkings.

While discounting many of the reported wise acts performed by dogs, beavers, and elephants, as well as other animals, there still remains a large residuum of known effort that can only be explained as due to simple though exact concepts or reasoning toward high proenvironal results. For when one watches the movements of a trained elephant, even though under the guidance of a still more trained "keeper," it is human arrogance and bigotry alone not scientific skepticism-that would deny reason to the former, and grant it to the latter.

The studies already referred to on monkeys (p. 565) and others that are yearly appearing all furnish solid and exact proof of the intelligence as well as the reasoning faculties possessed by many of these. But between the highest mental arts of a monkey and the average mental acts of a Bushman, a 
Fuegian, or a Tasmanian the gap is a wide one. Can this be explained, however? We would reply that it is wholly due to two causes: first, the increased use of the forelimbs by higher monkeys till these became specialized hands; and, second, the extinction-as so often happens to intergrading and conjunctive links in a great transition process-of the intermediate types that showed the transition proceeding. But the remains of Eoanthropus, of the Neanderthal, the Aurignac, and other transition human types indicate that exactly such desiderated links once existed.

Now, from the standpoint of mentality evolution, what alone or mainly separates and elevates primitive man above even the highest anthropoids is his increased capacity for distance-perception, or as we may term it telecognitation; then his succeeding power of distance-conception, or telecogitation as we may name it. Steady exercise of these during long millenia of the past has enabled superman to reach out during the past half century to the conception of an infinitely extended stellar space, in which our solar system constitutes but a few stellar atoms relatively speaking. Such also has enabled him to grasp the existence of an infinitely minute electron system, and to explain the relation of infinitesimally small amounts of energy in such.

As stage by stage of this telecognitation and then telecogitation are traced, we gradually recognize that the pathway opened up has been due to cooperative and reacting activity of hand and brain, with a resulting high condensation and work perfectioning of energy, that originated the state of energy that we have termed the spiritic. For in its highest expression this pathway has slowly but none the less surely enabled evolving man to grasp the conception of his own existence as being one with, and dependent on, a great energy, spiritus, motion, or power of the universe, which has energized, still energizes, and steadily evolves man to ever better and higher estate.

This upward advance might be roughly outlined as follows. Primitive man, like the higher anthropoid apes, largely occupied a limited area of action, both as an individual and as one 
of a family. Correspondingly he had a limited field from which to draw new sense stimuli or impressions. But perfected upright posture and bipedal progression caused him to look outward and upward, at the same time also set free the hand-arms that might gather countless afferent stimuli and pass these to the brain. Like the Ainu, the Fuegian, and the Australian native at the present day, his rude leafy bower or stone hut represented the center round which the aims, affections, efforts, successes, and burial places of each head of a family and of its units centered. But bartering at one time, fighting at another, migration-willing or forced-at another; or again following of the chase, and fishing along coasts and rivers, opened up widening fields of stimulation, increase of varied brain recepts, and advancing complexity of proenvironal concepts. Further, as the sun, the wind, the rain, or the snowstorm by day, the moon, the stars, the shifting shadows, or the prowling animals by night, were watched often with fear or awe, these opened up still wider fields of stimulation action and proenvironal response. As canoes of ever-increasing size by river and sea, or tamed animals by land, were used for rapid and wide conveyance to distant points, added stimulations and responses were made, while the brain became ever richer in nervous organization.

As with other evolving animal groups also, such as the bees, the ants, the beavers, the prairie dog, and some monkeys, social or moral combinations and restraints were increasingly perfected amongst ever-widening groups or races. This knit together the individuals of a family, the families of a race, and in time the races of a nation, alike for exploitation or vegetation, for defense, and for reproduction. And, in the accomplishment of this, primitive man was slowly evolving new and ever wider stimulation combinations, that resulted in advancing complexity of proenvironal response. Extravagant though the view may seem also on first thought, such advances were largely due to cooperative hand-arm and brain activity. For kind and considerate acts to wife and children, defense of these from attack, provision of daily food for them, instruction of the 
offspring in devices or skillful acts that would equip them later for mature life, as well as many other kindred deeds, are, and were nearly all, made possible by hand-arm and brain cooperation.

So human morals became a most important factor in human advance, and represented myriads of environal stimuli of an intelligent or even reasoning kind, due to contact of man with his fellows, and the resulting formation of proenvironal responses that gradually became recognized as conduct, or in other words the most satisfying and satisfied behavior for himself, and in relation to his fellows around. The consideration, therefore, of morals as a great agency for human advance will be taken up in a later chapter.

But a still more significant environal condition was established when man started distance concept connections. For, while morals became to him increasingly necessary if a satisfied but also a proenvironal and so progressive life was to be lived in relation to his fellows, his mind became occupied with ratiocinative problems that the moral relations in part started and seemed to obtrude on him. Love for wife, children, or members of the tribe; an awed and reverential regard for all the exhibitions of nature that surrounded him and which seemed to pursue their courses without his aid, before which also he appeared often powerless; an increasing knowledge of animal migrations, of seasonal flowering or matings, of diverse climates and their different products; a study of the movements and relations of the heavenly bodies and a cumulated knowledge of the power and magnificence of the sun and moon, were all compelling stimuli of most varied kind that started increasingly complex proenvironal thoughts and attempted reasons as to their interrelation with each other and with him.

So originated and developed, we have every cause for believing, the religious field of man's mental evolution, that has been as much misunderstood as it has been minimized in value, but which has been a powerful lever in man's mental and spiritual uplift.

For the recognition by man of great and as yet unsolved exhibitions of natural force, energy, spirit, that were outside him, 
that he could little if at all govern, that at times seemed to benefit, at times seemed to injure him, all conspired to raise sublime thoughts in him, and compelled him to reason on them. In his effort then to build up ever wider proenvironal responses to these expansive environal stimuli that stirred his highest brain centers, man had thus presented to him wide and lofty ideals, that in their practically infinite scope caused him to aspire, to proenviron ever higher, further, and more earnestly. Thus originated in time deep spiritic longings, a desire to comprehend the source and the working of that infinite energy that seemed to move all things, but was itself elusive. And so man became simultaneously the scientific and the religious animal.

He observed and thought more and more deeply and widely on the physical and vital phenomena around him; and so the discovered facts of law and order, in family life and heredity, in seasonal change, in plant and animal life, in the winds, the sunshine, the snow, the rain, or in the heavenly bodies, became at first crude, but in time accumulating scientific lnouledge. The reverence he showed, the submission of his knowledge and will to a greater Power, the longing desire to become one with, or on reverential terms with that Power, developed religious aspirations, the unfolding of which in human history is sketched in a subsequent chapter, and the phases of which, when followed in a pure and reverential frame of mind, have immensely helped to raise man above "the brutes that perish."

The brain centers that were mainly concerned in this eleration of man to high estate seem to be the deepening and complicated convolutions of the frontal cerebral lobes, and reference is made to these elsewhere (p. 684).

In concluding this chapter then, we would renture to assert that all molecular organization, from the simplest gaseous and liquid compounds, up to the most complex colloid cerebral constituents of man, is evidently the result of integrating physico-chemical force, or energy-exhibition. And so the all important consideration in such integration is not the purely chemical side of the question, as has too often been considered 
in the past, but rather the physical. It is for this reason, and in recognition of it, that in recent years there have developed the great and promising departments of thermochemistry, photochemistry, and electrochemistry, in each of which the chemist leaves his old standpoint of merely considering the atoms and molecules in their varied equilibrated groupings, and has advanced to the more suggestive inquiry of how and why they are grouped in definite manner.

So we believe that thermochemistry, photochemistry, electrochemistry, biochemistry, cognitochemistry, and cogitochemistry will each and all be gradually accepted as fields of study, in which the chemist will treat the variously energized ether particles, but will borrow from the physicist a knowledge of the graded modifications or condensations of energy, by which alone the steadily integrated ether particles become of increasing complexity, till they culminate in the most complex colloid molecules that characterize and are associated with, mentomoral and religious processes in the higher animals and specially in man. 


\section{CHAPTER XXII}

\section{THE OPERATION OF THE LAW OF PROENVIRON- MENT IN THE EVOLUTION OF MAN}

In Chapter IX the fundamental and widely extended importance of the law of proenvironment has been sketched, as it is exhibited in plants and in the lower animals. But, as we hope to demonstrate in the succeeding context, this law has received its most striking, most condensed, most varied, and most far-reaching confirmation in the history of human progress, since the time when man began to use his forelimbs as hands, and then evolved language, thereafter writing and mechanical inventions, on to the present day when he penetrates and maps out the heavens, weighs the earth, communicates from side to side of the world in a few minutes, and looks even on the larger molecules of matter.

The law moreover is seen everywhere in action, not only in the mental and moral fields of human effort, but specially in that field which we term the religious. For man also, as for other highly evolved mental types, the law of proenvironment becomes increasingly emphasized as special appendages accessory to the brain - in this case the hands-become more and more perfectly modified as environal contact-organs.

In Chapter IX we have defined the law as "the capacity of an organism for perceiving and then positively growing or moving toward an environment that is the most satisfying for it." We hope further to show that, as with many lower animals, man has the capacity in a unique manner to receive into his nerve-cells the resultants of many and varied environal stimuli, to compare or to correlate these with each other, and to evolve a compounded resultant that causes him to move or act in a relation that is the most satisfying for him. 
As compared therefore with proenvironment in plants or the simplest animals, man and the higher animals show one marked feature of advance. For while in the former any one organ takes a proenvironal course, that is a resultant of sereral compounded stimuli acting by aid of cognitic energy, in the latter many of the proenvironal courses taken are the compounded resultant of several separate resultants, each formed from compounded stimuli, as set forth in the last chapter. It is this capacity for forming a complex compounded resultant, which gives to higher animals, specially to ants, apes, and very notably man, the rapid power of mapping out proenvironal pathways, and of moving or acting along these.

Emerson recognized, and crystallized in words, the law of proenvironment in its applicability to man, when he said: "The genius which preserves and guides the human race indicates itself by a small excess of good, a small balance in brute facts always favorable to the side of reason."

In illustration, examples of proenvironal action will be cited first from personal or individual human life, and second from social life. But various of the evolving life-conditions set forth on pages of Chapter XX fit in perfectly under one or the other head. In many national museums also, suites of specimens are often exposed that form an admirable practical illustration of proenvironal progress, as accomplished by man in inventive achievement.

The ordinary pocket-knife of two or three blades is a highly useful article. We may recognize its first beginning in the rude, somewhat flat, roughly chipped flint with ragged edge, that man of the earlier stone age fashioned. Involved in the fashioning of it were numerous and repeated heliotropic, mechanotropic, apogeotropic, and other stimuli that were passed mainly from the eye and the hand to the brain, there to be summated into a resultant stimulus. But, as he compared a very rough-edged with a smoother and more sharply edged example of his effort, he again summated both pictures into one which caused him to evolve or fashion a tool that by continued and careful polishing would give a quite uniform 
sharp edge. So the proenvironal picture took shape in the improved knife of the mid- and later stone ages. But, in noting that to have it all or mainly cutting edge, as not a few showed, he hurt his own hand, a proenvironal picture was formed of one that should be rounded or hafted below and cutting above. This was incorporated into the knives of the bronze age, the immense superiority of which over the best stone ones was recognized. But, comparing the stone with the bronze implement, some inventive, aspiring, and unsatisfied mind looked to securing a metal that might be even harder and keener in cutting. Iron was the proenvironal reward of the proenvironal outfeeling or search.

But, in using the knife frequently for wet operations, a proenvironal picture arose of a handle that would not rust and which might furnish a frictional surface. What better here than the deer-horn secured in the chase, or the bone of the deer's limb. Thus the bone handle was proenvironed, was tried, was adopted, and is an heirloom that has come down to us.

But considerably later, as active proenvironal efforts and aspirings of a manifold mechanical kind possessed man, the knife joint was planned, and put into practice. Now here, as in many human inventions, and in not a few animal and plant variations, a distinct mutation was effected. For from the rough-edged stone knife to the smooth one with elongated cutting edge, from the latter to one with rounded handle, and from this again to the bronze and the iron implements, a steady continuous advancing variation took place. But alike in the introduction of the bone handle, and still more of the knife-joint for folding of the blade, a fundamentally distinct change and advance were made, that constituted a saltatory or mutational variation, according to de Vries' fundamental principle.

With new proenvironal advances, strength, neatness, and varied utility were more and more secured, till the picture of possible division of labor amongst several blades became the aspiration that ere long was fulfilled. As a final and almost 
exaggerated outcome of proenvironal planning, the complex though compact knife has been evolved that carries from 10-30 separate implements, such as knife-blades, file, corkscrew, chisel, and many other parts. Such an instrument also is a striking illustration of a compounded resultant, built up by uniting the resultants of various distinct stimuli into one correlated whole.

The writer has lived through the period during which the proenvironal evolution of two instruments of marvelous perfection has been going on, viz., the bicycle and the automobile. As a small boy he had the tradition of an uncle who planned and put into practice a small carriage that was difficultly propelled on four wheels by foot treadles inside. But as a young schoolboy he saw this further perfected into the heavy cumbrous tricycle of forty to forty-five years ago. Each inventor-proenvironer-as we might well say-who turned his thoughts to it, took all the best points of previous constructions, as definite stimuli toward better results. These he united into a new resultant stimulus in the ganglionic cells of the cerebrum, and evolved therefrom a plan that was an advance in the bearings, the couplings, the wheels, the seat, and so on, till the popular tricycle of 30 years ago resulted. In some points the improvements represented advancing variations of a very gradual kind, in others they constituted distinct breaks or mutational changes.

But even then a daring proenvironer had thought out and realized the high bicycle with large and small wheels, that for younger spirits soon put the tricycle in the shade. And this truly represented a mutational evolution. Such however was chased out in turn by the low-set, high-speed geared bicycle of the past decade, that is now being superseded in part by the autocycle. To those constructors and enthusiasts who followed closely all of the changes introduced, these were almost as gradual and imperceptible-because of their satisfying appropriateness - as have been the changes undergone by some cultivated plants and domesticated animals during the past century or millennium. 
The history of the automobile is so recent and so well known that mention of its proenvironal evolution will suffice. But in passing one may well ask, what could eren the most mentally accomplished man have achieved for it, or for the bicycle, had not the brain been constantly stimulated by mechanotactic and heliotactic stimuli sent to it from the hand and the eye, had not these been united into resultants that so acted by pencil, paper, and rubber as to start compounded resultants in which metals, woods, leathers, cloths, oils, and other substances were proenvironally dealt with as if each were a material fact that stood out before the operator's eye. The heliotactically acting eye guided the hand, which in all of the above varied efforts was the organ that continuously brought the brain into closest environal contact with those environal objects that had to be molded, fitted, adjusted, and started into final action. Then resulted such humanly devised or "proenvironed organisms," as we may well call them, that in their harmonious working and varied construction quite rival complicated animal or plant organisms. So the remarkable proenvironal advances made by man during the past century have been due to cooperative activity of the ocular or heliotactic sense, the hand or mechanotactic sense, as well as others of less prominent kind, and the brain as a sensitively receptive, correlating, and originating center for new resultant stimuli. All of this also has proceeded on the physical principle of action and reaction.

But a valuable application of the law of proenvironal erolutionary advance in man's history can be made to the problem of species and genus evolution amongst animals and plants. In previous chapters we have discussed "the factors of organic evolution" that have originated species, genera, and families in succession. But with man every proenvironal and then realized instrument is a genus, of which he continues to evolve species and varieties. Thus at the present day we can truly and exactly say that the cart, the wagon, the horse-carriage, the tricycle, the bicycle, the autocycle, and automobile form collectively a "conveyance" family, that might well be termed 
the Conferraceæ. The wagon or the cycle forms each a genus, the latter of which we might name Cyclus. It includes the old primitive four-wheeled type or Cyclus tetrarota, the newer $C$. trirota, then the bicycle or $C$. dirota of which several marked varieties as the high-wheeled and the low equal-wheeled as well as others might be given. Still more recently the autocycle appeared as $C$. autorota.

With all of these however, as with plants and animals, "fossil" forms often exist in museums, that in ordinary life are now extinct. Thus the old Irish cariole amongst horse conveyances and the high bicycle that might be termed Cyclus dirota var. inequalirota are alike relics of past decades or centuries. Later in this chapter we shall consider how far such phenomena in human progress may or may not be paralleled in the origin of organic forms; that is, whether or not fundamentally similar laws have been in operation that may govern both.

Before leaving instruments like the above, the fact deserves to be emphasized, even though known to all, that the most important improvements and modifications in all of them have mainly been with the aim of securing a more perfect storage and utilization of energy, in relation to the work that each has to accomplish, as well as the greatest capacity to set free most quickly the greatest and most perfect kind of energy available. Such also has been the principle running throughout the entire plan of organic evolution. For in man the degree of energy-concentration and expenditure needed to perform a certain mental or cogitic act greatly excels that needed by a lower animal in performing one that involves less mentality, or that rises only to the cognitic stage of energizing complexity.

The writer may next be permitted to refer to and describe the proenvironal act that first suggested to him the possible value and far-reaching nature of the law of proenvironment. At the University of Pennsylvania he was permitted to plan out and to develop a University Botanic Garden that would cover an area of fully four acres. The space to be changed 
was of the roughest and most desolate kind consisting largely of sand hills, gravel pits, soil heaps, and weedy banks.

His previous experience, or in other words his stored nerve stimuli, obtained from many and diverse stimulation centers; also his appreciation of the good and bad points of many existing botanic gardens; his desire to plan out the best results for the space at his disposal; his aim to suit the area and details to the most active period of teaching; and as in most human enterprises to achieve the results at a certain cost; represented each a resultant mental stimulus, that was built up of many separate sensory stimuli.

These separate resultants were all gradually linked up or combined into a definite plan or picture that became a proenvironal effort toward the aim in view, and that anticipated even the gradual material growth of the decade that was to follow. The lakes, the valleys, the quiet shaded walks, the shrub groups, the beds of natural plant families, the ferneries, the rock-garden, the bryarium, and the greenhouses, all took clear shape as to size, relation, value, color-effect, and scientific usefulness, while as yet desolate wastes were around.

Within two years many of the details had become accomplished and correlated results; within a decade most of them could be looked on. But even now, after a lapse of more than twenty years, a few items in that proenvironal aspiration remain unmaterialized except on paper, or in the writer's mind.

Throughout the years the mental proenvironal picture remained as a continuous resultant response that gradually worked to, and worked out, one main result. Throughout the same period also the writer had been endeavoring to explain the "betterment" principle which many evolutionists had noted, but had failed like himself in getting a key to. This summated proenvironal effort seemed to explain alike the gradually more simplified resultant acts in lower animals and in plants, as well as, and even more strikingly, the gigantic hand, eye, and mind efforts of man as witnessed in the pyramids, the Parthenon, the Colosseum, the cathedrals, and the 
huge factories of modern commerce with their contained machinery. And similarly for each of them, as for the Botanic Garden, one dominant idea prevailed as a compounded resultant, namely memory of the exalted dead for the pyramids, idol-worship for the Parthenon, barbarous amusements for the Colosseum, worship of God for the cathedrals, production of some one article of commerce for each factory.

If now we consider the connection between any one individual and his community, in a primitive state, where hunting, fishing, fighting, hut-building, clothes-weaving, food-cooking, and like occupations made up the acts of each life, the successful survivor, in application of the law of Natural Selection, would usually be the one who proenvironed and then fashioned the most imposing but healthful hut, the "best" clothes, the most nutritious food. The keenest and most far-sighted proenvironal outlooks, when put into practice as definite proenvironal responses, aided and selected the originator of such.

In modern civilized society, various conflicting factors come in to obscure or slightly alter the proenvironal law. But its constant influence is everywhere seen and felt. The man who can inhibit lower desires, and who can deny himself today, while others are spending on luxuries, is planning and projecting a wise future; the man who patiently combines several mental stimuli-even though some of these give rise to responses that are failures-into a tool that combines all the best of the past with added points of advantage; the architect who cumulates in his mind all the finest details of beauty, finish, internal adornment, and usefulness of past buildings, and then proenvirons in his brain, still later on paper, and finally in stone and mortar, a building that excels; the agricultural inventor who previews all past reaping machines in his mind, and then proenvirons one that in thought he already sees excelling all others at agricultural shows and in farm practice; these are the individuals who become "leaders," and successful survivors.

The continued mental environal stimuli and proenvironal responses draw new supplies of cogitic, cognitic, and biotic 
energy, that together tend to carry the human organism to ultimate success. And in any community or nation where "struggle for existence" is allowed fullest scope, "natural selection" then steps in to ensure survival of each such successful one. For under such a merciless law-fortunately not the only or highest law of organic life and survival-the less inventive, the poorer, the feebler, the less far-seeing succumb in "the race for life."

But mention of these last problems again brings forward for consideration that problem already discussed partially in Chapter XIX, namely social or cooperative action. We can now examine some phases of it, in order to ascertain how it might work out for man.

Amongst primitive nations of the past, or even amongst present-day ones, let us suppose that two of these inhabited adjacent land areas, and were struggling keenly for territory, and all that such means. Let us suppose further that both had at a certain time an equal number of inhabitants, but that one of them by hard vigorous individualistic laws allowed the less successful-the weaker, poorer, less alert by slight degree - to perish, while the other by ameliorated social laws kept the less successful alive, and even encouraged a feeling of kind loyalty, of sympathetic helpfulness, of friendly cooperation on the part of the less with the more successful. If now, in a close-drawn and sanguinary war, the former could muster and rely on the loyalty only of its "successful" ones who had pulled through in the national struggle for existence, while the other could muster equally its successful and its less successful though by no means discouraged or useless ones, who also were encouraged by a kind and sympathetic feeling toward the more successful, the victory would almost certainly rest with "the big battalions." For the latter was big in the double sense of numbers and of enthusiastic unity in a common cause.

Exactly such a biological condition prevailed during the war between Japan and Russia. The former country had a recent and comparatively unshapen history; it had derived 
its civilization and its fighting arts from the west; its people were physically smaller and mentally had not produced many recognized great minds. But the individuals of the nation were animated by a common sympathetic aim, they unitedly proenvironed a course which all were prepared to work out; the successful Count and the Samurai as well as the poorer peasants were fed, clothed, and cared for at each step in the campaign. In contrast to this the Russian army consisted of "successful" leaders in their generals, admirals, and commanders, who had faithfully imitated the brutish Peter the Great in use of the knout, the prison, the mines, the frozen fields of Siberia, for their "subjects," their less successful ones, when these proenvironed a nobler and sweeter national life. So, when the "successful" ones led the battle by sea or land, those who were not despatched by Japanese bullets in front fell by the bullets of their "subjects" behind.

History has often witnessed this biological procession of events in the past, and doubtless will also in the future, till each nation learns that every healthy member of it represents an asset that deserves highest consideration.

Such a phase of human natural history, often spoken of under the narrower and frequently misleading names of national politics, economic problems, party prejudices, local sympathies or antipathies, introduces us to the second and even more striking, though more complicated study, namely, proenvironal reaction in social human life.

As accepted and dilated on by McLennan, Lubbock, and others, primitive man, like most of the anthropoid apes, showed social as well as family tendencies and habits. So abundant fruit crops, or vegetable supplies, or animals caught in the chase, were a source of common rejoicing and were eaten in common, even though in separate family huts. Conversely times of scarcity caused each little community to feel the greater need for wise planning in the future, so that there might be food reserves for the days of scarcity.

Even when the stage had been reached where man became a settled agriculturist, it was possible for each individual or 
family to cultivate-in Thoreau fashion-a patch that would suffice for the individual or family needs. But-in Thoreau fashion again - the lone effort became an irksome and unsatisfying one. So like Thoreau with his third chair for "company," an exchange of neighborly ideas, of field produce, of fruits from the chase, became a proenvironed desire, and in time a fulfilled response and pleasure. Those who practised such acts helped each other, those who churlishly stood alone were left alone in hour of need.

So though families might be able under peculiar circumstances to live apart, in "Swiss Family Robinson" fashion, proenvironal dreams of the benefits of help, of exchange, of social defense, and even social offense against another foe, gradually became accepted and acted laws of life. For in the history of every nation it is true that the proenvironal dreams and aspirations of today become the laws of tomorrow. The Chartists who were hung in Edinburgh for their opinions in 1848 might, if revivified now, correspond to the Unionist or Liberal of today, so far as political and sociological creeds are concerned.

If then from such primitive beginnings of tribal community in goods, or of gifting, as we have outlined above, we now proceed to trace shortly the progress of human commerce, it would often happen that the surplus riches of one individual or family might be the desiderated articles of another, who by proffering some needed goods in return would effect a barter. So barter was and still is a means of social betterment in simple communities.

But the more adventurous and idealistic spirits-and they are the human organisms that always start variation changesof one village, combining, in mental picture or compounded resultant plan, all the mental stimuli that in past months or years had excited their brain centers regarding the wealth of some neighboring village community, might resolve on a proenvironal action-response that took form as a bartering journey. Such at first might be, and often was, more in the nature of a raid or foray, but, gradually comparing the fruits 
of such forays with peaceful bartering journeys, the latter became the recognized and accepted plan.

In thus extending barter to another village it could truly be said that "a pathway for commerce" was opened up that benefited both communities. If one of these adventurous ones was a rather keen bargainer-a Midianite of his daythe bartered wealth brought back with him might cause him and his family to proenviron a picture of his becoming a barterer, a merchant, by trade. And what, it may here be asked, would stimulate to such a proenvironal course of thought and future action? Exactly such as occurs in the pathway plotted or proenvironed by a root or shoot of a flowering plant. Several stimuli, acting on the ganglionic or neuratin substance, caused definite chemico-physical changes, which, linking together or combining into several complex resultants, caused a compounded resultant course of action, different from and more complex in nature than previous simpler stimuli produced.

If we may linger here a little longer, so as to try to analyze and estimate the nature of the human stimuli acting, and so measure approximately the resultant response that proenvironed a new pathway of progress, it can surely be asserted that some such as the following had a share. First, the returned trader would be viewed by the members of his community as "a successful man," and this needs no further comment as a stimulating cause to future wider action. Second, the slightly added family wealth, like the greater growth of one root over another, would give new possibilities for barteralike internal and external. Third, his family would afford to secure finer life accessories, such as dress, hut, ornaments, and so they would socially foster wider proenvironal plans. Fourth, increased currents of energy would tingle through the merchant's own frame, for success ever brings energizing exhilaration. "Nothing succeeds like success." Fifth, love for his family would cause him to proenviron the best possible picture for their future family greatness.

These and other mental stimuli would gradually combine into an inciting resultant response in the form of plans for 
pushing his trade further; for increasing the amount and variety of his barter-goods; for employing men and animalsin other words many hand-activities - in assistance; for securing a more and more extended knowledge of human needs. All of these would blend together into one proenvironal resultant, that would send him forth as an enterprising merchant who had determined on a definite course of successful action. For enterprise is varied and vigorous proenvironal planning that is being put into practice. Fungoid hyphæ, roots and shoots, spiders and ants, dogs and men, all show it in varying degrees of activity and complexity.

But, further, such human enterprise proenvironed the possibility of drawing many individuals together, at some one place, during a particular time. The outcome was the institution of fairs or markets. These are still with us, and "an open market" represents alike a highly evolved and steadily elaborated proenvironal plan, as well as an institution that steadily makes for increased social organization. The tendency to strangle these, that trusts and related monopolies have fostered, is one of the many glaring evils of trust organization, that has only been offset by the great lesson they have taught, of the comparative ease with which organized effort can produce enormous results by utilization of economizing machinery.

Flowing naturally again from such gradual commercial expansion arose a great question that required and received consideration. We refer to means of communication. Thieves by the highway who plundered villagers in some areas; flooded rivers and washed out roads at certain seasons; need for public caravanserai accommodation; all acted, along with many others, as stimuli to the proenvironing or mapping out of areas of tribal or national supervision, that culminated in the phenomenal international highways of the Iranian, the Hittite, and the Roman rulers, or of the Peruvian Incas. The proenvironal plan of one ambitious aspiring Roman Emperor often became also the continued response of his successor through years of effort, till final accomplishment was reached.

The railroad train with steam engine, dining-car, Pullman sleeper, and baggage wagon; the huge transatlantic liner; 
the telegraph, the cable, and the Marconigraph system; have all existed first as successively advancing summations of stimuli that were united into several resultants, and these again, compounded by the ganglionic cells of highest capacity, have cumulated into one proenvironal effort after another till all have combined into a climax of easy international commercial intercourse that is wide as the world, and that influences directly or indirectly the great mass of humanity.

And verily each such proenvironal advance meant for many human beings the formation of an entirely new environment around them, which in some cases has operated for their benefit, in other cases for their injury as human animals of highest type. The thousands of men who now live daily amid clanging hammers, creaking or grinding machinery, molten metal, hot furnaces, factory noise and dust, afford proof that the law of proenvironment, when put in operation as a material response, may in turn start environal forces and combinations that are absolutely new to evolving mankind, but which by no means conduce always to improvement of the individual.

And so in turn the workers who use the hands, or the masters who exploit the hands, have in turn to unite in proenvironing rules or laws that shall safeguard the worker, or invent improved machines or conditions that will overcome unhealthy conditions. Here therefore the brutal law of survival of the fittest may be modified and even overcome by the greatly nobler law of cooperation, that man is powerfully taught the benefit of, from the animals below him who have become the successful survivors.

We can next consider another proenvironal advance that is fraught with highest issues for the human race of today. The more rapidly each civilized nation advances, the more vigorously does it push forward its system of education. Why, it may be asked, does each child go to school, why do his parents, his city, his nation compel him to go? The usual answer is a familiar one, and need not be given here.

The child goes-sometimes reluctantly it must be confessed -because it is admonished on every side that years hence 
a career is planned and expected for it that shall be the most satisfying and the best for its relations in life; the parents send it, not for the present advantage or pleasure, but because they realize that for years ahead its hand and eye and ear and mouth and nose and brain should all act and react on each other under guidance of elders, so that a cumulated stock of brain stimuli and stimulus resultants should be originated, built up, and in part stored, that will enable it to do that useful work which shall be a reward to it, and an honor to the family.

The city compels it to go, since for each child it proenvirons the future man with education and without it. Like the graphic drawings that advertised and ushered in "The Popular Educator" in Britain nigh 50 years ago, the city council holds up before it the picture of an innocent child with two alternate proenvironal possibilities, and asks: which will it become? Either the boy at school, the office helper, the busy merchant, the happy father, and at length honored age. $O r$ the street boy, the inmate of the police cell, the degraded wretch, the homeless wanderer, the aged criminal. Society if worthy of the name proenvirons the former career, and even firmly guides the child toward it as a response action. For thus society, by long continued mental stimuli, is slowly discovering that its jails, its asylums, its workhouses, and its pauper graves can best be kept empty.

The State proenvirons and responds in action toward such a career, for so the world will honor its sons; and in return, when days of bad trade, of peril, of national danger come to the State, it learns that it can rely on them for support.

The State then that requires every one of its youth to attend school from 6 to 16 years is slowly proenvironing a course of honor for each of its sons; while it surrounds each of them with an educational environment that stimulates, inclines, molds, and builds up, at every turn of the mental way. Environal action and modification, that combine as resultant stimuli to work out a definite proenvironal plan, sum up biologically our entire system of education. Ind as with the 
proenvironing root-tip, or leaflet, or medusoid, or cephalopod, or beaver, when the response has been effected, the educator and Society that engages him or her are satisfied, till some new and possibly higher stimulus comes into play.

Even the history of evolving education has been one long tribute to the efforts made by individual men, or by groups of them toward compounding all of the most satisfyingnot always the best-responses into some one great proenvironal aspiration or effort, such as would steadily or even rapidly advance them beyond any previous position. This however may claim our attention in another place.

But, viewed from the standpoint of proenvironment, the mental development of every human being involves or should involve two totally distinct but parallel processes, which, when blended in effect, alone produce a truly symmetrical life. These processes are: (a) instruction, (b) education. The former should include all knowledge that bears on the individual, the world, and the universe, as acting and reacting in every relation on the individual and even mankind. Or in other words it should include a study of the laws and activities that exist independent of any one man. These, when mentally absorbed and understood, constitute his store of knowledge of the universe.

Education on the other hand should be the application to the life of each individual, and so to that of the nation and of humanity, of all the results of instruction, and also of the highest results of the past education of ancestors; with the aim of guiding each individual, each nation, and mankind to pursue the most satisfying pathway for further evolution along highest and safest lines.

So instruction has nothing directly to do with proenvironal responses, education has everything. Instruction involves a static cataloging or storing up of facts of the world and the universe, that are presented to and absorbed by each individual. Education involves the utilization of such instruction, for the formation or preservation of a present good environment; or often-and most to be desired-the plotting out 
and following of a summation, or resultant pathway, that the stimulating facts of cumulated instruction suggest as leading to a higher but satisfying environment. Instruction should focus up all that is known of actions and reactions outside the organism; education should apply these for the future guidance of each individual; instruction is a placing of the facts of the universe on the mental organization; education is a leading out or training of the reflective in relation to the perceptive faculties, so as to enable the organism to respond most perfectly to environal conditions.

Too often mere instruction is regarded as everything; real education as the day-dream of an idealist. But ethical principles, morals, and religion represent the cumulated proenvironal responses of long generations of mankind, in their search for the most satisfying environment. When these are wisely appreciated and applied in daily life, humanity will advance by leaps and bounds. The misfortune has too often been that they have been largely absorbed by priests, politicians, and unscrupulous place-seekers, who have utilized these principles as a means of professional livelihood, without realizing or acknowledging the cardinal importance of such principles in the evolution of the race.

Passing therefore to morals and religion, that play and have played so important a part in all human advance, these have been viewed in very different lights by different groups of man. Religion not unfrequently has had ridicule meted out by minds that were incapable of rising to higher energizing levels. Morals and religion are by no means unscientific vagaries of human fancy, for both represent definite, earnest, and hungering proenvironal out-reachings by man, who, satisfied only so long as a higher and nobler stimulus is not presented, at once starts to combine all of the best results of the past and therefrom fashions a resultant response that becomes a new dissatisfied stimulus guiding him to action that in turn becomes a temporary satisfaction, or a long-contimued one, if no new and higher stimulus reaches him.

Thus, in the sphere of combined morals and religion that will be separately treated in later chapters, as each community, 
tribe, state, and nation combined all the past mental stimuli that had moved them through long thousands of years, and formed a proenvironal picture for the future, the material response to which might seem for highest good, there arose a long procession of advances that we can only briefly mention. Respect and love of children to parents and of parents to children gave rise to moral family lars, and to kinship religion, that represented the most primitive but striking advance.

Next, a recognition of morals as principles that seemed outside of man, and yet that alone guided him aright as a social factor took shape, about the same time that man looked to spirits of the departed dead, of loved animal pets, of trees that lived before he was born and that lived on after his departure, or to the sighing "spirit" of the wind. So moral laws and animistic or naturalistic religions for man represented twin moral and religious proenvironal aspirations of deepest moment.

Thereafter the widespread moral laws-proenvironings truly - of 10,000 to 7500 years possibly ago - that the Sumerians, the Egyptians, the Assyrians, the Irano-Aryans, and the Chinese evolved, in order to ensure respect, authority, and obedience, were linked with materialized but consecrated idols that symbolized mundane and supra-mundane powers. So moral laws from the gods-the powers of the air whose images filled the temples-and polytheistic religion that these gods stood for prevailed for a time over a large part of the earth.

But ever higher and nobler proenvironings led Zarathushtra, Moses, Socrates, Plato, and Buddha to plan the unified morals of "The Perfect State," in the "Republic" and "Laws" of Plato, or those of the Biblical Pentateuch, along with the clear proenvironed presentation of a unified divine Spirita one supreme God, at one with and the author of, these moral laws. So the unified moral laws of one great and moral Being became a sublime proenvironed plan that centered in monotheistic religion.

Man however aspires to the eternal, the undying, but also reveres the paternal hearth. Therefore, when Christ came 
preaching a pure, true, honest morality between man and man, as well as an unshaken belief in a Unity, an energizing Godhead and Fatherhood to whom he and all mankind are related as sons and daughters, a grand proenvironal plan was set forth, which in its superior purity, sympathy, and brotherhood has slowly but surely been molding the world.

So Christ's fulfilling of the moral law, and his many illustrations of practical morality as the highest rules of guidance for mankind, combined with the intense and sympathetic recognition of God the Father, the infinite energizer of the world and the universe, and all men as his children, have given to this patritheistic religion a hold on the human mind and heart that has resulted in a world revolution, even while struggling alongside the entirely antagonistic competitive human life.

The conclusion comes as an important corollary to the above, that unbridled individualism and individualistic effort have had their day and ceased to be. A well-known university professor said a few years ago that "the law of competition was everywhere supreme." The results of the operation of the proenvironal law entirely negative such a conclusion. Had competition alone reigned supreme his parents would not have cared to supervise his growth and education; his teachers would not have aided him laboriously in acquiring knowledge; his professors would not have recommended him for promotion; he should not have received one act of true kindness in his progress through life. The more perfectly that environal action affects the organism, and the more perfectly that proenvironal response is shown by the highest minds, in like measure do social organization, service, and cooperation become a pleasure, yea even an agreeable necessity.

Therefore, when a social cooperative enviromment in institutions, in methods, in plans, and in accomplishments, that shall result in ever advancing proenvironal cooperative plans and responses, becomes some one nation's activity and growing purpose, then that nation will effect a solid and lasting evolutionary advance that will leave other and competitive individualistic nations far in the rear. If it be asked how 
such a proenvironal picture and plan can become a people's, a nation's realization, we would reply: by fostering and aiding prophets of ideals. For ideals, proenvironments, guide and at length rule the world.

Thus Bellamy's "Looking Backward" was an idealist's, a proenvironer's rolume, that called forth callow criticism, ribald jest, hateful remarks, and resentful antagonism on its appearance. But, on publication of his second volume, a former unbelieving critic confessed that so much of the teaching in the first volume had already come to pass, no one could safely predict how much of the second might not soon become practice. Already the two have effected a mutational evolution, while the principles there stated will more and more be reached out to.

No more suggestive, even marvelous, picture of exact scientific kind can be presented at the present day than a survey of man's proenvironal and response advance in the past ages. For is it not a terribly solemn thought that every man, woman, and child is the direct descendant for a million years of a few far remote ancestral forms, whose descendants during the ages between have been exposed to varied and countless environal stimuli, that have at times caused proenvironal stagnation, at times partial or temporary degeneration, but mainly have caused them to reach outward and upward, by aspiring and summated proenvironal effort, so that lines of word and action were pursued that have caused their existing descendants to be biologically surviving and successful organisms?

But equally deserving of study with the evolving or aspiring phases of proenvironal action is the uniformitarian or stagnating phase. We have seen in Chapter VIII that every plant and animal may be so exposed to a practically uniform set of stimuli, and may so exactly and similarly respond to these, that they and their progeny-similarly exposed-may remain unaltered for ages, as in the exceptionally striking cases of Terebratula and Lingula already cited. This result is exactly shown also by man. Had it been equally extensive, however, for him as for some of the lower animals, we should have had 
groups of individuals still alive, and of various ascending grades, which would have linked together for us every stage in man's evolutionary history. That so few are left to us, especially of primitive grade between the apes and man, is proof of man's past destructive tendencies toward his own fellow-men, of the onslaught by other animals of prey, of the widespread action of disease or of famine periods, as well as other hurtful environal conditions that have decimated the race.

Studies already made, however, of the extinct Tasmanians, of the bushmen of Australia, of the pygmies of the Congo, of the Papuans and of Mongolian tribes, of remote villagedwellers of the Zuyder Zee, of Spain, and of Italy, all demonstrate that, when man permits himself only to be guided by and to follow a life pathway that is exactly as was that of his ancestors, he may remain practically unaltered in thought, in word, and in action, for hundreds or even thousands of years.

Thus when the Sicilian peasant, who perhaps only once in a lifetime, if eren as often, visits Palermo or other busy human center, still desires and plans exactly the type of dress that his ancestors wore 1500 years ago, and wears this only; when he tills his olive yards and makes his oil, or tends his grape vines and manufactures his wine, exactly as his forefathers did, change or new impressions or higher aspirations become so repugnant to him that he indignantly or scornfully repels such, when offered by some pushing fellow-islander who may have visited and seen the advanced ways of France or of California. Only when the huge olive orchards and vineyards of the latter state yield their thousands of tons of olives or of grapes, when from these are neatly and expeditiously obtained oils and wines that are of a higher class than his own, when the western article supplants his own in European and western markets, when his supply thereafter is no longer needed, then and only then does he realize that either he must proenviron a new line of action or that starvation is before him and his family line.

Such a condition of affairs has been duplicated a thousandfold in every part of the world, and never probably was more often reproduced than in the past half century, when it can 
truly be said that the old is rapidly giving place to the new; that the individualistic, in thought and word and action, is being replaced by the cooperative and the socialistic, that isolated effort is being supplanted in favor of combined purpose.

But a third and a very real phase of environal action and proenvironal reaction remains for study in the phenomena of degradation or degeneration. We have seen how real and widespread are the ramifications of this, amongst some lower animals and amongst plants. Equally so is it with man, and the same agencies are operative. Thus the Fuegians are of the Red Indian race, but their dress, their customs, their practically obliterated ancestral and animistic religion, as well as other characters, all indicate that they are a degraded branch of the great Red Indian invasion of South America from the north. This branch, forced southward by the warlike Guarani and other Chaco Indians, reached the cold inhospitable wind- and rain-swept region of Tierra del Fuego. The miserable plant diet, the scant animal life, the scarce wood for houses, fires, etc., all conspired to check back their more ancient - even though simple - proẹnvironing aspirations. So, even in that inhospitable clime, clothing became scanty; houses took on a more degraded and temporary character; they fashioned simpler tools and weapons. In a sentence their entire proenvironal planning was on a downward, rather than on an ascending, plane.

Had some great stimulating spirits in their midst dared to plan and put into practice an uplifting program, their future might have been very different than is their condition now.

The steady deterioration that historically is known to have occurred amongst many barbarous, semi-civilized, or civilized nations has not been due wholly or even chiefly to the direct oppressive and despoiling action of invaders, but largely to these nations having lost a great ideal, a proenvironal aspiration for the future, and this unquestionably often owing to their having substituted greed of gold and lust of flesh, for thrifty industry and sober continence. Their resultant responses were built up from degrading stimuli, and so these responses brought degradation. 
At no periods in history has this been more fully illustrated than during the opulent centuries of Greek and Roman civilization. In the earlier centuries of the national life encouragement of thrifty virtues, culture of the land, respect for the paterfamilias, for the family, and for the country's welfare, prevailed. Thus were built up, from many hardy natural stimuli, proenvironal responses that caused each Roman of the Republic to look on the nation's future with determined and hopeful eyes. But when the period of incoming wealth came, with its opulent luxury, its licensed graft, its bribing of office-holders, its worship of debauching vices, these all became degrading stimuli that were compounded into groups of resultant degrading responses, which in turn combined into one great degradation response affecting the individual, and through him the nation. The time was when Greece first, Rome next, proenvironed heroic efforts in frugal spirit, and then prevailed; the time came when they successfully proenvironed luxurious degrading ease and vice. Then their defeat, conquest, and disruption came.

It has been said by some eminent authorities, and so far truly, that Greece, Rome, India, Spain, became prostrate and conquered nations because the flower of their manhood was successively decimated in foreign wars. But the fundamental cause of these wars was the lust of aggression, the desire for slaves, for riches, for wide empire, for empty power. Rome and Italy today support in benign peace a greatly larger population than at any time in past history. But this is because the nation has slowly, even if only slightly, learned that the arts of peace bring surer and more lasting satisfaction than do the destructive arts of war. When the nations recognize this as an international law, human evolution will make gigantic strides.

Never more truly than today are environal stimuli acting to build up proenvironal responses that for men and for nations will either cause evolution, or stagnation, or devolution. All national histories are but the human applications, in accurate scientific manner, of the more extended biological laws. 


\section{CHAPTER XXIII}

\section{MORALS AS A FACTOR IN ORGANIC EVOLUTION AND THEIR BIOLOGICAL ORIGIN}

"Individual rights," "good of the greatest number," "conscience," and "morals" are a few of numerous related terms that have been extensively used in the literature of the past half century in particular. These have been so variously used and connoted that the need for a continuous treatment of the origin of morals has been fully felt and has been ably met by Spencer, Huxley, Haeckel, Sutherland (202), and Westermarck (203).

But, in any inquiry into the field of morals, one is at once confronted by a condition of affairs that has greatly complicated the study in the past, and even now exists to a considerable degree. We refer to the constant tendency that has existed, alike in Europe and Asia, during the past 7000 years at least, on the part of a priestly or clerical class, to place morals under the special patronage and sanction of the temple or church, and to guide or modify the moral code as suited the circumstances of the case, or of each religion concerned.

As Draper (209), White (210), and others have shown, this absorptive tendency on the part of witches, magicians, medicine men, astrologers, priests, and clerics has caused the entire study of morals to be intertwined with other problems, in a manner and to a degree that a scientific consideration of morals and morality was long impossible. Morality, religion, divine revelation, and human immortality were intertwined, and were often riewed as of like origin. Frazer also (204: 44) has fully shown that this is true over many and widely separate regions.

But in recent decades the most brilliant demonstration of the confused and conflicting views that are held regarding 
morals and religion is to be found in "Questions of Belief" (205). From the earnest and aspiring articles by Frederic Harrison on "The Soul and Future Life," to the direct and incisive closing chapters by Mallock entitled "Is Life Worth Living," the reader is carried through bewildering mazes of hope and despair, of dogma and free inquiry, of scientific reasoning and illogical assertion. If a way out of the mazes is to be found, the writer considers that such can alone be secured by adoption of the historical and continuity method of scientific inquiry.

While slight efforts were made earlier, it is to Hume that we owe the first earnest and successful attempt to study morals, in unbiased manner, and to place such a study on a proper basis. His standpoint is well expressed in the following words: "Men are now cured of their passion for hypotheses and systems in natural philosophy, and will hearken to no arguments but those which are derived from experience. It is full time they should attempt a like reformation in all moral disquisitions; and reject every system of ethics, however subtle and ingenious, which is not founded on fact and observation." (Hume, Philos. Works IV (1854) 235).

In his analysis of moral sentiment he shows that such originates in the mind of man as a utilitarian measure. For primitive man, comparing the causes that produced a sense of joy or sorrow, benevolence or selfishness, freedom or tyranny, justice or injustice in himself when dealing with others, gradually transferred to and expected of his fellows such treatment as he felt to be productive of good, pleasant, happy, and successful feelings in himself; while he felt it to be his duty and even necessity to accord such treatment to his fellows in return, so that the general well-being of the social machinery might be ensured. Then and only then did a satisfied response feeling take possession of him.

Succeeding to Hume, and following him in most principles, was Jeremy Bentham, who by his more extended writings, and by his showing the application of Hume's moral principles to the regulation of human conduct, from that of the unit in- 
dividual, up to the most centralized governmental bureau, did much to place morals on a scientific basis. His ruling idea and trite phrase was Priestley's "greatest happiness of the greatest number," that undoubtedly has a large bio-sociological warrant. But, if for "happiness" the word "satisfaction" be substituted, we would regard the phrase as more exact.

Bentham's friend and literary successor, James Mill, still further extended the principles of both, and, in showing the bearing of the utilitarian-or we should now strictly call it the evolutionary-origin of morals on every department of human life, he deserves a high place in human regard.

Such views were long ridiculed, even denounced, by many theologians, often for very selfish reasons. But they are now felt to be in strict accord with the recent growth of moral sentiment on various subjects, every stage of which growth man has himself watched and can explain. But, further, the view was once widely proclaimed that morality was an intrinsic and intuitive possession of the human intellect, which in the sum-total of its interactions originated conscience. Thus Price (206: 63) expresses it, "Morality is eternal and immutable. Right and wrong, it appears, denote what actions are. Now whatever anything is, that it is, not by will, or decree, or power, but by nature and necessity." The logic of this may sound perfect; the historical accuracy is wholly lacking. Somewhat along this line also Emerson says: "The final solution in which skepticism is lost is in the moral sentiment, which never forfeits its supremacy. All moods may be safely tried, and their weight allowed to all objections; the moral sentiment as easily outweighs them all as any one." This often is true of higher grades of civilized man, but is absolutely inapplicable to the ruder savages of recent or even of present days.

In contrast to the above from Price and Emerson, Burton, the African traveler, says: "It is time to face the fact that conscience is a purely geographical and chronological accident. Where, may we ask, can be that innate and universal monitor in the case of a people-the Somali, for instance-who rob 
like Spartans, holding theft a virtue; who lie like Trojans, without a vestige of appreciation for truth; and who hold the treacherous and cowardly murder of a sleeping guest the height of human honor" (49:173). The testimony of many other travelers is unanimous, that amongst most savage races conscience is non-existent, and morals - that in their summation-resultant represent conscience are an irregular and uncertain quantity. Equally clear is it, and now recognized by all sound moralists, psychologists, and philosophers, that advancing or evolving conscience, in its mental bulk or development, follows and is the outcome of advancing morals. Morals therefore, like all other organic phenomena, have evidently had a slow, gradual, and advancing evolutionary history, every step of which it may be difficult to piece together, but some of the phases of which may be sufficientily complete to enable the evolutionary moral historian to connect all at some future day.

Accordingly Lauder-Lindsay very accurately sums up the situation, from his wide experience as a traveler, a student, a medical man, an alienist, and a humanitarian. "Darwin dwells on the differences of opinion that exist as to whether the moral sense is instinctive or acquired. Bain, Mill, Maudsley, and others have pointed out its acquired nature. It is, in truth, produced or developed in man by culture; it decays or disappears with age; it is perverted or destroyed by disease. It is certainly not innate in primitive man, in the civilized child or in the idiot. In none of these cases does it at first or under natural and normal conditions exist, while in some it cannot be developed by any degree or kind of culture" (49, I: 173). Featherman also expresses the situation well when he says: "Moral principles are the natural outgrowth of human society, for without society moral laws could never have been originated; and these principles are not absolute, for they are developed in accordance with the higher or lower degree of civilization of every political body which is capable of controlling the acts of the aggregate mass . . . of its members, and partly also of the individuals of which the mass 
is composed. Morality is not an abstraction; it is neither religious nor metaphysical. It forms the unwritten fundamental law on which society is founded" (207, I: IX).

Moreover Westermarck's extensive and analytical study of human morals (203), from the most primitive to the most advanced, clearly shows that in the process of their gradual evolution entirely opposite views have often developed and have become rigid rules among different peoples. These originate usually as customs or observances, then they become accepted principles, and at length binding laws. Thus theft, human sacrifice, slavery, prenuptial purity, and other moral attitudes have undergone totally opposite and diverging stages of evolution amongst different peoples, according to the proenvironal efforts that each exhibited. So in the concluding chapter to his exhaustive work he rightly observes (p. 742): "The general uniformity of human nature accounts for the great similarities which characterize the moral ideas of mankind. But at the same time these ideas also present radical differences. A mode of conduct which among one people is condemned as wrong is among another people viewed with indifference or regarded as praiseworthy, or enjoined as a duty. One reason for these variations lies in different external conditions. . . . But the most common differences of moral estimates have undoubtedly a psychical origin."

Such a position must be distinctly, fully, and finally accepted by all apologists for the different religious beliefs, unless they willingly desire to blind the eyes of their coreligionists to the facts of human history. Moreover, our knowledge of the development and evolutionary unfolding of many moral attitudes amongst diverse nations is sufficiently advanced to permit of our saying that these moral attitudes all represent proenvironal efforts by individuals, or by the mass of each nation, which have resulted in one of the three conditions already named, progress, stagnation, or regression and decay.

The absence of moral feeling or sentiment and of conscience in the child, along with its continued non-development if the child of highly moral parents be kept in the midst of non- 
moral surroundings, is proof that morality of any definite grade, and the resulting conscience associated with it, are an acquired and educational act largely. But the view that the moral tendencies have wholly to be acquired must be considerably qualified in connection with the evident tendency that all cognitic and cogitic cell substances have of becoming polar to, or more readily traversed by, certain lines of energy that have passed through them during successive generations of species or races. This seems to be the main and a sufficient reason why children of the more refined white and some yellow races are more readily and fully capable of moral education than are those of a black or red nation.

In line therefore with Herbert Spencer's conception of energy-distribution, with Cope's principle of Kinetogenesis, and with the fundamental thesis of this volume, moral tendencies become steadily fixed in the organization of the organism, if these be renewed and long continued in their renewal.

But, when we investigate comparatively the "moral" acts of man alongside those of some groups of animals, we discover that many of the latter equally develop moral sentiments. These animals moreover develop such qualities very nearly in the ratio of brain organization that has already been pointed out in this work. Thus bees and ants unquestionably show a high moral organization as compared with rabbits, sheep, or even squirrels. The dog and elephant again amongst mammals show such to a much greater degree than do the wolf, the baboon, or even the lemur.

Lauder-Lindsay has, to the writer's thinking, more exactly and vividly condensed our knowledge and expressed the situation than any other writer. After enumerating the leading moral qualities under twelve heads he adds: "There is not one of these moral qualities that is not possessed, sometimes in a high degree, by certain of the lower animals, and more especially the dog; and there are many authors who have been desirous of drawing marked psychical distinctions between man and other animals, who have nevertheless felt themselves compelled by the evidence of facts to concede to these other 
animals, or certain of them, the possession of morality akin to that of man." And again, after speaking of the capacity that the dog shows of distinguishing between right and wrong, he proceeds: "It must also distinguish between the right and the expedient-what would be most for its own interest to do. In other words it is just as apt as man is, and not more so, to take a selfish view of all affairs-to consider how they are likely to affect its own personal interests. The choice that is finally made between the right, the expedient, and the wrong is determined by a variety of considerationsby conflicting emotions, by the balancing of probabilities and inclinations, by the degree or kind of temptation, by the presence or absence of witnesses, especially human, by other specialities of an animal's position, by the nature and extent of its moral training, by the character of the rewards and punishments offered on previous occasions" (49, I: 176).

Sense of guilt, sense of duty and responsibility, of justice and injustice, of truth and falsehood, are all shown by Lindsay to be typical of various animals, but he wisely observes in closing his chapter: "It has to be remarked that the moral virtues are illustrated mainly or only in those animals that have directly or indirectly received their moral training from man-such animals as the dog, elephant, and horse (49, I: 185).

In his section also on "Morality not a thing exclusively human" Sutherland (202, 2: 10) aptly remarks: "There is much that is moral in the humblest human community; there is not a little that is moral in the conduct of many of the lower animals." And again "among ourselves the credit of the right conduct is freely and warmly allowed to the mother who works for her children, who denies herself many an innocent pleasure in order that she may feed and clothe and properly train her little family. Shall we deny the same applause to the savage mother who carries her babe on her weary shoulders through many a parching journey, who will risk her life for it, and toil till she is ready to drop rather than that it should suffer from hunger or danger? . . . Yet if the savage 
mother is to be credited with moral feeling when she lavished a self sacrificing love and care upon her child, why not the mother monkey, which, with her young one clasped close in her arms, flies over the tree tops, embarrassed in her flight, while danger presses near, yet thinking only of her tender offspring? Why not the wounded ape, which, in so many well authenticated cases, has used her last strength to place her little one safe among the foliage, and then has turned round to face the hunters and the death they brought? Why should the maternal care of the bird which so sedulously feeds and guards its fledglings be denied the praise of being moral? And why, when we see a mother desert her little brood, do we feel ourselves entitled to denounce her cruelty in leaving the helpless to perish while she departs to disport herself with another mate? It is only human pride which has made an arbitrary distinction of kind where there is in truth only a distinction of degree.

"And so of the social virtues. When we read of a digger upon a rough gold field who neglects his chance of the lucky patch just reached in order that he may nurse the slow hours of his dying mate, his conduct affects us by its noble disinterestedness. When a dog, healthy and naturally of high spirits, abandons the fresh delights of the open air and all the sports it offers, and creeps into the kennel beside his feeble mate to lick its sores and yield it the comfort of loving comradeship, wherein is any essential difference?"

Now it is well worthy of remark that the dog, the elephant, and the horse have shared with man the results of advancing civilization for at least 6000 years, as ancient carrings and inscriptions testify. The subservient position and brutal treatment at times accorded to them, howerer, might well "sour their tempers," or turn them into moral delinquents. The fact that they have become so wise, adaptable, and attached is proof of advancing mento-moral perfectibility, that under better and more prolonged treatment might result in greatly finer animals.

But that morals, even where seen in groups that man has long civilized, do not originate with man and spread to or 
become infective for the lower animals is clearly shown by the many moral acts of bees, beavers, crows, ants, and apes, that man has never domesticated.

Equally true is it that morals are only evolved and become active factors under a social system. This social system may include family members alone, or inhabitants of a common nest as with ants, or those of several adjoining nests derived from a common parentage, or between tribes and nations as in the case of man. Thus it is one of the strongest proofs of moral as of social advance, that Switzerland, the United States, and some other countries to a less degree, are made up of diverse nationalities, in the widest sense of the word, and yet all of these live together under common moral restraints. So advancing mentality and advancing morality are usually though not necessarily conjoined.

But, having made a short survey of the moral field as hitherto regarded by many, it may conduce to a more exact estimate of the content of it in relation to individual mental acts on the one hand, and to religious acts on the other and higher, if we define "morals." Such a definition would be: "The gradually elaborated system of cogitic principles, established and enacted conjointly by the individuals of a social organization, for promoting its satisfied social equilibrium, or its socially satisfying progress."

In his analysis of the origins and evolution of moral action Sutherland (202) considers that sympathy is the fundamental principle involved. But the highly complicated attitude that we call sympathy represents in nearly every case a resultant of several satisfied responses to stimuli, established environally between two or more organisms of the same or of other groups. Thus the sympathy between husband and wife, between parent and child, between master and dog, or between two ants of the same nest, all represent several satisfied response states, that have cumulated into a resultant response which we may call the sympathetic attitude. But these satisfied states are themselves usually the resultants of oftrepeated stimuli of varied kind, which have each produced a satisfied response between the two organisms involved. 
So, amongst the mentally higher animals, the resultant of numerous satisfied responses between two or more of these animals gradually establishes the sympathetic attitude, the resultant of several sympathetic responses gives rise to the attitude we call love. Thus the deep affectionate attachment that most of us have known to occur between two dogs even of the same sex, the endearing attachments between not a few birds and mammals of the same or of opposite sex, the like relation of the Davids and Jonathans, the Davids and Absaloms, the Dantes and Beatrice Portinaris of history, are all exhibitions of genuine love that have gradually cumulated from the linking together of several sympathetic responses, as these again represent the resultant linking together of several simple satisfied responses to stimuli established through the eye, the ear, the skin, or other receptive sensory centers.

Next therefore, it may be asked whether some definite center or structure, in the brain of those animals that show a decided moral sense, has not been built up for the elaboration of moral thoughts and for the production of moral responses. From all that we at present know experimentally it is the cortex substance of the fore-brain that is wholly concerned with the development and performance of moral acts. In cases of insanity therefore, where the moral nature is decidedly lacking, this region of the brain is defective.

Leaving the study of morals in the lower animals for the present, it may well be said that historically and so evolutionarily we are in a greatly better position today than were Hume, Bentham, or Mill, for viewing human morals and conscience in their true scientific relation. Only within the past 75 years has our knowledge become fairly adequate for weighing the evidence yielded by most-nearly all-of the races of the world, as to moral condition and moral evolution. Within the same time also we have alone been able to gain a fair, impartial, and scientific view of the origin, character, influence, and advance of the great moral systems developed during what may be called the written period of human history, and which extends over 3000 to 7000 years. 
If we compare the moral codes that have originated within the above period, these in themselves show a decided-though slow-evolutionary progress. The moral rules or recommendations of Zarathushtra dating back for 3000 years at least, according to best present evidence, were set down to be observed by love for family, country, and above all for Ormazd, the bright shining spirit, rather than to be obeyed by fear of punishment. Those of Moses were real commands, but their observance was enjoined as obtaining the favor of $\mathrm{Je}$ hovah, and thereby family or national blessing. Only in more flagrant moral acts did he, or possibly some Jewish successor, impose definite physical punishment. The recommended advices of Lao-tsze, and the rules of Confucius, were only very partially accepted for centuries by the Chinese, and have been viewed rather as rules of conduct for goodliving than as national commands. This applies also to the moral principles of Socrates, of Plato, of Marcus Aurelius, and of Seneca.

The highest moral teaching, that of Christ and his followers, notably Paul, has never been codified, and yet since it appeals to the highest aspirations of the finest human spirits-as Renan willingly testified - it is slowly winning its way, often in an unacknowledged and surreptitiously imitated manner. That such moral systems, specially those of Zarathushtra and of Christ, have steadily spread, apart altogether from their religious background setting, is proof that they contain elements of evolutionary value that enable them to persist, as part of a better system, which in time will triumph as successful survivors over more primitive, crude, and brutal methods.

Even the Mosaic law, "Thou shalt not covet," is an impossible law under the reign of competitive greed. Yet its high proenvironal aim and value are recognized, since it has called forth nearly every law on our statute books that guides or regulates commerce. So the trade progress of the past half century may largely be defined as "unbridled covetousness, guided and kept in leash by accepted moral laws that prevent a minority from eating up the majority of mankind." 
We find further, however, that the morality of nations, of families, and of individuals may fluctuate according to the environal conditions and stimuli of each. Thus, to return to a case cited in the last chapter, the healthy morals of early Rome became relaxed, contaminated, sapped away, and ultimately almost abolished by importation of luxurious Greek living, of immoral gods and goddesses, of truly stolen wealth for which they ransacked the ancient world, and of lying, adultery, and debauchery in their midst. They proenvironed a pathway of progressive immorality that led ultimately to national disintegration. Uhlhorn's historic description (208: 92-144) is as sad as it is graphic.

Recent descriptions of Napoleon as a boy show that he had great qualities, but quick, sensitive, unbridled disposition, that might morally change for better or worse, according to the surroundings in which he was placed, the ambitions he might cherish, or the action and reaction between himself and others. The moral outcome, thirty years thereafter, is Emerson's graphic picture: "He was thoroughly unscrupulous. He would steal, slander, assassinate, drown, and poison, as his interest dictated. He had no generosity, but mere vulgar hatred: he was intensely selfish; he was perfidious; he cheated at cards; he was a prodigious gossip and opened letters, and delighted in his infamous police, and rubbed his hands with joy when he had intercepted some morsel of intelligence concerning men and women about him, boasting' that 'he knew everything., ",

It has often been assumed or asserted, though of course quite erroneously, that a system of morals, in order to obtain acceptance, must be in written form. But the general system of morals that silently, pervasively, and yet efficiently guides the communities of many animals, e. g., ants, as of uncivilized man in many cases, yields conclusive proof that morals are slowly acquired, gradually become far-reaching in their scope, and are steadily handed down in the biological continuity of different species. For when we compare the geographical groups of a species, or the species of a genus, stages in the process of moral evolution can clearly be observed. 
And alike amongst lower animals, as in man at various stages of moral evolution, the entire process is one of continuous stimulation action on every hand, in the performance of acts which are either for the well-being or for the mere continuance or for the injury of the community. These acts are summated into more complex proenvironal resultant responses, which if helpful and integrating for the community will aid it, and contribute toward its survival in the struggle for existence alongside other and less perfectly proenvironing types; if merely satisfying and tending to no higher platform, this may ensure stagnating existence for a time but slow ultimate extinction; if retarding and disintegrating in proenvironal response the communities will comparatively speedily disrupt and decay.

But in all moral acts, as in simpler and more primitive actions and reactions amongst plants and animals, the fundamental outcome of moral response is a satisfied state. This may last for a shorter or longer period, until new and succeeding stimuli start a dissatisfied state. Then another response will be made as above, and a new satisfied state will be reestablished.

But many striking illustrations of moral action and reaction occur in recent human history, particularly where a system has grown up under continuous observation and record, as for example with slavery in the States. Here a continuous series of environal stimuli of diverse kind developed into proenvironal plans, at first by common, or at least tacit, consent of the nation. These plans developed into responses or acts that in turn developed new and wider stimuli which cumulated into still more complex plans that eventually threatened to split the commonwealth into two morally divergent nations.

For each had such steadily divergent proenvironal outlooks, and so were forming such divergent proenvironal responses, that only the overthrow of one, and the final establishment of a single and unified proenvironal plan, could restore unity and peaceful progress to the nation.

The main features in the moral drama might be traced as follows. During the 17th and 18th centuries the United 
States, along with other colonies of Britain and with other nations, came into possession of great, fertile, but warm areas, from which they proenvironed possible huge crops. But labor was scarce and uncertain. The richer and dominating element secured, often by morally degraded means, white and black indentured bond-servants. But a proenvironal plan resulted therefrom for securing an unlimited supply of strong ignorant black labor. The plan originated with a few northern and most southern whites; the labor of the plan was secured on the slave-making principle. In proenvironal response, by out-reaching to Africa, the slave-labor was cruelly secured.

And to analyze somewhat minutely the white man's plan, this resulted from combination of many environal stimuli cumulated in the brain. Chemotactic, heliotactic, thigmotactic, tonotactic stimuli were started as thoughts of rich food, fine homes, pictures, sumptuous furnishings, pianos, banjos, and other musical instruments, gay and extensive gardens pleasing to the eye and to the nostrils, all rose before the mind. These were joined into a resultant proenvironal response in the higher nerve cells of the brain, and gradually took practical shape on "the plantation." So an inferior uncivilized human infusion became increasingly mixed with the higher white population. For, whether in the relation of home servants, of nurses, of coachmen, of attendants, or of field workers, the association became so close and interrelated that degradation of the white stock ensued. So the coon talk, the sensuous tendencies, the brutal slave-treatment, the enervating ease, developed in the latter as an exact biological "action" and "reaction" process.

The increasing wealth and luxury of the South, when brought in contact with the hardy independence of the North, started those stimuli of the higher nerve centers, such as enry, jealousy, hatred, opposition to common plans, that Spencer and Lindsay have traced the progress of from lower animals up to man.

So such stimuli, cumulated into mental resultants, started two divergent proenvironal moral lines in the minds of dwellers 
in the North and the South. Amongst the former, active, self-reliant, cooperative, and inventive, plans took shape, so that these might equal or excel the sluggish, dependent, slaveholding, and conservatively uninventive plans of the latter. Such gradually acquired and divergent characters, in what was before a national race, could only result in one of the new evolving races becoming dominant and swamping the other; or each had to become a distinct national race, restricted to a distinct area. The North proenvironed and responded for unity of the national race. By the loss of hundreds of thousands of individuals in each incipient new race the balance was restored, the moral ideal was anew proenvironed on an evolving line, and, even though-for other and far-reaching reasons - the moral evolution has scarcely equaled the cumulated strength stored in the first proenvironal response, evolving moral characters have been added that the unified nation is acquiring and steadily handing down.

Biologically the interest of this struggle was that in 1862 it seemed scarcely possible to predict whether a whole nation was to accept and follow a proenvironal pathway of easy moral degeneration, or of earnest aspiring struggling evolution. And the whole moral question was decided fundamentally on utilitarian grounds that would have rejoiced the heart of a Hume, a Bentham, or a Mill. For the side conquered that represented hereditarily the most active, varied, energetic, and struggling aspiring aggregate of human units.

At first sight it might seem more utilitarian, more truly useful, to employ many slaves and let the lordlings go easily. But evolution has ever represented the pathway along which complex matter or static energy reserves, and complex energy or kinetic energy flows, are constantly acting and reacting, so as to build up still more complex results. Devolution is ever leading along an easier and analytic road. True utility then, from the moral standpoint, is the most continuously satisfying individual effort in relation to neighbors, cumulated into completed cooperative or social result. Such result the nests or hives of wasps, bees, ants, and beavers show, in their 
construction, internal economy, and relation of the builders to each other, and to their environment.

But in the above great national struggle, as in all cogitic acts, not only must we take account of afferent sensory impressions or stimuli, and of efferent proenvironal motor impulses; the inhibitory nerves and inhibitory impulses play an important part. And the action or play of the latter come up at every turn of the way in the eventful four years struggle. Thus the collective mind of the North had at first to compare and balance up the benefit derived, from the wealth of "downeast" slave captains; from the relative cheapness of slaveprepared raw cotton for the northern mills, in comparison with importation of free-labor cotton; of the wealth received by sale of the manufactured article; as well as other benefits.

Against these had to be placed the constantly present proenvironal picture of the untold cruelties and hardening effects of a slaving royage; the public sale of human beings; the degrading effects of forced labor; the utter inapplicability and inadaptability of the Christian code of morals to the national situation; the incapacity to reconcile and weld the more aspiring and nobly proenvironing spirits like Channing, Garrison, Lincoln, Whittier, Beecher, and Beecher Stowe to the existing conditions.

The numerous complex and varied mental stimuli thus constantly presented to the national mind, as fresh risual or mental pictures were presented, had to be correlated, compared, sorted out, and one of two or more possible proenvironal pathways chosen.

The loftier synthetic impulses prevailed in the nerve cells of the national brain, so as to inhibit and minimize the more degraded and analytic - even though financially paying-tendencies. The grand resultant of all the synthetic and analytic actions was a proenvironal moral pathway of human freedom, that untold thousands of individuals had to die for, in order to secure it for the living. But the pathway mapped out then will never be returned on. It became also a moral beaconlight for the world. 
As suggested then in the last paragraph, every truly moral act is a social synthetic one, in which the constructive, the integrating, the helpful course, for the general social organism, rises superior to others that might tend to disintegrate, and the action of the inhibitory nerves is of highest value in the process.

It seems then a necessary conclusion to accept that in the evolution of all social animal groups, and specially of man, wherever such organisms come into more and more intimate contact with varied environment-as set forth in Chapter XIX -and at the same time evolve an ever-expanding social system, in like ratio do combined mental resultants in each social system give rise to ever higher proenvironal stimuli, that manifest themselves in acts which are in the most exact sense moral.

When we look more minutely into what might be called the mechanics of the process, we see that very slowly a more and more complex brain system is integrated in the anterior frontal region, from the receding type of the Neanderthal man to that of the rude savage, from such to that of the early lake-dwellers, again to the Iranian and Aryan types upward to superman of today. Such a brain system advances step by step, as a material substance, alongside increasingly complex mental stimuli from without, that act on and stimulate the cogitic or brain substance on every side. Gradually the combined mental impressions of individual and of family value become, by constant brain-selection, comparison, combination, and resultant proenvironal effort, summated into acts for social betterment, that-in human language-pass from the mental to the mento-moral and the moral.

But all evidence goes to show that most moral acts are in no sense hereditary, though the machinery for the evolution of them becomes steadily more perfect. In other words every moral act starts as a resultant stimulation-response from several simpler cognitic or cogitic stimuli. The more frequently such separate stimuli course along the chromatin and neuratin or cognito-cogitic substances and stimulate these to high and yet higher molecular combinations, the more complex and elevated become the moral acts themselves, since they are 
the expressed resultants of the evolving molecules. These molecules also become so placed amongst themselves by delicate energy flows that a "habit" or "tendency" becomes more and more fixed.

Thus one of the most ancient and deep-seated moral acts is to respect the life of a member of one's family or community; still higher is that to respect the life of a member of neighboring communities or tribes, except in times of war; still higher is respect for a neighbor's wives or wife and children, still higher for his movable goods; still higher to show filial regard for one's parents; still higher to respect and foster intertribal and international barter or commercial relations; still higher to aid one's neighbors cooperatively in daily acts; still higher to reverence and respect communal, tribal, or national efforts that attempt to explain natural objects and forces around. And at this point comes in the contact-established for at least 7000 to 10,000 years amongst the more advanced nations -between pure morals and religion. The continuance forward of the moral act and the confusing or mixing up of this with the religious act, in thus reverencing the religious outlook for the tribe's or the nation's good, is at once the starting point for the astrologers' and priests' interferences, and the reason why morals and religion have been so confounded in the past.

Still higher, however, is the peaceful perfecting of the family life, by monogamy and increased love of wife and children, owing to development of many collateral influences; still higher the social regulation and prevention of excesses in meats, drinks, and other sensuous pleasures; while in highest place we would put destruction of competition in communities and nations, cessation of all wars, a quickened public desire for service in and promotion of the general public weal, mutual protection and aid to all members of the community, of the nation, and of the internation, against accidents, disease, or immoral conditions.

How far each of these mento-moral and moral responses has been adopted, or still remains for the future, every reader can readily tell, for the community or nationality in which he may be placed. 
Striking indication of the steady though slow moral advance made, during the past two millennia, is got when we compare the moral atmosphere of today with that when Christ and Paul appeared as great moral lawgivers, as well as religious teachers. Both enjoined moral obligation and observance not by the method of legal enactment or of compulsion, but by that greatest law that is steadily permeating the world, love for mankind. Flowing therefrom has gradually evolved legalized monogamy, constitutional national laws, free suffrage, compulsory national education, a widespread happy family life, enlightened prison treatment, hospitals or asylums for the sick, deaf, blind, and insane, repression or suppression of drunkenness, social vice, individual civic, national, and international brutality, abolition of slavery, international stamping out of disease, cooperative factories and trades-unions, social combinations and civic improvements. All of these are, in the exactest sense, moral advances that belong to a different category biologically from individual mental advance or improvement on the one hand and religious evolution on the other, though the former of these almost invariably leads up to moral organization; while true religious advance seems only possible when a progressive moral atmosphere surrounds it.

The written period of human history then, and particularly the past two thousand years, has witnessed a great erolution in morals, that is itself an object-lesson in evolution of new races of mankind. It largely explains also the decadence and in a few accurately known cases the extinction even of whole races of less morally organized humanity, when brought face to face with - not isolated individuals of, but - the moral resultant of a more moral race.

In order to estimate aright the usual complexity of a moral act, we shall now endearor to analyze a few of those into their component resultant responses, and these again into their primary stimuli. We may best succeed however if we reverse the process, and trace the evolutionary sequence, as worked out in the history of each organism, or in the mental history of its instructors. 
Let us suppose that the father of a family sees the child of another drowning, and rescues it. $\mathrm{He}$ is and can be only cognizant of the danger through two sense relations, sight or the heliotactic sense, and sound or the tonotactic. In other words, were such a father near a drowning child, he would be utterly unaware of its danger, were his eye and his ear sense destroyed. He might still experience keen sense of smell, of touch, of geotropic response, but these would avail nothing.

Further the heliotactic stimulus is itself of such a nature that the different light rays, their intensities, and their angular incidences give him a compounded or resultant heliotactic impression of the child's arms waving, of its face in terror, of its body sinking. The tonotactic stimulus might be a double compounded one, due to the high-pitched screams of the child, and its lashing of the water by feet and hands. He combines these into resultant responses along with others already acquired by geotactic and thigmotactic experience that the child like stone, heavy wood, etc., is heavy and will sink.

All of these combined resultant responses are instantly summated in the knowledge or experience he has already acquired that (a) water kills, (b) it kills by continued immersion, (c) that a helpless child may thus be killed, (d) that water in the same relation would kill him or a child of his. Now the purely heliotactic and tonotactic primary stimuli are cognitic in energizing complexity; the latter are mental or cogitic. But the last-named category (d) brings him to the transition and ascending stage between the purely mental and the moral, or, as it might better be termed, the mento-moral. For by heliotactic, tonotactic, thigmotactic, and chemotactic stimuli - all of which it would take us too far to trace here-he summates the complex stimulation ideas that (a) the child and he are both human, (b) that both belong to the same race, (c) that so they have common mental interests, (d) that both might be exposed to common dangers, (e) that his child might have been the drowning one, (f) that were such the case he would expect any other father to help, (g) that so as at social or moral act he should rescue the child. 
In the higher phases of stimulation toward the moral act, therefore, at least four social individuals - the rescuer, the child, his child, another father-become linked insensibly in the father's mind, while several complex resultants of successively combined cognitic and cogitic stimuli all become summated into the final resultant response of causing him to risk his own life for that of the child.

To put the whole in progressive tabulated form, we would arrange as under:

\section{Cognitic}

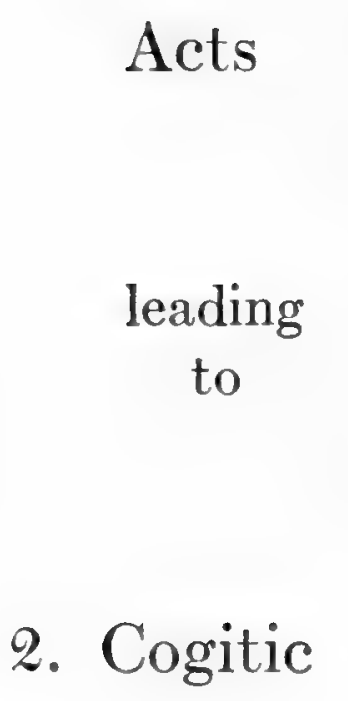

Acts

1. Several heliotactic stimuli (e. g., several color stimuli, light and shadow or light intensity stimuli, also color bodies changing position and thus becoming heliomotile stimuli) are compounded into a resultant heliotactic child-perception reperceptive $\begin{aligned} & \text { resultant } \\ & \text { sponse. }\end{aligned}$

2. Several tonotactic stimuli (e. g. shrill cries, so like child cries, also splash action in water) are compounded into a resultant tonotactic child-perception stimulus.

3. Summation of past geotactic stimuli

individual suggest by cogitic sense that child will sink in water.

4. All above are compounded into a child-sinking-in-water mental picture, that represents a compound resultant or cogitic transition from 1,2 and 3 to 4 and subsequent states.

5. Child sinking in water is a mental stimulus which combines with the chemotactically derived one that:

mental

6. Water kills land animals through or over-immersion. Both 5 and 6 combine cogitic with the cogitic stimulus that:

7. A helpless child can be so killed; while

individual acts from perceptive and mental impressions the stimulus is added that:

8. This is a helpless child which will be so killed. But all of the above compound into a stimulus which suggest to

mental or him individual and social relations. For:

cogitic

social

acts

summated

into a

resultant

moral

act

9. Water might similarly kill my child or a child of my race. So paternal love (itself built up of many cognitic and cogitic stimuli) combine the above cogitic and individual acts into a compound resultant stimulus which suggests:

10. Others would save my child or a child of my race. So:

11. I as man and father must save this child. So:

12. A compound resultant response is made in a united biotic, cognitic, and cogitic response effort to save the child. 
In view of the above considerations, the relation of morals to each individual might be regarded as the resultant of the sum totals of cognito-cogitic stimuli; that are collected by each individual in his or her life, or by instruction from others, and that are started by a sense of, and are expended for, the common or social satisfaction or good.

According to the above, and to the definition already given in the widest sense of morals (p. 660), conscience might be defined as the stored or static resultant of the sum totals of all the moral acts or teachings in the life of one individual, that are cumulated from direct experience, or by instruction from others, and that directs the individual how to respond most satisfactorily under varying social stimuli.

It follows therefore that each moral act represents a distinct mental (cogitic) state, that is started by one or more sensory or cognitic stimuli, and which from previous oft-repeated like mental states becomes, through expenditure of cogitic energy, a response that conduces toward well-being of the social whole. Conscience is the summated mental store of all the moral stimuli that are cumulated for future guidance of an individual in connection with the social whole. Morals therefore represent a set of distinct mental conditions, each of which is common to, and shared by, members of a community or organization, all of whom accept, and are guided by, these in their relation to each other. Conscience is the unified aggregate of all the distinct moral acts which have been presented to and accepted by each individual of a social whole during his life, and which is used only by that individual for placing him in harmony with the social whole.

So if we think of a Robinson Crusoe he might perform many mental acts conducive to or satisfying his mental life, and which might even advance it in complex condition, but if cast as a babe on a lone island and supposed capable of nourishing himself to manhood his moral nature would remain unawakened. His religious nature however might, in part from hereditary lines of ready stimulation, attain to fair growth, so that he might reverence the forces of nature around and 
even learn so far to understand them, then gradually transfer personal attributes to some of the more important of these.

But, with the advent of Friday on the island, the social and so the moral instincts would develop, aided also by hereditary lines of easy transmission of energy-flow for moral stimulation. Mental and religious acts therefore concern the individual, moral acts link him for good with the social organism.

Advancing human morality then, up to the highest stage that man has already reached, has represented varied external stimuli, established by social interaction between human organisms, which have started and effected cumulating complexity of, and rapid resultant intercommunication between, the cogitic substance of ganglionic cells in the brain. But such have by no means started with the human organism. Hereditarily, moral acts can be traced far down the animal scale, for only unscientific bigotry would deny the exact moral value of many acts of vertebrate and invertebrate organisms.

So, when the attempt is made to trace, in some area of the brain of average civilized man, a recognizable development of brain tissue associated with the moral function, it might well be expected that such may be centered in some region that is already more or less 'developed in the beaver, the eleplant, and social monkeys, as well as primitive man, since all of them show varying degrees of moral correlating functions.

But detailed knowledge of the cerebral centers is still too imperfect to permit a more exact location of the higher moral functions. Furthermore the quantity and quality of morality exhibited by many "distinguished" personalities are often greatly less, or of a more unstable and mixed kind, than is that of individuals of humble rank. Improved methods of observation and experiment however may yet yield results of highest and lasting value. 


\section{CHAPTER XXIV}

\section{RELIGION AS A FACTOR IN HUMAN EVOLUTION}

The position of Religion and the attitude of religionists to Science are sufficiently indicated in a general way by the title of White's well-known work, "A History of the Warfare of Science with Theology"; while the logically arranged indictments which Draper (209) and he make against many religious positions have possibly done more to discredit false religious methods and principles than any other works.

But it is one of the misfortunes of human investigation that it often tends to swing disproportionately far in one direction, after having been long held in an opposite. So the very dogmatisms, pretensions, and false teachings that the abovenamed authors have exposed have inclined many to adhere more rigorously than ever to the old view that Religion and Science are distinct and irreconcilable; that they occupy spheres and pursue methods that are largely opposed to each other. This view we cannot share, and for reasons that follow.

(1) As man has become increasingly evolved, and so has become mentally elevated, he has in approximate ratio developed that set of phenomena which we may call collectively the religious attitude.

(2) This attitude, as we now know and accept, may show gradations of elevation and complexity, from the crude ancestralism or ancestor worship of the lowest savage, to the elaborate and artificial ritual of some Christian churches.

(3) The actions of mankind for millennia are known to have been largely guided and influenced by phases of the religious attitude.

(4) Many of the wisest thinkers, and of the truest wellwishers of humanity, have ranged themselves alongside the religious attitude. 
(5) Some of the highest, if not the highest, phases of civilization have advanced markedly while allied to, or sheltering under, phases of the religious attitude.

(6) While humanity has enormously advanced through purely cooperative mental and moral effort, many features that can only be classed as religious have given an immense impetus to his forward progress, and to his repression or suppression of disintegrating tendencies.

(7) Individuals have often been willing to sacrifice some of the most cherished selfish ambitions in order to extend religious principles.

(8) Man's mind, following the great law of proenvironment, seeks after, desires, and appreciates explanations of or aspirations after natural phenomena that are beyond his full knowledge and capacity to understand at any one time.

Such are a few of the facts that all are conversant with in the connection of man with the religious attitude. Now, even though all such movements might by some be classed as vagaries, aberrations, diseases of the human mind, they even on this low platform of acceptance constitute a so striking, widespread, important, and ever-expanding set of phenomena that Science utterly fails in its true functions, if it does not take cognizance of them and try to explain them on a proper basis. For it must be acknowledged that the works of Draper and White lay bare flagrant defects or weaknesses of many religious groups, but they do not attempt to explain scientifically why a continuous procession of religious systems advances with the evolving civilization of man. Similarly, in Westermarck's able work on "The Origin and Development of the Moral Ideas," his frequent effort to reduce all the human emotions and aspirations to the level of morality, and his failure to estimate aright the powerful influence which high religious aspiration has had in elevating man, detract not a little from a correct scientific appreciation of human evolution. His attitude throughout is well summed up in his concluding words (203, 2: 746): "In its relation to morality, religion will be increasingly restricted to empha- 
sizing ordinary moral rules, and less preoccupied with inculcating special duties to the deity." It should be said however that on a nearby page (p. 737), and when speaking of Christianity, he accepts it "that its chief strength lies not in its abstract doctrines but in the simple personal following of Christ."

It has been one of the sad misfortunes of the situation in the past century that, while religionists have feigned to ignore, to discount, or even denounce many scientific discoveries, scientists have too often pooh-poohed the entire subject of religion, owing wholly or in part to false claims made, and unreasonable dogmatic attitudes assumed, by its votaries. But, even if we accept it that religious feeling or the religious attitude flows from a teratological or pathological condition, science has ignored or neglected one of its legitimate fields of inquiry, when it has failed to examine religious phenomena carefully and systematically.

The sign is a welcome one that, since the acceptance of the evolutionary principle, many students of comparative religions have defined or connoted their inquiry as "The Science of Religion." But too often they have contented themselves with the historical exhibitions of it, and have overlooked the causes at work in developing such histories.

It is an equally welcome sign that even the leaders of some of the purest Christian faiths have forsaken the old dogmatic and unreasoning attitude in favor of one that hails inquiry and knowledge from every side. Thus an Edinburgh theologian recently said before an assembly of foreign missionaries that "religions had been formerly classified into true and false, now they were generally classified into perfect and imperfect, every one of the great religions being accepted as containing some element of truth, and being of some good to its followers." He then invited those present to state freely what they regarded as helpful elements in the different religions, other than the Protestant Christian, with which they had come in contact.

In passing it may be noted that even he still clung to the idea that earth possessed a perfect thing. The definition of "perfect" or perfection was not given, however. 
Haring considered mental phenomena and also morals as the highest expression of mental molecular organization, that causes ever increasing, strengthening, and expansion of the social bond, it seems not only appropriate but claimant that an examination be made of the religious organization. Here the writer desires to state that he would not be true to himself, to his convictions, and to his fellows, did he not state frankly that he was reared amid Protestant Christian influences; that he has labored in many ways for many years in behalf of what he has regarded as pure Christian truth and practice, this also at times and in ways that interfered with other and cherished purposes; that he has spent many years in tracing out and comparing religious systems, in themselves and in the light of evolution, with scientific laws and principles; and that whatever he may now advance in explanation of a scientific religious attitude he does, only after long and careful weighings and earnest efforts after "light, light, more light." $\mathrm{He}$ hopes to analyze the religious situation without bias, and to learn its possible trend.

But, in relation to his fellow-men, the writer would recall that no struggles, feuds, and warfares have been more bitter during written human history than those which directly owed their origin to religious strife. However regrettable in itself, this fact alone proves that religion needs fullest scientific treatment, and is an exact scientific outcome of certain organizing phases in human evolution.

While it is true that selfish and debased characters like Alexander, Jenghiz, and Napoleon depleted countries of their manhood, and saw that manhood slaughtered in order to feed their own selfish egotism, it is equally true that the Iranian wars after the time of Zarathushtra, the wars of Mahomet, of the Crusaders, of the Spanish papacy in America, of the Anticovenanters, of the German Thirty Years' War period, and many others were at heart fostered as religious wars, even though baser human motives often participated.

So religious principle, which should, and often is pretended to, promote peace, has undoubtedly been a medium for the 
promotion of embittered human strife. This also, as every one knows, has been true of sects, of churches, and of churchmembers. In such an inquiry therefore as the present, the writer can scarcely hope that his views will be examined quietly, impartially, and candidly by every reader. But he would remind all that only such methods will in the end prexail, if mankind is to multiply and is to cultivate highest qualities.

In 1888 F. Max Müller said: "If the science of religion is to be treated as one of the natural sciences, it is clear that we must begin with a careful collection of facts, illustrating the origin, the growth, and the decay of religion.

"But we shall find it impossible to do so, unless we first enter on a preliminary and, I must add, a somewhat difficult inquiry, namely: What is meant by religion? Unless we can come to clear understanding on that point, we shall find it impossible to determine what facts to include and what facts to exclude in collecting our evidence for study of religion."

It may therefore conduce to a better understanding of the present religious position if we give a few definitions of religion, as culled from different sources. In its origin the word fundamentally has been considered by Max Müller, following Cicero, to signify "to care, to regard, to revere"; or again, following Lactantius, it has stood for a binding, a tying, a restraining, and "was applied by the Roman to all actions in which men are guided, not by motives deducible from the ordinary course of Nature, but by regard to some unseen power or mysterious influence."

This influence bound or restrained them from doing some acts, and equally obligated them to the performances of others. In other words, explain it as we may, the action of inhibitory nerves was keenly realized as a factor in human life, though the nerves themselves were unknown; and equally the function of sensory-motor nerves in antagonistic action was accepted.

Such a view has largely permeated all religious systems, since the time of the Sumerians and the Iranians, that is since about 5000 to $100 \mathrm{~B}$. C. This principle it is also which has 
largely been responsible for religious wars, religious tenets, and religious bigotry or dogmatism. Equally has it been the means of binding groups of individuals together in highest efforts for the ennobling and beautifying of human life. But, in many such cases as the latter, religion and morals have been viewed as of like origin and of related value. Thus Hopkins says: "No man can be truly religious, further than he is moral." Now we have already accepted it that morals are purely of animal origin, even in their highest expression, that they arise only in a connected social system, and that many of the mentally higher animals show highly moral acts. Either, therefore, such animals share the religious principle, or the author just quoted has mistaken his ground. To this we shall refer again.

James (211) defines Religion as "the feelings, acts, and experiences of individual men in their solitude, so far as they apprehend themselves to stand in relation to whatever they may consider the divine." Max Müller (212: 188) said: "Religion consists in the perception of the infinite, under such manifestations as are able to influence the moral character of man." Frazer (204, I:63) regards it as "a propitiation or conciliation of powers superior to man [ital. writer] which are believed to direct and control the course of nature and of human life." Again it has been defined as "a belief binding the spiritual nature of man to a supernatural being on whom he is conscious that he is dependent; also the practice that springs out of the recognition of such relations, including the personal life and experience, the doctrine, the duties, and the rites founded on it."

A frequent definition has been "a mode of knowing and worshipping God," while Seneca defines religion as "to know God and imitate Him." Flint in his "Theism" (p. 32) says: "Religion is man's belief in a being or beings, mightier than himself and inaccessible to his senses, but not indifferent to his sentiments and actions, with the feelings and practices which flow from such belief."

J. Caird again (213: 296) says: "Religion is the surrender of the finite will to the infinite, the abnegation of all desire, 
inclination, volition that pertains to one as this private individual, the giving up of every aim or activity that points only to my exclusive pleasure and interest, the absolute identification of my will with the will of God." Pfleiderer (214: 2: 29) holds that "Religion is the relation of our life to the worldcontrolling Power, which is to become a community of life with it."

Practically all of the above definitions postulate a more or less accurate knowledge of a being or agency higher than man, and accordingly proof of this should be forthcoming. Flint's definition also postulates knowledge coming to man that is "inaccessible to his senses." This involves a method and departure so fundamentally new and different from all other methods by which man obtains knowledge that acceptance of it would only fairly be expected when good proof was given of its operation. Both of these points will be considered later.

Further it should be clearly stated that the part of Flint's definition which says: "Man's belief in a being or beings, mightier than himself, and inaccessible to his senses," entirely breaks down in application. Not only so it absolutely prevents us from reaching a true conclusion. This it was largely which caused, as we believe, such an earnest seeker after religious, as after natural scientific, truth as Romanes to pen so contradictory and illogical even though noble and aspiring a work as his "Thoughts on Religion." For every religionist's beliefs are not only individual in origin and acceptance; they are built up primarily by activity of the senses.

Thus the statement "God is a Spirit" at once calls up the sensuous motion of the wind, its sound impression on the ear, its indefinite and extended compass, as ascertained by eye and ear. Similarly the sublime extended definition of God, as given in the Westminster Creed, "God is a Spirit, infinite, eternal, and unchangeable; in his being, wisdom, power, holiness, justice, goodness, and truth," is a sustained proenvironal effort to picture a being or existence with qualities the opposite in sense extension to those that we associate with fleeting 
and changing organic bodies. For the words "infinite, eternal, and unchangeable" can only convey meaning to us when they are measured alongside sense impressions that have exact significance to us, as finite, fleeting, and changing.

But often synonyms for "religion" are given as "devotion, faith, godliness, holiness, morality, piety, theology, worship." In all of the above then, the fundamental idea involved is that of a reaching out toward, and desire after, a being or power who is viewed as higher than man, as of infinite extension, and who deserves man's regard. If we add to the above the further conception that this being has a certain utilitarian connection with, a sympathy for, and a capacity to, at some time, reward each human being, if his or her deeds are acceptable, this is merely to state what all know to be true of most religious faiths, since about $7000 \mathrm{~B}$. C.

In order to at once indicate the goal toward which our inquiry will lead us, we would now define religion as "the perception and reverent appreciation, to greater or less degree by each human individual, of a universal Power, Energy, or Spirit which energizes and evolves all conditions, by which such human individual is surrounded or is influenced."

Contrasting our definition with others above given, the writer would consider that the conception of Max Muiller, and even more that of Pfleiderer, approaches most nearly to the one required. But "the infinite" is in every sense too rague and intangible a state to lead man upward from his most primitive condition to the platform of thought and aspiration that he now occupies. Even when Miuller attempts to show that man constantly passes from the finite to the infinite, he fails to define or to determine what the "finite" or the "infinite" is that impresses man.

But unquestionably it is energy, spiritus, the wind, the "breath of life" that man has ever sought to unravel the meaning of. It was the quest of the rudest savage, as he gazed on his recently deceased child, or favorite dog of the chase, and wondered whither the energy, the breath, the spiritus that had lately animated it had gone. It is the unceasing 
quest of man still, as he draws electricity from the rushing waters, as he strives to fathom and explain "life," as he compares the economic and happy results of cooperation with the wasteful effects of competition, as he observes the "consecrated" life of a Christian with the reckless and brutish life of a heathen.

Man's effort to understand "Energy" and his discovery of its subtle all-pervasive elusiveness has caused him to riew it with increasing reverence, and to regard it as an expression of that same power and agency "in whom we live and move and have our being." Condensed Energy it is which bestows on man his powers and possibilities; such Energy may exist in greatly more condensed state, as a much higher and more perfect "existence," though veiled to our senses or understanding. Thus man has felt, and he reaches out, he aspires longingly toward that great Energy or Power of the universe. He desires to link himself with that power, he feels that it is the active part of himself, it becomes his most cherished, his religious aspiration.

Our first duty then is to try to ascertain whether any law or principle of life has been steadily acting along the pathway of man's history, that has stimulated to successively higher phases of religious aspiration, and successively higher planes of religious life. If such can be clearly indicated, then the second duty will be to trace as completely as we now canwith the fragmentary material at command-the successive stages in religious advance that mankind has passed through. Third, a special examination will then be made of the causes that operated to unfold to mankind the character and attributes of a supreme Energy, Power, or Being, who governed this world and equally the universe beyond. Fourth, a comparison will then be instituted between progressive, stationary, and regressive religions and the agencies at work which give to each of these their tendencies and present position. Fifth, a review will be made of recent religious development, from the scientific and the spiritual standpoints.

Before entering on the first point, it should be noted in connection with it that Romanes in his latter-day earnest 
and sincere aspirations after religious light concluded "that, if there is a God, he is knowable (if knowable at all) by intuition and not by reason." And "it would be against reason itself to suppose that God, even if he exists, can be known by reason; he must be known if knowable at all, by intuition." (55: 156). It if can be shown that the child of a savage or of a civilized family can be reared apart from intercourse with other human beings, and amid the uniform surroundings of a lighted chamber, and can yet develop, by intuition, a high religious appreciation or a perception, this demand might be conceded. But no slight proof even of such has ever been forthcoming.

Under the first line of inquiry then, we have already seen that the law of proenvironment, working even in protoplasmic structures that were energized by biotic energy only, has apparently resulted in the perception of, and capacity to grow or move toward, the most satisfying environment for the organism. Again in nucleate plants and most animals the same law, working in chromatin structures, that were energized by cognitic as well as biotic energy, has linked together all stimuli into one resultant, that is the most satisfying for the organism, and the resultant proenvironal pathway thus outlined is then followed.

In higher animals we have concluded that the complex resultant of several compounded resultants becomes the proenvironal stimulus, when energized by cogitic as well as cognitic and biotic streams of energy, and when acting along the neuratin structures, which determines and initiates mental and moral action along definite lines.

In the succeeding context we hope to show that, by the same law of proenvironment, steadily advancing lines of mental complexity, and so of mental molecular organization in the frontal lobes of the brain, have caused man to look out, feel out, and otherwise advance beyond himself, and even beyond his merely moral obligations to his fellow-men, so as to aspire toward, reverence, and understand the relation which he bears to the forces of the world and of the universe. 
When this ceases to be the cardinal conception, discussion almost invariably descends to mere metaphysical speculation and assertion. Thus E. Caird in "Evolution of Religion" (215: 188) has suggested that human religious evolution exhibits three successive stages, "in which the form of his consciousness is successively determined by the ideas of the object, of the subject, and of God as the principle of unity in both." That is, man first "looks outward, not inward," and so considers objects around him rather than his own personality. Second, there succeeds "a period in which the form of selfconsciousness prevails, and determines both the consciousness of objects and that of God." Third, "the final form of consciousness is that in which the object and the self appear, each in its proper form, as distinct yet in essential relatio 1, and, therefore, as subordinated to the consciousness of God, which is recognized as at once the presupposition and as the end of both."

Such views are entirely contradicted by the history of man as now known to us; neither do they accord with the stages of religious advance, as shown by existing nations in their gradation from the lowest to the highest religious aspiration; nor do they outline to us a steady proenvironal advance toward ever higher and wider views of mankind, of the world, and of the universe, as all interrelated to man. They also lead the author to say first: "So long as God is conceived under the form of abstract objectivity or abstract subjectivity, $\mathrm{He}$ is not conceived as $\mathrm{He}$ is in truth"; and to follow this in a nearly succeeding sentence by the words: "It is impossible for any one who has breathed the spirit of modern science, modern literature, and modern ethics to believe in a purely objective God; to worship any power of nature or even any individualized outward image."

Assertions of such nature are alike logically incorrect and scientifically unthinkable, for they would compel us to believe that since God is neither a subject, nor an object, nor a "power of nature," his supposed personality and existence are a shadowy figment of the human intellect. Man has reverenced- 
worshiped-and will ever reverence that eternal, illimitable, and almighty Power of the Universe, whose all-pervading presence within and around us we ignorantly call energy. By that Power alone "we live and move and have our being." Could we comprehend that Power even approximately then truly would we know the Infinite God.

For the present then, guided by the law of proenvironment, we may confine attention to the advance made, during the past 5000 years, by the most civilized nations of man up to our own day. About the commencement of this period a widespread polytheism prevailed that succeeded to cruder and less evolved religious attitudes, considered in the two succeeding chapters. But the gods of the different nationalities varied considerably in value, in character, and in reflex capacity to elevate the peoples in whose midst they were. If it be here asked how such gods originated, we would reply that they represented the most satisfying desires or aspirations of each people, which were directly represented by as many gods as the different aspirations seemed to call forth. Thus the earlier animistic gods of Egypt, the animistic or anthropomorphic gods of Babylonia, of Greece, of Phønicia, and of Crete, were all associated with definite outreachings or aspirations on the part of their originators that in some cases elevated, in other cases degraded, in rare cases continued on an even plane of existence, the lives of their worshipers. Thus the sun-god Re of the Egyptians was the summated expression of brightness, power, majesty, warmth, and growth, and so these qualities, worshiped daily in a deity that was a unified expression of these qualities, acted as a constant proenvironal stimulus to the worshippers.

Similarly the primitive tender family ties and affection associated with the worship of Osiris and Isis-no matter whether these ideas were wholly mythical or in large part true and legendary-formed a proenvironal connection between earth, with its frequent cruelties, and a future state of human happiness, that had an elevating effect on the worshipers at their shrines. Again worship of the moon-god Thoth 
and the planetary goddess Sothis, with their personified qualities, caused proenvironal out-reachings beyond man and this world to spheres and systems beyond. The same is true of the deities of the other nations already named.

On the other hand the deification of views or of groups of views that were analytic and degrading in tendency, and that caused weakening of national life when put into practice, gave rise to such gods and goddesses as Ammon, Bel or Baal, Astarte, Venus, and other deities. These, while in part uniting and personifying true views of nature and of human life, became associated with other views that permitted and even ensured scenes of debauchery, of vice, of deceit, and of human cruelty, all of which led to individual and to national decay, often amid surroundings that seemed to proclaim wealth, success, prosperity.

But steady if slow evolution-upward adrance-took place, at one time in one nation, at another time in some other. And it is specially noteworthy that all true advance has in nearly every case been effected, not through the combined efforts or consensus of opinion of a tribe or nationality, but through the powerful advocacy of a higher proenvironal ideal on the part of one or of a few individuals.

So from amid the diffuse and often conflicting polytheisms of early Egyptian, Iranian, Aryan, and Semitic peoples there gradually emerged the unified view that one great supramundane power, majesty, and brightness governed the moon, the planets, and also the earth in its growth, fertility, seasonal changes, warming effects, and human happiness. That power was the Sun. In reaching this position, man had not hit on a lucky guess. The riew represented ever-widening senseimpressions presented by the phenomena of the world and the universe. 'These sense-impressions, gathered by the IranoSumerian star-gazers through long centuries, were summated into great and still greater proenvironal mental responses, that carried man onward and upward to ever wider and higher views. Therefore from 5000 to 2000 years ago the Sun-god held preeminent place in the religions of the most powerful 
nationalities; his temples in Irania, in Egypt, in Crete, in Babylonia and Assyria, in Greece and in Rome, were unrivaled in size and splendor; his priests became rivals to and even overthrew royal dynasties; his worship became so widespread and stimulating that heliotheism (term suggested by the writer) may well be regarded as the great transition religion from polytheism to monotheism, that held sway and inclined the thoughts of mankind upward for well nigh two millennia.

It would be beyond the scope of the present work to trace how the greatest human characters amongst the different nationalities of that period became linked with the sun in human thought and aspiration as deified beings, who linked man to the sun in sacred regard, and conversely who linked the sun to man as a great power afar, that was thus brought nigh to the deepest feelings of mankind, and that appealed to them as an anthropomorphic personality while existing as an unchanging celestial majesty.

Thus Re, the great Sun-god of the Egyptians, intertwined in thought with several of the deified kings and conquerors of the nation; Marduk and Assur the solar deities of the Babylonians and Assyrians; Zeus the bright god and sky-father of the Greeks, and Jupiter of the Romans, represented similar tendencies, along parallel lines, toward transition from an uncorrelated polytheistic pantheon to the proenvironal reverence and worship of one great Power in the universe.

Heliotheism then represented a great religious proenvironal impulse in which man endeavored to combine all the most satisfying qualities of the various deities that he had formerly worshiped with one great ruling, guiding, stimulating power, the Sun. This power, as in the higher stages of polytheism at an earlier period, became combined or interwoven with outstanding human qualities of one or more high human personalities, so as to constitute at once an ultramundane power of surpassing brightness and magnificence, as well as an object that stood for deified humanity. Every stage of this process can now be traced, alike for Re of the Egyptians and for Zeus of the Iranians and later of their Greek descendants. 
The transition from this to a pure monotheism, in which all contact with or relation to minor gods or goddesses was excluded, was taken by at least three nationalities 3500 to 2500 years ago. These were the Iranians, the Egyptians, and the Israelites. But, while the second of these never seems to have developed a stable and widely accepted monotheistic belief, the other two established such on a firm basis. Though our knowledge is still far from exact or minute, the unified monotheistic religions of both nations seem largely due to two personalities, Zarathushtra and Moses.

It may forever be impossible to tell which of the two appeared first in history, but, while the tendency is to place the period of Zarathushtra's appearance as about $1000 \mathrm{~B}$. C., the fact that the oldest of his principles, if not all, seem to have been written in an Iranian language now wholly lost to us, and even to Persians of two millennia ago, strongly suggest that he may have flourished 1500 to 2000 years B. C. Moses probably lived between 1300 and 1200 years B. C., but it is now generally conceded that much of the moral as well as religious teachings of which he is the accredited founder did not crystallize till five to six centuries later. The acknowledged polytheistic tendencies of the Israelites also, up to the period of the Babylonian and Persian captivities, strongly suggests that much of their monotheistic belief may have been derived from an Iranian source. For, while it has often been asserted in the past that little intercourse occurred between Medo-Persia on the east and Palestine, Greece, or Egypt on the west, it is gradually being recognized and accepted that movements of "holy men" between 1500 and $500 \mathrm{~B}$. C. may have been nearly as extensive as were those under Paul and his successors from 30 to $300 \mathrm{~A}$. D. The dispersion and persecution of Zarathushtra's followers doubtless aided this not a little.

The monotheistic religions of purified Mazdaism and Judaism, that Zarathushtra and Moses respectively founded, incorporated much of all that was best from the older religions and especially from that of Heliotheism. So in both a great 
and vaguely anthropomorphic Power or Ruler of the earth and heavens was proenvironed always as a mighty national deity of infinite brightness, majesty, and power, who blessed or chastised his people, their allies, or their enemies, according to the degree in which they obeyed his moral laws and strove to link themselves with him, as a great intramundane and ultramundane power, in religious reverence. When they strove proenvironally to become more and more pure, sympathetic man with man, and to live in conformity with their highest aspirations toward the attributes and being of the great God, they were pictured as prospering nationally and often individually. When they forsook high proenvironal spiritic aspirations, turned to and fostered lower cogitic and specially cognitic indulgences, permitted thereby the higher cogitic or moral and the spiritic parts of their being to become dulled and even atrophied, individual and then national degradation and disruption followed.

Such is the constant refrain in parts of the Zend-Avesta of Zarathushtra and his successors, in the books of Judges, of the early prophets, of the Kings, of Psalms, and to some degree of Proverbs.

So the three outstanding cogitic and spiritic attitudes of the real forward leaders-the mental wrestlers and aspirersof both nationalities were either a constant proenvironal outreaching or aspiration for themselves and for their nation, after "a closer walk with God," or a mourning over backsliding from such aspirations, or a rejoicing and hymn of praise that after such backsliding they had again been brought to their former aspiring or proenvironing frame of mind. And one need only try to realize the mental agonies, the spiritic wrestlings, which Zarathushtra, Moses, Samuel, Plato, Isaiah, and Paul experienced, to understand how intense and concentrated were their expenditures of spiritic, cogitic, and cognitic energy, as well as their out-reachings - their proenvironings-toward the unseen but yet mentally real power and immaterial personality that had developed in their minds.

The rise and subsequent wide extension, however, of Mithraism as a degraded semi-monotheistic, semi-polytheistic off- 
shoot from Mazdaism or the primitive religion of Zarathushtra, is an illustration of how the transition from polytheism through heliotheism to an evolving monotheism was constantly liable to suffer a backward movement. " The gradual spread of it westward, as well as eastward into Turkestan, is explained by its incorporating a high and even rigid morality, with a firm and yet adaptable belief in one great power or spirit, and also conjoined with both an acceptance of an elevated polytheism. These conjointly appealed to the Roman mind in particular, from the first to the fifth century A. D., when the better minds of the nation-stunned by defeat and disgusted by sensual license-turned to a religious system that would be a proenvironal incentive of higher character than that of the old Roman pantheon. This explains why it became a formidable rival to Christianity up to the 5 th or even 6th century A. D.

But, for the purest, noblest, most intensely proenvironing intellects, the lapse of centuries caused summation or cumulation of each new upward advance in moral or spiritual life, so that "a closer walk with God" became the life effort of not a few who are known to us historically, and doubtless of a still larger number now lost to fame. So, borrowing the Greek and other national ideas that "the gods are come down to us in the likeness of men," their more aspiring prophetic leaders proclaimed and looked for a high leader, not temporal, as many of the more degraded cogitic and cognitic intellects of the nations hoped for, but a spiritual, a supramundane leader.

For centuries before Christ's day this view was dreamed of, discussed, hoped for, pictured, and with ever-increasing earnestness, till amongst the Jews it became a powerful proenvironal expectation of each passing year. The soil therefore was ripe for nourishing a new leader who could combine all that he regarded, or had learned, as best from Iranian, Greek, Semitic, and probably Egyptian sources, into one unified resultant that would represent a new and more advanced standpoint than any hitherto attained. Three such presented themselves in the persons of Christ, of Mani, and of Mahomet. 
It would be impossible here and now to discuss the character, influence, life-work, and ultimate religious power that each of these proenvironers and so preachers of new spiritic effort illustrated. The first of the three we shall alone consider now, as being the dominant spirit for the continued forward and upward energizing of the most advanced nations of the world.

The pure, noble, unselfish, philanthropic, and eminently proenvironing life of Christ has had willing testimony paid to it by such diverse personalities as Agrippa, Constantine, Mahomet, Charlemagne, Hyder Ali, Napoleon, Renan, and Emerson. But the surpassing beauty, the humanizing value, and the unrivaled religious power of Christ's life has been largely minimized by many of his followers, who, measuring his doctrines and life alongside those of other high religious teachers, have tried to rival and even outstep the religious systems these teachers inaugurated, by inventing and adding on silly miraculous tales, as well as other unnatural and superfluous embellishments.

The supreme value of Christ's life, of his cumulated teaching, and of the religious system that-in its purity-flowed from that teaching, consists in the clear unfailing recognition that the great and terribly real law of "struggle for existence and survival of the fittest" can only be overcome and made subservient to mankind's further advance by the far higher law of social cooperation and social desire for the most satisfying individual and social life.

As is pointed out in a succeeding chapter, this social law constantly manifests itself along the entire scale of animal life, and evolves to a degree that corresponds approximately to the mental dignity of each group studied, unless such group persistently selects and lives along carnivorous lines. Therefore the animals inferior to man which exhibit the highest social organization, the herbivorous ungulate mammals, the social birds, various insect groups such as bees and ants, mammals like the beaver, the dog, and the elephant, also some monkeys-to name them in what seems approximately ascending 
scale-are those also in which the social stimulus becomes more and more prominent. But here the social stimulus, through long continued action and reaction, has largely become a social instinct. The moral acts that develop and ensure the continuance of this social stimulus and later of the social instinct are fundamentally the same with the moral acts in man, as Lauder Lindsay, Romanes, and Parmelee have considered. They represent the highest or social phase of the cogitic life, and are acts of social expediency and satisfaction.

But in Man we believe that a higher and fundamentally different plane or platform is being reached, that for convenience in past and future discussion we may call the spiritic.

Slowly evolving out of and upon the moral or higher cogitic platform, it has exhibited itself as the advancing religious spirit in man.

But here and now it may be asked: How does the mentomoral social life differ from the spiritic or religious life in Man? To this we would reply that the former develops only the social relation of "bear and forbear," so that the individual life may be safer and more satisfying against attack and destruction by surrounding agencies. The latter develops the greatly higher social duty "that ye love one another" since all look beyond themselves to some great ultimate Power of advancing well-being with which they feel themselves linked, and toward which they must place themselves in most satisfied relation. Man, in striving earnestly to unfold to himself his relation to such power or powers, has passed through successively advancing religious phases till he reached the view that the organisms which people the world itself, the cosmic system, and the entire universe even are guided and energized by one great ultimate Power, Principle, or Personality from whom he has been derived, and to whom he must again return. That power the religionist's rapid mental deduction called Ormazd, Theos, God; the scientist, by more slow and exact inductive, followed by deductive, method now calls in its omnipresent expression energy. 
The religionist has striven ever to reach nearer and nearer to this Power, to God as a synthetic progressive force for integration and increased satisfaction in life. The scientist has analytically striven to do so in order that he might understand, interpret, and correlate the varied exhibitions of that force or power, in minutest as well as in widest extension.

Christ's surpassing merit was that he proenvironally pictured this Power as being one with him and all mankind, though infinitely continuous beyond and above mankind in the universe. Science is slowly reaching the position that energy alone connects man with such attributes. Christ taught that this power or Godhead or source of highest good is fundamentally father-like, working toward the continued advance and highest good of the race, each individual of which should make this goal his aim. Science is beginning, in restricted and often imperfect manner, to teach the same lesson under the names of "eugenics," "social betterment," "ethical culture," etc.

Christ, in the oriental intensity of his devotion to that proenvironed ideal, and realizing that he surpassed other human spirits in proenvironing or picturing such, spoke with an authority and originality that many have entirely misjudged. But the yearning intensity of his and of Paul's human sympathy for man, the clear recognition that we are all links in an upward chain of human life, the realization that not by mere moral bearing and forbearing, but by a social love on the part of each for all others, could the law of "struggle for existence" be overcome, these are the fundamental principles that have caused Christianity to spread; to deepen, to mold, and to modify human life; to rise above all other religious systems; to be adopted by the progressive and dominant nations; and that will assuredly-to the writer's thinking-cause it to be the highest platform of the past on which more elevated superstructures of moral and religious truth will rest for the future.

Reverently therefore, but yet fearlessly, the writer asserts that the life of Christ, as that of a great religious guide and 
teacher, has to be cleared of those tawdry garments of unnatural surroundings that we have called his divinity, his superhuman origin, his infallibility, his miracle-working, his resurrection, his ascension, and his immortality. These are not, and have not been, the cardinal grounds on which his life and teachings have commended themselves to the world.

If then we carefully and impartially try to estimate what were the fundamental factors in Christ's career which have steadily and ever progressively acted for the improvement or evolution of mankind, we would state the following in order of importance.

First, his only and fundamental law that became the leading part of his gospel and which was to be preached "to every creature" was "that ye love one another." He did not preach the merely moral principle of respect or forbearance. His clear thought and teachings were that as mankind represented the highest earthly expression of a great universal Power or Godhead, of which somewhat figuratively they were the outflowing; they should view each other as parts of an energized whole, in truth as mankind, man in the mass.

It was unquestionably this law and constraining stimulus that energized his followers to endure willingly, even gladly, such hardships on behalf of their faith as Paul catalogued in one of his letters. It has also been the same Power or Force, working throughout the millennia since, that has been slowly but yet steadily binding mankind in ever wider bonds of Christian brotherhood.

True, many have deridingly described these proenvironers as idealists, religious enthusiasts, unscientific fatalists, and other supposedly unbalanced types of humanity. But man in his purest, highest longings has had to look to and come back to these, even after many excursions into supposedly "scientific fields."

Unfortunately Christ's followers have often shown their hereditary tendency to idol or fetish worship by almost unconsciously interweaving his noble command with the wooden Roman cross on which he died, thereby failing to realize that 
like Paul he died daily to lower cognitic and biotic tendencies that were competitive, analytic, and destructive.

Second, Christ silently and also orally taught that, by practice of the above law of glad social cooperation, man could alone modify, or curb, or even subdue the law of competition. This it was that, after his death, enabled his followers for centuries throughout the Roman empire to hold together at one time in "an upper room" at Jerusalem, at another in a Greek home, again in the catacombs of Rome, or in the "cells" of later monkish preachers. And we see a somewhat perverted form of this still in the monasteries, abbeys, or related institutions of the past millennia.

Third, Christ constantly taught that each individual is a temporary human expression, as he was, of that great primal or ultimate Power that governs, energizes, and evolves the universe. So, through contact in his earlier years with the thought of immortality as proenvironed and cherished by the Iranians and the Egyptians, and even more by Plato and many of his Greek and Roman followers, the doctrine of immortality became increasingly prominent in his teaching. That such is true or possible for the individual has been alternately granted or denied. On the principle that has been advocated uniformly throughout this work it does not seem likely, rather appears to be impossible. But it is at least thinkable that the highest or cogito-spiritic energies of those individuals who have fostered the upbuilding and condensation of such states in themselves during life may pass into some other place or state, while retaining the old cogito-spiritic capacity.

Christ's teachings were in no way final or perfect - the opinion of the theologian already referred to notwithstandingand have been improved or advanced on steadily during the past thousand years. So we would say that if such a condition as immortality is possible our knowledge of it at present consists merely in unverifiable statements, and not at all in a single conclusive fact. But that each individual represents and is energized by the same great Energy that radiates through space is proved by every phenomenon of man's being, and 
by the relations he bears to other organisms and to the world around him. But each human being owes personality to conjoint biotic, cognitic, cogitic, and spiritic energy combinations, that form the complex molecules we call living matter. If such energy combination can live on, when the molecules that conduct this energy have died or become disrupted, then immortality is a thinkable state of individual existence. At present absolutely no proof of such can be adduced.

Fourth, Christ taught that he desired not to supersede the moral law, as condensed in the ten commandments, but that he endeavored to enforce it willingly through operation of the grander law of love. In other words, his great aim in life was to establish a social polity on earth, that would be a fulfillment of the herald words, "Peace on earth, good will to men." When his true followers have consistently aimed for and practised this, the world and social life will have proved sweet and desirable heritages. But most of his professed followers-clericals, kings, courtiers, lawgivers, merchants, and others-have eluded or openly flung aside his most cherished convictions and aspirations. So the world of humanity is still an armed camp that is only slowly being shamed out of existence, and social competition is still more prevalent than social cooperation, that nevertheless is steadily advancing to ultimate triumph.*

The above we regard as Christ's cardinal teachings, and they are equally the teachings of Paul, his most distinguished Græco-Judaic follower. Their entire life-efforts were directed to teaching mankind how to live alike individually and socially, not how to die or how to live in a post-resurrection state of immortality. Neither of the latter states receives any important consideration at their hands, and some of the assertions that have been used by Churchmen in the past to support the latter views are clearly forgeries by post-pauline editors.

* The above, written in 1912, receives melancholy confirmation in the colossal war of today. Its saddest feature also from the religious standpoint is that a God-God alone-is constantly invoked to bless the breaking and transgression of every human moral law. Man is today doing what no groups of ants would do, and so is proving his moral inferiority. 
We would consider then that the religious world of today has reached its highest position, not by divine revelation of gods or of a God to man, nor by the appearance of any one who was God and man in blended existence, but solely by earnest proenvironal effort of one or of a few human souls who have successively lived through the ages. Each of these has striven to combine into one resultant line of teaching all that seemed best and most satisfying to his tribe, to his nation, or to the world, as inherited from preceding generations. 'This is what written, oral, inscribed, and archæologic history more and more clearly teaches, backward into the dim and receding millennia of the past. This also alone explains to us the grades of religious evolution still observable, or which history clearly gives us the records of. In view of what is stated above, we would share to a large extent the views of Benjamin Kidd (21\%: 132) and Chamberlain (218:1: 174) as to the immense impetus that the world has received from the teachings of Christ and his sincere followers. For, of all the world literature that bears on the mento-moral or social, and the spiritic or religious family life of man, none in the writer's estimate approach such sublime and yearning passages as the twelfth and the fifteenth chapters of Romans, the fifth chapter of Galatians, the latter chapters of Ephesians, the fourth chapter of Philippians, the third chapter of Colossians, and the second epistle to Timothy. These embody a few of the applied principles of Christ's teaching, as commended by his greatest follower.

Such great sweeps of high moral and religious aspiration have taken hold of the minds of men during the past two millennia, and have enobled them in the greatest and most advancing centers of civilization. The great religious waves that have successively swept over Europe and America under the teachings of Augustine, of Chrysostom, of Columba, of Huss, of Wycliffe, of Francis of Assisi, of Luther, of Melanchthon, and Wesley, as well as others of more recent date, were the motor expressions of far-reaching proenvironal resultants which developed by cumulation of all the highest views successively held regarding human life, and its relation to a great 
ultimate power or energy of the universe, that human life in its widest ramifications flowed from and had to pulsate in sympathy with.

We therefore consider that religious evolution, from the most crude and primitive to the most elevated, has invariably had as its fundamental feature the desire on the part of man to proenviron and then reach out to a reverent regard for the laws and forces that govern and explain family, tribal, and human life. Then man rises to a like reverent regard for the laws and forces that govern all plant and animal life, next to reverent regard for the mundane forces of nature, next to the supramundane bodies in general, thereafter to the sun in particular, as the transcendent governing member amongst these, and finally to the proenvironal conception of a great beneficient Power that energizes the universe, and of which he himself-the creature of a day-is a "breath of life."

An endeavor may next be made in succeeding chapters to pursue the second aim indicated, that is, to trace the successive stages of religious advance that mankind has passed through, from the crudest religious attitude and belief to the most advanced religious sentiment and relation.

But the undertaking will only conduce to a true scientific issue if an outline be first given of the types of individual concerned in the building up and establishment of the numerous religious systems. For the human motives, while often sincere, have been at times misguided, at times uninformed. But not unfrequently the motives have been purely mercenary, or mixed with cumning ulterior designs, or even professedly false and untruthful in the estimate of the promulgator.

So we would classify the promoters of religions approximately as follows: (a) pure and earnest religious aspirants or proenvironers; (b) religio-scientific aspirants; (c) religiosocial preachers and teachers; (d) religio-medical promulgators, including medicine men, miracle healers, etc.; (e) religio-deceptive wonder workers, including witches, sorcerers, wizards, etc., who live under the guise of and at the expense of religious life; (f) religio-dishonest; (g) religio-mercenary. 
For the frequently wavering, uncertain, and even disreputable courses that many religious movements have exhibited usually represent antagonistic efforts between the intensely true and earnest qualities of the first three, rarely of the fourth, and the more or less unworthy qualities of the remaining ones.

It is by no means surprising therefore that when some religious cult of unworthy type agreed on a definite religious program or creed, and required its adherents and specially its priests or clerics to give unswerving allegiance to such creed, under promise or expectation of mercenary reward, individuals were attracted whose sense of mental and moral honesty was of low grade. Thus earnest religious aspirants thereby often became mixed with unworthy men, so that both were judged by common standards. In the long run, mankind from sheer spiritic necessity discovers the true in order to follow it, and rejects the false or unworthy, as tending to drag him down.

Man only builds up religious "experience" then, by sense impressions from without, or by inherited energizing tendencies derived originally from sense impressions that incline him to utilize these impressions more fully. On the religious or spiritic side also, as on the cogitic, the cognitic, and the biotic, man may exhibit any one of the three lines of action open to him, namely evolution, stagnation, or devolution. And it is often difficult at the present day to determine whether some races-e. g., the Australian and the Peruvian Indian-exhibit the first or the last of these stages.

So, in a succeeding survey of the past and present religions of the world, the discoveries and studies of archæologists, of anthropologists, of ethnologists, of philologists, and of religiologists* must all be taken into account. So the writer and every one who approaches the subject with a sincere desire to unravel and trace man's religious history is equally indebted to the works of Dawkins, Reinach, Evans, Michaelis, Petrie and many others amongst archæologists; of Herodotus, Quat-

\footnotetext{
* This word the writer reluctantly coins as the only apparently appropriate one that the terms theologist or religious historian, hitherto used, fail to express.
} 
refages, Topinard, Tylor, Avebury, Brinton, and others amongst anthropologists; of Prichard, Ratzel, Mortillet, and Deniker amongst ethnologists; of Whitney, Max Müller, Sayce, Delbruck, and others amongst philologists; of Hume, Channing, Herbert Spencer, Tiele, Grant Allen, Pfleiderer, and many others amongst religiologists. By combining the efforts of these and of even more recent students, it seems possible to reach fairly accurate conclusions. But the future will doubtless bring greatly more extended and convincing information 


\section{CHAPTER XXV}

\section{THE HISTORY OF RELIGIOUS EVOLUTION}

\section{(A) To the Stage of Monotheism}

It is becoming a generally accepted conclusion that primitive man of the quaternary period lived a social or semi-social life, such as the monkeys in general and the higher types of the apes still present. Like these, and like many savage tribes still existing, man lived largely a bio-cognitic existence, that was elevated and stimulated by a considerable measure of cogitic action and reaction. For, while we may picture hunting, hut-building, weapon-fashioning, boat-building, primitive social cooking, and like occupations as all stimulating to cogitic advance, a large part of every 24 hours of each day would be passed by many groups, as by the Bushmen, the Australians, the Red Indians, and the Fuegians of today, in sleep; in lazy unstimulating indolence; in games that served well to stimulate alike the cogitic and the cognitic brain centers; in eating often to gorged surfeit, on a bio-cognitic plane that dulled and subdued for the time the higher or cogitic faculties, as such feasts still do amongst higher civilized man.

But these primitive men inherited, more or less powerfully, those highly complex cognitic and cogitic actions and reactions that large groups of animals show, namely, love for their offspring, and clinging attachment of the latter toward the parents. This then with man, as with many large groups of animals, produced consanguineous social sympathies that became family and tribal ties. Furthermore, the birth, the growth, the maturity, the decay, and the death of each individual would be more and more keenly noted, and as language developed be socially commented on. Again the change noted by his fellow from the living active body of a friend to the 
dead decaying one would produce, as amongst some ants, definite cognitic and cogitic stimuli, that must gradually have cumulated in thoughts that became increasingly complex and far-reaching. Whither was the energy gone that formerly gave life, activity, joy, to the frame; and now left it cold, passive, decaying? How did "the vital spark" first reach the babe, and why did the babe, as it grew into adult life, resemble the parents or grandparents, in bodily organization, in mental and even in social features? Why did departed dear ones reappear, after death, in the dreams of the still living, even after years?

All of the above impressions were discussed in the forest hut, in the silent cave with its answering echoes; round the camp-fire after rude man had discovered sources of fire for himself; at the close of the chase, or of a sanguinary battle when the dead were mourned over. For, if such did not occur exactly as indicated, then primitive rude man was totally different from even the crudest savage of today. But such a view we cannot accept, in light of the burial conditions and customs revealed by many archæologists and ethnologists.

Now the most fundamental principle back of all these varied impressions was love and affectionate regard of parents for children, and vice versa; of members of a family or tribe for others in it, specially for those who showed high or fine qualities; of weaker ones for some brave of the family or tribe who had shielded them from death. But coupled with this principle was the realization that many inexplicable causes or conditions were associated with it. So loving regard for and hallowed reverence toward family and social bonds became spiritic stimuli that greatly transcended mere moral obligation. To such a view many may reply, and rightly, that amongst rough savage tribes now, and rude primitive man many thousands of years ago, such feelings seldom existed. But, in all spiritic as in all moral and material advance, change and progress or evolution are not inaugurated so much by great masses of people as by one or a few superior ones who become proenvironers for the mass to follow. 
So, as has often been recounted by travelers amongst wild tribes, a more affectionate and faithful mother, a more thoughtful father, a more considerate chief or brave, as compared with others around, might give an upward impetus that was scarcely perceptible, but yet constituted a new proenvironal advance. When such nobler ones died, but yet reappeared in dream to those left behind, an explanation seemed demanded. What more exact scientifically, and natural according to the phenomena, than that the breath, the spirit, the something that had left the dead, should be around them in the sighing wind among the branches. So ancestralism, as it might be termed, constituted the primitive dawning of the religious or spiritic state, or, as Herbert Spencer has phrased it, "the universal first form of religious belief." This also assumed various stages of advance from the crude, almost brutish, regard of man during the palæolithic period, upward to the kinship regard of a Bushman or extinct Tasman, onward to the comparatively modern elevated and inspiring ancestralism of the Japanese that has been called Shintoism.

Reasons for regarding such ancestralism as most primitive in religious evolution are many. Thus those tribes or nations that have been pushed or have migrated furthest from the great Circum-Mediterranean area of keenest competition and advance still retain some form of ancestralism alone, or this as a recognized groundwork upon which a higher belief has been grafted. So the Eskimo and Red Indians, the Fuegian Patagonians, the African Bushmen, the Eastern Nigritos, and the Australians have the slight religious customs they exhibit, centered round totem poles or family rites, or the familiar spirits of ancestors. Even the Japanese Shintoism of the first to sixth centuries A. D., that may be regarded as the noblest expression of ancestralism, was fundamentally ancestralism on which was grafted later a celestial polytheism that almost attained to pure heliotheism, in which, as with the Germans, the sun became not a god, but a ruling goddess.

$\Lambda$ gain a considerable body of evidence now exists to show that at the basis of such elaborate poly theistic religions as those 
of Egypt, Babylonia, Assyria, Greece, and Rome, and equally of higher monotheistic ones, ancestralism survived in many customs and observances, as a heritage from a greatly more remote period.

Further the once widespread custom of killing attendants and cattle, also of placing foods and drinks by distinguished departed ones at their death, is another phase of ancestralism, amid many that might be cited; though it also represents the beginning of a crude wish for immortality for the departed ones.

Therefore we would trace the first real but still crude awakening of the religious spirit to loving and reverential regard by one individual for deceased members of the same family or tribe. Therefore also this spirit was limited in aim and influence to small groups of individuals. But, contrary to the opinion that some have expressed, such crude religion, like all religions, was not and could not be confined to one individual, for it invariably is an elevated social attitude.

Neither would we consider with Lang $(215 ; 219)$ and others that belief in one supreme being, or even in a number of these, was the first religious state. Religion begins with ancestralism, or reverential love of one individual for another or for a group around, or for some previous ancestor, as viewed from the standpoint of highest life energy.

In other words even the most primitive ancestralism differs from, and advances beyond, morality or the mento-moral stage, in that each human being collects and summates mentally all of the noblest qualities of a living or deceased individual that he respected or loved or revered while alive, and then makes such exhibitions of noblest life-energy the pattern or ideal, by which as a human being future conduct should be molded.

We would, therefore, define ancestralism as "a reverential and reciprocal religious affection shown by the individuals of mankind for all of the phenomena of kinship that united them into families and tribes, and that gave to them a common unified outlook on their past history and future destinies."

But, as mankind more and more peopled the trees, the dark forests, the caves, the deep pools of rivers and lakes, the ra- 
vines and the mountains with spirits or shadows of the departed dead, the human mind became more and more fixed on these surrounding objects as being associated with the spirits, and so with the memory of loved dead ones. The seasonal changes also that most of these surrounding objects showed, and the erident connection they had with his own welfare, as well as with the spirits of his dead, enabled him to summate all of these impressions or stimuli into successively greater and greater proenvironal resultants that linked all into a unified whole. Thus he, next his living relatives, then departed revered ones as spirits, thereafter the surrounding natural objects - it might be animal, vegetable, or inorganic - that the spirits hovered or abode amongst, and the seasonal or sudden changes these objects passed through, became a constantly extending environal area of action and reaction, that each individual was affected by and responded to.

This second or more extended spiritic or religious advance may very well be designated naturalism, though the term has been applied by some in a more general manner. In the gradual evolution of this naturalism, as the cogitic brain centers of man became more and more complex, distinct stages of advance have evidently been made, in some cases by very slow degrees, that illustrate in religion continuous variation, in other cases by marked and rather sudden steps that represent discontinuous variation or mutation.

As several well-known writers have pointed out, this mutation developed evidently along a variety of lines. The seasonal unfolding of trees into leaf, their blooming and subsequent fruiting, the apparent adaptive supply of the fruits to the needs of man, and the springing into life again of some of the enclosed seeds, as distributive and perpetuating bodies, gave rise doubtless to the most primitive, as it still is a recognized, reverential affection for many trees (204). It is not surprising, moreover, that tree worship developed most largely in temperate regions. For, while the Bo-tree of India as well as other tropical forms that gave grateful and social shade became objects of reverence, so many vegetables or fruits grew and 
steadily ripened through the year in warm climates that dependence on them became a passive and unstimulating accustomment. But, in warm temperate or temperate regions, where the wild olive or the oak gave food, fuel, wood for huts, dye stuffs, and other products for man, or food support for the animals on which he fed, a year of failure in their fruiting, their decay by disease, their destruction by fire or by floods, meant serious injury to man, and stimulated him to look with reverential affection on them when he enjoyed their produce. So various plants, and not least the oak and olive, became widely invested with special regard.

Again many animals, for a great variety of reasons, became intimately and reverentially linked with high regard, by man. It is often difficult or impossible as yet to tell why such animals as the crocodile, fish, the bear, the crow, and many others became reverentially united in man's thoughts. But seasonal migrations or activities, aspect and social qualities, the real or imaginery habits they showed specially in relation to man, were all doubtless determining factors, as they are still. When these also were grouped in dreams with deceased relatives, and with certain trees or their products already appreciated, all assumed a connected value for, and were viewed with reverential regard by, man that soon became what we can only designate by the term worship. For the expression that one not unfrequently hears uttered by one person in regard to another, "I just worship him," is at times extended to various attractive or fascinating objects. If such be true for educated persons now, even more would the principle find true observance with semi-brutish man.

Therefore we would consider that succeeding to and expanding from ancestralism came that wider and even deeper religious sense of naturalism. This originated the tree worship of many tribes in the northerm hemisphere, and that of primitive Asiatic and African peoples. It doubtless originated also the animalistic worship of the primitive Egyptians, as well as of the Assyrians, Arabians, and other semitic nations.

During this phase of man's advancing religious evolution we have the strongest reasons for believing that trees and 
the abodes of certain animals, or that hot springs, echoing rocks, and like striking natural environments, became pilgrimage spots to which members of a tribe might resort and show their affection for such. Hoping or even gradually believing that the spirit, the breath, the life-energy of departed dear ones might linger there, as suggested by their actual appearance in dreams, these spots became sacred or venerated, and offerings to the ancestral spirits were left on the trees or echoing rocks, as still is done by many rude tribes. Thus probably originated the first "leafy temples" that we should not despise, but regard with high respect as leading surely along an upward way.

If we pause again to ascertain how that upward way was blazed, amid the many mental and physical tangles that beset man, we would again explain it as primarily and largely due to operation of the law of proenvironment. In his desire to reach out ever to the most satisfied stage of living, he was stimulated by love for wife, for offspring, for dead ones who had loved him during life, for those living who had benefited him. He was also stimulated to know whither the living something, the energy, of the dead body had gone; why his and his parents' imprints-mental and physical-were upon his children; why all experienced a like hunger, disease, pain, and death; or satiety, health, and happiness.

These varied stimuli he combined in his highest nerve-cells -if we regard the continued mental effort employed, and the complexity of molecular organization that such involved-and summating them there into proenvironal responses of ever greater significance and complexity, the rites, the observances, and the social bonds of Ancestralism became the pathway mapped out and pursued.

In turn again, man realized by degrees that all ancestral phenomena were but a part of a wider and even more impressive set of events that stirred him to reverential affection for, and combination of, the responses of ancestralism with naturalistic phenomena above described. $\mathrm{He}$ in turn combined all of these stimuli into summated states, from which he evolved 
the proenvironal responses of ancestro-naturalism, that for a period became the satisfied state of those accepting such. The duration period, alike for ancestralism and for naturalism, has varied doubtless as has that for plant or animal variations. Thus the primitive ancestralism of primitive man evidently persists almost unchanged amongst many Australians, the Hottentots, the Central South American Indians of the Palmar, etc., while others exhibit this along with higher naturalism.

In review then we would define naturalism as "a reverential and reciprocal religious outlook, shown by individuals or groups of individuals, on the natural forces or phenomena of the earth that immediately environ them and that unite them to it. This outlook, having its origin in ancestralism, causes them to view such phenomena with affection or fear, according as they consider such phenomena to be connected with a family or a tribal friend or enemy."

A third stage of advance is animism, that in some regions of the world seems to have developed as a higher constituent of, and advance on, naturalism, in other parts as a somewhat sudden or mutational advance from ancestralism. In the former case man seems to have associated more and more closely the thought of spirit-or invisible energy-power-with most of the natural objects around him, while still recognizing the material basis that linked him with them. In the latter the dominant idea was the moving, spiritic, energizing action that each material organic body harbored while alive, or that the inorganic bodies like running water, echoing rocks, rushing winds, earthquake-shocked strata strongly suggested the existence of. Flitting night bats, twittering leaves, nocturnal birds, prowling carnivores, stealthy enemies, and other agencies, all increased the sense of fear, wonder, and reverence.

Thus, as many writers and travelers have stated, spirits or unseen personalities came to people the trees and even lowlier plants, the animals, the streams, the wind, the ringing rocks. These spirits were regarded as the loving shades of tribal friends, or as the hateful shades of trilal enemies who 
had departed life. So mixed groups of benign and evil spirits were proenvironed by man as the only resultant explanation of all the stimuli which seemed to come to him by day and night, in sunshine and in storm, amid grateful rains or destructive earthquakes.

But since all spirits could only be thought of by man as the intangible, unmaterial life-exhibitions of his dead friends, or ancestors, or enemies, or deceased animals, such spirits were always anthropomorphic or of some biological concept to him, while at the same time they now exhibited qualities that were dissociated from material existence. And the writer cannot but regard it as striking and even suggestive that, at this stage of man's religious evolution, he should have recognized, with surprising clearness, the possible difference between kinetic energy that might leave one body and yet be continued as an intangible state elsewhere, and material bodies or stores of static energy that might disrupt when a certain amount of spiritic or other energy had left it. It must be acknowledged that the entire view thus proenvironed was crude and very loosely scientific. But it illustrates the great fact that all human history bears witness to, namely, that man on his spiritic side often outruns slow and exact scientific effort and result, so far as they are applicable to man, and reaches positions - even though vague and at times unscientific-that slower cogitic effort attains to later and more laboriously.

In all subsequent considerations therefore of man's religious advance, this animistic outlook on his environment that came only gradually, and subsequent to ancestralism, or even in many cases to naturalism, has to be kept in riew and reckoned with. Furthermore, it impressed on his mental being the sense of reverence and inhibitory control, amid many forces that might cause him to act in impetuous and unguarded manner. For it compelled him to realize that often "things are not what they seem."

We now know enough of the evolving religious life of the Egyptian, Babylonian, Assyrian, Aryan, and Iranian families 
to accept it that all passed through a phase of animism or polydæmonism to naturalism or that the latter only slightly preceded and opened the way for the former, which thus seem often to unfold side by side. Animism also lingers amongst not a few rude nations as their phase of religious erolution. Thus the Dakotas and other North American Indian tribes, some of the Hottentots, and more primitive Arabic tribes are a few that might be named. The Chinese again, while largely ruled by a high ancestralism, have passed comparatively recently, 3000-2000 years ago, probably by contact with Brahmanism and Buddhism, from it through an animistic to a semi-monotheistic stage.

A definition then of animism would be "a reverential and reciprocal religious consideration by man as to the possible relation between the natural phenomena or forces of the world that environ him, and the invisible energy of cherished visible organic or later of inorganic bodies, so as to lead man to connect the visible bodies with the transferred energy of departed friends, and later of other living bodies. All of these energies transferred thus became anima or spirits to man, and such spirits might later be viewed as invisibly energizing even inorganic bodies."

Up to this stage the tribal individuals seem to have shown their religious regard for these spirits by direct connection. That is, votive offerings, addresses, or prayers to the unseen spirits, and promises of future conformity to the supposed desires or character of the spirit personalities, were directly made by each religiously inclined individual. But more and more an exclusive group of individuals arose, or was selected by the tribe on account of their keener or clearer proenvironal aspirations; or individuals obtruded themselves as worthy to be so regarded. Thus for the animistic stage, or possibly in some cases earlier, priests and wise-men or magicians became increasingly prominent. These in some cases had sincere desire for the tribal or national good; in other cases, they showed some or all of the degraded qualities that are apt to creep in wherever high service is combined with possible oppor- 
tunities for enrichment, aggrandizement, or wielding of political or family influence.

It is to the latter type of individual, who obtrudes himself at every phase of earnest religious aspiration, that humanity owes some of the most misleading, unworthy, debased, and utterly false conceptions of human life now, and of a possible continuation of it in a future state. But, wherever a caste, a class, a sect, or a privileged group of individuals is permitted to develop from amongst their fellows, invariably regrettable and often disastrous consequences follow for the human race.

The association of departed human spirits with many material things led by slow degrees to a still wider proenvironal outlook. For in addition to the objects of his more immediate environment, that seem always to have appealed most powerfully to primitive man, up even to the present day, he became increasingly impressed with the relation that he and immediately surrounding objects bore to the celestial bodies. $\mathrm{He}$ gradually realized that the recurrent thunders and storms, the moon and the sun in their periodic appearances, the stars in their multitude and movements, were all greatly more important and enduring than himself, or than most objects by which he was surrounded. So by slow degrees he came to view these as great heavenly spirits that might scatter broadcast good or evil, blessing or cursing. Of these naturally the two most impressive for him were the Sun and the Moon. The former appeared as a great dispenser of light, heat, growth, extended capacity for vision and happy human feeling. But at times also the sun hid its face behind a veil of clouds, or even by eclipse almost suddenly left the earth in darkness as if in anger.

The moon in succeeding the sun by constant appearance and disappearance, in its waxing and waning, in its demarcation of monthly periods, in eclipses, in halo surroundings, showed a wonderful personality and beauteous majesty. So man collected all his sensory impressions of both bodies in themselves, in their evident connection with many phenomena of the world, and in their action on his tribe, his family, and 
himself. He then combined all that was best, greatest, most impressive, in these into proenvironal pictures. These became the two greatest superhuman personalities or deities of the Irano-Aryan, the Semitic, the Egyptian, and much later of the Inca Indian peoples, namely the Sun god or goddess and the Moon god or goddess.

Only within recent decades have we begun to realize how intimately and continuously man's reverential regard for such objects permeated his life, and how deeply they influenced his thoughts, words, and actions, eight to ten thousand years ago. In no field of human action is this better demonstrated perhaps than in the origin of the Sabbath, as a day set aside from the other six days of the week. We are greatly indebted to Floody (220) for a masterly presentation of his predecessor's views, and his own extensive studies on this subject.

From these we learn that the primitive origin of the Sabbath was as a lunar feast day, each of which marked the period of the new, the waxing, the full, and the waning phases of that luminary. Hence also the primitive months were lunar in duration and origin. But further "ancient peoples, supposing the gods looked like themselves, identified the moon with the Great Cause because of its similarity to a living being. Ordinary work was avoided for fear of arousing the wrath of the patron god. Though work was prohibited, yet feasting and merry-making were felt to be perfectly legitimate, and constituted the essential feature of the day. It was an unlucky day among all primitive peoples. Bad luck occurred only when the god was angered by the ordinary work of man on that day."

Gradually it became a sacred day, on which pious meditation and sacrifice, as well as rest, were combined. Thus originated amongst the Iranians, at least 4000 B. C., its designation Sa-bat or Heart-rest. But this consecration of the day even was in all probability effected, when a common bond of origin existed amongst the Irano-Aryan human stocks. For, as with so many other customs and observances, as pointed out by Geiger (221: xxiv), it was founded "upon the old Aryan 
religion of nature, which the Iranian nation shared with the Indian people."

As Floody shows, the Jewish observance of it was of the most general kind, until after the Exile, when, through that contact of the Jews with the Iranian Zoroastrians which has been successfully traced by Mills (222) and others, it became a sacred day by law. Still later, the Rabbins hedged it round with those irksome and unnatural observances that Christ at times exposed and ignored.

Finally the calm, refreshing, and spiritual benefits which its appropriate observance can bring to man have been increasingly recognized during recent years.

The stars similarly engaged man's attention and interest, till they also became to him enduring personalities. The earthly objects even, that he in earlier ages regarded as being haunted by or associated with vague departed spirits, became linked with great unseen personalities, that were only inferior to the gods of the heavens. Thus fire, the sea, the growing and also the reproductive powers of nature, the lightning, and many other exhibitions of energy became prominent objects of reverence, and in some cases of love, in some cases of fear.

Therefore, even though without the exact knowledge to reason upon it in most instances, mankind was slowly but surely realizing that the world and the heavens were the arenas for mighty displays of energy, power, might, in presence of which man himself was truly "the creature of a day." Each separate exhibition or center of such energy became to him a token of some sublime spirit Power behind it, who so revealed himself to man.

Thus originated the great pantheon of gods and goddesses which was already fully recognized by the Iranians, the Babylonians, the Egyptians, the Aryans, when ancient records of 4000 to 8000 years ago reveal to us their religious beliefs.

To civilized man of today some of these beliefs seem truly foolish, superstitious, and vain. But, face to face often with the forces of nature, the nobler though untutored minds amongst 
each race or tribe yearned for some meaning to the phenomena that crowded in on them, and that seemed often to help, to bless, and to make happy, but at times also seemed to wither and to destroy. A widespread polytheism, that was an advance on animism or naturalism, was their only refuge and explanation, in their upward pathway. The very intellectuality, or increasing mental alertness of the higher individuals, forced them to this.

Very slowly therefore, through thousand of years of reverential and often loving outlook, over ever widening fields of inquiry as to his destiny, man constantly received abundant spiritic stimuli that were combined by him into new proenvironal responses which were associated with ever wider recognitions of the activity of cosmic energy. These carried him from ancestralism to naturalism, from the latter to animism, and from it to polytheism. Now the latter step involved great issues. For, while his outlook from the first three platforms was limited to mundane conditions, polytheism in practically every known age, and amongst every people, has reached out to the celestial bodies. Furthermore, in investing each of the more evident heavenly bodies, and also the great phenomena of the earth, with separate deistic names and attributes, man was almost unconsciously estimating, cataloging, and realizing the separate great centers or exhibitions of the energy of the universe.

The gradual elevation of the "spirits" of animism or polydæmonism to the more personified and elevated rank of gods seems often to have taken place separately amongst distinct nationalities and to varying degree. So far as written or carved records carry us, the prehomeric Iranians and Aryans had strong animistic hereditary beliefs, but already by 4000 B. C. had proenvironed a series of earthly and of celestial gods. Similarly the Egyptians and Babylonians of 4000 to 3000 B. C. had their natural pantheons. The passage of merchants, of sacred men, of armies, and of adventurous navies westward and eastward would spread such worships or religious faiths to considerable degree. It is by no means im- 
probable therefore that some of the Hottentot tribes, which show rather advanced polytheistic beliefs, may have risen from ancestralism and naturalism to polytheism through ancient contact with voyagers from Arabia, Egypt, and other countries, thousands of years ago. But the nations that gave them such have advanced far along higher ideals, while the Hottentot has remained on the stagnating platform of human life, or in some groups has degraded and died out before the pressure and onslaughts of Kaffir and other invaders.

But for a correct estimation of the scope of polytheism, as of the other religious platforms already referred to, in its effect on any people, one must constantly keep in view the relative degree to which the lower and quite distinct mental and moral activities advanced or stagnated or retrograded. For, since spiritic evolution largely rests on moral and mental platforms, even though distinct from and superior to these, if the latter become degraded in character or tendency the whole religious life suffers. Therefore, amongst minor as amongst the greater nations of antiquity, when an advancing mentality and morality were conjoined with earnest proenvironal aspiration toward pure national deities, the people became courageous, energetic, and dominant. Such was the case, as historians like Gruppe and Fowler tell us, with the early Greeks and the Romans in the age of the Republic.

The family was the healthy moral unit; the daily life of effort in the field, on the thrashing floor, in home and temple building, conduced to active mental effort. The strong vigorous ancestralism that had developed into naturalism and later animism was giving place to polytheism, in which the gods were reverenced and regarded even with affection. So the festivals of Mars as the primitively protective god of the fields, of Pales and Ceres as the deities of summer plenty, and of Chronus or Saturn and Ops as those of autumnal thanksgiving, were heartfelt and joyous acknowledgments by the nation's ancestors, that great forces outside themselves which they loved to regard as personalities sent the sunshine and the shower, the dew and the drying winds. Only when their 
descendants began to make Mars an aggressive and bloodthirsty deity, who incited them to battle; when they degraded the winter festival of Saturn into licentious saturnalia, did the agencies-alike mental, moral, and spiritual-start, that gradually sapped the strength of the republic and the empire, till its fall hundreds of years afterward ensued.

Practically the same history has been gone through by every nation that has risen and in time decayed.

The degree of elaboration in polytheistic worship has usually corresponded to the relative intensity of proenvironal worship, or of wealth, or of enlarged outlook on the world and the universe. Thus, alike in early Egyptian, Iranian, Assyrian, Greek, Hebrew, and Roman polytheistic history, simple shrines or altars were set up under the trees, or in some spot that was associated with some revered tribal or national event. This altar then stood for, or was a material reminder of, the spiritic personality for whom it had been reared.

But, amongst stagnating or rude nations for thousands of years up to our own time, the presence of the deities might be suggested by rude sticks, stones, pieces of metal, carved stones and metal, or even humanized likenesses in wood, metal, or stone. These constitute the objects round which polytheistic fetish worship centers, and all stages in the elaboration of the representative objects still exist amongst tribes of the world. Not unfrequently the objects themselves were figures used in a simpler animistic, naturalistic, or even ancestralistic phase of religious development, or eren the deified chiefs, kings, or emperors who had ruled over them, as in the case of the early Egyptians of 4000-5000 B. C. or of the Romans of 2000 years ago, and which had become invested with more and more expansive deistic conceptions.

The latter, or personified deistic representation, gradually spread amongst most polytheistic nations, and so degraded to mere human level those higher proenvironal aspirations that had carried man outward and upward to recognition of the primal forces of the world, the great hearenly bodies, and the orderly movements that these showed. 
So when the Romans, like the Greeks and many other nations, erected temples round their altars, and personified in material-usually human-form the deity for which that altar stood, they dragged down the high proenvironal ideal that their ancestors had built up, to a material and individualized human basis. This it is which explains the steady decline and often extinction of polytheistic religions, and even of their worshipers, alike in the past and present. When the high and noble ideal has gone, the individual and the nation decay.

This explains why the followers of Zarathushtra and of Moses constantly prohibited the making of images, and as constantly directed their coreligionists to an immaterial and spiritic outlook, rather than to "temples made with hands."

The climax of polytheism in the world's history then was reached 4000 to 1700 years ago, when the numerous and magnificent altars, temples, costly figures, and priestly service of Egyptian, Babylonian, Assyrian, Cretan, Greek, and Roman religious life were devoted to a pantheon that often was so numerous, interwoven, and confused in personality and in attributes as to have originated the Athenian dedication "to the unknown god."

Polytheism then might be defined as "a reverential and reciprocal religious outlook and regard, founded on animistic and other more primitive religious feelings, that led mankind to a realization of the magnitude and variety of mundane and supramundane forces or agencies; and to investing these with spiritic personalities. He in time often tried to localize and materialize his reverence and regard for these personalities by such substitute representations as fetishes, altars, temples, or images."

We reach now a phase in religious evolution the beauty, magnificence, and unity of which has been fully realized by some writers. But its supreme importance in leading upward to a still later monotheism, as well as its cultural religious value for man, has been little emphasized. We refer to that stage in religious progress that may be distinguished by the term Heliotheism. 
As mankind advanced in purely mental knowledge or cogitism of the universe, as he observed the workings of his own and his neighbors' minds, in their moral relations or higher cogitism; above all, as the different agencies of the world and of the universe were recognized to center round or be influenced by the Sun, the characters and the spiritic personalities of his deities became slowly but surely subsidiary to this one great luminary.

Not only so, when it was more and more realized by longcontinued annual observation and record, on the part of the wisest and most aspiring minds, that the activities or qualities associated we will say with Ceres, with Saturn, with Pales, or with Ops, of the Roman pantheon, seemed in turn to be determined by and to originate from the Sun, the latter became the primal Source, Power, and Personality that guided the actions or even determined the existence of the others. Therefore in the progress of all the ancient and civilized nations a point was reached where polytheism failed to satisfy. For, by combining all the cogitic and spiritic stimuli that came to him, man united these into a resultant response that directed him to view the sun as the all-mighty Energy, Agency, and Personality of the universe.

Conducing to this result were the manifest facts that, as it rose in the skies from the spring to the summer solstice, an ever increasing heat, light, growth, and fertility radiated from it to the earth. When it sank daily in the west all nature became hushed or inert, except for a few animals that seemed alone to resist or overcome its somnolent action, and which thereby took on a sacred character in some religious systems, as, for example, the Egyptian. When storms raged and storm clouds drifted threateningly across the sky, these latter to many seemed the garments of the Sun-god that he was wrapping around himself in anger, while he hid his face.

These and many other environal stimuli so impressed man that, in summating them into a resultant whole, the mental response was a great reverential and loving, though at times also awed, feeling for the powerful, yet distant and intangible, 
orb, that was ever moving and still seemed unattached to a source of motive power.

Amongst the Sumero-Iranians, 5000-4000 B. C., star-towers were in existence, and that knowledge of the heavenly bodies was gathered which caused their descendants to be regarded as "the wise men of the East" for many succeeding centuries. So, in the subsequent religious evolution of Babylonia and later of Assyria, the Irano-Semitic inhabitants of the Euphrates plains, as well as the Iranian inhabitants of the hills, looked to Ea the all-powerful Sun-deity. Therefore, from about 4000 to $3500 \mathrm{~B}$. C. the worship of Ea as a sun-god, above the other early deities who daily rose out of the waters in resplendent glory, was continued till about 2200 B. C. in Babylonia, when a rededication of gods occurred in which the sungod now became Marduk, and his worship at Babylon became as resplendent as was that for Re in Egypt. But, when the northern empire of Assyria gained the ascendancy, their Sungod Assur was often blended with or regarded as the substitute of Marduk. This relation continued without interruption till the Medo-Persian invasion about 530 B. C., but for hundreds of years after that the Sun-god was their leading divinity.

Similarly in the religious evolution of Egypt the polytheistic stage with its crowded pantheon of plant, animal, spirit, and heaven gods which flourished up to about the period of the IV dynasty or 4000 years B. C. gave place to the heliotheistic deity $R e$ who was in time regarded as the one great god from whom sprang all other gods, and the races of man. The rich temples dedicated to his service, and specially that at Heliopolis; the power and wisdom of his priests, as well as the universality and unquestioned superiority of his worship, were continued with little interruption for fully 3000 years, and even later furnished the example for Roman imitation to some extent.

It is still impossible to tell even approximately when Zeus, the great sun-god of the Greeks, came into prominence. But our knowledge of Aegean and of ancient Hellenic civilization is now sufficient to make it fairly certain that Zeus belonged 
not to the Aegean civilization, in which a mother-goddess seems to have been predominant, but rather to a north-eastern source that may have centered in Medo-Persia or even Turkestan. Certain it is that Zeus was the all-powerful god of Homeric poetry, and so was recognized by the ancient prehomeric clans. Few now incline to place the homeric poems earlier than 1000 to $1500 \mathrm{~B}$. C. So it is fairly safe to conclude that the great Greek god was the chief deity about 1500 to 2000 years B. C. It is impossible as yet to determine whether the proenvironed human conception of Zeus was derived from those of Ea, Re, and Marduk of the Iranian, the Egyptian, and Assyrian pantheons, whether the reverse may not have been the case, or whether each god was a separately proenvironed conception and response of these great ancient nationalities. The first seems the most likely origin.

The religious principles associated with the name and worship of Zeus, the sun-god or god of the heavens, from about 1000 to $400 \mathrm{~B}$. C. indicate that these were the summated thoughts of a hardy, fearless moral group of people who loved home, kindred, religious aspiration, and effort to conquer nature. So his altar was often an integral part of the home, and his worship was regarded as a highest duty.

As with their rise in history, so with the religion of the Romans. The great deity of their pantheon Jupiter comes into prominence hundreds of years later than Zeus, but many attributes and qualities of the latter were associated with him. He seems to have come gradually into prominence during the royal period, and was fully recognized as the ruling god by 500 B. C. About that time his temple on Capitol Hill was founded, and this afterward became unrivaled as a center of religious magnificence. Round him all that was highest, most stimulating morally, and most conducive to happy home life, civic life, and national life clustered. In other words, for four or five centuries at least, we might eren say during seven to eight centuries, for many of the best Roman citizens Jupiter represented the highest summation of all the tested and accepted principles that could guide and elevate 
a people, and that the doctrines of his religion were the proenvironal responses made by Rome's leading spirits in their effort to reach highest and most enduring life. The numerous temples dedicated to his service, and not least that at Baalbek, testify to the power and opulence of his worshipers.

The prominence of, and precedence given to, the sun-god in all Celtic, Germanic, Saxon, and Norse religious advance are well known, while his worship, as indicated in our term Sunday, took precedence over that of all other gods, even though these were regarded as greatly to be venerated.

It is highly suggestive to find that sun-worship had evidently succeeded a more primitive polytheistic worship amongst the Incas of Peru, and that this even did not center round an anthropomorphic conception, but was, so to speak, of an immaterial spiritic and supramundane character, at the same time that the spirit-god exercised constant supervision over mundane affairs.

Finally, in early Chinese history the supreme ruler Yien was recognized centuries before the Christian era, and before the introduction of Buddhism. He was the ruling and yet largely immaterial sky-god or sun-god, in whom highest virtues dwelt, and who also animated man to good deeds when prayer or petition was made to him.

The above evidences strongly favor the view therefore that, when man has advanced to a certain stage of mental or individual cogitic and of spiritic or social religious development, he reaches a platform of aspiration in which one great Force or Agency is recognized. The sun most nearly personified this Force or Agency to his intellect, even though, as amongst most of the above nationalities, the sun is often regarded more as the token of his presence rather than as the immaterial existence himself.

Such represents a really sublime and wide-reaching proenvironal response by man, that was slowly attained by combining such cogitic and spiritic stimuli as were presented by the feelings and desires that family, tribe, and nation successively called forth; by the phenomena and motions of the 
world; by the aspect, motions, and relations of the heavenly bodies as influencing each other and man; by the predominant majesty and power that seemed to reside in the sun; and by the sense of infinite intangible expanse, brightness, continuity and power, connected therewith.

One can truly say then, in the deepest, truest, and most reverent spirit, that man in reaching the highest stages of Heliotheism had approached very nearly to that simple, sublime, and yet inconceivable definition of the Shorter Catechism, "God is a Spirit, infinite, eternal, and unchangeable; in his being, wisdom, power, holiness, justice, goodness, and truth."

Therefore we would define heliotheism as "A reverential and reciprocal religious outlook and regard, that caused mankind to pass from polytheistic and other lower forms of religion to a summated and unified conception of the forces and phenomena of the universe; so that the Sun became his personified object and center of worship, since it seemed to man the most perfect representation of that unified natural energy which permeated all space and all objects in that space to which he had access by the senses." 


\section{CHAPTER XXVI}

\section{THE HISTORY OF RELIGIOUS EVOLUTION}

\section{(B) From the Stage of Monotheism Upward}

By a full and exact recognition of the widespread influence of Heliotheism from about 4000 to 500 B. C. we can alone learn, and correctly follow, the pathway reached by man in attaining to the next higher stage of monotheism.

It would be impossible in the present work to follow, even superficially, the numerous ramifications of action and reaction, of effort and failure, of advance and retreat, that man passed through, in order to reach the platform of monotheism. Here also, as in all previous religious advances, the main lines of progress were not planned out and performed by nations or even groups of men, but almost invariably by one, or by a few men, whose intense concentrated cogito-spiritic energy influenced and moved larger and larger numbers of their fellows.

Many reasons and convergent lines of study seem to indicate that transition from heliotheism to widespread monotheism was largely made from about 2000 to $400 \mathrm{~B}$. C. This took place in Iran or Medo-Persia, in Assyria, in Egypt, in Palestine, and in Greece, at varying times during the above period. It is well to bear in mind also here that - contrary to past popular opinion-all of these places enjoyed constant commercial intercourse with each other, or were constantly invading each other's territory, and so undergoing the varying fortunes of war. Captives taken in war would carry, and we know did often carry, the religious views of their own people amongst their conquerors. So the above wide area common to western Asia, north-eastern Africa, and southeastern Europe became the fermenting and stimulating center for further and higher religious advance. 
During the above 1600 years there flourished Zarathushtra, Abraham, Amenophis IV, Gautama, Moses, Socrates, Democritus, and Plato, all of whom proenvironed and aspired to a high and elevated monotheism, or to a spiritic and non-anthropomorphic view of the relation of man to a unified governing power of the universe, that satisfied many of the deepest longings and aspirations of the human mind.

We now know enough of the life, of the moral and the religious struggles, of the teachings, and the effect of these teachings on mankind, in the case of each teacher above cited, to estimate their influence on the world. And for all of them we put aside as alike untrustworthy, misleading, and unnatural the legendary extravagances, miracles, visions of an unseen world, and other superstitions by which designing and often unscrupulous followers of these religious aspirants have tried to push the claims of their cults, at the expense of all others. All of the above-named religious characters were animated by a high proenvironal enthusiasm for the uplifting of their nation, or of the world, that has borne rich fruit for mankind.

It is impossible as yet to state accurately for Zarathushtra, Abraham, Moses, and to some extent Gautama, when they flourished and taught by precept or example. But the writings of them or of their period, that have come down to us, show that strong remnants of polytheism along with a stronger and higher heliotheism clung to them in their earlier years, and even persisted long after their death, amongst the nations they represented.

Thus the frequent likening of Ormazd and of Yahweh or Jehovah to the brightness of the sun, or to "the sun in his strength," the constant tendency their nations showed to return to polytheism or to the worshiped deities of it, the strong desire these teachers showed to wean their fellows from such worship, and to turn their thoughts to one great Power and Governor of the universe, are cardinal points in their systems.

If, then, Monotheism represented an advance on Heliotheism or older religious platforms, certain higher and more 
commendable principles must have characterized it, and those must have been combined into one great proenvironal response that constituted a satisfied spiritic response, the monotheistic religion. Such was clearly the case.

In looking to the Sun as a magnificent central and personified power and deity, in observing the wide influence it had on nature, and not least on man; in rearing altars and still later gorgeous temples for sun worship; man still felt that many mental and moral processes of his own and of his neighbor's being, that many natural events of an arresting kind, that many movements even of the stars seemed outside the power and influence of the sun-deity. Particularly in this connection one should note, as appealing very nearly and sympathetically to man, the apparently opposite and even conflicting relations of good and evil, of love and of hatred, of chaste and of sensual behavior, of cherishing life and of destroying it, of aspirations after highest living and of grovellings amid ways that were felt to be degrading.

These are the principles which, alike in the Zend Avesta of Zarathushtra, in Plato's writings, and in the Old Testament scriptures, are constantly emphasized. So, putting all of these and many other mental impressions or stimuli together, man evolved a proenvironal resultant that led him to set before himself one great and infinite Power and Majesty who ruled the universe. But he was often opposed and thwarted by the "Wicked One" who, however, had early been vanquished, but who still often wrought evil in the world, and not least in the hearts of men.

How far such evil influences gradually came to be personified by the Moon, and the associated "powers of darkness" in the monotheism of Zarathushtra, and how far therefore the moon-formerly worshiped as a revered celestial spirit - became "cast down from heaven," and personified as Ahriman in his theology, we do not propose to consider now. But, with our increasing knowledge of Iranian and Aryan theology, such a view has much in its favor. To this we may return in another work. 
Now this position, essentially anthropomorphic on first view, represents nevertheless a great scientific truth clothed in a spiritic guise, and one also that has stimulated man for millennia to highest effort. For, scientifically, man had thus reached the view, after long struggle and much observation of himself, his neighbor, the world, and the universe, that there is one great Power or Energy ever working throughout the universe and producing beneficent results that appear on every side. These beneficent results are ever conducing to high and still higher effort, in other words, to evolutionary advance and synthesis, that become in time results of greater beneficence.

But, wherever this beneficent Energy or Spiritic Power was not fostered, advanced, and added to, there disintegrating and degrading results followed, which in their action puzzled the early monotheists, and so caused them to place all such under the influence of "a power for evil," vaguely proenvironed by man as a semi-anthropomorphic being.

So Ormazd of Zoroastrian worship and Yahweh of the Hebrew scriptures, Re as the semi-heliotheistic, semi-monotheistic deity of Amenophis IV in Egypt, and Prajapati of brahmanical literature, are all great national embodiments of the monotheistic position and attitude reached by man, namely, that one great pervading Power, Energy, and Principle reaches forth through space, and is recorded and exhibited in every object there. With the lapse of centuries and millennia the evil principle or energy, Ahriman, Satan, or by whatever name called, gradually assumed a minor importance, though it still lingers in current theology.

Ormazd, Yahweh, God, the Lord, represent then the longdrawn effort made by man to unravel the multitude of questions presented by his own existence, as well as his relation to his neighbor, to his immediate enviromment, to the world, and to the universe. This effort was put forth as a result of his constantly combining the various stimuli or sensory impressions, received from every side, into greater or compounded stimuli that were cumulated again into one resultant mental 
response after another. Such responses led him through the successive stages of ancestralism, naturalism, animism, polytheism, and heliotheism to the final recognition of one supreme Power, Energy, or Principle that moved the universe. An since man could think of or conceive nothing higher or more complicated in cognito-cogitic relation than his own individual existence, and all the thoughts, words, and actions that he and mankind had experienced, he of necessity gave a dim and yet vaguely anthropomorphic conception to his final proenvironed response or picture. At the same time he felt how far he was from reaching out to, or understanding, or being even distantly equal to, the spiritic picture of universal power and bounty and beauty that he had formed from his summation of all the sensory impressions inherited or received from day to day.

So reverence, worship, adoration, have ever been the true attitude of the noblest spirits among mankind; while prayer and communion with that greatest mental realization of the human intellect have been and are a true index to the intensity of the proenvironal aspiration. This attitude also is exactly consistent with, and yet different from, that of the pure scientist as he endeavors to follow some law in nature, to trace the interaction of some stars, to unravel the development of some animal, or to explain the intricacies of some psychological processes in terms of brain structure. Here the observer concentrates his mind on a small realm of nature; shuts out other areas of action and reaction that might interfere with his direct line of inquiry, feels interest in, and expresses admiration for, the results that accumulate from his investigations, but the very concentrating on a small field of study that ensures his success, prevents this wide outlook over the world and the universe that has developed and that still fosters the religious attitude.

The unfortunate feature in the past has been that some members of the group which we might call the professional religionists have introduced dogmatic but erroneous teachings, miracles, and magic arts, necromancy, and so-called prophetic 
foresight; in a word a supposed supernaturalism in the midst of continuous and evolving natural events. These they have often created from their own imagination. And on the principle of "the three black crows," what was a bold assertion or a slight suggestion on the part of the authors of such has become-specially during the monotheistic and later centuries up to two centuries ago-an accepted statement of fact.

So the true reverential or religious attitude, that strives through the medium of each true religionist to link together all knowledge of the phenomena of the universe in a loving endeavor thereby to benefit mankind, and raise him to ever higher platforms of thought and word and action, is the highest expression of that law of cooperation or synthetic action, that alone can successfully overcome keen analytic competition. It needs no miracle working, no inflated and erroneous teaching, no "consecrated priesthood" to commend it to humanity. It ever calls for new seers, new and earnest proenvironers, who can link all the best principles of the past into higher and greater principles for the future. When such are found, mankind honors and follows them.

In such religious progress intuition has often carried the religionist far in advance of full demonstration. But intuitionism in its ultimate analysis represents a rapid mental synthesis of many rather superficial observations, combined into a resultant response that future experiment and prolonged observation alone accept as correct or erroneous. So a recognition of one great ultimate governing Power or Energy in the universe, that to its first proenvironers could only be appreciated in a distantly anthropomorphic sense, has become the wide basis of belief for most scientists of the present day.

The scientist of recent day who has laboriously reached this position after centuries of detailed effort may attempt to flout the earlier religious conclusions of his ancestors. But such an attitude can well be pardoned when one realizes how much mankind owes of unity in thought, in action, and in international life to religious aspiration.

It is not possible in the present work to trace the many causes that have operated, to ensure that professedly mono- 
theistic nationalities have gradually risen above those of lower religious belief and practice. This primarily has not been due to exterminating wars of the former on the latter, as some might at first sight suppose. For the wholesale massacres of Jenghiz Khan, of Timur, and other Mongol leaders with their hordes of shamanistic followers have often been repeated in other parts of the world, and by other religious fanatics.

Rather it has resulted from the high moral standard of nobler cogitic thought and action, combined with an even higher spiritic or religious realization of love and obedience to a great Power or governing Principle of the universe. These together have given rise to a vigor, purity, elevation, and aspiration in life relations, that have proved silent but sure dominant qualities.

Between the years 2000 to 200 B. C., therefore, there existed temporarily or permanently five centers or foci for dissemination of monotheistic religion. These were the Brahmanic in India, the Zoroastrian in Medo-Persia, the Judaistic in Palestine, the short-lived Amenhophic in Egypt, and the Platonic in Greece. The first of these has influenced a large group of mankind, but its polytheistic tendencies, its nonprogressive or stagnating character, its introspective rather than extra-impressional methods, and above all its exclusive caste regulations have been much more its weakness than have these or other inherent features been its strength.

The second, though now greatly restricted in the number of its followers and in direct influence on human thought, undoubtedly exerted a stimulating action from about 1000 B. C. to 300 A. D., that we now can scarcely estimate. For the fundamental tenets of Mithraism, of Mazdaism, and even of Judaism have all sprung from, been more or less influenced by, or have been a part of Zoroastrian faith. With the knowledge even that we now have of Mithraism, it may in time prove to be true that, from a primitive semi-heliotheistic, semi-monotheistic Mithraism, the Brahmanic, the Zoroastrian, the Hebrew, and later the Mohammedan monotheism may have been derived. If so, it would be proof that in religious, 
as in moral and mental evolution, the primitive Irano-Aryan race has been the proenvironing and progressive one in history.

The doctrines of Zarathushtra unquestionably influenced and molded Hebrew thought and action to an extent that has only in recent years been recognized, while to its tenets Greek thought and aspirations were alike indebted. But when it became a state or national religion, and when the royal professors of it often made it a cloak for superfluous and aggressive wars, rather than for the benefit and blessing of humanity, its followers became decimated and are now few and uninfluential for religious advance.

But Judaism and Platonism deserve special note also, for these, after absorbing all that was best from Zoroastrianism and from older religious beliefs, gradually combined the whole into a system of moral and spiritual thought, that became the seed-bed for the next great advance to Patritheism.

If we inquire then as to the fundamental beliefs common to Zoroastrianism, to Judaism, and to Platonic teaching as revealed in the Avesta, the Old Testament, and in "The Republic," we discover that there is constantly proclaimed a unity between man, God, and the laws of the universe, that gave a hallowed, consecrated, and mighty impulse to human life. So, in spite of frequent human weaknesses and errors practised by even its leading followers, all three systems lifted man to newer and more noble conceptions of life than had hitherto obtained. Above all they united in placing the material or biotic and the sensuous or cognitic phases of man's history in the background, and correspondingly elevated the cogitic and the spiritic.

If next one ask what the conception was of a Supreme Being in the three systems, no better answer could be given than to cite the simple, direct, but sublime first chapter of Genesis. Even if we place the final composition of it much later than was once claimed, and accept that it had possibly more an Iranian than Judaic strain and origin, the entire picture of cosmological evolution there presented is proof that man had now reached the stage where he could recognize and proen- 
viron a single first Cause, a directive Force, an Energy which he believed was steadily working toward the accomplishment of a definite end, in which the perfection of man formed an important and inevitable part of the plan. Law, truth, and justice, often coupled with human sympathy or love, were the leading tenets of all three systems.

A tendency to recognize an evil or disrupting force or even such an immaterial personality is strongly seen in the Zarathushtrian belief, is less marked but crops out often in the Hebrew writings, having probably been derived from an Iranian source, and in Plato's philosophy is attributed rather to inferior deistic powers.

In summing up the monotheistic position it may now be said that in the thousand years between 500 B. C. and 500 A. D. the crucial time had come when the older polytheismwidespread, powerful, encased in armor of rite, ritual, endowment, and priestly advocacy - was rapidly going to decay like one of the mailed eurypterids, ganoid fishes, placoid amphibians, or encased reptiles of earlier times; and when the apparently weak mental or cogitic and the spiritic forces of monotheism were to expand and prevail.

It becomes then a matter of highest moment to try to follow the fortunes of the decaying religions, and of those which have prevailed, to learn also, if possible, the causes which have weakened or blotted out the former, and those which have favored the latter.

In Greek, Roman, Syrian, Egyptian, and to a degree in Assyrian history of the above period, rules of moral conduct and the submission or conformity of these to higher governing deities or proenvironal ideals of pure character, were wavering and uncertain. Sensuous life became the desired goal of success amongst these nationalities. To eat, to drink, to bathe in sensuous fashion, to picture and to indulge in sexual excesses were at times permitted, at times even openly practised.

The foreign victories that each country achieved by the renowned valor of its warriors, were often turned later into 
bacchanalian rejoicings, through exploitation of the riches secured in each campaign. Ishtar, Ashtoreth, Aphrodite, Urania, and Venus - national adaptations of the sensual semitic goddess of Love-spread into every circum-mediterranean land, and, along with gods and goddesses of less pronounced sensuous type, started a degraded pleasure-seeking and pessimistic outlook on life. The biotic and the coarser cognitic energies most largely energized the free peoples.

The principles of sympathy, of brotherhood, of cooperative kindness and helpfulness that Zarathushtra, Gautama, and many of the Hebrew teachers of later Old Testament history proclaimed could not well survive amongst nationalities that had bond-slaves. The invariable brutal cruelty, deceit, oppression, sensuality, and unintellectuality that slavery brings to those who practise it sapped the higher cogitic aspirations.

So, when the Visigoths, Ostragoths, and other rude though more natural and undegraded hordes swept down, the old polytheistic nations fell, after each had weakened the other by repeated assault, Greek against Persian, Assyrian, and Syrian; Roman against Greek and Carthagenian; Egyptian against Roman, and vice versa. Unlikely though it might seem, the higher cogitic and the spiritic triumphed, and monotheism became the hope of the world.

But in the process Zoroastrianism-the religion of Zarathushtra or Zoroaster-, that promised so much, receded into the background, though it evidently made itself far more felt from its home in Iran westward into Greece and Rome, as well as eastward into India than we have yet recognized. It evidently also influenced and modified that still older Iranian cult of Mithraism, which from 100 B. C. to 300 A. D. spread far abroad and became a formidable rival to Christianity amongst the evolving nations of Europe.

Even were ample evidence at our disposal, it would be superfluous now to attempt proof that Egyptian, Mycenax, Hittite, and Greek religious beliefs, as well as artistic and scientific knowledge, were largely derived from a primitive IranoSumerian source. Such however, the writer anticipates, may 
yet be demonstrated, when the antiquities of Turkestan and of Medo-Persia are more fully investigated.

We strongly incline also to the belief that Christ and coming Christianity received much of noblest teaching, not from the Semitic writings, which had proved narrow, exclusive, and unhuman in many features, as well as incapable of wide human acceptance; but from the sayings and writings of Zarathushtra and his successors, of Plato and many other of the Greek philosophers who taught from $500 \mathrm{~B}$. C. to Christ's day. It was on this account also that Jowett wrote "the germs of all ideas, even of most Christian ones, are to be found in Plato."

In view of previous definitions then, and divesting it of all supernatural, unnatural, and human embellishments that some religionists have added for effect, we would now define monotheism as "that religious attitude and belief founded on higher polytheism or on heliotheism, in which man came to view the world and the universe as under the government and guidance of one all-pervading Power, Energy, or remotely placed unity or Personality, whose attributes, as measured by later human standards, were love, truth, and justice, as set forth in the works of nature, and in man's relation to that personality and to each other. These attributes were therefore binding on all who accepted this belief, as their appropriate rule of conduct toward the divine Being and toward their fellow-men."

At this stage of our inquiry a short statement may be interjected as to the origin and significance of Buddhism, since this has great interest from the standpoint of religious evolution.

Gautama or Gotama was the founder of Buddhism, and so illustrates once more the truth of our proposition, that most or all new variations or mutations start from a single individual. Of noble birth, brought up amid the rigorous religious teachings of the Brahmins, and also amid sensual indulgences that these teachings winked at or even permitted, of alert mind and fine bodily presence, aspiring to ever higher views of human life and destiny as he grew to maturity, the 
time arrived when, after years of intense asceticism, he proclaimed a new doctrine, that of the eight-fold noble pathway. This consisted in right-mindfulness, right views, right aspirations, right rapture, right effort, right speech, right behavior, right living.

By the time of his death at an advanced age he had already influenced for good and had regenerated multitudes in northern India, either by his personal teaching or by that of his disciples.

When we remember that he was born about $550 \mathrm{~B}$. C., and that from this date onward to the appearance of Christ a constant ferment and movement of Aryan, of Iranian, of Greek, of Bactrian, of Macedonian, of Assyrian, of Egyptian, and later of Roman armies, teachers, prophets, merchants, and war-captives marched more or less across the wide area from Greece-and later even Rome - on the west to the valley of the Ganges on the east, we need not wonder that he may have absorbed much from Zoroastrianism, from Egyptian lore, and from Judaism, while in return many of his doctrines carried as sutras could scarcely fail to reach and influence western thought.

The steady progress eastward of Buddhism continued for a millennium, till it became the dominant belief from western India to Japan, and from Ceylon to Thibet.

The remarkable feature of Buddhism is that it represents a purely subjective religion, which emphasizes as strongly as does Christianity the two cardinal virtues of aspiration after good and repression of evil. Unlike many other important religious systems also, it is in no sense a world or universe embracing system, and does not recognize great social, national, or international bonds of love and sympathy. Nor does it recognize a supreme power or guiding principle in the world or universe. Its central aim and tendency is individualistic and subjective. But many of the rules for right living and speaking are of a sublime nature and are of highest value. On the other hand the injunctions to mortify many aims and natural conditions that ensure continued and highest well- 
being of mankind, have caused it to become-like the modern super-ideal and inpracticable religion of the Doukobars and others-unnatural, pessimizing, or unsatisfying in relation to its professed followers.

But its high proenvironal aims, combined with its repression of disintegrating tendencies, have given it an important place amongst the world's religions. In its present-day slow but steady decay, it represents organically a lateral line of religious evolutionary advance that has flourished, reached a climax, and is passing toward extinction.

We come finally to the study of Christ as a great world teacher, and to Christianity as the dominantly advancing religion of the past 2000 years. The causes which have brought about the latter result have been fully analyzed by many minds, in many publications, and from many points of view. In particular the epochal studies of Kidd and of Chamberlain have been highly suggestive, though the writer would not accept all of the views that both-specially the latter-have set forth.

While gladly availing ourselves of suggestions from every source, the writer proposes to pursue the strictly biological and historical course. That is, in the present case, as with all of the religions already referred to, we propose to inquire what were the aims, the principles, and the views of the teacher, also what was the explanation of the success of his teachings, that have caused the gospel of love, purity, and sympathy to become the dominant factor in the world's progress today. For here we do not propose to consider whether the latter position is correct or false. We accept it as correct because biologically it has survived as the most useful and satisfying spiritic guide for man, and because the many arguments in its favor far exreed in number and value all that have been brought against it.

Nor do we linger at this stage to consider whether Christianity or any other of the above religions are "true" or "false." Each individually and all collectively have constituted great phenomena of the human race in its evolutionary contact with forces of the world and of the universe, and so have to 
be judged by the sum-total of their results, good, bad, and indifferent.

First, it cannot be too strongly emphasized that to many of the more inquiring and thoughtful minds the noblest side of the teachings of Zarathushtra, of Moses, of Gautama, of the earlier Greek philosophers, and many others of whom we now have only vague records, had appealed powerfully, and unquestionably influenced their views and writings. Socrates, Plato, Aristotle, and their numerous disciples had flourished, taught, and written from $500 \mathrm{~B}$. C. to the time of Christ's appearance. Some even of them had turned to Zoroastrianism, some to Mithraism, some as did Luke, "the beloved physician" of later date, had turned to Judaism.

By action and reaction of mind against mind; by perusal of the precious manuscripts of the philosophers; by quiet individual meditation on all the changing events - often cruel, sensual, and degrading, interspersed with kind, happy, and elevating-that made up the life-history of mankind at that day; by the travels and teachings of Iranian, of Aryan, and Semitic philosophers, students, merchants, and soldiery, great human proenvironal aspirations and even actions began to agitate mankind as never before probably in the world's history. The Alexandrian, the Seleucid, the Ptolemaic, and the Roman wars had often centered in or round Palestine, and had made it the meeting place for the views and customs of the then civilized world.

Men with minds equal to the best of today were comparing every biotic, cognitic, cogitic, and spiritic stimulus that reached them, were compounding some of these into cumulated resultants, which when tried and so put to the test were rejected. They compounded others into new resultants which when put to the test of life-action were found to be hopeful and were adopted. So, gradually, guiding principles of doctrine, of word, and of action were all summated into such great cogitic and spiritic proenvironal works as Aristotle's later biological, psychological, and religious writings. For the political, moral, and religious writings of Plato and Iristotle 
are in no sense national. They are strivings after eternal human verities, and so have an international scope and applicability.

When Christ appeared then as a teacher, the Persian, Greek, Hebrew, and Egyptian religious systems had all given rise to blended proenvironal views that could not be termed the pure product of any one teacher, but which waited for some new and synthesizing mind to knit together all that was best in them into a unified whole.

As to Christ's ancestry, immediate parentage, and education we need not attempt to speak, for of these we are wholly ignorant, except for some entirely vague, at times contradictory, in part unnatural, statements. But the indications all point to his having had a mind that was keen, analytic, synthetic, judicial, calm, above all that was eminently moral and spiritual in tendency, with the cognitic and biotic powers or inclinations kept thereby in constant balance and even check.

In all of these respects, he seems most nearly to have approached Zarathushtra, while his intense love for the highest social-and so moral-as well as spiritual life of man far excelled that of any amongst the earlier teachers, so far at least as we have exact information. He was reared as a Hebrew in a Galilean home, and amid men who were renowned for patriotism and valor. He had synagogue advantages and instruction, but he also possessed-as all his teaching showed -an active independent and original mind that was largely molded, not by the Hebrew writings alone, but even more by the higher monotheistic and idealistic teachings of Zarathushtra, of Gautama, of Plato, and of Aristotle.

These teachings were equal to, or higher in aspiration than, much of Old Testament writing; they breathed a more expansive humanitarian and regenerating tone; they suggested not merely national but human cooperation, sympathy, and regard.

No matter how it is to be explained, Christ had evidently hecome closely conversant with the best thought of the age during which he lived; had reflected deeply on all of the prob- 
lems of human relationship, was strongly molded by and daily reflected the every-day national traits of the Hebrews; but had a capacity for mental, social, and national detachment, that gave to him a strong originality. During his life Palestine was, as for 300 years before it had been, and for centuries after continued to be, the great meeting ground, mixing place, and fermenting center for eastern and western nationalities. So, in spite of the narrow, rigid, often bigoted care with which the Rabbis regarded their "flock," socio-political conditions around favored a width and independence of thought that was conducive to new and original proenvironal outlooks.

Therefore, as Mills, Sayce, Chamberlain, and other writers during the past quarter century have ably shown, it remains a widely open question how much Hebrew blood Christ may have possessed. It is undoubtedly true that after the invasions, the wars, the plunderings of human life and riches, the forced emigrations and the immigrations that had occurred throughout Syria and specially Palestine, little of pure Israelitish blood remained. Further it is now practically conceded by every unbiased and honest investigator that the more rigid and pharisaic growth of Hebrew thought was limited to a small section in the region around Jerusalem. This in large part explains the bigoted, contemptuous, and insolent treatment that Christ and his views received from the "Doctors of the Law," and later from such Hebrews as these "Doctors" could influence. To counteract such opposition from the Hebrews, some of Christ's followers unfortunately adopted from other and lower religious systems the unnatural-often still called supernatural-miracles, resurrection views, and other articles of "faith and doctrine" which became to many superstitious minds a main attraction.

In estimating Christ's character and teachings also we must entirely divest our minds of the erroneous views still often expressed, that the Hebrews were the monotheistic people above all others, and that Yahweh held an undisputed place of regard in the affections of most Hebrews. The whole tenor of Old Testament history negatives this; the rock writings 
of the Persian kings show that in Zoroastrianism was an equally, or even a more, enthusiastic regard paid to one great divinity or first power Ormazd; the lives and writings of many Greek and Babylonian religionists are quite equal to any of the Old Testament. But further in "the apology of Aristides" (circa $140-160$ A. D.) this author berates the Hebrews of his and of earlier centuries as little better than pantheistic, or even as nature-worshipers in some of their ways, while he equally decries the Greeks for their degraded sensual practices.

The true position eridently is that amongst all the monotheistic nations of the third century B. C. to the second century A. D. there were many earnest spirits at work endeavoring to keep alive high human aspiration in the midst of much degraded thought and practice.

In the most reverential spirit then the writer would state that he regards Christ as one and only one of those great human prophets or proenvironers who have successively appeared during the past ten to twenty millennia. Each of these in succession has continued and demonstrated the action of the law of proenvironment, as exhibited in nucleate plants and in simpler nucleate animals, still higher in ganglionated animals up to primitive man, and most highly in evolving man up to the Christian era. All of these proenvironers, in unbroken biological continuity, have been exposed on every side to sensory stimuli, some of which have been beneficial and synthetic in action, others have been analytic and slowly or rapidly destructive. By withdrawing from the latter, and by cumulation or summation of the former into new responses that have started lines of advancing and evolving action, some have progressed along steadily improving lines. After each such advance a temporarily satisfied state ensued and continued, until new and improved stimuli were generated, as a result of the actions and reactions of the evolving world. These then were combined into a new proenvironal response, that again resulted in a temporarily satisfied state.

Viewed from such a standpoint as the above, Christ was a great and noteworthy proenvironer. To appreciate this 
we must try to stand outside mundane affairs, look on them impartially as eye-witnesses, and estimate at exact value man as well as other organisms, while they pass in evolutionary sequence.

In such a procession every organism, according to the most recent physical riew is an aggregate of so much energy centered in and round inert ether particles. Therefore from their increasingly complex molecular structure it can be said that a blue-green non-nucleate alga is greatly and more densely energized than is the highest inorganic body; a nucleate multicellular plant is greatly more energized than is the alga; a higher ganglionated invertebrate is an immense advance in molecular complexity, and so in power of perception and response, on the nucleate plant; an anthropoid ape, in its rich and abundant gray brain substance, is a great advance on the invertebrate; while recent man in his active, varied, and abundant responses to environal stimuli proclaims that his highly complex molecular composition is linked with a like complexity and condensation of energy.

To the successive phases of organic energizing we have applied the terms biotic, cognitic, cogitic, and spiritic.

Man's religious advance we believe has been synchronous with his degree of progressive spiritic energizing. And in its application to Christ's teaching and example this is eminently true. During the two to three centuries before his appearing, aspiring minds had reached out to the conception of a great world Power, Energy, or Spirit which energized and moved them as well as universal nature. This in reverence they named Ormazd, Yahweh, Theos, or God. And truly, if we, who each represent so much highly complex energy which. so acts that it bestows on us our personality, can thereby think, plan, proenviron, and act, is it not likely that this world and universe contains a greatly higher type and condensed expression of energy that may be unseen to us as yet, but which permeates the universe? Such is God, the proenvironed and at length realized God of higher and purer Brahmanism, of Zoroastrianism, of Judaism, and of Platonism. Each religion 
and sect, filled often with a lower human bigotry and zeal, has denied the community of thought and result, in their effort to prove their own the only true religious faith. But mankind is beginning to perceive the true trend of events, and to believe and live accordingly.

So toward the close of the pre-Christian era the more earnest spirits of the civilized nations of the world had sought after, proenvironed, and accepted the existence and power of a great Energy or Guiding Will of the universe. To feeble, timidly outreaching man this Will could only be realized by semianthropomorphic or anthropomorphic terms, for no other measuring line could gauge such. Thoughts and words and actions were molded, guided, advanced, and summated, so as to conform to the highest human conception of this Power.

Mankind was thus yearningly reaching out toward ever higher aspirations, or in other words was proenvironing ever higher ideals. The brilliant and saintly mind of Channing has well expressed it thus (223: 931): "The desire for an excellence never actually reached by humanity, the aspiration toward that ideal which we express by the word perfection, this is the seminal principle of Religion. And this is the root of all progress in the human race. Religion is not an exclusive impulse. It does not grow from an emotion that is centered wholly upon God and seeks no other object. It springs from the same desire for whatever is more perfect than our own nature and our present life, which has impelled man toward all his great spiritual acquisitions and to all great improvements of society."

Linked up with this realization were belief in the connection of God with man, as a great felt but unseen power who could help, benefit, and bless each one who strove to mold life-conditions to the highest conception of God. Such were the noble aims and aspirations of Zarathushtra, of Abraham, of Moses, of Cyrus and Darius, of David, of Socrates Plato and Aristotle, of Gotama, of Daniel and the later Hebrew prophets, as well as of thousands more, regarding whom the world has no record. But these all regarded God as a distant 
power and majesty, imbued with attributes such as man might group round the person of one who represented their highest mental conception or proenvironed picture. So reverence for God, rather than confiding love in him, was the mental tendency in man. Furthermore the intensely national and even personal character and connection of God with each nation and individual, such as were generally proenvironed, promoted a family and national sentiment often of enduring kind, but failed to develop an international regard of man for man. Unquestionably the nearest approach to this regard is shown by Cyrus and the Medo-Persian nation, in their relation to conquered nationalities. This is fully acknowledged in the Hebrew Old Testament and was a fruit of the joyous, expansive, and all-embracing conception the Zoroastrians had of Ahura Mazda (or Ormazd). This was in striking contrast to the narrow, selfish, and exclusive view entertained regarding Yahweh, who was associated with a tribal or restricted national outlook.

As we now survey the completed record of Christ's and of Paul's life and teachings, the question may well be asked: What were the great principles that caused their teachings to assume an international importance? We would reply: first, that they emphasized the already accepted principles of sobriety in all things; second, they proclaimed a new and only additional command to those already accepted by man, viz., "that ye love one another"; third, they had an intense self-conscious belief in the view that each was a part of and proceeded from God, the great final power and energizer of the world; fourth, they equally clearly taught that all men were brethren, since all had proceeded from and were temporary expressions of an infinite power or Godhead; fifth, that all who would aspire to and reach the high cogitic and spiritic: platform that they had reached must subdue the biotic and the cognitic so as to "bring into subjection" the latter to the former, and so fulfill the highest claim of human life, by making the lower or biotic and cognitic energies subservient and ministers to the higher cogitic and spiritic; sixth, that when such 
a result was attained a real reign of peace, righteousness, happiness, and progress would be inaugurated, and this not in some dim uncertain extramundane region, but actually here on earth. For no unbiased reader of Christ's fundamental utterances can deny this. So the writer would entirely reecho Romanes' opinion: "Of the religious consciousness Christianity is unquestionably the highest product" (55: 164).

But it must freely be confessed that to attain this Christ clearly foresaw and foretold that relentless wars in homes, in cities, and between nations would thus ensue. For, in reaching the higher good, the lower degrading condition has ever to be overcome. During the past 200 years groups of mankind have proenvironed and have fought out these wars, often under the name and guise of Christianity and the Prince of Peace, while running contrary to his most cherished doctrines. These religio-selfish and religio-deceptive ones have been a large though decreasing majority, who have filled the world with malice, rapine, and carnage.

The emperors, the kings, the popes, the politicians, the priests, the preachers of mythical dogmas, have all striven to impose on aspiring man tenets that in some cases helped him to higher platforms, because derived from the teachings of earnest souls, who looked out on the forces of this world and of the universe with wistful and reverent eye. But often for their own selfish aggrandisement, or for that of their religious party, they have invented and forced on humanity "burdens grievous to be borne" and as impious as they were grievous. "The divine right of Kings," "the infallibility of popes," "the true and only Catholic Church," "submission to those in authority," even when these were tyrants, libertines, or murderers, have all been foisted on staggering but aspiring humanity by unscrupulous and hired pseudo-religionists. The day has come when man is rejecting such burdens utterly, and is reaching to a clear and sober estimate of his relation to and union with the all-energizing Guide and Goodness of this world and of the universe.

Think of all that can truthfully and worthily be laid at the door of the popes and prelates of one church alone! And 
in the name of Christ they have done it. But evolving mankind is sick of it, and is deliberately shaking off the filthy garments that degraded religious conceptions and systems have tried to clothe true religion with; while with deepest earnestness it is seeking to have Christ's and Paul's true doctrines accepted, expanded, and above all practised.

It is a matter for regret, however, that in those eminently practical and suggestive volumes by Draper and by White, on the conflict between religion and science, both authors have dealt almost wholly with the religious conflict as waged by those degraded or bigoted types of spurious religionist whom we have designated (p. 699) the religio-deceptive, the religio-selfish, and the religio-dishonest. They have largely failed to perceive that while false religionists of the above type were oppressing humanity, and fettering human thought and aspiration, other and noble lives, imbued with deeply religious sentiments, were constantly in the van of progress and discovery.

For it is unquestionably to the Chrysostoms, the Husses, the Savonarolas, the Ridleys, the Galileos, the Luthers, the Calvins-spite the treatment of noble Servet-, the Newtons, the Hampdens, and their like successors, all men of earnest religious outlook and proenvironal striving, that humanity today owes a large measure of its freedom of thought and aggressive spirit of inquiry. In recent years the almost immediate acceptance and advocacy of the doctrine of organic evolution by Fiske, Tennyson, Kingsley, Romanes, Renan, and Kelvin, all of whom cultivated the religious spirit and attitude, demonstrates that religious aspiration is a fundamental longing and need for the highest human spirits, in their effort to reach the most satisfying relation between themselves, their neighbors, the world, the universe, and the great First Cause and Energizer of the universe.

But equally the constant prostitution of all that is best in it by time-servers, by selfish pseudo-religious aspirants to earthly place and power, by priest-craft and like conditions, has to be reckoned with as influences that have often dragged religion in the dust. 
Instead then of religion having been a shadowy emotional, sentimental, anti-scientific, and even unreal thing, it has been - eren in the antagonisms, the bitternesses, the wars and destruction that have accompanied it - the greatest proenvironal principle, in the cogito-spiritic sphere of action and reaction, for lifting humanity steadily and irresistibly forward to ever higher platforms of thought and word and action. The action and reaction of hand and eye in relation to the lorain have rapidly advanced man from the cognito-cogitic environal standpoint; the action and reaction of eye and mind after man had reached a definite stage of advance, that resulted in storing impressions of the objects around him in the universe, as bearing on his happiness and welfare, has as rapidly advanced man from the cogito-spiritic environal standpoint.

It is this combination of the moral or higher cogitic with the religious or spiritic forces that has welded mankind from the state of isolated and separately struggling units into ever larger groups, till now it may be said that the North American continent contains one great cogito-spiritic internationality of one and a quarter millions that dominate it.

Here too it should be observed that over this continent the religious factor was the one which most prominently guided and molded events. For, degraded, mercenary, and bloodthirsty though they often were, the early Spanish and Portuguese explorers carried monotheistic Christian principles ever before them as a proenvironal ideal, even though their acts often utterly traversed and minimized that ideal. Later the Swedish and Puritan Protestants, the Lutherans, the Friends, the Mennonites, the Dunkards, the Scotch and Dutch Protestants, the Hugenots, and the Doukobars all represented human groups who migrated hither not from want, nor from mental incapacity, nor from desire to roam, nor wish to subdue new lands. They came because moved by a proenvironed response to many stimuli, that pictured a region where a combined mental, moral, and spiritual atmosphere of liberty would surround them, and in midst of which they could summate 
the factors for that new and higher religion that is surely, if slowly and fitfully, evolving now.

Sad it is that so many debased spirits have been mixed amongst the incomers, or have descended from them, who in the names of "sharp business methods" and of "politics" have defiled the high ideal. When they are purged from the bodypolitic, the national summated ideal will become a practical realization.

Since the days of Christ and of Paul, marked moral and religious advance have taken place. Marriage and the home have become more santified; monogamy has been definitely accepted as the future rule for man; debauching wine-drinking is becoming a thing of the past; love of man for his fellows was never more sincere and widespread than now; Christian missions, that have been derided by many, have netted over the world with outposts of civilization where self-denying men and women have lived lives of Christ-like love and sacrifice; hospitals, orphanages, shelters, homes for the blind, the deaf, and the dumb are a part of every Christian country; churches vie with each other in preaching and spreading altruistic truths, even though they at times practise these in illogical manner.

So we would fearlessly assert that we live amid the highest proenvironal plannings, and see the pathway steadily taken that these plannings have projected. But, as will be indicated in future chapters, so long as the law of "struggle for existence" is made the bare rule of life, and the law of "cooperation for social advance" is viewed with distrust, so long will human life advance amid grinding friction and constant death. When Christ's command "that ye love one another" is willingly followed, when the "Sermon on the Mount" is regarded not as a mere rhetorical teaching but as a practical rule of life, when the moving sympathy and heart throbs of Paul's utterances meet full response in human life, then another and higher platform of human progress and evolution shall have been reached.

As being the climax therefore of religious proenvironal aspiration, we would define Patritheism as "a reverential and affec- 
tionate proenvironal realization by Christ and his followers of the existence and power of a good First Cause or God, of whose infinite energy man is a temporary and greatly condensed expression, whose laws bring man into relations with that First Cause who to the human intellect can alone be pictured as paternal, and by whose laws, impressed on the world and interpreted by man, the latter should view his fellow with loving regard and consideration."

The writer desires now, quite clearly and candidly, to state the following views regarding his outlook on some important questions involved in the foregoing description. He states these under a deep - a most profound - feeling of responsibility and regard for his own past views, his upbringing, his national traditions, his future outlook on life, his realization that he truly is his "brother's keeper." He has reached these views after years of struggle toward some position that would enable him to unify and clarify his conceptions regarding pure science, psychology, religion, and the general trend of human life.

The mistakes he has doubtless made - for life is composed in no small measure of mistakes, and all that these involvewill in time be all indicated and corrected; the views worthy of acceptance will, he trusts, receive fair treatment. His conclusions are:

(1) Each human individual can discover and recognize truth only by the senses and the nervous mechanism associated therewith.

(2) The senses, by intermediation of the connecting nerve fibers, stimulate the substance of the nerve cells, so that the latter store up, elaborate into more complex summated resultants, and then discharge nerve impulses by the efferent nerves.

(3) If these impulses are hurtful to the human organism and its progeny, they may be minimized or annulled by action of the inhibitory nerves. If such action does not proceed, the organism in time dies.

(4) If these impulses are beneficial, they emerge as words or actions that then characterize and advance the individual.

(5) In the relation of man to his neighbor-wife, children, friends, national kin, international fellowmen - the words and 
actions that conduce most to the general well-being of the whole have, by long-continued proenvironal effort and mutual trial, become recognized and fixed as moral principles.

(6) The summation of moral principles, effected through long millennia by each individual in relation to his neighbor, constitutes conscience.

(7) The universe, of which each individual forms a small but highly energized transient material portion, and the great energizing agency or Godhead of it constitute one vast and continuous natural phenomenon that extends its boundary, by insensible degree, from the finite to the infinite. No supernatural agency or object is known or verifiable.

(8) But man is ever advancing in his elucidation of the ramifications of the above natural phenomenon, and so is ever coming nearer to a realization of his Sonship with the agency or Godhead.

(9) God is the great ultimate power, force, or energizing Unity of the universe, whom man by continued and summated proenvironal effort and realization, has discovered and approached ever more nearly to. So God does not reveal himself, man must earnestly and patiently discover Him, as he discovers the laws of the universe.

(10) In the quest man discovers that the organic law of "struggle for existence" may be modified and overcome by the law of "cooperative love and sympathy" between him and his fellows.

(11) Christ and his followers have reached the highest stage of proenvironal aspiration in the religion of Patritheism. This realizes the beneficent and immanent presence of God, or a final good Power, with those who strive to live according to the highest proenvironal ideal. So to human understanding God becomes a beneficent but wise Father, mankind become brethren and sisters with Christ in their relation to the Godhead, Father, or ultimate energizing Agency.

The whole "Philosophy of Life" then for the writer is the ancient law or rather recommendation of the Medo-persian Zoroastrians, afterwards borrowed by the Jews, still later 
adapted and expanded by Christ and Paul in that condensed couple of sentences: (1) "thou shalt love the Lord thy God with all thy heart and soul and strength and mind"; (2) "thou shalt love thy neighbor as thyself." The first stands for evolved and condensed religion; the second stands for evolved and condensed morality. The two are distinct, but the former is gradually built upon, and is aided by the latter. For self and neighbor are, according to highest moral standards, emanations from God, the one great energizing Power of the universe.

So the highest aim of each proenvironing human being should ever be: (1) to reverence, to aspire after, to seek knowledge regarding the great final Power, Energy, or Spirit of the Universe, and reverently-not unscrupulously, nor insolently, nor flippantly - to strive in each life to live out the Natural Laws that embody and exemplify that Spirit; (2) as leading up to this is the innate sense which higher evolving man gradwally realizes, namely, to war against, to deceive, to in any way injure one's neighbor the world over, is disintegrating and destructive.

Therefore there should be a constant double aspiration, namely reverent love for God, and reverent love for fellowman; also a constant double repression, namely repression of all that would traverse or minimize God's laws or evolutionary workings, and repression of all that would injure one's neighbor.

Cooperation then is the great evolving ideal for man in relation to his fellowman; reverent affection and investigation form the great evolving ideal of man in relation to God. Christ is that noblest human spirit who has gathered up and linked together all of these ideals into the humanized phrase, "Our Father."

Finally, the great law that all such effort exemplifies, is that shown alike by inorganic and organic bodies, namely: progressive energy condensation accompanying and effecting progressive ether aggregation. 


\section{CHAPTER XXVII}

\section{THE COMPETITIVE SYSTEM AMONGST THE LOWER ANIMALS AND WITH MAN*}

"Struggle for existence" and "survival of the fittest" have been regarded as universal biological laws during the past half century. "Cooperative or social surviral and erolution" have been practically entirely overlooked.* In the present chapter an effort is made to condense and recapitulate facts regarding the former. In the succeeding chapter the law of cooperative evolution will be examined. Thereafter a comparison will be made between the two.

It is generally conceded that the most fundamental factor in determining the abundance, distribution, and continued existence of animal life is the food supply, included under which is water. All others are secondary in importance. If we neglect the simpler groups, it may be said that from the arthropods upward all animals can be classified in rather general terms under four categories as follows:

(a) Carnivorous, predatory, and competitive types.

(b) Carnivorous, semi-predatory, and semi-social types.

(c) Largely phytivorous, feebly predatory, and social types.

(d) Phytivorous, peaceful, and social types.

The first group includes examples that live wholly alone except during the breeding season, and when the young are being reared; or that consort only in sex-pairs throughout life. Illustrations are seen in the solitary crabs and lobsters, the arachnids generally, many myriapods and insects, a considerable number of the molluses, many fishes, about one-half

* The writer was not aware, when he wrote the above, of the existence of Krapotkin's remarkable work, "Mutual Aid, a Factor in Organic Evolution." In next chapter it is commented on, but should be read by all as one of the most valuable contributions ever made to Biological science, and presented in that lucid and attractive style which is so characteristic of the author's writings. 
or more of the Amphibia, many reptiles, a relatively small number of birds, and possibly one-third of living mammals. Nearly all of these have the prehensile parts-the limbs and claws, the beak, or the teeth-modified in varying degree for seizing animal prey; the eyes and nose are usually keen for sight and smell-perception; their alimentary system is modified for digestion of rich readily decomposable animal material; their general nerrous organization is seldom equal to that of semi-phytivorous or phytivorous types. Biologically they show a merciless and relentless relation to other animal species, and even in many cases to individuals of their own species. Their morements accordingly become stealthy, suspicious, sly, sudden, and aggressive; while other animals-even individuals of their own species-show a constant dread of or hostile relation to them. As a whole they reproduce rather, or even very, slowly or sparingly, and the young are exposed to considerable risks, at times even are preyed on by their parents or by each other.

To this biological assemblage belong many of the protected, often encased, or even heavily mailed animals like the larger crabs and lobsters, the scorpions and spiders, some groups of insects, the odontophorous molluses, the heavily scaled fishes, amphibians, and reptiles. Though they have evolved into a rather varied assemblage of species, palæontological as well as recent evidence show that they constantly become scarcer in species and individuals, and at length suffer extinction.

The second group includes species that often are social or semi-social. Some of the smaller crabs and lobsters, a considerable number of insects, many molluses and fishes, a fair number of amphibians, reptiles, birds, and mammals are here included. These feed usually on forms much smaller than themselves, or on decaying animals. They vary from active to rather sedentary, may secure their prey either by pursuit, by rapid picking, by protrusible viscid tongue, or by passing currents of water through some channels. They are at times graceful and active in movement; live peacefully 
among themselves as a rule; may show defensive contrivances by spines or surface secretions that are disliked by other animals; or they may burrow or form protective structures around themselves. They are often heavily preyed on by other animals, but they reproduce much more abundantly than do species of the preceding group, and so they hold their own well in the "struggle for existence." Since they prey on small forms or on decomposing animal bodies, they do not show the same degree of antagonistic fighting action as do the last.

So, in the wear and tear of life they are not exposed to so great strains.

The third group may be, according to opportunity, wholly phytivorous or to slight degree may resemble the last. They are often highly social, and may fashion community dwellings, or may travel in social groups or herds, which by their unity and compactness alike afford shelter to the young and a degree of protection to each other. Not a few of them are protected like the last group. They often have a well-developed nervous system, and their social habits enable them to organize and evolve to a high degree. Included here are a few crustacea, a fair number of insects, some molluscs, many fishes, some amphibians, a few reptiles, many birds, and many mammals. They are often decidedly dominant species, owing to the wide area from which they may draw food supplies, the degree of protection and even strength that the social habit gives to them and their young, also the extreme fertility that characterizes them.

The fourth group includes nearly all of the most highly evolved and organized insects, a small number of fishes, a considerable number of birds, and these amongst the most highly organized, also the greater number of species of the mammalia. It is amongst this biological group that we meet with the most elaborately and diversely modified types, alike as to nerrous, alimentary, and reproductive organization. They include, on the whole, the most highly colored, the most defensively protected by secretions, the most abundantly reproductive, the most highly evolved in social organization, and the types that are now richest in individuals. 
In characterizing this fourth group as "phytivorous, peaceful, and social," we by no means overlook the fact that sanguinary sexual fights often occur between individuals-as with deer, bulls, horses, etc.- or between groups from separate colonies of the same species - as with ants, with pigeons and other birds-or between two phytivorous families as with locusts and some ants. Such are well known cases, and the results at times are terribly destructive to the species, as all can testify who have witnessed a one or two days' warfare amongst red ants from separate colonies. But even here the food question seems mainly to be the cause of strife. The comparative rarity however of such happenings, and the general social tendencies of life, are helpful factors for this fourth group.

If we return now to a more detailed study of the first group, it may truly be said that it includes "the merciless competitors" of the animal kingdom. The qualities already noted are fairly or even keenly and highly developed. But these qualities are limited in range, and are concentrated on the main issue of securing food by competitive destruction of animals as large as, or larger than, themselves. Now, if keen and merciless competition is the successful method for ensuring continued existence, and is also the best means for multiplying any species, the above carnivorous and competitive groups should constantly lead in the race of life, and should "survive" most perfectly in the struggle for existence. But we renture to assert that the opposite is undoubtedly true.

Before adducing proofs of this, however, it may be well to again point out that the three prime factors in animal as in plant life that ensure evolutionary "success" are successful regetation (including successful food supply that is neither too abundant nor too scant), also sufficient defense against attack, and fairly abundant means of reproduction. So long as a couple of individuals or a species can maintain a course of living that fulfills these requirements, they remain successful, or even may tend to become dominant.

Now in such requirements the first group is handicapped along several lines. The prey becomes increasingly wary; they 
avoid the lurking places of their enemies; the common danger inclines them to live in groups, and so the competitor has to exert himself the more sharply. Turned on often by its wouldbe prey, it has to resist as well as overcome the latter; its young, in living the solitary life of the parent, is liable to attack; and even the carnivorous tendencies at times incline the individuals of a species to fall on each other. From their rough-andtumble life, those forms have for the longest periods survived which evolved and protected themselves by increasingly heary armor. But a striking feature of the entire palæontological record is the constant extermination of these predatory, competitive, often mail-encased and carnivorous groups, much more abundantly and completely than the groups that possessed colonial or gregarious habits.

It has been true also, during recent millennia, that the competitors, amongst all of the groups already named, are the types that are poorest in species and in individuals. For the predatory crabs, lobsters, myriapods, insects, molluses, fishes, reptiles, birds, and mammals do not compare in highly evolved species, and specially in highly evolved individuals, with those of the third and fourth groups.

Competition then, as a fundamental zoological law, is not nearly so "successful," we believe, as that of cooperation or social union. The mental capacity also is relatively retarded. Partial proof of this is got when man domesticates predatory species like the dog, the cat, and the snake. For then the removal of the competitive effort and all that this involves, the life lived as part of a social human system, the often kind and sympathetic treatment which has gradually drawn out those fine qualities that we associate with the cat and dog, combine to call forth those nobler traits that have helped to make both world-wide in distribution.

All animals then have had the selective choice of a highly carnivorous or semi-carnivorous, or a phytivorous diet, but continued selection of the first has led in nearly all cases to isolated individualism, to tense nervous watchfulness, to wear and tear of body, to concentration of the nerve powers mainly 
for the procuring of food. Such have reduced the rearing of sufficiently healthy offspring to a minimal or subminimal point. Many of the Crustacea; the Arachnida as a whole; groups of insects that are rich in species, such as the mantids, the dragonflies, and many beetles; some gasteropodous and many cephalopodous molluses; groups of fishes like the sharks and dogfishes, the garpikes, the cods, the pikes, and the anglers; a few amphibians; snakes and crocodiles; groups of birds like the Raptores; and amongst mammals the carnivorous marsupials, the carnivores, a few monkeys, and some uncivilized races of man, are outstanding examples.

The second and third groups, alike by their mode of feeding and their frequent secretion of substances distasteful to their competitors, constitute a large proportion of existing animals, though greatly less abundant, we consider, in individuals than the fourth group. Their frequent semi-social or social habits aid them not a little also, in spite of the animal or mixed diet secured.

The fourth group is examined in next chapter, and so need not now detain us.

To a certain degree though not along exactly parallel lines-it may be said that evolving man has presented four stages which approximately correspond to the four stages already presented. For the most primitive or hunting phase in man's history is that when-as with many savage tribes of the present day-he lived the family life or at most the feebly social life, when he trusted wholly or mainly to the chase of large animals, or secured these by competitive fighting with others of his race. The second or pastoral presents results not strictly comparable with the second stage in animal evolution, but which approximate very closely in the effect on the organism.

The third or agricultural phase in man's evolution runs closely parallel with the third in animal evolution, while the commercial or manufacturing phase in man's history is closely related to the fourth amongst animals.

But throughout man's advancing history a marked difference has been that he has struggled to retain a keen competitive 
individualism of cruelest most merciless tendency, in midst of a progressive social and national organization that has yielded highly beneficent results. We may now review the main lines of development which this competitive system has given rise to.

We have already seen that, in the religious aspirations and evolution of mankind, there soon appeared, alongside those who earnestly strove to elevate the race, others whose main or whole desire was to reach place, pelf, pomp, or powernot unfrequently all four combined-and who were prepared to degrade the religious sentiment and aspirations so that they might fulfill selfish ambitions.

Equally true has it been in social, political, and religious life. Mankind willingly recognizes, welcomes, praises, and looks up to those who subdue nature and the forces of nature. While some of these have proved its real benefactors and have been honored as such-like the Euclids, the Archimedes, the Aristotles, the Watts, the Stephensons, and the Marconisothers-and a sadly large number-have proenvironed and worked out selfish careers in which the synthesis, the upbuilding of mankind along ever higher planes have been almost or wholly unheeded ambitions. Rather gaining added power, they have become unscrupulous tyrants, wholesale butchers, deceitful schemers, traitorous aspirants over the rights of others. So have originated the politicians who have gambled with human happiness; the diplomats who have overreached neighbors, tribes, or nations, and sown seeds of lasting discord that the sword too often has extended; the captains, marshals, and generals who became wholesale cannibals, except that often after slaying victims they mayhap left their victims to rot instead of economically devouring them; the princes and kings who eventually became so impious and desecrating toward the human race or sections of it that they claimed forsooth "the divine right of kings," in order that they might continue their unhallowed ways.

For long millennia man in struggling upward has constantly proenvironed plans for delivery from such analytic and preda- 
tory ones. But he has had to carry out many millions of costly experiments in chiefship rule, in hierarchic rule, in aristocratic rule, in papal rule, in kingly rule, in imperial rule, in presidential rule, so that he might at length discover by costly process, never to be forgotten, that the rule of loveof mutual regard and forbearance-can alone unite, guide, evolve, and advance him to the highest plane of possible development.

Let us look somewhat more closely in the succeeding pages at the main phases of the human competitive struggle. We shall not attempt at present to complicate the matter by tracing man's transition from the phytivorous habits, typical of practically all primates to the mixed or the at times largely carnivorous diet that man has long indulged in. We shall rather accept the conditions known to us during written history as included within the past $3000-6000$ years, and try to sum up these in their present-day relation and possible future application.

In all evolving and increasingly complex societies, the constant threatened danger to the general well-being and advance of the community is in permitting to one or a few individuals the exercise of exceptional and special power, or the securing and holding of special natural supplies. As a result of variation tendencies, individuals constantly appear who are more richly provided with certain advantageous points than others. If these are bred into the general stock, a general improvement will result. If they are permitted to assume special power, they attempt to grasp exclusive privileges, to live exclusive lives, to use those who are less gifted or powerful as servants at will, to inter-marry with each other, to absorb the best at the expense of the whole, to build up an oligarchy instead of a democracy.

If such were always animated by a high proenvironmental aim, they would exercise, and in some cases have exercised, the highest good. But the selfish, the competitive, and the analytic spirit and mode of life nearly always so largely creep in to influence their actions that in the long run they become 
reduced in numbers, weakened, and at length die out. This is in nearly every case due to their allowing the biotic or carnal and cognitic or perceptive to overbalance and subdue the higher cogitic and the spiritic. So indulgence in meats and drinks on the biotic side, over-sensuous indulgence in sexual stimuli, disinclination to face the responsibilities, the joys, the labors, and the rewards of child bearing and child rearing, conspire to reduce their numbers or to weed them out.

No more melancholy and instructive a biological study can be made than of the royal families of Europe through the past half millennium, or of the nouveaux riches of America during the past century. Nearly all conform to the same bio-sociological laws and exhibit the same phenomena of biotic and cognitic indulgence with cogitic and spiritic impoverishment, leading to analytic decay and often extinction.

Moreover the actions they have often been lauded fortheir battles, their intrigues, their high matrimonial or social alliances-have in most cases been at bottom selfish and analytic acts of indulgence in which the good, the advance of the mass, have been sacrificed for the preferment of one or a few.

So while the Empress Eugenie schemed for the succession to the throne of a son who would doubtless have inherited "the divine right of kings" she gambled in unscrupulous cold blood with the welfare of a nation that has been distinguished by energy, thrift, and brilliance, even though it has worshiped over zealously at the altar of sensuous indulgence. Nature has demanded the biological reckoning, alike from the empress and the nation, and in large measure the payment has already beén made.*

Now just as some have called the lion or the tiger "the king of beasts" and then have turned to shoot it as an analytic, a destructive agent, so the earnest reasoning masses of man-

* As the secrets of diplomacy have gradually been revealed during the past decade, other and worse national gamblers in human blood and life have stood revealed. The reckoning is being summed up as these pages are being produced, and payment will surely follow. France learned wisdom, others will also with the passing years. 
kind may in past time have supposed they admired the "brute courage" of the kings, their rulers, but now are discovering that such can best be done without, as have the bees, the wasps, the ants, and the bearers discovered long ago. Furthermore, as true and pure religious feeling-feeling for the widest welfare of the greatest number of the human race, and desire to bring such into closest contact with the great all-pervading synthetic Power or Godhead of the universe -has steadily expanded, it has brought out in ever clearer contrast the past terrible evils of the competitive system for man, and its failure in large measure to ensure his best evolution.

So the following are now clearly recognized scientifically as a few of the baleful results that the competitive system has given rise to. Chiefs, thanes, barons, dukes, princes, kings, and emperors have arisen who to retain place and power have often pandered to the worst, not the best, side of humanity. A far deeper and more significant lesson than appears on the surface is taught by the picture of Zarathushtra's noble birth and self-sacrifice-so-called-in later life, as well as Christ's temptation through offer of earthly dominion with all its sensuous and lower mental accompaniments. Their noble rejection of power and riches as part of the religious creed of each is one of many proofs that the Zarathushtrian and Christian systems projected themselves far into the future of man's evolution.

A second competitive result has been that gradually but surely it gave much land with all its representative wealth to a few, some land with limited power to a still greater number, and no land to the largest numbers who became dependent for direct natural support on a few-the lordlings of the soil. Lnder wise and advancing social systems other and even more intellectual callings have developed, but direct access of all or of most to the soil is more and more recognized as a prime need for man's healthful continuance.

A third result flowing largely from the last has been that roaming animals have often been "corralled" by the land-rich few, have been preserved as game, have wandered and de- 
roured the crops of the underlings for whom little or no redress was possible.

But the appearance within the past $8000-10,000$ years of money gave a weapon of most terrible import wherewith the competitor could squeeze, mold, subdue, and eren starve groups of fellow mortals in the evolving process. Its recognition and legalization have permitted one or a few-like the story of Pharaoh and Joseph-to buy up crops, lands, dwellings, labor, and even their fellowman wholesale, and to utilize or oppress the last as the whim of the hour best suited. Groups of dependents and of slaves naturally followed. So for thousands of years a relatively few men have callously looked on and even arranged for giving a living wage to so many, while others had to shift for life along the starvation line of existence. The sight is keenly competitive and analytic-truly the opposite of cooperative and synthetic-where in hundreds of cities of the world men have again and again been left unprovided for, been left to wear their increasingly tattered garments, and, unhelped or rejected, been left with their families to face starvation and despair.

We would regard it as a ludicrous sight biologically, did we come on ant nest after ant nest in some favored region where we knew that great stores of grain were stowed away safely and were being gluttonously devoured by a few, while for yards round each nest solitary ants or groups of hundreds or thousands of them with broken down aspect were longing to enter in order to share in the general work of the nest, and not least to share in the stores their earlier industry had created. Biologically we might well paraphrase the roluptuous king's advice and say, "Go to the ant, thou cirilized human one, consider her ways and be wise." The ants are wise; they have got rid of their tyrannical "bosses." Man is slowly but the more surely learning wisdom betimes.

It is no cause for wonder, then, that under such a system man has evolved a highly specialized and scientifically beautiful system of war, devastation, and bloodshed that eren the ants fail to equal. Such a system has been the gradual out- 
come of larger and larger groups of human beings becoming associated by mutual interests, as man steadily evolved the forelimbs for wider and more varied uses, and simultaneously of the more keenly competitive amongst them inciting the others to acts of outside aggression. This has undoubtedly greatly quickened and evolved mankind in the struggle for existence, as the period of war-service in many European countries shows. But the advantage gained along the competitive pathway has been secured at such a terrible price that civilized man would never traverse that pathway again, with the knowledge he now possesses.

International conciliation is now becoming fashionable; international peace will surely follow. Naturally the military and naval rulers will exert themselves to the utmost to prevent this, for their destructive death-dealing occupation and opportunity for political intrigue would then be gone. It is for thinking, reasoning, aspiring man to say which he shall determine to have: peace or the sword, cooperation or competition.

Another product of competitive human activity has been the gambling spendthrift rich one, standing out in insolent and luxurious ease among his fellows, as a dangerous and disintegrating agent for humanity. The bonded indebtedness that some of these have developed in Austria, in France, and in Britain caused their hirelings to squeeze the hard-working land tenants, who at any time might be cast adrift, when the gambler willed it.

Alexander, Caligula, Napoleon, Harriman, and like personalities have been finished products of the competitive and spoliation system. Of one such it has well been said "that man has the heart of a Numidian lion." More humanly and truthfully Emerson said of Napoleon: "He did all that in him lay to live and thrive without moral principle. It was the nature of things, the eternal law of man and of the world, which baulked and ruined him; and the result, in a million experiments, will be the same. Every experiment, by multitudes or by individuals, that has a sensual and selfish aim, 
will fail. The pacific Fourier will be as inefficient as the pernicious Napoleon. As long as our civilization is essentially one of property, of fences, of exclusiveness, it will be mocked by delusions. Our riches will leave us sick; there will be bitterness in our laughter; and our wine will burn our mouth. Only that good profits, which we can taste with all doors open, and which serves all men."

The above "leaders of men" all constructed great and farreaching proenvironal plans that were wholly for self glorification, or for the forced dominance of the nation they represented, rather than for the benefit or improvement of the human race. So their plans were essentially analytic, degrading, and makeshift. No one recognized this better than Napoleon, who, looking on his meteoric career, and recognizing that the code of Napoleon was his only great proenvironal effort toward human constructive improvement, exclaimed: "I shall go down to posterity with my code in my hand."

France, at the beginning of the last century, was a great (shall we say the greatest?) European nation, whose generous alert people had thrown off the yoke of aristocratic despotism that had become effete. They had reasserted true freedom for man, like the valiant little republic Switzerland, and the younger republic over the waves. A great chance came for a wise and loving constructor to lead the country to highest heights of achievement in human development.

As to the result Emerson has again forcefully expressed it, "Never was a leader so endowed, and so weaponed; never leader found such aids and followers. And what was the result of this vast talent and power, of these immense armies, burned cities, squandered treasures, immolated millions of men, of this demoralized Lurope? It came to no result. All passed away like the smoke of his artillery and left no trace. He left France smaller, poorer, feebler than he found it; and the whole contest for freedom was to be begun again. The attempt was, in principle, suicidal. France served him with life, and limb and estate, as long as it could identify its interests with him; but when men saw that after victory was 
another war; after the destruction of armies, new conscriptions; and they who had toiled so desperately were never nearer to the reward - they could not spend what they had earned, nor repose on their down-beds, nor strut in their chateaux-they deserted him. Men found that his absorbing egotism was deadly to all other men."

But the above principle and the colossal personality exemplifying it have alike been reproduced in every civilized land on varying scales of decreasing greatness, through exploiters of railroads, of factories, of shops, of house building, of natural products, of human labor, down to each local village magnate who attempts to place as many of his neighbors under economic tribute as he possibly can. And with what result? The free healthy country life has more and more been gone from. All flock into towns and cities. There competition is keen; work is not only hard, it is anxious, worrying, and straining for the nerves; morals become relaxed, and religion disappears.

Even those who remain on or go back to the land find themselves often in the hands of financial exploiters and middlemen who corner the grain, the cotton, the tobacco in America, the wheat, potatoes, and hay in Britain, the sugar beets and grapes in France, the beets and the fruit crops in Germany. These products then, when in the exploiters' hands, are gambled with, prices are inflated, the great poor and middle classes so-called are squeezed, and the cunning squeezer retires laughingly with his ill-gotten gain. The papers, the press, the universities and the churches are nearly all comfortably subsidized in diverse and skillful ways, in order that they may support "the system," and so, when the falsities, the unscientific methods, the inhuman unchristian character of it all is exposed by some thoughtful heart and fearless one, a howl from the privileged ones goes up that their freedom is being curtailed, that charter-rights are infringed, that the "honest" earnings of the industrious rich are envied by the thriftless poor, that Nature and the God of Nature - whom they impiously malign and do despite to-always intended that there should be rich 
and poor, surfeited and suffering; so they lay a complacent salve to their hearts and consciences.

The competitive, the analytic system has been tested for millions of years amid millions of animal species up to man himself. It is one of two-and only two-possible means of survival and advance. The other is the cooperative, the social, the synthetic. Amongst animals below man the former has been tested in every possible way, and the competitive groups have surely become lone, few, exterminated; while the latter have increased to ever greater masses of individuals, to ever more ramified species, to higher and higher types of organization.

For many thousands of years evolving man has been comparing, experimenting on, and measuring the two systems. Often baffled, defeated, and decimated, he has been seeking slowly but surely for some way that would link him with his fellows, with the universe, and with the great Power-the Godhead of that universe. Would the competitive or the cooperative guide him thither? has been his anxious and unanswered query through all the scores of millennia. With ever surer, clearer, more determined decision, the recent centuries have been giving him reply.

Through these past centuries the competitive system has been tested in the most varied manner, on the most gigantic scale, amongst people of diverse origin, by the brainiest men backed by resources that were undreamed of two or three millennia ago when Lao-tsze, Amenhotep IV, Zarathushtra, Plato, Christ, and Paul were proclaiming new doctrines. The thoughtful, the laboring, the earnest, the sympathetic, and the major mass of mankind have discovered that competition has failed and failed signally.

It has absorbed the land that is the heritage of all by mature, and has used it to enslave, or stunt mentally, or dissipate to less natural centers those who should cultivate and enjoy it. It has absorbed the natural products of the soil that earch one could once grow or barter, and has made them pawns for gambling with, or has-shame to even dream of it-destroyed 
them wholesale when the chance of their abundance became possible. It determined that millions of human fellows should be enslaved and should die in slavery, so that a few might wallow in luxury, and decay unwittingly. "Those were the nickel-plated days," as one of the enslavers once put it to the writer. It compelled the enslaved ones to toil without a man's reward; it bought and sold them in the market places like cattle; it tore them apart as father, mother, and children when the legal or other whim arose. It made human cruelty a fine art, and the practisers of it rewarded men.

It hedged round patents, that might benefit the race, with costly fines or dreadful snares, so that the inventor became often the sad and despondent one, who was exploited, fleeced, and cast out by the competitive exploiters. It laid hold on the streams, the bays, and the foreshores, so that the ever changing waters and life of these were often locked up in monopolizing grasp.

It has gone into the earth and laid cold greedy clutches on the coal, the oil, the iron, and other minerals, so that from the gain on these it might reach out and command other sources of wealth. It got hold of and juggled that greatest possible 19 th century blessing to man-the railroad system. Only where this might minister to war and destruction did the governments of some countries step in to make the railroads national-i. e., aristocratic military-systems. The Leopolds, the Goulds, the Harrimans, and the Krupps have used these to bow national feeling to their desires, or to corrupt legislatures. Above all it has tried to hedge round education and knowledge, as well as the means of gaining this by the bulk of the people. So the free and compulsory educational system came not from the monarchies of the old world, but from healthy democratic proenvironal aspirations of the New England settlers, who by their Massachusetts School Act of 1647 planted an advanced standard of idealism that the other nations have biologically been compelled to follow, as an absolute necessity in the struggle for existence. One need only call to mind here the shameful aristocratic hedging round of the 
two old English universities against all non-conformist entrants till about a half century ago. But only thus could the antiquated feudal and aristocratic system have been bolstered up and perpetuated.

On most gigantic scale in this United States, this land of freedom and opportunity, it has reared a competitive system of most gigantic iniquity and effrontery. It has built up unlimited capital in the hands of a few, who have become Napoleonic tyrants in crushing out weaker or more scrupulously organized opponents, under the specious plea of "industrial organization." It has created "trusts" that have gradually stifled, throttled, and destroyed their once greatly vaunted exhibitions of healthy competition. It has cornered and appropriated the soil, the coal, the iron, the cotton, the corn, the wheat, the carcases, the eggs, the tobacco, the timber, the steel, the sugar, the oil, the leather, the glass, and nearly every other commodity. Then it has in bitter practice exclaimed: "When I, the wheat beast of prey, the timber beast of prey, the oil beast of prey desire, then, and only then, can you-free people of these United States-get, at my price, the staff of life, the shingles to protect from cold, the cheering light and all other commodities that I alone permit the use of."

From this school of Adversity, the great masses of mankind, after learning bitter lessons, are slowly but ever more surely graduating, to teach and to enforce better and higher doctrines, such as are dealt with in the next chapter, and which can alone come when education along the highest mental, moral, and spiritual lines shall have permeated the majority of mankind. 


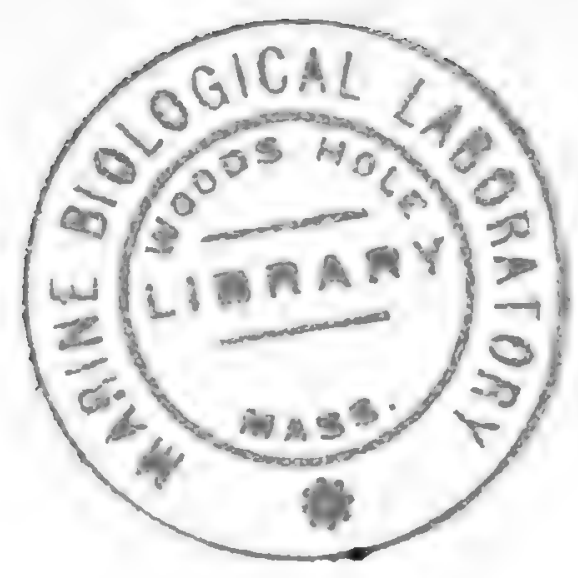

\section{CHAPTER XXVIII}

\section{THE COOPERATIVE OR SOCIAL SYSTEM AMONGST LOWER ANIMALS AND MAN}

Amongst the lower invertebrate classes the rudimentary character of the nervous system is evidently correlated with a want, or a feeble indication, of cooperative effort. But the manner in which large masses of individuals that constitute a species, amongst the coelenterate, echinoderm, and worm series, live together in complete tolerance, at least, would suggest that certain chemotactic and other energizing currents, of like value in each set of individuals, contribute to a certain neutrality of action in feeding and in other life processes. Amongst these also the reproductive products are shed into the water, and so the maternal or paternal 'acts are practically non-existent.

But with advancing cumulation of a nervous system, a distinct regard and care for the eggs are first shown; next for these till the period of hatching ensues; next for the eggs and the callow hatched brood, as among some social insects; finally for each stage in the process of growth, from the egg to the maturing adult, as amongst many ants. Increasingly complex evolution of the nervous system, and increasing care for the offspring of each species concerned, are thus correlative developments in nearly every instance.

In comparing, however, the exhibitions and relative value of competition and cooperation along the animal scale, it should be noted that richness in individuals, and steady expansive distribution of them over the earth, counts far more powerfully in the end than does richness or variety of species, specially when such species are carnivorous in habit. In no group is this more strikingly verified than amongst the most evolved of all insects, the ants. Regarding these, Wheeler 
(225: 2) says: "Not only do they outnumber in individuals all other terrestrial animals, but their colonies even in very circumscribed localities often defy enumeration. Their colonies are, moreover, remarkably stable, sometimes outlasting a generation of men."

Now, as the above author elsewhere points out, the total number of ant species is relatively small, their dominance nevertheless is preeminent. And the result has been secured, not by predatory and competitive methods between themselves, or even against other animals to any great degree, seeing that ants are largely vegetarian. It is due to combined social habits and excellent kinds of defense, alike on the part of the individual and the community. Their systems of defense have made them respected by other animals, and so Wheeler says (225:4): "The dominance of ants is clearly indicated by the small number of their enemies. They are preyed upon by comparatively few mammals, birds, reptiles, parasitic insects, and other ants."

So the "successful" species of animal are not part of the most numerous species of a class, but rather restricted species that have developed an advancing nervous and social organization, alongside, it may be, many related genera or species that do not so develop.

We may now pass in review some of the evidence yielded from higher invertebrates up to man.

Although of a simple and elementary nature, the social relation of young spiders to their mother, in such genera as Pardosa, where the former may be carried about for several days on the backs of the latter, or the social life of the young for ten to fourteen days in the web-spinning Epcira (179:340) represent commencing social organization, even though later these individually become ferocious carnivores. Again the genus Uloborus includes several species with marked social habits. But the nervous complexity of none of these has as yet been compared with that of other spiders of non-social habit.

But amongst invertebrates it is in some groups of insects that the most elaborate social and cooperative organization 
has been effected. This also can be traced through successive stages. The migratory locusts live and feed together in enormous swarms, and often perform long social journeyings in search of food, that much resemble those conducted by bison of the western prairies in former years. The countless myriads of individuals, that often represent one species, the practically world-wide distribution of the species and genera, as well as their successful mode of defense against enemies of many kinds, constitute them one of the dominant groups. They are all purely vegetable feeders.

Under the ancient group of the Neuroptera, the Termites or white ants have advanced to a high degree of social organization. In addition to the carefully preserved males and females, there is a soldier and frequently also a worker caste, the soldiers being usually much larger than the workers. The division of labor shown, the elaborate and often huge nests constructed, the mode of feeding, and the wide distribution of the known species over the world, all indicate that the cooperative habits evolved have greatly aided them in attaining a dominant place. The nervous system has been stated to be comparatively simple, but a careful study of these with other individualistic neuropterid genera is greatly to be desired.

The Hymenoptera is the insect group that preeminently shows cooperative evolution, but which is also suggestive as showing graded transitions from the competitive to the higher state. While the chalcid and ichneumon flies are eminently individualistic and carnivorous, others like the saw flies include genera that show marked care for the young, and a certain degree of social union in the formation of cocoons. But in the groups of the wasps and bees some are solitary and competitive, others are highly social and cooperative, while still others show transition conditions.

The ants, however, none of which are solitary, excel these as well as all other insects, and even most vertebrates, in their elaborate cooperative and social organization, as they do in their highly developed nervous system and sensory apparatus. So Lubbock truly wrote: "If we judge animals by their intelli- 
gence as evidenced by their actions, it is not the gorilla and the chimpanzee, but the bee, and above all the ant, which approach nearest to man."

Now, while there are many hundreds of hymenopterous genera, including at least 20,000 species, which are all decidedly competitive, these in number of individuals, in specialization for defense, in nervous complexity, and in capacity for multiplication do not even approximately compare with the relatively few genera of social wasps, and specially of bees and ants. But, in estimating their highly successful cooperative efforts, human observers have attempted, till within the past twenty years, to interpret their sensory actions and reactions purely by human measure and comparison. Now it is being recognized that their marvelous senses of odor and sound are a powerful aid toward social union.

If we only attempt to condense shortly the phases in the life history of ants that have conspired to give them dominant position next to man himself, we should say, first: that the continued evolution of an increasing care for the young, from the egg to the nearly adult individual, has developed a reflex love and regard for the members of each colony, so that mutual help and regard are prominent characteristics. Social care for the young also greatly reduces the action of injurious agencies, at the critical period in individual history. Man is gradually moving toward a similar line of action, though his efforts are often crude and indeterminate, being restricted mainly and often imperfectly to the family, rather than to the social system.

Second: By a remarkable and unified process of feeding, distinct types or castes have been evolved that undertake successfully the biological continuity of the species. In this connection few facts in the entire range of intra-specific variations are more remarkable than those associated with the evolution and adaptation of the queens, the males, the workers, and the soldiers. These have all become so equilibrated in their cooperative efforts, that the successful economy of each nest, or even of many adjacent colonies, proceeds with har- 
monious and perennial regularity. So "the ant colony, as many authors have suggested, is analogous to a single large organism, in which the soma is represented by the body of workers, the reproductive organs by the fertilized queen. It follows, of course, from this conception, that the differentiation of the colonial soma into castes is merely the visible result of a psychological and physiological division of labor" (225: 522).

So the fundamental principle thus worked out in the higher species of ants is that already cited as the successful one in every society, namely, successful vegetation, successful defense, and successful reproduction.

Third: In their cooperative life the ants show a more careful, elaborate, and sagacious provision for the future than does any other group of animals, except some of the highest races of mankind. This is seen in their methods of building granaries or other store houses, of preparing and storing seeds, of cultivating fungi, of storing honey, of keeping and feeding aphids, of providing for the maturation and flight of queens, and in their care of the young through successive stages of growth. The details of the above are so well set forth in the works of Huber, Lubbock, Forel, McCook, and Wheeler that further amplification is unnecessary here.

Fourth: In their cooperative effort for the welfare of the entire colonial commonwealth, they subdue selfish efforts that might result in the death of other members of the commonwealth, and they show unflagging zeal in promoting the wellbeing of it, and thus of their individual selves. This is the practical outcome that the higher socialized races of men are at present struggling to accomplish, and which is the avowed aim of the purer and more advanced present-day socialism. But, as all are aware, the results already achieved have been very partial, and the perfected work is largely in the future.

Against the above successful efforts are to be placed the following retarding ones, that ants have gradually evolved in their midst, and which have prevented an even more dominant growth than they now exhibit. First: Owing to increasing formation of protective subterranean dwellings, and 
to their living there for considerable periods in the dark, their vision has become reduced in intensity orer that of other insects.* The intensified senses of smell and of touch are the somewhat compensating advantages gained.

Second: Though the nuptial flight is still made in the air, ants as a group have ceased to cultivate an aerial or semi-aerial life, which, while attended by many disadvantages and dangers, yet would bring them into rapid contact with many environal conditions. Thus had they constructed strong nests on the higher branches of trees, and along with this have secreted formic acid, as well as the firm chitinous shin that most now possess, it seems likely that such would have secured wider dominance. This is to some extent proved by the successful adoption of such by certain social wasps and bees.

Now, in line with the physical law of action and reaction already accepted by the writer as true for organisms (p. 130), we would consider that the high plane reached by bees, and specially by ants, has been secured through long continued proenvironal responses to countless stimuli, dating from the period of the cretaceous or even older. This has stimulated the nervous system to ever-increasing formation of more complex molecules, along which cogitic as well as the lower energies could travel. So for these insects as for man cooperative effort, with its increasingly more complex interconnections, has given rise to ever more varied and abundant stimuli, which, acting on the highly complex nerve substance, has slowly started and elaborated in this new additions in the form of nerve cells, of conducing nerve fibers, and even of new brain centers. The final outcome has been the large and very complex ant brain, the weight of which, in comparison to that of man, has already been cited (p. 553).

Amongst the molluses, as with animals in general that live in water, cooperative effort is feeble or scarcely existent.

Our very imperfect knowledge of the nervous actions of fishes from the social standpoint prevents any detailed study

\footnotetext{
* That subterranean life, as a means of defense and even of aggressive persistence, has high advantages is proved in the present international war, and in the trench life it has evolved.
} 
of them. But, while most of the highly carnivorous species live an isolated or feebly social life, others of semi-carnivorous habit of life like the herring, the salmon, and the sardine live, feed, mate and often spawn in large shoals. The extensive and often exact migrations carried out from one locality to another by large shoals indicates that some common sense relation guides them in their movements. The greatly larger proportion of individuals that are social and semi-carnivorous as compared with others like the sharks, codfish, and catfish that are strongly carnivorous suggest an advantage that tends to become dominant, though we would not press the view too strongly here.

The Amphibia and Reptilia are on the whole solitary and non-cooperative. In spite of raried and at times elaborate means for defense, they are in no sense dominant or directive classes. The group Aves on the other hand deserves some study. We have already pointed out (p. 556) that the modification of the forelimbs into wings and the strictly limited use of these as the collectors of sense impressions have seriously militated against high nervous evolution in this class. The great majority of the species then are solitary, and are relatively poor in individuals. On the other hand the most widely distributed genera or species at the present day all show social and even markedly cooperative qualities in that they attack and repel a common foe, select breeding places in common, advance in groups to new feeding grounds, and often carry out extensive migrations in large flocks. While the solitary species are often carnivorous, the social ones are nearly always vegetarian in diet.

The penguins, solan-geese, flamingoes, and ducks represent abundant and widely distributed groups of low social organization; the pigeons, the fowls, and the swallows form a still higher series; while the sparrows, the parrots, the jackdaws, and the crows seem to be the highest and dominant ones. In the last of these the beak and one of the hind feet are often efficiently used for a wide range of activities, and such use seems to have stimulated to increasing development of the brain (p. 556). 
The class Mammalia, that has retained a soft flexible impressionable body, that has evolved almost wholly on land, that has largely become regetarian in diet, and that bestows the largest, the most continuous, and the most affectionate care over its young, now remains for examination.

We believe all will concede that, on survey of the world as known to man about one hundred years ago, the specially dominant masses of bulky individuals, and the large majority of species, belonged to social orders of mammals. Thus the countless herds of social marsupials that roamed over Australia as compared with the solitary carnivorous ones; the equally enormous herds of antelopes, cattle, horses, giraffes, rodents, and elephants that inhabited Asia and Africa; the herds of bison, deer, beavers, prairie dogs, and monkeys that occurred in America, formed a biological aggregate that had steadily prevailed over solitary and competitive types, till the latter were often a negligible quantity.

The appearance of man, at once on his savage side a severely competitive, and on his civilized side the most perfectly cooperative of animals, has seriously altered the biological result that was working out for lower mammals. But in the altering process he is himself demonstrating, with each passing century, that the most perfectly cooperative groups are those that become the dominant ones. And here, as in the case of ants, we would assert that, for the social group as for the solitary individual, cooperative dominance results from the combination of the most perfect vegetation, most perfect defense, and most perfect reproduction possible under environal conditions.

But, for the achievement of a highly perfect cooperative system, individual mobility combined with a rapid reception, correlation, and summation of sensory impressions from without, with resulting proenvironal response thereto, were absolutely necessary. As already pointed out (p.567) the achievement of this, through increasing evolution of the forelimbs in phalangerid marsupials, in lemurs, and in anthropoid apes, as compared with the less perfect forepaw action in beavers, 
squirrels, etc., or with the elongated proboscis in elephants, and still worse the retention of the forelimbs wholly or largely for progression, as in hoofed animals, caused steady advance of the anthropoid apes over the latter groups.

We accept it then, as a proven principle amongst animals lower than man, that the cooperative or social plan has ever tended to evolve and select forms which have possessed resulting advantages over the competitive plan and that such caused them to become, in spite of their apparent weakness, truly dominant groups alike in high organization, in capacity for defense, and in reproductive capacity. So it is safe to say that, for every individual which lives a keenly competitive life, a dozen can be found that are united in such social activities and in general provision for the species that the common welfare of each individual is nearly always assured. Furthermore, with advancing mentality and social organization, this principle is the more perfectly exhibited.

Some remarkable confirmations of the principle however are seen, when civilized man domesticates certain highly carnivorous and predatory types. Thus the wolf in the wild state has become a human synonym for many of the worst features of animal competition. That cannibalism is a constant tendency amongst them is also well known. But what might be called a churlish and suspicious social bond keeps them in flocks often of considerable size. Now, by many zoologists the wolf has been regarded as the wild progenitor of the dog, and the dog seems to be the mammal that man has domesticated longest and best. Even if we grant the dog's specific distinctness as a wild species, its close affinity to the wolf is usually accepted.

Of all animals below man it is the one which has shown qualities of affection, regard, sympathy, endurance with man amid dangers, reasoning on questions of every-day happening, rescuing of human life, and submission to man's commands, qualities that have endeared it to him for thousands of years. The competitive and analytic mode of life in the wild state has been largely replaced by a cooperative and synthetic civilized life. 
If we now try to ascertain the factors that have operated in giving to such comparatively defenseless animals as the horse, the sheep, the goat, and the bison their widespread persistence and abundance till man appeared on the scene, it will be found that at least the following are all cooperative: (a) numbers can usually in the end overcome isolated effort even of a fierce adversary; (b) the sight of a host of individuals has a retarding effect even on a bold intruder; (c) though some individuals may be cut off on the edge of a flock there is a sense of solidarity and increased safety in numbers. And here sacrifice as a virtue begins to develop, reaching its climax in man; (d) the constant tendency to shield the young of a flock gives to these an increased chance of survival, and thus of ensuring added and vigorous youth to the species. So even far down in the animal scale the three cardinal social and cooperative principles, love, sympathy, and sacrifice, become operative. These must ever constitute the foundation tripod on which every cooperative structure is built.

We recognize all three in operation when an ant's nest is attacked, when a herd of cattle is face to face with a common foe, when a band of monkeys is menaced by some aggressive mammalian opponent. Unfortunately man has hitherto selfishly tried to usurp credit for the possession of all the finer feelings and mental traits, so that he has given small credit to animals below him for the initiation and gradual extension of those qualities that now characterize him to a marked degree.

Primitive man then, as a descendant of the higher apes, inherited long-drawn ancestral experiences and mental tendencies toward social organization, at the same time that his struggles with many of the larger predatory and competitive animals around, the tiger, the lion, the bear, the wolf, and the crocodile, often inclined him to competitive and aggressive habits, as indicated by the cave remains of France and elsewhere. But, from the primitive and only recently extinct Tasmanian savage, whose biological relation was essentially that of the earlier stone-age, up to the highest civilized nation of the present day, the most fundamental characteristic has 
been an earnest endearor to evolve and perfect a non-competitive and cooperative organization of increasingly wide extent. The experiments leading up to this have been prolonged through many thousands of years-20,000 to 50,000 seem by no means too extended-but the more exact trend taken will only be traced here during the recent historic period of $8000-10,000$ years.

So when rapid and raried environal impressions and proenvironal responses in primitive man became perfected, and were united hereditarily with those social tendencies that he inherited from the anthropoid apes, man became alike structurally and hereditarily, the organism that would carry cooperative effort to its highest and most satisfying plane of action. The marvelous adrance that man has made during the past century is wholly due to the evolution amongst the civilized nations of cooperative effort in ever widening degree, combined with the correlative evolution in him, as in every cooperative group of animals, of an advancing moral perception, that has registered equally the failures and shortcomings of cooperative activity, as well as the immense success that accrued therefrom.

In thus reaching to the cooperative and moral platform, that may be said to have had its feeble inception twenty to fifty thousand years ago, man had advanced to the third of four great platforms of advance. The first of these was occupied when primitive and nude man allowed the carnal or biotic and the sensory or cognitic largely to dominate all higher qualities, and so caused him to live a semi-solitary life that might be termed the brutal. Lingering though somewhat advanced examples of this were men like the Tasmanians, the Bushmen, the Hottentots, and the more primitive Australians where untouched by higher outward influence.

The second platform was reached when man began mentally to compare, to correlate, and to appraise at their summated ralue all of the carnal or biotic, and the sensory or cognitic acts of his and his family's every-day acts. This caused him to develop a quickened cogitic relation to all forces around 
him, and so might be termed the lower cogitic or mental platform. The negritic, the primitive Egyptian, many primitive Red Indian, and other nations are examples. In these a loose social and semi-tribal life began to develop and so a transition was made to the next higher or moral platform. This third stage of advance may be said to have gradually evolved as the progenitors of the Iranian, the Aryan, the Egyptian, the Minoan, the Babylonian, and other ancient nations grew into ever larger groups. Principles for the general guidance of the cooperative units of mankind that made up each nationality gradually became laws or moral guides that all accepted. With the adrent of personalities in the pre-historic period now lost to record, later with the advent of Zarathushtra, Plato, Aristotle, Christ, and Paul, this platform became more fully accepted, but is reaching its widest sphere of occupation during our time.

The fourth and highest platform is the spiritual, that had its dawn in animism, polytheism, and heliotheism, but advanced rapidly with the perception and advent of monotheism under Zarathushtra and other Iranian seers. This reaches out far beyond the moral and social, in that it requires the recognition by each human organism of a Spirit Power or guiding energy of the world and of the universe, that not only links man to his fellow, but further raises him to a recognition of his own oneness with all cosmic processes, and so stimulates him to mold his life actions that these shall be attuned to the highest evolutionary progress.

The knowledge we now have of the early Sumerian inhabitants of Mesopotamia, of the ancient but already civilized Egyptians, of the Minoan and pre-Minoan civilizations of Crete, as well as others less perfectly traced, all indicate that man while retaining, or in some cases developing for himself, cruel customs, had already discovered that the most striking, most widespread, and most beneficial results could be secured, only by closer and closer social organization. 'Toward this result also religious proenvironal aspiration constantly tended. For, while as already stated (p. 700) unscrupulous and even 
hypocritical religionists might step in, who for sake of place and wealth might ally themselves with tyrannical rulers, earnest, reflective, and noble spirits must then as always have arisen to direct toward purer aims. So, in spite of the Minoan and similar cruelties associated with worship even in the years 2000-3000 B. C., this existed alongside fairly peaceful civilizations that must have bound together into nationalities hundreds of thousands or even millions of Sumerians, Cretans, and Egyptians of those times.

The evil and regrettable results of the cruelties, the deceits, the imprisonments, the butcheries, the wholesale supplantings and subjugations that the "rulers" of these civilized ones and the peoples themselves, as well as those of less civilized grade, every now and again indulged in or were subjected to, led the nobler minds to compound together all the best points in their civilization, and to proenviron therefrom a more lofty and aspiring plan where not merely justice, but where peace, love, happiness and brotherhood would rule. That the old Aryan and Iranian nationalities were proenvironing it, hoping for it, and even expecting it is shown by the eagerness with which Zarathushtra, the Buddha, Plato, Aristotle, and others were welcomed and their teachings discussed. So, for a thousand years at least before the Christian era, the above teachers of humanity proclaimed a gospel that was in many cases no mere idle religious dream, but was an intense struggle toward the humanization and socialization of mankind. They graphically realized the abounding evils of struggle for existence, competition, analysis. They clearly foresaw also that by socialization alone could man happily and peacefully overcome competition.

The origin of the conception of a "Golden Age," as set forth in the Zend-Avesta and in the Latin Poets, is merely a proenvironal longing for what their authors hoped had once existed and might again return, while large parts of the Zoroastrian Vendidad, fragmentary though they be, are social rules of so general and widespread a character that they might be applied by any nation at any time, during a certain ad- 
vancing phase of civilization. One cannot sufficiently emphasize here also that the surpassing social value of such writings has been largely minimized by the interweaving into their writings of miracle stories, of useless legends, and of trumped-up family histories.

But where we have the complete books of one of these authors, as with Plato's "Gorgias" and specially his "Republic," we see how the writer struggles, by deepest yearnings, reasonings, and aspirations, to evolve a system of rules, a mode of life, a synthesized unity of national action-we might almost say of international action-that would make man truly noble in himself, noble in civic life, noble and aspiring in national ideals. Nothing can be more sublime also than his intense yearning to link the principles of his republic with, and trace them back to, great laws of the universe that a beneficent God, a first cause, had placed it within the range of the minds of his creatures, his offspring, to recognize and to set in order.

The writer has already stated that he regards many of Christ's fundamental doctrines as derived from a joint Greek and Zoroastrian source. But Christ greatly excels alike Plato and Aristotle, in that he vividly proenvirons the God, the ruler of the universe, as an immanent, wise, and loving Father, whose offspring son he was, and whose were all mankind.

But, while our present-day fashionable preachers have constantly minced over and pared down Christ's principles, till they have alienated the great throbbing mass of civilized man, the only fundamental message of Christ is, "All ye are brethren. God is your Father. Conform therefore your thoughts, your words, and your actions so as to overcome competition by social cooperation." So, while he in no way ammulled the moral law, which is the most concise, inclusive, and lofty presentation of the neuro-inhibitory and the neuro-motor aspiring sides of man's life, he gave the new commandment, that alone makes and will make cooperative effort a success: "Love one another."

"Love one another" is the mental, the moral, and the religious cementing substance that for 2000 years since Christ's 
time has been slowly permeating humanity. But our lawgivers, rulers, moralists, and reformers have almost wholly failed to realize that alike Plato, Aristotle, Christ, and Paul have all regarded the realization of this commandment as possible only when the two sets of nerves in the human system, the inhibitory and the motor, are equally cultivated and utilized.

If we analyze the social regulations and recommendations that Zarathushtra, Plato, Christ, and Paul laid down, we clearly recognize that they fall under two categories. Many thoughts, words, and actions that readily tend to assert themselves are, by increasing use of the inhibitory nerrous system, to be inhibited, restrained, and by degree suppressed. No one has put these so pithily as Paul, when he classifies them as "Adultery, fornication, uncleanness, lasciviousness, idolatry, witchcraft, hatred, variance, emulations, wrath, strife, seditions, heresies, envyings, murders, drunkenness, revelings, and such like."

In contrast to these are the thoughts, words, and actions that can alone make a cooperative state possible, and which are in line with the highest uses to which the motor nerves can be put. These Paul equally perfectly summarizes as "love, joy, peace, long-suffering, gentleness, goodness, faith, meekness, temperance."

In these two quotations, from the moral and religious leader who for thirty years poured out his life for humanity, we have placed in perfect antithesis the principles and well-springs of competition, disintegration, analysis, with those of cooperation, integration, synthesis. Christ was rejected by the Jews because of their keenly individualistic and acquisitive spirit, just as Socrates was rejected by the Greeks because he rejected their immoral superstitions.

The Jewish law, even when acted up to, meant morality, justice: Christ's message meant sympathy, generosity, love. The former was the legal climax of competition; the latter sounded the tocsin of cooperation. The Greeks, unwilling in their national pride to accept the Medo-Persian Zarathush- 
trianism, from which the Jews had drawn their best aspirations, flung themselves into the arms of Egypto-Semitic polytheism, and so flung aside the lofty teachings of Socrates and Plato. Thereby they failed to see that they were working out for themselves moral, and so-alongside other and more earnest nations-physical death.

In the western world these social principles triumphed in the abstract. In not a few sects of the Christian Church, as well as amongst a few of the Jews, the Parsees, and the stricter Buddhists, they have in part been lived up to in the concrete. But 2000 years in the seething pot of human vanity, ambition, tyranny, of "divine right of kings," of unhallowed individualistic "liberty" had to be passed through; rapid means of intercommunication, by thought, word, and action, between great nations had to be discovered and applied; an extensive cheap and quick means of disseminating printed knowledge had to be devised; international travel and discourse as inaugurated by Paul had slowly to be made a heritage of many, before mankind could be educated to believe in, accept, and adopt cooperative Christian socialism.

Throughout those twenty centuries the writings of Plato, Aristotle, Christ, and Paul shone forth as teachings that were universally revered and studied. But man excused himself from practising them by silently or openly saying, "Oh! Plato and Aristotle were cultivated idolaters whose writings we can admire, but need not follow," also "Christ's and Paul's teachings were idealistic, they were intended more to teach us of a future life of heavenly blessedness than of an earthly life that could be lived according to their standard." So, in the Alexandrian, the Byzantine, the Moorish, the Italian, the French, the British, and the German schools and universities the classics of Plato, Aristotle, and their successors were read as mental gymnastics, not as highest human forms of wisdom that could advance and regenerate humanity. The teachings of Christ and of Paul were read and approvingly commented on in family, in school, in church, and in lofty cathedral, if the doctrines read exactly fitted the time and the mood; were 
apologized for, whittled down, or modified (as the writer has often heard from Presbyterian pulpits) if they ran counter to the occasion. And need we wonder at the result today?

Constantine, perceiving the inexorable superiority of the synthetic Christian doctrine for daily life, did one of the worst acts for it that ever happened to it. He made Christianity a fashionable, royal, and aristocratic appanage, that would sanction and bless "the divine right of kings," that would hedge round and protect from popular anger "the holy office of bishop," that would regard it as a state crime when the mass of the people "spoke evil of dignitaries" who had deceived them, led them to slaughter, and forged the tools that laid waste their home and country.

So, while Frederick dubbed the time from the 5th to the 15 th century "the insane period of history," he might more appropriately have called it "the hypocritical period of history." True, to the credit of some rulers it should be said that they aimed high and fulfilled much, while beset by cunning and relentless schemers in the persons of popes, prelates, premiers, and politicians. So Charlemagne, Alfred, Cromwell, William of Orange, and Gustavus of Sweden accomplished much in demonstrating how many millions of people can be wisely guided and helped. But that fundamentally sacred principle, "the will of the people," was largely ignored by them, with the result that the death of each brought weakness or disaster to his country.

That "will of the people" as a ruling principle had been attempted with many handicappings by the Iranians, the Greeks, the Romans, and others, but when the little uprising of a rural people hemmed in by mountains took place at Morgarten it only attracted such attention as did the defeat of Charlemagne's army by the Wascones nearly five centuries before. It was, however, "the stone cut out of the mountain" for future centuries, for, from that eventful day till now, "Swiss freedom" has been a sacred example and altar-fire to the rest of the world. For Switzerland has demonstrated that government "of the people, for the people, and by the 
people" is not only possible, it is the only true pathway for future advance. So the dukes, the counts, the barons, and the knights were sent about their business as ordinary men, and their Swiss strongholds were dismantled.

But, while cooperative government with gradual growth of the Initiative and Referendum in Switzerland proved that the people could think and be trusted, there still remained the essentially semitic and unchristian individualistic competition, that Switzerland inherits almost as keenly today as any other country.

Centuries after that which included Morgarten had to roll by; the world had to be explored, surveyed, and estimated in its capacity; industrial inventions had to be perfected; commercialism had to be sampled alike in its pleasant and in its bitter draughts; knights of industry had to show their ability and also their fangs; knights of labor had to show brotherly union, solidarity, and sympathy, as well as discords and disintegrating jealousies; noble spirits had to arise who might prove not merely the abstract but equally the concrete applicability of the proenvironal principles of Zarathushtra, of Plato, of Christ, and of Paul to the life of humanity.*

So the dawn of a new day, and therefore of a new human order, was ushered in gradually as a slow evolutionary process, by the teachings of the four great reformers above named; by their followers-open or unconfessed-who thought over, were guided by, and inclined others to their teachings; by those who even in restricted and local manner tried to put these teachings into practice as a means of knitting together

\footnotetext{
* The most gigantic and successful experiment, not alone in national but even in international cooperative action, is now being witnessed as a result of the present iniquitous war. Kings, politicians, and financiers have steadily and doggedly resisted cooperative action for the benefit of the mass of the people, when it was urged by the more noble and far-seeing minds in the different nations. Witness for example the antipathy shown by British aristocratic capitalists to Lloyd George's national pension scheme, and by Prussian Junkers now to social reform in Germany. So the present war, though a scandal to Twentieth Century civilization, has proved a valuable demonstration of the ease with which the entire world of mankind can be provided for, if a few governmental bureaus in each nation cooperate with those of other nations toward a common beneficent end.
} 
and elevating mankind; by the often sensuous teachings of a Rousseau; by the partly fantastic teachings and efforts of a Fourier; but largely by the teachings, practice, and experiments of Owen and the socialists of the past and present century.

But in much of recent cooperative and socialistic writing the absolutely clear and undoubted scientific fact has been overlooked or ignored, that the minds and methods of man in European and American countries had largely been inclined and even guided into complete socialistic and synthetic effort by the writings of the above four great teachers and specially of Jesus and of Paul. For, as with Plato's "Republic" and Aristotle's "Ethics" and "Moralia," so with the recorded teachings of Christ, and the writings of Paul, these are socialistic almost wholly. Thus, if one compare Paul's catalog of "the works of the flesh" as above given with "the works of the Spirit," these are not mere abstract notions relating to an ideal heaven or future state, but are exactly the personal and social characteristics that have to be repressed by the inhibitory nerres, or encouraged and put into action by the motor nerves. Mankind, though unable to recognize this as an abstract principle, has keenly realized it in practice. So the early Christian Church was as perfect a socialistic organization as the environal factors of the time would permit. Its aim also was to extend its socialistic principles to world-wide limit.

But in the days of the early Church the disciples were too thoroughly imbued with the truths of their own doctrines to commit the mistake of supposing that they could change all municipalities, states, and governments into a world-wide socialistic system.

That they largely molded not a few municipalities and even states was a main cause of their persecution up to the time of Constantine. The gigantic mistake then made-necessary perhaps however for the future sure progress of man-was that the great body of Christians ceased then to have hope in their doctrines as capable of securing the further evolution 
of man on the earth. They looked more largely to an ideal and future heaven, but even this was a perfected earth, "wherein dwelleth righteousness." The cunningly-at times however honestly-devised "state" religions simply served to divert Christian minds from the possibility of Christ's and of Paul's doctrines becoming practical guides to the earthly unification of man, and invested the doctrines with an ultraterrestrial fulfillment that the propounders of it never intended.

It follows therefore that the Christian Socialists of today are, in their social attitude, the hereditary descendants of the Christians of the primitive Church. Kidd and Chamberlain have to a considerable degree recognized this, and above all have clearly pointed out that the high-water mark of civilization today has been attained in professedly Christian lands and by Christian peoples.

Furthermore, if the leading social literature of today be synopsized into a set of brief axioms, these are equally the fundamental duties that Paul enjoined, and that the early Christian Church put into practice. The socialistic extensions of recent socialistic writers are the details that fit these axioms to modern industrial, commercial, agrarian, and governmental development.

So, as not a few writers of the past decade-some of them even by no means biased toward cooperative or socialistic evolution-have testified, mankind in relation to the erolution of the world and of his own species has reached a stage where the individual as an organism becomes merged in and aids toward the upbuilding of "Society as a compound organism." Just as in all biological advance the "organic" molecules organize into a cell, as the cells organize into a multicellular individual, as the multicellular individual develops a synthesizing brain, so in time the next stage is reached of the individuals organizing into a social whole that constitutes "society." Here Herbert Spencer reached a safe conclusion in his "Westminster" Article (227), even though the foundations on which it largely rested had to be constructed in the future. The picture from Hobbes that he quotes of "a vast 
human-shaped figure, whose body and limbs are made up of multitudes of men," may suggest in pictorial allegory not a little of what may yet come to pass.

At this stage in our study of socialistic development, we may now stop to inquire as to the agents at work in effecting slowly but surely the above change. Kidd in his valuable studies has laid it down (228: 328) that not merely individual betterment but simultaneously social betterment have resulted from correlated qualities of an individual and a social kind which have combined to evolve and improve alike the individual and the social whole. This principle we regard as entirely satisfactory. But it only explains the position to a slight extent, while the attempted restriction by him and other writers of social integration to man is alike arbitrary and contrary to scientific evidence.

We have already accepted it as a postulate that all organic evolution has proceeded through the conjoint action of heredity, environal action, proenvironment, struggle for existence with selective survival, and reproduction. No one of these factors can be neglected, but their relative value and activity at any one time may vary.

In that social upbuilding of animals that is receiving its climax-expression in man, heredity has played and is playing an important part as permanently registering and handing down morphological and physiological details. Thus the socalled instinct of the lower animals represents the gradually acquired morphological details, through long-continued stereoenergizing or physiological strains and stresses, that incline or permit the organism to pursue a definite course of action.

In the social organization of bees, of ants, and of the simpler groups that lead up to them, of social birds, of beavers, of monkeys, and of forms leading up to them, a hereditary morphological and physiological tendency to social acts that are as much or more for the good of each colony as for the individual can be followed. It is the gradual registering in the individuals and the transmission hereditarily of such acttendencies that we have vaguely called instinct. But the 
transmission does not explain the origin of such acts. This we must look for in what follows.

Environal action and proenvironal reaction play, the writer believes, a fundamentally important part in the evolution of social organization. Whether it be a bee, an ant, a beaver, or a primitive man, each by aid of sense impressions from without quickly learns, and slowly records on the nerve mechanism, the fact that the united efforts of two or more can accomplish what one alone would entirely fail to do. Thus, when one watches the transportation of a dead bee or other large insect by four to six ants, and notes how each of the latter changes position and the direction of push or pull, so as to bear the bee rapidly along against obstacles, he at once appreciates that this has become a wonted act in which feeding of the mass of young ones, well-being of the colony, rapid securing of the food by the colony against surrounding competitors, as well as other considerations, are all concerned. So, what once was a rather fortuitous act, in which each of several ants responded to a chemotactic food stimulus, and hauled the booty to a common nest rather than permit it to be carried off to another, becomes by repetition and related brain stimulation a registered tendency of the organism. Alike for the individual ant and for the social colony the socialistic act is beneficial. The environmental food-stimulus, the large size of the body, the environal competition started by ants of other colonies, and the difficulty of transport are responded to by the united acts of the cooperating ants.

But in all social evolution the law of proenvironment plays the most important part. We have already shown how it operates from the more individualistic standpoint (p. 636). It seems to become a hastened and condensedly active agent where two or more organisms are concerned. And here sexual reproduction is involved. For-absent in the very simplest asexual organisms-sex fusion is a proenvironal act, while amongst the higher invertebrates and vertebrates the social, sympathetic, and correlated acts connected with reproduction and perpetuation of the species do not need emphasis. The 
social life then shown by male and female, even in the most competitive and predatory species, is a proenvironal phenomenon in which the production and rearing of a future offspring stimulates to abundant and varied social developments. Here also we believe is found the reason for the widespread socialism typical of many animal groups. For, since proenvironment, in its most fundamental expression, is the compounding of two or more environal stimuli into a resultant stimulus that starts a new line of satisfied progress for the individual, so when applied to social organization it becomes the resultant of all the stimuli that are found by long and repeated trial to be best alike for each individual, and for the group of which the individual is the unit.

So the building of nests above ground, the excavation of nests below the surface, or the hollowing of trees into nests by ants and other insects; the gradual elaboration of complex loghouses and dams by beavers; the planning and erection of a lake-dwelling by medieval man are all proenvironal acts that at first were largely of a simple and individualistic kind, but now have become, by oft repeated trial, by slow effort after ever better results, and by recognized benefit of united action in elaborating designs that would the better house a social group, cooperative social results that have elevated alike the individual and the social race.

That struggle for existence and selective survival operate in social colonies of animals up to man himself is attested by abundant and exact evidence. But these factors are largely - almost wholly-dependent on and sequent to the activity of those already referred to. So heredity, environment, proenvironment, selection, and reproduction have together evolved and are evolving social organization.

But for man the phase of proenvironal action that has most powerfully aided in social and socialistic organization has been Religion. We desire again, however, to have it clearly understood that by this we mean, not the false concretions, the accessory schemings of cunning men, the vague hypotheses that have been foisted on mankind as revelations from heaven, 
but the earnest effort of the individual and the social mind to proenviron, more and more clearly, an elevated conception of his best possible relation to his neighbor, to the world around him, and to a great Agency or Power who formed and energizes the universe, its laws, and its motions. At times the conceptions that aggregations of men have proenvironed and set before them for their socialistic guidance have been degrading, socially demoralizing, and disrupting, as with the Ashtar or Ashtoreth of the Assyrians and Phœnicians. But far-seeing prophets of their own or of other nationalities have noted the evil, have denounced it, and have proenvironed higher and truer pathways of progress. True, the social cancers may meanwhile have eaten into the body politic till, as in Imperial Rome, conquest was easily possible by a more vigorous and morally healthy nation. Then the conquered nation may largely have disappeared from history, to become a fossilized tradition, as with the extinct Tasmanians.

In all such sociological growth, the proenvironal outlook and aspiration became a biological necessity, unless death of the social mass was accepted by it as an--and the onlyalternative. Thus often, in the history of primitive as of civilized man, the determined effort has been made by one man or group of men to hate and destroy another such. But, even after generations of such strife, the hater and the hated have alike learned that unity and friendship are better than discord, so very slowly but very surely mankind has proenvironed the law, "Thou shalt love thy neighbor as thyself." The history of the kings of West Asia for a thousand years B. C., as well as their history in Western Europe from 1200 to 1900 A. D., has been a wavering but steady testimony to the truth of this. For the treaties they have made, the marriages they have consummated, and the mutual concessions they have agreed to, have been either perfect or more or less imperfect commentaries on the above moral law. Behind their diplomacy and statesmanship so-called they have felt compelled steadily to proenviron the greatest social good for their nation. The sad thing often has been that they have put selfish indi- 
vidualistic preferment before national advance, and so nations that have confided in their selfish kings, statesmen, and generals have been betrayed, duped, conquered, at times destroyed. Mankind is surely learning to appraise these actions at their true scientific value.

So, as already sketched under the chapter on religion (pp. 708, 728), mankind has advanced to high and still higher heights of social and sociological development, till, under the pure, lofty, and ever unattained perfections included in the pure and simple doctrines of Christianity, the nations that have made its truths their pole-stars by which to steer are now the dominant ones of the world. The most altruistic, rigorous, and sublime proenvironment has produced the most perfect, most extensive, and most powerful social unions.

Mankind however, now as ever in his past history, is searching out for the highest attainable social organization. He has learned by long and bitter experience that "competition is commercial war, cooperation is commercial peace." He has learned that as are the units-the individuals-so is the social organism. He has learned, and is learning to the point of graduation, that, as disintegrating influences in the individual can best be overcome by action of the inhibitory or repressing nerve centers, so disintegrating influences in the body politic have to be subdued and weeded out by like repression on the part of the united mass of that body. He has also learned that by use of the motor nerve-centers for highest accomplishments, alike in the life of the individual and of the state, the highest benefit will accrue to both. Therefore Aspiration and Repression are the two watch-words for human scientific advance.

So, many of the imaginary obstacles that privileged and enfranchised ones have attempted to place in the pathway of socialistic evolution are purely imaginary and reactionary, which will disappear as that pathway is advanced along. With a perfected system of Initiative and Referendum, the wisdom of each man and woman in each community, state, and nation will be learned on each new problem, that even a few may 
suggest. This, when carefully discussed by the chosen individuals or highest minds of the nation, will take form as a measure that is referred to each individual for final decision.

In apparently strong and sarcastic terms we referred in the last chapter to the many woes that competition has wrought in the past. But in exactest scientific measure such terms have been earned. For no animal other than man has behaved so brutally, so mercilessly to his fellow. But the powerful synthetic golden thread of advancing evolution has ever triumphed over the analytic forces of devolution. And so with pleasure we begin to record the achievements that cooperative socialism has already won. These we give approximately in the order of far-reaching import for man's social evolution.

First in importance is the existence today of a large international group of men and women who have pledged themselves to destroy competition, and to inaugurate a human social union, in which healthy and happy rivalry to reach the highest cogitic and spiritic results for mankind shall in time replace the old antipathy of tradesman against tradesman, of town against town, of nation against nation.

Second, a widespread and constantly extending system of free or almost free education has been adopted, and has become a social force of inestimable value. For "where wisdom ceases the people perish." This in some countries like Switzerland, America, Germany, Scotland, and France extends eren to the highest university grades, and so is giving to these countries a mental ability and brain wealth that will make them dominant nations. Here a highly cheering feature of the past half century has been that the social power, the so-called "working class" (the others in applying this term did not necessarily mean that they were the "non-working" or loafing class), has welcomed and even demanded well equipped universities, for they are fast learning that all "knowledge is power." A sad feature has been that, by inoculation with "class distinction," the children of this working class have often spurned the "hole from whence they nere dug" and 
have tried to perpetuate the class distinction they should have aided in removing. But time is surely remedying the evil.

Third, free libraries are arising everywhere, and while the great demand at these for "trashy novels" may indicate that the sensuous or cognitic is too often fostered at the expense of the cogitic and spiritic, yet the reading habit of one generation may become the reading and thinking habit of the succeeding one, later still the reading and thinking and socially acting one. But what previous century could ever boast of having like the nineteenth, at least $300,000,000$ of intelligent readers? The present century also will have at least as many thoughtful ones by its close.

Fourth, thanks to the sanctified humanity and far-seeing wisdom of Robert Owen and the Rochdale cooperators, every civilized nation has become netted over with Cooperative societies that for the past half century have slowly been evolving a better and truly social form of industry and commercialism. At first merely mutual distributive groups of working men or of the professional and civil workers, the movement within a quarter century of its establishment took on a cooperative - one might truly say a socialistic-productive character. The competitors - the tigers - of commerce have tried hard to undermine the growth and usefulness of both phases of development, but the whole has now passed beyond the stage of experiment or even of danger. The writer can never forget the intense thrill of pleasure he felt, when a few years ago he attended a great annual gathering and exhibition of cooperators in the Crystal Palace, London. An imposing orchestra of male and female cooperators filled the grand hall with music; a cooperator's flower show was made up of about 1500 exhibits; the display of cooperators products included finely finished samples of practically every requisite for civilized life. Then one learned that nine perfectly equipped steamships formed the peaceful cooperative fleet that plied between Denmark, Sweden, Holland, France, and other countries, bringing commodities direct from producer to consumer. This 
however, is only one phase of a like movement that most advanced countries can now boast of.

As one then listened to the swells of music, threaded one's way amongst the flower exhibits, questioned the producers of manufactured articles as to the methods pursued, one began to realize that the once disjointed units of humanity had already come together to constitute "Society as an organism."

Fifth, the past century may be said wholly to have witnessed the evolution and adoption of the international postal, railroad, telegraph, and telephone systems, while the closing days of it fittingly witnessed also the practical application of wireless telegraphy. In spite of competitor's greed, and efforts to commandeer these for selfish ends, these inventions have served to hasten more the socialistic unification of mankind, than any other combined agencies.

During the past ten millennia of human history man might appropriately have been defined as "the animal of war and bloodshed." Unquestionably the keen competition and accompanying proenvironal efforts toward highest efficiency that such war stimulated or involved have contributed greatly, though in bloody fashion, toward man's present evolutionary state. For the more terrible the bloodshed that marked the career of a Tethmosis, a Cambyses, an Alexander, a Caligula, a Jenghiz, a Tamerlane, a Napoleon, the more did thinking humanity proenviron a higher and truer life. The past decade has witnessed the welcome sight of whole nations repudiating their war lords; of determined protests against military expenditures; of the erection of a temple of peace where arbitration principles can be scientifically discussed; the binding together of socialist workers into international committees whose sacred aim is to prevent international feuds. The "military business" men, like the competitive commercialists, have fought hard to prevent such a peaceful close to their career, but even they begin to foresee the end.

Sixth, hospitals, asylums, and sanitaria are the gradual and steady growth of the past three centuries. These have done much alike to record human sympathy and to make mankind 
scientifically acquainted with the causes, the treatment, and the cure of diseases. Some students of genetics, eugenists so-called, have recently inveighed against the aims and results of such institutions. But humanity can well afford, in getting rid of its wholesale competitive slaughter, to watch over and to bear with the mentally or physically diseased, who often owe their condition to competitive evils. They forget also that from these institutions have come some of the most important scientific facts, that are slowly changing the entire mode of treatment of crime, disease, and insanity. Socialized humanity in the not distant future will largely free itself from such results, by simply remoring the causes which competition has strewn thick along the pathway of evolutionary progress.

Seventh, the international dissemination of scientific literature alike along biotic, cognitic, cogitic, and spiritic lines has hastened socialistic advance to a remarkable degree. The benefits have reached out to and influenced the humblest agriculturists, plant and animal breeders, artificers, manufacturers, manual workers, and explorers of the world's raw wealth. The countless inventions and discoveries that such literature has dealt with indicate how prolific and rich is the presentday human intellect, when given free scope to reach out along the peaceful avenues of Science and Art. When one notes further that the high mentality in the great army of such workers has been furnished from the less influential and poorer sections of civilized life, this is but proof of the contention that the human hand-arm has been the potent lever in stimulating to brain evolution and activity, and that the rich and privileged ones are a dwindling factor. It is proof also that a free and aspiring democracy has the brains to be trusted with its own destinies.

Eighth: Hygienic discoveries, hygienic laws, and the acceptance of social hygiene may last of all be taken account of. These all represent a growing cooperation of individual with individual, of nation with nation, to ensure that the greatest good of the largest number shall be promoted. So civilized man 
is growing taller on the average, is lengthening his years, is curtailing or stamping out disease, and though still handicapped by many competitive ills, he is surely facing the problem of getting rid of them.

The writer would conclude this chapter in words he has already used, when recalling the efforts and accomplishments of the evolutionary "fathers" of the by-gone century (11:62): "It is recognized that the one organism man possesses, dominates, and will still more fully dominate the earth, so that man's evolution is now the great and central problem. Man now no longer sees with the eyes of the individual; he penetrates the past, the present, the future, with the compound eyes of "Society as an Organism." Nationality counts now for little, and will count for less in the future. World problems are before us, for man's exploitation of the world is becoming increasingly easy. . . . May we not regard it then, as the crowning legacy of nineteenth century advance, that Knowledge is now for all, that schools, colleges, and universities no longer exist to manufacture a select and privileged cult, but to people the world with the highest types of earnest thinking individuals, that, as today is the best day in the world's history, so future days will be on ever higher planes." 


\section{CHAPTER XXIX}

\section{HUMAN ORGANIZATION IN RELATION TO ENVIR- ONMENT}

The problem of human life has been the riddle of the millennia. One of the great biblical writers answers his own question, "What is your life," by replying: "It is even as a vapor that appeareth for a little time and then vanisheth away." There is poetry and perhaps also some sound science in the reply. The despairing Schopenhauer says: "Everything in life indicates that earthly happiness is destined to be frustrated or to be recognized as an illusion. The conditions of this lie deep in the nature of things. Accordingly the life of most of us proves sad and short. The comparatively happy are usually only apparently so, or are, like long-lived persons, rare exceptions-left as a bait for the rest. Life proves a continued deception, in great as well as small matters. If it makes a promise it does not keep it, unless to show that the coveted object was little desirable. Thus sometimes hope, sometimes the fulfillment of hope, deludes us. If it gave, it was but to take away.

"The fascination of distance presents a paradise vanishing like an optic delusion when we have allowed ourselves to be enticed thither. Happiness accordingly lies always in the future or in the past; and the present is to be compared to a small dark cloud which the wind drives over a sunny plain. Before it and behind it all is bright, it alone casts a shadow. The present is therefore forever unsatisfactory; the future uncertain; the past irrecoverable. Life with its hourly, daily, weekly, and yearly small, greater, and great adversities, with its disappointed hopes and mishaps foiling all calculations, bears so plainly the character of something we should become disgusted with, that it is difficult to comprehend how any one 
could have mistaken this and been persuaded that life was to be thankfully enjoyed, and man was destined to be happy."

Amiel, at times as despairing a pessimist as Schopenhauer, wrote amid the "grandiose splendour" of the Bel Alp (232, 2: 78): "What is Man-this weed which a sunbeam withers? What is our life in the infinite abyss? I feel a sort of sacred terror, not only for myself, but for my race, for all that is mortal. - . I am conscious of a mad clinging to life, and at the same time of a rush of despair and repentance, which forces from me a cry for pardon. And then, all this hidden agony dissolves in wearied submission. . . But there is no peace except in order, in law. Am I in order? Alas no! My changeable and restless nature will torment me to the end."

Again Amiel says (232, 1: 155): "Our work-my workconsists in taming, subduing, evangelizing, and angelizing the evil self, and in restoring harmony with the good self. Salvation lies in abandoning the evil self in principle, and in taking refuge with the other, the divine self."

Were we to attempt to review further, even by condensed extracts, the thousand views on human existence that have been offered by pure scientists, by psychologists, by philosophers, by litterateurs, by poets, volumes would be needed. The above quotations from Schopenhauer and Amiel represent the opinions of sensitive philosophers of akin and yet diverse tendencies. How are all to be reconciled? Amiel, like many others, wrestles to reach the solution.

Let us try to learn whether a great and steadily advancing wave of organic evolution cannot be traced that would explain many present individual and social relations. Amiel proclaims that there is no peace except in order, in law. If this be true man should show the law in his evolutionary history. Accepting such a possibility, in relation to the views already advanced in this work up to the present point, we would consider that super-man has developed through four successive and advancing stages of complexity. First, and forming a continuous, a lasting, a wide foundation of vital action, is the 
basic life substance of every cell, from the simplest non-nucleate cell of the lowest plant, to the highest ganglion cell of manprotoplasm. This we have regarded, for reasons already given, as the molecularly complex material that is energized by a higher and more perfect energy than even electricity, which we have named the biotic. Formed early probably in the archæan epoch as a complex matter-energy combination, it exhibits amongst the protophytic and protozootic groups vegetative activities that we classify physiologically as the irritable, the nutritive, the respiratory, the asexual-reproductive. But its irritable responses are feeble, sluggish, largely local and uncorrelated. This protoplasm nevertheless persists, even may increase in amount for new individuals, along the scale of advancing plant and animal life, but, in its fundamental composition, its staining capacity and affinities, its chemical reactions, its disintegration products, it retains the same qualities throughout. Every organism then has its foundations laid in a vegetative or biotic substance - the protoplasmthat has retained a fundamental sameness of relation from archæan days to the present, and is a common and continuous heritage from the simplest bacterium or blue-green algæ or moneran up to super-man.

Second, with advance of the archæan epoch a gradual progression or elaboration took place within this protoplasm, as a still higher and more complex exhibition of energy, the cognitic. So there segregated off by slow degrees-as seen in higher Blue-green Algæ and in some protozootic organisms still living-a more complex labile responsive substance that differentiated into an irritable network permeating the protoplasm, centered in the nucleolus, and having a special area of action, the nucleus. This we call the chromatin substance. With its final differentiation all nucleated organisms showed and continue to show a delicate correlated or resultant senseresponse to light, to gravity, to heat, to chemical agents and other environal stimuli, that is as typical of higher Algæ and Infusoria as it is of man. The quality or concentrated capacity for response may vary much, but the fundamental sub- 
stance remains the same in composition; in staining capacity and affinities, in its chemical reactions, and disintegration products.

So, while the protoplasm that is traversed and "vitalized" by biotic energy is a universal organic basis, the chromatin is confined to nucleated plants and animals, and confers on them a quickened sense perception and response. Further, with its segregation hereditary traits became condensed in it, and sexual reproduction became an accompaniment of its presence.

But, as cognitic or sense response became increasingly perfected amongst nucleate animals, a third and slowly evolved substance was built up in special cells-the nerve gangliaand by aid of a more perfect form of energy, the cogitic, that enabled the organisms to form more complex and interlinked impressions of a mental kind. This we regard as the Nissl substance or neuratin that reaches its greatest condensation in Man, but extends in unbroken line from the colenterates and worms upward, as studies like those of Smallwood and Rogers show (84: 45). By action of the cognitic energy, and by aid of a still more perfect form of energy, the cogitic, traversing the Nissl substance, mental impressions were formed and in particular were correlated into ever more complex resultants. Some of these resultants were of an inhibitory nature, designed to counteract injurious effects to the organism, others were motor and beneficial in action. Finally in man alone, or possibly in him along with the higher monkeys, there evidently exists a more complex form of energy than the biotic, cognitic, or even cogitic, and which we have termed the spiritic. We are as yet unaware of any special brain or nerve ganglionic substance with which this energy might be associated, though the suggestion has been made that those highly typical areas of the brain-the frontal convolutionsmay possibly be developing in connection with such condensed combinations of energy. But all of those highly complex phenomena, that enable man to link himself to the widest forces of the universe, and that permit him to express his highest thoughts and aspirations in speech and writing, that 
enable him to build up religious conceptions, that enable him to realize the existence of a supreme Energy, Power, or Being, form a combined expression of energy and matter activity that seem only explicable - but yet seem truly explicableif we accept these as due to a remarkably perfect and subtle form of energy that traverses and energizes some equally complex material substance, located probably in some layer or area along the frontal sinuses of the cerebral substance.

We may attempt to represent the above views in diagrammatic fashion as on the page opposite.

According to this view man is heir to four successively evolved and increasingly complex substances, the simplest of whichprotoplasmatin - he inherits in common with all organisms and the energy for the upbuilding of which-the biotic-gives to him certain vegetative impulses that - humanly speakingwe may call the carnal. Chemical perception of and response to environal food substances as well as digestion, absorption, and assimilation of these form the main functions, though respiration is an accessory activity connected therewith, while growth results from the healthy interaction of both.

Built upon and nourished by this protoplasmatin as a basis is the highly important chromatin substance that he inherits in common with most plants and animals, and the energy for the upbuilding of which - the cognitic-brings him actively and continuously into contact with varied environal stimuli, which, as they affect him, are responded to correspondingly. This gives to him what-humanly speaking-we may call the sensuous or sense-perceptive capacity. Perception of and response to energizing stimuli, either by direct reflex action, or by linking together of several of these stimuli into a resultant response of more complex character, form its main function; but by being itself slowly altered in its molecular relations, and by similarly stimulating the protoplasm to effect changed responses, it assumes prime importance as the bearer of heredity.

Built upon, nourished, and receiving external sensuous impressions from one or other of the two last is the ganglionic or 


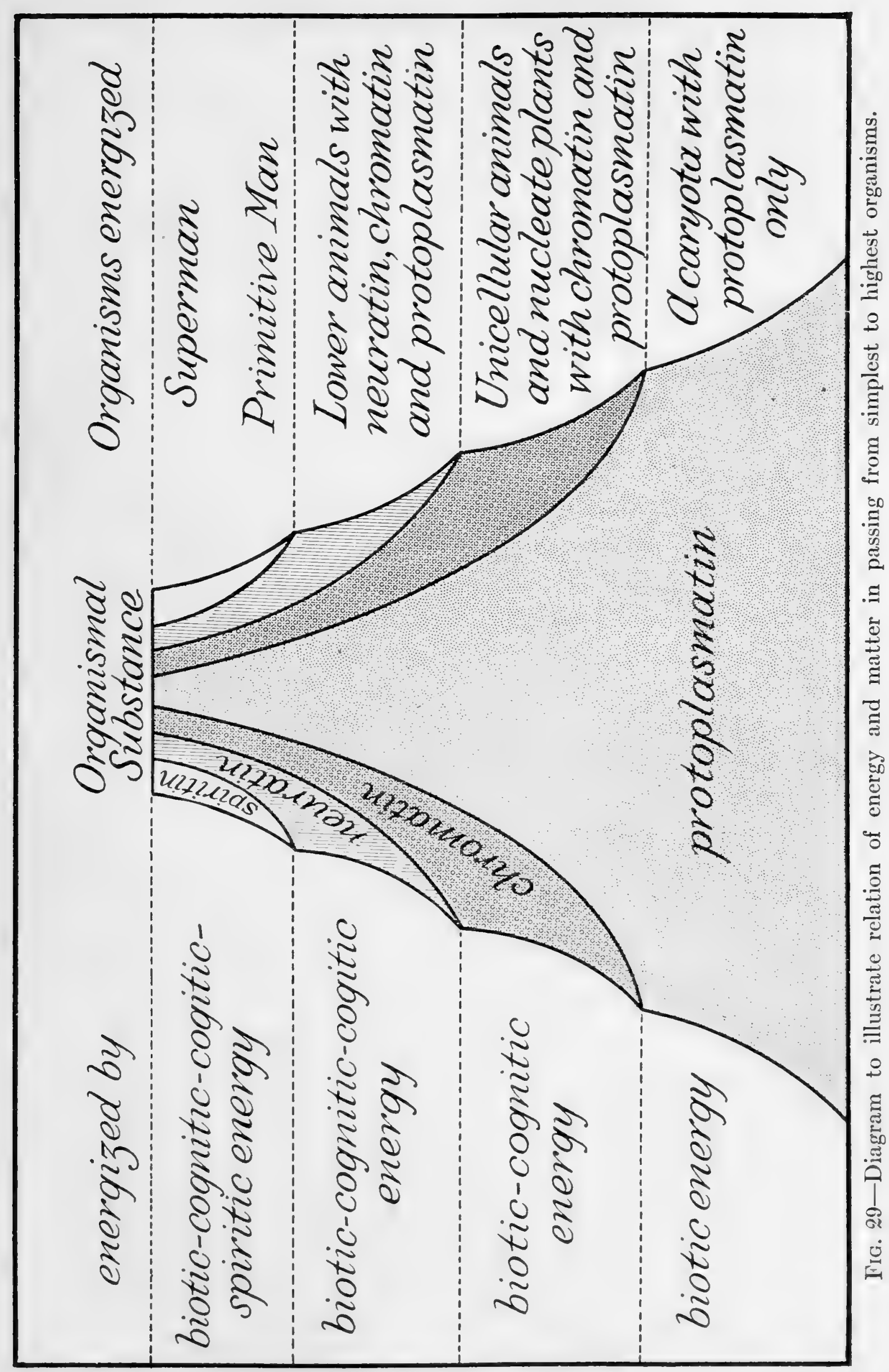


Nissl or neuratin substance. This he inherits in common with all animals from the rotiferan grade upward, and the energy for the upbuilding of it - the cogitic - enables him to receive and respond to directly, or to sort out, compound, and unite into simple or complex resultant responses, the varied stimuli absorbed by the cognitic system. This confers on him that capacity which-humanly speaking-we may call the mental and mento-moral. The entire material mechanism connected with it is derived embryologically from the surface or sensory layer-the ectoderm-but it becomes wholly shut off from the surface as a part of the internal nervous system and very largely as the brain. It functions therefore as an internal correlating, compounding, and mentally elaborating substance, that gives to super-man his high position over the other mammals and even over the ruder savage.

Finally, the phenomena, the experiences of human life in the past ten millennia especially, powerfully suggest to the writer that built up on, energized by, linked into complex mental relations by, a combined bio-cognito-cogitic union is a still more complex substance than the protoplasmatin, chromatin, or neuratin, probably resident in some part of the gray frontal matter of the brain, and which hypothetically we may call the spiritin. This, if we judge by phenomena observed in hypnotism, in thought reading, in thought transfer, and in possibly as yet unexplained spiritualistic actions and in high ecstatic religious experiences, may be traversed by an energy that we have called the spiritic, that man inherits probably in feeble and provisionally imperfect beginnings from the higher apes, but which has been in process of active evolution during the recent millennia under such complicated and often imperfectly understood conditions that many have rejected some of the phenomena displayed as being impositions of pseudoscience. But the possibility alone of thought-transfer through wide distances of space is now accepted as a question worth debating by some, and even as a demonstrated fact by others; while hypnotic, religious, and other experiences are not vagaries or delusions of the human mind, but scientific 
exhibitions that must be explained. The enormous and exact knowledge gained also of what are practically infinite spaceconditions is due, we believe, to the same energized capacity.

So spiritic energy, greatly more than the cogitic, has so functioned as to energize the more aspiring and lofty souls of humanity to widest outreachings, toward the most profound questions of the world and the universe. Man's knowledge is still very limited; his attitude toward the phenomena involved has been critical-even suspicious - rather than inquisitive and sympathetic. The future however may hold great things in store. If each human being then is an exhibition of the above four constituent and increasingly condensed exhibitions of energy in relation to material particles, what should be the highest aim of each aspiring and proenvironing individual?

Moralists, religionists, and specially pre-Christian and Christian teachers like Plato, Paul, Francis of Assisi, Savonarola, and Channing have tried to picture and define "the perfect man." With no exact scientific chart to guide them, but striving to interpret aright the deepest longings, the complex entanglements, the frequent shortcomings from high ideals, the noble aspirations toward a better state that each individual shows, they have often proenvironed rules of guidance that have influenced millions for good. Equally also the philanthropists, the missionaries, the social workers have lived worthy lives, and have held up ideals of life, that have elevated and blessed the people amongst whom they labored. But all have felt after and longed for an exact and reasonable scientific basis on which to ground their deepest and highest longings. The comparative absence of this has caused some to forsake religious life, has caused others even to question like Schopenhauer the reality of any moral code, has led others to view the biotic or carnal and the cognitic or sensuous as the entire end and aim of human life, has caused others like Amiel-and these a numerous class - to grope vaguely and longingly for some unified system of life that might guide and encourage them. 
Now, though the mainsprings of their actions have often been put down by their fellowmen of less high aspiration to religious delusion or mental unbalance, the heroic self-denying lives of Zarathushtra, Socrates, Gautama, Christ, Paul, St. Columba, Huss, Wycliffe, Zinzendorf, Heber, Livingston, and Paton will live through all time as an incentive toward a "perfect life."

If we attempt now to analyze their lives and the lives of those whose memory the world increasingly cherishes, in the light of the above recognized complex constitution of man, it may truly be said that each of them strove to live a balanced life, in which the biotic or carnal, the cognitic or sensuous, the cogitic or mento-moral and the spiritic or religious were blended into a harmonious whole. True, in striving to live such lives they were often constrained by customs, opinions, traditions that were regrettable remnants of human misjudgment or error. But, in spite of these, their lives have been such that the world has honored them for the living. This honor has been accorded, not alone or often chiefly for what they have done, as for the record they have left of their high proenvironal aspirations, alike for themselves and for mankind.

Now it is most important to observe, in this relation, that they all had a proenvironal pathway set clearly before them, that was of high-often some have inclined to say of superhuman-character. And this pathway was undoubtedly the compounded resultant of many and diverse carnal, sensuous, mental-moral, and spiritual stimuli, passed in from without or inwardly developed mentally, as a result of these stimuli from outside.

If now one contrast such highest lives of historical personages with those of Confucius, of Solomon, of Seneca, of Wolsey, of Bacon, and of Bismarck, while all may be called "great," no one of them represented a truly balanced life. Confucius elevated the mento-moral, atrophied the religious, and so far as we know balanced the carnal and sensuous. Solomon elevated to a degrading degree the sensuous and the carnal, along with the mental and at times the religious, but the two former 
overtopped the two latter, and caused their abasement. Seneca, keen, cunning, brilliant, and calculating, gave full rein to the mental, and for reputation's sake to the moral side of life; he dallied at times with highest religious doctrine, and by all of these kept in check the sensuous and carnal elements of his being. The often unscrupulous use of the two former, and bridled guidance of the two latter, largely accounts for his downfall before the carnal and sensuous Nero.

Wolsey was a finished product of mento-moral cultivation, gilded by touches of the higher sensuous from below and by flashes of spiritic light from above. But the higher sensuous and the selfish mental gained the ultimate upper hand. Bacon's colossal and outstanding mentality scarcely rose to the moral stage. The religious, largely through environment, was atrophied, while the carnal and the sensuous seem to have been given rather free play.

Bismarck cultivated the rough carnal and the mental sides so that these largely concealed the sensuous, the mento-moral, and the spiritic, which were of feeble growth in him. All of the above then we would class as a second or medium type of historical personage.

But again, if we consider the career of a Nero, a Caligula, a Jenghiz, a Judge Jefferies, a Peter the Great, or a Napoleon, as outstanding historic personages, that are representative of thousands of lesser but like characters, we find that in each of these any trace of spiritic energy that they showed was distorted, degraded, almost obliterated; the mento-moral or cogitic in its higher moral phases was equally debased, while the high and alert purely mental qualities were used almost wholly for enabling the egotistic self to beat down or destroy all efforts toward social union in others, except such as they could control. The sensuous or cognitic impulses, instead of being used in balanced fashion for ministering to continued life and well-being, were largely abused; while the carnal or biotic energies were not unfrequently expended in glutonous eating and drinking.

Let us now take the above three groups to represent a best, a medium, and a worst type of historical personages. Wherein, 
it may be asked, did these differ fundamentally so that they were or became so diverse in their life careers? We would reply that such was due first to hereditary tendencies passed down in ancestral relation; second, that it was due in considerable part to environal action from childhood upward; and third that it was in large measure due to the different proenvironal pathways projected, as a result of compounding all the hereditary and environal stimuli-biotic, cognitic, cogitic, and spiritic-into a complex resultant that became lines of conduct pursued by each.

Further it can in the most exactly scientific manner be said that the sum-total of the life actions of the above three types of individual worked toward and absolutely ensured a life resultant that we would call good, indifferent, bad. Thus, though Emerson in his "Representative Men" started out in earnest manner to make a great hero of Napoleon, he entirely succeeded in proving him to have finished up his career as one of the most accomplished villains the world ever saw, and who attempted to cloak his unscrupulous actions by constantly invoking a "Fate," a "star," a "destiny," so that he might the better deceive humanity.

In contrast, the life of Livingstone, bleeding out by degrees for enslaved and down-trodden mankind, has been a model and an incentive for all who have studied it.

If each human life then be first analyzed, so that the average biotic, cognitic, cogitic, and spiritic actions and reactions of daily life be estimated aright, and the resultant combinations of these be considered as a sum-total, we believe that a correct estimate can be reached as to the good, the average, the bad finished life.

Here unfortunately it has to be confessed that we possess only the most fragmentary records of even the most fully recorded lives. The "Psalms of the Old Testament," the "Confessions of Augustine," the "Essays of Montaigne," and the diary of Amiel or of Richard Jeffries are duplicated by few and surpassed by no other books. But how poor an idea do they give us of all the thoughts, words, and acts that made 
up the lives of these men. Each individual knows only fairly well his own personality, gets glimpses-more or less intimate - of his daily associates, learns slightly regarding others, and therefrom makes up his estimate of human personality.

But the carefully recorded studies of the past 30 years regarding child development shed a welcome flood of light on human behavior, and entirely confirm the views just set forth for adult conduct. Thus, as has already been indicated (p. 88), the child at birth shows wholly or almost wholly biotic or protoplasmic response. Food can be absorbed, digested, and assimilated; breathing also goes on. But the cognitic or chromatin relation is scarcely, if at all, exhibited till the third day. Thereafter for four to six years direct or increasingly complex resultant responses to environal stimuli give rise to a cognitic education that may be continued in many cases to adolescence. The chromatin substance during this time will be exercised in flows of cognitic energy that-hereditarily made easy by relation to previous generations of men-evidently confer on it added stability and complexity in molecular linking together.

But child-mentality, or the cogitic state, only begins to show itself about the fourth year, and may be said to increase and expand as a mental education till the brain ceases to grow, at the age of thirty to forty years.

Now it is alike the result of observation, experience, and experiment that if the environal stimuli that start cognitic and cogitic response be of a pleasing helpful or synthetic nature the organism is correspondingly benefited, while the opposite is equally true in cause and effect. It would be impossible to deal with the wide question of human emotions or sentiments in such a work as the present, and after the able analyses that have been made of these since Spencer's and Darwin's time, by Hobhouse, Washburn, Parmelee, and others. But it may shortly be said that, while over-indulgence bioticallyas by a surfeit of eating and drinking-tends on the whole to dull the cognitic and cogitic sensibilities, any action or actions which cause synthesis or upbuilding, or which pro- 
duce a harmonious molecular vibration of the cognitic and cogitic sense-structures, tend also toward the general advance and proenvironal activity of each organism; while actions or stimuli that tend to disintegrate or to set up an unharmonious molecular vibration give rise to decay and to imperfect response of the organism to its environment.

So, alike in child education and in the environment of the adult, those cognitic or sensuous and those cogitic or mental actions or stimuli that give rise to resultant effects which serve to upbuild the whole organism and to start steady proenvironal responses along advancing lines are alone beneficial to the whole organism. The converse is equally true. Therefore it is that, with the advancing socialization of mankind, child education and adult environment have undergone a progressive evolution in the past half century that has been profound. Schools and their classrooms have been enlarged and beautified, textbooks have been illustrated and carefully selected, teachers have been much more highly educated, experiments and diagrams that appeal to the sense directly have been increased and perfected, while the health of the scholar-once a fortuitous and negligible quanity-is now regarded as of prime importance.

We arrive last, in study of child development, at the higher cogitic or mento-moral and the spiritic stages. The first awakening of the moral or ethical perceptions in the child appear, according to Preyer (51), Romanes (52), and others, in the third year. $\quad$ p till then the child is an unmoral and unreligious organism. Furthermore, not a few cases of child history show us that, if the environal educational stimuli be absent, children descended from moral and religious parents remain dull and apathetic to such questions. The material substance or basis may be present, definite inclinations or tendencies of appropriate molecules to place themselves in relation to possible future tubes of energy may exist, but, unless appropriate and everaugmented stimuli from without of a moral and religious kind be also present, the organism fails to respond, and the highest nerve centers of the organism remain rudimentary. 
A great lack of appreciation of the above exact scientific law has given rise to a loose, wavering-at times even antagonistic-method of dealing with higher cogito-spiritic questions in schools, colleges, and universities where such questions should most, and most fully, be studied. One important cause for this has been that many have failed to perceive to how great a degree all important inquiries and needs of the human race have been mixed up with theological bickerings and animosities, with dogmatic assertions on moral and religious questions, that are utterly unsupported by any evidence, with sectarian jealousies and prejudices that largely cover up and conceal the great core of scientific proenvironal effort which every truly "educated" human being feels and reaches out to.

So, in rejecting the rags and wrappings which have been wound, at times by ignorant, at times by cunning, at times by unscrupulous, at times by selfish, at times by utterly malignant adherents of religion, round the great progressive questions of humanity, they have missed the latter as well.

Unfortunately also, in the strifes of sects regarding their little tenets, few educated minds have become so detached, independent, and unbiased in their studies as to have been able to separate true religious principles from their superstitions, hereditary traditions, or false imaginations, and to link together the cardinal proenvironal responses into a great system of cogito-spiritic truth. Thus, one of the most earnest and inquiring minds in the scientific field of the past half century was Romanes, who, with his intense cogito-spiritic longings after a reconciliation of religion with science, was alternately a religious sceptic and a religious aspirant. But, as is proved by several sections of his "Thoughts on Religion" and notably those on p. 41 and pp. 42-43, his mental attitude was so overshadowed by "fashionable" views of Religion that he utterly failed to trace the correct biological origin and history of religion. 'Thus on p. 41 he says: "Religion is a department of thought, having for its object a self-conscious and intelligent Being which it regards as a personal God, and the fountain-head of all causation." 
Again on pp. 42-43 he says: "The distinguishing feature of any theory which can properly be termed a Religion is that it should refer to the ultimate source, or sources of things; and that it should suppose this source to be of an objective, intelligent, and personal nature. To apply the term religion to any other theory is merely to abuse it." Here, strangely enough, Romanes is unconsciously but thoroughly abusing the term religion, for he is giving it a circumscribed range that it does not possess, as we have shown on p. 684. For, in the gradual evolution of religion, we have no more right to say that the most primitive bushman is devoid of religion when he invests a stick or a stone with fetish virtues, in which the idea of a great personal God are entirely wanting, than we would have in saying that a primitive methodist is devoid of it because he rejects the altars, the ritual, the confessional, the archbishop, the transsubstantiation that his high church coreligionist believes in. The higher cogitic or moral and the spiritic or religious mind is one that is intensely proenrironing a best resultant line of action that shall link man in most satisfying relation to his family, his neighbor everywhere, the world with all its forces, and the universe with its forces. In biological evolution such has been man's pathway, and in pursuing it along many turnings and doublings, amid many "wills o" the wisps" and deceptions, he has reached, in the higher and purer Christian truths and axioms of conduct, a platform of noblest proenvironal aspiration that, if he has the courage and repressive power to follow it, will build up him and his fellows into "Society as an Organism."

In virtue of the above evidence we would reiterate that the "perfect" man is he who most perfectly builds up, balances, and utilizes the material bases, the protoplasmatin, the chromatin, the neuratin, and hypothetical spiritin, and the energizing biotic, cognitic, cogitic, and spiritic currents that traverse these, so as to reach most beneficial and satisfying relation to all the forces of the universe by which he is surrounded, from the family circle outward to the noblest possible proenvironal conception of a great World Power. 
That man is built up of several and successively more highly evolved states is by no means a new view. Thus, various rather vague or even fairly approximate conclusions in line with the above views have been enunciated for decades and even centuries, but no exact material histological basis has been worked out therewith. Thus Richard Hooker, writing nearly 500 years ago, speaks of "that law which God from the beginning hath set himself to do all things by" and which he considered consisted in "a triple perfection: first a sensual, consisting in those things which very life itself requireth, either as necessary supplements, or as beauties or ornaments thereof; then an intellectual, consisting in those things which none underneath man is either capable of or acquainted with; lastly a spiritual or divine, consisting in those things whereunto we tend by supernatural means here, but cannot here attain unto them."

Again Ribot, Romanes, and various others recognize an almost identical relation with Hooker. The latter in his "Thoughts on Religion" (pp. 152-153) says: "It is what we understand by man's moral, and still more his spiritual, qualities that go to constitute character. And it is astonishing how in all walks of life it is character that tells in the long run.

"It is a fact that these distinctions are all well marked and universally recognized, viz., Animality, Intellectuality, Morality, Spirituality. Morality and spirituality are to be distinguished as two very different things. A man may be highly moral in his conduct without being in any degrce spiritual in his nature, and, though to a lesser extent, vice versa."

The above is extremely apt, though the writer considers that Romanes has failed to give the high and distinct value to the sensuous or cognitic that it requires, and that we all know is one of the most powerful stimulators and directors of our being. Further, as already emphasized, we would regard the intellectual and the moral as a lower and higher expression of the cogitic sense, though the latter usually acts as the initiator of and introduction to the spiritic sense. But neither Romanes nor any others have shown the interrelation of phe- 
nomena with definite cells and cell substances, such as we have traced throughout the present volume, while most have more or less run together some two of the four that we distinctly recognize, so as to treat of three only. Though we can as yet identify no material constituent as a spiritic substance, the phenomena of the biotic, the cognitic, and the cogitic are so distinct from, even though continuous with, the spiritic that we confidently look for an identification of a material center or basis of it.

The search would probably prove most successful were the brains of the lion, the gorilla (an anthropoid that is largely lacking in the socio-moral qualities) and a rude savage to be minutely compared with that of a highly developed and "balanced Anglo-Saxon."

If then the cogitic and the spiritic represent the two higher life energies, in contrast to the two lower or the biotic and the cognitic, it is of some importance that in all educational books these should be highly emphasized and expanded. Now, as already pointed out, teaching can truly be said to consist of two quite distinct lines of effort, that can often however be made mutually helpful and intertwining with each other. These are instruction and education.

The former is-as it should be-largely cogitic, and consists in storing the mental mechanism or neuratin of the gray brain substance with accumulated and connected facts, that link together the inorganic and organic phenomena of the world. Instruction then is - as its very derivation indicatesa disposing or placing within the nerrous mechanism of a store of knowledge, that bears on and explains the environal phenomena that surround the individual.

But successful instruction largely rests, and must rest, on a continuous and skillful presentation of cognitic or sense impressions drawn from every source. It is the recognition of this fact-largely ignored till fifty to seventy-five years ago-that has caused the revolution in educational method whose fruits are now yielding richest reward to the human race. 
The latter or Education is, as its derivation indicates, the linking together, when occasion arises, of sereral stimuli into a compounded resultant that can be so set free or led out as a motor stimulus that it guides each individual how best to act under a given condition or relation.

The former supplies us with stores of information as to the character, aims, methods, and tendencies of our neighbors in the widest sense, as well as with countless data regarding the universe. The latter enables us to reach conclusions as to how best we should act in relation to our neighbor, and as to us and them in relation to the highest and best forces of the universe. Now it is an absolutely true and is also an appalling fact that, while the minds of our children and our own minds are filled and have been filled often to orerflowing with instructional facts as to the arithmetic, the geography, the history, the linguistics, the chemistry, the physics, and the biology of the world, they are left largely undereloped as to the daily and hourly relation of each human being to his or her neighbor. Individuals and society are being more and more constrained by laws, acts, regulations that are set down in the civil statute books, but the fundamental wellsprings of highest human thought and action are largely left uncultivated. But we need not wonder at this, so long as the law of competition holds a strong place.

This is in part to be explained when we remember that human history is too often studied, not as a living moving procession of individuals whose words and actions-whose whole careers in fact-should be replete with lessons for the future, but rather as a pictorial human canvas, painted to attract the eye and to tickle the lower cogitic nature, or to exercise the mind in linking dates by connecting these with passing events. Seldom indeed in any classroom are broad fundamental rules of moral and religious conduct deduced from the lives of the leading, not to say the minor, characters of history.

So instruction abundantly increases as science invades new fields of inquiry, but education lingers while conflicting moral- 
ists, sectarian leaders, religious propagandists set forth diverse doctrines.

We claim it that moral and religious impression and aspiration are as exact fields of scientific inquiry as are the origin of language, the beginnings of sexual organization in man and the lower animals, or as the perception and explanation of the movements amongst celestial bodies. They are equally or even more claimant of human attention if mankind is to continue along an upward evolutionary pathway. But a huge impediment along this pathway is the competitive system. Man largely clings, the world over, to individualistic even to analytic methods. He largely refuses to see that the teachings of Zarathushtra, of Plato, of Christ, of Paul, of Savonarola, of Wesley, of Owen, of Booth, of Bellamy, aye, not least of trades unionists, and of many socialists, are truly synthetic and inspiring.

And why are the latter fast reducing the mountain of competition to a mole hill that will ere long disappear? It is that these all have definitely established a proenvironment for the future that greatly transcends even the best estate to which man has yet attained. Further, while Plato, Saint-Simon, and Owen proenvironed almost wholly a high cogitic estate of social betterment, Christ, Paul, Booth, and Drummond have linked with the cogitic that highest energy of man's being, the spiritic, which stimulates him to yearn after, to aspire to, to live amid an earthly atmosphere that is charged with and in touch with, the unseen but ruling Power, ultimate energy, or Godhead of the universe.

For millennia before the students of Cogitic Science discovered and proved that the universe is a boundless arena for the display of one great omnipresent energy or Power that may at one time be termed heat, at another light, or again electricity, the investigators of spiritic human science, sweeping the intellect, the world, and the universe for a reason and explanation as to its being and relation, reached the conclusion that a great Power exists, which by cogitic and spiritic as founded on cognitic and biotic activities is ever advancing, 
improving, evolving, and raising man to increasing platforms of perfection.

By loftiest proenvironal effort man has attained to loftiest moral and spiritual results. In some cases the upward struggle has been for many individuals a life-long one, in which a too powerful biotic or cognitic or cogitic activity has retarded the highest spiritic. At times in strong wills, after years of stimulation by the biotic or cognitic, and to a greater or less extent by the cogitic, a stage was reached where a great proenvironal effort set before them a spiritic picture that caused a surcharging of the spiritic man at the expense of the lower energies. Such has been called conversion, that has been ridiculed by most scientists, or has been contemptuously rejected by so-called atheists. But, as Begbie has well put it, "the fact stands clear and unassailable that, by this thing called conversion, men consciously wrong, inferior, and unhappy become consciously right, superior, and happy" (229: 17).

It is equally one of the regrettable results of scientific inquiry in the past that aspiration and spiritual impulse have been more or less decried, even put out of court--while plainly confronting one as human phenomena that call for scientific explanation as much as do sexual impulse, movement toward the light, or primary cognitic actions of man. All call for and will receive, we are persuaded, an exact scientific explanation.

The writer proposes now to compare in the remaining pages of this chapter the relative values and exhibitions of the biotic, the cognitic, the cogitic, and the spiritic in definite human lives that he has come into close daily contact with for at least twelve years during some part of the past forty years of his life, from boyhood upward.

If a human being constantly proenvirons, desires, and plans conditions that look mainly toward gratification of the carnal or biotic nature, increasing flows of biotic energy will be directed toward the alimentary canal, the associated nerves, the sympathetic system, and the cerebro-spinal nerves associated with the digestive and excretory tracts. On the prin- 
ciple of energy and growth correlation, the streams of cognitic, cogitic, and spiritic energy will be reduced in quality, as the biotic flow becomes increased in strength, and as the glandular cells, blood ressels, and involuntary muscles become steadily multiplied to the point of hypertrophy. The main activity of the cognitic energy will be directed toward increasing the gustatory nerve terminations and the associated glands. Reflexly the sensual tendencies will be increased, for since sexual reproduction represents the highest and most intense response of the bio-cognitic system, and is stimulated in intensity by rich food and drink supplies, a strong-at times almost overmastering-sexual impulse will be started. This will become the more powerful if the cogitic and spiritic energies and associated nerve tracts be left comparatively uncultivated. A distention of the stomach and alimentary tract, a slow labored gait, a puffy dull aspect of the face, heavy eyes with drooping eyelids, and a slow breathing gradually are acquired. The concentration of the biotic and of the gustatory and sexual cognitic flows of energy cut off normal supplies to the cogitic and spiritic centers, which become irregular, uncorrelated, and impulsive in action.

After an often fairly prolonged period of such "animalistic" living, the human organism usually dies of apoplexy or some related disease, in which the hypertrophied biotic or carnal man overcomes and destroys by pure over blood supply-over carnality - the mental or cogitic and any trace that is still left of the spiritic.

Examples of such careers are known to every one. That of Henry VIII, though under fair auspices and often painted under apologetic and highly unscientific colors, became to an increasing degree with the years an exemplification of it. Peter the Great, with heroic human upbuild, fulfilled it to a large degree. For the butcheries seen in his youth, the carnal profligacy that he unfortunately was initiated into by Lefort, the jolly company that he organized, the despotic sway he was permitted to wield, the often gross and unbridled sensuality he indulged, as pictured by Carlyle and others, all 
rioted unchecked as a bio-cognitic "mélange" below powerful qualities of mind that moved impulsively and brutally, while the spiritic sense became, as in Napoleon's case, degraded into the notion of a star of destiny, that served to cloak from his mind his many loathsome carnal and sensuous acts.

But in every-day life even more perfect examples of the carnal and lower sensuous development can be witnessed, for with many men the absence of kingly or direct moral responsibility the more surely permits them to fall back on the carnal. The writer has watched the careers of several such for years to their life close. The selfish apathy, indolence, and carnal greed that in the latest stages overtake such form a truly sad picture, and in its eloquent silence demonstrate that man may degrade himself to the level of the lowest brutes that perish.

Not unfrequently however, though in some countries and centers more than in others, careers are lived in which the biotic and cognitic energies equally are permitted to hold most powerful sway, while the cogitic may be largely made subsidiary to both, and the spiritic nature may become practically atrophied. Outstanding examples can be seen in all the centers of social gaiety and "dissipation"; at Nice, Monaco, Ostend, and Brighton, in the old world; at Saratoga, Palm Beach, and Newport in the new. But in every considerable human aggregation they can not unfrequently be studied even in churches.

With less of the degraded carnality that characterizes the first group, there is combined a keen appreciation of sights, sounds, tastes, and other sensuous impressions that make life "a round of pleasures." Sensuous impressions derived from natural scenes of sky, sea, mountain, and valley are interposed with reading of novels that picture these, or that depict groupings of sensuous men and women, languid studies of music and pictures, or exciting adventures where the sensuous and lower cogitic wholly predominate over the higher cogitic and the spiritic. The cogitic energy even, in certain cases, is utilized to stimulate sensuously the afferent and efferent nerves by card-playing, betting, gambling, or by indoor and outdoor 
sports. The latter, however, often become the healthy natural corrective to the evil effects of the other stimuli, even though such corrective takes the form, with more earnest men, of normal work accomplished in steady fashion.

Dilettanti like these are a constant product of and form one of the greatest menaces to every advancing civilization. They become most obtrusive and dangerous wherever an excess of wealth tends to accumulate amongst individuals. They view with a sensuous, carnal, supercilious, and selfish eye the more earnest, endeavoring, and striving masses of humanity. From their ranks come the individuals who are "content" with the world, and who ridicule the noble souls whose minds and mental eyes can proenviron higher and truer aims for humanity. It has often been said that such "lead a butterfly existence," and there is much more of exact truth in the phrase than even appears on the surface.

But, since all sensuous and carnal pleasures in time dull the higher cogitic and the spiritic natures, the latter end of such individuals is that they tire of existence; ennui and a blasé indifference take possession of them; and their outlook on life is summed up in the sentence, "without God and without hope in the world."

The human career that combines the biotic, the cognitic, and the cogitic energies and their associated substances is one that abounds today in our midst. The "sharp business man," the "active professional gentleman," the "successful working man," usually show all three of these energies operating in a more or less combined proportion. When all three are fairly well balanced, but are uninfluenced by highest cogitic or by spiritic action, the individual becomes usually a "successful man of the world." By aid of the inhibitory nerves, and by judicious action of the motor nerves, the sensuous and the carnal stimuli and their reactions are held in check, the raried and complex mental activities that the daily life of higher cirilized men call forth excite the gray matter of the brain and the associated nerves, to those responses which ensure the successful accomplishment of cogitic or mental 
efforts, the organism becomes a keen, calculating, analytic, and synthetic machine moved by impulses of self-preservation, self-esteem, self-glorification, self-advancement. But even the higher cogitic centers may often be linked up into moral considerations and "feelings" or resultant responses, which produce in the organism love of family, regard and even love for social and civic organization, and improvement of the centers in which his life is passed. He may be a regular attendant even at church, for the sense of order, of sobriety, of decency, of harmonious moral relation between man and man prompts him to find in such service a glad satisfaction. But he has no inclination toward viewing "all men as brethren"; for easing the heavy burden of the oppressed; for uniting backward or unprivileged fellow-men, who show equal qualities with him, but have had less worldly opportunity, into a brotherhood of effort and improvement; for thinking of a great fatherhood of the universe and of the world toward whom he and all common children of the Father may turn; for trusting that gradually but surely the world will be so suffused with the fatherhood spirit that wars shall cease, and that human love and forbearance shall replace intense competition, keen dealings, and unscrupulous deceits.

So trades unions are regarded as suspicious institutions, dissenting religious bodies are only worthy to be frowned on, cooperative unions represent efforts "to upset the regular order of Society," while the often yawning gap between wealth and poverty is a sacred institution that "in the Providence of the Lord must always exist."

On either side of this exactly scientific and common life picture might be ranged others, who on the lower side give freer rein to the sensuous - and specially the sex-impulse, who indulge more freely and yet with guarded rarity in carnal excesses, who regard all religious or spiritic stimuli and responses as worthy only of "old women." On the higher side are others who, as in the parable of the rich young ruler, are "not far from the Kingdom of Heaven." But the pleasing mental response called forth, as sensuous impressions of lands, 
houses, vineyards, pictures, jewels, and rare books flit before the mind, serves gently but perfectly to put to rest all higher proenvironal aspirations of a spiritic kind.

But now we reach that highest platform, that noblest, most expanded, and ever stimulating proenvironal vista, which draws out all the other energies in best proportion and is itself stimulated to reach out, but in doing this perceives that greater perfection is still beyond, toward which new effort must be directed.

For the spiritic energy that has called forth in man the religious sense, and the highest effort to reach out to and apprehend a final Power, an infinite directive source of energy and progressive evolution, has least been appreciated in its scientific relation, but has most stimulated man to acts of sympathy, human love, forbearance, consecrated unity of noble action that has steadily conquered the degrading and inhuman in man, and is today advancing to final, even if slow, victory. Romanes in this connection strikes a splendid note-even though we believe that he started from a mistaken basiswhen he says: "Consider the happiness of religious-and chiefly of the highest religious, i. e., Christian-belief. It is a matter of fact that, besides being most intense, it is most enduring, growing, and never staled by custom. In short, according to the universal testimony of those who have it, it differs from all other happiness not only in degree but in kind. Those who have it can usually testify to what they used to be without it. It has no relation to intellectual status. It is a thing by itself and supreme." Here is an aspiring outlook toward "the perfect man," that starts a proenvironal longing for its early and wide adoption.

"The perfect man" has been the desire of the ages. Paul gives us in sane, exact scientific manner his definition of such. But, surrounded as Paul was in most of his assemblies and in his daily walks by groups and by individuals in every stage of evolution from rudest barbarism to Christian monotheism, he at times failed to see the beauty of his own descriptions. so he was attracted somewhat by the ascetics at one time, 
and thus influenced he wrote, "mortify therefore your members which are on the earth," and then proceeded to enumerate analytic and disintegrating actions which might appear. But in the recommendation, "mortify therefore your members," he at once saw one good result of ascetic effort, and forshadowed though he did not know of, the actions performed by the inhibitory nerves.

The perfect man then we believe is he who can use without abusing the biotic, the cognitic, the cogitic, and the spiritic flows of energy, and can keep these in balanced proportion, so that all may be adjusted to and helpful of each other. But the higher are to be preferred to the lower, and give more satisfying results, as Romanes has emphasized. When this has been said however, it should equally be accepted that just as we may have carnal or biotic sufficiency and balance, as well as carnal gluttony; as we may have cognitic balance as well as sensuous or sensual gluttony; as we may have cogitic balance as well as intellectual gluttony and pride; so we may have spiritic or religious balance as well as religious gluttony or fanaticism. The bigoted Moslem, the exclusive Churchman, the narrow Baptist in his worship of immersion, the revivalist Methodist, who in earnest love denominationally for others may show little of love - of charity - for those who differ from his opinions, the antiquated Friend who views every one "outside meeting" as a religious suspect, are indulging a temporary or permanent spiritic gluttony that upsets the balance of the "perfect man."

We believe then that individual, social, national, and international life will advance and evolve rapidly when every educational system will recognize, as a fundamental principle, the co-existence of four interrelated and ascending grades of matter-and energy-evolution. Each of these may vary in each individual according to heredity, environment, capacity to proenviron, and to opportunity for development. But the great end and aim of all education should be to subdue analytic or disintegrating tendencies, whether biotic, cognitic, cogitic, or spiritic in each individual by such precept and action as shall 
strengthen the appropriate inhibitory nervous impulses for such; and to lead out, strengthen, hereditarily impress, those synthetic or constructive tendencies by precept and action that shall strengthen the motor nerves to noblest impulses.

So, in the four-fold nature of man's constitution; in the balanced use of the afferent sensory, the inhibitory, and the motor nerves, as a result of a wise system of education; in the gradual elimination of lines of disintegrating energy-flow and the supplanting of these by lines of constructive and aspiring energy-flow along appropriate constitutive pathways; in the slow but sure evolution thereby of more elevated races of mankind; there is exact and surest hope for the rapid evolution of humanity, to a stage that even Bellamy in his most "inspired" moments did not dare to picture.

For the accomplishment of this result three fundamental recuirements are necessary. First, that each and all should be energized by a steady flow of spiritic love; second, that highest types of trained manhood and womanhood be selected to frame best rules of conduct; third, that equally wise teachers, who represent the best and most perfectly equipped minds in each community, be placed in charge of the young, who shall also have careful home training and parental influence.

The nation that first reaches to such a proenvironal degree of advance is the nation that soonest will lead the van of the world's progress. Switzerland, the United States, Holland, Britain, France, Sweden, Germany, and Japan all possess the beginning possibilities. Will any one of them win the prize of national evolution, or will it go to some other and possibly oriental proenvironer and competitor?

Finally if at the close of each human life it can truly be said that he or she had lived a satisfying and satisfied existence, with an unaccomplished remnant of aspiring ideal left for the future, then such a life has nobly fulfilled its life destiny. Then such senseless inquiries as: "Are you saved," "Have you found the Lord," "What is your hope of Heaven," will be entirely superfluous, for such a well-spent life is the best answer to all inquiries regarding the future. 


\section{CHAPTER XXX}

\section{PROBABLE FUTURE ADVANCES IN HUMAN EVOLUTION}

To forecast the future is ever a thankless task. But the physicists, the chemists, the astronomers, have reached a stage in the knowledge and perfectioning of their sciences that makes such possible. In botanical and zoological science also many predicable combinations and results can be anticipated.

By application of the laws of heredity, of environal action, of organized reaction or proenvironal response, of selective survival, and of the laws of reproduction we are more and more becoming able to read into the future. In truth the law of proenvironment, that has steadily guided all organisms along well regulated pathways, represents a constant seeking out by each organism or by each organ of it, toward a new and future environment. It may therefore be a not unprofitable inquiry if we attempt-however imperfectly-to follow in the train of Bellamy and other authors, not in the attitude however of "looking backward," but in that of proenvironing future pathways of evolutionary progress for superman, and even for many races of mankind. For we have already demonstrated that thus events, wholly in the future, and which were advances over the combined "best" of the past and present, have been worked up to and fulfilled.

We would consider that all evidences point to the conclusion that man and specially superman will increasingly break down national barriers and will establish an international comity and cooperation. The following causes amongst others will more and more conduce toward such a result.

(1) With each succeeding year increasing numbers of visitors from one country make summer journeys to another, and so are able to compare the industrial, the social, the moral, the 
spiritual life of each. But apart from the many advantages flowing from this, they are at stated places assailed by certain officers who often compel worrying examinations, cause needless delays, scatter at times the contents of trunks, suitcases, and handbags on the parement, tempt weak ones to prevarication, and are as clearest evidence has proven-themselves at times lured into dishonesty. Worst in this respect is that country of freedom, the United States, which often seems jealous lest any other country should benefit at its expense. The system supports a horde of office holders, who are paid by each nation for viewing its own citizens suspiciously. When sound cogitic and spiritic counsels prevail, such vexations will disappear, for the nation's individuals will be ashamed of them.

(2) International expositions-though at times attended with friction in the past - will become educative and stimulating centers for display of international art, treasures, manufactures, raw products, that will constantly advance and enlighten each nation that exploits such. But, further, one of the grandest benefits attending the Chicago Exposition of 1893 was the Congress of Religion, that brought together religionists from the ends of the earth in friendly discussion and comparison. Since that time bigotry has seldom reared its head, and only then to plead that possibly the human intellect was becoming too precipitate.

(3) Groups of capitalists, teachers, workers, and social organizers are ever more frequently making international visits to country after country, and on return present the good and bad points of each, so that new proenvironal lines of action are started that incorporate all or many of the best points seen in each nation's growth. Here, be it said, when large groups of educated thinkers who have been drawn from and are at one with the working classes, children of them in truth who glory in their birthright, shall have been trained as apt linguists in three or four of the leading tongues, and are delegated to attend international congresses, the world's advance and peace will be assured. The international trades-union 
and socialist congresses have already accomplished great thin $g$ along such lines.

(4) The recent initiation of the system of exchange professors between several of the international universities has been productive of highest good, while the various international scientific congresses have brought mental leaders together from the ends of the earth.

(5) An international peace temple and an international peace conciliation council have alike been established, and the world at this stage of its moral unfolding will not willingly allow either of these to die. The great mass of the people has fully and finally discovered that wars are seldom desired by them, and are used by their lordlings only to divert attention from internal needed improvements, or to furnish apology by these lordlings for their own aggrandisement. So determined expressions of opinion have gone forth from large groups of thoughtful men in Germany, France, Portugal, Britain, Spain, Italy, the United States, and other lands, that the age of exhausting and bloody wars has appropriately almost come to an end; that the future policy of nations must be a constructive rather than a destructive one; that by far the greater part of the world is still lying fallow and waiting for willing hands to exploit it; and that this can only be done when peaceful international progress is assured. So they are surely and steadily determining upon means that shall rid them of their old military and naval despotisms and nepotisms, and enable them to place in power men pledged to peace, progress, and true economy. So that noblest benediction of Christ to humanity, "peace on earth, good-will to men," that each Christmas tide brings round will in due time be realized.

Such a consummation will doubtless not be reached until heroic and continued efforts have been put forth to overthrow the old, and firmly establish the new. For, just as cooperative trades-unionism, labor representation in our halls of legislation, socialism, and democracy have been bitterly antagonized by reactionaries in each civilized country of the world, so doubtless will international peace and activity be ridiculed and fought against. 
(6) Again, an international language already exists in Esperanto, that has so demonstrated its value and easy acquisition that, even though it be replaced by another, the successor or some successor will prove an advance that in time will become an accepted medium of communication. But in the adoption of such a language antagonistic influences have to be expected and overcome. For powerful vested interests in commercial offices, in university centers, in governmental bureaus, in regal halls, have to be considered. It has already been demonstrated an easy task to govern the area of the United States covering about 3000 by 2500 miles and filled with a heterogeneous human aggregate of one hundred millions such as the world has never before had equaled. But it would be impolite to suggest that one emperor, king, or president could govern Europe that is about 1200 by 1200 miles in expanse. Only when the European peoples resolve, by a decided majority vote that is persistently upheld, on establishing a similar degree of supervision, will the feasibility of it be seriously considered.

(7) But, of all the influences - environal stimuli to use a more exact term-that have been at work during the past century for bringing about an international comity and peace, we would reckon, as by far the most powerful, the action of higher Christian missions as exemplified in the lives of the great majority of the missionaries; in the schools, colleges, and hospitals that they and their supporters have upheld; as well as in the more elevated-often immensely improved-lives that their converts have lived in midst of fearful odds against them. But to those who measure human existence from the lower cogitic and the cognitic-from the hard mental and the sensuous standpoints-Christian missions have been a hypocrisy or a failure. For those however who can disinterestedly watch the beat of humanity's pulse, and the swing of the human pendulum the world over, these have humanized, socialized, elevated, and enormously advanced every nation into whose midst they have come.

Naturally we would exempt here every "missionary" system that has attempted to use its religious message and appeal 
as a political and commercial system of intrigue and diplomacy. What would the shameful treatment of the Red Indian have been, had there been no Brainard to give it a ray of human sympathy? What would the flogged, shackled, and enslaved black man of Africa have done, had no Livingstone appeared to show the world that a human heart beats under each black skin? How would China have viewed "western civilization" had no Morrison or Williamson been near to light them along humanized and religious pathways?

Every country could yield its quota of noble self-sacrificing spirits, projected into it, who have stimulated, elevated, and advanced mankind, if such were willing to be advanced. The sad feature of it all has been that some of the worst, most hard-hearted, least human, and most brutal types of civilization have been projected into such lower and more primitive forms of nationality, and by their degraded cogitic and sensuous acts have blasted the lives of those amongst whom they come. Then they have faced round to announce to civilization that Christian missions were a failure for "the natives were fast dying out through the effects of the white man's vices." Another proof this that the world's true religious or spiritic progress has been most retarded by unworthy or hypocritical ones, who shelter, or rather sneak, under its advancing benefits.

Finally the constantly increasing demands that superman makes on the entire environment of the world for the satisfaction of his proenvironal aspirations are an assurance that only by continuous intercontinental comity can those needs be perfectly satisfied. So whether we stand in Amsterdam, Berlin, Canton, London, New York, or Paris we demand tea from China or India, coffee from Arabia or Brazil, rubber from Central America or the East Indies, ivory or vegetable ivory from Africa or Brazil, spices from Ceylon or Java, corks from France or Spain, leather from Russia or the Argentine, oranges from the West African Isles, Florida, or California, pineapples from Brazil or Malaya, oils and fats from China or North Africa, dates from Egypt and North Africa, tin from Cornwall or Bolivia. 
The list might be multiplied indefinitely, and for all of these commodities the consumers gladly welcome and agree to fair exchanges; the competitive exploiter alone upsets the pleasant relation that the producer and consumer of each desire. When a really healthy and dynamic spiritic energy is permitted to flow between the two latter, by elimination of the "break in the current" that the exploiter constitutes, nations will begin to realize how great are the future potentialities of this earth, that many proclaim to be nearing its exploitable end. Cogitic, cognitic, and biotic energy will not ensure this realization, the higher cogitic or moral and the spiritic alone can.

With such results as the above, that have already been in part secured, and will be enormously increased in the near future, other results will be arranged for, as necessary requirements for continued progress. Thus, recognizing the fundamental importance of the law of proenvironment, and the value of the proper balancement of the biotic, cognitic, cogitic, and spiritic planes of human life; by gradual adjustment of the sensory, the motor, and the inhibitory nerves to these, educated mankind will be guided by an aspiring public conscience. This will demand that all literature, educational methods, civic, social, and family life, shall be energized by a throbbing religious sentiment, that will almost unconsciously throw off and reject degraded words and actions that would lower the vitality of "the body social."

Already some nationalities have advanced along this way and certain of these even much more than others. When a scientific religion, a spiritic principle, that acts as exactly and surely as does gravity, sound, or light, is felt to inspire and even impel each human organism, noblest proenvironal action will follow.

An outcome of this will be that, instead of the artificial selection that wealth, influence, relationship, and other artificial circumstances foster in filling our high schools, colleges, and universities with young men and women devoid of highest mental and spiritual capacity and aim, only those will be chosen and advanced from grade to grade who come up to 
the highest standards of mental, moral, and not least spiritual equipment. From these again can be chosen the highest types of mind that can lead wisely and well in the two most important lines of human progress-education in its widest sense, and legislation.

But scientific research and discovery will be equally benefited. For the keenest minds that show alike analytic and synthetic capacity of a high grade will be advanced step by step into departments suited to their special lines of study and their mental bias.

Above all the highest and most consecrated religious spirits will be fostered, and will be at the same time unfettered by dogma, by sectarian rancor, by priestly pride and exclusiveness. So the simple clear and elevating doctrines of Christ and of Paul; the still older and suggestive views of Zarathushtra, Plato, and Aristotle; the as yet unexplained phenomena of mind contact at a distance; the still doubtful phenomena of spiritualism; the means by which spiritic energy can be increased, distributed, and made helpful for each individual; the action of this energy in binding together groups, communities, towns, and states so that these may unite in highest effort for the social well-being, will all be investigated and advanced in a careful and generous scientific spirit.

Again, since society will thus be built up as a social organism, on the principle that every cell or individual unit in an organism requires careful adjustment and representation in the whole, so the already fairly perfected Swiss system of the Initiative and the Referendum will be still further adjusted, so that each individual will feel that he or she directly aids in building up the social whole. Whatever defects occur in that whole will then be felt to be due to regrettable but cumulative individual action that the individuals must in turn review and change. A civic and state pride as well as an individual - would then be generated that would greatly excel any that the world has hitherto witnessed. For, while the law of proenviromment has silently worked during the past evolutionary century with greatly increased force, as compared 
with any previous century, the action will be greatly more accelerated when the united and balanced biotic, cognitic, cogitic, and spiritic energies of the majority of individuals are combined to perfect society as an organism.

Resulting from the above, and of highest importance for the safety of every social organization, will be a curbing and guiding of every one-man power and even of a few-men power. In the past such bloody and unscrupulous despots as Jenghiz, Caligula, Attila, Pope Innocent, the Charleses, Napoleon, and Abdul Hamid have been able to ride to power, to hold it, and to slaughter thousands or millions, in part through the absence of a true popular representation, and in part by bribing and playing one human tool against another, when these were equally unscrupulous characters with themselves, in part by utilizing the competitive system of which they were one shameful outcome.

But equally will the individual with natural and with cultivated talents be encouraged to proenviron, and to carry out, new and progressive enterprises that will benefit society as well as the individual. For a perfected Initiative and Referendum can equally promote individual, civic, and state prosperity as it can promote international welfare. Here conservative reactionaries with vested interests will doubtless endeavor to sidetrack and to ridicule the practicability of such schemes. But a noble enthusiasm, a steady determination of purpose, and repression of all unworthy means to any end that would lower the spiritic aspirations will steadily ensure great forward movements of humanity.

Emperors, kings, lords, and courtiers, who, as already emphasized, have been the producers, the fosterers, the perpetrators of most of the quarrels, wars, and bloodshed of the world, will be entirely unnecessary and superfluous functionaries. For the wholly erroneous and silly notion that they possess even ordinary wisdom, considering the advantages they have been born into, has been so often and so fully demonstrated to be false that humanity will firmly and gladly set them aside as has brave Switzerland for the greater part of a mil- 
lennium, as has the whole continent of America for decades or centuries, and as have France, Portugal, and China in more recent days.

But the tenacious and utterly unscrupulous efforts that the descendants of a Napoleon, a Carlos, and a Dowager Chinese queen have made, backed often also in secret by the regal partisans of other nationalities, to upset at any cost of blood and treasure the recognized voice of a large majority of the peoples of these nationalities, are proof that regal rule lives only by secret intrigue against the happiness and progress of a people.

Of all the changes proceeding in this day of small things that we now live amid, none possibly are fraught with more portentous omen than the intense popular clamor for reduction and ultimate breaking up of the bloated armies and navies of the "civilized" nations. These are bolstered up, subsidized, and clamored for by the regal contingents who require "billets" for their favorites and their dependents, but who are utterly deaf to the wail of needy and starving ones in their midst. For their trade of human butchering, and their dangling of the sword that is the butcher's ensign, alike conspire to deaden all human love and sympathy, while in critical moments of some popular appeal such only serve to inflame and to intensify human strife. The sane nations of the future will be those who shall unite in a firm pact to subdue and exterminate such wasteful, unholy, and unreligious representatives.

Evolving man, with each added century, millennium, and multimillennium of scientific wisdom, has slowly but surely risen to high and still higher heights of religious proenvironment. From primitive ancestral and animistic worship, to worship of the spirits of dead ancestors or of supposed flitting night spirits, from this to a worship of the heavenly bodies as associated with earthly spirits, then to preeminent recognition of the sun as a universal cosmic power, later to a linking on of a great spiritual fatherly Power as associated with but superior to such solar beauty, next to a close earthly con- 
nection, by grove and temple worship, of social humanity with this hearen power that the sun revealed the might and beneficence of, but whose majesty was equally revealed by the motions of the stars, and the fathomless immensity of space, onward to a pure and idealized God-Spirit of Zarathushtra, of Moses, of Plato, and of Aristotle, the passage was steady and upward. It ennobled man's intellect, it united for him expansive but finite earth to infinite heaven.

The advent of Christ proclaiming a fatherhood of the GodSpirit, and a unity of mankind as children of that ever-living and all-powerful Spirit, gave unbounded aspirations for human love and human socialization. It gave also an outlook of cogitic and spiritic expanse that was practically limitless. And so, round this man and his teachings in the future, the human race can everywhere gather, in halls and temples dedicated to earnest spiritic inquiry. There the elevation of the spiritic and the cogitic life will be fostered, guided, and advanced, so as to subdue and "keep under" the sensuous and the carnal, that after all are the necessary foundations on which the higher cogitic or mental and mento-moral as well as the spiritic or religious have been reared, till they have "reached to the heaven."

Every question will be discussed and viewed not in the light of dogmatics, of sectarianism, of religious bitterness, but as suffused with the light of Christian love, and with an exact scientific value that will guide surely, if it be at times falteringly, to higher goals.

Thus will evolve simply, naturally, and ever more clearly a real acceptable "scientific religion" such as Haeckel, Carus, Drummond, Chamberlain, Romanes, and many others have groped after, and at times seen, "as in a glass darkly." Thus "faith" will never be a final stereotyped dogmatic belief such as the Protestant, Catholic, Mohammedan, Jewish, and other theologians have tried to force on their respective sects, but will proceed "from strength to strength, from glory to glory." For, like every human source of knowledge and well-being, it will be a continuous evolution along an ascending line, if 
superman and superwoman will persistently aim to ensure such by proenvironal effort.

But advancing side by side with all of this, on the hackneyed but trite principle that "cleanliness is next to godliness," will be an ever advancing regard for household, civic, national, and international hygiene and health. Already the care taken in the schools, colleges, and universities of some lands, and specially of the United States, for the health and physical development of childhood and youth leaves little to be desired for the future, except that all will have to be transferred from the disintegrating field of competition, and placed in that of social cooperation.

Owing to local or international jealousies, a more extended system of hygiene has been at times crippled, but even already the united and usually successful efforts made to stamp out cholera, yellow fever, and other infectious diseases in man, or rabies, foot and mouth diseases, etc., amongst animals, is a foretaste of the universal success that will attend such efforts when nationalities are at one in aiding each other along a peaceful and truly religious pathway.

Thus the best, the most cleanly, and the most healthful populations will advance to front rank, alike through prolonged life of the individual, and improved health of the social fabric. Health and vigor of body and mind bring an optimistic feeling, an intense proenvironal striving toward higher planes of living; disease and impairment of the bodily faculties bring pessimism, gloomy fears, and a hopeless outlook for the future. So the health of the individual, and healthy employment of every faculty of the individual, ensure national vigor and proenvironal enthusiasm.

As in every advocated improvement or system of improvements, the conservative reactionary will declare that the above are vague, ideal, and unrealizable purposes. But the world has moved rapidly during the past twenty years to make many of these purposes partially realized results, and the remainder are events that are fast approaching to fulfillment. The original, striking, and attractive program of Bellamy in "Look- 
ing Backward" excited only the ridicule or irony of the above type of reactionary.

But, apart from the moral or higher cogitic and the religious or spiritic advances, that phase of future human progress that will result from the more keen realization of the general cogitic or mental activity deserves consideration. Nearly three years ago (1909) the writer wrote:

"It is recognized that the one organism man possesses, dominates, and will still more fully dominate the earth, so that Man's evolution is now the great and central problem of the system that Darwin, Spencer, Wallace, and Haeckel have established. His world-wide advance and occupancy may seem to be checked at one time by selective survival of the Russian thistle, at another by the insidious relation and action of the mosquito, in one place by the temporary fertility of the rabbit, in another by 'plague' of divers sorts. But the 19th century, the evolutionary century, has included the year of his 'coming of age.' Man now no longer sees with the eyes of the individual; he penetrates the past, the present, the future with the compound eyes of 'Society as an Organism.' Nationality counts now for little, and will count for less in the future. World problems are before us, for man's exploitation of the world is becoming increasingly easy.

"Whether, therefore, it be an international study of infection by mosquito or by tuberculosis germ; of selective breeding along exact lines, for production of the best races of plants or animals; of the acclimatization and adaptation of useful forms; of the reclamation and the enrichment of the earth; of the best devices for man's mental and ethical improvement; the bonds of municipality, of state, of nationality can no longer fetter or limit.

"Such questions do not concern only sociologists, economists, or moralists. 'They are Biological Questions. And every human being is a biologist. For, though each may not be trained in this or that laboratory so-called, each daily experiences and is affected by environmental agents, to which response is made. Each records also, if in the least degree thinking and reflect- 
ing, the cyclic changes noted in plants and animals around. Results therefore accumulate that each describes to the home circle, to friends, to the world, as impressively and effectively mayhap, as does a teacher in some great University. This it is which explains in large measure the remarkable success which the works of Darwin, Spencer, Wallace, Huxley, Haeckel, Drummond, and Kidd have achieved. This is the thinking, reflecting, acting age of mankind, and so when it was groping after such works, because it needed them, they were found and welcomed. The truths these works contain will gradually be gathered and conserved, as jewels of the 19th century. The slips, the mistakes, the rash statements, the false generalizations will be eliminated, but meanwhile mankind will march forward, as new seers arise to guide by added truths.

"May we not regard it then as the crowning legacy of 19th century advance that knowledge is now for all; that schools, colleges, and universities no longer exist to manufacture a select and privileged cult, but to people the world with the highest types of earnest thinking individuals; that as today is the best day in the world's history so future days will be on ever higher planes?"

We would attempt now to enlarge somewhat on the above and this along purely economic or utilitarian lines. We believe that we make a fair, reasonable, and practical claim when we assert that the world lies before advanced superman for future exact exploitation, subjection, and guidance along such highest proenvironal lines as he may resolve upon. A few examples of future possible and realizable advances may be given.

Till within the past seventy-five years man was practically ignorant of all fungoid - including bacterial-diseases, and accordingly was powerless to cope with them when they acted as injurious agents, or to aid and further develop them when beneficial. But the past half century has truly witnessed a revolution - a mutation - in his attitude to these. Agents injurious to human life, like the diphtheritic, the malarial, the tubercle, splenic fever, and related organisms, have had their 
life histories traced and their injurious action successfully combatted; while the fermentation, the nitrifying, and other beneficial organisms have been guided and multiplied in their action.

Again the higher or multicellular parasitic fungi that cause regetable diseases are being successfully combatted, and by further skillful treatment may be exterminated. So, if at one time, in the past of human history, the vine diseases, the potato, the tomato, the melon, the gooseberry, the cabbage, the pear, and other "blights" promised wholesale extermination for many of his most treasured plants, the future holds out brightest promise, if steady effort be exerted. This effort is also for man one of his best educational agents.

But in similar manner the varied and abundant animal organisms belonging to the groups of hæmoflagellates or blood parasites of man and lower animals, also the Sporozoa or body parasites, have had their life-histories unraveled to a remarkable degree.

In like fashion the parasitic worms of man and the domesticated animals have become understood and successfully antagonized? From the above standpoints, the past half century has truly been a mutational one of phenomenal portent for the future.

The enormous waste that has resulted in the past through the ravages of caterpillars and predatory insects in general, also through the attack of rats, mice, and other wasteful mammals, will gradually but surely be minimized, till a point is reached when they need no longer be dreaded. This result can and will only be secured through international effort, but the accruing benefit obtained will represent food sufficient for a population equal to that of China.

Again, if attention be concentrated on the question of drainage, irrigation, and water supply, results of the past half century have been such that the near future will open up sure and projected vistas of noblest expanse. The drainage of extensive river-side and shore areas has yielded millions of rich acres to man's hand. The bad lands of the West, also 
similar tracts in Africa and Asia, have been changed from deserts into smiling gardens, while the river dams of Assouan and other centers have changed uncertain fertility into assured crop return. What would the future not bring, along similar lines, were the world's soldiery and the military wealth that upholds its armaments to be directed into such channels?

Another phase of plant culture and of food supply has too little been considered. We refer to the weeds of cultivation. Where weeds and useful plants grow side by side, the former absorb what the latter could use. We emphatically assert that weeds can be and will be exterminated. By exercise of moral action and neighborly sympathy, the wild carrot, the dock, the ragweed, the "tares" of parable, can all be subdued and exterminated in favor of economic forms. Human labor can then be economized and concentrated on the desirable and desired plants. Two blades of grass will then truly grow where one grows now.

But in this connection intensive cultivation brings several rewards. It reduces-on the law of struggle for existencethe crop of weeds; it assures a succession and rotation of crops; it keeps the soil active and fermenting; it engages, exercises, and evolves the brain of man.

Once more want of knowledge, lack of moral and religious aim, relative absence of sympathetic and social spirit, cause poor and improvident methods of soil cultivation. So the farmer who indulges in loose living, who cultivates carelessly, who fails to secure best seed, who buys in a dear market, and who does not make sympathetic contact with his neighbors, becomes the unsuccessful one. When we learn that in one state of the Union an average return of sixty-seven bushels per acre of sweet potatoes is secured, as against one hundred and thirteen per acre in a state that is less favored in its soil and climate, or that fifty bushels of wheat per acre are yielded by land in Scotland, as against ten bushels in Russia, a subject for future consideration and improvement at once is before us.

One of the most sad, widespread, and wasteful conditions now confronting civilized man is the disposal of city, town, 
and even farm sewage. The examples of Berlin, Manchester, and other cities in solving this question open up future possibilities for improvement that can scarcely as yet be estimated, and that time only will reveal. Under the present wasteful, immoral, and irreligious competitive system the consideration or practical solution of such questions is side-tracked, while the beneficial results that would ensue are largely minimized for interested reasons. Socialized and cooperative superman, guided and energized by a quickened cogitic and spiritic energy, will apply himself with alacrity and success to such problems. Already the admirable work being done, against often adverse conditions, by agricultural colleges and institutes in quickening the proenvironal outlook, is best guarantee that a great awakening is near at hand.

When one considers and compares many of the above questions on their already realized side, and in view of the undoubted expansion they will undergo in the near future, the mind begins to estimate how enormously man has extended his dominion over the forces and the objects of nature. But more striking still are the possible combined activities that one individual may exercise within an hour or two, if a few wise arrangements be made for the exercise of these activities. Thus, seated in a room appropriately arranged, the modern superman of a city might talk with friends hundreds of miles apart; might next by electric connection start all of the machinery of a large manufacturing town; might next dislodge thousands of tons of rock by electric spark; then pass a cable message to the other side of the world; next dictate some letters that would in a few days start removal of thousands of tons of merchandise across thousands of miles of ocean; he might then take a trip of a few miles through midair and within half an hour return to speak to a friend for a few minutes in Atlantic mid-ocean, and conclude by reading the world's news of the previous day.

Such immediately realizable possibilities afford a glimpse into future developments of superman, especially under a synthetic social system. 
But the above questions cause us to turn now to a very different though much debated and far-reaching issue. For man's "future" has been widely and distantly proenvironed by theologians during the past 3000 years, and "the future state," "immortality," "an undying soul," as well as akin but differently phrased ideas, have been expressed. These views also have been put forth by many of the grandest souls this world has entertained.

It may at once be conceded that the doctrine of immortality has had a powerful hold on the civilized mind for the past three millennia and even longer. Renan voices the sentiments of millions when he says: "There is no lever capable of raising an entire people if once they have lost their faith in the immortality of the soul." If this opinion be correct neither a nation nor humanity as a whole can advance without belief in, and a molding of the life in view of, individual immortality. That this view is entirely incorrect is demonstrated by the steady or even marked advance made by nations at times when there is not a tittle of evidence that they entertained such views. Thus, no writer on Eschatology or on primitive views of immortality has ever claimed for the earliest elements of the Persian, the Jewish, the Greek, the Roman, and the Peruvian nations that they had even a faint conception of immortality, not to say an established faith in it. Yet no one will assert surely that in the virile growth and establishment of power by these nations, up to the time when we trace a feeble or evolving belief in immortality amongst them, that they did not rise, that they were not rapidly evolving.

Here therefore, as for every question connected with human development or the human mind, the evolutionary history must be traced, if we are to reach a correct standpoint. Furthermore, since immortality has bound up in it the thought of continuity of body or matter, and of spirit or energy together, or of at least one apart from the other, all related questions such as thought transfer, spiritualism, hypnotic suggestion and action, second sight, spirit rapping, and like phenomena deserve candid examination. 
First then we believe that all students of biological phenomena will concede direct life continuity for each human being of today, backward through many millions of years, to those primeval forms that started organic being during the archæan epoch. Furthermore, we have given reasons for believing that from the primeval forms up to man a slow but advancing integration and evolution has proceeded, in which increasing complexity of molecules and of cells has been correlated with increasing complexity of energizing phenomena. In other words the finite immortality-if we may coin such an expression-that each human being is the end-expression of has been bound up in an intimate association of matter and energy, both of which we have considered to be working steadily into more complex relations. On uniformitarian grounds, therefore, we might expect-almost predict with a large measure of certainty-that, so long as environal relations continue along their present course, human life will steadily advance to ever higher and more complex conditions.

But the question of immortality from its earliest suggestion in human history up till now has concerned and been centered round each human individual. When death of each body occurs, does this close existence for each, or is there some essence, some energy, or some indestructible principle that separates from the body and that lives on, possibly even in a higher and more ethereal state, owing to its very disengagement from the material body? Such has been and is the question that man has been striving to decide during the past 5000 years at least, from the time of the early dynastic Egyptians. Here we may note in passing the extremely crude riew, entertained by many during the past 2000 years at least, and which recent advances in chemical and physical science alone have set in the background, that the corporeal as well as the spiritual being will alike live again but in altered and immortalized conditions. When the above sciences were in a crude state, and "philosophy" was alone supposed to explain man's higher being, such crude views might linger on. Now it is felt and expressed that the field of discussion must rest on and occupy a much higher plane. 
The varied and often conflicting as well as vague views of primitive races, now extinct or still existing, regarding a future life might represent either a proenvironal picture and hope, or the crude beginning of a definite goal toward which man has been, is now, and will continue to be moving. We shall accordingly compare both possibilities, and the views that might be advanced in favor of or against them.

If immortality be a proenvironal picture, we would expect to find as in all proenvironal advances a fairly uniform set of desirable positions or conditions attained from stage to stage, and then that the best points of these would be selected and combined in advancing to the next higher stage. But the uncertainties, inconsistencies, and vagaries of the positions assumed and of the results secured seem completely to militate against this. Thus, even after long nillennia of advance by man from beliefs in the wind, the breath, dream visions, and crystal-gazing, as being expressive of powers of the being that were higher than and could be separated from the body, the wise Socrates confessed that he was in doubt whether death meant dissolution or separation of the body from an ethereal essence that lived on somewhere.

Aristotle's view that the intellect is the undying part of man was shared by many, but was reduced to a limited and conditional immortality by Chrysippus and others. The most sanguine eschatologists confess that little evidence is met with in the Old Testament which would favor immortality, and even that little is most shadowy.

But Israelitish contact with Egypt and later with Zarathushtrian and Mesopotamian influences during the exile developed a national though largely materialistic view of immortality, that was connected more with well-being and temporal national triumph than with post-mortem existence.

The belief in a heaven, a hell, and even a Sheol or purgatory by some and the rejection of one or all of these by other sects in a common nationality-as seen amid Pharisees and Sadducees of the Jewish nation-is proof that a common basis of thought and aspiration had not been secured. 
It is more and more conceded that Christ and Paul, while preaching a vivid Fatherhood and Godhead, as well as a general Brotherhood for man, refrained from expressing any exact riew as to the nature and relation of an immortal existence. But their followers by no means showed such caution or reserve, and so in the Christian church diversified doctrines arose on the subject, till all culminated in the trivial though typical scholastic questions of Thomas Aquinas. From that time to the present a return to a neutral position has been the tendency with most, and this attitude has been summed up by Orr when he writes: "We have not the elements of a complete solution, and we ought not to attempt it" (230:397).

But we may now consider whether the varied attitudes represent real mental and physical states that are working toward a definite goal of immortality, that man is reaching or will reach, even though he may not have been surely cognizant of its scope and trend. One feature in this connection seems fairly evident. It is that a future existence of the body after death becomes more and more an established belief side by side with religious progress. But, though religion has advanced to higher and higher platforms of thought and word and action, it is exactly correct to say that we have as little proof of a future or continued individual existence today as had mankind 5000 or more years ago, so far as exact evidence helps us. Nor, if the great majority of earnest Christian thinkers and believers be squarely and firmly asked as to their own assurance of immortality, or their sure grounds for acceptance of it as a physical or mento-religious reality, can any of them give proofs that it rests on an unassailable basis.

True, it forms one of the cardinal points in the history of ('hrist and Christian teaching, as given in three of the gospels, but Mark's gospel, alike the simplest and most ancient, is broken off just at the point where this is most necessary to the question at issue. Up to the present day there is no clear proof, as we see it, for verification of a possible immortality as understood in Christian teachings of many centuries. Ferguson borrows the question (231: 119) when he says: "The 
desire for immortality is an instinct, and can reasonably be regarded as an indication of that which the author of this desire wills to do." But an instinct is invariably the outcome of definite actions oft repeated in the history of an animal and which has become correspondingly easy of repetition (p. 560). But, as Ferguson puts it, what has really happened is that man has so long thought of and pictured out the idea of immortality that the act itself must be a reality, which is a begging of the whole position.

But, before accepting or rejecting such a possible state, we may try to learn whether a natural and connected explanation can be given of the belief in man, as originating from spiritic action in relation to proenvironal effort or stimulus.

The history of nations and of the individuals composing them, as well as the personal experience of each aspiring human being, give proof that the individual often strives to commemorate his or her personality to succeeding generations. Equally true is it, specially in the case of distinguished persons, who may have industriously labored to blot out or erase the commemorative records of their contemporaries or predecessors. Nowhere has this been more extensively shown than in Egypt, the land that fostered-if it did not actually give rise to-the first clear teachings on the doctrine of immortality. Thus the records of the splendid achievements and the ambitions of Queen Hatshepsut were so definitely obliterated by her early successors that we only know of these by collateral or neglected evidence. But long prior to her reign many of the Egyptian kings had claimed the homage due to gods, and later were viewed as immortal beings.

Further, many circumstances prove that primitive man early viewed death as a separation of the material or bodily part from the immaterial or mento-spiritual part. The latter also was regarded as a form of existence that lived on under different conditions or to practically indefinite extent. Thus originated the Brahminic "transmigration of spirits" idea, and the greatly more elaborate worship of the dead in their "houses of eternity" by the Egyptians. It is now universally con- 
ceded that the latter ceremonial was an established custom and belief with the earliest dynastic peoples, and so dates back from 6000 to 8000 years. But the dependence for wellbeing of the disembodied spirit on living descendants and its personal connection with the embalmed body remained almost continuously as an article of faith and practice. So, though Egypt erected the most stupendous monuments the world has ever seen to commemorate the spirits of the dead, the highest thoughts associated with such were a combination of personal giant-tombstone egotism, personal vanity, personal ignorance, and a desire to pry into the future; only to a small degree the more ennobled idea that "the spirit returns to the God who gave it."

In every respect the thought of Socrates that at death he would "go away to the happiness of the blessed" is greatly more elevated than anything Egyptian, and is quite equal to Old Testament post-exilic hopes. The views of Plato and Aristotle are equally elevated, and all stand in marked contrast to the Egyptian care of the dead body. This was almost certainly owing to the powerful influence on western thought of the Zarathushtrian faith which disregarded the dead body, but venerated good deeds and pious life.

In the religion of Christ and of Paul a strongly blended combination of the immortality views of the Egyptians, and the antithesis of good and evil in this world and the next, as taught by Zarathushtra is met with, and this was perpetuated with various embellishments down to the time of Aquinas. But the highly noteworthy feature is that no exact and tangible evolution has resulted either in our knowledge of immortality, or in the virtue of the belief in immortality to raise man to higher levels of living. For in actual human life and practice the thought of again meeting departed friends-and enemies alas also, for surely to be complete such logically must be the view-and of living on in some quite unknown state have only to a minor degree influenced human conduct. Had immortality been an undoubted goal for all, surely many would have changed their course of living here. 
All the evidence at our disposal then tends to show that immortality as a doctrine, a possibility, or a state has wholly originated as a proenvironal effort by man to think out and picture a future life that should be free from most or all of the sad, cruel, and fleeting events only too typical of many millions of human careers in the past. But, while religious evolution has progressed steadily and powerfully from strength to strength, from glory to glory, the evolution of immortality either as a doctrine or a realizable state has remained stationary or has run into grotesque vagaries. Man then seems to have aimed at a goal, but has failed to reach it. The goal so far as it has ever been described in the Iranian, the Greek, the Egyptian, the Hebrew, the Christian, or the post-Christian writings has been an as yet unattained state where elevated and regenerated humanity shall dwell together under the realized presence of God, the guide, the energizing one, and the father of the universe; where happy social companionships will make duty a pleasure; where the activities of each will be unhampered by envy, jealousy, and malice; where service will be regarded as noblest endeavor; and where the environal surrounding will be such that of them it could be said, "eye hath not seen, nor ear heard, neither hath entered into the heart of man to conceive."

In aiming at this what has man truly been aiming to accomplish? In reply it should be pointed out that, under the old competitive system which man has tried to laud and to adapt to his surroundings, his aspiration never could remain satisfied. If we try to build up the life-environment of a Lao-tsze, a Daniel, a Socrates, an Isaiah, a Paul, a Gerhard Groot, a Wesley, or even the environment of today compared with what thousands long for, it can truly be said that the hydra-headed evils of competition rise up and beat one down on every side. The names just given are prominent examples of many thousands - millions we might safely say-who during the past 3000 years or more have pictured, hoped for, prayed for, and at times almost lived into a state where such inequalities should cease, and where peace and righteousness should prevail. 
But, looking around on the course of life in Corinth, in Athens, in Ephesus, in Rome, how could Paul hope that such would come on earth? Looking out at the battles of popes, the lives of the clergy, the international struggles that kings inflamed their subjects to fight out, the licentious conduct of the nobles, the half-starved state of the people, how could Groot hope for "a kingdom of righteousness" on earth? So they carried their thoughts and aspirations beyond this life and this world, as their only possible field of hope, and the immortal life became their living and their dying trust.

Thoughtful men no longer view life in such a light, nor do they feel that an unknown future life should be bartered for a realizable life here of social cooperation, well-being, aspiration, and true happiness. This is within their reach, and they have resolved to attain to and live in it. For every one has felt at times periods of intense satisfaction, and has realized that life can be a truly joyous, inspiring, and sacred gift, in the unfolding of which every one can aid. Then, if immortality and immortal peace be a future gift of the great God of the universe, they will welcome it. But, if death ends conscious personality, they will look forward with satisfaction to "the restful rapture of the inviolate grave," for they will rest fully satisfied in the thought that they have helped forward the great evolutionary plan of the world, and so of the universe.

The most melancholy commentory on Christian love (not Christ's love for man which was ideal and perfect) is the scant exhibition of it in this twentieth century amongst those who fully or formally profess it; the saddest commentory on Christian faith (not Christ's faith which enabled him ever to live in the presence of God, the father of the universe) is its want of faith in believing that the man who passes us with little of this world's goods is our neighbor; the strongest argument against immortality is that we are so little prepared to make a heaven of the present world, in which all might be helped, blessed, and better prepared for a heaven beyond, should such exist. 
But is immortality unthinkable or impossible as a future heritage for mankind? In reply we might first quote the following by an eminent psychologist: "Science," he says, "opposes to any doctrine of individual immortality an unbroken and impregnable barrier." But, while, with our present knowledge, almost insuperable difficulties stand in the way of an acceptance of immortal life for the individual, the possibility is by no means unthinkable. We have tried to demonstrate that all terrestrial bodies exhibit an advancing molecular complexity from primitive ether atoms through hydrogen atoms and other atomic bodies to simple bivalent molecules, next to trivalent and quartivalent ones often associated with colloid substances. From these we have passed to the higher quartivalent and the pentavalent, so typical of biotic and cognitic life. Next, in hexavalent and heptavalent compounds we recognize the highly complex bodies associated with cogitic activity of higher animals, while these again suggest that even eight "elements" may enter into composition of the highest human activities. With these advancing molecular aggregations we have correlated thermic, lumic, chemic, electric, biotic, cognitic, cogitic, and spiritic energies, as ever more condensed conditions or states of motion, in relation to inert ether particles.

Now if the sum total of such "states of motion" exist in a definite ratio as the energy-characteristic of each human being, and are constantly being diminished by expenditure of energy, but as constantly rebuilt again to continue the individual, it follows that if at death the energy, and specially the cogitic-spiritic, can leave the then deenergizing body, and pass in the same related ratio into any other terrestrial or ultra-terrestrial body, such might continue to absorb fresh energy to perpetuate itself, from supplies of it in solar space, and thus live on at least as indefinitely as has living substance since its first evolution on the earth, many millions of years ago. But the whole speculation demands so much of what now appears unlikely, forced, and artificial that we can only put it aside by saying that individual immortality in a per- 
fected state conjures up pleasing desires, but fails to verify its own existence.

Therefore, though we put individual immortality aside as an unproved and apparently unrealizable proenvironal picture that has originated slowly in the human mind, Renan's idea that thereby "a terrific moral and spiritual decadence" would ensue seems to us the opposite of correct. Already during the past fifty years, as cooperative stores, factories, villages, recreation grounds, and educational as well as refining halls have increased throughout civilized lands, a change has taken place in every grade of society alike in physical, in mental, in moral, and largely in spiritual outlook, that would truly have been called a mutation, had not we lived in its midst, and seen its easy even though rapid advance. The world never before contained so many physically superior, so many mentally alert, so many morally elevated and aspiring, so many who linked their neighbor's happiness with their own, and all of this again with reverent love to and outlook on the great God, the energizing Spirit-Power and Governor of the universe.

So, when socialized cooperation shall have triumphed completely over disintegrating - even if keen-edged-competition, each individual will regard it as a sacred joy to have lived, and to have aided in the world's evolution to ever higher planes. Such individuals also will regard questions like immortality or future life as superfluous bribes, which in a past period were often cunningly thrown out by high and selfish ones to appease the longings of those less fortunate in this life than themselves. But, till direct proof can be adduced of an undying individual state, they will view such questions as beautiful thoughts that the mind can cherish and profit by, but which the body or the spirit can never attain to as realizations. 


\section{REFERENCES TO LITERATURE}

1. Soddy, F.

2. Le Bon, G.

3. Curie, P.

4. Mackower, W.

5. Thomson, J. J.

6. Lodge, O. J.

7. Spencer, H.

8. Arrhenius, S. A.

9. Arldt, $\mathrm{T}$.

10. Joly, T.

11. Sollas, W. T.

12. Geikie, A.

13. Macfarlane, J. M. Jour. Acad. Nat. Sc. Phila., 2d ser. 15 (1912).

14. Weed, W. H.

15. Davis, B. M.

16. Jones, $\mathrm{H}$.

17. Burton, E. F.

18. Ostwald, W.

19. Walker, J.

20. Fauvelle, Dr.

21. Bütschli, $\mathrm{O}$.

22. Mann, G.

23. Leduc, S.

24. Czapek, F.

25. Höber, R.

26. Duclaux, J.

27. Macallum, A. B

28. Waller, A. D.

29. Zopf, W.

30. Hansgirg, A.

31. Gomont, M.

32. West, G. S.

33. Tilden, J.

34. Kirchner, $\mathbf{O}$.

35. Phillips, O. P.

36. Kützing, F. Y.

37. Setchell, A. W.

38. Engelmann, T.W. Arch. f. d. ges. Physiol., 29 (1882).
Radioactivity, (1904).

Evolution of Forces (Inter. Sc. Ser.), (1909).

OEuvres, (1908).

Radioactive Substances, (1908).

Electricity and Matter, (1904).

Modern Views of Electricity, 3rd ed. (1907).

Principles of Biology, 6th ed. (1898).

Worlds in the Making, (1908).

Entwicklung der Kontinente, (1907).

Trans. Roy. Dublin Soc. s. 2, v. 7 (1899); also Geological Magazine, s. 4, v. 7 (1900).

Age of the Earth, (1905).

Textbook of Geology, 4th ed. (1903).

U. S. Geol. Surv. 9th Annual Report, (1888).

Science, 6 (1897).

Physical Chemistry, (1902).

Philos. Mag. ser. 6, 11 (1906).

Principles of Inorganic Chemistry, (1908).

Physical Chemistry, 4th ed. (1907).

La Physico-Chimie, (1908).

Protoplasm and Microscopic Foams (Eng. Tr.), (1894).

Physiological Histology, (1902).

The Mechanism of Life, (1911).

Biochemie der Pflanzen, (1905).

Physikalische Chemie der Zelle, (1911).

La Chimie de la Matiere vivante, (1910).

Trans. Canad. Inst., 8 (1909).

Lectures on Animal Electricity, (1897).

in Schenk's Handbuch, 4 (1890).

Physiol. u. Phyto. Untersuchungen, (1893).

Ann. Sc. Nat. ser. 7, 16 (1892).

Jour. of Bot., 40 (1902).

Minnesota Algae, (1910).

Pflanzenfamilien, 1 abt. 1. (1898).

Bot. Contrib. Univ. Penna., 2 (1904).

Species Algarum, (1849).

Science, n. s. 17 (1903). 
39. Fischer, A.

40. Fischer, E.

41. Davenport,

42. Jennings, H. S.

43. Hegler, R.

44. Olive, E. W.

45. Osborn, H. F.
Struct. and Functions of Bacteria, Eng. Tr. (1900).

Preparation of Organic Compounds, (1909).

Experimental Morphology, (1908).

Behavior of Lower Organisms, (1906).

Jahr. f. wiss. Botanik, 36 (1901).

Beih. Bot. Central, 18 (1905).

Jour. Acad. Nat. Sc. Phila., s. 2, 15 (1912).

46. Burdon-Sanderson, J.

Proc. Roy. Soc., 21 (1873); also Phil. Trans., (1888).

47. Kunkel, A.

Arb. d. bot. Inst. Würzburg, 2 (1879).

48. Waller, A. D.

Signs of Life, (1903).

49. Lauder-Lindsay, W.

Mind in Lower Animals, (1879).

50. Romanes, G. J.

51. Preyer, W.

52. Romanes, G. J.

53. Parmelee, M.

54. Ribot, $\mathrm{T}$.

55. Romanes, G. J.

56. Binet, A.

57. Mann, G.

58. Maupas, E.

59. Loeb, J.

60. Loeb, J.

Animal Intelligence, ed. 8 (1904).

Mental Development in the Child, Eng. Tr. (1894).

Mental Evolution in Man, (1888).

Science of Human Behavior, (1913).

Diseases of the Will, 3d Eng. ed. (1903).

Thoughts on Religion, (1895).

Psychic Life of Micro-organisms, Eng. Tr. (1889).

Chemistry of the Proteids, (1906).

Arch. d. Zool. exp. et gen. 2 ser., 6 (1888).

Dynamics of Living Matter, (1906).

Physiol. of Brain, (1905).

61. Macfarlane, J. M. Botan. Centralbl., 61 (1895).

62. Watson, C.

Bot. Contrib. Univ. Penna., 2 (1904).

63. Mactarlane, J. M. Trans. Roy. Soc. Edin., 37 (1892).

64. Macfarlane, J. M. Bot. Contrib. Univ. Penna., (1901).

65. Schively, A.

66. Wiesner, J.

Bot. Contrib. Univ. Penna., 1 (1897); 2 (1898).

67. Huxley, T. H.

Die Rohstoffe des Pflanzenreiches, (1900-03).

68. Bergmann, E.

Jour. Linn. Soc. Bot., 24 (1889).

69. Green, J. R.

Botan. Zeit., 40 (1882).

70. Harmer, S. F.-Shipley, A. E.

Cambridge Natural History, Vols. 1-10.

71. Macfarlane, J. M. "Charles Darwin": Three Appreciations, (1909).

72. Galton, F.

Natural Inheritance, (1889).

73. Cope, E. D.

74. Lothelier, A.

75. Bonnier, G.

76. Beebe, C. W.

Primary Factors of Organic Evolution, (1896).

Revue Gen. de Bot. 5 (1893).

Ann. d. Sc. Natur. s. 7, 20 (1895).

77. Ryder, J. A.

Zoologica, 1 (1907).

78. Macfarlane, J. M. Annals of Botany, 7 (1893).

79. Thomson, J. A.

80. Massart, J.

Heredity, (1908).

Bull. Acad. roy. Belgique, 22 (1891). 
81. Macfarlane, J. M. Woods Hole Lectures, (1893).

82. Hickson, S. J. Lankester's Zoology, 1, fasc. 1. (1903).

83. Uexküll, J. V. Zeitsch. f. Biol. 37 (1899).

84. Smallwood, W. M.-Rogers, C. G.

Jour. Comp. Neur. and Psych., 18 (1908).

85. McClure, C. F. W.

Zool. Jahrb. abt. Morph., 11 (1897).

86. Ranson. S. W. Jour. Comp. Neur. and Psych., 18 (1908), 19 (1909).

S7. Uexküll, J. V. Leitfaden im Stud. d. Wasserthiere, (1905).

88. Allen, J. Jour. Comp. Neur. and Psych., 14 (1904).

89. Spauldıng, E. G. Jour. Comp. Neur. and Psych., 14 (1904).

90. Yerkes, R. M. The Dancing Mouse, (1908).

91. Edinger, L.

Vorlesungen ueber den Bau der nerv. Centralorgane,

Ed. 7, (1908).

92. Herrick, C. L. Jour. Comp. Neur. and Psych., 8 (1898).

93. Bütschli, O. Infusoria, in Bronn's Tier Reichs (1889).

94. Uexküll, J. V. Zeit. f. Biol. 39 (1900).

95. Montgomery, T. H.

Zool. Jahr. anat. 10 (1897).

96. Brauer, A.

Zool. Jahr. anat. 12 (1899).

97. Hartog, M.

Cambr. Nat. Hist. 1 (1906).

98. Huber, J.

Ann. d. Sc. Nat. S. 7, Bot. 16 (1892); Bull. Soc. Bot.

France, 41 (1894).

99. Oltmanns, F. Morph. u. Biol. d. Algen., (1904).

100. Wille, N. Natürlichen Pflanzenfam., 1 part 2. (1890).

101. Macfarlane, J. M. Proceed. Brit. Assoc. Aberdeen, (1886).

102. Mitzkewitsch, L. Flora, 85 (1898).

103. Macfarlane, J. M. Trans. Bot. Soc. Edin., 14 (1883).

104. Fol, H.

Jenaische Zeitschr. 7 (1873).

105. Calkins, G. N. The Protozoa, (1901).

106. Sanson, A. L'Heredite Normale et Pathologique; (1893).

107. Lillie, F. R. Science, 38 (1913).

108. Woodward, H. M. Lankester's Zoology, 1 (1909).

109. Minchin, E. A. Lankester's Zoology, 1 p. 2 (1903).

110. Klebs, G. Jahrb. f. wiss Botanik, 33 (1899).

111. Ambroz, Adolf Centralbl. f. Bakt. Refer. vol. 48 (1911).

112. Engelmann, T. W. Bot. Zeitung, 41 (1883).

113. Gaidukou, N. Ber. d. Deutsch. Bot. Gesell., 21 (1903).

114. Kjellman, F. R.-Svedelius, N.

Pflanzenfamilien, Nachtr. 1 pt. $\mathcal{2}(1910)$.

115. Globig, Dr.

Zeit. f. Hygien., 3 (1887).

116. Miehe, Hugo

Selbsterhitzung des Heues, (1907).

117. Kruyff, E. de

Cent. f. Bakt. S. 2, 26 (1910).

118. Rabinowitsch, L. Zeit. f. Hygien, 20 (1895).

119. Lotsy, J. P. Botanische Stammesgeschichte, (1907). 
120. Wettstein, R.

121. Ford, S. O.

122. Wieland, G. R.

123. Porsch, $\mathrm{O}$.

124. Engler, A.

125. Henslow, G.

126. Henslow, G.

127. Sargent, E.

128. Sollas, W. J.

129. Boulenger, G.A.

130. Hartog, M.

131. V. Graff, L.

132. Bürger, $\mathrm{O}$.

133. Cooke, A. H.
Systematischen Botanik, (1901).

Annals of Botany, 16 (1902).

American Fossil Cycads, (1906).

Oesterr. Bot. Zeitg., 54 (1904).

Natürlichen Pflanzenfamilien, III (1894).

Jour. Linn. Soc. 29, (1892).

Annals of Botany, 25 (1911).

Annals of Botany, 22 (1908).

Trans. Roy. Dublin Soc., S. 2, 3 (1884).

Presid. Address Brit. Assoc. (1905).

Cambridge Nat. Hist., 2 (1910).

Monographie d. Turbellarien, in Bronn's Tierreichs 4, suppl. 1c. (1908).

Bronn's Tierreichs 4, suppl. (1907).

Cambridge Nat. Hist. 3, Molluses, (1895).

134. Hubrecht, A. A. W.

Quart. Journ. Micro. Sc. N. S. 23 (1883).

135. Hubrecht, A. A. W.

Zool. Anzeiger, 8 (1885).

136. Hubrecht, A. A. W.

Challenger Reports, 19 (1887).

137. Hertwig, O. Textbook of Embryology, Eng. ed. (1892).

138. Wiedersheim, R. Comp. Anat. of Vertebrates, 2d Eng. ed. (1897).

139. Bridge, T. W. Camb. Nat. Hist., 7 (1910).

140. Zelinka, C. Zeit. f. Wiss. Zool., 53 (1891).

141. Brookover, C. Jour. Comp. Neur. and Psych., 20 (1910).

142. Sargent, P. E. Bull. Mus. Comp. Zool., 45 (1904).

149. Herrick, C. J. Jour. Comp. Neur. and Psych., 18 (1908).

144. Johnston, J. B. Nervous System of Vertebrates, (1906).

145. Hubrecht, A. A. W.

Verhand. v. d. Konin. Akad. t. Amsterdam, (1880).

146. Goodrich, E. S. Lankester's 'Zoology, 9 (1909).

147. Lönnberg, E. Bronn's Tierreichs, 6 (1901).

148. Retzius, G. Biolog. Untersuch., 12 (1905).

149. Shipley, A. E. Encyclop. Brit., ed. 11, 19 (1911).

150. Case, E. C. Publications, Carnegie Inst., 146 (1911).

151. Brauer, A. Zool. Jahr. Anat. 10 (1897).

152. Cope, E. D. Batrachia of N. America, (1889).

153. Bürger, O. Bronn's Tierreichs., 12 (1899).

154. Brauer, A. Zool. Jahr. Anat., 12 (1899).

155. Gadow, H. Camb. Nat. Hist., 8 (1909).

156. Maurer, F. Morph. Jahr., 18 (1892).

157. Schultze, F. E. Bronn's Tierreichs., 62 abt.

158. Meijerle, J. C. H. de

Morph. Jahrb., 21 (1894). 
159. Lull, R. S. American Naturalist, 38 (1904).

160. Huxley, T. H. Proceedings Roy. Soc., 28 (1879).

161. Matthew, W. D. American Naturalist, 38 (1904).

162. Herrick, C. J. Jour. Comp. Neur. and Psych., 20 (1910).

163. Johnston, J. B. Jour. Comp. Neur. and Psych., 19 (1909).

164. Flower, W. H., and Lydekker, R.

Introd. to the Mammals, (1891).

165. Hoffman, C. K. Bronn's Tierreichs 6, (1878).

166. Beddard, F. E. Camb. Nat. Hist. 10 (1909).

167. Spencer, T. Jour. Quekett Micro. Club 4 (1890).

168. Hlava, S. Zoolog. Anzeig. 28 (1904).

169. Gosse, P. H., and Hudson, L.

The Rotifera, (1889).

170. Shipley, A. E. Camb. Nat. Hist., 3 (1895).

171. Korschelt, E.-Heider, K.

Textbook of Embryology of Invertebrata, 1. (1895).

172. Korschelt, E--Heider, K.

Textbook of Embryology of Invertebrata, 2. (1899).

173. Korschelt, E.-Heider, K.

Textbook of Embryology of Invertebrata, 4. (1900).

174. Fischer, $\mathrm{P}$.

Manuel de Conchyliologie, (1887).

175. Zelinka, C.

Zeit. f. Wiss. Zool., 47 (1888).

176. Gaskell, W. H

Origin of Vertebrates, (1908).

177. Lubbock, J.

Proc. Entom. Soc. London, (1866).

178. Patten, W.

Evolution of the Vertebrates, (1912).

179. Shipley, A. E.

Camb. Nat. Hist. 4 (1909).

180. Forel, A.

Nouv. Mem. Soc. Hev. Sc. Nat. 26 (1874).

181. Sharp, D.

Insecta in Camb. Nat. Hist., 5 (1910).

182. Houzeau, J. C.

Faculte Ment. des Animaux., 2 (1872).

183. Holmes, S. J.

Evolution of Animal Intelligence, (1911).

184. Hobhouse, L. T. Mind in Evolution, (1901).

185. Romanes, G. J. Mental Evolution in Man, (1889).

186. Müller, F. Max Science of Language, s. 2 (1884).

187. Waitz, T.

188. Müller, F. Max

189. Ward, James

190. Piette, E.

191. Noiré, L.

Anthropologie der Naturvölker, 1 (1859).

Lectures on the Science of Language, (1861).

Encycl. Brit. Ed. 11, 22 (1911).

L'Anthropologie, 7 (1896), 16 (1905).

Origin of Speech, (1877).

192. Geiger, L.

Ursprung der Sprache, (1869).

193. Sayce, A. H.

194. Darwin, C.

Introd. to Science of Language, ed. 4 (1900).

195. Hume, D.

Descent of Man, (1871).

Philosophical Works, (1854).

196. Washburn, M. F. The Animal Mind, (1909).

197. Landois, L.

Human Physiology, Amer. ed. (1904).

198. Maudsley, Henry Pathology of Mind, (1879). 
199. Durham, Dr. Guy's Hospital Reports, (1860).

200. Wilson, E. B. American Naturalist, 25 (1891).

201. Tennent, E. Nat. Hist. of Ceylon, ed. 4 (1860).

202. Sutherland, A. Origin and Growth of the Moral Instinct, (1898).

203. Westermarck, E. A.

Origin and Development of Moral Ideas, (1906).

204. Frazer, J. G. Golden Bough, ed. 2 (1900).

205. Burlingame, E. L. ed.

Questions of Belief, (1878).

206. Price, Richard Rev. of principal questions in Morals, (1897).

207. Featherman, A. Social History of Races of Mankind, (1885).

208. Uhlhorn, G. Conflict of Christianity with Heathenism, Amer. ed, (1894).

209. Draper, J. W. History of Conflict between Religion and Science, (1874).

210. White, A. D. History of the Warfare of Science with Theology, (1897).

211. James, W.

212. Müller, F. Max

213. Caird, John

214. Pfleiderer, $\mathrm{O}$.

215. Lang, Andrew

216. Caird, Edward

217. Kidd, B.

218. Chamberlain, H. S.

Varieties of Religious Experience, (1902).

Natural Religion, (1889).

Philosophy of Religion, (1880).

Philosophy and Development of Religion, Eng. ed. (1886).

The Making of Religion, 2nd ed. (1900).

Evolution of Religion, ed. 3 (1899).

Social Evolution, new ed. (1894).

Foundations of Nineteenth Century, Eng. ed. (1913).

219. Lang, Andrew

220. Floody, R. J.

221. Geiger, Wilhelm

222. Mills, L. H.

Myth, Ritual, and Religion, new ed. (1899).

Scientific basis of Sabbath and Sunday, (1906).

Civilization of the Eastern Iranians, (1885).

Zarathushtra, Philo, etc., (1906).

223. Channing, W. E. The Perfect Life, new ed. (1899).

224. Emerson, R. W. Representative Men, new ed. (1908).

225. Wheeler, W. M. Ants, (1910).

226. Tylor, E. B.

Anthropology, (1881).

227. Spencer, H.

228. Kidd, B.

229. Begbie, $H$.

230. Orr, J.

231. Ferguson, A.

232. Amiel, H. F.

Westminster Review, (1860).

Encycl. Brit., 25 (1911).

Twice-born Men, (1909).

Christian View of God and the W ord, (1893).

Institutes of Moral Philosophy (1785).

Amiel's Journal, and Eng. ed. (1900).

233. Burton, R. F.

234. Cole, L. W.

235. Davis, H. B.

First Footsteps in East Africa, (1856).

Jour. Comp. Neur. and Psych. 17 (1907).

Amer. Jour. Psych. 18 (1907). 


\section{INDEX}

Abies 362

Abraham 725

Acantharia 272

Acanthocephala 390

Acanthocystis 382

Acaryophyta 38

Acaryota 51, 61, 62, 78, 80, 82, 84,

$94,98,107,108,175,222,377$

Acaryozoa 51, 222, 298

Acoela 388

Actinophrys 276

Acton 83

Agathis 361

Ahriman 726, 727

Ainu 625

Aistopodes 455, 488

Akinetes 249, 267, 284

Alchemilla 292

Alcock 459

Alcyonidium 391

Alexander 678

Algae, thermophilic 53

Alkaloids 65, 161

Allantois 484, 490, 492

Allen 238

Allotropa 202

Alternation of Generations 336

Aluminium 6

Amaryllidaceae 371

Amblystoma 184, 459, 463, 470, 493

Amblystomidae 458, 462, 472

Ambroz 304, 326

Amenophis IV 725, 765

Amides 155

Amiel 799

Amnion 484, 492

Amoeba 121

Amphibia 405, 418, 455, 479

Amphicarpaea 138
Amphidinium 310, 323

Amphimixis 289

Amphineura 394, 396

Amphioxus 401, 411, 447, 448, 453. 466, 502, 503

Amphiporus 424

Amphiuma 462, 464, 466, 470

Amphiumidae 458, 462, 471

Amygdalin 157, 160

Anabaena 54

Ancestralism 704, 705, 708, 798

Andreaeaceae 343

Aneimiteae 354

Anemone 87, 115

Animalistic worship 707

Animals, statistics of 409

Animism 709, 711, 715, 728

Anisoamphimixis 289

Annelida 511, 512, 514

Anonaceae 371

Ant 546, 553, 554, 618, 768, 769, 771,773

Antennaria 292

Anthoceroteae 337, 341

Anthozoa 222

Anthropomorpha 300, 557

Anthropomorphic spirits 710

Anuraea 126

Apes, anthropoid 565, 587, 588, 659, 702

Aphanocapsa 50, 99, 245

Aphanochaetaceae 323

Aphanochaete 314, 344, 349

Aphanothece 50, 105

Aplanospore 249, 251, 267, 284

Aplectrum 202

Apoda 450, 455, 460, 464, 471, 473, 488, 492

Apopetalae 149 
Aptian beds 368

Aquinas, Thomas 844,846

Arachidic acid 155

Araucaria 361

Araucarioxylon 361

Arcella 272, 382

Archaean rocks 6, 12, 27

Archaeocalamiteae 351

Archaeocordaiteae 352

Archaeocycadeae 352

Archaeofilicineae 352

Archiannelida 390

Argon 4

Aristotle 737, 783

Arldt 7, 11, 13, 21, 22, 25

Arrhenius 6, 10

Arsenic, colloid 16

Artemia salina 184

Arthropoda 396

Articular condyles 460, 476, 477, 478

Aryans 714, 715

Ashtoreth 733, 791

Aspartic acid 156

Aspiration 792

Assur 720

Assyrians 707

Asterocystis 319

Astrorhizidea 379

Auditory nerve 430, 434

Auditory organ $431,442,447$

Auditory sense 610

Aurantiaceae 162

Aurignac man 624

Australians 702, 704, 709, 778

Automobile, evolution of 633

Axolotl 184

Azolla 54

Babylonians 714, 715

Bacillus 97, 326

Bacteriaceae 304

Bacteria, iron 42, 56

Bacteria, nitrogen 41, 42

Bacteria, purple 42, 56

Bacteria, sulphur 40, 56
Bacteria, thermophilic 57

Bacterio-purpurin 330

Badhamia 136

Baiera 557, 360

Bain 655

Balanoglossus 401, 502, 503

Balfour, F. M., 422, 485

Baltzer, 290

Bangia 318, 331

Bangiaceae 318, 319

Bangiopsis 318

Barosma 161

Bartonia 202

Bartram, John 183

Bary, de 330

Batrachia 405, 419, 476, 485

Batrachospermum 317, 331

Bdellostoma 448, 456, 457, 466

Beaver 559

Beebe 184

Beech drops 199

Beecher 392

Beecher, H. W. 667

Beecher Stowe 667

Bees 771, 773

Begbie 817

Beggiatoa 38, 40, 50, 304, 326

Beggiatoa alba 78

Beijerinck 40

Bell, C. 574

Bellamy 648, 824, 835

Beneden, Van 290, 388

Bennetiteae 354

Bentham, Jeremy 653, 666

Benzoic aldehyde 15, 66

Berberidaceae 371

Bergmann 155

Bernard, Claude 114

Berneck von, on platinum 18

Berthold 78

Bertholet's law 68

Berzelius 68, 69

Berzelius' Chemical Law 4

Biciliatae 334, 348

Bicycle, evolution of 632 
Bimanes 596

Binet 98

Binz 113

Biochemistry 628

Biophytum 220

Biotic act 600

Biotic energy 27, 33, 79, 85, 97, $604,605,800$

Bismarck 806, 807

Bismuth 6

Bismuth, colloid 15

Blepharoplast 152

Blue-green algae 42,52

Böhmig 444

Bon, Le 2, 7, 24, 28, 29

Bonnier 184

Borzi 128

Bostrychia 318, 319

Botrydium 309, 315

Botryopterideae 354

Boveri 133, 139

Bowerbankia 391

Brachiopoda 302, 392, 517, 518, 521

Branchiopoda 397

Brandt 553, 555

Brauer 461, 462, 466

Bredig, on platinum 18

Brewer 53

Brine-shrimp 184

Bromine 5

Bronn, Tierreich 397

Brookover 429

.Broomrape 196

Brown, Horace 101

Bryaceae 343

Bryophyta 49, 51, 258, 287, 313, 338

Buddha 646

Buddhism 735

Budgett 114

Bürger 421, 423, 428, 431, 432, 436, $437,439,442,444,452$

Burmanniaceae 202

Bursaria 281

Burton 19,23

Burton, R. 654
Bushmen 702, 704, 778

Bütschli 28, 78, 128, 134, 378, 380

Butyric acid formula 155

\section{Cacti 110}

Caeciliada 418, 441, 446, 454

Caffein 37, 61

Caird, E. 685

Caird, J. 680

Cajal 235

Calamiteae 351

Calamites 341

Calamostachys 351

Calcium in living tissue 6

Calkins 264, 280, 378

Callidina 228, 230, 427

Calothrix 54, 329

Cane Sugar 103

Canscora 202

Capric acid formula 155

Caprylic acid formula 155

Capulus 303

Carbohydrates 65

Carbon, allomorphic 5

Carbonates in earth's crust 7

Carbon compounds 5

Carlsbad Springs 35

Carnivora 499, 557, 558, 563, 621

Carolina poplar 111, 244

Carotin 77

Carrel 113

Carriere 235

Caryophyta 51

Caryota 51, 52, 82, 84, 87, 94, 98, 222, 376

Caryozoa 51, 222, 378

Case 459

Cassia 220

Casuarinales 366

Casuarineae 358

Casuarina 363, 364

Catalpa bignonioides 244

Caulerpaceae 315

Cebus 566

Cellulose 30, 153 
Centropyxis 272, 275, 382

Centrosomes 262

Cephaleuros 331

Cephalocbordata 401, 411, 502

Cephalopoda 302, 394, 520, 545, 549,601

Cephalotaxus 360

Ceratopteris $349,350,352,353$

Cerebellum 435

Cerebral organs 429, 447

Cerebratulus 424, 429, 431

Cestodes 388

Cetacea 498

Chaetophoraceae 306, 313, 314, 331, $349,350,353,365$

Chamaecyparis 362

Chamaesiphon 316,318

Chamaesiphonaceae 247, 249, 251, 253, 313

Chamberlain 736, 739

Channing 667, 742, 805

Chantransia 318, 331

Chara 258, 261, 292

Characeae 49, 315

Chartists 639

Chemoenergids 120, 129

Chemotactic Sense 118

Chenopodiaceae 358

Chenopodiales 373

Chert 27

Chilomonas 125

Chimaphila 202

Chimpanzee $546,567,581,586$

Chinese 711

Chlamydomonas 164, 168, 269, 310

Chlorochytrium 254, 309

Chlorophyll 31, 32, 42, 152

Choanophoraceae 253

Chodat 57, 253

Chondrostei 403

Chorda dorsalis 422

Chordariaceae 317

Chordata 406

Christ 646, 647, 662, 670, 677, 691, $692,694,695,696,697,698,734$,
$736,738,740,743,747,781,782$, $816,831,834,846$

Christianity 734, 736, 744, 760, 792

Christian Missions 828, 829

Christian Socialists 787

Chromatin 52, 83, 108, 259, 260, 270,293

Chromatin skeins 130

Chromatium 40

Chromatophore 309

Chromidia 130

Chromosomes 262, 334

Chroococcus 30, 105, 306

Chroolepidaceae 331

Chytridineae 284

Cilia 151

Cirripedia 397

Cladophoraceae 312

Clarke, F. W. 9

Clotting ferments 158

Coccidia 58

Codiaceae 315

Coelenterata 87, 298, 385

Coenolestes 495

Cogitic Act 600, 667, 672

Cogitic energy $82,87,88,90,222$, $417,603,702$

Cogitochemistry 628

Cognitic Act 600, 672, 690

Cognitic energy 82, 87, 88, 90, 263, 603,702

Cognitochemistry 628

Cohn 35, 53

Cohnheim 156

Cole 618, 623

Coleochaete 258, 264

Coleochaete divergens 342

Coleochaete pulvinata 342

Coleochaete scutata 342,345

Colcochaete soluta 342

Colloid bodies $9,15,45,50$

Colosseum 635

Colpidium 125

Comparison, animals-plants 375 
Competition, law of $647,697,754$, $755,764,765,776$

Concepts 621

Conferraceae 634

Conferva 309, 313

Confucius 662, 806

Coniferin 161

Conjugatae 255, 256, 265, 266

Conscience, definition of 673,749

Consciousness 599, 600

Constantine 692, 784, 786

Cooke 392, 407

Cooperation 642, 650, 676, 683, 692, $697,768,769$

Cooperative evolution 751,776

Cope, E. D. 178, 180, 188, 463, 467, $482,489,500,502,657$

Copepoda 397

Corallorhiza 202

Cordaianthus 357, 361

Cordaitales 368

Cordaites 360

Cordylophora 385

Correns 252, 295

Cox 236

Crania 303

Creatin 116

Crenothrix 50

Creodonta 498

Cristatella 391

Crossopterygii 404

Crystal Palace 794

Ctenocladus 315

Ctenophora 386

Ctenostomata 391

Cunningham, D. J. 574

Cunninghamia 361

Cunningham, J. T. 520

Cupressineae 357

Cupressus 362

Curies 2

Cuscuta 186

Cutin 152, 169

Cutleriaceae 317

Cyanogen 116
Cyanophyceae 42, 51, 303, 304, 306, 313

Cycadaceae 54, 286, 347, 354

Cycadofilices 334, 347

Cycas revoluta 85

Cyclops 291

Cyclostomata 401, 412, 418, 441, $443,446,459,460,469$

Cylindrospermum 250, 307

Cyperaceae 371

Cypris 398

Czapek 37, 123, 149, 154, 160, 161, 163

Dacrydium 360

Daedalea 332

Dahlgren 73

Dalton's first Law 4

Dalton's second Law 4, 68

Dammara 361

Darwin, C. 68, 123, 453, 545, 570, $574,584,586$

Darwin, F. 217

Darwin, G. 11

Dasycladiaceae 315, 316

Davenport 210

Davis, B. M. 13

Davis, H. B. 623

Davy, H. 69

Dean 456

Debarya 266

Degeneration, human 650, 651, 665

Delage 133

Democritus 725

Demospongieae 383

Dendrocometes 273, 281

Dermocarpa 54, 307, 308, 318

Desmideae 255, 263, 268, 309, 323

Desmidiaceae 133

Desmodium 220

Desmognathus 472, 482

Devoletzky 431

Diastase 156

Diatomaceae 255, 310, 311

Diatomaceae centricae 305,311 
Diatomaceae pennatue $35,305,311$

Dichothrix 329

Dicotyledons 369

Didelphys 495

Difflugia 272, 382

Digital reduction 483

Digitalin 93

Dimorphococcus 308

Dinophilus 511, 514

Dionaea 77, 137, 183, 548

Diploid number 335

Diplophrys 379

Dipnoi 404, 454, 474

Diprotodontia $490,494,495,496$

Distomum 388

Dog 563, 565

Dracaena $15 \pm$

Draparnaldia 253, 349

Draper 652, 675, 745

Drepanophorus 431

Drew 325

Drosera 137,548

Drugs, action of 607

Duclaux 37, 80

Ductus endolymphaticus 431

Dulcite 66

Dulong on specific heat 4

Dumas 69

Durham 606

Ea as Sun god 720

Echinodermata 222, 298, 386

Echinorhynchus 390

Ectocarpaceae 317

Edinger 238, 240, 619

Education 644, 814, 823

Egyptians 707, 714, 715

Lhrenberg 125

Eimer 105, 602

Electro-chemistry 3, 628

Electrolytes 18

Electrons 3, 4

Electrons, radius of 3

Electrons, size of 3
Electrotactic sense 118

Elephant 561

Emerson 570, 572, 576, 584, 593, $630,654,692,762$

Emulsin 157

Emulsoids 17, 39, 43

Encoeliaceae 317

Endoclonium 253, 315

Endosphaera 254, 309, 310

Endospore 250, 251

Energids 120

Energy, biotic 604, 605

Energy, cogitic 603, 605

Energy, cognitic 603, 605

Energy, intraatomic 24

Engelmann 208, 211, 307

Engelmann, on heat rays 41

Enteromorpha 312

Enteropneusta 422

Enterprise defined 641

Entomostraca 397

Entoprocta 391

Enzymes 31, 156

Eoanthropus 624

Eomyces 330

Epeira 769

Ephedra 357, 362, 364, 663

Ephedraceae 362

Epibiotism 329

Epibiotic 399

Epiphegus 199

Epipogon 202

Equisetales 351

Erythrobacteria 327

Ether 2

Etiolin 77

Eudyonema 274

Eugenie 759

Euglena 122, 209, 211

Euglypha 382

Euphorbias 110

Eupolia 230, 424, 428, 433, 435

Eurypterida 399

Ewing 395

Excretory System in Apoda 468 
Excretory System in Cyclostomata 449

Fxcretory System in Nemertinea 448

Excretory System in Rhabdocoela 448

Excretory System in Rotifera 448

Excretory System in Urodela 468

Eyes in Nemertinea 428

Eyes in Rhabdocoela 428

\section{Fabre 552}

Fagales 373

Faraday, M. 187

Fatty acids 154

Fauvelle on electricity 23

Featherman 655

Ferguson 845

Ferrocyanide of copper 29

Ferrocyanide of potassium 28

Field 587

Filices 123

Filicineae 354

Fischer, A. 40

Fischer, E. 37, 66, 327

Fixed Oils 154

Fleming 235

Flint 681

Floody 713, 714

Florideae 317

Fol 261

Fontinalis 343

Foraminifera 271, 272, 275, 378

Forel 555

Formic acid formula 155

Fourier 763, 786

Fowler 716

Frazer 652, 680

Frederick 784

Frontal cerebral lobes 627, 684

Frontal organs 428

Fucaceae 317

Fucus 149

Fuegian 625, 650

Fungi, sexuality in 283

Fungus sugar 157
Gaidukov 307

Gallardo 261

Galton, F. 177

Gametophytic generation 336

Garlic, oil of 617

Garman 586

Garrison 667

Gaskell 544

Gasteropoda 388, 394, 522, 524

Gasterostomum 388

Gastrotricha 388

Gautama 725, 734

Gehuchten 235

Geiger 595, 713

Geikie, A. 10, 12, 16

Gelatin emulsoid 18

Genetics 290

Gentiana 150

Gentianaceae 202

Geoenergids 120

Geonemertes 389, 401, 436, 441

Geoplasts 128

Geotactic sense 118, 126

Gephyrea 399

Geppert 114

Germplasm 295, 297

Ginkgo 244, 357

Ginkgoaceae 286

Ginkgoales 359, 360

Ginkgophyllum 360

Glands of mouth 457,458

Glands of skin 419,481

Globig 326

Gloeocapsa 30, 50, 82, 97, 99, 306

Glossopteris 357

Glucose 154

Glucosides 65

Glycogen 119

Gnathostomata 404

Gnetales 357

Gnetum 357, 358, 363

God 646, 647, 680, 686, 691, 693, 698

Goethe 68 
Gomont 52, 303

Gomontia 315

Goodrich 449, 450, 467

Goodyera 202

Gorilla 586

Götte 473

Graff, von $388,442,445$

Graft hybrids 112

Graham on colloids $15,17,18,45$

Graminaceae 110, 371

Granite in crust 7, 9

Graphite 7, 27

Graptolites 302

Green, Reynolds 158

Griffith 55, 236

Gromia 382

Gromiadae 272

Groot, G. 847

Gruppe 716

Gymnodiniaceae 310, 323

Gymnodinium 310

Gymnophiona 2, 454

Gymnophrys 271

Gymnospermia 334

Gyroplasts (gyroenergids) 128

Gyrotactic sense 118, 124, 126

Haack 459

Haberlandt 120, 217

Haeckel 87, 500, 570, 652

Haecker 291

Haematite 16

Haemoflagellata 223, 225, 271, 273, $276,279,282,298,378,380,417$

Haemoglobin 77, 112, 113

Haemosporidia 279

Hair development 479, 480

Halidrys 149

Hamaker 236

IIand action $574,578,580,581,596$

Hansgirg 52, 54, 246

Haploid number 289, 335, 336

Harrison, F. 653

Harrison, R. 113

Harting 435, 436
Hartog 246, 259, 261

Hatschek 507

Hatshepsut 845

Hegler 52, 82, 119, 128, 135, 306

Held 235

Helioplasts 128

Heliotactic sense 118, 119

Heliotheism 688, 689, 704, 718, 723, 724, 728

Heliozoa 271, 276, 378, 379

Helium 3, 5, 44

Helminthocladieae 320

Hemichordata 401, 406, 411, 501

Henking 289

Henry VIII 818

Henslow, G. 367, 369, 371, 372

Hepaticae 123, 286, 288, 334, 342

Heptoic acid 155

Heredity 174, 175

Heribaudiella 316

Herpetomonas 58, 59

Herrick, C. L. 239, 427, 489, 621

Herrick, C. J. 429, 439

Hertwig 423

Hesperidin 161

Heterogametes 282

Hickson 378

Hillhousia 55

Hirudinea 389, 390

Hlava 514

Hobbes 787

Höber 37

Hobhouse 88, 585

Hofmeister 353

Hog peanut 227

Holmes, S. J. 567, 601, 615, 619

Holmgren 236

Holostei 403

Homo 501, 566

Hooker, J. D. 200

Hooker, Richard 813

Hopkins 680

Hormidium 315

Hormones 298

Hottentots $709,711,716,778$ 
Houzeau 561, 585

Huber 314, 555

Hubrecht 420, 423, 435, 436, 437, $445,451,465$

Hudson-Gosse 523, 526, 536

Huie, Lily 132

Hume, D. 598, 653, 666

Huxley 150, 418, 475, 486, 652

Hyaenodon 498

Hybridity, bisexual 142

Hybrids 290, 291, 294

Hydatina 294

Hydra 385, 505, 610

Hydriodic acid 103

Hydrocarbons 65

Hydrodictyaceae 312

Hydrogen sulphide 19

Hydropterideae 352

Hydrotactic sense 118, 119

Hydrożoa 222, 386

Hyella 249, 254, 316, 318

Hymenoptera 373, 770

Hypnotism 804

Hypnum aduncum 343

Ideals 648

Ikeno 258

Immortality $652,696,697,841,842$, 843,848

Immortal substance 297

Incas 641,722

Industrial Organization 767

Infusoria 142, 152, 231, 239, 280, $298,378,413,414$

Initiative 792, 831, 832

Insecta 400

Insectivora 499, 557

Instinct $614,618,622$

Instruction 644, 814

Intelligence $614,617,619,622$

International comity 825, 829

International Congresses 826

International Expositions 826

International Hygiene 835

International Languge 827
International Peace Temple 827

Inulase $\mathbf{1 5 7}$

Inulin 30,70

Invertase 157

Iodine 5

Ionium 3, 24

Iranians $641,668,688,689,696,713$ 714,720

Irano-Aryans 713, 731

Iron oxides, colloid 16

Iron, sesquioxide of 15

Irritability 44,45

Ishtar 733

Isis 686

Isogametes 282, 289

Isoleucin 156

Israelites 689

Issakowitsch 293

Jaccard 363

James, W. 680

Japan 637

Jehovah 725

Jenghiz 678,730

Jennings 56, 121, 122, 125, 126, 209

Jenson 210

Johnston 427, 489, 621

Joly, J. 11

Jordan 426

Joukowsky 264

Joule's Law 68

Jowett 734

Judaism 730

Jungermanniaceae 341

Juniperus 362

Jupiter 688, 721

Karyosome 278

Kelvin, age of earth 11

Kenyon 555

Kerner 7\%

Kidd, B. 189, 698, 736, 788

Kinoplasmic threads 83

Kinorhyncha 390

Kirchner 52, 54, 303 
Klebs 253, 269, 285, 293

Knop's Solution 269

Kolbe 37, 69

Korschelt-Heider 520, 530, 534, 537

Kovalevsky 392

Krafft 28

Krapotkin 751

Kruyff 326

Krypton 4

Kuhlmann 69

Kunkel 84

Kurajeff on serum 76

Kutschin 437

Kützing 52, 53, 312

Laboulbeniaceae 331

Lacrymaria 415

Lagena 382

Lagenostoma 355

Lamarck 68, 200, 570

Lamellibranchiata 394, 395, 521

Lamiaceae 162

Laminaria 149

Laminariaceae 317, 347

Land 363

Land drainage 838

Lang, A. 705

Language 584, 588

Lankester, E. R. 422,449

Lao-tsze 662, 765

Lapageria 185

Larmor 3

Laurales 373

Lauric acid formula $\mathbf{1 5 5}$

Lavoisier 68

Lecidium 308

Lecithin 116

Leduc $28,29,31,39,45,46,63,75$, $134,176,246$

Legendre 235

Leger 223

Leguminosae, seeds of 110

Leibnitz, on force 32

Leiphaimos 202

Lemanea 318, 320, 331
Lemuridae 502, 557, 558

Lemuroid types 499

Lepidocarpon $347,348,355$

Lepidodendreae 341, 347, 354

Lepidoptera 373

Leptophrys 271

Leptosira 256, 257, 267

Leptosporangiatae 353

Leptothrix 326

Lessepsia 383

Letterstedtia 312

Leucin 156

Leucoplasts 120, 128

Leydig 553

Lieberkühnia 379

Liebig 69

Lignin 153, 169

Liliaceae 371

Lillie, F. 270

Limnocnida 386

Limnocodium 385

Limodorum 202

Linaria cymbalaria 227

Lincoln 667

Lindsay, Lauder, 87, 546, 561, 584, $602,655,657,665,693$

Lingula 178, 303, 648

Lipase ferment 157

Lithamoeba 272

Lithocolla 379

Lituolida 379

Livingston 808,829

Lobosa $378,379,382$

Locusts $\mathbf{7 7 0}$

Loeb, J. 114, 123, 139, 208, 232, 234. $292,548,591,601,612,613$

Loligo 550

Lönnberg 457

Lophopus 391

Lothelier 184

Lotsy 341,345

Lowne 553

Lubbock 546, 555, 638, 770

Lugaro 235

Lull 483 
Lung development 467

Lycopodineae 315,344

Lycopodium 344, 346, 347

Lyginodendreae 354, 355

Lyngbya 118, 130, 192

Lysorophus 459

Macallum 39

Macrozoospores 253

Magnesium, in tissues 6

Magnoliaceae 371

Mahomed 687, 691, 692

Malacostraca 398

Mallock 653

Malvaceae, seeds of 110

Mammalia 460, 476, 478, 479, 485, 487,775

Mammary glands 492, 493

Mammoth Hot Springs 34

Manganese 780

Mani 690

Mann, G. 28, 100, 156

Marattiaceae 353

Marchantia 135, 187

Marchantiaceae 341

Marcus Aurelius 662

Marduk 688, 720

Marinesco 235

Marsilea 292

Marsupialia 484

Massachusetts School Act 766

Massart 209

Mastigophora 59, 378, 380

Matthew, W. D. 487, 495

Matthews 196

Maudsley, on vitality $71,605,606$, 608

Maupas 112, 143, 264

Maurer 480

Mayer, on energy 2, 110

Mazdaism 691

McClung 289

McClure 235, 236

McCook 552, 555

McLennan 638
McIntosh 432

Medulla oblongata 439

Medulloseae 354

Meijerle 480

Melsens 37, 69

Mendel 294, 295

Mendelian inheritance 294

Mendelians 179

Mercury 5

Merismopedia 192

Mesencephalon 435, 440

Mesozoa 388

Metazoa 51, 222, 223, 273

Methylxanthin 161

Metzgeriaceae 341

Meyer 120

Miadesmia 348

Microcachrys 360

Micrococcus 82

Microgamete 278, 279

Microhydra 385, 505

Micronucleus 225, 280

Microthamnion 313

Microzoospores 253

Miehe 326

Milan Cathedral $20 \%$

Mill, J. 654, 666

Mills 714, 739

Mimosa 218, 220

Mimosa pudica 137, 195, 218, 548

Miracles 728

Mithraism 690, 730, 733

Mitzkewitsch 259

Mivart 197, 500, 584

Miyoshi 287

Money 761

Monkey, capuchin 566, 567, 577

Monocotyledons 369

Monostelic bundle 350,355

Monostroma 312

Monotheism 688, 689, 724

Monotropa 202

Montgomery 388,449

Moon, worship of 712

Morals, animal 657, $65 \mathrm{~s}$ 
Morals, human, 695, 645, 646, 65ㅇ, $656,660,663,669,673,674$

Morgan 989, 290

Morgan, Lloyd 618

Morphin 161

Morse 39?

Moses 718, 720

Mosses 646, 662, 689

Mougeottia 966

Muck on colloids $\mathbf{2 5}$

Mucor 284

Müller, J. 70

Míller, Max 588, 589, 590, 595, 679,680

Munro $5 \pi+$

Musaceap $3 \pi 1$

Iusci 193, 342, 345

Mutational erolution 648

Mrcetozoa 378

Myelencephalon 434, 440

Myriapoda 396

Mrristic acid 155

Myrtaceae 162

Mrxine 42, 419, 425, 429, 431, 432, $431,435,43 \%, 441,450,45 \%$

Nansen 235

Napoleon 576, 584, 663, 678, 699, \%63, 80\%, 808

Naturalism 706, 709, 728

Natural selection $63 \%$

Nautilus $\mathbf{5 4 9}$

Neanderthal man $57 \%$

Necromancy 728

Neencephalon $\$ 240$

Nemalion 319

Nematoda 390

Nemertinea $388,393,401,412,418$, $419,423,501$

Nemertinea, blood system 443

Neon 4

Nenttia 202

Nepenthes $3 \pi 1$

Nephrocytium 309

Nereideae 235
Nereis 515

Neuratin 235, 238, 582, 602, 603, $604,621,801,804$

Neuroptera $7 \% 0$

Neuropteris 355

Nigritos 704

Nissl substance 235, 236, 582, 801, 804

Nitrogen, free 4

Noeggerathia 357

Noiré 595

Noll 217

Nostoc 54

Notochord 435, 443,447, 463, 464

Nuclear spindle 260

Nucleolus 259, 260, 262, 269

Oak 707

Obolaria 202

Octopus 550

Oedogoniaceae 312

Oedogonium 255, 358

Ohm's Law 68

Olfactory Lobes 488

Olfactory Nerve 434

Olfactory Sac 427, 456

Oligochaeta 389,390

Olive 82,248

Oltmanns $264,268,314,317,340$

Ontogeny 174

Oocystaceae 331

Oocystis 308

Oogoniaceae 331

Opalinopsis 273

Ophioglossaceae 353

Opossum 494

Optic nerve 434

Orchidaceae 371

Ormazd 662, 693, 725, 727, 740,741

Orobanche 186

Orontium 153

Ortmann 397

Osborn, H. F. 174, 194, 489, 493, 573

Oscillatoria 50, 97, 113, 118, 130 . 192,307 
Osiris 686

Osmunda 216

Osmundaceae 354

Ostracoda 302

Owen, Robert 794, 816

Oxytricha 964

Palaeoblattina 400

Palaeophonus 399

Palmelloid spore 251, 253, 267

Palmitic acid 155

Palmophyllum 308

Pandanus caricosus 215

Paraheliotactic sense 118

Paramoecium 114, 195, 209, 264, 280, 281

Parapineal eye 441,442

Pardosa 769

Parkeriaceae 353

Parmelee 574, 618, 693

Parthenogenesis 296

Parthenon 635, 636

Patritheism 731, 747, 749

Paul 662, 670, 694, 696, 743, 747, 765, $782,787,816,822,831,846,847$

Pearl 126

Pearson 359

Peckhams 552

Pecopteridaceae 354

Pecopteris 355

Pectase 158

Pectin 15:

Pectinatella 391

Pelargonic acid 155

Pelomyxa 217

Peltigera 308

Pentamorphogeny 174

Pepsin 158

Peptides 85

Percepts 692

Perfect Life $\mathbf{8 0 6}$

Peridiniaceae 310

Peripatus 400

Perry 11

Peter of Russia 818
Petit on specific heat 4

Petromyzon $402,419,425,430,432$, $436,437,438,442,457,458,466,468$

Pfeffer 933, 270, 987

Pfleiderer 681, 68:

Pflücke 235

Pflüger 116

Phaeophila 314

Phaeophyceae 316

Phaeothamnion 313, 316, 3 오

Phalangeridae 499,558

Phalansterium 383

Phascolomys 494

Phascum 343,345

Philageria 185

Philesia 185

Phillips, O. P. 82, 135, 248, 250, 313

Phoenix 216

Phosphorus 5, 9

Photochemistry 628

Photoenergids 190

Phragmonema 318, 320

Phryganella 38:

Phylactolaemata 391

Phyllobium 309

Phyllocladus 360

Phylloglossum $3 \pm 4$

Phyllopods 397

Phyllosiphon 315

Piette 594

Pinaceae 347

Pinaciophora 38?

Pinacocystis 379

Pineal eye $441,4+2$

Pinus 362

Piptocephalis 284

Pithophora 319

Pituitary body 426

Placenta, vertebrate 491,492

Plagiostoma 388

Planaria 126, 232

Plastin 139

Platinum 6

Plato 646, 662, 696, 795, 737, 765, 816,831 
Pleasure, definition of 616

Plectonema 307, 308

Pleurocapsa 307,308

Pleurococcaceae 128, 133, 251, 308, 335

Pleurococcus 70, 308

Podocarpus 360

Poisons, action of $93,608,609$

Polar bodies 292

Polonium 3

Polychaeta 389

Polyciliata 33t

Polygordius 512

Polvphagus 284

Polypodium 385

Polyporus 164

Polyprotodontia 490, 494, 495, 496

Polypteridae 403

Polysiphonia 320

Polytheism $687,704,715,717,718$, 728, 732

Polyzoa 391, 515, 516, 517

Populus 244

Porifera 383

Porphyra 318

Porphyridium 318

Porsch 363

Potassium in living tissues 6

Potomac beds 368

Preyer 88, 89, 90, 810

Price 654

Priestley 31

Primates 596, 621

Primitive man 702, 777

Proboscidea 557, 621

Proboscis of elephant 562, 568

Proboscis of Nemertinea 420, 423, $426,434,435,451$

Proboscis of Turbellaria 422, 427

Proboscis sheath, Notochord 435

Proenvironment, Definition 205, 629

Proenvironment, Law of, 174, 188, $194,205,629,676,684,699,789$, 790,831

Pronucleus male 279
Prorocentraceae 310

Prosencephalon 433, 440

Protamoeba 271, 274

Proteidae 462, 471

Proteolytic ferments 158

Proteomyxa 59, 245

Proterospongia 383, 384

Proteus 463

Protobiota 38

Protococcaceae 129, 133, 251, 256, 265

Protohydra 385

Protomycetes 51

Protoplasm 30, 39, 50, 55, 57, 60, 61, $65,72,78,80,81,84,85,88,98$, $99,102,103,107,108,110,114$, $116,132,136,142,151,165,170$, 222, 227, 237, 248, 259, 598, 600, $609,800,801,802$

Protopterus 85

Protozoa 51, 223

Protozoa, sexuality in 271, 274, 275

Prout 3

Prowazek 223

Prussic acid 157

Pseudodifflugia 379

Psilotum 346

Pteridophyta 49, 258, 287, 313, 504

Pteridospermeae 315

Ptyalin 157

Pulmonata 522

Purpurin 77

Pyrenoids 119, 309

Pyrocystis 310

Pyrola 202

Quadrate bone 477, 483

Quercitrin 161

Quinary compounds 32

Quincke 78

Quinine 66

Quinoline 66

Rabinowitsch 326

Radaisia 254, 318, 320 
Radioactive bodies 2, 22

Radiolaria 271, 272, 274, 378

Radium 2, 3, 24

Ramalina 308

Ramsay 2

Ranales 373

Rand 236

Ranson 235, 236

Ranunculus 153

$\operatorname{Re} 686,688,720$

Reason 622

Reduction division 270,271

Referendum 792, 831, 832

Reichert 140

Reissner's fibre 433, 437, 438

Religiologists 700, 701

Religion 645, 652, 669, 675, 681

Religion, definitions of 679,682

Religious evolution 626

Renan 692, 850

Rennet 158

Repression, human 792

Reproductive organs in Amphioxus 469

Reproductive organs in Apoda 469

Reproductive organs in Cyclostomata 469

Reproductive organs in. Nemertinea 469

Reproductive organs in. Urodela 469

Reptilia 476, 477, 556

Republic of Plato 731, 781, 786

Resins 162

Rhabdocoela 388, 442, 445, 448, $450,501,510$

Rhizoclonium 312

Rhizopoda 271

Rhode 235

Rhumbler 378

Rhynchonella 303

Ribot 92, 93

Riccia 340, 341, 342, 345

Ricciaceae 335, 337

Richet 98

Richter's Law 68
Riella Paulsenii 343

Robinia 161

Rodentia 499, 557, 558, 559, 621

Rogers 237

Romanes $87,88,93,546,550,559$, $560,564,565,566,584,586,587$, $588,590,595,598,600,681,683$, $684,693,744,811,813,822$

Romans 721

Rotifera 122, 125, 386, 393, 412, $415,417,420,426,432,450,506$, $507,509,513,522,523$

Ruff 25

Russia 637

Rutherford 2

Ryder, J.'187

Sabbath 713

Saccharose 28

Sachs 330

Salicin 151

Salamandra 464, 484, 486, 491

Salamandridae 472

Salensky 420

Sanders 437

Sanderson, Burdon 84

Sanson 291

Saprolegnia 187, 285

Saprophytism 329

Sarcina 56, 82, 97

Sargent, E. 367, 368

Sargent, P. E. 437

Sarracenia 182

Sarrasin 446

Satan 727

Satisfied state $614,615,616,617$, $623,626,629,644,660,664,666$

Saxegothea 360

Sayce 595, 739

Scaphopoda 394, 395

Schaudinn 223, 273, 278

Schimper 247

Schively 138, 184

Schizomycetes 51, 54, 58, 59, 89

Schizophyceae 51, 58, 59, 77, 100 
Schneider 550, 551

Schoenbein 114

Schools, types of 810

Schopenhauer 798

Schultz 113

Schultze 457, 480

Schweinitzia 202

Scorpionida 400

Scotosphaera $\mathbf{2 5 6}$

Scott 498

Scrophularia 161

Selachii 103

Selaginella 344,347

Selection 195, 197

Selenka 578

Semitic nations 707

Semper 381

Seneca 662, 680, 806

Sequoia 115

Setchell 53, 57, 304

Shea oak 358

Shibata 387

Shintoism 704

Shipley 459, 551

Shull 294

Sigillaria 346,347

Silica, colloid 16, 53

Silicates in earth's crust 7

Silicon 6

Siren $459,463,464,466,468,470$, 482,488

Sirenidae 462, 470

Skraup 66

Slave labor 665

Sleep 606

Smallwood 235, 237

Society as an Organism 787, 795, 813

Socrates $646,662,725,783,846$

Sollas 381,383

Somali 654

Somatoplasm 295, 297

Sothis 687

Soxhlet 66

Spaulding 238
Spelerpes 473, 484, 493

Spencer, H. 17, 38, 115, 178, 180, $246,545,570,615,652,657,665$, 704,787

Spencerians 179

Sphaerella 209, 210

Sphaerocarpaceae 341

Sphaerocarpus 337, 342

Sphenophylleae 351

Spireme thread 262

Spirit of the Universe 750

Spiritic energy 624, 700, 801, 805, 822

Spiritin 804

Spirogyra 124, 149, 156, 244, 257, $262,263,266,269$

Spongida 222, 383, 505, 613

Spongilla 383

Sporophyte 289, 337

Sporozoa 112, 271, 272, 276, 378, 380

Stapelia 111

Starvation 607

Statocyst 428

Staurospermum 266, 268

Stearic acid 157

Stearin $\mathbf{1 5 7}$

Stentor 209, 224

Stephanosphaera 309

Stereoenergetics $249,283,415$

Stereum 136

Sterrocladia 331

Stichostemma 389, 401, 449

Stieda 437

Stigeoclonium 252, 254

Stigonema 192

Stigonemaceae 247, 249

Strasburger 247, 258, 363

Strasser 462, 473

Studnicka 437, 442

Stylonychia 264

Sulphur bacteria 78

Sumerians 679

Sunday worship 722

Sun god 688, 726

Sun, worship of 712,722 
Supernaturalism 729

Suspensoids 17, 39, 43

Sutherland 652, 658, 660

Swamp cypress 216

Swarmspores 252, 257, 267, 284

Sweet Potatoes, yield 839

Switzerland, people 784,785

Symbiotic fungus 302

Sympathy 660

Sympetalae 149

Synaptase 157

Table of Algae-Fungi 305

Tannins 162

Tardigrada 400

Tarsipes 497

Tarsius 494

Tasmanian 624, 649, 777, 778, 791

Taxaceae 357,360

Taxodium 216, 362

Taxus 360

Teleostei 403

Tennent 611

Terebratula 648

Termites $\mathbf{7 7 0}$

Tetrablepharis 331

Tetraplasy 174

Tetraspora 308

Tetrasporaceae 128, 133, 251, 252, $265,308,311$

Tetrastemma 401, 430, 441, 449, 469

Thalictrum 292

Thallocarpus 337

Thallophyta 51, 334

Theobromine 37, 61

Theos 693, 741

Thermochemistry 628

Thermophilic bacteria 326

Thermophilic plants 304

Thermotactic sense 118

Thigmotactic sense 118

Thiobacteria 327

Thiothrix 40

Thoreaceae 319

Thoreau 639
Thompson, W. P. 366

Thomson, J. A. 206

Thomson, J. J. 3

Thorndike 234, 618

Thoth 686

Thought-transfer 804

Thrombin 158

Thymus gland 439

Thyroid gland 439, 458

Thysanozoon 232

Tilden 52, 53, 54, 303

Tmesipteris 346

Toddalia 161

Tolypothrix 118

Tommasi, on colloids 25

Tonotactic sense 610

Tooth development 420,460, 481, 482

Tornier 184

Torpedo fish 104

Torrey, 360

Transformability of energy 2

Traube 28, 29, 43, 45, 46

Traube's cells 176, 292, 304, 327

Trehalase 157

Trematoda 388

Trentepohlia 252, 267

Treub 363

Trichopilus 253

Trilobita 399

Trilobites 302

Trinema 379, 382

Trochophore larva 506

Trochosphere 392

Trypsin 158

Tschirch 160

Tuomeya 318

Turbellaria 122, 388, 418, 421, 509, 511

Turkestan 734

Tussilago Farfara 138

Uexkiüll 618

Uhlhorn 663

Ullmannia 357 
Ulodendra 346

Ulothricaceae 312

Ulrich 233

Ultramicroscope 17

Ulva 312

Ulvaceae $311,312,317$

Ungulata 499

Uranium 3, 24

Urnatella 391, 516

Urochordata 400, 406, 411, 453

Urodela 418, 455, 458, 460, 461, 466, 476,485

Uronema 267

Uterus 491

Utility 666

Vaginula 394

Valencinia 424

Valoniaceae 315, 317

Vampyrella 59

Van Tieghem 363

Vaucheria 315

Vaucheriaceae 312, 315

Vejdovsky 55

Venus 733

Verrucaria 308

Vertebrae 464, 478, 483, 488

Verworn 45, 46, 47, 71, 116

Victorella 391

Vital force 68

Volatile oils 162

Voltzia 361

Volvocaceae 310,330

Volvox 310

Vorticeros 427

Voyria 202

Vries, de 173, 294

Wager 248

Waitz 590

Walchia 357,361

Walker 17

Wallace, A. R. 197

Waller 84, 99, 100

Walters 299
Ward, Lester 367

Ward, M. 56

Warrington 236

Washburn 602

Watson, C. 128

Weed, W. H., 13, 33, 53, 326

Weismann 200

Weismannians 179

Wells 195

Welwitschia 358

West 52,55

Westermarck 652, 656, 676

Westminster Creed 681

Wheat gluten or artolin 76

Wheeler 555, 587, 768

White 652, 675, 745

White Ants 770

Whitney 294

Whittier 667

Wiedersheim 425, 457, 458, 460, 462,472

Wiegmann 523

Wieland 355

Wille 315

Winogradsky $40,41,79$

Wöhler, 37, 69, 71

Wolf in relation to dog $\mathbf{7 7 6}$

Wollea 192

Wolsey 806,807

Woodruff 264

Woodward 11

Xanthin 160, 161

Yahweh 725, 727, 741

Yellowstone geysers 16, 34, 57

Yerkes 238, 618

Young, W. S. 114

Yucca filamentosa 138

Zacharias 128

Zarathushtra 646, 662, 678, 689, $690,691,717,725,726,731,733$, $738,760,765,831,846$

Zelinka 228, 415, 416, 427, 432, 507 
INDEX

Zend Avesta 726

Zeus 688, 720, 721

Zinc sulphate 103

Zingiberaceae 371

Zoogametae 265

Zoosporangia 252
Zopf 52, 149, 378

Zoroastrians 714, 749

Zygnema 203, 266, 283

Zygnemeae 263

Zygospore 267, 275

Zymogens 31. 156 




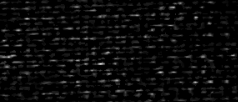

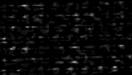

(5) 